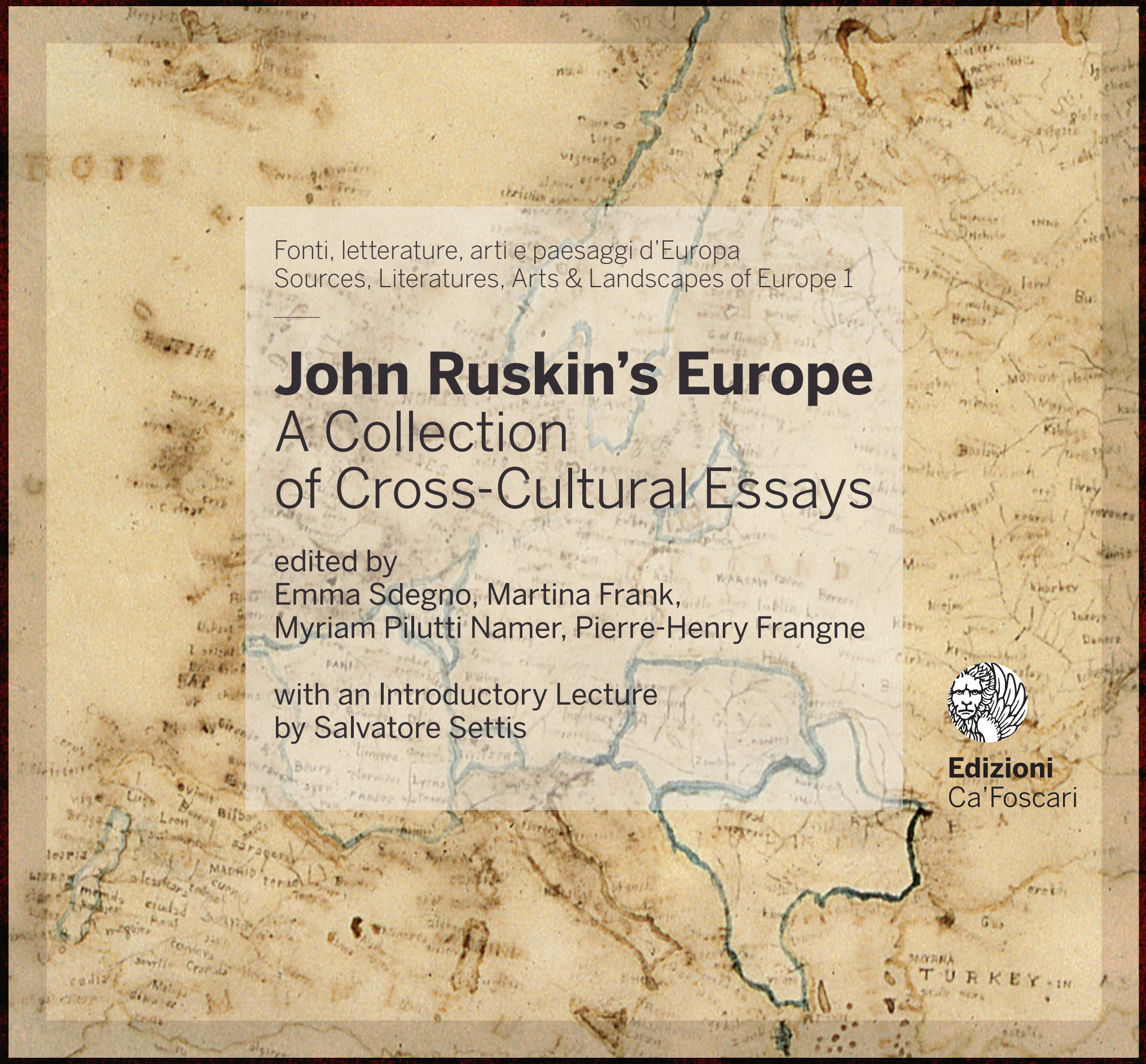


John Ruskin's Europe

\section{Fonti, letterature, arti e paesaggi d'Europa \\ Sources, Literatures, Arts \& Landscapes of Europe}

Collana diretta da $\mid$ A series directed by

Martina Frank

Myriam Pilutti Namer

Emma Sdegno

1

Edizioni

Ca'Foscari 


\section{Fonti, letterature, arti e paesaggi d'Europa Sources, Literatures, Arts \& Landscapes of Europe}

\section{Direzione scientifica}

Martina Frank (Università Ca' Foscari Venezia, Italia)

Myriam Pilutti Namer (Università Ca' Foscari Venezia, Italia)

Emma Sdegno (Università Ca’ Foscari Venezia, Italia)

\section{Comitato scientifico}

Malcolm Andrews (University of Kent, UK)

Juan Calatrava Escobar (Universidad de Granada, España)

Kathleen Christian (Humboldt Universität zu Berlin, Deutschland)

Dede Fairchild Ruggles (University of Illinois, USA)

Marco Fernandelli (Università degli Studi di Trieste, Italia)

Jorge García Sánchez (Universidad Complutense de Madrid, España)

Marko Marinčič (Univerza v Ljubljani, Slovenia)

Cédric Michon (Université Rennes 2, France)

María del Valle Ojeda Calvo (Università Ca' Foscari Venezia, Italia)

Caterina Parigi (Universität zu Köln, Deutschland)

Fabio Saggioro (Università degli Studi di Verona, Italia)

Valentina Sapienza (Università Ca' Foscari Venezia, Italia)

Luigi Sperti (Università Ca' Foscari Venezia, Italia)

Francesco Vallerani (Università Ca' Foscari Venezia, Italia)

\section{Direzione e redazione}

Università Ca' Foscari Venezia

Dorsoduro 3246, 30123 Venezia

e-ISSN 2784-8507

ISSN 2724-6620

URL https://edizionicafoscari.unive.it/en/edizioni/collane/fonti-letterature-arti-e-paesaggi-deuropa/ 


\section{John Ruskin's Europe A Collection of Cross-Cultural Essays}

edited by

Emma Sdegno, Martina Frank, Myriam Pilutti Namer, Pierre-Henry Frangne

with an Introductory Lecture by Salvatore Settis

Venezia

Edizioni Ca' Foscari - Digital Publishing 2020 
La collana accoglie studi capaci di offrire un approccio innovativo e originale su generi editoriali della tradizione umanistica europea quali la biografia intellettuale, la storia della letteratura, delle arti maggiori e minori, dell'archeologia e del collezionismo pubblico e privato, o in grado di rispondere a sentite esigenze di ricerche puntuali sulla storia dell'ambiente e del paesaggio. Caratteristiche principali di questo progetto editoriale sono il dialogo profondo tra lingue e discipline, in prospettiva inter- e transculturale, e l'interesse per la documentazione inedita e poco nota, sia che si tratti di ricerche archivistiche sia che riguardi saggi e opere d'autore per i quali si propone la traduzione. La collana nasce con vocazione apertamente internazionale e si offre di divenire il punto di riferimento per ricerche originali su tematiche che, per quanto centrali nella cultura europea, faticano a trovare spazi editoriali che diano loro voce e adeguata visibilità.

The series comprises a set of perspectives that bring a fresh eye to the main genres of the European humanistic tradition, such as intellectual biography, the history of literature, of major and minor arts, of archaeology and of public and private collections. It will provide a base for new research, including on the history of the environment and of landscapes. By focusing on unpublished and little-known works, ranging from archival source material to essays, scholarly works, also in new translations, this project brings to life the dialogue between cultures and disciplines. The series has an openly international outlook and is intended to be a point of reference for original research on themes that are central to European culture and require adequate editorial recognition and visibility. 
L'opera di Ruskin s'inscrive con forza nel grande contesto europeo, segnando un momento importante del movimento di costituzione di una cultura e di uno spirito comunitari. I saggi qui raccolti intendono porre al centro della riflessione critica il tema del rapporto fecondo e imprescindibile di Ruskin con l'Europa, presentandosi come occasioni di approfondimento e di confronto su questioni attinenti all'estetica, alla tutela del patrimonio materiale e immateriale, alla memoria culturale e letteraria. Portando all'attenzione della comunità scientifica i molteplici aspetti - geografici, storico-artistici, critico-estetici, letterari, socio-politici - dell'opera di Ruskin secondo prospettive inter- e transculturali, il volume si propone di (ri)scoprire un Ruskin deliberatamente europeo e di stimolare nuove rotte di ricerca.

L'œuvre de Ruskin s'inscrit avec force dans le grand contexte européen, marquant ainsi un moment important du mouvement de constitution d'une communauté culturelle et spirituelle. Les contributions présentées ici se proposent de mettre au centre de la réflexion critique la question de la fécondité et du caractère essentiel du rapport de Ruskin avec l'Europe, et elles sont autant d'occasions d'approfondir et de confronter des problématiques touchant à l'esthétique, à la protection du patrimoine matériel et immatériel, à la mémoire culturelle et littéraire. En attirant, selon des perspectives interculturelles et transculturelles, l'attention de la communauté scientifique sur les multiples domaines - géographie, histoire de l'art, critique d'art, littérature, sociologie, politique -, dans lesquels se déploie l'œuvre de Ruskin, ce volume vise à faire (re)découvrir un Ruskin délibérément européen et à ouvrir ainsi de nouvelles voies à la recherche.

Ruskin's work is strongly embedded in the broad European context, marking an important moment in the movement for the establishment of a community culture and spirit. The essays collected here intend to place the theme of Ruskin's fruitful and vital relationship with Europe at the centre of a critical reflection, opportunities for an indepth study and a discussion on issues related to aesthetics, the protection of tangible and intangible heritage, cultural and literary memory. By bringing to the attention of the scientific community the multiple aspects - geographic, historical-artistic, critical-aesthetic, literary, socio-political - of Ruskin's work from inter- and transcultural perspectives, the volume aims to (re)discover a deliberately European Ruskin and to stimulate new research paths. 
John Ruskin's Europe. A Collection of Cross-Cultural Essays

Emma Sdegno, Martina Frank, Myriam Pilutti Namer, Pierre-Henry Frangne (edited by)

(c) 2020 Emma Sdegno, Martina Frank, Myriam Pilutti Namer, Pierre-Henry Frangne for the text

(c) 2020 Edizioni Ca' Foscari - Digital Publishing for this edition

\section{(1)}

The texts of the essays here collected are licensed under a Creative Commons Attribution 4.0 International License

I testi dei saggi qui raccolti sono distribuiti con Licenza Creative Commons Attribuzione 4.0 Internazionale

\section{()) $\Theta \Theta$}

The images in the Open Access digital edition of this work are licensed under a Creative Commons AttributionNonCommercial-NoDerivatives 4.0 International License.

Please refer to the "List of Figures" section in order to know the credits of the images here published.

Le immagini pubblicate nella edizione digitale Open Access sono distribuite con Licenza Creative Commons AttribuzioneNon commerciale-Non opere derivate 4.0 Internazionale.

Si rinvia alla sezione "List of Figures" per consultare i crediti delle immagini qui pubblicate.

This volume includes a collection of papers presented at the conference A Great Community: John's Ruskin Europe, held at Ca' Foscari University of Venice, 7-9 October 2019. The volume has been refereed by selected subject-matter experts appointed by the Editorial Board.

Questo volume include una raccolta di contributi presentati al convegno A Great Community: John's Ruskin Europe, tenuto alla Università Ca' Foscari Venezia, 7-9 ottobre 2019. Il volume pubblicato ha ottenuto il parere favorevole da parte di valutatori esperti della materia selezionati dal Comitato editoriale.

Edizioni Ca’ Foscari - Digital Publishing

Fondazione Università Ca’ Foscari Venezia | Dorsoduro 3246, 30123 Venezia

http://edizionicafoscari.unive.it|ecf@unive.it

la edizione dicembre 2020 | 1st edition December 2020

ISBN 978-88-6969-487-5 [ebook]

ISBN 978-88-6969-488-2 [print]

Printed on behalf of Edizioni Ca' Foscari - Digital Publishing, Venice

in April 2021 by Skillpress, Fossalta di Portogruaro, Venezia

Printed in Italy

Published with the contribution of Unité de recherche Histoire et critique des Arts of Université de Rennes 2

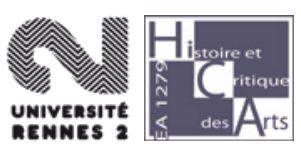

John Ruskin's Europe. A Collection of Cross-Cultural Essays / Edited by Emma Sdegno, Martina Frank, Myriam Pilutti Namer Pierre-Henry Frangne - 1. ed. - Venezia: Edizioni Ca' Foscari - Digital Publishing, 2020. - 448 pp.; $22 \mathrm{~cm}$. - (Fonti, let terature, arti e paesaggi d'Europa| Sources, Literatures, Arts \& Landscapes of Europe; 1). - ISBN 978-88-6969-488-2.

This work is fully available in Open Access PDF ebook format

La presente opera è integralmente disponibile in formato ebook PDF Open Access

URL http://edizionicafoscari.unive.it/it/edizioni/libri/978-88-6969-488-2/

DOI http://doi.org/10.30687/978-88-6969-487-5 
A Collection of Cross-Cultural Essays

edited by Emma Sdegno, Martina Frank, Myriam Pilutti Namer, Pierre-Henry Frangne

\section{Table of Contents}

John Ruskin: un paysage moralisé per il nostro tempo

Salvatore Settis

\section{Foreword}

Emma Sdegno

\section{Introduction}

Pierre-Henry Frangne

RUSKIN: A EUROPEAN AESTHETIC?

John Ruskin, un œil européen

La photographie, la peinture, l'écriture et l'énigme de la visibilité

Pierre-Henry Frangne

Osservazione e comprensione dal rudere al paesaggio

Unità morfologica e verità estetica negli scritti di John Ruskin

Emanuele Morezzi

«Aratra Pentelici» di John Ruskin

Insegnare l'arte greca dopo Winckelmann

Myriam Pilutti Namer

La corrispondenza epistolare come rete di conoscenza, dibattito e azione Le riflessioni sulle arti e sulla tutela di Philip Webb, Giacomo Boni e John Ruskin

Andrea Paribeni, Silvia Pedone 
Ruskin's Ontology of Architecture

Pedro Marques de Abreu

ntermezzo

Amelia Sarah Levetus (1853-1938) e il John Ruskin Club di Vienna dalla sua fondazione fino alla Prima Guerra Mondiale

Martina Frank

THE CENTRES OF RUSKIN'S EUROPE

Ce qui commence à Calais : l'Europe, terrain de jeu de Ruskin

André Hélard

Division, Juncture, System: Bridges and Bridge-Building in the Work of John Ruskin

Paul Tucker

John Ruskin and the Europe of Cathedrals

Claude Reichler

Il mercato antiquariale nella Venezia di Ruskin

L'arte medievale in Germania

Michela Agazzi

John Ruskin and His 'Witch of Sicily', Amy Yule

Stephen Wildman

Songlines: Ruskin and the Roads of Europe

Howard Hull

LITERARY INTERSECTIONS

\section{Ruskin, Dante e l'Europa romantica}

Giuseppe Sandrini

The Stones of Venice: Lady Augusta Gregory and John Ruskin 
From Ruskin's Amiens to Proust's Venice

Reflections on the Diapered Screen

Emily Eells

Edited by Ruskin: Francesca Alexander's Roadside Songs of Tuscany

Emma Sdegno

Ruskin's Islamic Orient and the Formation of a European Ideal

Mujadad Zaman

intermezzo

John Ruskin and Kenji Miyazawa

An Idea of Nomin-Geijutsu (Peasant Art) and its European Legacy

Yasuo Kawabata

CULTURE AND SOCIETY IN EUROPE ACCORDING TO RUSKIN

Tra nostalgia preindustriale, ghildismo e rinascita nazionale

Il pensiero sociale di Ruskin nel dibattito culturale italiano

Laura Cerasi

Ruskin in Translation: Versions of Unto this Last in a Few Europeans Languages Toward a Reception History of John Ruskin's Social Thought

Jean-Yves Tizot

The Apostle of Beauty: Some Turn-of-the-Century Perceptions of Ruskin in Central and Eastern Europe

Stuart Eagles

Index of Names

List of Authors 



\title{
John Ruskin: un paysage moralisé per il nostro tempo
}

Salvatore Settis

Scuola Normale Superiore di Pisa, Italia

\author{
Caro Guardian Grando di questa Scuola Grande di San Rocco \\ Magnifico Rettore \\ Care Consorelle e Confratell \\ Care Colleghe e Colleghi \\ Signore e Signori
}

È per me un vero privilegio prendere la parola in questo luogo prezioso, per la prima volta da quando la Scuola Grande di San Rocco mi ha fatto l'onore, davvero immeritato, di eleggermi Confratello d'Onore. E sono lieto di parlare, oggi, in una circostanza tanto rilevante come il convegno sull'Europa di John Ruskin, intesa come una 'grande comunità'.

Come i veri studiosi di Ruskin presenti in questa sala sanno bene, io non sono né specialista né comunque esperto di una figura complessa e ancora attuale come la sua; né, d'altra parte, oserei mai avventurarmi in approssimazioni che pretendessero di presentarne, a un pubblico come questo, l'opera e il pensiero. Se, tuttavia, ho accolto con gioia e riconoscenza l'invito a parlare qui rivoltomi da Emma Sdegno e Myriam Pilutti Namer, è per l'urgenza, che certo non sono il solo ad avvertire, di una riflessione sui rischi che incombono nel nostro tempo sul paesaggio, sul patrimonio culturale, sull'ambiente. Una riflessione nella quale emergono di volta in volta aspetti politici, economici, naturalistici, estetici, ma in cui troppo raramente (così a me pare) si dà il giusto rilievo alla natura e alla forza propriamente etica che fu all'origine delle prime perorazioni in favore della conservazione dei paesaggi storici, e dunque anche delle leggi di tutela oggi vigenti in Europa e nel mondo. Perciò ho fatto ricorso, nel titolo di questa lezione, alla dizione, che può parere arcaica o leziosa, 'paysage moralisé'. Una scelta che ritengo, al contrario, pienamente adatta a desi- 


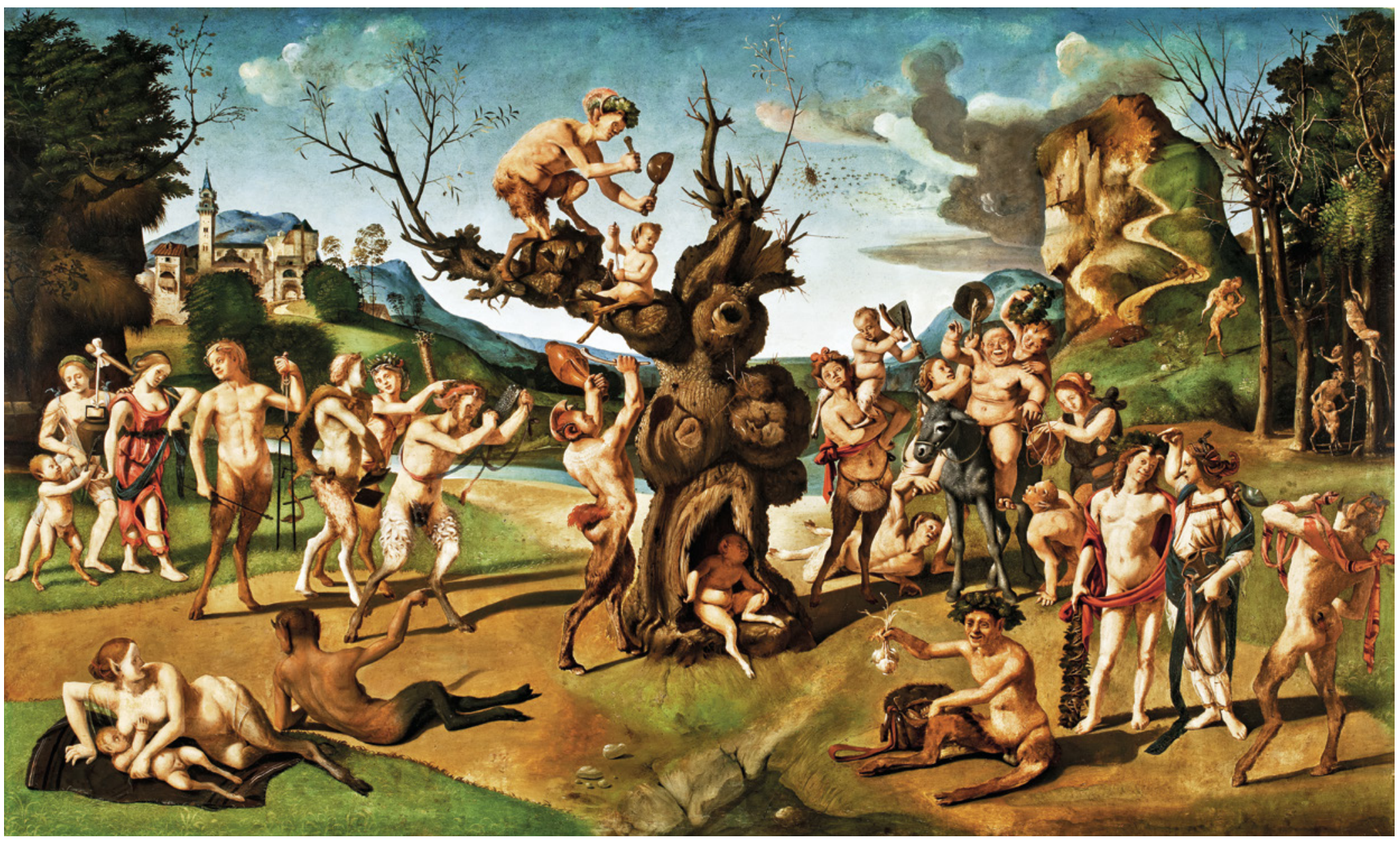

Figura 1 Piero di Cosimo, La scoperta del miele. 1505-1510 ca. Olio su tavola. Worcester (MA), Worcester Art Museum. @ Worcester Art Museum / Bridgeman Images 


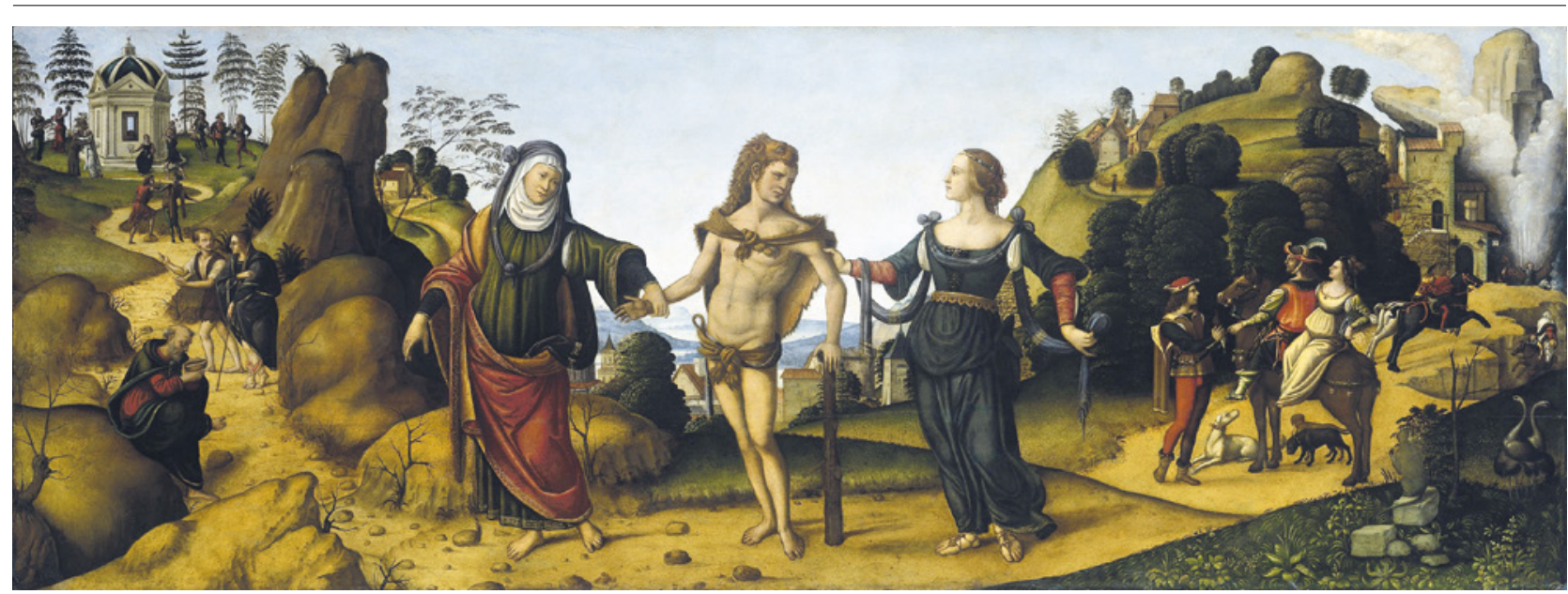

Figura 2 Nicolò Soggi, Ercole al bivio. 1510 ca. Olio su tavola. Berlino, Bode Museum

gnare un orizzonte drammatico come quello del nostro tempo e ad indicare norme e traguardi di impegno morale di cui è più che mai necessario prendere coscienza e assumere responsabilità. In questo cammino verso una piena consapevolezza dei nostri problemi e dei nostri doveri verso le generazioni future ritengo che John Ruskin possa esercitare ancora quella funzione di directeur de conscience che gli veniva assegnata da Marcel Proust nel suo necrologio. Un 'direttore di coscienza', questo può ancor oggi essere Ruskin, per ripensare il paesaggio nel nostro tempo, che ne ha disperatamente bisogno.

L'espressione 'paysage moralisé' fu coniata nel 1937 da un grande storico dell'arte, Erwin Panofsky, che si era rifugiato negli Stati Uniti per sfuggire alla persecuzione nazista, e fu poi divulgata nei suoi celebri Studies in Iconology, che sono del 1939. Panofsky introdusse questa formula per descrivere una particolare modalità della rappresentazione non tanto di paesaggi in senso stretto, ma di quelli che fanno da sfondo alla pittura di storia.
Per esempio in un dipinto di Piero di Cosimo, La scoperta del miele [fig. 1], basato sulle Metamorfosi di Ovidio, l'albero disseccato al centro divide lo spazio in due metà, caratterizzate l'una da una via piana che conduce a una remota città, l'altra da un erto sentiero che porta in cima a un monte coperto di nere nuvole. Secondo Panofsky è questo un «espediente, comune nella pittura tardomedievale e rinascimentale, di dividere lo sfondo paesaggistico in due metà, di carattere simbolicamente contrastante», che qui rappresentano l'antitesi fra «la spietata durezza della barbarie immitigata e la felicità innocente di una civiltà pastorale». In altri dipinti studiati da Panofsky e dedicati al tema di Ercole al bivio [fig. 2], il paysage moralisé può mettere in scena, invece, il contrasto fra la facile vita di chi si adagia nei piaceri e quella, ardua ma nobile e meritoria, di chi si dedica all'esercizio delle virtù. Ma io vorrei piuttosto fare appello alla vostra memoria per evocare, nel nostro contesto, quel sensazionale paysage moralisé che è il ciclo di Ambrogio Lorenzetti nel Palazzo Pubblico di Siena, dove nell'ampia veduta della 


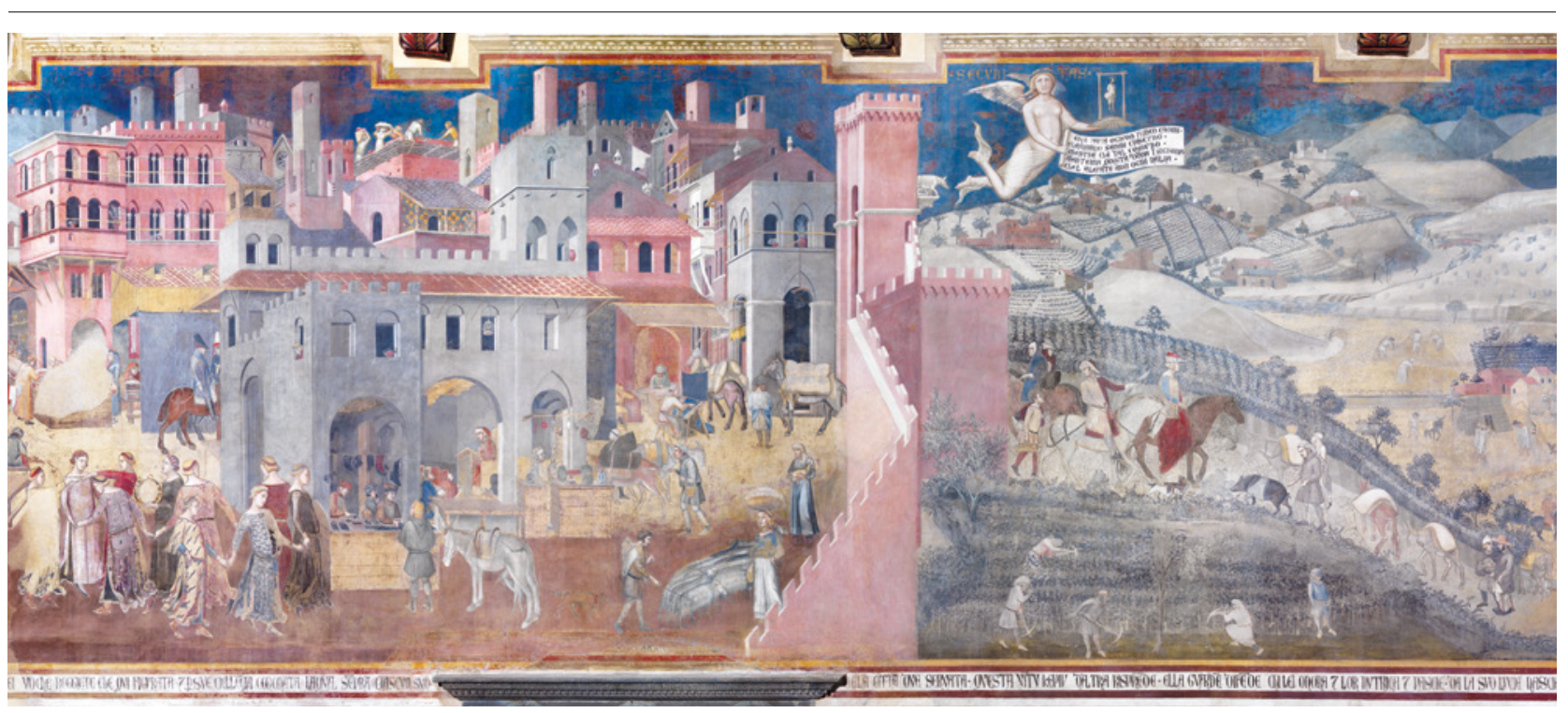

Figura 3 Ambrogio Lorenzetti, Gli effetti del Buon Governo, città/campagna (dettaglio). 1338-1340. Affresco. Siena, Palazzo Pubblico. (C) Roberto Testi, Comune di Siena

città di Siena e del suo contado si contrappongono le allegorie del Buon Governo e del Cattivo Governo, ma soprattutto la rappresentazione dei loro effetti sulla vita dei cittadini. Da un lato la prospera città con le sue case, i monumenti, le chiese, la ferma cinta delle mura e la distesa dei fertili campi coltivati con amore e costanza [fig. 3]; dall'altro, la desolazione di un suolo arso dagli incendi e dalle guerre, devastato dall'incuria, prostrato dagli odi intestini, popolato di monconi di case, macerie miserande, alberi bruciacchiati [fig. 4]. Quel che fa la differenza tra l'uno e l'altro è precisamente l'ethos dei cittadini, da intendersi come scelta morale, ma anche come comportamento pratico.

Ho citato alcuni possibili esempi di quel paysage moralisé in pittura che Panofsky mise a fuoco con la sua brillante e fortunata invenzione verbale. Ma questa formula convenzionale della storia dell'arte deve gran parte della sua fama a un grande poeta, Wystan Hugh Auden, che nel 1945, ripubblicando un suo poema di qualche anno prima (1933) vi appose il titolo Paysage moralisé, prendendolo a prestito da Panofsky. Leggiamone insieme i primi sei versi:

Sentimmo dire di messi rimaste a marcire nelle valli, Vedemmo in cima alla strada montagne sterili, E scafi concavi incombere sull'acqua; Sapemmo i naufragi di chi puntava sulle isole. Onoriamo chi ha fondato queste città affamate: Il loro onore è figura del nostro dolore. 


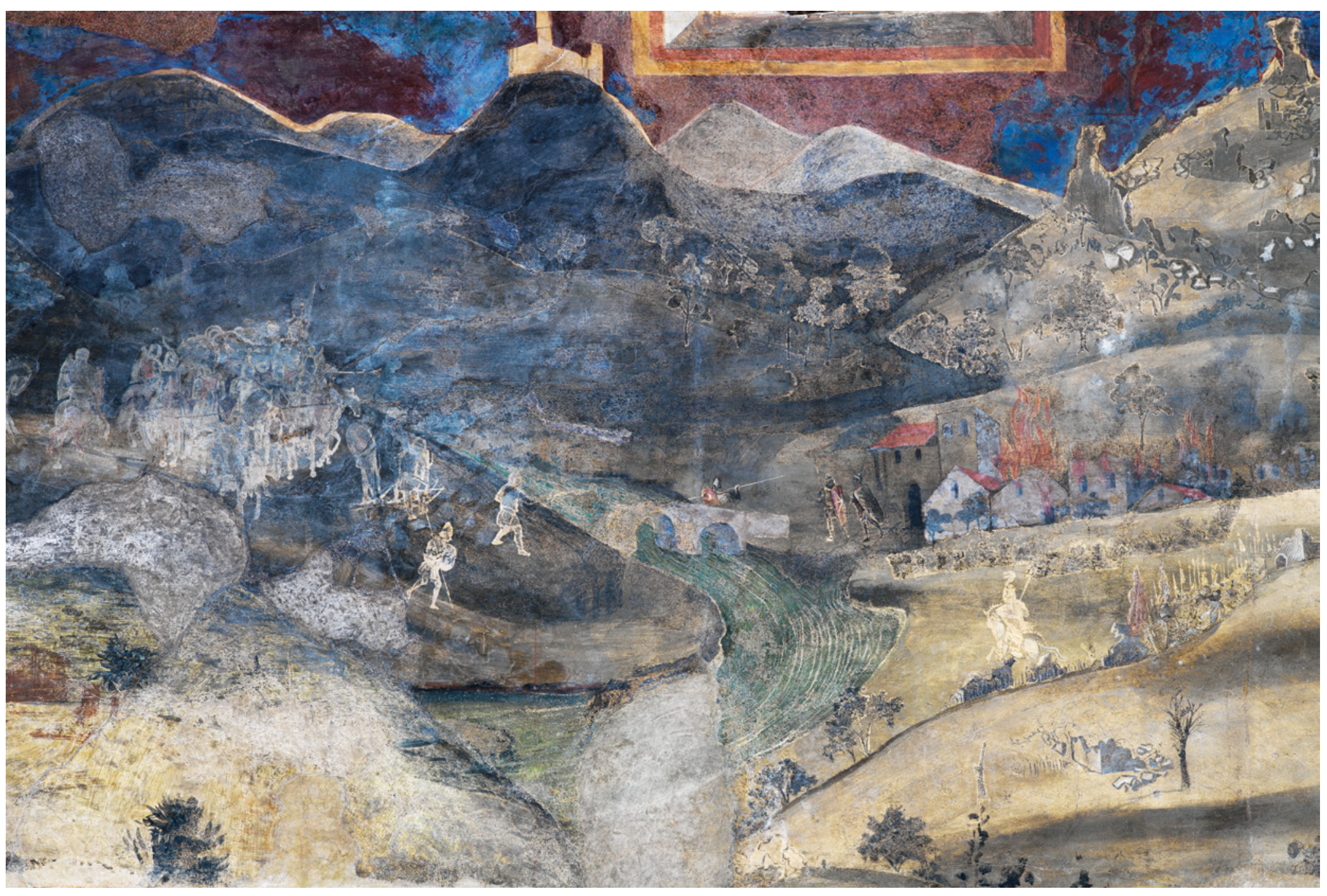

Figura 4 Ambrogio Lorenzetti, Gli effetti del cattivo Governo, campagna (dettaglio). 1338-1340. Affresco. Siena, Palazzo Pubblico. @ Roberto Testi, Comune di Siena 
Questi versi segnano una svolta importante nell'uso e nel senso dei paysages moralisés, dal paesaggio dipinto con la sua densità figurale e allegorica al paesaggio reale, vissuto o poeticamente immaginato. Una transizione che avviene, è vero, attraverso la dimensione metaforica, ma che si estende in profondità, come la lettura integrale del testo potrebbe mostrare, dando al paesaggio vero (quello di valli abbandonate e aride montagne, di città infelici e isole remote, di notti e d'albe) la funzione poetica di riflettere i più intimi pensieri di quegli afflitti viandanti, di quei naviganti sull'orlo del naufragio.

Il paesaggio reale, insomma, quando venga moralisé (cioè letto in chiave etica) traduce paesaggi interiori, e in qualche modo equivale ad essi, perché li popola e li ispira. Un'immedesimazione, una fusione emozionale che si compie specialmente nel dolore, come nei versi finali del poema di Auden:

Questo è il nostro dolore. Si scioglierà? Ah, l'acqua Potrebbe sgorgare, scorrere, far verdeggiare questi [monti e queste valli,

E noi ricostruire le nostre città, non più sognare le

[isole.

Il paesaggio vissuto come il riflesso e l'identico dell'ethos interiore: in questo gioco di echeggiamenti, anzi nella triangolazione fra emozioni dell'animo, paesaggio vissuto e paesaggio dipinto, incontriamo fatalmente la figura di John Ruskin, in alcune delle sue più felici espressioni. Come sappiamo, egli dedicò straordinaria attenzione al paesaggio nei suoi celebrati scritti d'arte, ma coltivò anche un appassionato interesse per la storia naturale e per la critica della società del suo tempo, anche perché riteneva il quadro socio-politico essenziale per intendere scopi e sviluppi dell'arte. La sua visione utopica (da cui nacque perfino una piccola città, Ruskin Colony, che ebbe breve vita fra Tennessee e Georgia) assegnava all'arte un ruolo centrale nella società, quasi un antidoto (educativo, rigenerativo) all'industrializzazione galoppante che stava trasformando l'Inghilterra, e poi l'Europa.

Si può dire che per Ruskin il paesaggio dipinto culmina e condensa la storia, l'essenza e le finalità spirituali della pittura [fig. 5]; ma per lui il paesaggio vissuto va inteso in piena continuità con quello esplorato da poeti e pittori, e anzi accomuna chi è artista e chi non lo è, ed è dunque un ingrediente essenziale della vita culturale e sociale delle comunità, anzi ne forma la trama. Perciò non basta né ammirare i quadri di paesaggio né leggerne le descrizioni di letterati e poeti, se non sappiamo rivolgere eguale attenzione ed eguali cure ai luoghi reali che ci circondano, e senza i quali né quei dipinti né quegli indugi ecfrastici sarebbero mai nati. Il paesaggio vissuto è fonte di intense esperienze etiche ed estetiche per il singolo (anche per chi non è né pittore né poeta), ma per la sua vastità rimanda alla maestà della natura; inoltre, la stessa sutura fra paesaggio urbano, periurbano e non urbano, coi suoi diversi livelli di antropizzazione, implica un livello più alto di quello delle emozioni individuali. Nel gran teatro della natura, il vero 'titolare' del paesaggio, e dei valori che gli sono associati, è la collettività dei cittadini, ed è ad essi (potremmo dire: alla società) che il paesaggio, che in certo senso si identifica con la natura, impartisce con la stessa sua esistenza una forte lezione morale. Perciò il paesaggio richiede di essere conosciuto, descritto, dipinto, protetto: perché la pratica stessa dell'arte converge con la bellezza dei paesaggi più vicini allo stato di natura, e dunque fonda e impone la necessità di preservarne l'aspetto, arrestando la devastazione delle campagne prodotta dall'industrializzazione e dall'urbanizzazione forzata. Per Ruskin insomma, il paesaggio riflette e determina l'ordine morale, e perciò è il luogo-chiave della responsabilità sociale: in esso le istanze sociali e politiche del presente sono obbligate a misurarsi con i valori della natura, della bellezza e della memoria, né possono rinunciarvi senza tradire se stesse. Alcuni quadri baroc- 
chi come quello esposto di seguito [fig. 6] anticipano immaginosamente questa identificazione, propriamente fisiognomica, fra il volto degli esseri umani e quello della natura che li circonda, li plasma e ne è plasmata. Vediamo qui un paesaggio enfaticamente antropomorfo, dove un colle assume l'aspetto e il profilo di un uomo barbuto. Fessurazioni e caverne si aprono sulla bocca e sul naso, un lago sorge nell'occhio, alberi e cespugli circondano l'ampio prato della guancia, su cui continuano i lavori agricoli in corso nella valle sottostante.

Questa immagine che (lo confesso) ho usato qualche anno fa come copertina di un mio libro, si presta, come altre simili, a commentare una famosa metafora, che con il nome di Ruskin ha avuto un'enorme fortuna in Italia e altrove: il paesaggio come volto della patria. A dire il vero, la fortuna di Ruskin in Italia non fu immediata, ma fiorì poi in modo assai notevole, non solo per le sue frequentazioni e i suoi scritti di tema e ambiente italiano (non ho certo bisogno di ricordare qui The Stones of Venice), ma anche perché le sue idee incrociavano preoccupazioni e dibattiti in corso nell'Italia di quegli anni. L'inglese vi era, in quel tempo, assai meno praticato del francese anche nelle classi colte, e si capisce perciò come mai la conoscenza di Ruskin presso molti italiani si fondasse sull'esposizione del suo pensiero che si poteva leggere in alcuni libri di Robert de la Sizeranne, soprattutto Ruskin et la religion de la beauté (Paris: Hachette, 1897). Caratteristico della temperatura culturale di quegli anni è che questo libro sia stato reso subito noto in Italia da un'ampia recensione di Ugo Ojetti, che comparve nella Nuova Antologia, anzi nello stesso fascicolo che conteneva anche un intervento di Luigi Rava, deputato della sinistra liberale, per la salvezza della pineta di Ravenna minacciata di distruzione. Un tema di forte attualità politica si affiancava dunque, nelle stesse pagine, alla conoscenza (organica anche se indiretta) del pensiero di Ruskin sui valori etici del paesaggio. Una giunzione piena di conseguenze, non solo per la legge a protezione della pineta di Ravenna, approvata nel 1905, ma perché lo stesso Rava, divenuto ministro della Pubblica Istruzione del III governo Giolitti, avrebbe poi varato nel 1909 la prima legge fondamentale dell'Italia unita per la tutela del patrimonio culturale. Il pensiero di Ruskin, per come riassunto da de la Sizeranne, e gli inizi della discussione politica sul paesaggio in Italia si intrecciarono dunque da subito.

$\mathrm{Fu}$ in quel contesto che prese piede, e venne citata spessissimo fino a diventare quasi lo slogan della tutela in Italia, una frase tolta da Ruskin et la religion de la beauté e attribuita senz'altro allo stesso Ruskin: «Il paesaggio è il volto amato della Patria». Invano l'ho cercata, con l'aiuto di Donata Levi e Paul Tucker, negli scritti di Ruskin che conosco e in quelli che non conosco, che sono i più. Mi pare dunque probabile che in questa forma l'aforisma 'di Ruskin', pur vicino al suo pensiero, non sia in realtà una vera citazione 'autentica' (e del resto de la Sizeranne non la mette tra virgolette). E tuttavia queste parole ben corrispondono, per trarre dalle sue pagine un solo esempio, allo spirito delle Lectures on Art (1870), in cui Ruskin sottolineava, per i suoi studenti di Oxford, che

sanno godere il paesaggio solo le persone colte, educate dall'arte che li circonda (musica, letteratura, pittura) ma anche dalla pratica di azioni significative. Essi troveranno un piacere intenso nel paesaggio del loro Paese per il suo valore di memoria, perché lo vedranno come sigillo e premio della persistenza di un'alta vita nazionale.

Perciò,

una nazione merita il suolo e gli scenari che ha ereditato solo se, con tutte le sue azioni e le sue arti, si preoccupa di renderli ancor più gradevoli per $\mathrm{i}$ propri figli.

17 Fonti, letterature, arti e paesaggi d'Europa S Sources, Literatures, Arts \& Landscapes of Europe 1

\begin{tabular}{l|l}
$\mathbf{1 7}$ & $\begin{array}{l}\text { Fonti, letterature, arti e paesaggi d'Europa } \\
\text { John Sources, Literatures, A }\end{array}$ \\
\end{tabular} 


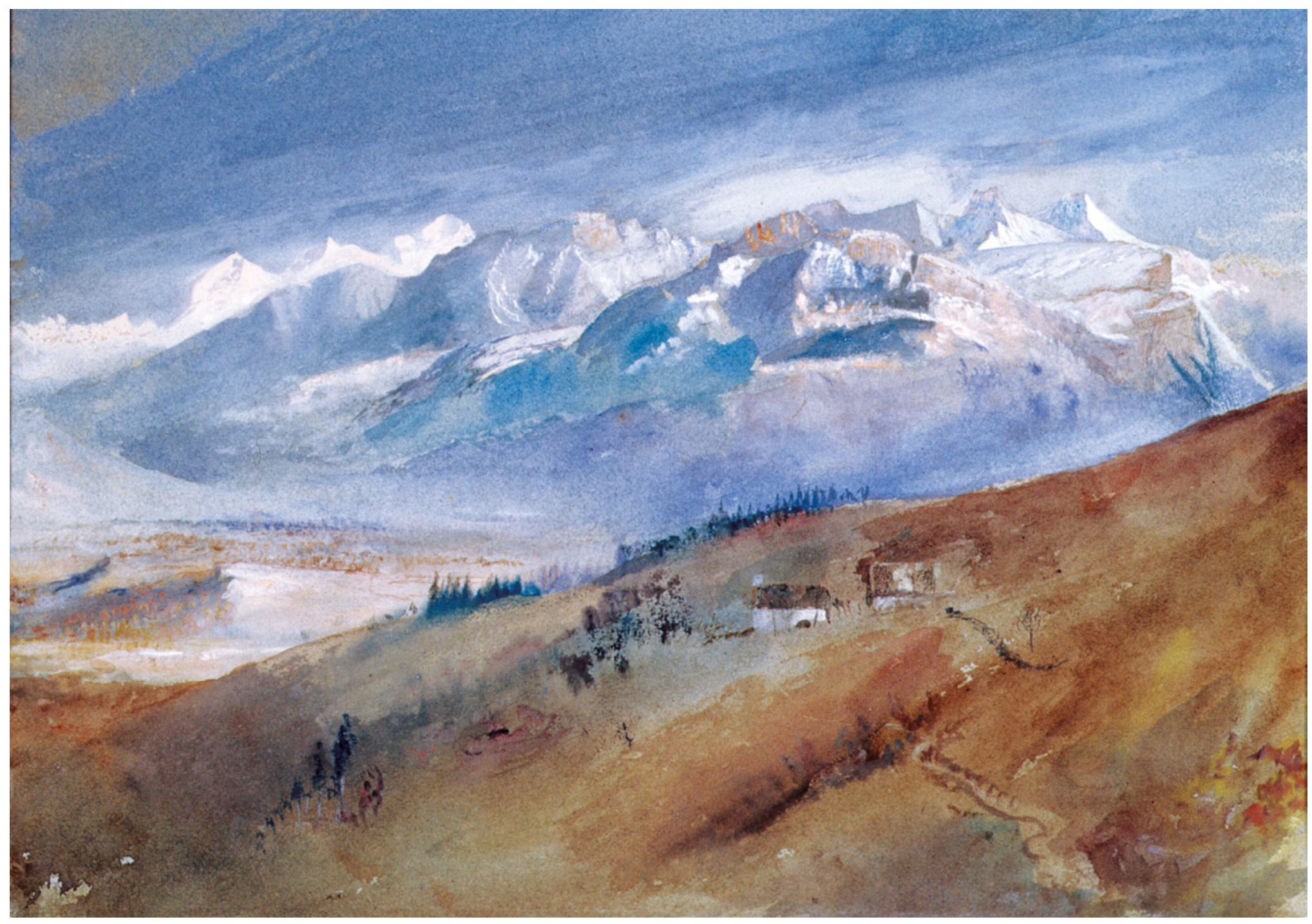

Figura 5 John Ruskin, Chamonix: vista dalla mia finestra a Mornex. 1862-1863 ca. Acquerello. Kendal, Cumbria, Abbot Hall Art Gallery. (c) Abbot Hall Art Gallery / Bridgeman Images 


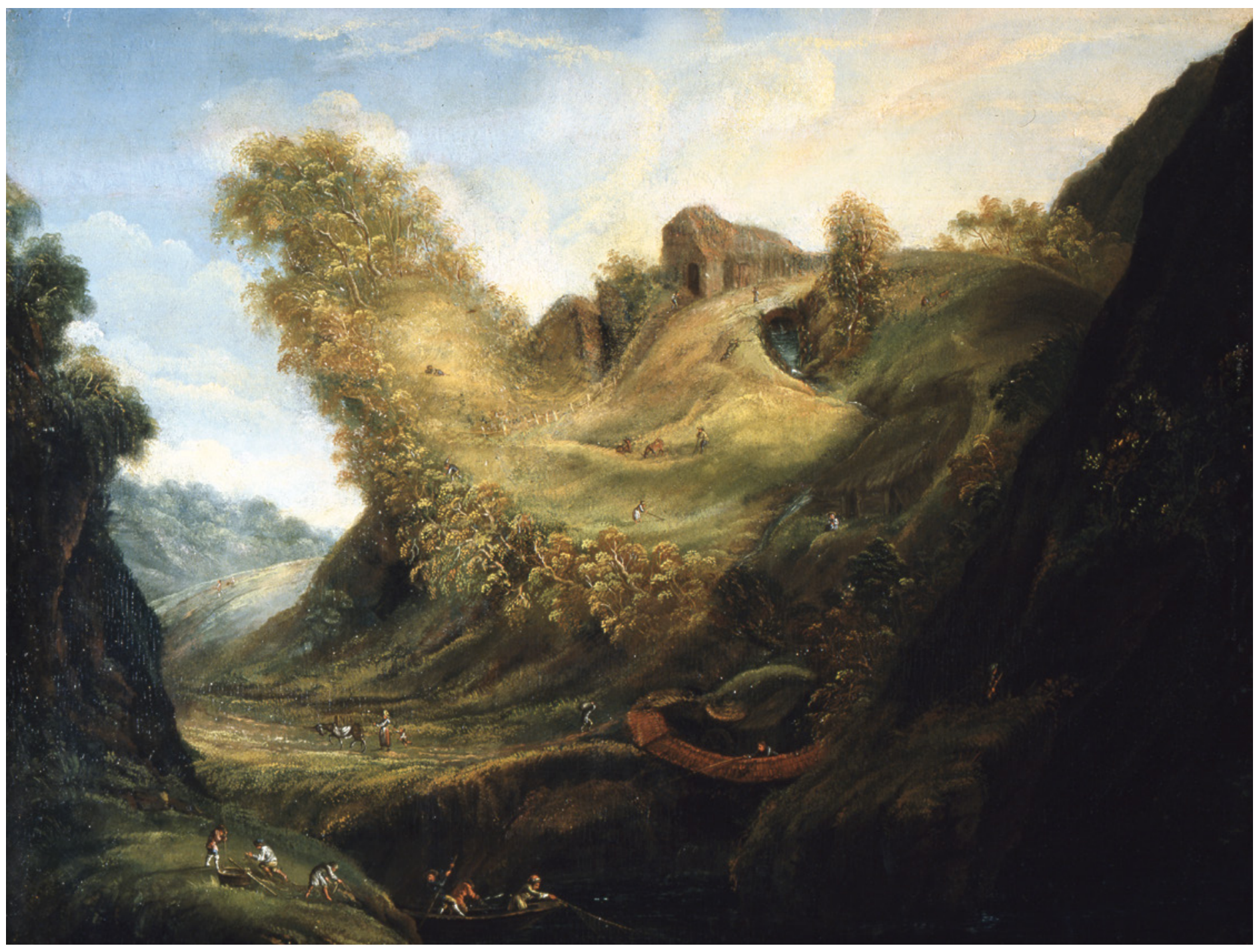

Figura 6 Paesaggio antropomorfo, pittore tedesco (?) del primo Seicento. Collezione privata 
Ma la popolarità di quella frase attribuita a Ruskin si radicò rapidamente in Italia, anche perché già l'anno dopo compariva la prima traduzione italiana degli Elementi del disegno e della pittura (Torino: Bocca, 1898), seguita poi da altre traduzioni negli anni a seguire.

«Il paesaggio è il volto amato della patria»: per dar solo veloce e superficiale conto della fortuna e dell'incidenza di questa frase nella discussione italiana del primissimo Novecento, mi limiterò qui a due esempi. Il primo è un giovanissimo giurista abruzzese, Nicola Falcone, che prima di cadere sul fronte italo-austriaco nel 1916 pubblicò un libro assai originale e importante, Il paesaggio italico e la sua difesa. Studio giuridico-estetico (Firenze: Alinari, 1914), nel quale la frase «Il paesaggio è il volto amato della Patria» viene citata alla lettera, e senz'altro attribuita allo stesso Ruskin, che Falcone definisce «il più eletto glorificatore della natura». Il mio secondo esempio è assai più rilevante, perché riguarda un personaggio di primissima fila, Benedetto Croce, e perché lo riguarda quando, nella sua veste di ministro della Pubblica Istruzione nel V governo Giolitti, Croce varò la prima legge italiana di tutela generale del paesaggio.

La legge del 1905 a tutela della pineta di Ravenna, che ho ricordato poco fa, riguardava solo una piccola porzione di territorio, e si fondava sulla storia del sito e sulle sue memorie, da Odoacre e Teodorico alla «divina foresta spessa e viva» di Dante, a Dryden, a Byron, a Garibaldi. Presentando la legge alla Camera, Luigi Rava sostenne che

il culto delle civili ricordanze va esteso ai monti, alle acque, alle foreste, a tutte quelle parti del patrio suolo, che lunghe tradizioni associarono agli atteggiamenti morali e alle vicende politiche di un grande Paese.

In Italia, dove l'industrializzazione procedeva con ritmo assai più lento che nell'Europa del Nord, la discussio- ne politica e culturale sul paesaggio si svolgeva soprattutto a livello di casi singoli (come Ravenna), anche se gli spiriti più avvertiti già sentivano la necessità di una legge generale di tutela. Lo stesso Rava provò a inserire la tutela del paesaggio nella sua legge sul patrimonio culturale del 1909; ma l'articolo che includeva fra le cose da tutelarsi «giardini, foreste, paesaggi, acque, e tutti i luoghi ed oggetti naturali che abbiano interesse storico, archeologico o artistico», dopo essere stato approvato dalla Camera, venne cancellato dal Senato.

E su questi antefatti che s'innesta la relazione di Benedetto Croce al Senato (25 settembre 1920), che avrebbe portato due anni dopo all'approvazione della prima legge italiana di tutela del paesaggio (l. 778/1922, poco prima dell'avvento del fascismo). I principi ispiratori di questa legge sopravvivono ancora, attraverso le trasformazioni che essa ha subito fino al Codice dei Beni Culturali e del Paesaggio oggi in vigore (D. Lgs. 42/2004, modificato con D. Lgs. 156 e 157/2006 e con D. Lgs. 62 e 63/2008). La relazione di Croce, Per la tutela delle bellezze naturali e degli immobili di particolare interesse storico, merita ancor oggi di esser letta come il testo-cardine di una svolta politica significativa, il culmine di un lungo processo che aveva mobilitato associazioni e politici, giornali e opinione pubblica, attraversando non meno di cinque legislature, e che nella ferma volontà di Croce trovò il suo acme.

Che una legge in difesa delle bellezze naturali d'Italia sia invocata da più tempo e da quanti uomini colti e uomini di studio vivono nel nostro Paese, è cosa ormai fuori da ogni dubbio.

Così esordì Croce, ricordando i due voti formulati in tal senso dalla Camera (1905) e dal Senato (1909), la legge sulla Pineta di Ravenna, quella su parchi e giardini del 1912 e la proposta del deputato Rosadi del 1910, caduta nel vuoto. Occorre dunque una legge che 
ponga, finalmente, un argine alle ingiustificate devastazioni che si van consumando contro le caratteristiche più note e più amate del nostro suolo.

Tanto più che già nel governo precedente il presidente Nitti aveva affermato che la necessità di «difendere e mettere in valore, nella più larga misura possibile, le maggiori bellezze d'Italia, quelle naturali e quelle artistiche» risponde ad «alte ragioni morali e non meno importanti ragioni di pubblica economia».

Un identico, «altissimo interesse morale e artistico», prosegue Croce, «legittima l'intervento dello Stato» a protezione di monumenti e di paesaggi: poiché il paesaggio

altro non è che la rappresentazione materiale e visibile della patria, coi suoi caratteri fisici particolari, con le sue montagne, le sue foreste, le sue pianure, i suoi fiumi, le sue rive, con gli aspetti molteplici e vari del suo suolo, quali si sono formati e son pervenuti a noi attraverso la lenta successione dei secoli.

Com'è subito evidente, si nasconde qui una citazione della celebre formula attribuita a Ruskin, secondo cui il paesaggio è «il volto amato della Patria». Citazione, peraltro, appena velata, dato che subito dopo Croce menziona espressamente Ruskin:

Il movimento a favore della conservazione delle bellezze naturali rimonta al 1862, allorquando John Ruskin sorse in difesa delle quiete valli dell'Inghilterra minacciate dal fuoco strepitante delle locomotive e dal carbone fossile delle officine, e si diffuse lentamente ma tenacemente in tutte le nazioni civili, e specie in quelle in cui più progredite sono le industrie e i mezzi di locomozione. Infatti questi mezzi, togliendo più facilmente gli uomini all'affannosa vita delle città, per avvicinarli più spesso alle pure gio- ie dei campi, han diffuso questo anelito, tutto moderno, verso le bellezze della natura, mentre le industrie, fatte più esigenti dalla scoperta della trasformazione della forza, elettricità, luce, calore, attentano ogni giorno più alla vergine poesia delle montagne, delle foreste, delle cascate.

Con movimento argomentativo che di lui era caratteristico, Croce fondava dunque la propria proposta di legge al tempo stesso sull'etica e sulla storia, e subito passava a conferire al movimento per la tutela del paesaggio una dimensione latamente europea:

Queste idee, del resto, sono da tempo presso tutti i popoli civili il presupposto di ogni azione di difesa delle bellezze naturali, azione che, in Germania, fu appunto detta di difesa della patria (Heimatschutz). Difesa, cioè, di quel che costituisce la fisionomia, la caratteristica, la singolarità, per cui una nazione si differenzia dall'altra, nell'aspetto delle sue città, nelle linee del suo suolo, nelle sue curiosità geologiche; e da alcuni si aggiunge, (dai tedeschi stessi e dagli inglesi) negli usi, nelle tradizioni, nei ricordi storici, letterari, leggendari, in tutto ciò insomma, che plasma l'anima dei popoli, o meglio ha influito o maggiormente influisce allo sviluppo dell'anima nazionale. Si è insomma compreso come non sia possibile disinteressarsi di quelle peculiari caratteristiche del territorio, in cui il popolo vive e da cui, come da sorgenti sempre fresche, l'anima umana attinge ispirazioni di opere e di pensieri.

Sto indugiando su questa presenza di Ruskin sulla bocca di Benedetto Croce e nell'aula del Senato del Regno nel 1920 anche perché, se non ho visto male, anche nell'utilissimo volume a cura di Daniela Lamberini su L'eredità di John Ruskin nella cultura italiana del Novecento (Firenze: Nardini, 2006), nato da un convegno al Gabinetto Vieusseux, questo aspetto è rimasto in ombra, e Croce 
lettore di Ruskin vi è stato visto piuttosto sotto l'angolatura delle concezioni estetiche.

La combattuta introduzione in Italia di norme a tutela del paesaggio ebbe dunque, provo a dirlo riassuntivamente, quattro distinte ma convergenti radici. In primo luogo, e di questo posso qui solo far cenno, la secolare tessitura di norme degli Stati italiani preunitari, che già si erano dotati di magistrature e regole per la gestione dei loro paesaggi (basti menzionare quel che Venezia seppe fare per la Laguna e più in generale per il regime delle acque anche in Terraferma; o, all'altro estremo d'Italia, l'Ordine del Real Patrimonio di Sicilia del 1745 a tutela dei boschi ai piedi dell'Etna e del teatro di Taormina). In secondo luogo, il vicino modello della Francia, dove la legge Beauquier del 1906 sulla protezione del «paesaggio e dei siti storici, pittoreschi e leggendari» offriva un modello giuridico specialmente utile, data l'affinità di ordinamento con quello italiano. In terzo luogo, i Paesi di lingua tedesca coi loro vasti e variegati movimenti di Heimatschutz, che trovavano marcate affinità anche nei Paesi nordici, forniva un ingrediente per dir così patriottico, che in Italia andava a integrarsi con la fierezza municipale per i grandi artisti e monumenti di una storia specialmente ricca. Infine, la cultura inglese, massimamente rappresentata da Ruskin (di cui vediamo un autoritratto [fig. 7]): i suoi scritti ispirati e intensi, connettendosi a una produzione letteraria variegata di cui pochi conoscevano l'estensione e le tematiche, ebbero in Italia un effetto profondo su almeno tre fronti. Ispirarono, sull'esempio del National Trust, la fondazione di associazioni protezionistiche nell'Italia dell'ultimo Otto- e del primo Novecento; fornirono, come dalle stesse parole di Croce abbiamo visto, il linguaggio e la chiave di lettura per evocare i paesaggi naturali come il contraltare di una industrializzazione che «attentava alla poesia» di una incontaminata natura. Infine, identificando con il paesaggio il volto collettivo «della patria» o della nazione, o comunque della collettività dei cittadini, le concezioni di Ruskin, per come semplificate e divul- gate, donarono alle discussioni politiche e culturali in corso in quegli anni un prezioso ingrediente etico, un forte richiamo alla responsabilità degli individui, delle istituzioni, della società.

Non era certo la prima volta che un'alta e ben formulata concezione estetica si faceva strada non solo per l'eloquenza di chi andava perorandola, ma anche per il suo più o meno esplicito messaggio educativo. Per citare un precedente solo, ma indubitabile, un secolo prima di Ruskin il tedesco Winckelmann, nella sua prodigiosa Geschichte der Kunst des Alterthums (Dresden: Georg Conrad Walther, 1764) seppe non solo lanciare una sorta di profezia sull'arte greca che servisse di manifesto a un'arte nuova (che sarebbe stata il Neoclassico), ma parlare al cuore delle élite d'Europa. La narrazione storica dell'arte greca venne da lui calata entro una visione nutrita di valori attuali, e perciò assurse a sorgente di un programma educativo diretto a ogni uomo colto. Nelle pagine di Winckelmann, traguardo etico e ideale estetico si fondevano in uno, in una visione secondo cui il Bello, e in particolare l'arte classica, potesse generare una metamorfosi degli animi umani, donando loro, per illuminazione e per disciplina dell'intelletto, una vita più piena, una più ricca interiorità. In quel contesto Winckelmann evocava anche il paesaggio greco, che peraltro non vide mai; ma l'idea che dal paesaggio potesse venire una qualche moralità gli restò sostanzialmente estranea.

La valenza etica del paesaggio (cioè la necessità che noi lo guardiamo come un riflesso di noi stessi, della nostra società) è un tema del nostro tempo, e come ogni richiamo alla responsabilità morale presuppone e risponde al suo contrario, la devastazione dei paesaggi innescata dall'industrializzazione, con la conseguente urbanizzazione, che tende ad annullare l'equilibrio città-campagna, e infrange ogni codice storico-culturale dello spazio. In questo processo, in cui l'Inghilterra di Ruskin si lanciò precocemente, e servì presto di modello a una arretrata Italia, s'intrecciarono i luoghi di abitazione e le fabbri- 


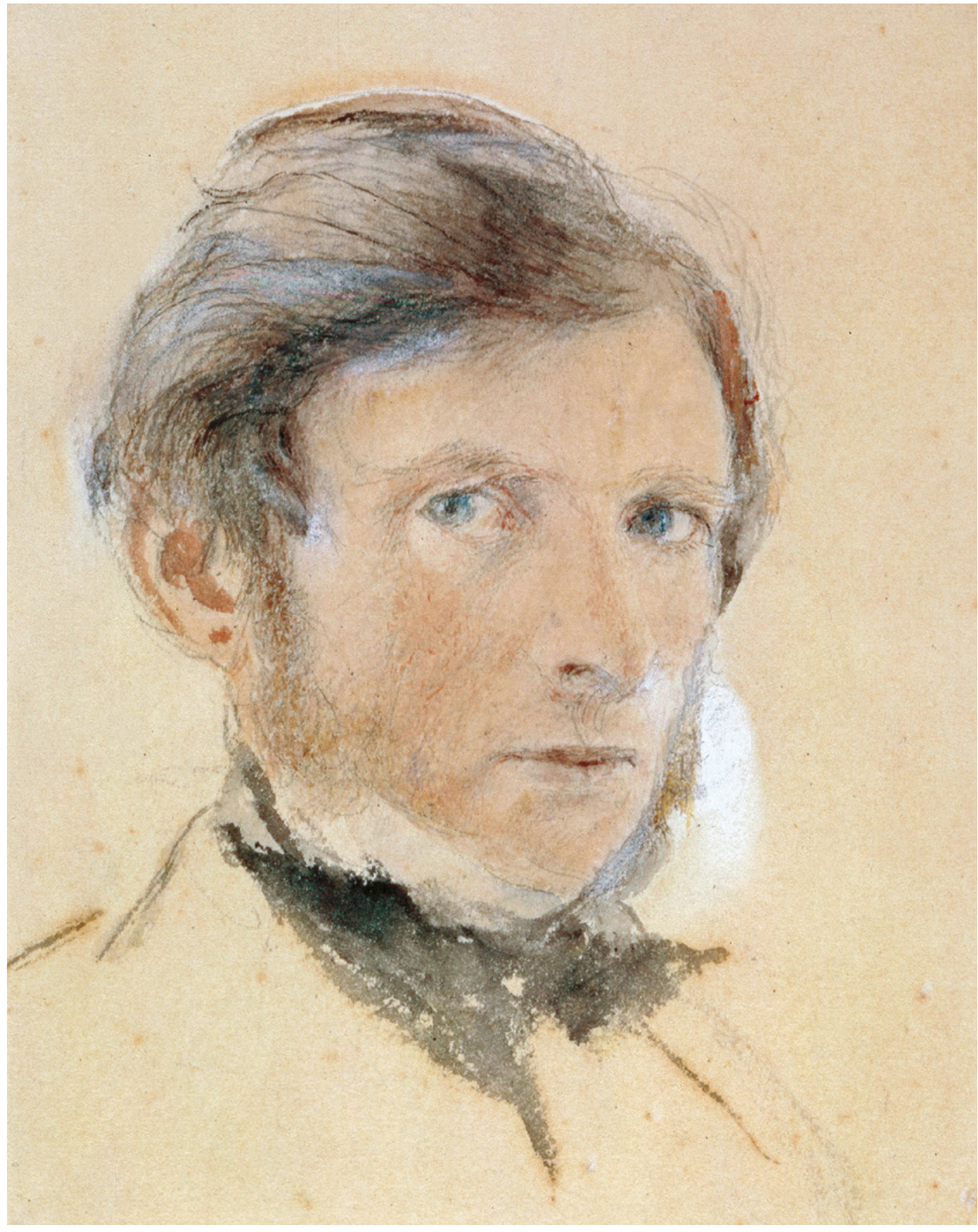

Figura 7 John Ruskin, Autoritratto. 1861. Matita e acquerello. New York, The Morgan Library and Museum 
che, compenetrando l'una dell'altra l'industria e la città. La città storica finì col subordinarsi al nuovo paesaggio industriale, e l'idea o il sogno di un paesaggio 'modernamente' invaso da ciminiere fumanti contagiò il paesaggio reale, penetrando le coscienze e guidando i desideri. Perciò nel 1910, lo stesso anno della prima traduzione italiana delle Pietre di Venezia (Roma: Ulisse Carboni) di Ruskin, i futuristi propugnavano in questa stessa città l'immediata erezione di «opifici chiomati di fumo». In sintonia con tali tendenze, nasceva, e si è ormai fortemente radicata, la voluttà dell'alveare, la voglia di imbrancarsi nella plebe urbana, l'autoriduzione a ingranaggio produttivo, l'autodissoluzione in un habitat anonimo purché 'moderno'. Le antiche modalità del vivere con il loro prodigioso equilibrio fra natura e cultura finivano col cedere a nuovi rituali entro spazi sempre più alieni a chi li abita; la felice familiarità del proprio spazio 'indigeno' finiva con l'essere spazzata via da una voluta estraneità. Si perdeva nel vuoto la terra natale.

\section{E ora cito:}

La città si dilata, la città si estende. Una speranza colorata di certezza, una solleticazione aritmetizzante, una disposizione emulatrice: arriveremo anche noi ai tre milioni di Parigi, ai quattro di Berlino, agli otto di Londra: e via via. Nelle acropoli e nei turriti municipi della provincia codesto civico solletico è addirittura frenesia.

Parole, queste, di Carlo Emilio Gadda, sul Politecnico del 1955. Ma se volessimo invece, come amava fare Ruskin, dar sostanza a questo discorso con un passo biblico, ecco a soccorrerci Isaia:

Guai a voi, che ammucchiate casa su casa e congiungete campo a campo, finché non rimanga spazio e restiate i soli ad abitare la terra! Ha parlato alle mie orecchie il Signore degli eserciti: «Edificherete molte case, ma resteranno deserte per quanto siano grandi e belle, e non vi sarà nessuno ad abitarle!». (Isaia 5: 8-9)

Parole che paion scritte per il nostro tempo, in quel davvero profetico contrasto fra la terra coltivata e le case degli umani.

Dallo stravolgimento di quegli antichi equilibri, dal danno inflitto alla nostra memoria culturale, insomma dai traumi che abbiamo vissuto e viviamo, nascono le perorazioni in favore della conservazione delle città e dei paesaggi storici. Ma da un trauma subito e dal timore di mali ancor peggiori nasce, egualmente, il sistema giuridico delle norme che mira ad arginare devastazioni e barbarie. A mostrare questa sintonia fra le esortazioni di intellettuali e poeti e tendenze del diritto valgano tre esempi.

Il primo è l'invenzione e la fortuna dell'idea di paysage moralisé: come abbiamo visto, l'invenzione spetta a Panofsky verso il 1939, il suo riuso poetico per parlare del presente a W.H. Auden nel 1945. 1939, 1945: sono le date estreme di una guerra distruttiva, che sfidava le coscienze a interrogarsi sulla natura e la moralità degli uomini e delle nazioni. Secondo la storica dell'arte americana Patricia A. Emison, la formula introdotta da Panofsky fu sempre meno utilizzata dagli storici dell'arte a partire dagli anni Sessanta: forse dovremmo chiederci se quell'ansia di moralità che accomunò Panofsky e Auden in quegli anni difficili non si sia poi diluita in un'epoca di benessere, che è ancora la nostra.

Consustanziale per temperie e per data è il mio secondo esempio, l'ingresso della tutela del paesaggio al più alto livello del diritto, quello costituzionale. A molti dei presenti è certo noto che la Costituzione italiana fu la prima al mondo a porre questa prescrizione tra i principi fondamentali dello Stato. Ė l'articolo 9, «il più originale della nostra Costituzione» secondo Ciampi: 
La Repubblica promuove la cultura e la ricerca scientifica e tecnica. Tutela il paesaggio e il patrimonio storico e artistico della Nazione.

Eppure esso ha un significativo precedente tedesco, l'articolo 150 della Costituzione della Repubblica di Weimar (1919), secondo cui

i monumenti dell'arte, della storia e della natura, così come il paesaggio, godono della protezione e della tutela dello Stato; è compito dello Stato impedire l'esportazione del patrimonio artistico tedesco.

La Costituzione di Weimar fu espressamente richiamata durante le discussioni dell'Assemblea Costituente che portarono al testo del nostro articolo 9. Non fu invece citato un altro precedente, la Costituzione della Repubblica Spagnola del 1931, dove l'articolo 45 dette a «tutta la ricchezza artistica e storica del Paese», inclusi i «luoghi notevoli per bellezza naturale o per riconosciuto valore artistico o storico», il rango di «tesoro culturale della Nazione».

Le date di queste tre Costituzioni europee incitano a una riflessione: la Costituzione di Weimar è del 1919, all'indomani della prima guerra mondiale, quella italiana del 1947, all'indomani della seconda: nell'un caso come nell'altro, la consapevolezza della propria memoria culturale s'intensifica col trauma della guerra e della sconfitta. Anche in Spagna, la Costituzione del 1931 corrisponde a un periodo di crisi, che avrebbe condotto alla guerra civile. Come nella vita individuale, così anche nelle comunità i traumi generano consapevolezza, innescano meccanismi di difesa, costringono a ripensare il passato. Il dolore della perdita, come quello della morte, è un momento di focalizzazione suprema dei meccanismi sociali, mette allo scoperto quel che prima era celato e lo proietta oltre l'ostacolo, verso il futuro. Come ha scritto Orhan Pamuk nel catalogo del suo Museo dell'innocenza,
A quanto pare non è possibile scoprire il segreto delle cose senza avere avuto il cuore spezzato. Dobbiamo umilmente sottometterci a questa definitiva, segreta verità.

Il terzo esempio che scelgo a illustrare la sintonia fra le esortazioni di intellettuali e poeti e le tendenze del diritto parte dallo stesso Ruskin. Negli stessi anni in cui egli è nel Regno Unito uno dei principali animatori della riflessione sul paesaggio e contribuisce a fondare la Commons Preservation Society (1865) e il National Trust (1895), e in cui Charles Dickens denuncia nel suo Hard Times le conseguenze dell'inquinamento a Coke Town, vengono emanate le prime norme intese alla tutela, a partire dall'Ancient Monuments Preservation Act (1882), poi gradualmente esteso ad altre tipologie di beni, come gli edifici religiosi e storici.

Chiediamoci dunque, mentre questa lezione si avvia alla chiusura: di quale paysage moralisé ha bisogno il nostro tempo? E vi è forse, nell'intenso e visionario pensiero di Ruskin, qualche ispirazione che possiamo trarne?

Come Ruskin fece con Venezia, è sulla città che dobbiamo far centro. La città è la più radicale e promettente creazione della civiltà umana, perché comporta uno scambio di esperienze e di emozioni che avviene grazie al luogo e non grazie al prezzo; ma lo stesso può dirsi del paesaggio naturale, se concepito e preservato come necessario contraltare agli spazi urbani. E però questa complementarietà è sempre meno vera nel mondo che ci circonda. L'armonica integrazione fra spazio urbano e paesaggio naturale (forgiato esso stesso dall'uomo) è ora in grave pericolo, ed è a tal pericolo che deve reagire un nuovo paysage moralisé da costruire con la saggezza della storia e la fantasia della volontà. Cresce in ogni dove la corsa verso un'omogeneizzazione del mondo, apparentemente inesorabile, e specialmente evidente nella forma delle città. Questa omogeneizzazione è fatta di tre processi convergenti: la verticalizzazione delle architet- 
ture (i grattacieli); la megalopoli, espansione urbana indefinita e senza confini esterni che divora le campagne; infine, le nuove barriere intraurbane fra quartieri riservati ai più abbienti e ghetti urbani per i meno fortunati.

La forma-grattacielo, nata in America intorno al 1900, è oggi in forte espansione perché consente il massimo sfruttamento degli spazi in aree urbane preziose (come Manhattan) o soggette a forti speculazioni (come Dubai). Si è così scatenata la corsa al grattacielo più alto del mondo: il Royal Hotel Clock Tower alla Mecca, copia ingigantita del Big Ben di Londra (2010, $601 \mathrm{~m}$ ) è stato subito sorpassato Burji Khalifa di Dubai (2011, 828 m). L'esibizione della ricchezza s'intreccia con la retorica delle altezze, le prodezze tecnologiche, i giochi della finanza.

Crescono intanto, a spese dei paesaggi extra-urbani, le megalopoli (città con oltre 20 milioni di abitanti). La percentuale della popolazione mondiale che vive in città era del 3\% nel 1850, oggi è del 54\% e sarà del $70 \%$ nel 2030: i due terzi dell'umanità. Nel 1950 solo 83 città in tutto il mondo superavano il milione di abitanti, oggi almeno 500 città hanno varcato questa soglia, e almeno quindici hanno superato i 20 milioni di abitanti.

In questa mercificazione degli spazi (in orizzontale e in verticale) si diffondono i confini intra-urbani. Vi fu in tempo in cui la cinta delle mura segnalava, come nel Buon Governo di Siena, il chiaro confine fra la forma urbana e la campagna. Ora non più. Un settimo della popolazione mondiale (un miliardo di esseri umani) vive in bidonvilles o shanty towns che di città non meritano il nome: fra megalopoli e baraccopoli si è creata una perversa continuità. Al polo opposto si situano le gated communities, quartieri per benestanti cinti da mura spesso sorvegliate da guardie armate. I confini della città tendono a sparire, e nascono invece nuovi confini nella città, che materializzano l'auto-segregazione delle classi abbienti dagli altri strati sociali, come nella favela di Paraisópolis a São Paulo.

Gli strumenti concettuali del nostro tempo consentono di misurare con nuovi metri la dimensione del nostro vissuto. Nuove ricerche di sociologi, psicologi, antropologi definiscono lo spazio in cui viviamo come un formidabile capitale cognitivo che fornisce coordinate di vita, di comportamento e di memoria, costruisce l'identità individuale e quella, collettiva, delle comunità. Il grado di stabilità dei luoghi in cui viviamo è in diretta proporzione a un senso di sicurezza che migliora la percezione di sé e dell'orizzonte di appartenenza, favorisce la produttività degli individui e delle comunità, innesca la creatività. Per converso, la frammentazione territoriale, la violenta e veloce modificazione dei paesaggi, l'obesità delle periferie provocano severe patologie individuali e sociali. Due formule vengono alla mente: 'angoscia territoriale' e 'dismorfo-fobia'. Angoscia territoriale non più nel senso di sradicamento dell'emigrante strappato ai propri orizzonti (De Martino, Il mondo magico, Torino: Bollati Boringhieri, 1972), ma come ansia di chi resta nei propri luoghi e non li riconosce più perché devastati da mostri di cemento o da montagne di detriti che ne annientano la familiarità. La nozione complementare di dismorfo-fobia descrive bene il passaggio dalla dimensione individuale a quella collettiva: nella pratica psichiatrica essa definisce i disturbi psichici di chi non accetta il proprio corpo come è, e lo vive come una 'forma distorta' (questa l'etimologia greca di 'dis-morfo'). Ma anche la forma distorta della città e dei paesaggi provoca sofferenze individuali e disturbi del corpo sociale. Più grave di ogni dismorfo-fobia individuale è la dismorfo-fobia delle comunità.

Mentre la crisi della città storica e la contaminazione dei paesaggi si diffondono come una peste, il maggior insegnamento che ci viene da Ruskin può essere anche l'indicazione della strada per un riscatto. Come ha scritto Tomaso Montanari,

l'intuizione fondamentale del capolavoro letterario e storiografico di Ruskin (The Stones of Venice) riguarda il nesso strettissimo che unisce le pietre di Venezia al suo popolo: nulla si può capire dell'urbs, della

Fonti, letterature, arti e paesaggi d'Europa | Sources, Literatures, Arts \& Landscapes of Europe $1 \mid$ 


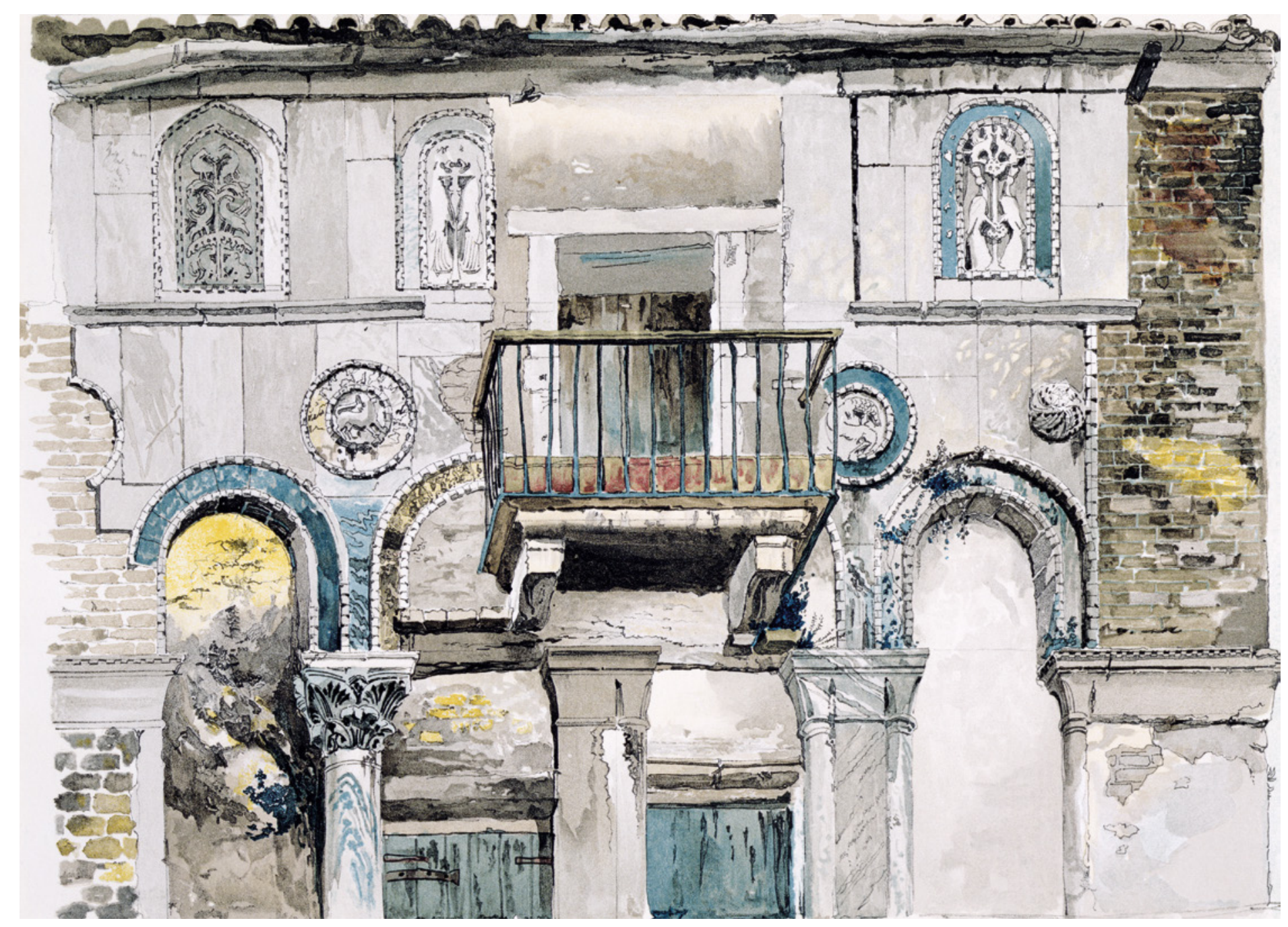

Figura 8 John Ruskin, Fondaco dei Turchi. 1853 ca. Acquerello su carta. Coniston, Cumbria, Ruskin Museum. ๑ Ruskin Museum / Bridgeman Images 
città materiale, se non la si mette in connessione con la civitas, la città degli uomini, cioè la società.

Perciò non possiamo accontentarci di una concezione puramente estetica del paesaggio (urbano e non urbano); dobbiamo anzi interrogarci sulla natura e sulla funzione della bellezza, respingendone un uso meramente estetizzante che facilmente traligna in una sorta di evasione dalla realtà, tendenzialmente 'anestetica' (in senso propriamente medico), abbassando la soglia della nostra coscienza.

Dobbiamo, al contrario, interrogarci sulle devastazioni a cui troppo tempo assistiamo impotenti, chiederci quale sia il modo di combatterle. Non parlerò qui di Venezia, massimo esempio di svuotamento e profanazione di una città storica: tutti i presenti ne sanno certo più di me. Citerò invece, e a proposito del paesaggio non-urbano (che esemplifico sullo schermo evocando la strage di nove milioni di ulivi nel Salento), un grande poeta, Andrea Zanzotto. In un'intervista di qualche anno fa (Finnegans, 21, 2012, 26-8) egli disse che

Un bel paesaggio una volta distrutto non torna più, e se durante la guerra c'erano i campi di sterminio, adesso siamo arrivati allo sterminio dei campi: fatti che, apparentemente distanti fra loro, dipendono tuttavia dalla stessa mentalità. Queste modificazioni violente del paesaggio generano l'assenza stessa di orizzonti, il colore dello spaesamento, lo smarrimento interiore che assale chi tenti di guardare oltre il fragile paravento del paesaggio.

Quando Zanzotto parla di 'campi' intende in generale i paesaggi non urbani, dalle montagne a questa Laguna, e le sue parole di poeta non solo veneto, ma italiano, valgono egualmente per Venezia e per le campagne del Salento. Non c'è bellezza senza consapevolezza del passato e responsabilità verso le generazioni future. La bellez- za di cui abbiamo bisogno non è evasione dal presente: non c'è bellezza senza storia, senza una forte responsabilità collettiva. Il grande messaggio di Ruskin, dal quale dobbiamo ripartire se vogliamo pensare un paysage moralisé per il nostro tempo, è l'invito a ricostruire, facendo convergere memoria culturale e forza delle emozioni, il nesso fra uomo e natura. Vorrei dirlo con un passo, a me caro, del saggio di Susan Sontag sulla bellezza («An Argument about Beauty», Daedalus, 131(4), 2002, 21-6), in cui mi pare di avvertire una qualche sintonia con questi pensieri:

La risposta alla bellezza nell'arte e la risposta alla bellezza nella natura dipendono l'una dall'altra [...]. Quel che è bello nell'arte ci ricorda la natura in quanto tale, ci richiama alla mente quel che sta oltre l'umano, oltre l'artefatto, e in tal modo stimola e intensifica il nostro senso della diffusione e della pienezza della realtà (inanimata o palpitante) che ci circonda. Se questa intuizione ha qualcosa di vero, essa ha una conseguenza positiva: la bellezza riconquista la propria solidità e inevitabilità, diventa un valore necessario per dare un senso a gran parte delle nostre energie e affinità, ai nostri sentimenti di ammirazione; e le nozioni che hanno usurpato lo spazio del 'bello' si rivelano risibili.

Queste parole penetranti di una grande testimone del nostro tempo giocano la loro forza di persuasione su un'intuizione interamente laica, che per impulso etico e poetico reagisce al processo di marginalizzazione della bellezza (a cui quel saggio di Sontag è dedicato). Eppure, per quante affinità con Ruskin possiamo scorgere o immaginare, non fu questo il registro emotivo su cui egli volle muoversi. Le sue pagine (in particolare in quelle di The Stones of Venice) sono intrise di citazioni bibliche, che conferiscono al suo argomentare una speciale, quasi religiosa eloquenza. Ogni rivendicazione della bellezza si accompagna in lui alla consapevolezza del suo decli- 
no, ogni dolore ispirato dalle rovine innesca una speranza di riscatto. Come ha scritto Michael Wheeler (Ruskin and the Environment, Manchester: Manchester University Press, 1995, 168 s.), dobbiamo

riconoscere l'importanza che ebbero per lui alcune parole-chiave della Bibbia, come ruin (nel senso di fall, 'caduta') e restoration (come si riferisse a Israele) [...]. Per lui San Marco è un simbolo di mediazione fra la terra e il cielo, e di una speranza del tempo presente per una futura restoration: idee che vogliono echeggiare le 'mura in rovina' di Gerusalemme, le 'splendide pietre' del Tempio che diventano quelle di Palazzo Ducale, e l'esortazione di San Paolo ai Romani di 'trasformare se stessi' [sulla base del Bene, e senza conformarsi all'opinione generale: Rom. 12.21]. Perciò il restauro, terribilmente rozzo, perpetrato a San Marco (che fu oggetto di una delle sue campagne di protesta più riuscite) è ancor più importante, se consideriamo il peso morale e religioso che l'edificio ebbe per lui. La chiesa che egli aveva scelto come il più intenso simbolo di salvezza dalla rovina veniva sottoposta non a un processo di restoration, ma a una nuova e peggiore rovina.

Un'ultima notazione: per Ruskin quella di Venezia-città è una bellezza che prende forza dal fatto stesso di aprirsi alle bellezze naturali che la circondano, e in certo senso di abbracciarle e includere in sé [fig. 8]. Perciò, leggiamo verso la fine dei Modern Painters, [a Castelfranco] lo sguardo di Giorgione, gli occhi limpidi e indagatori della giovinezza, si aprirono su un mondo di vita vigorosa, dalle montagne fino alla costa, di meravigliosa vita quando andò alla città di marmo e cominciò ad ardere per essa. [...] Il mare incontaminato respirando profondamente formava, avanti e indietro, i suoi vortici di onde verdi. E intorno, fin dove l'occhio poteva arrivare, il dolce movimento di acque incontaminate, orgogliosamente pure; non il fiore, non la spina o il cardo potevano crescere nei campi. Eterea potenza delle Alpi, come un sogno, che svanisce oltre Torcello; isole blu delle colline padovane, sospese nell'occidente dorato. In alto, venti liberi e nuvole infuocate [...] nella luce illimitata del cielo e del mare circostante. Tale fu la scuola di Giorgione, tale la patria di Tiziano.

Venezia, la Laguna, il suo mare e il suo cielo, la natura che la circonda, fino al Cadore di Tiziano.

Anche per questa intima unione, non dimentichiamolo, la Venezia di Ruskin è «l'asse del mondo», il centro e il culmine della creatività di ogni civiltà. Perciò, per Ruskin, la bellezza di Venezia non è solo la condensazione e il simbolo della bellezza del mondo. Come in un paysage moralisé essa può essere, anche in un tempo di profanazioni e di rovine, promessa di riscatto, di piena ricreazione di una civitas che faccia tutt'uno con la bellezza dell'urbs [fig. 9]. Questo, io crederei, per Ruskin. E per noi?

Venezia, 7 ottobre 2019

$29 \quad$ Fonti, letterature, arti e paesaggi d'Europa | Sources, Literatures, Arts \& Landscapes of Europe 1 Fonti, letterature, arti e paesaggi d'Europa| Sources, Literatures, A
John Ruskin's Europe. A Collection of Cross-Cultural Essays, 11-30 


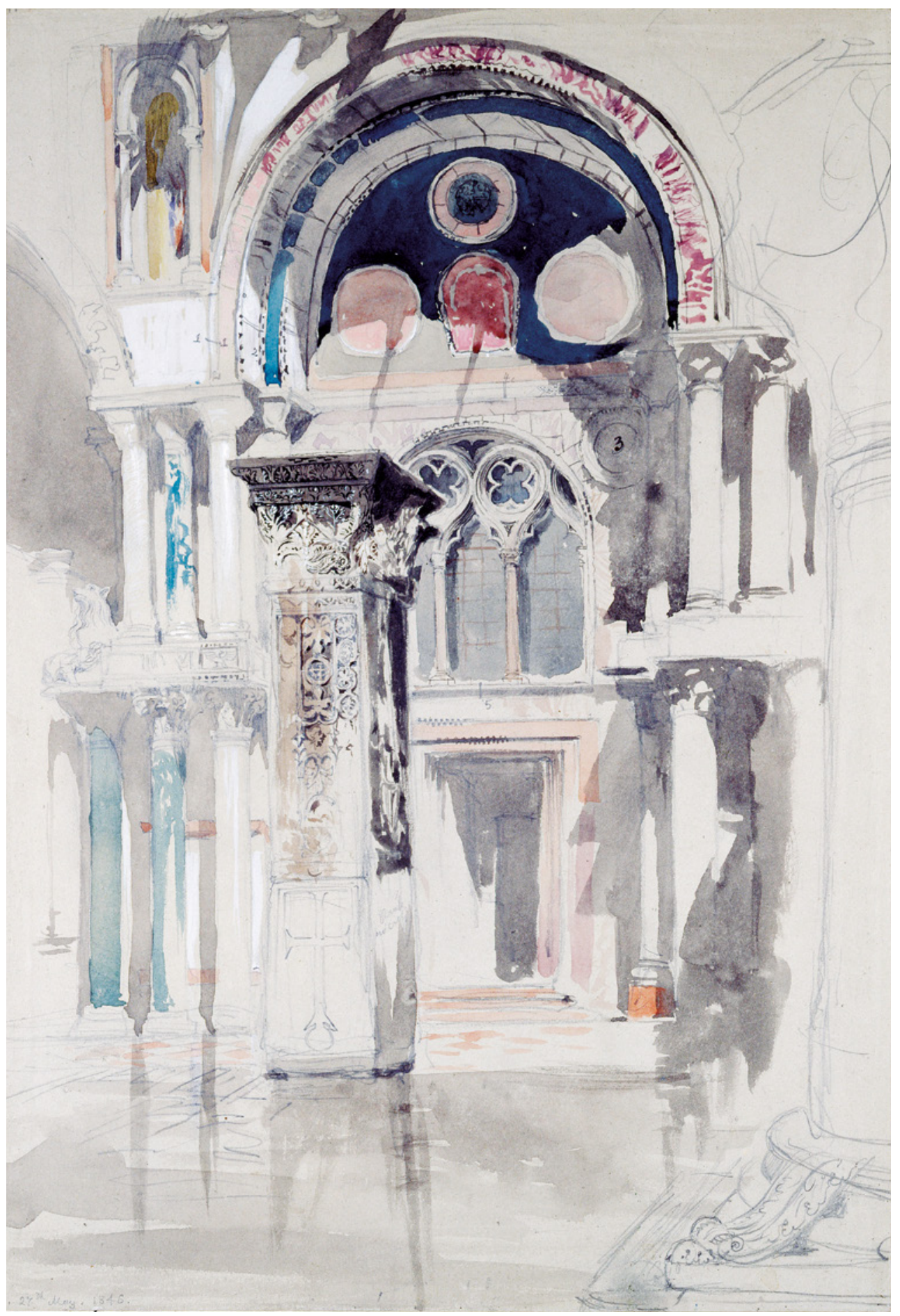




\section{Foreword}

Emma Sdegno

Università Ca' Foscari Venezia, Italia

This volume is a record of the bicentenary conference held in Venice on 7-9 October 2019, a conference organized by the Departments of Linguistics and Comparative Cultural Studies (DSLCC) and of Philosophy and Cultural Heritage (DFBC) of Ca' Foscari University Venice in partnership with the Department of Art History and Archaeology (UFR ALC) of the University of Rennes 2 . The occasion came at the end of a particularly generous and festive year, with events following one after another in Pau (France), in London, Venice, Verona, Florence, Lucca and Rome, and closing in December at the Huntington Library, San Marino (California), among them two major exhibitions, one in the Doge's Palace in Venice (MarchJune 2018), the other at Two Temple Place in London (January-April 2019). All this took place just before the coming of the pandemic that has tragically changed our habits and desires, establishing other priorities and putting more urgent concerns on the agenda. The essays gathered here were delivered in a period in which scholars could still travel freely, talk to unrestricted audiences, and get together in regenerating conviviality. In collecting and reorganizing them for publication, our intellectual effort and excitement at re-encountering familiar thoughts and provocative insights are inextricably intertwined with a host of recollections that the present situation renders at once remote and even more urgent and timely.

The topic of the conference was conceived in the aftermath of Britain's divisive vote to leave the European Union, and when the date of the British departure still looked as though it would proceed straightforwardly and imminently, which it did not. It was these expectations that inspired our call for papers for a conference whose title "A Great Community: John Ruskin's Europe" echoed a sentence in a 1857 lecture later published in a volume entitled A Joy For Ever. In that lecture Ruskin energetically defended what he saw as a distinctive European culture and spirit, a defence that in his later works would become more and more forthright and complex. It was this challenge that our bicentenary celebrations in Venice aimed to meet. Like individual nations, Europe is an "imagined community", "constructed through 
widely-accepted 'narratives'”. ${ }^{1}$ In the map of the continent Ruskin drew and re-drew repeatedly in the course of his life, boundaries shift continuously, so that - as Denis Cosgrove has brilliantly pointed out - he sometimes presents Europe as "a single cultural entity against which England and Britain is contrasted, sometimes focusing on its diverse and conflicted 'nations', sometimes making Europe stand for the whole 'globe of earth', across which world historical forces contend and collide". ${ }^{2}$

In the impressive map Ruskin drew as a ten-year-old child in 1829 [fig. 1], Britain appears hazy and faraway, while Europe is fairly neat and in close focus, bearing names written clearly and encircled within the blue lines he particularly enjoyed drawing, as he recollected in old age. ${ }^{3}$ To the young John Ruskin Europe was mainly a place of romantic poetry and picturesque views, a place of imagination and desire. In 1825, when he was aged six, his family had ventured to travel as far as Paris and to Belgium to visit the field of Waterloo; his second journey to the Continent, the first to comprise also Germany, Switzerland and Italy, would take place in 1833. Twenty-two more visits to the Continent followed, the last, that of 1888, ended in Venice, where the last words of his diary were written.

It was to discuss this both dim and detailed map that scholars from different disciplines, countries, outlooks were invited to meet in Venice in October 2019. All readers of Ruskin feel at times the frustration of meeting with the limitations of their fields, and a major aim of the conference was to favour interdisciplinary dialogue. If a community of scholars can be beneficial for the study of Ruskin, this is especially so as regards Ruskin's Europe. A variety of competences and approaches was thus thought to be a vital starting point for an enquiry into his polymath European researches. This collection of essays is therefore both a celebratory occasion and a challenge, as challenging are all Ruskin's works. Programmatically multi-disciplinary as whole, it is composed of papers that variously explore relationships, ranging from comparative translation (Eells, Tizot), to history of reception (Cerasi, Eagles, Frank, Kawabata, Pilutti Namer, Remport), and of collectionism (Agazzi), to Ruskin's relations with contemporaries (Paribeni and Pedone, Sdegno, Wildman), to his own reception of European culture (Sandrini), encompassing Islam (Zaman). Through this variety of perspectives and field-crossing a Ruskin eminently relational stands out, also in the sense of establishing spatial connections between places, ${ }^{4}$ through an ever-expanding comparative method (Hélard, Reichler), where the road (Hélard, Hull) and the bridge (Tucker), prove to be intriguing and as yet utterly unexplored icons. The net of relationships becomes even more closely interwoven on the theoretical level, as Ruskin's thought enters wider western discourses involving aesthetics (Frangne), and the theories and practice of architecture and conservation (Abreu, Morezzi). That this multivocal approach may open new paths in Ruskin scholarship is among the auspices of this volume.

In 2018 Europe was among the core concerns of a series of studies recognizing the crucial importance of place,

1 Anderson, B. Imagined Communities: Reflections on the Origin and Spread of Nationalism. London; New York: Verso, [1983] 2006. Heffernan, M. The Meaning of Europe. Geography and Geopolitics. London; New York: Arnold, 1998, 2.

2 Cosgrove, D. "Ruskin's European Visions". Geography and Vision. Seeing, Imagining and Representing the World. London; New York: I.B. Tauris, 2008, 136 .

3 Works, 35: xx; Works, 13: 502-3.

4 A contribution to this line of research is Jeanne Clegg's "From Dead End to Central City of the World: (re)-locating Rome on Ruskin's map of Europe", a lecture given at the British School at Rome on 30 October 2019, forthcoming in Papers of the British School at Rome 2021.

Fonti, letterature, arti e paesaggi d'Europa | Sources, Literatures, Arts \& Landscapes of Europe $1 \mid$ 


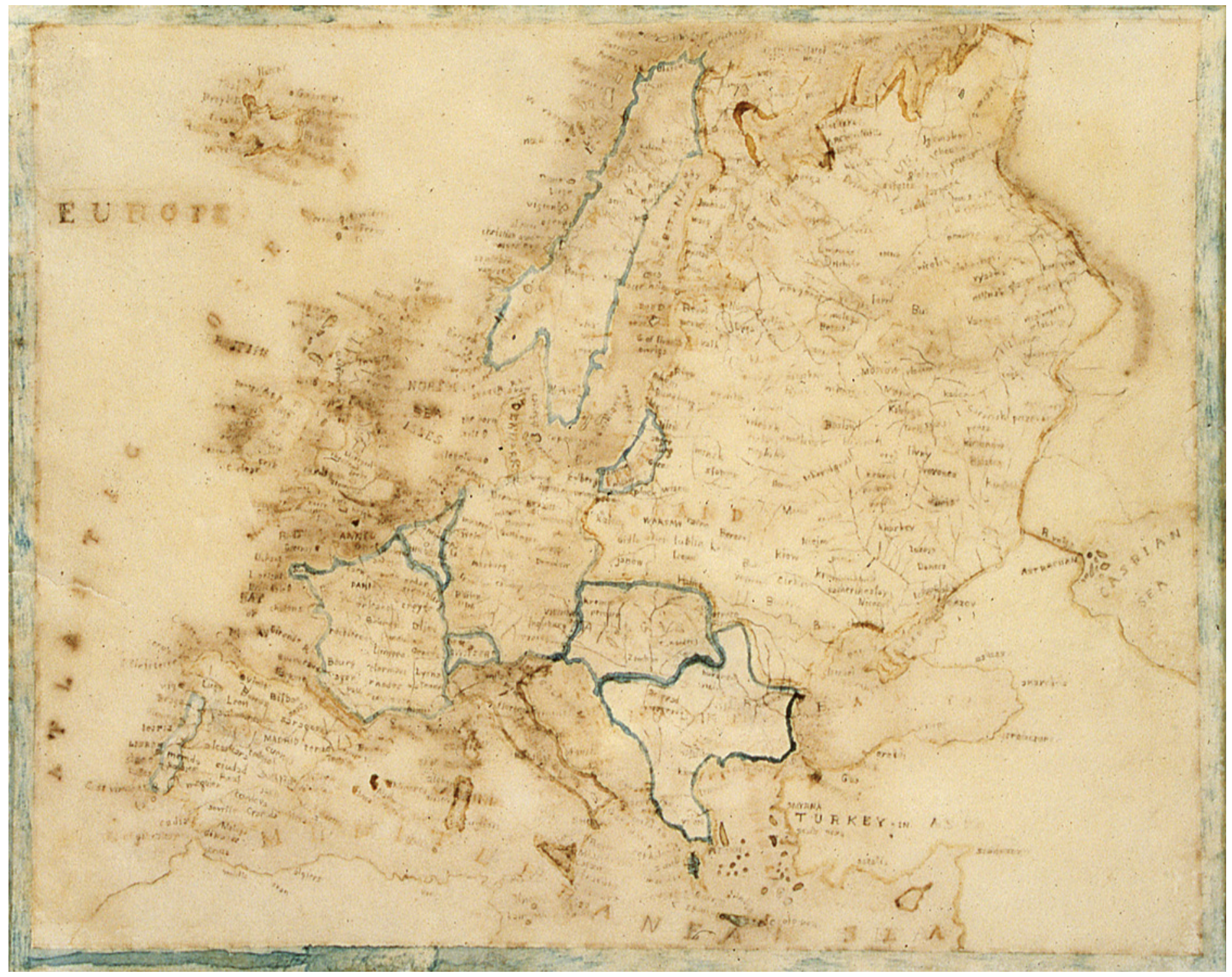

Figure 1 John Ruskin, Map of Europe. 1829 ca. Drawing. CONRM.1989.540 Map of Europe H Res 3937. Coniston, The Ruskin Museum. "I began to learn drawing by carefully copying the maps in a small quarto Atlas of excellent old fashioned type, the mountains well marked (but not blackened all over like those in the modern Geological Survey), the names clear, not crowded - above all, not run across each other, not to be gleaned, a letter at a time, when one can pick them up. A map of France, and a map of Africa, are examples of many done by the time I was ten years old. These maps were of great delight to me; the colouring round the edges being a reward for all the tediousness of the printed names; the painting, an excellent discipline of hand and eye; and the lines drawn for the mountains and sea a most wholesome imitation of steady engraver's work" (Works, 13: 502-3). This is a note occurring in the catalogue of the exhibition of Ruskin's "handiwork" held in London at the Fine Art Society in 1878, in which the maps of France and of Africa were exhibited 
of myth, and of image in the construction of a European fabric. ${ }^{5}$ Within this significant plethora of works Salvatore Settis's particularly authoritative voice is the one that most directly shares Ruskin's ethos in that it combines high scholarly research with a public engagement in our cultural and environmental heritage on the national and international scales. The convergence of aesthetics, ethics and constitutional legality Settis advocates in Architettura e democrazia. Paesaggio, città, diritti civili ${ }^{6}$ was the inspiring motif of the wonderful lectio magistralis he delivered at the Scuola Grande di San Rocco on the first day of the conference, and which we are honoured and grateful to be able to publish at the opening of this volume.

Young people are the direct addressees of most Ruskin's works, and as a University event, our conference aimed at directly involving students. Under the excellent guidance of Sandra Zodiaco, language students helped with the organization of the conference, keeping in constant and highly appreciated contact with speakers from abroad. Collateral but closely pertinent to the conference spirit was the event involving secondary school students from the Liceo Artistico "Michelangelo Guggenheim" in Venice-Mestre. In the days preceding the conference British artists Kate Genever and Steve Pool held workshops with these students in which they imagined the future of Europe, making works which were exhibited at the University's Cultural Flow Zone on the Zattere during the conference itself. A few of these are reproduced and commented on in the appendix of this volume, which comprises the collage drawings Kate made during the talks, her unique visual record of the conference (see Appendix). We cannot conclude this foreword without presenting our warmest thanks to Architetto Franco Posocco,
Guardian Grando of the Scuola di San Rocco, and the Cancelleria for hosting us in the inspiring space of the Sala Capitolare. We also wish to thank our directors of Department, professors Valle Ojeda Calvo and Antonio Trampus (DSLCC) and Giuseppe Barbieri (DFBC), and the headmistress of the Liceo Guggenheim, professor Cecilia Martinelli, for their help and active support. Special thanks are also due to the administative staff of the Departments of DSLCC, DFBC, and UFR-ALC for their untiring helpfulness; and to the director and the great team of Edizioni Ca' Foscari for producing this volume so efficiently and smoothly. Many people were involved in the conference and in producing this volume, as speakers, participants, discussants, reviewers, museum curators, and we wish to express warmest thanks for material help as well as intellectual support to Dinah Birch, Luigino Bruni, Enzo Buvoli, Juan Calatrava Escobar, Alexandra Cheira, Jeanne Clegg, Jim Dearden, Irene Favaretto, Sandro Franchini, Flavio Gregori, Pamela Hull, Mary Ann Lancaster, Geraldine Ludbrook, Rosella Mamoli Zorzi, Franco Marucci, Vito Mistretta, Anna Ottani Cavina, Louise Pullen, Sarah Quill, Jolanda Ramos, Laurence Roussillon-Constanty, Baldine Saint-Girons, Jim Spates, Bianca Tarozzi, Liz Waring, Clive Wilmer. The project and this book took shape in many mutual exchanges, marked by intellectual enthusiasm and generosity, among colleagues of different backgrounds and countries: a small and very real European community. My final most heartfelt words of gratitude are for André Hélard, who sowed the first seed of the project, and the co-editors of this volume, Martina Frank, Myriam Pilutti Namer, Pierre-Henry Frangne, who helped bring it to fruit.

5 Ossola, C. Europa ritrovata. Geografie e miti del vecchio continente. Milano: Vita e Pensiero, 2017. Published in French as Fables d'identité. Pour retrouver l'Europe. Paris: PUF, 2018. See also: Charle, C.; Roche, D. (éds). L'Europe. Encyclopédie historique. Paris: Actes Sud, 2018.

6 Torino: Einaudi, 2017 


\section{Introduction}

Pierre-Henry Frangne

Université Rennes 2, France

Summaire 1 Un élargissement du regard. - 2 La crise de la recherche philosophique de la vérité. - 3 Soi-même comme un autre. - 4 Une pensée incarnée et vivante 'à la surface de la terre'.

Puisqu'il m'est fait l'honneur d'introduire ces actes du colloque sur l'Europe de Ruskin, sur Ruskin l'européen, je voudrais commencer par remercier ceux qui l'ont rendu possible : l'Université Ca' Foscari au premier chef parce qu'elle nous accueille bien sûr par l'intermédiaire de ses deux départements de philosophie et d'études de linguistique et de culture comparées ; la Scuola Grande di san Rocco qui tisse depuis longtemps avec ces départements de solides partenariats ; l'Université de Rennes 2 en France, enfin, que je représente et qui est heureuse et honorée à la fois de pouvoir mettre en œuvre une collaboration qui sera, à n'en pas douter, fructueuse. Je voudrais remercier également tous les participants, tous ceux qui ont écrit un article dans l'ouvrage qui s'ouvre ici, tous ceux qui viennent souvent de loin, de nombreux pays européens mais aussi du Brésil et du Japon. Ils mettent en œuvre par leur présence et leur travail une communauté de recherches, une communauté spirituelle qui est le reflet de cette 'grande communauté' européenne que Ruskin et les hommes du XIXe siècle ont aidée à constituer et qu'ils nous ont léguée comme un travail à faire ; un travail qui n'est pas achevé parce qu'il est sûrement infini comme le disait Husserl en 1935 et 1939 dans sa conférence viennoise sur « La crise de l’humanité européenne 
et la philosophie ${ }^{1}{ }^{1}$ puis dans La crise des sciences européennes et la phénoménologie transcendantale.

Si ce travail est infini, c'est parce que la grande communauté européenne est une communauté de cultures qui s'unissent en reconnaissant dans le monde et l'histoire communs qu'elles construisent leur spécificité, leurs différences mais aussi leur nécessaire circulation et leur impérieux dialogue. C'est ce dialogue, permis par «la merveilleuse distance » comme disait Montaigne dans ses Essais $^{2}$ sur laquelle ce dialogue est greffé, qui garantit (comme tout dialogue humain d'ailleurs) la présence, la différence et l'équivalence des cultures qui lient leur destin. Cette présence, cette différence et cette équivalence sont celles de leurs multiples cultures, c'est-à-dire de leur multiples langues en lesquelles elles s'incarnent, et qui doivent passer les unes dans les autres par la traduction tout en reconnaissant ce que Barbara Cassin appelle le risque de leurs intraduisibles, comme par exemple, en allemand aufheben (supprimer-conserver, relever) en portugais saudade (mélancolie et nostalgie solitaire d'un ailleurs où l'on a pas vécu), en italien sprezzatura (désinvolture comme summun de l'art), en anglais design (dessin et dessein) et care (soin, souci, attention), en grec logos (ratio et oratio) ou aidos (pudeur et respect). C'est parce que « la langue de l'Europe c'est la traduction $»^{3}$ selon la magnifique expression d'Umberto Eco, c'est parce que son transit plein de doute entre les langues est constitutive de l'identité européenne, que nous avons voulu que chaque auteur écrive ici, et autant que faire se peut, sa langue maternelle, comme je le fais ici même en ce lieu qui veut éviter le surplomb du global English. Car le globish est fait pour les moteurs de recherche et pour le discours des experts. Comprendre, au contraire, un écrivain quel qu'il soit, c'est-à-dire prioritairement un style, c'est éviter ce langage de pure communication, de pure assimilation, ${ }^{4}$ en son simple fonctionnement de "numéraire, facile et représentatif » comme a dit Stéphane Mallarmé, qui s'y entendait en poésie, en style, en écriture, en traduction lui qui était professeur d'anglais et qui fit en français (en 1894) deux conférences mémorables à Oxford, l'université de Ruskin, puis à Cambridge, intitulée La Musique et les Lettres. C'est la fonction numéraire qui rend le langage aussi plat qu'une feuille de journal, qu'une pièce de monnaie ou qu'un billet de banque passant de mains en mains.

C'est donc en français que je voudrais remercier mes amis et mes collègues avec lesquels nous avons pensé le colloque Ruskin, l'Europe de Ruskin ; l'Europe de Ruskin qui est justement celle où, en plein XIXe siècle, les écrivains se font, non par surcroît mais dans le mouvement même de leur œuvre et de leur création littéraire, traducteurs. Ce faisant, ils produisent ce que Malraux a appelé « un échange horizontal entre les Européens substitué à l'échange vertical avec les Anciens », ${ }^{5}$ échange horizontal qui accompagne « la conscience du fait poétique lié au fait pictural » : pour la France, je pense à Nerval, à Vigny, à Baudelaire, à Mallarmé et à évidemment à Proust par lequel Ruskin est venu parler la langue que j'écris en ce moment. Ces collègues que je remercie tout particulièrement sont Martina Frank, Myriam Pilutti Namer et Emma Sdegno. Je les remercie avec d'autant plus de chaleur et d'insistance que je connais la difficulté de l'organisation d'un colloque et d'un ouvra-

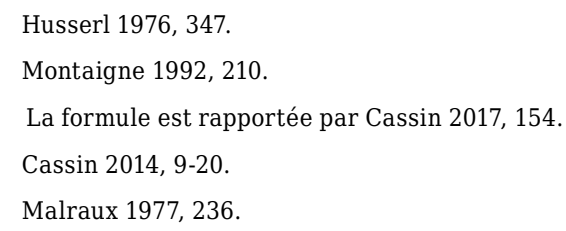


ge véritablement, et je dirais substantiellement, internationaux. À cette équipe à laquelle j'ai l'honneur d'appartenir, je voudrais associer mon ami André Hélard de Rennes parce qu'il a aussi une place éminente dans la conception de notre rencontre et de notre livre, et parce qu'il faut le considérer comme le grand traducteur actuel de Ruskin en français en ce qu'il a déjà donné sa voix aux textes ruskiniens, notamment sur les Alpes et sur la critique ruskinienne du Tintoret. Il a mis en valeur plus qu'un autre ce que Mikhail Bakhtine a appelé le « chronotope $»^{6}$ ruskinien se déployant entre les pierres de la montagne du massif du Mont-Blanc et les pierres de Venise. Ce chronotope il est celui de « l'Europe des cathédrales » (Georges Duby), des cathédrales des hommes comme celles de la terre qui sont, pour Ruskin, tout à la fois naturelles (la croisée d'ogive serait calquée sur la forme des plantes), culturelles (la cathédrale est une forme symbolique et la pétrification de la Somme théologique de Thomas d'Aquin), scientifiques (Viollet-le-Duc), mystiques (Victor Hugo), locales (Amiens, Rouen, Venise, Vérone, Florence etc.) et universelles (comme nous l'a montré récemment le grand retentissement de l'incendie de Notre-Dame de Paris en 2019).

\section{Un élargissement du regard}

Le premier principe que nous voudrions faire jouer est celui d'une Weltliteratur telle que Goethe en parle, et, après lui, Milan Kundera. Ce dernier considère «qu'il y a deux contextes élémentaires pour situer une œuvre d'art, ou bien l'histoire de sa nation (appelons-le le petit contexte) ou bien l'histoire supranationale de son art (ap-
On aperçoit alors la logique - toute ruskinienne et toute européenne - que notre ouvrage voudrait explorer : la logique de l'opposition voire de la contradiction par laquelle les choses ne vont pas de soi, mais sont, au contraire, dans leur opacité, mystérieuses, problématiques ou aporétiques. "Nous autres Européens » comme dit si souvent Nietzsche, nous savons " aujourd'hui que c'est un signe de culture que de pouvoir supporter la contradiction ». Et il poursuit : « Mais quant à savoir contredire, quant à maintenir la bonne conscience acquise dans l'hostilité à tout ce qui est habituel, traditionnel, sacré - voilà ce qu'il y a d'essentiellement grand, nouveau, étonnant dans notre culture, voilà le pas suprême de l'esprit libéré : qui donc en est capable aujourd'hui ? ». Je répondrais à Nietzsche : John Ruskin bien sûr ! Pour le montrer, pour le démontrer, notre livre voudrait mettre l'accent sur quatre principes qui le gouvernent. Quatre principes ou quatre modalités d'un même arrachement constitutif de l'esprit ruskinien et de l'esprit européen : 1) un élargissement du regard ; 2) la crise de la recherche philosophique de la vérité ; 3) soi-même comme un autre ; 4) une pensée incarnée et vivante 'à la surface de la terre'.

pelons-le le grand contexte) $\gg .{ }^{8}$ Or, pour Kundera, seul ce qui « permet d'embrasser le grand contexte de la Weltliteratur est capable de faire apparaître la valeur esthétique » d'une œuvre parce qu'elle la déporte et la dépayse en dehors de sa langue, de ses goûts, de ses préjugés qui la fixe et l'identifie ostensiblement au petit

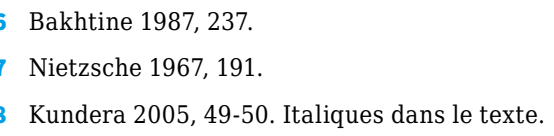


contexte national. Tel est le risque, le beau risque dirait Platon, que nous voudrions courir : envisager l'œuvre entier de Ruskin dans un mouvement d'élargissement du regard de façon à faire apparaître des aspects inconnus ou des formes nouvelles par un décentrement de l'analyse qui suit exactement le décentrement que Ruskin a lui-même voulu mettre en œuvre pendant toute sa vie.

\section{La crise de la recherche philosophique de la vérité}

Le second principe consiste alors à mettre sous leur lumière réciproque et à articuler : a) l'identité plurielle de l'œuvre ruskinienne ; b) l'identité de la pensée ruskinienne dans son mouvement de confrontation avec l'espace et le temps de l'Europe ; c) l'identité de la réception européenne de son œuvre ; d) enfin, l'identité de l'Europe ou de la pensée européenne elle-même qui enclot les trois identités précédentes mais qui se laisse aussi de construire par elles. Or, ce cercle de cercles, si je puis dire, il est mouvant et ouvert parce que s'y déploie une commune circulation par laquelle les quatre cercles, les quatre identités, font foncièrement l'épreuve de l'autre, de l'altérité, de l'étranger et même de l'étrangeté de l'étranger. L'identité de l'œuvre ruskinienne et l'identité européenne peuvent se refléter les unes dans les autre parce qu'elles sont le contraire d'une identité fermée et immobile au sein de ce que l'on pourrait appeler une mêmeté. L'identité de l'œuvre ruskinienne et celle de Europe relèvent de ce qu'on appellerait plutôt une ipséité qui fait entrer ce qui n'est pas soi dans la définition de soi. Elles sont une identité ulysséenne en ce que, jamais complètement accomplie ni fixe, elles sont un processus ; elles sont un voyage et une conquête à l'intérieur et en face de l'extériorité du monde naturel et culturel. La curiosité universelle de Ruskin, son souci de tout (de ce qui est le plus grandiose comme de ce qui est le plus minuscule et le plus humble), comme son souci du tout, se reflètent bien dans la volonté européenne tout à fait vive en son temps et dans le nôtre de produire comme un arraisonnement et un inventaire du réel. Ce réel il est conçu comme une Lebenswelt à partir de ce qu'Husserl nommait, dans les textes que j'ai évoqués plus haut, un monde ambiant ${ }^{9}$ qui est une erlebt Welt, un monde vé$\mathrm{cu}$, vécu «comme une formation spirituelle en nous et dans notre vie historique ». Or, ce qui caractérise « la figure spirituelle de l'Europe », son «idée philosophique immanente », c'est « l'attitude » qui «traverse » toutes les nations européennes ${ }^{10}$ et qui consiste en la volonté de "vivre dans la libre formation de son existence, de sa vie historique, par les idées de la raison, par des tâches infinies ». ${ }^{11}$ J'ai ainsi la conviction que cette attitude traverse aussi le projet ruskinien tant il est spectaculairement à la fois scientifique, littéraire, artistique, esthétique, philosophique, anthropologique, politique et apologétique, dans une liberté de ton, de méthode et d'écriture constamment adossée à la devise kantienne de l'Aufklärung : Sapere aude, ose savoir. De Modern Painters, Tim Hilton a écrit :

C'est de la philosophie et de l'esthétique, et beaucoup 
plus que cela. C'est de la poésie. C'est de la prose. C'est un traité. C'est un grand pamphlet. C'est une défense ou plutôt un règlement de comptes. C'est un sermon. C'est de la critique d'art, de l'histoire de l'art, un commentaire d'expositions récentes, une introduction à certaines collections. C'est une méditation sur le paysage, et un exercice pour apprendre comment les yeux doivent regarder la nature..$^{12}$

Tel est aussi l'œuvre entier de celui qui dans sa jeunesse signait ses premiers articles du pseudonyme grec et aris-

\section{Soi-même comme un autre}

Troisième principe alors : tout l'œuvre de Ruskin peut être pensée comme un moment de reprise à l'époque contemporaine de l'arrachement constitutif de l'Europe si l'on prend soin de rappeler que 'le vieux continent' porte le nom d'un rapt ou d'un enlèvement incessant : enlèvement d'une princesse phénicienne telle que Véronèse par exemple la représente en 1580 (palais des Doges), arrachement du logos par rapport au mythos, arrachement du droit par rapport au cosmos grec, arrachement des nations naissant (c'est le même mot) et se différenciant, arrachement de l'individu à l'époque de l'humanisme renaissant puis du libéralisme des XVIIe et XVIIIe siècles, arrachement aux rivages de l'Atlantique comme de la totélicien de Kata physin afin de montrer que, sans être philosophe au sens disciplinaire ou académique, il voulait philosopher, c'est-à-dire connaître, c'est-à-dire encore s'étonner, penser systématiquement mais sans système, penser par principe et non occasionnellement que le monde est contradictoirement à la fois admirable et inquiétant ; et qu'il est à connaître. La vérité est son but : Of Truth of Colour, Of Truth of Space, Of Truth of Skies, Of Truth of Clouds etc. La crise de l'examen (krinein), de l'analyse, est son chemin.

Méditerranée vers toutes les cultures de la terre. Pour le meilleur de la liberté et de l'universalité de la raison, et pour le pire du colonialisme, de l'impérialisme, de la guerre, de l'esclavage et de la profanation de la nature, l'Europe est un grand vent qui envisage toujours l'autre pour se fuir elle-même et pour se saisir dans la grande fugue polyphonique qu'elle voudrait être et continuer. Ruskin est notre contemporain parce qu'il a saisi cette logique ambivalente de l'aliénation et qu'il lui a donné (qu'il nous a donné) des formes littéraires et imagées diverses, contradictoires mais toujours engagées, c'est-àdire toujours vivantes.

\section{Une pensée incarnée et vivante 'à la surface de la terre'}

Quatrième principe enfin. Ces formes artistiques, critiques, théoriques etc. ne sont jamais séparées d'un expérience singulière et individuelle, une Erlebnis comme disent les philosophes allemands dont l'écriture ruskinienne déplie sans cesse les mille et un aspects ou les mille et un secrets. Si l'Europe a scindé le sensible de l'intelligible, si elle est née de cette séparation qu'elle a voulu combler ou réparer, Ruskin a toujours fait de 
même. Proust est celui qui le dit le mieux dans son introduction à la traduction de La Bible d'Amiens :

Car la pensée de Ruskin n'est pas comme la pensée d'Emerson par exemple qui est contenue tout entière dans un livre, c'est-à-dire un quelque chose d'abstrait, un pur signe d'elle-même. L'objet auquel s'applique une pensée comme celle de Ruskin et dont elle est inséparable n'est pas immatériel, il est répandu çà et là à la surface de la terre. Il faut aller le chercher là où il se trouve, à Pise, à Florence, à Venise, à la National Gallery, à Rouen, à Amiens, dans les montagnes de la Suisse. Une telle pensée qui a un autre objet qu'ellemême, qui s'est réalisée dans l'espace, qui n'est plus la pensée infinie et libre, mais limitée et assujettie, qui s'est incarnée en des corps de marbre sculpté, de montagnes neigeuses, en des visages peints, est peut-être moins divine qu'une pensée pure. Mais elle nous embellit d'avantage l'univers, certaines partie nommées, de l'univers, parce qu'elle y a touché, et qu'elle nous y a initiés en nous obligeant, si nous voulons les com- prendre, à les aimer. / Et ce fut ainsi, en effet ; l'univers reprit tout d'un coup à mes yeux un prix infini. ${ }^{13}$

L'impureté d'une pensée incarnée qui se jette dans le monde, qui fuit toute identité narcissique mortifère, qui ne sépare jamais « la beauté des cathédrales du charme de ces pays d'où elle surgirent, et que chacun de ceux qui les visite goûte encore dans la poésie particulière du pays et le souvenir brumeux ou doré de l'après-midi que Ruskin y a passé » $:^{14}$ telle est bien la pensée de Ruskin explorant impatiemment l'Europe naturelle et culturelle, s'enracinant toujours dans le lieu et le moment vivants où elle se constitue, et, comme Ulysse revenant chez soi, c'est-à-dire à soi, se fait lui-même, par l'écriture, la photographie et le dessin, un immense auteur. Notre ouvrage collectif voudrait recueillir la puissance, l'écho et la limite de son autorité, de son auctoritas, afin de la comprendre et de comprendre (c'est sans doute là l'essentiel) en quoi elle nous donne, ou peut nous donner, le sens du présent de nous-mêmes et de notre futur.

\section{Bibliographie}

Bakhtine, M. (1987). Esthétique et théorie du roman. Paris : Gallimard.

Cassin, B. (2014). Philosopher en langues. Les intraduisibles en traduction. Paris : Éditions rue d'Ulm.

Cassin, B. (2017). «La langue de l'Europe ». Po\&sie, 160-161, 154-9.

Hélard, A. (2005). John Ruskin et les cathédrales de la terre. Chamonix : Éditions Guérin.

Hilton, T. (1985). John Ruskin, The Early Years. New Haven : Yale University Press.

Husserl, E. (1976). La crise des sciences européennes et la phénoménologie transcendantale. Trad. par G. Granel. Paris : Gallimard.

Kundera, M. (2005). Le Rideau. Paris : Gallimard.

Malraux, A. (1977). L'Homme précaire et la littérature. Paris : Gallimard.

Montaigne, M. de (1992). «Des Cannibales ». Les Essais, t. 1, livre 1, ch. 31. Paris: PUF.

Nietzsche, F. (1967). Le Gai savoir. Ed. par G. Colli et M. Montinari, trad. par P. Klossowski. Paris : Gallimard

Proust, M. (2011). «Préface ». La Bible d’Amiens. Paris : Rivages poche, 11-105. 


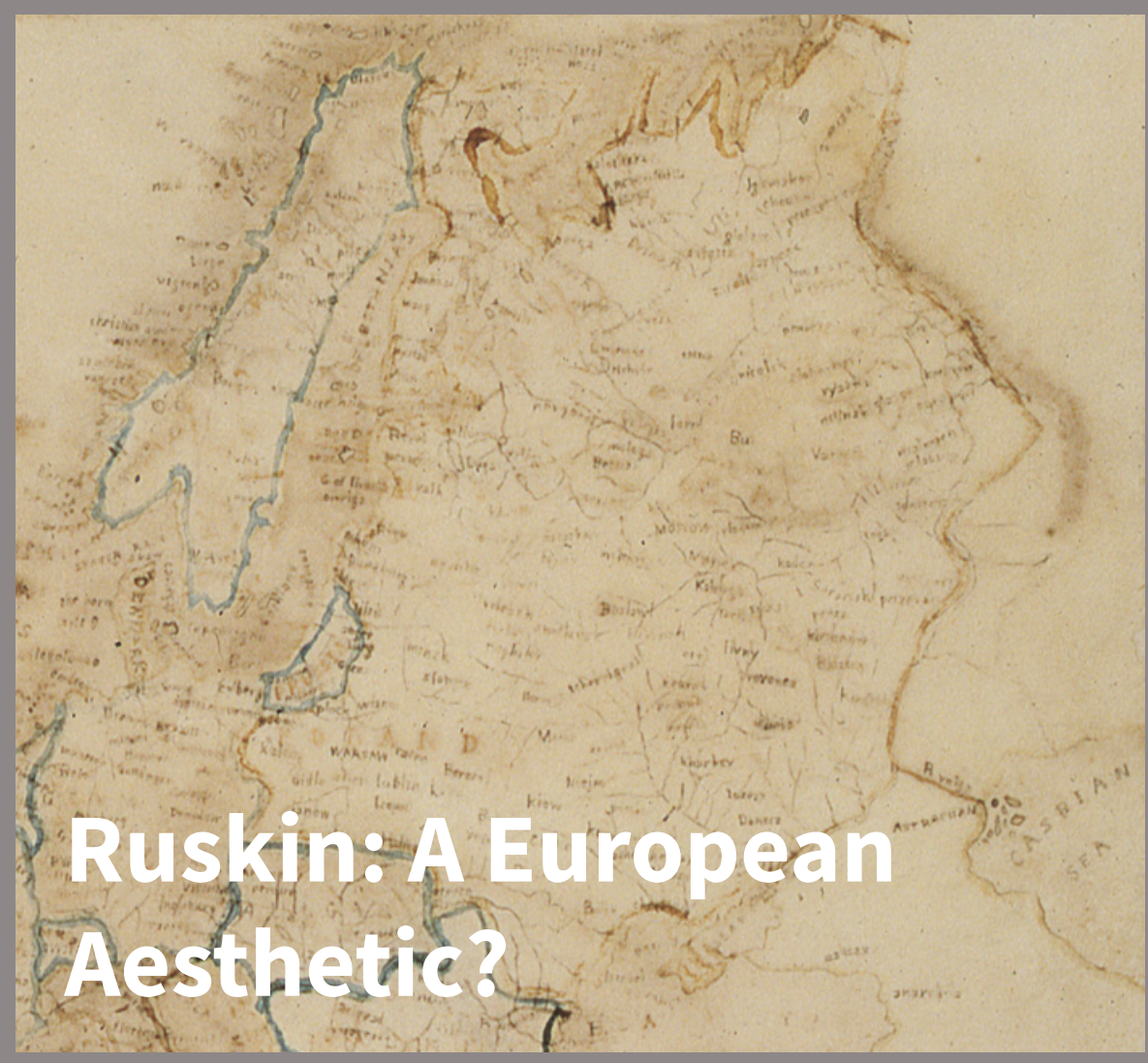





\title{
John Ruskin, un œil européen La photographie, la peinture, l'écriture et l'énigme de la visibilité
}

Pierre-Henry Frangne

Université Rennes 2, France

\begin{abstract}
My analysis of the question of sight, vision, gaze and the visible in John Ruskin's work will consist of three moments: 1) the delimitation of the problem I propose to address, 2) the photographic temptation that was Ruskin's, 3) the question of the innocence of the eye and the pure visibility that makes Ruskin a central maillon in the European aesthetics of the 19th and 20th centuries. The aim is to show how the English writer plays an important role in contemporary thinking about visibility in its artistic, scientific, aesthetic and philosophical implications. Since for Ruskin, «seeing involves the whole man».
\end{abstract}

Keywords John Ruskin. Optical thinker. Photography. European aesthetics. Aesthetics of the 19th and 20th century.

Sommaire 1 Le problème d'un optical thinker. - 2 La tentation photographique. - 3 L'innocence de l'œil et la pure visibilité. - 4 Conclusion.

À la mémoire de Marie-Claude que je ne reverrai plus

\section{Le problème d'un optical thinker}

Limage photographique sur feuille de papier (William Henry Fox Talbot), sur plaque de verre (Nicéphore Niépce) ou sur plaque de métal (Louis Daguerre) est une invention européenne contemporaine de la jeunesse de Ruskin. Créée par un scientifique anglais, physicien et chimiste (Talbot), par un expérimentateur « demi savant » selon l'expression de Gisèle Freund (Niépce) et par un artiste, peintre décorateur (Daguerre) ; annoncée et présentée au monde par l'astronome François Arago lors d'une séance de l'Académie des sciences de Paris le 9 janvier 1839 puis à la Chambre des députés du 3 juillet de cette même année, ${ }^{1}$ l'image photographique est, dans la complexité

1 François Arago, « Rapport à la Chambre des députés », in Rouillé 1989, 36-43.

Fonti, letterature, arti e paesaggi d'Europa | Sources, Literatures, Arts \& Landscapes of Europe 1 ISSN 2724-6620 e-ISSN 2784-8507 
de son invention, de sa nature et de ses opérations optiques, chimiques et mécaniques, à la fois un complet accomplissement et un considérable bouleversement. En 1900, l'année de la mort de Ruskin, Félix Tournachon dit Nadar l'écrit très clairement en commençant Quand j'étais photographe :

Quant le bruit se répandit que deux inventeurs venaient de réussir à fixer sur des plaques argentées toute image présentée devant elles, ce fut une universelle stupéfaction dont nous ne saurions nous faire aujourd'hui une idée, accoutumés que nous sommes depuis de nombreuses années à la photographie et blasés par sa vulgarisation. [...] L'apparition du daguerréotype [...] ne pouvait donc manquer de déterminer une émotion considérable. Éclatant à l'imprévu, au maximum de l'imprévu, en dehors de tout ce qu'on croyait connaître et même le supposable, la nouvelle découverte se présentait assurément, comme elle reste, la plus extraordinaire dans la pléiade des inventions qui font déjà de notre siècle interminé le plus grand des siècles scientifiques - à défaut d'autre vertus. ${ }^{2}$

Puis, après avoir énuméré l'invention de la machine à vapeur, de l'électricité, du téléphone, du phonographe, de la médecine de Pasteur, des découvertes astronomiques de Laplace, il conclut :

Mais tant de prodiges nouveaux n'ont-ils pas à s'effacer devant le plus surprenant, le plus troublant de tous : celui qui semble donner enfin à l'homme le pouvoir de créer, lui aussi, à son tour, en matérialisant le spectre impalpable qui s'évanouit aussitôt aperçu sans laisser une ombre au cristal du miroir, un fris- son à l'eau du bassin ? L'homme ne put-il croire qu'il créait en effet lorsqu'il saisit, appréhenda, figea l'intangible, gardant la vision fugace, l'éclair, par lui gravés aujourd'hui sur l'airain le plus dur ? ${ }^{3}$

Ce qui s'accomplit c'est le programme que s'est fixé l'ensemble de l'art occidental et européen depuis les Grecs et qui se déploie entièrement sous la double exigence de la mimésis et du naturalisme. Ce qui s'accomplit c'est le rêve narcissique d'une image-reflet mais délivrée de sa puissance de mort ; c'est le pouvoir de fabriquer une image mais cette fois-ci si tangible et si reproductible qu'elle n'est plus vouée à l'éphémérité d'une apparition mais, bien au contraire, qu'elle accède à une maîtrise d'elle même comme du monde dont elle capte impersonnellement les aspects infinis.

Cet accomplissement est aussi un stupéfiant bouleversement. Car l'invention d'une nouvelle image - et bientôt d'un nouvel art - désorganise les notions d'art, de création, d'imagination, de représentation, comme ils dérange brutalement le rapport entre les arts et les sciences ainsi que la relation entière entre l'homme et la réalité : face à l'homme en effet, la réalité que la photographie enregistre et authentifie semble désormais lui apparaître dans une présence réduite à celle d'un objet, d'une complète extériorité et d'une pure visibilité. De la nouvelle image photographique, de la nouvelle image-machine quasi perceptive « dépouillée des idées qui l'accompagnent » selon l'expression de Fox Talbot ou « exigeant le moins d'Homme possible » (Valéry en 1939), de ce reflet « qui conserve toutes les empreintes » (Jules Janin en 1839) et qui est « sans code » comme l'écrira bien plus tard Roland Barthes, on peut dire, on doit dire :

Là, ni fantaisie, ni supercherie, la vérité nue. ${ }^{4}$

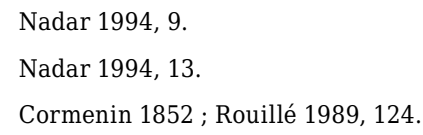


La vérité nue puisque la photographie est l'écriture même de la lumière ; puisqu'elle est aussi héliographie, c'est-à-dire l'écriture même du soleil, ou encore The Pencil of Nature, le crayon même de la nature d'où se sont retirés par son anonymat, son automatisme, son mutisme, sa platitude et sa fixité, l'évocation, la suggestion, l'imagination, le symbolique, le poétique, le rêve et la fiction en sa triple signification de feinte, de monde imaginaire, de travail d'élaboration d'une forme, de façonnage et de manipulation.

John Ruskin fut l'un des premiers - peut-être le premier - à réaliser cette contradiction entre continuité et rupture, entre accomplissement et bouleversement que produisirent ce qu'il a appelé lui aussi, dans Praeterita, " the sun drawings ». ${ }^{5}$ Quand je dis réaliser, je veux renvoyer aux deux sens que le verbe possède en français et qu'il superpose : le sens théorique de réfléchir, de comprendre ou d'entendre d'une part ; le sens poïétique d'effectuer ou de rendre réel d'autre part, puisque Ruskin prit, dès 1846 avec le concours de ses deux valets, John Hobbs (dit George) puis de Frederick Crawley, quelques centaines de daguerréotypes donnant à voir les Alpes ainsi que l'architecture italienne, française et suisse. Devant les pierres naturelles du ' terrain de jeu de l'Europe ' comme devant les pierres culturelles de Fribourg ou de Rheinfelden, de Venise, de Vérone ou de Turin, devant ' les cathédrales de la terre ' du massif du Mont-Blanc comme devant les cathédrales de l'humanité d'Amiens ou de Rouen, Ruskin éprouva - comme les frères Bisson à la fin des années 1850 - la même puissance d'observation, d'enregistrement et de description des images machinales qu'il produisait où qu'il achetait avec enthousiasme durant ses nombreux voyages européens. Et par éprouver, il faut aussi entendre le double sens que le verbe recèle en français : d'une part, le sens de ressentir et d'expérimenter, mais d'autre part, le sens de mettre à l'épreuve de façon critique afin que la photographie fasse sa preuve, afin qu'elle fasse la démonstration ou la vérification de sa valeur, de ses capacités, de ses limites, mais surtout de ses vertus, c'est-à-dire de ses forces.

Cette mise à l'épreuve Ruskin la fit au début de façon admirative à cause de la nouvelle puissance de captation des infinis détails que le daguerréotype permettait ; à cause de sa puissance d'exploration et de notation de la complexité et de l'infinie richesse du visible, que ce visible soit celui des infimes fissures et taches d'une pierre, d'un arbre ou d'un tableau du Tintoret. C'est cette mise à l'épreuve qui fait de Ruskin un auteur moderne (et non un auteur vieilli ou même dépassé comme on l'a trop longtemps considéré) parce qu'il met au centre de lui-même la question de la vue, du regard ou de l'œil et qu'il se constitue comme essentiellement un optical thinker. Cette question de la vue, elle est sûrement de toutes les cultures et de tous les temps. Cependant, et quoiqu'anthropologique et anhistorique, elle prend une importance capitale au sein de la culture scientifique, philosophique et européenne d'origine grecque (socratique et platonicienne) tant cette culture se veut réflexive et théorétique (theoria), ${ }^{6}$ et tant elle entend déployer sa théorie de la connaissance (et même sa théorie de l'art) sur le principe d'une équivalence entre le voir et le savoir, entre la lumière et la pensée, entre l'œil et l'es-

5 Works, 35: 372. Toutes les traductions françaises sont de moi et de mon ami André Hélard. Je le remercie beaucoup de m’avoir aidé dans cette tâche, lui qui s'y entend magistralement dans l'art de rendre si bien en français l'écriture libre et «nonchalante » (au sens de Baldassare Castiglione. Voir en fin d'article) de John Ruskin. Je nous revois tous les deux pendant un long après-midi dans son bureau, ouvrant et refermant à grand-peine les 39 lourds volumes de la monumentale et magnifique Library Edition, soupesant chaque mot de Ruskin et admirant, tous les deux ensemble, l'aspect tout vivant et pénétrant de son style qui fait comprendre et qui fait voir.

6 Works, 29: 576 ; Ruskin 2013, 79.

$45 \quad$ Fonti, letterature, arti e paesaggi d'Europa | Sources, Literatures, Arts \& Landscapes of Europe 1

John Ruskin's Europe A Collection of Cross-Cultural Essays, 43-58 
prit. Quoique grecque et européenne depuis 2500 ans, la question est aussi tout à fait actuelle et contemporaine. À quatre titres :

- parce que, scientifiquement, notre époque multiplie les instruments optiques d'observation et, selon l'expression de son contemporain, poète français et professeur d'anglais Stéphane Mallarmé, « les engins de captation du monde moderne $;^{7}$

- parce que, socialement ou politiquement, «la multitude réclame de voir avec ses yeux $» ;^{8}$

- parce que, artistiquement et esthétiquement, la photographie y acquiert le rôle de modèle pour la littérature (de Flaubert à Raymond Roussel et à Proust) et les arts plastiques contemporains ( $\mathrm{Du}$ champ, Warhol, Stella) ;

- parce que, philosophiquement enfin, notre pensée s'attache à comprendre d'abord l'expérience concrète, la vie ordinaire, la phénoménalité par laquelle le monde nous est présent et par laquelle nous sommes présents à lui.

Sous tous ces aspects, Ruskin se met à l'origine de nousmêmes en ce qu'il considère que «le monde est ce que nous voyons et que, pourtant, il nous faut apprendre à le voir » comme l'écrit Merleau-Ponty à l'entrée du Visible et de l'invisible au tout début des années $1960 .{ }^{9}$ Mais ce qui est crucial et intéressant chez Ruskin est que cette position théorique et historique il la prend de façon étonnée, de façon critique, distanciée et problématique au point qu'il abandonna la photographie après 1858 au profit exclusif du dessin et de la peinture, et aussi de l'écriture. Qu'est-ce qui se joue alors dans ce moment de dix années de ce qu'il faut bien appeler une traversée de la photographie ? Qu'est-ce qui se joue dans cette aventure et cette hésitation, puis, finalement, dans ce renoncement?

Mon hypothèse est la suivante. Par delà ou en deçà de cette hésitation et de cette tension sises au creux de la vue (et qui se révélèrent à lui dans sa relation à la photographie), mais qu'il a apprises d'abord devant Turner comme Merleau-Ponty l'apprendra plus tard devant Cézanne (comme Baudelaire devant Delacroix, comme Mallarmé devant Manet), John Ruskin «ne célébra jamais d'autre énigme que celle de la visibilité $»{ }^{10} \mathrm{Or}$, cette énigme que Turner et l'image photographique lui permettent de célébrer Ruskin voulut la mettre au cœur de son écriture au point de devenir peut-être ce que Théophile Gautier appelait, ${ }^{11}$ en se nommant lui-même en 1843, un "daguerréotype (ou daguerréotypeur) littéraire ». Cela ne voudrait-il pas dire qu'il faille pour Ruskin reprendre à la photographie son bien : le bien de la photographie mais aussi le bien de l'écriture qui est le sien propre et que la photographie a d'abord volé ? Quel est le mystérieux pouvoir de la photographie alors ? Quelle est aussi selon lui, sa limite qui fait qu'elle doit être dépassée, non pour être détruite et complètement oubliée, mais pour être comme reprise, pour être sauvée, rédimée ou exhaussée, à un niveau supérieur?

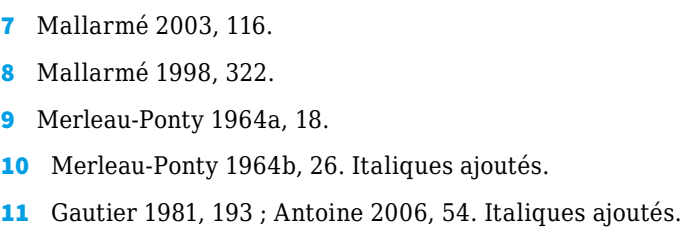




\section{La tentation photographique}

Si Ruskin a vécu la tentation photographique, c'est de toute évidence parce qu'il est fondamentalement un penseur du voir et que toute son écriture est orientée par la nécessité de voir et par le devoir de voir :

Tous les grands hommes voient ce qu'ils peignent avant de le peindre - le voient d'une manière parfaitement passive - et ne pourraient s'empêcher de le voir même s'ils le voulaient ; que ce soit avec l'œil de l'esprit ou celui du corps ne change rien... ${ }^{12}$

\section{Ou encore :}

Il est toujours faux de dessiner ce que l'on ne voit pas. Cette loi est absolue. Cependant, il y a des gens qui ne voient que des choses qui existent, et d'autres qui en voient qui n'existent pas, du moins apparemment. Et s'ils voient vraiment ces choses non apparentes, ils sont parfaitement en droit de les représenter. ${ }^{13}$

Sans doute y a-t-il plusieurs vues ou plusieurs visions (celle de l'œil intérieur - theoria - ou celle de l'œil charnel - opsis - comme disait Plotin puis les philosophes médiévaux et même les romantiques allemands) ; sans doute y a-t-il plusieurs regardeurs. Mais, qu'ils soient voyants (comme Turner, des choses qui existent) ou bien visionnaires (comme Blake, des choses qui n'existent pas), les hommes et les artistes sont comme des « télescopes $»^{14}$ plus ou moins puissants, oscillant sans cesse entre la nécessité due à leur nature anthropologique de voir et l'exigence morale qui en découle, celle de donner à voir. À des étudiants oxoniens, Ruskin a dit :

Croyez-moi messieurs, vous ne développerez pas votre faculté de voir les montagnes par vanité, la curiosité ni l'amour de l'exercice physique. Cette faculté ne dépend que d'un instrument à affûter : la vue. ${ }^{15}$

Et l'on peut considérer que toute la vie de Ruskin fut vouée à cette pratique de l'affûtage qu'il l'exerça devant les spectacles de la nature (et de la montagne spécialement) ou devant les spectacles des arts en tant qu'ils sont eux-mêmes visuels, en tant qu'ils voient, qu'ils s'offrent à la vue et qu'ils sont des instruments qui font voir. Comme Baudelaire son contemporain, Ruskin a eu en conséquence la passion des images. Il aurait pu dire comme dans « Mon cœur mis à nu » : «Glorifier le culte des images (ma grande, mon unique, ma primitive passion ${ }^{16}$ et, comme l'écrit Proust, l'iconographie - l'écriture des images, le dialogue du texte et des images -, est toujours une heureuse « idolâtrie ». Pourquoi heureuse ? Selon Proust, pour trois raisons :

a. d'abord, parce que cette idolâtrie est en perpétuelle combat contre elle-même au nom d'un amour plus grand que celui des images et qui est l'amour du monde et de la vie, l'amour de ce qui est réel et qui est admirable ;

12 Works, 5: 114. Italique ajouté.

13 Works, 6: 28.

14 Works, 36: 213

15 Works, 26: 103. Rapporté par Kenneth Clarke dans son introduction à Praeterita et cité par Shama 1999, 578.

6 Baudelaire 1975, 701

$47 \quad$ Fonti, letterature, arti e paesaggi d'Europa | Sources, Literatures, Arts \& Landscapes of Europe 1

John Ruskin's Europe. A Collection of Cross-Cultural Essays, 43.58 
b. ensuite, parce que les images ou les vues que prennent les artistes et lui-même sont des instruments de connaissance de cette réalité qui est étonnante (au sens philosophique, d'admirable mais aussi d'inquiétant et de problématique), qui ne va pas de soi, qui, par son opacité, résiste à son observation et son intelligence, et qui par-là, est un monde extérieur, une contrée (au sens étymologique) à conquérir, à explorer dans sa mystérieuse étrangeté ou sa muette altérité ;

c. enfin, l'idolâtrie ruskinienne est heureuse parce que, non seulement, elle s'oppose à toutes les projections et séductions narcissiques des images que forge le moi intérieur, non seulement elle refuse de retrouver dans le monde ce que le moi intérieur a commencé par y mettre afin de se voir lui-même, mais parce que - dans cette effacement ou dans cette "vaporisation » pour utiliser encore une fois un mot baudelairien ${ }^{17}$ - cette idolâtrie est le principe d'une expulsion au dehors, d'une aliénation et d'un constant dépaysement, c'est-à-dire d'un voyage et même d'une aventure au sens strict de ce qui incessamment advient. Proust écrit admirablement ce qui est bien un œil européen qui aurait le pouvoir, par décentrements successifs, de devenir l'œil de la terre entière :

Car la pensée de Ruskin n'est pas comme la pensée d'Emerson par exemple qui est contenue tout entière dans un livre, c'est-à-dire un quelque chose d'abstrait, un pur signe d'ellemême. L'objet auquel s'applique une pensée comme celle de Ruskin et dont elle est insé- parable n'est pas immatériel, il est répandu çà et là à la surface de la terre. Il faut aller le chercher là où il se trouve, à Pise, à Florence, à Venise, à la National Gallery, à Rouen, à Amiens, dans les montagnes de la Suisse. Une telle pensée qui a un autre objet qu'ellemême, qui s'est réalisée dans l'espace, qui n'est plus la pensée infinie et libre, mais limitée et assujettie, qui s'est incarnée en des corps de marbre sculpté, de montagnes neigeuses, en des visages peints, est peut-être moins divine qu'une pensée pure. Mais elle nous embellit d'avantage l'univers, certaines partie nommées, de l'univers, parce qu'elle y a touché, et qu'elle nous y a initiés en nous obligeant, si nous voulons les comprendre, à les aimer. / Et ce fut ainsi, en effet ; l'univers reprit tout d'un coup à mes yeux un prix infini. ${ }^{18}$

C'est ce mode exploratoire et comme cynégétique de la vue en perpétuel éveil ( « il faut aller chercher l'objet de la pensée là où il se trouve », il faut le débusquer donc, pour le prendre et le mettre sous les yeux) qui est constant chez Ruskin et qui l'amène à parcourir l'Europe mais surtout à ne pas séparer :

la beauté des cathédrales du charme de ces pays d'où elle surgirent, et que chacun de ceux qui les visitent goûte encore dans la poésie particulière du pays et le souvenir brumeux ou doré de l'après-midi que Ruskin y a passé. [...] Ce n'était donc point seulement dans ses dessins que Ruskin mettait les églises au bord des rivières et qu'il associait la grandeur des cathédrales gothiques à la grâce des sites français. Et le charme in- 
dividuel, qu'est le charme d'un pays, nous le sentirions plus vivement si nous n'avions pas à notre disposition ces bottes de sept lieues que sont les grands express. ${ }^{19}$

Le dessin ou la description d'un motif d'architecture, d'une cathédrale, d'une pierre ou d'un sommet de montagne ne sont jamais "des exemples abstraits ${ }^{20}$ coupés, ni du paysage comme espace environnant ou comme paysage (urbain, naturel, social, historique), ni du moment temporel singulier où le dessin et la description sont faits c'est-à-dire sont pris : «I got a view » écrit souvent Ruskin à propos de ses dessins réalisés dans la journée à Chamonix..$^{21}$

Telle est la logique photographique et telle est la tentation ruskinienne de la photographie qui tient, non sans ambiguïté, dans ce texte de Praeterita :

Ce fut probablement durant mes derniers jours à Oxford que M. Liddell, le Doyen actuel de Christ Church, me parla des expériences originales de Daguerre. Mes amis parisiens m'obtinrent les meilleurs échantillons de ses résultats; et les plaques qu'ils m'envoyèrent à Oxford furent certainement les premiers exemples jamais vus là-bas de dessins tracés par le soleil, et, je crois, les premières envoyées en Angleterre.

Étant à l'époque totalement insoucieux de la précision des détails, je ne vis dans le Daguerréotype rien qui pût m’aider, ou m'inquiéter; et je ne cher- chai pas à en savoir davantage, jusqu'au jour où, justement à Venise, je trouvai un artiste français produisant des petites plaques (environ quatre pieds carrés) d'un brillant exquis, contenant, sous une lentille, le Grand Canal ou la Place Saint Marc comme si un magicien avait réduit la réalité pour l'emporter bien loin dans un pays enchanté. Ces petits bijoux de peinture coûtent un napoléon pièce ; mais pour deux cents francs j'achetai le Grand Canal depuis la Salute jusqu'au Rialto ; et j'en fis un paquet que j'expédiai avec un sentiment de triomphe irréfléchi. ${ }^{22}$

La logique photographique tient rapidement en 4 points.

1. Elle est d'abord celle d'une captation, d'une capture et de l'enregistrement d'une trace du réel qui est là, authentifié et présenté dans une transparence de la représentation tendant à s'effacer au point de donner le sentiment que, dans une photographie, c'est le soleil lui-même qui dessine les aiguilles de Chamonix ${ }^{23}$ ou qui fait le portrait du Cervin (le premier sun portrait de l'histoire). ${ }^{24}$ C'est le Cervin et le Grand canal eux-mêmes que nous tenons!

2. La logique photographique est en conséquence celle d'une exploration du réel, de sa transformation en documents ou en archives « dignes de confiance » et qui permettent de "vérifier les dessins ${ }^{25}$ et même de s'en passer comme Ruskin le dit par exemple dans The Stones of Venice : le daguerréotype donne « la possibilité

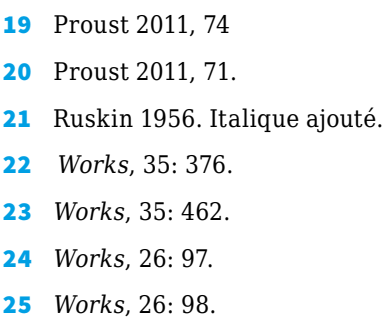

49 Fonti, letterature, arti e paesaggi d'Europa | Sources, Literatures, Arts \& Landscapes of Europe 1 
d'obtenir une certaine vérité dans la représentation des choses matérielles et tangibles [...] qui se trouvent maintenant entre les mains de tous les hommes pour ainsi dire sans effort $» .{ }^{26} \mathrm{Il}$ permet de réaliser, chez Ruskin comme chez tous les européens du XIXe siècle, l'inventaire du monde : « autant de traces mémorables, memoranda, des faits de la nature que souhaite le lecteur $» .{ }^{27} \mathrm{~A}$ cet égard, et comme tous ses contemporains, Ruskin est frappé par la puissance de monstration des mille détails que la photographie inscrit dans l'image : "Le plus grand service qui puisse être rendu aujourd'hui à l'architecture, c'est que le détail des cathédrales (Vérone, Venise, Florence, Notre-Dame de Paris) soient rendu si méticuleusement par les moyens de la photographie $» .{ }^{28}$ Alors que le grouillement du détail inquiète beaucoup toute son époque - parce qu'il produit « l'émeute » (Baudelaire) ou « l'ennui » (Delacroix) d'une image chaotique, sans choix ni art, qui transgresse les règles de la beauté et même de la visibilité, Ruskin admire la précision « pierre par pierre, sculpture par sculpture » des édifices dont il voit le moindre fragment, «les fractures et les taches » "sans erreur dans les proportions », c'est-à-dire dans l'ensemble.

3. Pour que le lecteur possède le monde « entre les mains », il faut le parcourir avec la conscience, non seulement d'une proximité avec lui, mais surtout de notre immersion et même de notre enga- gement à l'intérieur de lui. Pour le photographe et pour le regardeur des photographies qui font tous les deux exister les images sur le mode de la démultiplication et même de la dispersion (alors que la peinture existe sur celui de l'unicité et de l'originalité), le monde est beaucoup plus qu'un paysage vu à distance. Il est, comme l'écrivait Proust, un milieu auquel nous sommes présents, au cœur duquel nous existons. La nature indicielle de la photographie fait exister l'image sur un mode métonymique et non sur un mode métaphorique de transport, c'est-à-dire de construction et d'interprétation. C'est en ce sens que la photographie est un art du prolongement : prolongement des choses et des corps matériels et sensibles dont elles sont faites ; prolongement de leur présence et de leur contact; émanation de leur être même auquel ils sont profondément arrimés. Et c'est ce prolongement métonymique que Ruskin envisage quand il note que l'acte photographique est celui d'un très grand rapprochement et d'une miniaturisation de la réalité ${ }^{29}$ permettant ce que l'on pourrait désigner aujourd'hui comme sa mise en abyme ${ }^{30}$ au sein de ce que Claudel appellera « un carré durable, portatif, quelque chose désormais et pour à jamais à notre disposition $»{ }^{31}$ Ruskin compare la photographie de paysage à un jouet (a toy). Ce jouet devient cependant un sérieux et «précieux document his-

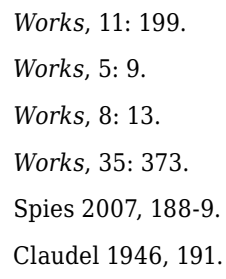


torique » quand ce qui est photographié est un monument gothique. ${ }^{32}$

4. Et puis, la photographie repose sur une dernière opération portant, non sur l'espace, mais sur le temps. La photographie est un art de la surprise, de l'inattendu et donc de la nouveauté. À trois titres principaux : a) sa précision automatique fait surgir des détails que l'on ne verrait pas sans elle; b) comme elle garde nécessairement la trace du moment de sa prise, elle n'est pas seulement le memorandum des faits de la nature ; elle est surtout la trace fixée de l'apparition fulgurante et jaillissante ${ }^{33}$ de ces faits sur le regardeur. C'est cette apparition fulgurante qu'atteste l'écriture de Ruskin constamment (comme celle de Gautier d'ailleurs) grâce aux « voilà ! », aux « voici! », à toutes les exclamations spontanément et librement notées devant le surgissement instantané d'un objet; devant ce qu'il faut bien appeler une épiphanie (au sens païen mais aussi au sens chrétien) devant l'instant d'une rencontre. c) Comme l'enregistrement photographique est impartial et sans choix, il fait monter à la surface de l'image l'inattendu du prosaïque et du hasard que l'art classique a tendance à occulter quand il n'est pas celui du Tintoret replon- geant sans cesse la présence éternelle du sacré dans la temporalité événementielle de la laideur du banal. Art de l'événement, la photographie fixe des moments et des êtres singuliers dans la nouveauté de l'instant de l'émergence qui est celle de leur être et de leur apparaître. Cette nouveauté a quelque chose de solaire, de glorieux, de radieux bien sûr. Elle a aussi quelque chose de ténébreux et de tragique dans la mesure où l'instant nouveau et le détail dont elle fixe l'apparition, ils ne reviendront plus, emportés qu'ils sont par le passage du temps. L'œil photographique nous jette au visage à la fois le temps de la génération et le temps de la corruption : de la corruption de Venise " cité moribonde et magnifique dans sa decrépitude $»^{34}$ ou "fantôme sur les sables de la mer, dans l'ultime période de son déclin $»^{35}$ et qui " est en train de disparaître, à peu près aussi vite qu'un morceau de sucre dans une tasse de thé » $;^{36}$ de la corruption moins apparente mais tout aussi certaine des montagnes soumises à leur statut de ruines. En ce sens, le black art qu'est la photographie, cette « noble invention quoiqu'on en dise » selon le mot d'octobre 1845 nous met sous nos yeux complètement décillés « les cadeaux de la poussière ». ${ }^{37}$ 


\section{$3 \quad$ L'innocence de l'œil et la pure visibilité}

La pratique photographique de Ruskin s'est arrêtée vers 1858, l'auteur corrigeant finalement son sentiment de triomphe irréfléchi. À la réflexion donc, Ruskin s'aperçoit des limites de l'image machinale. Affinant et exerçant son regard, il s'aperçoit que la photographie est souvent trop sombre dans le rendu des couleurs (le rouge, le bleu). Dans Modern Painters IV, il note que si « la photographie rend les subtilités de forme qu'une main humaine ne peut accomplir », " elle exagère les ombres ou perd les détails dans la lumière. Elle manque les subtilités des nuances de la nature que Turner a cherché à rendre $» .{ }^{38}$ Mais surtout :

les photographies ne l'emportent pas sur l'art, le bon art, car ce qu'elles ont en commun avec la nature c'est un caractère parcimonieux qui fait qu'elle ne vous donne rien de valable si vous n’avez pas travaillé pour. Car l'art c'est le travail humain réglé par le dessein [design] humain [human labor by human design]. Et ce design qui est l'évidence d'un intellect dont l'action consiste à choisir et à disposer est la part essentielle de ce travail. L'art ne peut être remplacé par une machine [a mechanism]. Les photos ont une valeur inestimable pour enregistrer les faits et les dessins des grands maîtres mais, dans une œuvre photographiée, vous ne verrez rien de plus que ce que contient l'œuvre; dans une photographie de dessin, vous ne verrez aucune bonne vérité que celle que vous verriez dans les choses mêmes et qui vous paierait le prix de votre attention. Les photos de paysage ne sont pas vraies ; elles sont un paysage gâté, la nature ratée [spoiled]. Il y a plus de beauté dans un talus de chemin que dans tous les papiers noircis par le soleil que vous pouvez collectionner dans votre vie. Allez regarder un paysage réel et prêtez-lui toute votre attention. ${ }^{39}$

Bref, l'évidence ou la transparence du daguerréotype réapparaissent dans la brûlure et dans la noirceur de la lumière qui faisaient déjà dire à Ruskin que Canaletto ne " professe rien d'autre qu'un daguerréotypisme coloré » alors même « qu'il a vu des daguerréotypes dans lesquels chaque figure et rosette, chaque lézarde, tache et fissure est reproduite à une échelle d'un pouce, là où Canaletto les a peintes à une échelle de trois pieds ${ }^{40}{ }^{40}$

Ruskin se rangerait-il alors du côté des thèses de Charles Baudelaire dans son Salon de 1859, et son expérience photographique serait-elle devenue vaine ? La critique de la photographie, contemporaine de son éloge, au sein de ce texte de 1843, permet de répondre par la négative. C'est dans cette hésitation et dans le suspens même qu'elle suppose que se poste la conception ruskinienne de la vision. Celle-ci obéit à l'idée que dira Heinrich Wölfflin en 1915 selon laquelle « le voir inclut l'homme tout entier $»^{41}$ Celle-ci obéit au principe que nous venons de voir à l'œuvre : celui d'une oscillation entre la transparence et l'obstacle, l'immédiateté et la médiation, la nature et l'artifice, la lumière et l'obscurité, le visible et l'invisible, oscillation qui est, pour la modernité artistique, critique et philosophique, constitutive de la vision envisagée d'un point de vue radicalement esthétique au sens grec d'aisthétique. 
L'oscillation princeps est celle qui consiste à dire, comme Schopenhauer, que "l'artiste nous donne des yeux pour regarder le monde ${ }^{42}$ ce qui en langage ruskinien s'écrit : "Au grand peintre d'imagination, nous devons dire : viens te mettre entre cette nature et moi - cette nature qui est trop grande, trop merveilleuse pour moi ; modèle-la, interprète-la pour moi ; laisse-moi la voir par tes yeux, entendre par tes oreilles $\gg .{ }^{43}$ Mais dans le même temps où l'artiste est un médiateur, cette médiation nous délivre paradoxalement de toutes médiations par un regard pur, immédiat, libre ; un «premier regard » qu'il faut entendre en plusieurs sens superposés : a) un regard neuf, nouveau qui n’a jamais été posé : original ; b) un regard régressif et dépollué ou purifié des constructions et des préjugés de la culture : originaire ; c) un regard spontané sans crier gare, en commençant ; comme quand on dit « au premier regard » : originel.

L'artiste est donc un « écran transparent » selon la célèbre comparaison de Zola dans sa lettre à Valabrègue du 18 août $1864 .{ }^{44} \mathrm{Et}$, comme depuis le romantisme allemand d'un Runge, d'un Carus ou d'un Friedrich, le tableau n'est plus une fenêtre ouverte sur l'histoire selon l'expression d'Alberti (avec son histoire, ses histoires, ses allégories et ses symboles), mais sur la « création » (Zola) ou sur la nature, ce qui fait que la peinture moderne repose sur le primat du paysage : «les abstractions périssent, tout est plus aérien et plus léger que jadis, tout aspire au paysage ${ }^{45}{ }^{45}$ C'est la clarté et l'immédiateté de la vision qui font l'originalité du tableau ; sa virginité, comme retour à « l'enfance de l'art », à une période « naïve » qui fait des œuvres authentiques « capables d'en revenir au monde originel de l'innocence ${ }^{46}$ aux " inclinations spécifiques de l'enfant », à une vision émancipée des conventions et des écoles. Ruskin est de plain-pied dans cette tradition moderne que l'on retrouve à l'époque de l'impressionnisme et jusqu'au XXe siècle d'un Matisse. Si l'artiste est un « tempérament » par l'intermédiaire duquel passe et se réfracte la réalité, si le regard artistique et esthétique suppose encore une conversion (épistrophè) de l'esprit, l'œuvre d'art et le regard que l'artiste possède et qu'il offre au spectateur ne sont pas une fuite dans un monde différent ou dans la contemplation de choses différentes ou dans l'exercice de facultés différentes. Tout est là devant nous, dans notre perception, notre sentiment et notre attention originaires. Tout est là mais perdu, oublié par et dans le cours de nos vies ordinaires. Le paysage peint c'est la profondeur de l'évidence, la vue de ce que nous ne cessons pas de regarder mais que nous ne voyons pas au sein de notre affairement, de la recherche de ce qui nous est utile ou de significations que l'éducation nous a apprises. Mallarmé, en 1874 et devant la peinture de son ami Manet, ne dira pas autre chose. L'Olympia est la peinture d'un nu, " non le nu conventionnel de la tradition $»,{ }^{47}$ mais le nu d'une femme nue et aussi la nudité de la peinture elle-même qui se montre, à même « les couleurs et les onguents »: une vue et non une vision; une vue de ce qui est dans le monde, et non une vision de qui est au-delà du monde. Manet, pour Mallarmé, est comme Turner pour Ruskin : non un visionnaire, mais

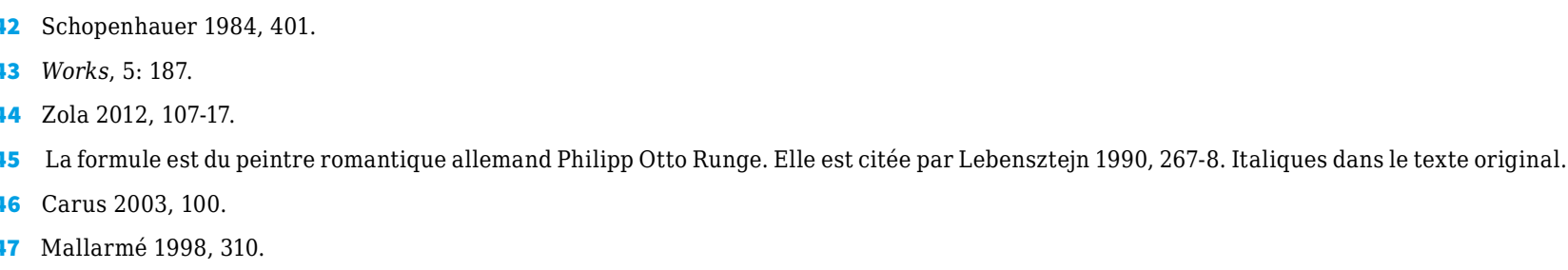


un artiste des temps modernes marqué par «la sincérité, la simplicité et d'un charme comme d'enfance $»^{48}$ Mallarmé écrit :

À cette heure critique pour l'espèce humaine où la nature désire fonctionner pour elle-même, elle exige de certains de ses amants - des hommes nouveaux et impersonnels, en communion directe avec l'esprit de leur temps - de dénouer les entraves de l'éducation et de laisser la main et l'œil agir à leur guise, afin de se révéler par leur entremise. ${ }^{49}$

L'originalité du peintre c'est « d'abdiquer sa personnalité ${ }^{50}{ }^{50}$ 'est « l'absence [paradoxale] de toute intrusion du moi dans l'interprétation ${ }^{51}{ }^{51}$ c'est « cette isolation en soi-même $»^{52}$ lui permettant d'avoir un œil qui « doit oublier tout ce qu'il a vu ailleurs et réapprendre à partir de ce qui le confronte. Il doit rompre avec la mémoire, ne voyant que se qui s'offre au regard comme pour la première fois $\gg{ }^{53}$ C'est ce que réclame Ruskin dans les Elements of Drawing de 1857 :

La perception des Formes solides relève entièrement de l'expérience. Nous ne voyons pas autre chose que des couleurs plates [flat colours] ; et ce n'est seulement par une série d'expériences que nous découvrons qu'une tache de couleur, noire ou grise, indique le côté sombre d'un corps, ou qu'une teinte pâlissante signale l'éloignement de l'objet sur lequel elle apparaît. Toute la force de la technique picturale dépend de la possibilité pour nous de retrouver ce que l'on pourrait nommer l'innocence de l'œil ; c'est-à-dire une sorte de perception enfantine de ces taches plates et colorées, vues simplement en tant que telles, sans aucune conscience de leur signification - comme un aveugle les verrait si la vue lui était subitement rendue. [...] Nous croyons toujours voir ce que nous ne faisons que savoir, et nous n'avons aucune notion de l'aspect réel des signes que nous avons appris à interpréter. Très peu de gens réalisent que l'herbe au soleil est jaune. Or les artistes accomplis se sont toujours rapprochés autant que possible de ces conditions de la vue enfantine. ${ }^{54}$

Le texte, si célèbre, n'est pourtant qu'une note de la lettre 1 et, en plus, à l'intérieur de cette lettre, il n'est qu'un exercice que le lecteur peut sauter s'il le veut, mais qui lui est simplement conseillé de lire s'il est « incrédule ou curieux ». Le texte n'exprime donc pas une doctrine et encore moins une « théorie » comme l'écrit Ernst Gombrich au chapitre 9 de L'art et l'illusion intitulé « Analyse de la vision artistique ${ }^{55}$ Son statut est bien plus prudent et modeste que celui de la critique mallarméenne ou de la philosophie bergsonienne des années 1889-1899 développant l'idée selon laquelle « l'art entre en communication immédiate avec les choses et avec nous-mêmes » parce qu'il soulève « le voile épais de l'utilité », voile qui a été

48

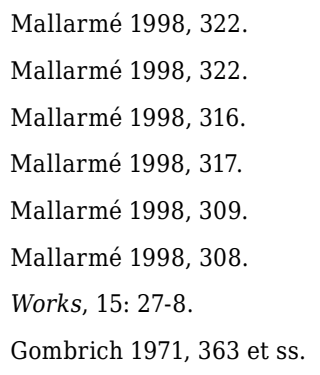


jeté sur les choses par notre intelligence et le langage, asservis qu'ils sont à la nécessité d'agir et de vivre. ${ }^{56} \mathrm{Le}$ but de l'art selon Bergson c'est de nous ramener à une vision virginale, immédiate " détachée » des intérêts, des symboles, des généralités que la société nous apprend pour son propre fonctionnement et pour le nôtre. La vocation de l'art est bien d'en revenir aux données immédiates de la conscience qui ne sont jamais immédiatement données mais qu'il faut apprendre ou réapprendre tant nous avons été institués et transformés. Alors, la mission de la peinture et du dessin est celle d'une heureuse régression, d'une heureuse déconstruction, dirions-nous, dans un langage contemporain, celui d'un oubli comme «faculté active », cette faculté que revendique aussi Nietzsche dans une toute autre perspective à l'entrée de la seconde dissertation de la Généalogie de la morale ${ }^{57}$ Et cette mission serait de voir ; de voir véritablement ce qui suppose l'apprentissage d'une technique négative ou soustractive de désencombrement. Ruskin écrit :

Je crois que la vue est une chose plus importante que le dessin ; et je préférerais enseigner le dessin grâce auquel mes élèves apprendraient à aimer la nature, plutôt que d'enseigner le regard sur la nature qui leur apprendrait à dessiner. ${ }^{58}$

Il s'agit donc d'apprendre à voir, à aimer voir, mais surtout à aimer la nature ; à aimer la nature en la voyant et à la voir en la dessinant. Le dessin et la peinture ne sont qu'un instrument pédagogique et non une fin en soi. Ils ne sont qu'un processus susceptible de progrès, d'affûtage, c'est-à-dire d'affinement de la vue du monde, de ce monde dont Konrad Fiedler dit dans les années 1880 « que l'artiste peint en le voyant et voit en le peignant $»{ }^{59}$ Ce processus est difficile. Il est une conquête de ce que Benedetto Croce, lisant et critiquant Fiedler ${ }^{60}$ (le formalisme de Fiedler), ${ }^{61}$ appellera « la pure visibilité » que semble aussi chercher Ruskin. Dans le texte de la « childish perception », Ruskin montre que le regard par la peinture apprend à voir sans savoir. Il apprend aussi à voir sans toucher. Proust cite cette anecdote narrée par Ruskin lui-même selon laquelle Turner aurait répondu à un officier de marine protestant contre l'absence de sabords dans une peinture de navire : «Oui, je le sais, mais mon affaire est de dessiner ce que je vois, non ce que je sais $\gg{ }^{62}$ Cette anecdote renvoie cette autre fort connue de la vie de Gustave Courbet demandant à son ami Francis Wey (nous sommes vers 1869) d'aller regarder « là-bas ce que je viens de faire. Je n'en sais rien du tout ». Francis Wey (membre de la mission héliographique et l'un des premiers critiques de la photo) écrit que «c'était un bloc grisâtre dont, à distance, je ne me rendis pas compte; mais jetant les yeux sur la toile, je vis que c'était un massif de fagots. “ je n'avais pas besoin de le savoir, dit Courbet, j'ai fait ce que j'ai vu sans m'en rendre compte" $\gg .{ }^{63}$

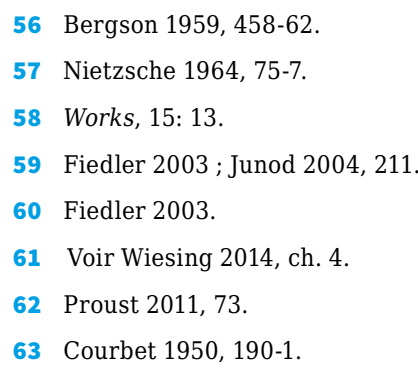


Qu'il soit Turner ou Courbet, le peintre ne sait pas ce qu'il peint au sens d'un savoir théorique identifiant un objet en le séparant de son apparaître. Selon Ruskin, il voit comme un aveugle-né qui recouvre la vue et découvre le monde pour la première fois. Se référant cette fois implicitement au problème de Molyneux dont s'empara John Locke, ${ }^{64}$ Berkeley et Diderot, Ruskin soutiendrait l'idée selon laquelle l'aveugle de naissance qui distinguait un cube et un globe au toucher, ne sera pas capable de le faire à la simple vue récemment retrouvée parce qu'il n'aurait pas encore appris par l'expérience qu'un globe est « un cercle plat diversement ombragé, avec plusieurs niveaux de luminosité et de brillance parvenant à l'œil $»{ }^{65}$ De même que la vision est un acte spécifique et n'est donc pas un toucher, de même l'ombre possède une couleur propre et n'est donc pas, pour le regard enfantin du peintre, une teinte plus sombre qui aurait en elle plus de noir : c'est une autre couleur qualitativement distincte de toutes les autres.

\section{Conclusion}

Tel est alors et sans doute la vertu et la limite du regard photographique qui tenta Ruskin et qu'il abandonna. La vertu est de donner à voir une vue que l'expérience et l'histoire n'abîment pas dans des conventions, des canons ou des stéréotypes. La vertu est de permettre de voir sans regarder, si par regarder on entend re-garder, c'est-à-dire voir ce que l'on a déjà vu, et surveiller ce que l'on voit par des catégories conceptuelles qui permettent de savoir, de savoir d'avance, avant d'avoir vraiment vu. ${ }^{66} \mathrm{La}$ vertu du regard photographique est qu'il est idiot, un peu comme celui d'un enfant sauvage, celui de Gaspar Hauser ou de Victor de l'Aveyron, un peu comme celui d'un fou ou bien d'une bête qui en reste à la notation plutôt qu'à la représentation, à la matérialité contingente de ce qui est plutôt qu'à la spiritualité harmonisée de ce qui devrait être. Pour le photographe, pour l'œil de son objectif, la nature « ex-iste » au sens où elle se tient-là, devant nous, grandiose ou banale, mais donnée une fois pour toutes et qui nous confère la tâche infinie de la voir et de la comprendre parce qu'elle est notre séjour.

Mais la photographie possède cependant un vice congénital qui fait qu'elle gâte sa découverte. Son voir est en effet machinal et ne relève donc, ni du travail ni du design humain au double sens de dessein et de dessin. Dans la mesure où pour Turner, pour Manet, pour Courbet et pour Ruskin lui-même, il ne s'agit de voir avant de peindre ou avant d'écrire, il s'agit pour eux tous de voir en peignant et en écrivant, dans le même temps de ces activités et dans le même geste qu'elles supposent. Promener son daguerréotype dans toute l'Europe ce n'est donc que prendre des vues, ce n'est que poser par-ci par-là un œil, qu'avoir un œil (un œil mécanique, optique et chimique) avec soi. Mais l'ambition ruskinienne de l'art est plus haute et plus difficile : il s'agit d'être un œil. D'être un œil dans le mouvement même - énergétique, laborieux et labile - de la main et de la pensée. C'est ce que l'image achéiropoétique de la photographie ne saurait accomplir. 
Il lui manque ce que Ruskin appelle « the skill », l'habileté, la dextérité ${ }^{67}$ et que Manet indique d'une formule dont se souvient Mallarmé :

l'œil, une main... Cet œil [...] gardait naguère l'immédiate fraicheur de la rencontre, aux griffes d'un rire du regard [...]. Sa main - la pression sentie claire et prête énonçait dans quel mystère la limpidité de la vue y descendait. ${ }^{68}$

Selon Ruskin, l'énigme de la visibilité qui fut toute sa vie durant son principal objet, son principal souci, ne sera pas partiellement éclaircie par la seule imitation ou par la seule reproduction du visible. Il ne s'agit pas seulement pour lui de prendre le visible comme le fait la photographie que la nature chasseresse met toujours en éveil, c'est-à-dire sans cesse aux aguets de toutes les occasions qui s'offrent au marcheur. Il s'agit, plus fondamentalement, de « rendre visible » selon la fameuse formule de Paul Klee dans son Confession créatrice de 1920, c'està-dire de travailler à montrer la genèse de la première fois d'un voir sans revoir, d'un voir dans la pure visibilité que les artistes et théoriciens du XIXe et du XXe siècles européens ont essayé de mettre en œuvre et d'explorer.

Or, ce voir " est un avoir à distance ${ }^{69}$ une possession qui n’a rien de tactile parce que si c'était le cas elle serait celle d'un aveugle. La main du peintre n'est pas celle qui tâtonne ; elle est celle qui fait et qui, en faisant, donne existence à distance comme le dit Merleau-Ponty à « la voluminosité du monde ». ${ }^{70}$ Pour ce faire, le geste manuel du peintre est apte à rendre le temps des choses que la photographie immobilise de l'extérieur en détruisant « le dépassement, l'empiétement, la 'métamorphose' du temps que la peinture rend visible au contraire ${ }^{71}{ }^{71} \mathrm{Ce}$ sont cette voluminosité à distance et ce temps énergétique intérieur des choses qui frappent Ruskin devant Long Ships Lighthouse, Land's End de Turner (tableau de 1834) en citant Wordsworth :

'Soyez une présence ou un mouvement - une chose et une seule, parmi toutes celles qu'il y a ici ; tandis que les brumes qui flottent et les vapeurs de pluie font surgir des formes et des fantômes des rochers et de la terre, aussi vite qu'un musicien fait s'éparpiller les notes avec un instrument'. Il n'y a que Turner et la nature qui en soient capables. ${ }^{72}$

La lecture de Ruskin critique d'art, diariste, poète, orologue, daguerréotypeur littéraire, donnerait à voir qu'il est aussi capable lui-même de cette présence en mouvement. Capable d'être un œil - un œil européen, j’ai essayé de le montrer - par le maniement, subtil, vivant, libre, léger, désinvolte au sens de Baldassare Castiglione, ${ }^{73} \mathrm{de}$ l'appareil le plus léger : « l'appareil du scribe ». ${ }^{74}$

67 Works, 20: 96.

68 Mallarmé 1998, 325

69 Merleau-Ponty 1964a, 68.

70 Merleau-Ponty 1964b, 27.

71 Merleau-Ponty 1964b, 80-1.

72 Works, 3: 405

73 Le mot de Sprezzatura (la dissimulation de l'art comme le degré le plus haut de l'art) est forgé par lui. Voir Castiglione 1991.

4 Mallarmé 2003, 171

57 Fonti, letterature, arti e paesaggi d'Europa | Sources, Literatures, Arts \& Landscapes of Europe 1

John Ruskin's Europe A Collection of Cross-Cultural Lssays, $43-58$ 


\section{Bibliographie de John Ruskin}

Les références aux œuvres de Ruskin renvoient au volume et à la page de:

Cook, E.T.; Wedderburn, A. (eds) (1903-1912). The Works of John Ruskin. Library Edition, 39 vols. London: George Allen.

https://www.lancaster.ac.uk/the-ruskin/the-complete-works-of-ruskin/.

Works, 3-7: Modern Painters I-V. | 9-11: The Stones of Venice I-III. | 15: The Elements of Drawing, The Elements of Perspective, The Laws of Fésole. | 17: Unto this Last, Munera Pulveris, Time and Tide. | 18: Sesame and Lilies, The Ethics of Dust, The Crown of Wild, Letters on Public Affairs (1859-1866). | 20: Lectures on Art, Aratra Pentelici. | 26: Deucalion. | 29: Fors Clavigera, Letters 73-96 (1877-1884). | 35: Praeterita, Dilecta. | 36: The Letters of John Ruskin I (1827-1869).

\section{Bibliographie générale}

Antoine, P. (2006). « Ceci n'est pas un livre. Le récit de voyage et le refus de la littérature ». Sociétés et représentations, 21, 45-58.

Baudelaire, C. (1975). « Mon cœur mis à nu ». Euvres complètes, t. 1. Paris: Gallimard.

Bergson, H. (1959). « Le Rire». Euvres, édition du centenaire. Paris: Presses Universitaires de France.

Carus, C.G. (2003). Neuf lettres sur le paysage. Trad. par E. Dickenherr, A. Pernet et R. Rochlitz. Paris: Klincksieck.

Castiglione, B. (1991). Le livre du courtisan. Trad. par A. Pons. Paris: Flammarion.

Claudel, P. (1946). L'œil écoute. Paris: Gallimard.

Cormenin, L. de (1852). La lumière, 25, 12 juin.

Courbet, G. (1950). Courbet raconté par lui-même et par ses amis, vol. 2. Genève: Pierre Caillé éditeur.

Fiedler, K. (2003). Sur l'origine de l'activité artistique. Trad. sous la dir. de D. Cohn. Paris: Éditions Rue d'Ulm.

Gautier, T. (1981). Voyage en Espagne. Paris: Gallimard

Gombrich, E. (1971). L’Art et l'illusion. Trad. par G. Durand. Paris: Gallimard.

Junod, P. (2004). Transparence et opacité. Essais sur les fondements théoriques de l'art moderne. Nîmes: Éditions Jacqueline Chambon. Lebensztejn, J.-C. (1990). L'art de la tache. Montélimar: Éditions du Limon.

Locke, J. (1972). Essai philosophique concernant sur l'entendement humain. Trad. par M. Coste. Paris: Librairie J. Vrin.

Mallarmé, S. (1998). Écrits sur l'art. Paris: Flammarion.

Mallarmé, S. (2003). Euvres complètes, t. 2. Paris: Gallimard.

Marion, J.-L. (2014). Courbet ou la peinture à l'œil. Paris, Flammarion.

Merleau-Ponty, M. (1964a). Le Visible et l'invisible. Paris: Gallimard.

Merleau-Ponty, M. (1964b). L'œil et l'esprit. Paris: Gallimard.

Nadar, F.T. (1994). Quand j'étais photographe. Paris: Le Seuil.

Nietzsche, F. (1964). La Généalogie de la morale. Trad. par H. Albert. Paris: Gallimard.

Proust, M. (2011). « Préface ». Ruskin, J., La Bible d'Amiens. Paris: Rivages poche, 11-105.

Rouillé, A. (1989). La Photographie en France, textes et controverses, une anthologie, 1816-1871. Paris: Macula.

Ruskin, J. (1956). The Diaries of John Ruskin. Edited by J. Evans and J.H. Whitehouse. 2 vols. Oxford: Clarendon Press.

Ruskin, J. (2013). Écrits sur les Alpes. Textes réunis et présentés par E. Sdegno et C. Reichler, trad. par A. Hélard. Paris: PUPS.

Schopenhauer, A. (1984). Le Monde comme volonté et comme représentation. Trad. par A. Burdeau. Paris: PUF.

Shama, S. (1999). Le paysage et la mémoire. Paris: Le seuil.

Spies, W. (2007). L'œil, le mot. Trad. par F. Joly et J. Torrent. Paris: Christian Bourgois éditeur.

Wiesing, L. (2014). Le visibilité de l'image. Histoire et perspectives de l'esthétique formelle. Trad. par C. Maigné. Paris: Librairie J. Vrin. Wölfflin, H. (1997). Réflexions sur l'histoire de l'art. Trad. par Cl. et M. Raymond. Paris: Flammarion.

Zola, É. (2012). Correspondance, choix de lettres. Présentation, notes, notices, bibliographie et index par Alain Pagès. Paris: Flammarion. 


\title{
Osservazione e comprensione dal rudere al paesaggio Unità morfologica e verità estetica negli scritti di John Ruskin
}

Emanuele Morezzi

Politecnico di Torino, Italia

\begin{abstract}
The paper proposes a reflection on the theoretical activity of John Ruskin towards the ruins and aims to analyse if these ideas find a legacy and a connection today in the criticism of the current conservation and architectural policies. For this purpose, my paper will analyse the ideas of the English critic related to ruins, studying how his approach was not of an aesthetic nature, but rather ethical. From this, his hostility to restoration will be better understood, as actions of mystification not of protection, and it will be possible to underline his attention to the conservation of cultural heritage with a more authentic approach. Finally, the paper will analyse some contemporary researches and activities attributable to the thoughts of Ruskin, to reiterate the relevance of the theories of the English critic even in different contexts and arts.
\end{abstract}

Keywords Ruins. Observation. Perception. Abandonment. Legacy. Cultural heritage.

Sommario 1 Introduzione. - 2 Ruskin, ruderi e immaginario del XIX secolo. - 3 II rifiuto del restauro dei ruderi come lotta per la loro conservazione. - 4 (Molteplici) eredità di Ruskin nella contemporaneità. - 5 Conclusioni.

\section{Introduzione}

Let us trace the genealogy of this fear. The art of seeing in a normal and natural way is acquired unconsciously during infancy and childhood. Then, owing to physical disease or, more often to mental strain, good seeing habits are lost; normal and natural functioning is replaced by abnormal and unnatural functioning.

A metà del secolo scorso, Aldous Huxley $(1942,99)$ trattava del timore di perdere la vista perché essa costituiva l'unico senso in grado di consentire una reale e autentica percezione del mondo sensibile. Queste teorie sono state citate, oltre che da più recenti studi di 
critica dell'architettura, ${ }^{1}$ da Roberto Di Stefano ${ }^{2}$ quando analizzò l'opera e il pensiero di John Ruskin attraverso l'osservazione e la percezione che il teorico inglese adottava nei confronti del costruito. La percezione in Ruskin, infatti, assume un ruolo di primaria importanza e rappresenta l'elemento primario di analisi del mondo basato soprattutto sul senso della vista e sull'osservazione diretta dei fenomeni. ${ }^{3}$ La sequenza Sensazione, Selezione, Percezione, che muove dall'occhio alla mente, rappresenta anche il passaggio cruciale dalla semplice osservazione del mondo sensibile alla sua più profonda comprensione. L'osservazione e la vista sono quindi canali che consentono una duplice conoscenza. Una prima permette la comprensione dell'aspetto figurativo de mondo, della dimensione paesaggistica, architettonica e di dettaglio, utile alla restituzione attraverso il disegno, mentre una seconda conoscenza riguarda l'aspetto intrinseco delle cose, non rispetto al loro carattere morfologico, ma piuttosto al loro valore etico e morale. Osservare, per Ruskin, non significava solamente comprendere le peculiarità di un oggetto, ${ }^{4}$ ma anche e soprattutto, capire le ragioni storiche, fisiche, geologiche ed etico-morali che avevano causato quel determinato fenomeno. ${ }^{5}$ Questo obiettivo appare evidente se si analizza l'intera produzione bibliografica del critico inglese capace di raggiungere elevate vette teoriche e speculative partendo ogni volta dalla semplice osservazione di un dato sensibile, sia esso l'erosione di alcuni tubi di piombo, come in uno dei suoi primi articoli scientifici, ${ }^{6}$ o i dipinti di pittori dei secoli precedenti come Claude Lorrain o Salvator Rosa, o il sistema geologico delle Alpi. $^{7}$ L'osservazione quindi è, per Ruskin, la chiave della comprensione fisica e spirituale del mondo: solo osservando è possibile avvicinarsi al concetto di Natura da lui teorizzato e proprio lo sguardo consapevole appare come l'unica via alla reale comprensione del mondo utile anche per un idoneo intervento architettonico. La tensione verso la bellezza, per Ruskin, deve passare dall'imitazione di Natura e tale imitazione sarà possibile solo dopo aver compreso appieno il mondo naturale:

For whatever is in architecture fair or beautiful, is imitated from natural forms; and what is not so derived, but depends for its dignity upon arrangement and government received from human mind, becomes the expression of the power of that mind, and receives a sublimity high in proportion to the power expressed. ${ }^{8}$

L'importanza dell'osservazione quindi, non va intesa come un atto contemplativo, finalizzato all'indagine fenomenologica delle cose e senza un ritorno in termini operativi o propositivi, ma, al contrario, è solo il primo passo verso una interiorizzazione dei concetti appresi e una loro successiva divulgazione. Da una corretta percezione delle cose dipenderà la bontà del lavoro artistico e architettonico dell'osservatore. Il legame tra osservazione

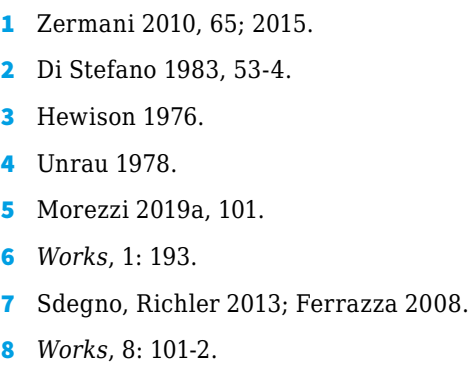




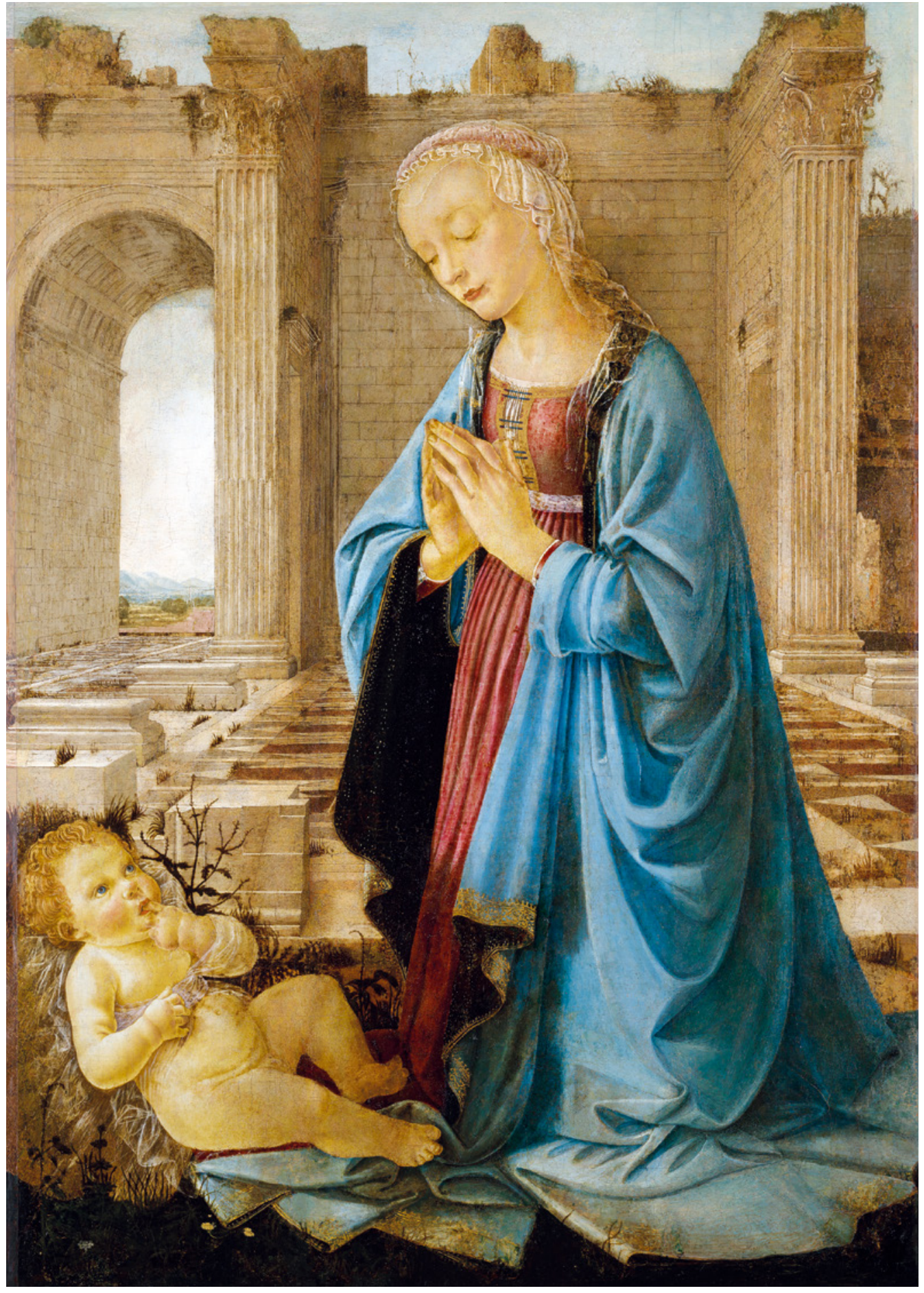

Figura 1 Autore Sconosciuto (attribuito ad Andrea del Verrocchio e bottega) (1435-1488), Vergine in adorazione del Bambino ('The Ruskin Madonna'). 1470 ca.

Tempera e olio su tela. Questo dipinto, caratterizzato da rovine classiche sullo sfondo, apparteneva alla collezione privata di John Ruskin. National Galleries of Scotland, accession number NG 2338. Acquistato con l'ausilio di Art Fund and the Pilgrim Trust 1975 
e concetto di Natura/Naturale è infatti evidente anche nella già nota polemica che si pone alla base del lavoro di scrittura del primo volume di Modern Painters. ${ }^{9}$ Come già analizzato, ${ }^{10}$ la primaria necessità di difendere Turner dalle accuse di aver dipinto «out of nature» pone Ruskin di fronte alla possibilità di realizzare una ben più ambiziosa opera in più volumi che possa esporre le tematiche della pittura contemporanea in relazione ai Maestri del passato. Proprio da questa prima opera emergerà in maniera chiarissima l'esigenza di porre l'attenzione sull'osservazione e sulla comprensione del mondo e dei suoi valori più profondi. ${ }^{11}$ Da ciò, il testo diverrà presto non solo un punto di riferimento della pittura ottocentesca, ma anche uno strumento di propaganda, utile a spiegare come l'arte, l'architettura e il paesaggio siano portatori di un valore intrinseco che solo attraverso una opportuna percezione può essere compreso e difeso.

And, first, observe that the charm of romantic association ( $(14)$ can be felt only by the modern European child. It rises eminently out of the contrast of the beautiful past with the frightful and monotonous present; and it depends for its force on the existence of ruins and traditions, on the remains of architecture, the traces of battle-fields, and the precursorship of eventful history. The instinct to which it appeals can hardly be felt in America, and every day that either beautifies our present architecture and dress, or overthrows a stone of mediæval monument, contributes to weaken it in Europe. Of its influence on the mind of Turner and Prout, and the permanent results which, through them, it is likely to effect, I shall have to speak presently. ${ }^{12}$

Uno degli aspetti più interessanti di Modern Painters risiede infatti nella sensibilità con cui il critico inglese riesce a mettere in relazione la comprensione della Natura, la pittura e la percezione dei valori morali. ${ }^{13}$ In tal senso, appare di particolare importanza analizzare le idee e le teorie che Ruskin espose in merito alle rovine e ai ruderi architettonici. La scelta di orientare le analisi verso questa specifica categoria di beni può risultare utile a comprendere come, sebbene una deteminata storiografia ${ }^{14}$ abbia voluto archiviare il teorico inglese come semplice ruinista, la sua attenzione nei confronti dei ruderi architettonici risultava molto più complessa e affascinante. Ciò che si cercherà di studiare è il rapporto con la rovina non come semplice luogo dell'abbandono, ma piuttosto come entità architettonica che, sebbene in avanzato stato di degrado, appare ancora portatrice di valori morali ed etici e quindi degna di una particolare attenzione.

9 Works, 3.

10 Hilton 1985; Hewison 2000.

11 Hewison 1976.

12 Works, 5: 369.

3 Leoni 1987, 21-49.

4 Caperna 2017, 250 


\section{Ruskin, ruderi e immaginario del XIX secolo}

Le rovine rivestono un ruolo di grandissimo valore per tutto il XIX secolo, sia nel mondo dell'arte che in quello dell'architettura, in ambito inglese e non solo. Già dai secoli precedenti, molti pittori come ad esempio i noti $\mathrm{Hu}-$ bert Robert e Giovanni Paolo Pannini, avevano elevato i ruderi a protagonisti della loro arte, dichiarando un crescente interesse verso il passato. ${ }^{15}$ Ma questi resti acquistano una importanza ancora maggiore alla fine del XVIII secolo quando il Gothic Revival, eleva gli edifici allo stato di rovina a simbolo della decadenza del passato. Come ricorda Clark $^{16}$ in merito alla genesi di Strawberry Hill e al movimento ruinista, dopo il 1750 si ricorre alle finte rovine per avere 'stimoli per la fantasia'. In modo analogo, anche le raffigurazioni della prima metà del XIX secolo si popolano di macerie in tutta Europa e non solo in Inghilterra dove comunque è riscontrabile una naturale coda artistica che, dalle prime manifestazioni ruiniste del secolo precedente, prosegue anche in pieno clima vittoriano. Un esempio piuttosto noto in tal senso, è rappresentato dall'acquarello di Gandy, commissionato da John Soane, in cui viene dipinta la banca d'Inghilterra in stato di rovina nel $1830 .{ }^{17}$

Tra le altre, anche le opere di Turner, tra la fine del Settecento e l'inizio dell'Ottocento, si popolano di ruderi e di resti medievali. ${ }^{18}$ Osservando con attenzione i dipinti del periodo, si può osservare evidentemente come esistano due aspetti sempre presenti nelle rappresentazioni di questo genere: il legame del rudere con la Natura, intesa come vegetazione lussureggiante, che ha invaso il bene architettonico, e la presenza dell'uomo. Sintetizzando e riprendendo Clark ${ }^{19}{ }^{19}$ sembra ipotizzabile leggere queste composizioni come il tentativo di porre in equilibrio tre aspetti fondativi dell'esistenza attraverso il sistema dei simboli: la vegetazione come metafora della Natura; il rudere come simbolo del passato e di memoria dell'antico; l'individuo come simbolo dell'umanità al cospetto del sublime e del pittoresco. ${ }^{20}$ Questo aspetto è riscontrabile soprattutto in quei dipinti, realizzati in Inghilterra a cavallo tra i due secoli, da artisti quali Turner, de Loutherbourg, Constable che scelgono consapevolmente di elevare i ruderi di antiche abazie a soggetto dei loro dipinti. Da questa serie di opere, in chiaro stile romantico, nascerà poi l'affezione verso beni prima sconosciuti e abbandonati come la Tintern Abbey, rappresentata sia da Turner sia da de Loutherbourg, la cattedrale di Llaandaff o l'abbazia di Melrose. Sebbene siano riscontrabili, negli scritti di Ruskin, molte citazioni riferibili ad «abbey in ruins», questo genere di dipinti richiama un tiepido coinvolgimento del critico inglese: Ruskin appare, al contrario, più concentrato sulla dimensione ontologica della rappresentazione piuttosto che rivolgere i propri studi verso lo spirito dei ruderi architettonici nella pittura romantica.

Forse proprio partendo da questo aspetto si può comprendere meglio il pensiero di Ruskin nei confronti delle rovine e anche il motivo della stigmatizzazione del

$63 \mid$ Fonti, letterature, arti e paesaggi d'Europa | Sources, Literatures, Arts \& Landscapes of Europe 1 John Ruskin's Europe. A Collection of Cross-Cultural Essays, 59-84 


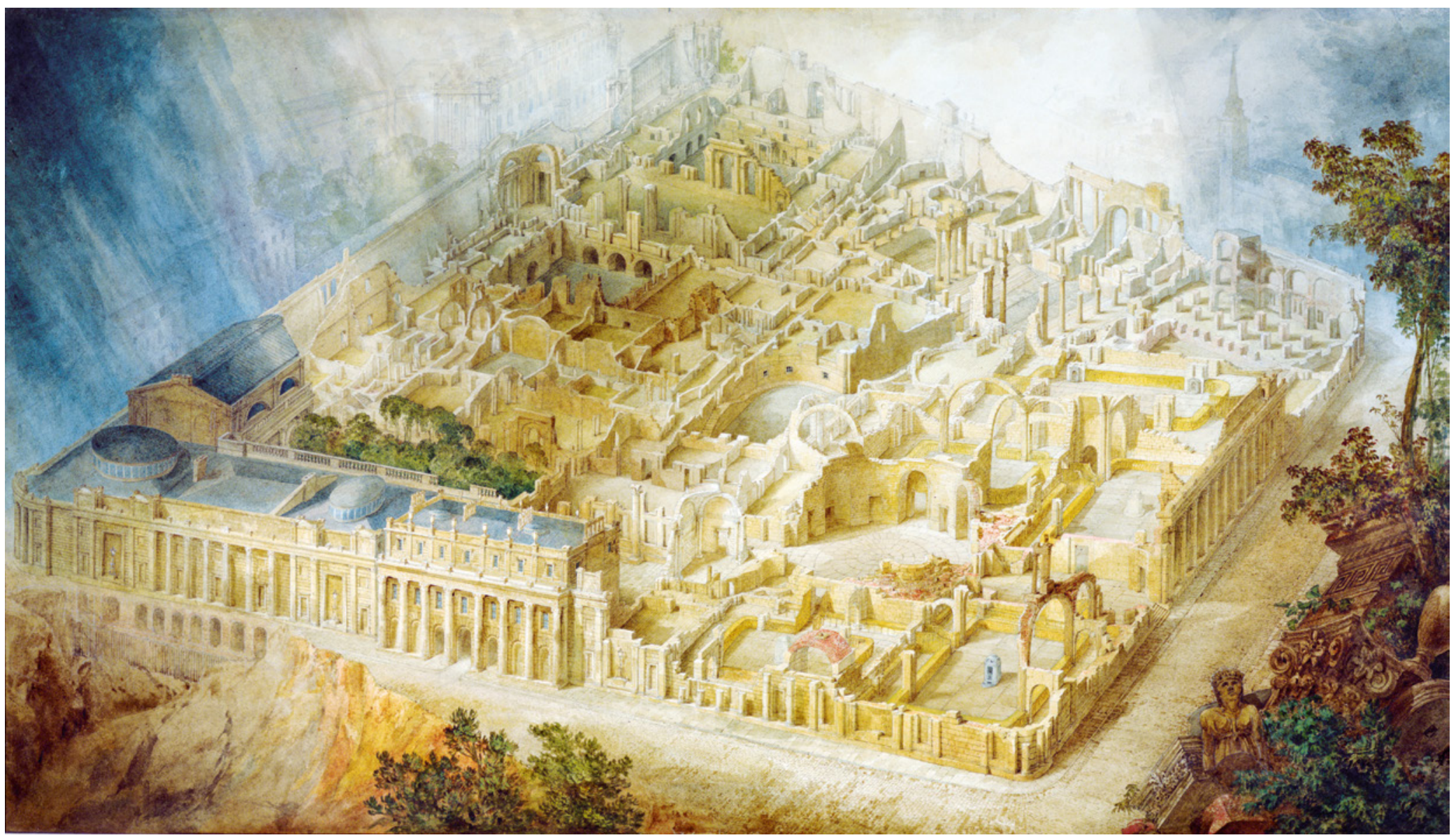

Figura 2 Joseph Michael Gandy (1771-1843), A Bird's-eye View of the Bank of England. 1830. Acquerello su carta. Museum number: P267. @Sir John Soane's Museum, London 


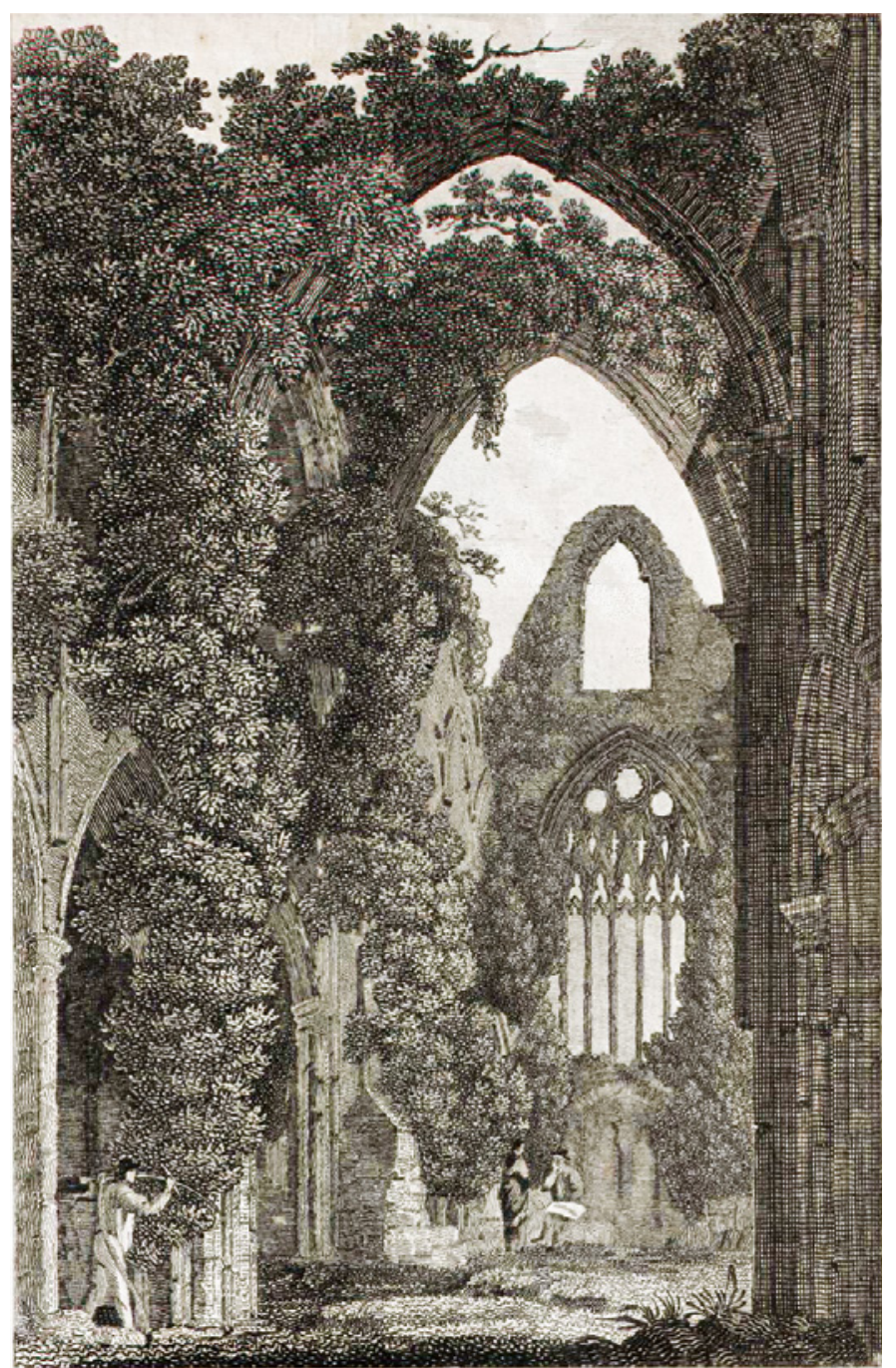

Figura 3 John Le Keux (1783-1846), Tintern Abbey, Monmouthshire, Looking West. 1809. Incisione. London, Tate Gallery. CC license

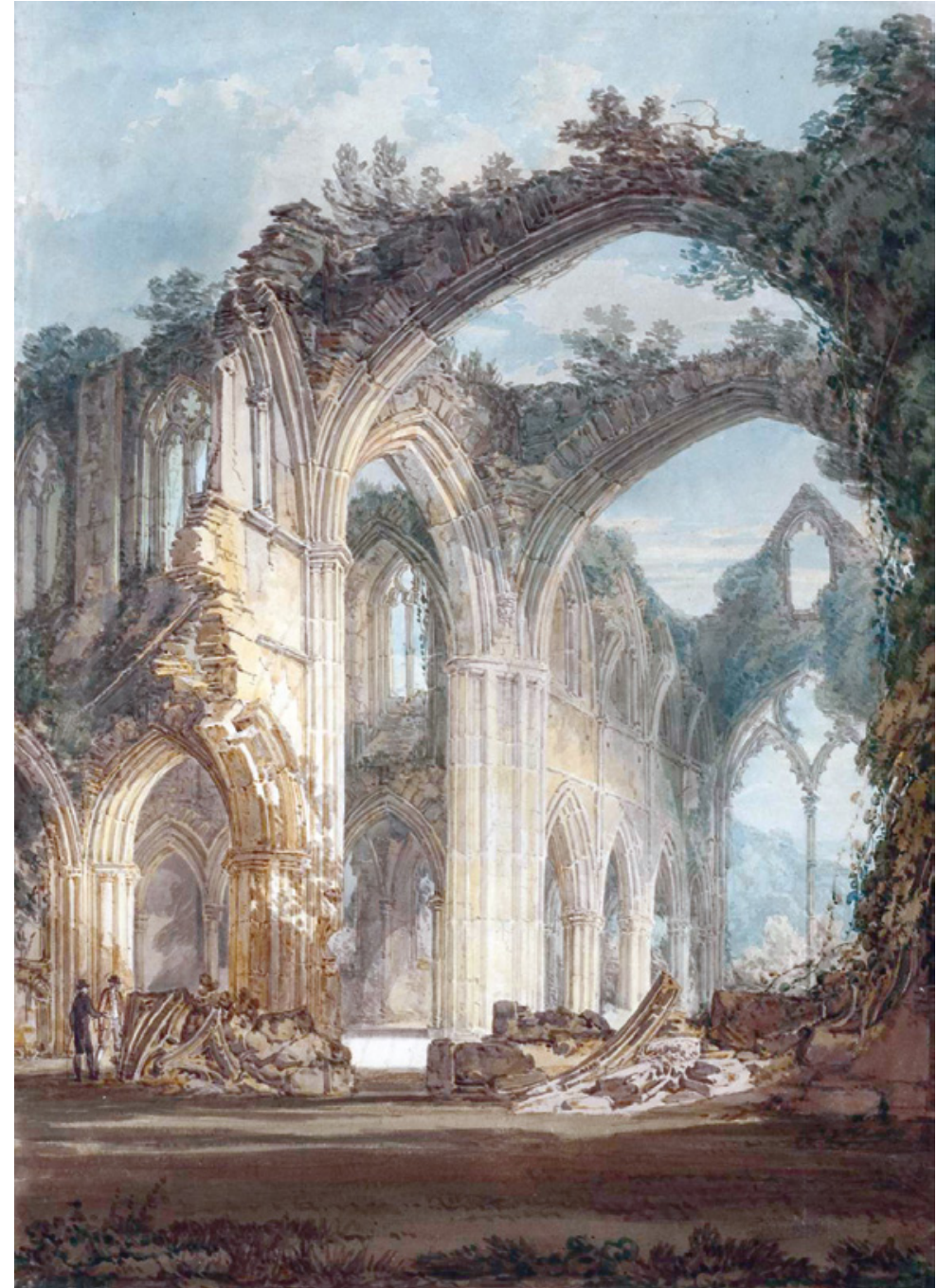

Figura 4 Joseph Mallord William Turner (1775-1851), Tintern Abbey: The Crossing and Chancel, Looking towards the East Window. In Watercolours and Studies Relating to the Welsh and Marches Tours, 1794. London, Tate Gallery. CC license 
Figura 5 John Constable (1776-1837), Netley Abbey by Moonlight. $1833 \mathrm{ca}$. London, Tate Gallery. CC license

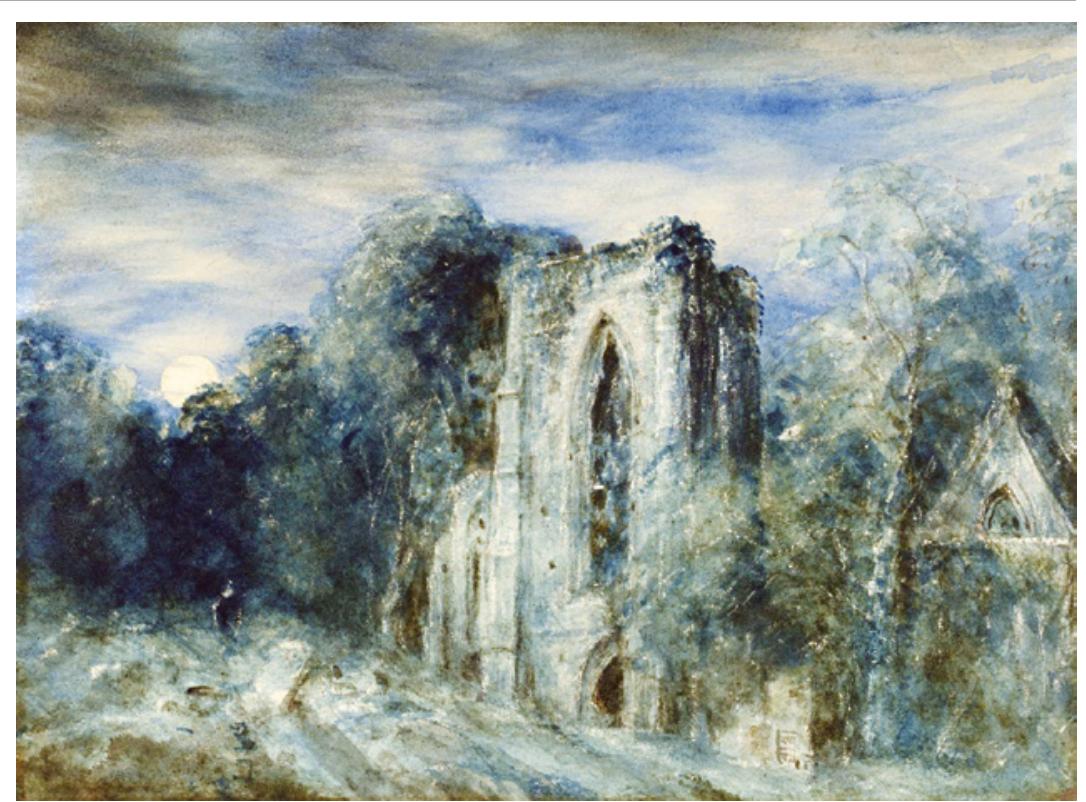

critico inglese a mero ruinista. Anzitutto va riconosciuto come, nonostante il periodo storico lo permettesse, e in un certo senso lo consigliasse, negli scritti di Ruskin non appare mai alcuna particolare attenzione o fascinazione nei confronti dei ruderi. ${ }^{21}$ Piuttosto è vero l'esatto contrario: le rovine non suscitano in lui, almeno nella prima parte della sua vita, nessuna emozione particolare. Ciò è vero, come ha recentemente analizzato Romeo, ${ }^{22}$ nel corso dei primi viaggi in Italia, quando i suoi diari si popolano di pessime impressioni nei confronti dei ruderi classici. Roma e Paestum rappresentano per il giovane Ruskin, in viaggio con i genitori, motivi di grande de- lusione e quasi repulsione, manifestata facendo spesso ricorso a parole quali «sgradevole, desolazione». Ė ipotizzabile che l'aspettativa nei confronti della penisola italiana fosse diversa dal reale stato di conservazione dei luoghi. Le tavole e le illustrazioni dell'Italy di Rogers, ${ }^{23}$ realizzate da Turner, rappresentano infatti per Ruskin il mezzo attraverso il quale lui conosce l'Italia e grazie al quale inizia ad apprezzare il paesaggio e l'architettura, ma anche il loro valore morale ed etico:

Nevertheless, Mr. Telford had a singularly important influence in my education. By, I believe, his sisters' ad-

21 Ricercando il termine «ruins» all'interno dei suoi scritti si nota che tale vocabolo viene usato sporadicamente e spesso con un significato metaforico (Sdegno 2004).

22 Romeo 2019, 134.

23 Rogers 1822. 
vice, he gave me, as soon as it was published, the illustrated edition of Rogers's Italy. This book was the first means I had of looking carefully at Turner's work: and I might, not without some appearance of reason, attribute to the gift the entire direction of my life's energies. $^{24}$

Oltre all'importanza del volume nell'educazione del piccolo John però, occorre evidenziare come, anche in questo fatto biografico, sia evidente un'associazione capace di legare l'osservazione delle tavole di Turner, la visione del monumento dal vero e la comprensione dei valori di queste due (diverse) immagini. La bontà della comunicazione delle tavole di Turner è certa e ribadita:

But it is the great error of thoughtless biographers to attribute to the accident which introduces some new phase of character, all the circumstances of character which gave the accident importance. The essential point to be noted, and accounted for, was that I could understand Turner's work, when I saw it;-not by what chance, or in what year, it was first seen. ${ }^{25}$

La comunicazione dell'immagine (sebbene retorica e di carattere paesaggistico) sembra essere anche più efficace dal punto di vista divulgativo rispetto alla visione dal vero: lo stesso Ruskin, di fronte alla Piramide Cestia, sentenzia di preferirne la rappresentazione che ne ha dato Turner. ${ }^{26}$ Da tale asserzione possiamo forse comprendere come il processo di identificazione dei valori dell'architettura allo stato di rudere abbia subito un'evoluzione all'interno del pensiero ruskiniano. Dalle giovanili brusche considerazioni, sebbene condizionate da molti fat- tori esterni, verso le rovine di Roma ad una maggiore introspezione e volontà di comprendere quale sia il fascino dell'edificio abbandonato. Lontano da un cedimento emotivo per il senso di pittoresco o sublime del paesaggio con ruderi, Ruskin tenta di analizzare razionalmente il contesto architettonico e costruttivo in decadenza per poterne comprenderne i valori e le prerogative più profonde. Sembra quasi ipotizzabile che il giovane Ruskin, durante il suo primo viaggio italiano, non avesse ancora ultimato le proprie riflessioni nei confronti e dell'architettura nella sua complessità e del significato dei ruderi immersi nel paesaggio. Tale processo critico ed analitico trova un momento di particolare importanza all'interno di Modern Painters, quando, trattando il tema della Vital Beauty, il teorico inglese compie una interessante riflessione interrogandosi in merito al fascino che rivestono $i$ ruderi e cercando di razionalizzare l'interesse suscitato da questa specifica categoria di beni. Egli indaga quanto l'assenza di una specifica funzione, per questi edifici, consenta di contemplarli come mera bellezza, priva di ogni accezione funzionalista. Il loro essere edifici depotenziati dall'aver perso uno scopo abitativo o sacro, li apre ad una interpretazione che è puramente estetica: l'aver smarrito il loro uso ha regalato loro una bellezza privata dall'essere stati trasformati in oggetti architettonici e scultorei.

The bending trunk, waving to and fro in the wind above the waterfall, is beautiful because it is happy, though it is perfectly useless to us. The same trunk, hewn down, and thrown across the stream, has lost its beauty. It serves as a bridge,-it has become useful; and its beauty is gone, or what it retains is purely

24 Works, 5: 28-9.

5 Works, 5: 29.

Romeo 2019; Brilli 1985.

67 Fonti, letterature, arti e paesaggi d'Europa | Sources, Literatures, Arts \& Landscapes of Europe 1

John Ruskin's Europe. A Collection of Cross-Cultural Essays, 59-84 
typical, dependent on its lines and colours, not on its functions. Saw it into planks, and though now adapted to become permanently useful, its beauty is lost for ever, or to be regained only when decay and ruin shall have withdrawn it again from use, and left it to receive from the hand of nature the velvet moss and varied lichen, which may again suggest ideas of inherent happiness, and tint its mouldering sides with hues of life. There is something, I think, peculiarly beautiful and instructive in this unselfishness of the Theoretic faculty, and in its abhorrence of all utility to one creature which is based on the pain or destruction of any other; for in such services as are consistent with the essence and energy of both it takes delight, as in the clothing of the rock by the herbage, and the feeding of the herbage by the stream. ${ }^{27}$

\section{Il rifiuto del restauro dei ruderi come lotta per la loro conservazione}

Sebbene la metafora del tronco si allontani solo apparentemente dalla nostra analisi in merito agli edifici, il concetto qui espresso da Ruskin consente di cogliere perfettamente la maturazione delle sue riflessioni in merito non tanto alla componente figurativa di dettaglio che segue l'osservazione, quanto piuttosto alla comprensione più profonda del valore del mondo naturale. Se applicato all'ambito architettonico si può evidenziare come, per un edificio, il decadere ad uno stato di rovina, non rappresenti unicamente la perdita di una funzione, ma anche la riconquista del suo semplice valore estetico come pura manifestazione dell'opera dell'uomo. ${ }^{28}$ Letta in questi termini, l'osservazione di Ruskin sembra straordinariamente anticipatoria di tutto il dibattito (e scontro) tra forma e funzione che costituirà il tema principale della teoria architettonica della prima metà del secolo successivo. Attraverso il rudere, o meglio, attraverso il vuoto che la perdita di funzione ha lasciato, Ruskin intende leggere quella che era una architettura come una opera d'arte figurativa, libera da obblighi funzionali. ${ }^{29}$
In più, appare utile muovere un'ulteriore riflessione per analizzare sia il pensiero teorico originale di $\mathrm{Ru}$ skin sia l'interpretazione storiografica. Se applicate al caso specifico dei ruderi, e quindi agli edifici incompleti che hanno perso la loro integrità, ${ }^{30}$ le teorie ruskiniane sul non-restauro, a volte considerate eccessivamente integraliste, appaiono perfettamente comprensibili e condivisibili.

Il pensiero di Ruskin sul restauro e sulla sua totale negazione di ogni forma di intervento sui beni monumentali emerge in maniera chiarissima in Seven Lamps of Architecture, in cui però la storiografia si è affrettata a ribadire come tale negazione fosse da intendersi come chiara risposta ai restauri stilistici che già avevano avuto luogo in Inghilterra e che stavano prendendo sempre più piede in Francia. Le parole di Ruskin sembrano confermare apertamente tale concezione:

Do not let us talk then of restoration. The thing is a Lie from beginning to end. You may make a model of a 
building as you may of a corpse, and your model may have the shell of the old walls within it as your cast might have the skeleton, with what advantage I neither see nor care: but the old building is destroyed, and that more totally and mercilessly than if it had sunk into a heap of dust, or melted into a mass of clay: more has been gleaned out of desolated Nineveh than ever will be out of re-built Milan. ${ }^{31}$

Risulta però doveroso ribadire come, sebbene la presa di distanza dai restauri stilistici sia evidente in Ruskin, ciò che lo getta in un odio così radicato nei confronti del restauro non sia tanto la modalità di intervento sul patrimonio, quanto piuttosto l'azione stessa di modifica del bene. Ciò si deve all'implicita alterazione che, insieme all'azione di ricostruzione e restauro, avrebbero subito anche i valori e il significato del bene medesimo. Intervenire sulle componenti morfologiche dell'edificio e su quelle costruttive non significava per Ruskin solamente creare una copia del passato, ma distruggere la componente di valori di cui i beni, ruderi compresi, erano ancora portatori. Attraverso la metafora del tronco e anticipando altre riflessioni successive, ${ }^{32}$ egli ha spiegato come vi fosse una sorta di scelta aprioristica tra bellezza pura e funzione, e che, anche ricorrendo a questa seconda scelta, attraverso il ritorno ad uno stato primigenio di rovina, la bellezza originaria fosse riconquistabile, lasciando questo compito all'azione del tempo e della Natura. In tale ottica, l'intervento dell'uomo attraverso il restauro annienterebbe questa opportunità, tentando una azione opposta di riproposizione funzionalistica per un monumento che non può più essere ri- portato alla configurazione precedente, condannandolo alla perdita sia dell'aspetto funzionale, sia di quello formale, dell'istanza estetica e di quella storica.

Neither by the public, nor by those who have the care of public monuments, is the true meaning of the word restoration understood. It means the most total destruction which a building can suffer: a destruction out of which no remnants can be gathered: a destruction accompanied with false description of the thing destroyed. Do not let us deceive ourselves in this important matter; it is impossible, as impossible as to raise the dead, to restore anything that has ever been great or beautiful in architecture. That which I have above insisted upon as the life of the whole, that spirit which is given only by the hand and eye of the workman, can never be recalled. Another spirit may be given by another time, and it is then a new building; but the spirit of the dead workman cannot be summoned up, and commanded to direct other hands, and other thoughts. ${ }^{33}$

Ruskin dimostra di aver sondato non solamente il valore estetico del paesaggio e dell'architettura, ${ }^{34}$ ma di essersi interrogato a fondo sulla comprensione e sui valori di cui tale patrimonio è simbolo. Il critico inglese intende già le manifestazioni dell'architettura come «manifestazioni di civiltà», simboli non solo del passato gusto artistico o architettonico, ma veri monumenti ricchi di valori immateriali non modificabili. Il concetto è espresso, ancora una volta, nella Lamp of Memory, senza possibilità di errate interpretazioni:

\footnotetext{
1 Works, 8: 244.

Fiorani 2009

Works, 8: 242

Leoni 1987, 24-6; Forti 1983.
}

\begin{tabular}{l|l} 
Fonti, letterature, arti e paesaggi d'Europa | Sources, Literatures, Arts \& Landscapes of Europe 1 \\
John Ruskin's Europe. A Collection of Cross-Cultural Essays, 59-84
\end{tabular} 
"See! this our fathers did for us." For, indeed, the greatest glory of a building is not in its stones, nor in its gold. Its glory is in its Age, and in that deep sense of voicefulness, of stern watching, of mysterious sympathy, nay, even of approval or condemnation, which we feel in walls that have long been washed by the passing waves of humanity. ${ }^{35}$

e ancora:

it is in that golden stain of time, that we are to look for the real light, and colour, and preciousness of architecture; and it is not until a building has assumed this character, till it has been entrusted with the fame, and hallowed by the deeds of men, till its walls have been witnesses of suffering, and its pillars rise out of the shadows of death, that its existence, more lasting as it is than that of the natural objects of the world around it, can be gifted with even so much as these possess, of language and of life. ${ }^{36}$

L'avversione nei confronti del restauro nel pensiero di Ruskin trova le proprie ragioni nella necessità, ancora una volta di carattere etico e morale, di conservare i beni architettonici del passato e il loro più profondo significato, il valore che essi rappresentano. Ogni modifica costruttiva e morfologica all'edificio va esclusa perché andrebbe a corrompere il significato intrinseco del bene che, come già affermato, sta proprio nella patina, nella vetustità dell'opera.
I do not at this moment recollect a single instance of any very fine building which is not improved, up to a certain period, by all its signs of age; after which period, like all other human works, it necessarily declines; its decline being, in almost all ages and countries, accelerated by neglect and abuse in its time of beauty, and alteration or restoration in its time of age. [...] And I have never yet seen any restoration or cleaned portion of a building whose effect was not inferior to the weathered parts, even to those of which the design had in some parts almost disappeared. ${ }^{37}$

Questa sua posizione risulta confermata per tutta la sua esistenza. A confermarcelo una specifica lettera che invia al direttore del Kidderminster Times da Brantwood, il 24 luglio 1877, per richiamare la sua attenzione sui restauri a suo modo di vedere per nulla condivisibili, della Ribbesford Church. ${ }^{38}$ Appare di elevato interesse sottolineare come la disaffezione per il restauro e la modifica dell'esistente non coincida, nel pensiero teorico del critico inglese, con il rifiuto di alcun tipo di intervento o manutenzione. Per Ruskin restaurare significa la peggiore delle distruzioni, perché, come visto, significa modificare il valore di un bene, ma, al contrario, la conservazione è sempre raccomandabile e utile a preservare il messaggio dell'opera architettonica. Oltre a ribadirlo in maniera esplicita all'interno delle sue opere, ${ }^{39}$ l'incontro con le teorie di Boni a Venezia ${ }^{40}$ riempirà Ruskin di gioia, perché gli consentirà di condividere con lui la medesima visione nei confronti dei beni culturali. 


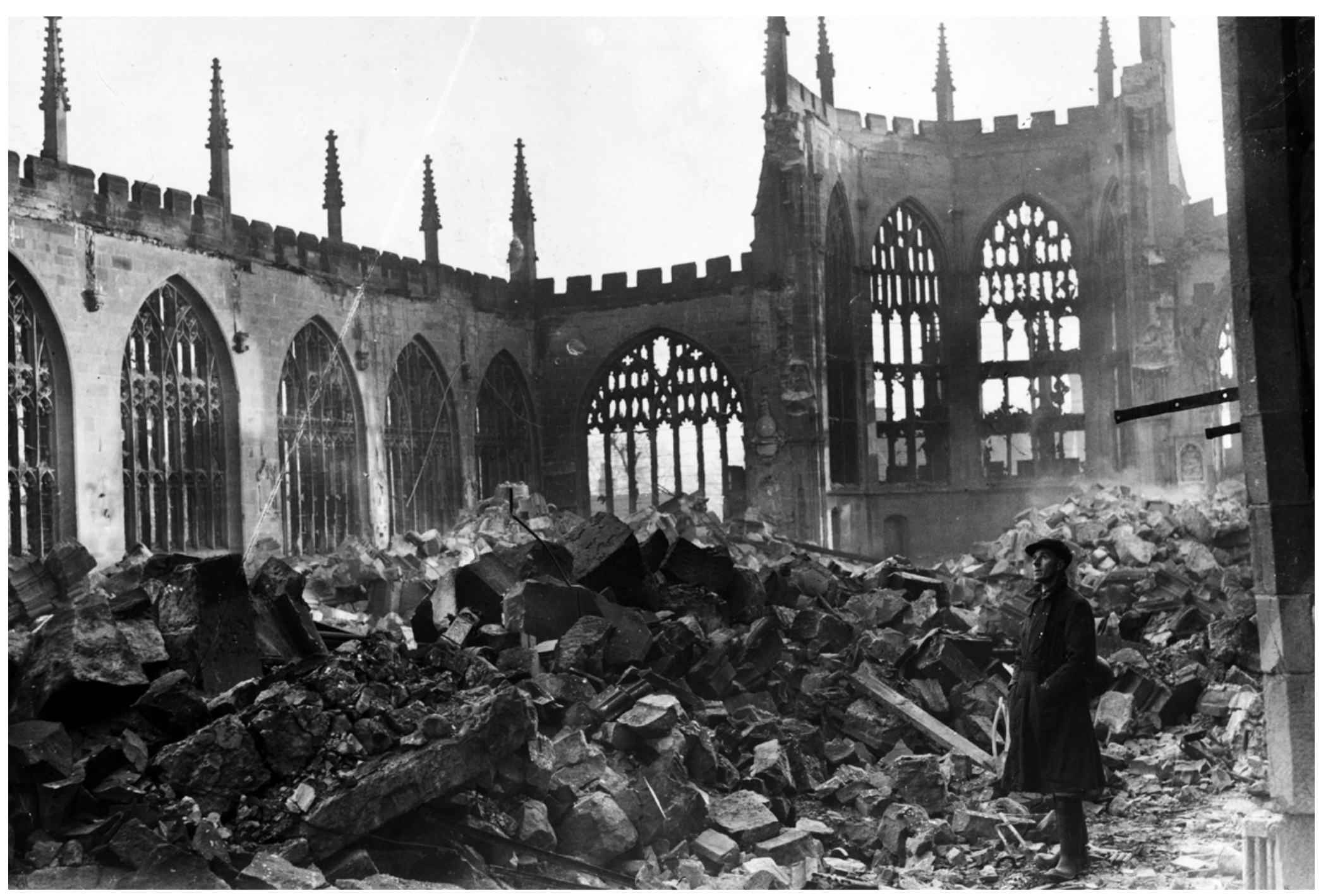

Figura 6 Ruderi della cattedrale di Coventry dopo l'attacco aereo dell'esercito tedesco, 16 novembre 1940. Fotografia di George W. Hales/Fox Photos/Getty Images. 


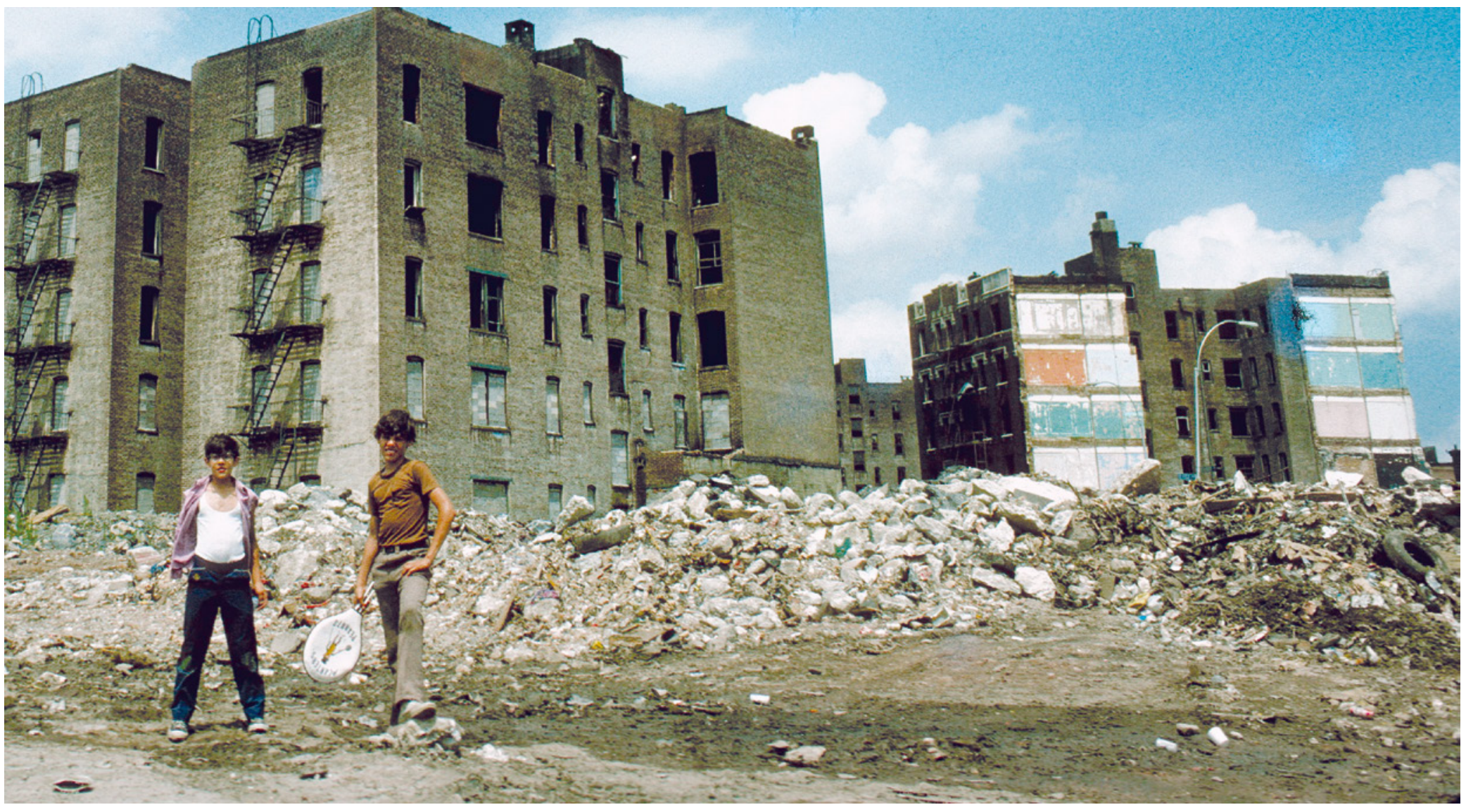

Figura 7 Steven Siegel, New York in the 80s n.94. Charlotte street, the Bronx. 1980 ca. Courtesy of the Author 


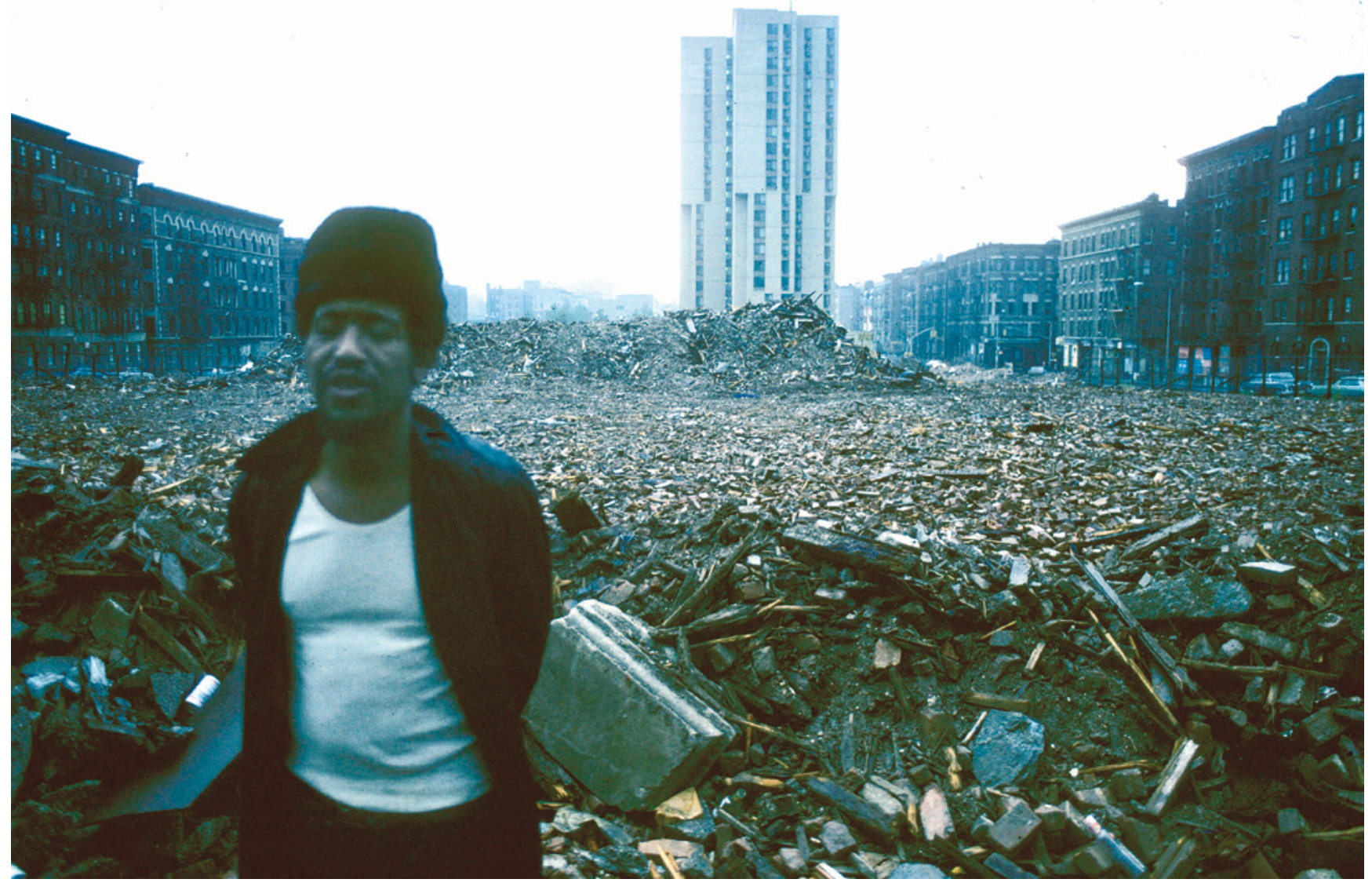

Figura 8 Steven Siegel, New York in the 80s n.190. Central Harlem. Primi anni Ottanta. Courtesy of the Author 


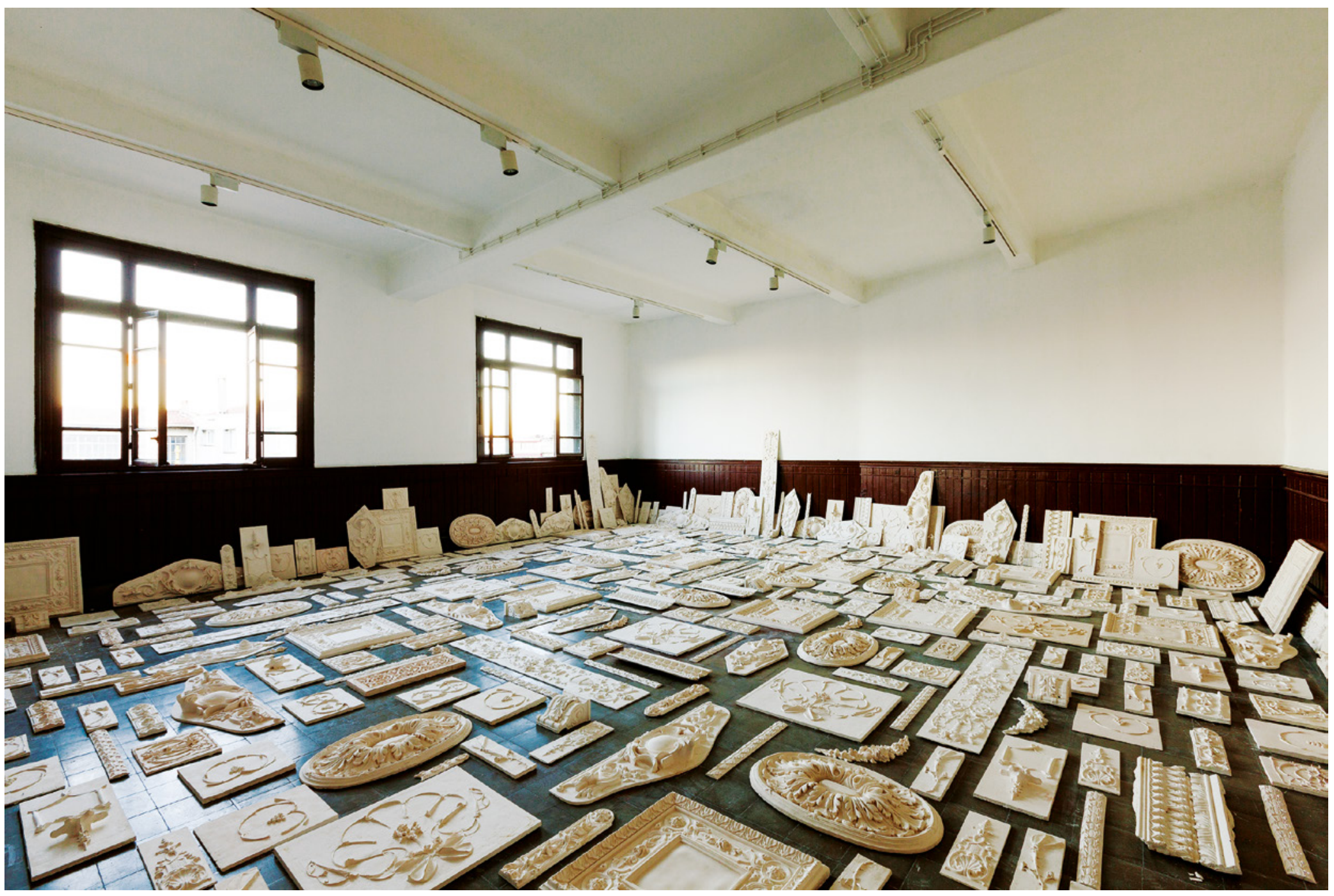

Figura 9 Michael Rakowitz, The Flesh is Yours, the Bones are Ours. 2015. Allestimento per la Biennale di Istanbul, 2019 


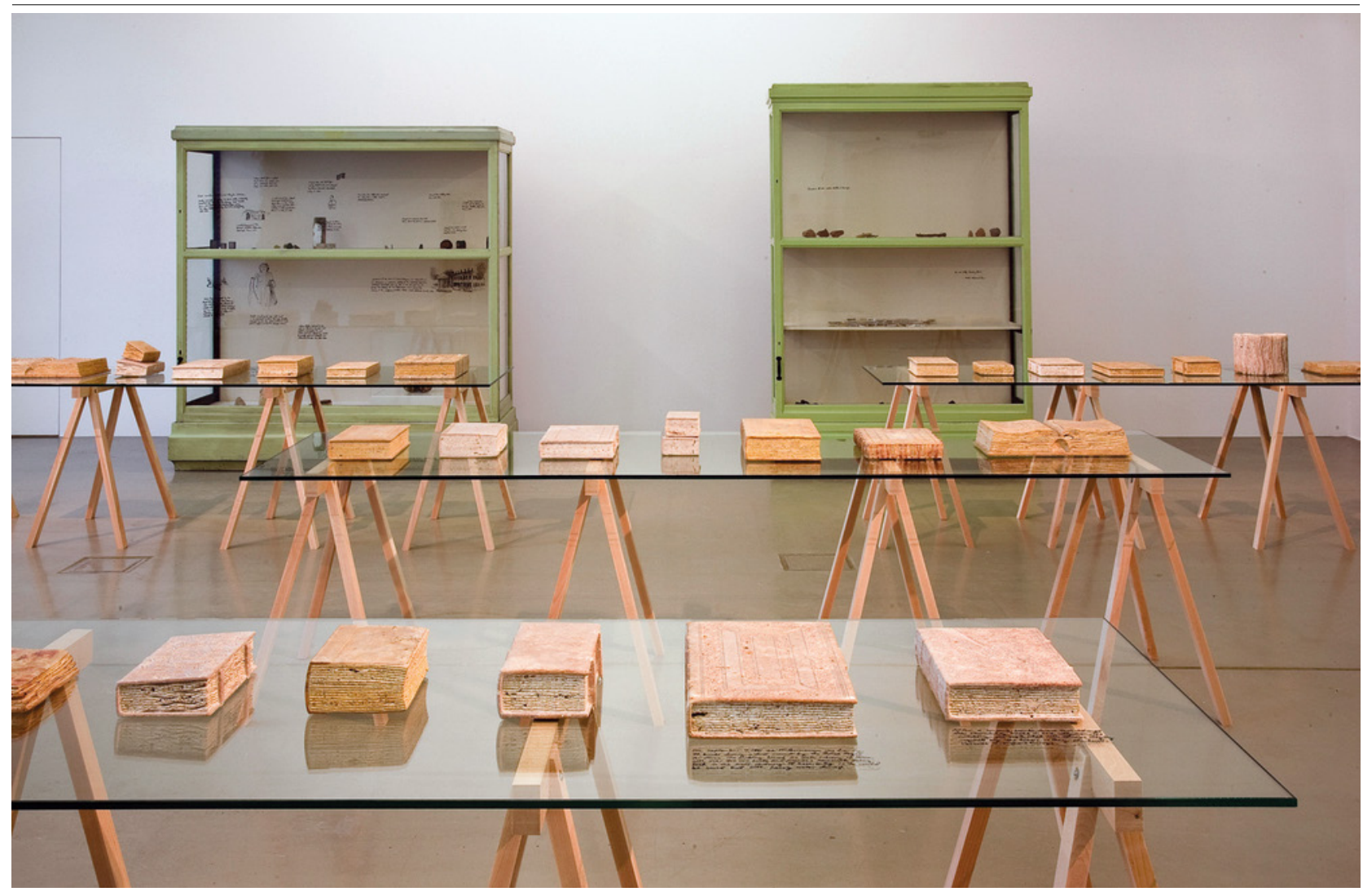

Figura 10 Michael Rakowitz, What Dust Will Rise?. 2012. Courtesy of the Author

The devoted enthusiasm of this architect (Boni) who interpreted "restoration" as preservation, not destruction, was very pleasing to Ruskin. I do not know whether the studies in archaeological research and excavation, by which Commendatore Boni is now so well known, owed anything to him; but certainly Ruskin urged him to classical studies, and sent him various books. ${ }^{41}$
«'Restoration' as preservation» è la chiave di lettura più efficace per comprendere le istanze di Ruskin e le sue raccomandazioni: attuare una manutenzione costante a tutti i beni culturali e procedere con opere di consolidamento puntuali allo scopo di allungare il più possibile la vita dell'edificio e, contemporaneamente, preservarne i valori autentici più a lungo nel tempo. 


\section{4 (Molteplici) eredità di Ruskin nella contemporaneità}

Da queste raccomandazioni appare possibile comprendere come le teorie ruskiniane siano state interpretate nei decenni successivi e quali ricadute abbia avuto il pensiero del critico inglese sul mondo della conservazione. Sembra lecito quindi porsi la medesima domanda che altri si sono posti in passato:

What's a Ruskinan architecture? An architecture inspire BY Ruskin, yet NOT Ruskin's, and, in fact, derived partly through misinterpretation - a problem that plagued him in all his endeavors. ${ }^{42}$

Forse appare opportuno accettare che, soprattutto da un punto di vista novecentesco, quindi più consapevole, dell'evoluzione del dibattito sulla conservazione e tutela in Europa, esistano architetture 'ruskiniane'. Seguendo i dettami emanati negli scritti, alcuni edifici sono stati sì conservati allo stato di rudere e quindi cercando di mantenere il fascino della bellezza dell'opera senza adeguarli a una funzione, senza ricorrere ad un ripristino stilistico.

Questo fenomeno, diffuso soprattutto, ma non solo, nel Regno Unito, ha permesso la sopravvivenza di alcune realtà che sono state conservate allo stato di rudere e che costituiscono oggi interessanti casi studio di osservazione e comprensione della valenza del patrimonio. Per una circostanza storiografica e non puramente casuale, molte delle abazie citate in precedenza, come la Tintern o la Melrose Abbey, presenti come ruderi nei dipinti di Turner o de Loutherbourg, sono state oggetto delle raccomandazioni ruskiniane e si presentano ancora oggi in rovina. L'aspetto ruskiniano delle rovine in questione non dipende però dalla sola esclusione di interventi stilistici di completamento dell'edificio, ma anche, e soprattutto, da un'azione costante di monitoraggio, consolidamento e manutenzione, proprio come raccomandato dal critico inglese nei suoi scritti.

Da ciò è possibile individuare una prima eredità del pensiero ruskiniano con ricadute dirette nel mondo del restauro: paradossalmente, ma solo per chi ne ha tratto una lettura superficiale, i testi di Ruskin non si pongono in antitesi ai restauri in genere, ma piuttosto alle mistificazioni. La scelta di conservare a rudere alcuni edifici che avevano perso le loro funzioni e si presentavano allo stato di rovina appare l'unica strategia possibile per mantenere il giusto grado di autenticità della struttura. Questa modalità di intervento, appare giusto sottolinearlo, non vide la luce solo negli anni contemporanei agli scritti del pensatore inglese, ma anzi, fu largamente adottata anche nel corso del XX secolo. Sembra possibile leggere un filone di intervento caratterizzato proprio dalla volontà di conservare le rovine senza aggiungere nuovi apparati architettonici, allo scopo di mantenerne invariato l'aspetto simbolico. ${ }^{43}$ Tale atteggiamento è stato adottato per i casi citati di edifici religiosi quando persero la loro funzione, ma è stato perseguito anche in altre regioni d'Europa e del mondo, per conservare traccia di un disastro naturale (ad esempio Igreja do Carmo, Lisbona) o di un bombardamento bellico (Cattedrale di Coventry, Genbaku Dome di Hiroshima). ${ }^{44}$ Il restauro di queste ultime architetture «ruskiniane», sebbene sia stato attuato nel XX secolo, appare conforme alle disposizioni teoriche del pensatore ingle- 
se, sia nella strategia di intervento, sia nell'esigenza di una manutenzione costante.

Allo stesso modo, l'attualità della linea segnata da Ruskin nei confronti del restauro è riscontrabile in una seconda eredità immediatamente contemporanea al proprio tempo, leggibile già attraverso le prime raccomandazioni delle Carte del Restauro. Già alcuni estratti dei documenti del 1882 sembrano concettualmente vicini alle teorie ruskiniane, adottandole quasi completamente:

Le disposizioni per lo studio dei restauri mirano ad ottenere, che si conoscano bene i monumenti, e si sappiano evitare gli errori in cui ora per lo più si cade, ricorrendo a rifacimenti non indispensabili, che spesso non rispettano né per forma né per sostanza l'antico, a ripristinamenti per cui si sopprimono ricordi storici o elementi di costruzione, o decorativi, e che hanno qualche importanza per la storia o per l'arte, a completamenti non studiati a sufficienza, che impongono interpretazioni discutibili, le quali possono forse essere dimostrate erronee. ${ }^{45}$

Allo stesso modo, si richiama implicitamente Ruskin nell'articolo nr. 7 della Carta di Atene del 1931 che raccomanda all'attenzione verso l'ambiente e la cura dell'elemento vegetale, specie quando è parte integrante del monumento, dimostrando un particolare interesse non solo per l'oggetto architettonico ma anche per il legame che esso ha costruito con l'ambiente circostante nel tempo. In ultimo, gli sforzi del critico inglese per la comprensione e la comunicazione dei valori intrinseci dell'architettura e dell'arte alle generazioni future ${ }^{46}$ attraverso gli aforismi della Lamp of Memory ${ }^{47}$ e a testi come The Bible of Amiens ${ }^{48}$ sembrano sorprendentemente ricollegabili alle ben più recenti affermazioni della Convenzione di Faro: «una comunità di eredità è costituita da un insieme di persone che attribuisce valore ad aspetti specifici dell'eredità culturale, e che desidera, nel quadro di un'azione pubblica, sostenerli e trasmetterli alle generazioni future». ${ }^{49}$

Appare quindi possibile leggere una molteplicità di eredità del pensiero di Ruskin nella contemporaneità: una legacy riguardante l'attenzione per le rovine, che non sembra essersi esaurita nel periodo vittoriano, ma piuttosto costituisce un filone di indagine e ricerca ancora di grande attualità.

L'interesse per i ruderi ha coinvolto molte discipline soprattutto nella seconda metà del XX secolo, quando il pensiero versoi resti archeologici si è trovato a sovrapporsi con quello delle rovine postbelliche, ${ }^{50}$ in una crisi psicologica di identità già indagata, non solo nel mondo del restauro e della conservazione. L'istanza psicologica, teorizzata da Roberto Pane ${ }^{51}$ ha potuto completare alcune riflessioni che già Freud aveva compiuto nei confronti delle rovine e del patrimonio del passato. ${ }^{52}$

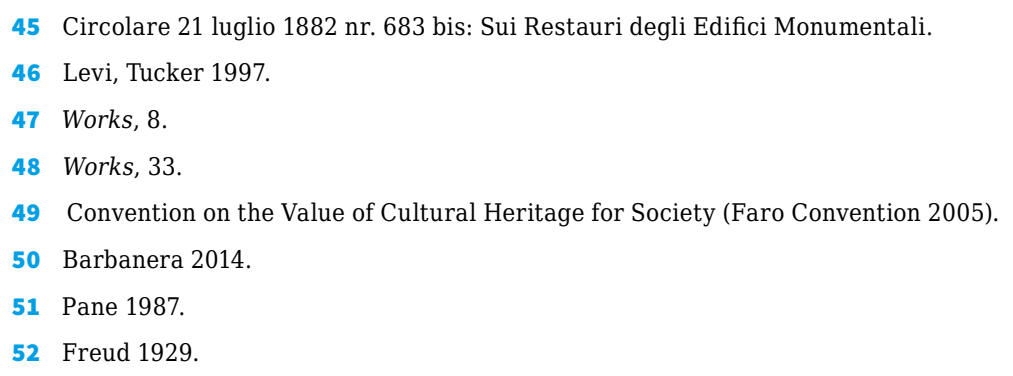

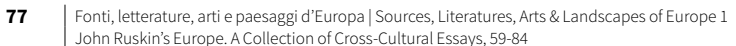




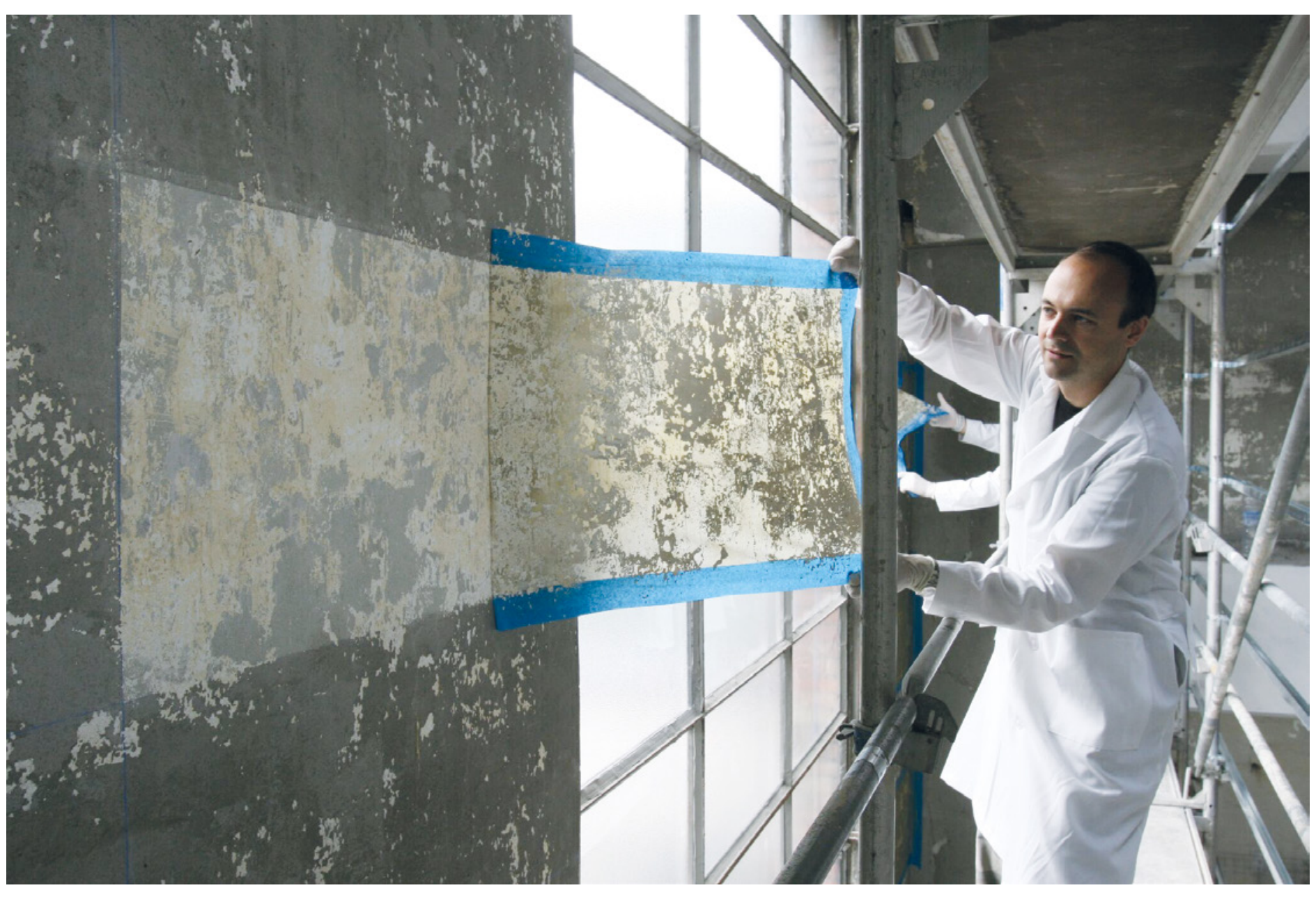

Figura 11 Jorge Otero-Pailos, The Ethics of Dust. 2008. Manifesta, Bolzano. Courtesy of Patrick Ciccone 


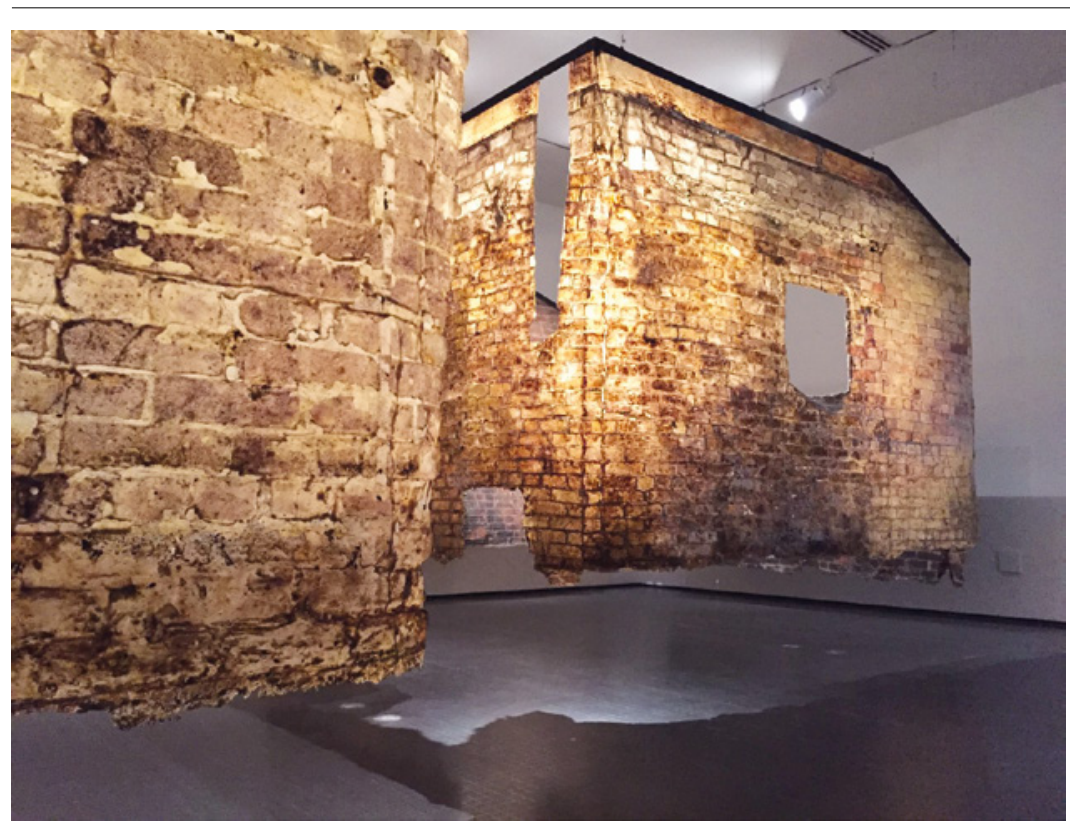

Figura 12 Jorge Otero-Pailos, The Ethics Of Dust: Old United States Mint. 2016. Mostra allestita nel 2016 a San Francisco, Museum of Yerba Buena. Courtesy of the Author

Questo legame fra rovina classica e rovina postbellica, all'interno di una ben più ampia bibliografia di riferimento, ${ }^{53}$ appare chiaramente sottolineato da due volumi differenti, ma entrambi legati alle teorie ruskinane. Il primo è Viaggio in Italia di Guido Piovene, ${ }^{54}$ che, soprattutto nei capitoli dedicati al Mezzogiorno, compie un'interessante descrizione capace di sovrapporre la memoria storica del mondo classico che ancora è possibile osservare tra i ruderi di templi e teatri, con le macerie e le rovine della Seconda guerra mondiale. Un secondo caso letterario, di carattere più europeo, è rappresentato da Pleasure of Ruins ${ }^{55}$ nel quale, dopo una lunga trattazione sul significato e sulla morfologia dei resti architettonici delle antiche civilità, l'autrice dedica il settimo capitolo A Note on New Ruins, alla descrizione e all'interpretazione critica dei nuovi monumenti in stato di rudere, fra cui la Cattedrale di Coventry. Entrambi questi volumi, molto differenti negli intenti editoriali, nell'ambito disciplinare di provenienza e nella prosa, sembrano mantenere un legame molto chiaro con le idee di Ruskin, riuscendo a comprendere il valore universale e a-temporale dei ruderi e il fascino che essi esercitano sull'uomo, al di là di interpretazioni storiche o delle ragioni che hanno causato l'abbandono 0 
la distruzione. Allo stesso modo, e in tempi più recenti, il concetto teorizzato da Carmassi ${ }^{56}$ di 'ruderi ricostruiti' per descrivere il palinsesto storico del centro urbano di Pisa, sembra legarsi alla lettura storica del manufatto in rovina e alla sua valenza nelle epoche passate. In maniera solo apparentemente opposta si pone invece il concetto di 'ruderi di nuova generazione' proposta da Andrea Bruno dove l'architetto ribadisce:

Ho scelto come titolo per questo intervento I ruderi di nuova generazione; il rudere non ha dimensione, può essere sia il più piccolo frammento sia la grande statua del Buddha. Il rudere è il risultato della volontà di distruzione dell'uomo o di cause naturali, è comunque un qualche cosa che, indipendentemente dalla sua dimensione e dalla sua forma deve essere protetto. ${ }^{57}$

La rovina contemporanea, intesa come maceria da $\mathrm{Au}-$ gè ${ }^{58}$ risulta alla base di altre manifestazioni culturali contemporanee che, in accordo con le disposizioni di Ruskin, mirano a privilegiare la dimensione estetica del bene incompleto. Dal Dopoguerra ad oggi l'interesse per le rovine è cresciuto esponenzialmente, toccando anche luoghi privi di memorie architettoniche di età classica. Le arti figurative degli Stati Uniti, ad esempio, hanno messo il rudere al centro di una serie di ricerche e sperimentazioni, dalle indagini sulle abitazioni abbandonate di Gordon Matta-Clarke, alle fotografie delle rovine della periferia di New York di Steven Siegel (1971-), alle prime sperimentazioni sulla land art con i Dialectical
Landscapes di Stevenson, fino alle ultime opere di Michael Rakowitz inerenti i resti degli enormi Buddha di Bamiyan in Afghanistan con What dust will rise? e le antiche statue votive dell'Iraq con The Ballad of Special Ops Cody. ${ }^{59}$ Fra le altre arti visive, la fotografia si è negli utlimi tempi avvicinata ai ruderi con manifestazioni varie, fra cui la più rilevante è associabile al movimento UrbEx o Urban Exploration che, nato in tempi recenti, si è diffuso rapidamente. L'esplorazione e la riconquista di luoghi abbandonati e inaccessibili, senza voler richiamare precedenti più aulici come le esplorazioni settecentesche del Grand Tour, è vicina alle riflessioni ruskiniane e anzi ne costituisce una ulteriore eredità contemporanea. La ricerca in questo caso, infatti, non si limita all'effetto estetico dell'abbandono e del conseguente scatto fotografico, ma è orientata anche verso una indagine nei confronti del recente passato industriale e architettonico e quindi verso i valori che sono ancora presenti all'interno dei ruderi. In maniera analoga, negli ultimi anni hanno visto la luce in Italia alcuni progetti interessanti, capaci di fondere differenti discipline e competenze con l'unico obiettivo comune di indagare i ruderi e le rovine contemporanee. ${ }^{60}$

Anche la ricerca architettonica statunitense, sebbene apparentemente libera dalle necessità della ricostruzione postbellica, si è misurata con il tema della rovina e del ricordo. Già negli anni Settanta, Venturi e Rauch avevano manifestato attenzione non tanto verso il concetto di rovina o di riproposizione stilistica di ciò che è perduto, quanto di riconfigurazione dei semplici valori
56

57 Bruno 2006

58 Augè 2003.

59 Blazwick, Christov-Bakargiev 2019.

60 Nella vasta bibliografia di riferimento, questo atteggiamento è riscontrabile sia in Incompiuto, la nascita di uno stile (Alterazioni video, 2018) sia nel progetto Recycle-Italy coordinato da numerosi Atenei italiani nel 2017. 
di un bene architettonico scomparso, arrivando a progettare a Philadelphia le Ghost Houses che tentano un superamento dell'idea ruskiniana di morte dell'edificio: consci della perdita e dell'impossibilità di ripristinare un bene del passato, la ricerca architettonica propone una nuova visione simbolica del bene, privando l'architettura della quasi totalità della componente formale ed elevandola a simbolo dell'antico, un fantasma del passato. L'architettura contemporanea, nonostante le enorme potenzialità scientifiche e tecnologiche, non sembra

\section{Conclusioni}

Oltre alle doverose considerazioni sul ruolo delle rovine nella contemporaneità appare, forse per una irrisolta comprensione dei manufatti, inquietantemente insoluta la strategia di intervento sul rudere. Come ricorda Giz$\mathrm{zi}^{63}$ le due polarità restano ancora distinte: la prima, di stampo ruskiniano i cui prodromi sono da ricercarsi nelle riflessioni di Schelling e Burke, suggerisce la sola conservazione, mentre la seconda raccomanda l'intervento diretto e la modifica del bene. Nonostante tale dualità, la disciplina del restauro ha indagato a fondo la tematica, misurandosi costantemente con il pensiero del critico inglese. La reale consapevolezza dell'importanza delle ricadute del pensiero di Ruskin sulla teoria della conseva- aver abbandonato il proprio rapporto con il passato; anzi, è possibile cogliere molte tendenze capaci di elevare la rovina a topos centrale nella progettazione del nuovo. Eclatanti in tal senso le parole di Zumthor ${ }^{61}$ che, interrogandosi sull'atmosfera dei luoghi, si chiede se sia proprio la «patina del tempo» (di ruskiniana memoria) a conferire una aggettivazione a spazi altrimenti asettici, così come la ricerca vicina alla teoria del progetto contemporaneo si è recentemente interrogata in merito al significato delle rovine contemporanee. ${ }^{62}$ zione attuale risulta evidente, non solo dalle recenti celebrazioni per il duecentennale della nascita, ${ }^{64}$ ma anche dal moltiplicarsi di riferimenti e rimandi che il restauro non può negare.$^{65}$ Le eredità ruskiniane hanno importanti ricadute nelle attuali strategie di conservazione di ruderi dal punto di vista teorico, ${ }^{66}$ storico ${ }^{67}$ e psicologico. ${ }^{68}$ Se è però vero che la polarità di cui si è detto resta alla base delle speculazioni attuali, è anche possibile andare oltre. Seguendo le riflessioni di Ruskin, la ricerca contemporanea ha ipotizzato di seguire fedelmente le estreme conseguenze del pensiero del critico, ipotizzando la necessità di preferire la demolizione, la distruzione di un bene, al suo abbandono o ad un suo pessimo restauro.

61 Zumthor 1998, 18.

62 Nicolini 2014; Ferlenga 2015; Menzietti 2017.

63 Gizzi 2006, 23; 2012, 5.

64 Caccia Gheradini, Pretelli 2019.

65 Bellini 1984; La Regina 2006; Carbonara 2019; Dezzi Bardeschi 2019.

66 Picone 2012, 27-39.

67 Pretelli 2010; Ottani Cavina 2018.

68 Napoleone 2019, 316-21. \begin{tabular}{l|l}
81 & $\begin{array}{l}\text { Fonti, letterature, arti e paesaggi d'Europa } \\
\text { John Sources, Literatures, Arts \& Landscapes of Europe } 1\end{array}$ \\
\end{tabular} 
Tali teorie hanno trovato spazio sia in ambito europeo ${ }^{69}$ sia nel contesto statunitense, dove le prime riflessioni di Lynch e Southworth ${ }^{70}$ hanno avuto seguito in più recenti studi, tesi ad abbracciare la possibilità, di fronte all'impossibilità di poter conservare tutto, di dover rinunciare almeno a parte del patrimonio esistente. ${ }^{71}$ Queste riflessioni, sebbene disegnino uno scenario estremo, sembrano essere coerenti con l'integralismo di Ruskin che costringeva ad una presa di posizione netta tra l'eliminazione di un edificio e la conservazione del valore autentico di un bene, senza compromessi di alterazione o mistificazione. In maniera analoga, l'influenza di Ruskin appare evidente anche nel lavoro artistico e di ricerca di Jorge Otero-Pailos che, rimandando esplicitamente al ruolo della patina espresso da Ruskin, rinforza l'eredità teorica trasformando i precetti dello storico inglese in performance in cui il 'mezzo pollice' di deposito superficiale è enfatizzato nella propria dimensioni estetica e documentaria. ${ }^{72}$
Tali molteplici eredità, come si è visto, appaiono di diretta derivazione dal pensiero di Ruskin e ne costituiscono ipotetiche prosecuzioni speculative. L'importanza e la vastità delle teorie del critico consentono e anzi invitano ad un approfondimento e un loro superamento. Occorre ribadire però come tale superamento si potrà rivelare possibile solo attraverso un opportuno esercizio di osservazione prima e di comprensione del patrimonio poi. Appare giusto quindi suggerire un completamento ipotetico all'iter concettuale Sensazione, Selezione e Percezione: solo attraverso una profonda Comprensione si potrà avere un corretto intervento su di un rudere. Saper vedere (e non solo guardare) le cose prima di volerle conservare, imparare a capirle prima di trasformarle:

Ruskin's mission in life, he used to say, was to teach people to see. ${ }^{73}$

\section{Bibliografia di John Ruskin}

I riferimenti alle opere di Ruskin rinviano al numero di volume e alle pagine di: Cook, E.T.; Wedderburn, A. (eds) (1903-1912). The Works of John Ruskin. Library Edition, 39 vols. London: George Allen. https://www.lancaster.ac.uk/the-ruskin/the-complete-works-of-ruskin/.

L'abbreviazione utilizzata è la seguente: Works, [vol.]: [p.].

Works, 1: Early Prose Writings. |3-7: Modern Painters I-V. | 8: The Seven Lamps of Architecture. |33: The Bible of Amiens, Valle Crucis, The Art of England, The Pleasures of England. |34: The Storm-cloud of the Nineteenth Century, On the Old road, Arrows of the Chace, Ruskiniana. Works, 36: The Letters of John Ruskin I (1827-1869).

69 Choay 1996

70 Lynch, Southworth 1990.

71 Cairns, Jacobs 2014.

72 Ebersberger, Zyman 2009; Burgio 2011, 95-106.

73 Works, 1: xxxix. 


\section{Bibliografia generale}

Alterazioni video; Fosbury Architecture (a cura di) (2018). Incompiuto: La nascita di uno stile. Milano: Humboldt books.

Assmann, A.; Gomille, M.; Rippl, G. (Hrsgg) (2002). Ruinenbilder. Wilhelm Fink Verlag: Munchen.

Augè, M. (2003). Les temps en ruines. Parigi: Galilee.

Barbanera, M. (2014). «Osservazioni marginali sul destino degli edifici antichi in rapporto alla modernità». Capuano, A. (a cura di), Paesaggi di rovine, paesaggi rovinati. Macerata: Quodlibet, 118-29.

Baridon, M. (1985). «Ruins as a Mental Construct». Journal of Garden History, 5, 84-96.

Bellini, A. (1984). «Riflessioni sull'attualità di John Ruskin». Restauro, 71-72, 63-84.

Blazwick, I.; Christov-Bakargiev, C. (a cura di) (2019). Michael Rakowitz = Catalogo della mostra organizzata dalla Whitechapel Gallery, London e dal Museo di Arte contemporanea di Rivoli, Torino. Milano: Silvana Editoriale.

Brilli, A. (a cura di) (1985). John Ruskin. Viaggi in Italia 1840-1845. Firenze: Passigli.

Burgio, V. (2011). «Dal dagherrotipo di John Ruskin all'impronta di Jorge Otero-Pailos: The Ethics of Dust: Doge's Palace, Venice, 2009». Migliore, T. (a cura di), Sulla 53 Biennale di Venezia. Quaderni della Biennale. Milano: Edizioni Et Al., 95-106.

Caccia Gheradini, S.; Pretelli, M. (a cura di) (2019). Memories on John Ruskin. Unto this Last (Florence, 29 November 2019). Firenze: Firenze University Press.

Cairns, S.; Jacobs, J. (2014). Buildings Must Die. A Perverse View of Architecture. Cambridge: MIT Press.

Caperna, G. (2017). «Sulla storiografia del restauro». Fiorani, D. (a cura di), Questioni teoriche: storia e geografia del restauro. Roma: Edizioni Quasar, 250-9.

Carbonara, G. (2019). «L'eredità smarrita di John Ruskin». ANANKE Quadrimestrale di cultura, storia e tecniche della conservazione per il progetto, 86, 6-8.

Cecchi, R. (2006). «Rudere e Carte del restauro». Billeci, B.; Gizzi, S.; Scudino, D. (a cura di), Il rudere tra conservazione e reintegrazione = Atti del convegno internazionale (Sassari, 26-27 settembre 2003). Roma: Gangemi, 107-10.

Choay, F. (1996). «De la démolition». Fortier, B. (éd.), Métamorphoses parisienne. Paris: Editions du Pavillon de l'Arsenal-Piere Mardaga Editeur. Clark, K. (1962). The Gothic Revival. Londra: John Murray Publisher.

Clark, K. (1964). «A Note on Ruskin's Writings on Art and Architecture». Ruskin Today. London: Murray, 133-4.

Cupperi, W. (a cura di) (2004). "Senso delle rovine e riuso dell'antico». Annali della Scuola Normale di Pisa, Quaderni 14.

Di Stefano, R. (1983). John Ruskin interprete dell'architettura e del restauro. Napoli: Edizioni scientifiche italiane.

Dezzi Bardeschi, M. (2019). «Vogliamo ravviare queste tremule, smarrite Seven Lamps?». ANANKE Quadrimestrale di cultura, storia e tecniche della conservazione per il progetto, 86, 2-3.

Ebersberger, E.; Zyman, D. (eds) (2009). Jorge Otero-Pailos: The Ethics of Dust. Köln: Walther König.

Fancelli, P. (2006). «Tempo, natura, rudero». Billeci, B.; Gizzi, S.; Scudino, D. (a cura di), /l rudere tra conservazione e reintegrazione = Atti del convegno internazionale (Sassari, 26-27 settembre 2003). Roma: Gangemi, 125-54.

Faro Convention (2005) = Council of Europe Framework Convention on the Value of Cultural Heritage for Society. Faro, Portugal, 27.10.2015.

Favaretto, I.; Pilutti Namer, M. (a cura di) (2016). Tra Roma e Venezia. La cultura dell'antico nell'italia dell'unità. Giacomo boni e i contesti. Venezia: Istituto Veneto di Scienze.

Ferlenga, A. (2015). Città e memoria come strumenti del progetto. Milano: Marinotti.

Ferrazza, M. (2008). Cattedrali della Terra. John Ruskin sulle Alpi. Torino: Cda e Vivalda editori.

Fiorani, D. (2009). «Architettura, rovina, restauro». Barbanera, M. (a cura di), Relitti riletti. Metamorfosi delle rovine e identità culturale. Torino: Bollati Boringhieri, 339-55.

Forti, L.C. (1983). John Ruskin: un profeta per l'architettura. Genova: Compagnia dei librai.

Freud, S. (1929). Das Unbehagen in der Kultur. Wien: Internationaler psychoanalytischer verlag.

Gamble, C.; Pinette, M.; Wildman, S. (2003). Ruskin - Turner: dessins et voyages en Picardie Romantique. Amiens: Musée de Picardie.

Garrigan, K.O. (1973). Ruskin on Architecture; His Thought and Influence. Madison: The University of Wisconsin Press.

Ginsberg, R. (2004). The Aesthetic of Ruins. New York; Amsterdam: Rodopi.

\begin{tabular}{l|l}
83 & Fonti, letterature, arti e paesaggi d'Europa | Sources, Literatures, Arts \& Landscapes of Europe 1 \\
John Ruskin's Europe. A Collection of Cross-Cultural Essays, 59-84
\end{tabular} 
Gizzi, S. (2006). «ll rudere tra conservazione e reintegrazione». Billeci, B.; Gizzi, S.; Scudino, D. (a cura di), Il rudere tra conservazione e reintegrazione = Atti del convegno internazionale (Sassari, 26-27 settembre 2003). Roma: Gangemi, 23-50.

Gizzi, S. (2012). «ll senso delle rovine oggi. Intervista a Giuseppe Galasso». Confronti. Quaderni di restauro architettonico della Soprintendenza per i beni architettonici, paesaggistici, storici, artistici ed etnoantropologici per Napoli e provincia, 0. Napoli: Arte'm, 5-16.

Hewison, R. (1976). Ruskin and the Argument of the Eye. London: Thames and Hudson.

Hewison, R. (2000). Turner Ruskin and the Pre-Raphaelites. London: Tate Modern Press.

Hilton, T. (1985). John Ruskin: The Early Years 1819-1854. New Haven; London: Yale University Press.

Huxley, A. (1942). The Art of Seeing. New York: Harper \& brothers.

La Regina, F. (2006). «ll rudere come oggetto e come evento: considerazioni critiche». Billeci, B.; Gizzi, S.; Scudino, D. (a cura di), Il rudere tra conservazione e reintegrazione = Atti del convegno internazionale (Sassari, 26-27 settembre 2003). Roma: Gangemi, 191-200

Lamberini, D. (a cura di) (2006). L'eredità di John Ruskin nella cultura italiana del Novecento. Firenze: Nardini.

Leoni, G. (a cura di) (1987). John Ruskin. Opere. Roma-Bari: Laterza.

Levi, D.; Tucker, P. (1997). Ruskin didatta. Venezia: Marsilio.

Lynch, K.; Southworth, M. (1990). Wasting Away. San Francisco: Sierra club.

Macaulay, R. (1953). Pleasure of Ruins. London: Walker and company.

Menzietti, G. (2017). Amabili resti d'architettura. Frammenti e rovine della tarda modernità italiana. Macerata: Quodlibet.

Morezzi, E. (2019a). «La percezione del paesaggio attraverso la visione di Turner. Riflessioni sull'idea di Etica e Natura in John Ruskin». Caccia Gheradini, S.; Pretelli, M. (a cura di), Memories on John Ruskin. Unto this Last (Florence, 29 November 2019). Firenze: Firenze University Press, 100-7.

Morezzi, E. (2019b). «Destruction/(Re)construction. Process and Strategies of Intervention in Historial Cities». Morezzi, E.; Haj Ismail, S. (eds), Post-War/Disaster Recovery of Historical Cities and Cultural Heritage Sites. Ankara: Aybu publishing, 13-88.

Napoleone, L. (2019). «L'attualità di John Ruskin: architettura come espressione di sentimenti alla luce degli studi estetici e neuroscientifici». Caccia Gheradini, S.; Pretelli, M. (a cura di), Memories on John Ruskin. Unto this Last (Florence, 29 November 2019). Firenze: Firenze University Press, 316-21.

Nicolini, R. (2014). "L’oro della memoria». Capuano, A. (a cura di), Paesaggi di rovine, paesaggi rovinati. Macerata: Quodlibet, 214-19.

Ottani Cavina, A. (2018). «John Ruskin, ritratto d'artista». John Ruskin. Le pietre di Venezia = Catalogo della Mostra omonima (Venezia, Palazzo Ducale, Appartamento del Doge, 10 marzo-10 giugno 2018). Venezia: Marsilio, 25-48.

Pane, R. (1987). «L'antico dentro e fuori di noi». Civita, M. (a cura di), Attualità e Dialettica Del Restauro. Chieti: Marino Solfanelli editore, $230-7$. Picone, R. (2012). «Il rudere architettonico nella storia del restauro». Confronti. Quaderni di restauro architettonico della Soprintendenza per i beni architettonici, paesaggistici, storici, artistici ed etnoantropologici per Napoli e provincia, 0. Napoli: Arte'm, 27-39.

Pilutti Namer, M. (2019). Giacomo Boni. Storia, memoria, archeonomia. Roma: L'Erma di Bretschneider.

Piovene, G. (1957). Viaggio in Italia. Milano: Mondadori.

Pretelli, M. (a cura di) (2010). Il riposo di San Marco. Santarcangelo di Romagna: Maggioli.

Rogers, S. (1822). Italy, a Poem. London: Cadell.

Romeo, E. (2019). «John Ruskin e l'architettura classica. La rovina nei contesti medievali come accumulazione della memoria». Caccia Gheradini, S.; Pretelli, M. (a cura di), Memories on John Ruskin. Unto this Last (Florence, 29 November 2019). Firenze: Firenze University Press, $134-41$.

Sdegno, E. (2004). Saggi su Ruskin. Stile retorica traduzione. Venezia: Cafoscarina.

Sdegno, E.; Reichler, C. (2013). John Ruskin, Écrits sur les Alpes. Textes réunis et présentés par E. Sdegno et C. Reichler. Traduction de A. Hélard. Venise: Marsilio.

Settis, S. (1986). «Continuità, distanza, conoscenza. Tre usi dell'antico». Settis, S. (a cura di), Memorie dell'antico nell'arte italiana, III. Dalla tradizione all'archeologia. Torino: Einaudi, 375-486.

Unrau, J. (1978). Looking at Architecture with Ruskin. London: Thames \& Hudson.

Zermani, P. (2010). Il muro di gomma. Parma: Diabasis.

Zermani, P. (2015). Architettura: luogo, tempo, terra luce, silenzio. Milano: Mondadori Electa.

Zumthor, P. (1998). Pensare architettura. Milano: Mondadori Electa. 


\title{
«Aratra Pentelici» di John Ruskin Insegnare l'arte greca dopo Winckelmann
}

Myriam Pilutti Namer

Università Ca' Foscari Venezia, Italia

\begin{abstract}
This research paper offers a preliminary analysis of the volume by John Ruskin "Aratra Pentelici" (1872), which contains five of the six lectures on sculpture he gave at Oxford in 1870. The article begins with an investigation of the structure of the volume, focusing on Ruskin's views on the arts and customs of the ancient Greeks. The essay continues with an attempt to enucleate the influence of Winckelmann's thought on Ruskin and the spread of the work of the English art historian in German culture, with particular reference to the work of the intellectual Charlotte Broicher. The article concludes by outlining a future study perspective, never attempted before, on Ruskin, as an original interpreter of the art of ancient Greece.
\end{abstract}

Keywords John Ruskin. Aratra Pentelici. Queen of the air. Johan Joachim Winckelmann. Carl Justi. Charlotte Broicher. Reception of classical antiquity. Reception of ancient Greek art.

Sommario 1 «AratraPentelici»: linee guida perla lettura di un trattato poco noto. -2 Ruskin lettore di Winckelmann? - 3 L'influenza del pensiero di Ruskin in Germania. - 4 Si può parlare di pensiero originale di Ruskin sull'arte greca?

\section{1 «Aratra Pentelici»: linee guida per la lettura di un trattato poco noto}

È senza nascondere un certo imbarazzo che Ruskin si accinge a fungere da «apologist for Greek Art». ${ }^{1}$ E si accosta al compito, richiesto dal suo ruolo di professore ad Oxford, intitolando il volume tratto dalle sue lezioni «Aratra Pentelici», ' 'i solchi del marmo pentelico', dove aratra implica l'azione del vomere che permetterà la semina, la maturazione delle se-

\footnotetext{
1 «I cannot easily express how strange it seems to me that I am obliged, here in Oxford, to take the position of an apologist for Greek Art» (Works, 20: 250).

2 Il volume, pubblicato nel 1872, verrà qui citato nell'edizione delle opere complete di John Ruskin a cura di Cook, Wedderburn 1903-1912, vol. 20 (1905) con la sigla «AP» (Works, 20).
} 
menti, il raccolto. Ne nasce una bella metafora che vede il marmo pentelico (elemento-simbolo dell'arte greca e romana) 'dare frutto' attraverso l'insegnamento e il corrispettivo apprendimento. Non è il solo testo dove Ruskin tratta o cita l'arte dell'antica Grecia; scrive infatti Charles Eliot Norton nell'introduzione all'edizione di Queen of the Air (1869) a sua cura, edita a New York nel 1893:

The study of Greek myths for the purpose of ascertaining their moral and spiritual significance, and their true relation to the religion of the Greeks, had been, for many years, a favourite pursuit of Mr. Ruskin. ${ }^{3}$

Aratra Pentelici è però interessante perché lo studio dell'arte greca vi è affrontato in forma organica; la nota critica all'edizione completa delle opere tramanda che le lezioni si tennero secondo questa partizione:

I. The Division of Arts (November 24); II. Imagination (November 26); III. Likeness (December 1); IV. Structure (December 3); V. The School of Athens (December 8); VI. The School of Florence (December 10). $(« \mathrm{AP} », 185)$

Nell'autunno del 1871 Ruskin ultimò per la pubblicazione, avvenuta nel 1872, sotto al titolo Aratra Pentelici, le prime cinque lezioni, dividendo la seconda in due capitoli (Idolatry e Imagination). ${ }^{4}$

Nell'indagare un soggetto per cui lo studioso sembra avere un interesse marginale, come l'arte greca, ${ }^{5}$ pare opportuno procedere, anzitutto, con una descrizione sin- tetica della struttura del volumetto. Com'è noto, le lezioni sulla scultura seguono le Seven Lectures on Art: scrive Ruskin stesso che le prime quattro erano state «wholly prefatory», le altre tre erano servite soltanto «to define methods of practice». Le lezioni sulla scultura erano intese come l'inizio di «systematic analysis e progressive study» della materia («AP», 199). Il progetto complessivo di Ruskin avrebbe implicato, inoltre, che a queste sarebbero seguiti un corso su «elementary characters on Architecture» e una terza serie di lezioni sulla scultura cristiana; progetto reso complicato dal suo sforzo «to direct the attention of the resident students to Natural History, and to the higher branches of ideal Landscape» $\mathrm{e}$ al quale Ruskin non diede seguito in questa precisa forma («AP», 196).

Nella prima lezione, Of the Division of Arts, Ruskin si preoccupa di definire agli studenti l'oggetto di studio, perché anche

painting and sculpture, no less than language, or than reasoning, have grammar and method - that they permit a recognizable distinction between scholarship and ignorance, and enforce a constant distinction between Right and Wrong. («AP», 193)

Lo studioso rifiuta la distinzione tra arti maggiori e minori, mentre propone la suddivisione dell'Arte in tre elementi:

We have simply three divisions of Art - one, that of giving colours to substance; another, that of giving

3 Ruskin 1893, v.

4 La sesta lezione (The School of Florence) rimase inedita e fu pubblicata per la prima volta nell'edizione delle opere complete. Come precisato dai curatori, che l'hanno correttamente espunta dopo che era stata inclusa in alcune edizioni di Aratra Pentelici, la lezione che tenne nel giugno del 1871, The Relation between Michael Angel and Tintoret, pubblicata da Ruskin nel 1872 con il titolo Seventh of the Course of Lectures on Sculpture, Delivered at Oxford, 1870-1871, non presenta connessioni stringenti con le lezioni pubblicate in Aratra Pentelici.

5 Sull'interesse di Ruskin per la mitologia greca si rimanda a Birch 1981, 1988. 
form to it without question of resistance to force; and the third, that of giving form or position which will make it capable of such resistance. («AP», 200-1)

cui si deve aggiungere anche l'osservazione che «generally speaking, Painting and Sculpture will be imitative, and Architecture merely useful» («AP», 204). La pittura e la scultura, infatti, «simply graphic», rispondono alle parole della Politica di Aristotele $(8,3)$ che Ruskin traduce come «having capacity and habit of contemplation of the beauty that is in material things», più oltre spiegate dal filosofo col concetto di chairein orthòs, «to have pleasure rightly» («AP», 209), che per Ruskin implica la ricezione da parte dello spettatore di una corresponsione. ${ }^{6}$ L'architettura, invece, «and its correlative arts, are to be practiced under quite other conditions of sentiment» («AP», 202).

Dopo aver accennato alla scienza della disposizione dei colori e della combinazione di linea e forma («AP», 209) Ruskin specifica ulteriormente quel che intende per scultura:

sculpture is essentially the production of a pleasant bossiness or roundness of surface; [...] the pleasantness of that bossy condition to the eye is irrespective of imitation on one side, and of structure on the other. («AP», 214)

\section{L'oggetto delle lezioni sarà la scultura imitativa,}

that being defined as the art which, by the musical disposition of masses, imitates anything of which the imitation is justly pleasant to us; and does so in accordance with structural laws having due reference to the materials employed. («AP», 218)
La prima parte della seconda lezione, pubblicata con il titolo Idolatry, pone la questione di quale debba essere il soggetto da scolpire, che Ruskin intende come subordinata alla domanda su quale dovrebbe essere l'oggetto preferibile della nostra immaginazione («AP», 221). Ruskin procede con lo stabilire le caratteristiche che una nazione (l'autore utilizza sempre questo termine) debba avere, vale a dire

Instincts of Mimicry, Idolatry, and Discipline, meaning, by the last, the desire of equity and wholesome restraint, in all acts and works of life. («AP», 229)

L'idolatria, per Ruskin «the desire of companionship with images», non è come le altre due naturale e giusta. Va compresa, indagata, definita. Giunge così a dividere le tre fasi delle «greatest races» in infanzia, età adulta e l'età della scienza e della razionalità, piena di rischi, dove la corruzione può annidarsi se non vi è dirittura morale («AP», 231-3), come riteneva avvenisse nella cultura a lui contemporanea («AP», 238-41) per la 'frivolezza' della gioventù e la sua sottomissione al denaro come a un dio («AP», 240). Continua quindi nella seconda parte della seconda lezione a trattare di immaginazione. L'idolatria imperante era per lui delle più servili e, accostata all'incapacità di esercitarsi in una immaginazione fertile, portava Ruskin a constatare, ribadire e confermare l'abissale differenza tra il suo tempo e il tempo dei Greci («AP», 244).

The entire Greek intellect was in a childish phase as compared to that of modern times. Observe, however, childishness does not necessarily imply universal inferiority: there may be a vigorous, acute, pure, and solemn childhood, and there may be a weak, foul, and ridiculous condition of advanced life; but the one is still

6 «So much as there is in you of ox, or of swine, perceives no beauty, and creates none: what is human in you, in exact proportion to the perfectness of its humanity, can create it, and receive» («AP», 209).

\begin{tabular}{l|l}
87 & $\begin{array}{l}\text { Fonti, letterature, arti e paesaggi d'Europa| Sources, Literatures, Arts \& Landscapes of Europe } 1 \\
\text { John Ruskin's Europe. A Collection of Cross-Cultural Essays, } 85-98\end{array}$
\end{tabular} 
essentially the childish, and the other the adult phase of existence. You will find, then, that the Greeks were the first people that were born into complete humanity. All nations before them had been, and all around them still were, partly savage, bestial, clay-encumbered, inhuman; sill semi-goat, or semi-ant, or semistone, or semi-cloud. [...] Fix your mind on this as the very central character of the Greek race - the being born pure and human out of the brutal misery of the past, and looking abroad, for the first time, with their children's eyes, wonderingly open, on the strange and divine world. («AP», 248-9)

Nella terza lezione, Likeness, Ruskin tratta del soggetto da scolpire, che deve essere «the spiritual power seen in the form of any living thing, and so represented as to give evidence that the sculptor has loved the good and hated the devil» («AP», 277). Il fine deve essere «to produce something which shall look as like Nature as possible» («AP», 284), qualcosa che sia comprensibile a tutti («AP», 287), «nothing but what is honourable» («AP», 291). E conclude:

1. Not only sculpture, but all other fine arts, must be for the people; 2 . They must be didactic to the people, and that as their chief end. The structural arts, didactic in their manner; the graphic arts, in their matter also; 3 . And chiefly the great representative and imaginative arts - that is to say, the drama and sculpture - are to teach what is noble in past history, and lovely in existing human and organic life; 4 . And the test of right manner of execution in these arts, is, that they strike, in the most emphatic manner, the rank of popular minds to which they are addressed; 5 . And the test of utmost fineness in execution in these arts, is that they make themselves be forgotten in what they represent; and so fulfill the words of their greatest Master, The best, in this kind, are but shadows. («AP», 300)
Quanto alla quarta lezione, Structure, vi si trovano qui descritte le leggi che devono regolare la composizione artistica: anzitutto, «to be beautiful, our structure must be produced with tools of men», e, in secondo luogo, «it must be composed of natural substances» («AP», 304); ancora «to exhibit the virtues of those materials, and aim at no quality inconsistent with them»; infine, «its temper is to be quiet and gentle, in harmony with common needs, and in consent to common intelligence» («AP», 309). Quanto alle tipologie esecutive, Ruskin ne indica quattro: «Flat Relief, Round Relief, Edged Relief, Full Relief» («AP», 323-4).

La quinta lezione, The School of Athens, ultima del volume che Ruskin licenziò, è dedicata a tracciare le principali differenze tra l'arte greca e l'arte fiorentina, anche se a partire dalla considerazione che fossero entrambe di «equal rank, as essentially original and independent» («AP», 333). Ma l'arte greca, come per Ruskin dimostra l'esempio di una Afrodite rappresentata su vaso, che paragona con una Venere di scuola fiorentina da incisione («AP», 336-7), «never expresses momentary passion and personal character», lì dove l'arte fiorentina sceglie proprio entrambe queste caratteristiche come obiettivo e tratto distintivo di bellezza («AP», 339-40) [figg. 1-2]. Infine l'arte greca non esprime «refined or naive beauty» («AP», 342), «divine strength» 0 «divine sadness» («AP», 345); un giudizio piuttosto severo, anche se temperato da alcune considerazioni conclusive:

It may not be sublime, nor beautiful, nor amusing; but it will be full of meaning, and faithful in guidance. [the Greek] will give you clue to myriads of things he cannot literally teach; and, so far as he does teach, you may trust him. Is not this saying much? («AP», 347)

He made as many [mistakes] as we do ourselves, nearly; - he died of his mistakes at last - as we shall die of them; but so far as he was separated from the herd of more mistaken and more wretched nations - so far 


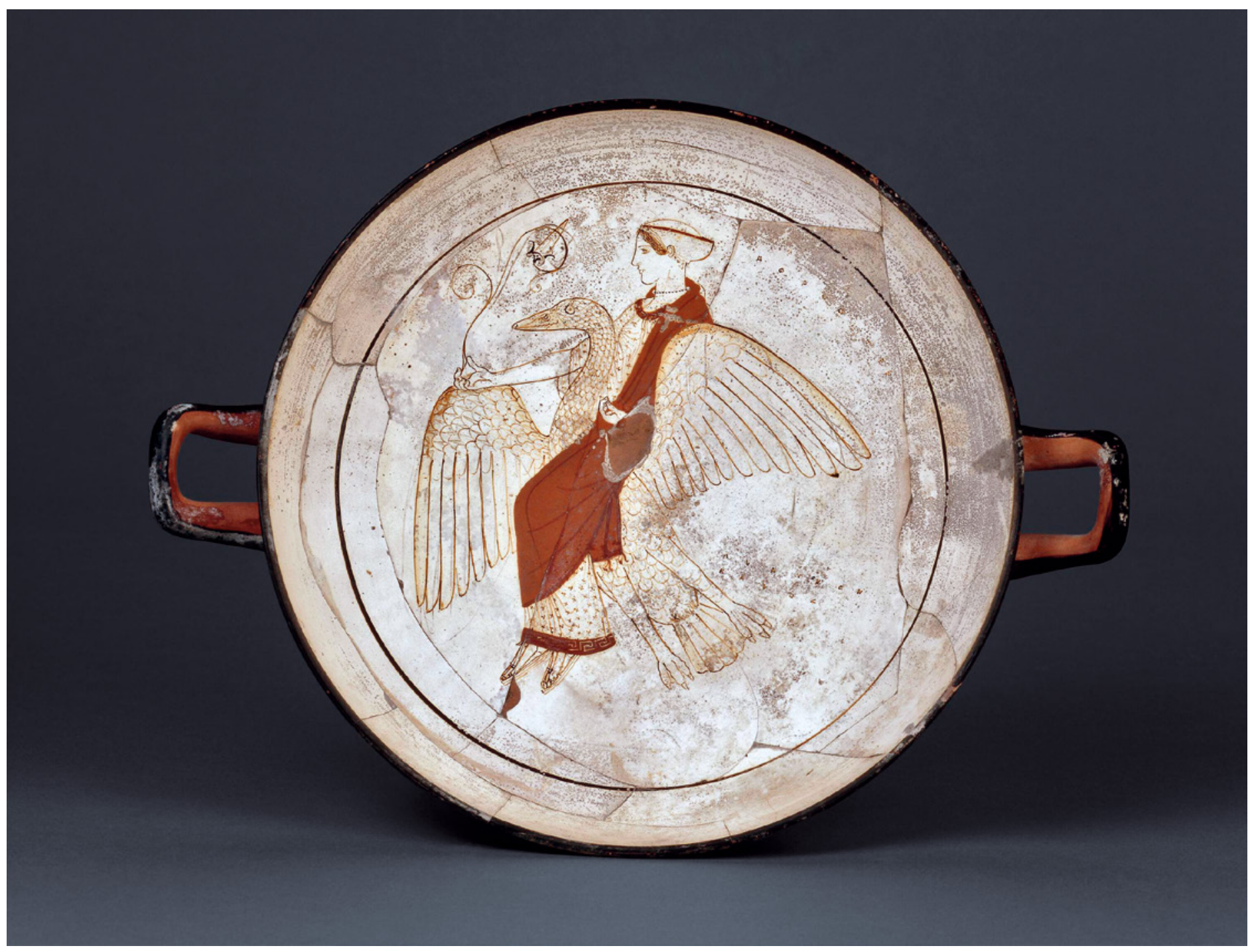

Figura 1 Afrodite assisa su un cigno. Tondo da una kylix a figure rosse a fondo bianco, dalla tomba F43 a Kameiros (Rodi). Nell'opuscolo «Aratra Pentelici» è indicata come «Aphrodite Urania. Photogravure from a Greek vase» («AP», 337-8). (c) The Trustees of the British Museum 
Figura 2 Venus. Quarantatreesima carta dei cosiddetti 'Tarocchi del Mantegna', qui nell'incisione di Hans Ledespelder, con annotazioni, conservata presso il British Museum e datata tra il 1530 e il 1561. Nell'opuscolo «Aratra Pentelici» è indicata come «Aphrodite Urania. Photogravure of an early Florentine engraving» («AP», 336-7). ( $)$ The Trustees of the British

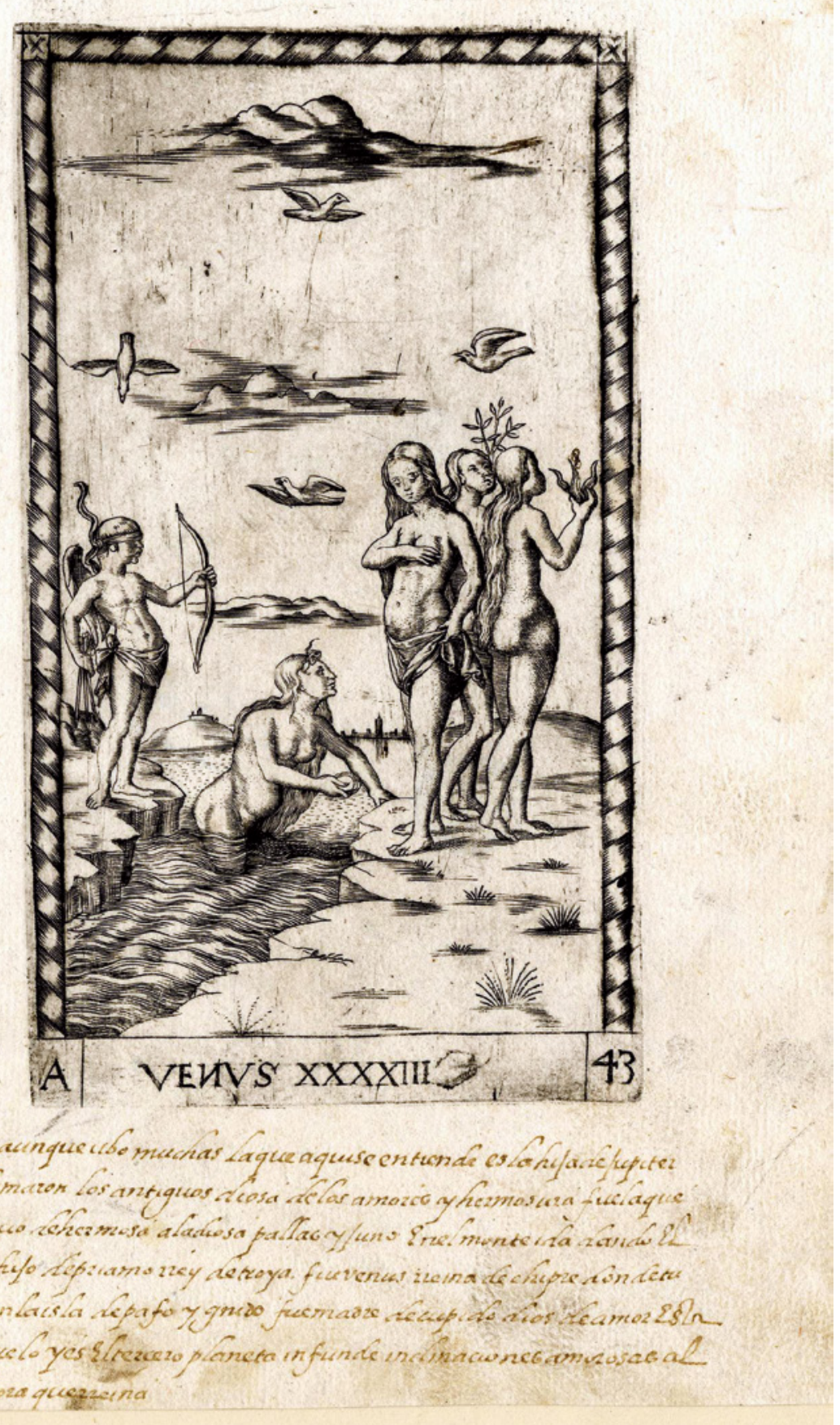


as was Greek - it was by his rightness. He lived, and worked, and was satisfied with the fatness of his land, and the fame of his deeds, by his justice, and reason, and modesty. He became Graeculus esuriens, little, and hungry, and every man's errand-boy, by his iniquity, and his competition, and his love of talk. But his Graecism was in having done, at least at one period of his dominion, more than anybody else, what was modest, useful, and eternally true; and as a workman, he verily did, or first suggested the doing of, everything possible to man. («AP», 351)

Abbiamo visto che l'argomentazione del trattatello è piut- tosto complessa: Ruskin prova una percepibile ammirazione per i Greci e più in generale per la cultura classica sia per il ruolo che vi ebbe la presenza del mito, ma anche nella filosofia e nella poesia, come dimostrano all'interno del testo le frequenti citazioni di Aristotele, Platone, Luciano di Samosata, Virgilio, Orazio, Pindaro. Assenti sono invece in Aratra Pentelici i drammaturghi greci (Eschilo, Sofocle, Euripide) e rare sono anche le citazioni omeriche. Ma si intuisce che il dover trattare della scultura greca fosse una scelta obbligata per un professore di Oxford che insegnava in un contesto europeo dove la scultura classica era ancora tratto distintivo di un partecipato milieu culturale.

\section{$2 \quad$ Ruskin lettore di Winckelmann?}

Nel considerare questo elemento il pensiero non può che rivolgersi al grande critico dell'arte classica la cui immensa fama si diffuse fino a tutto l'Ottocento: Johan Joachim Winckelmann (1717-1768). ${ }^{7}$ Ruskin non dice esplicitamente di averne lette le opere, ma almeno in un punto il riferimento pare evidente.

There is a school of teachers who will tell you that nothing but Greek art is deserving of study, and that all our work at this day should be an imitation of it. Whenever you feel tempted to believe them, think of these portraits of Athena and her owl, and be assured

that Greek art is not in all respects perfect, nor exclusively deserving of imitation. ${ }^{8}$

A Ruskin del resto non interessa discutere criticamente dell'arte greca: dà per assodata una cronologia tripartita [fig. 3] e la classificazione delle opere in senso evoluzionistico («AP», 231-2), dove l'arte ellenistica è interpretata come espressione di un periodo di decadenza. E non è questo l'unico tratto comune con il pensiero di Winckelmann: una fondamentale analogia vi è anche nell'impostazione didattica del discorso sull'arte greca, vale a dire nella tradizione di un insegnamento che deve essere

7 Negli anni 2017 e 2018 si è celebrato il doppio anniversario della nascita e della morte con ampia diffusione europea, portando all'edizione di numerose pubblicazioni, qui elencate in un'appendice bibliografica dedicata, alle quali si rimanda per ulteriori letture. Il filosofo inglese B. Bosanquet (1848-1923) accosta esplicitamente Ruskin a Winckelmann nella sua storia dell'estetica (1892, 445-54): «It is not too much to say that he like Winckelmann has given the mind a new organ for the appreciation of beauty»; la citazione è tratta da Works, $38: 162$.

8 «AP», 254. Il riferimento alle immagini di Atena con elmo raffigurate su monete è alle figg. 10-12 («AP», 252-3). Anche in Modern Painters, in un suo commento sul Laocoonte, Ruskin fa riferimento all'opera di Winckelmann senza citarla esplicitamente, ma è interessante notare, come già Cook e Wedderburn, che tralascia del tutto - o ignora - il Laokoon di Gotthold Ephraim Lessing (Works, 3: 121 e nota 4).

$91 \quad$ Fonti, letterature, arti e paesaggi d'Europa | Sources, Literatures, Arts \& Landscapes of Europe 1

John Ruskin's Europe. A Collection of Cross-Cultural Essays, 85 -98 
trasmesso agli studenti, e in particolare ai giovani artisti, per educarli; ${ }^{9}$ nonché sull'importanza che entrambi gli studiosi attribuiscono al 'sentimento', che però i due studiosi declinano in modo diverso: nei Gedanken Winckelmann sostiene, come ampiamente noto, che si può raggiungere il 'pensiero originale' (se possibile) attraverso l'imitazione' delle opere d'arte greca, ${ }^{10}$ Ruskin sostiene invece che bisogna scolpire 'quel che si sente' ispirandosi alla natura:

You must carve only what you yourself see as you see it; but, much more, you must carve only what you yourself feel, as you feel it. You may no more endeavour to feel through other men's souls, than to see with other men's eyes. Whereas generally now, in Europe and America, every man's energy is bent upon acquiring some false emotion, not his own, but belonging to the past, or to other persons, because he has been taught that such and such a result of it will be fine. Every attempted sentiment in relation to art is hypocritical our notions of sublimity, of grace, or pious serenity, are all second-hand: and we are practically incapable of designing so much as a bell-handle or a door-knocker, without borrowing the first notion of it from those who are gone - where we shall not wake them with our knocking. I would we could. («AP», 292)

La marginalità o la centralità della natura sono quindi l'elemento di discrimine tra Winckelmann, l'ispiratore della stagione del neoclassicismo europeo, e Ruskin, una delle personalità più influenti del romanticismo inglese. Se Winckelmann crede nella selezione già operata dai Greci delle opere meritevoli di essere imitate, quindi sostiene l'importanza del canone a discapito dello stu- dio della natura, Ruskin suggerisce che gli artisti studino 'anche' l'arte greca - 'anche', non esclusivamente. Entrambi coltivano la speranza che gli artisti possano divenire 'originali', vale a dire che entrambi si pongono anzitutto come educatori, condividendo il disprezzo per l'erudizione e l'accademismo. Sembrerebbe pertanto di poter dire che, a prescindere dal contesto (Winckelmann ebbe familiarità prevalentemente con l'arte antica e con i maestri del Rinascimento romano, Ruskin con l'arte medievale e il Rinascimento veneziano e fiorentino) e dagli interessi di studio e didattici (Winckelmann fu un indiscusso appassionato di valori plastici, mentre Ruskin preferiva la grafica, il colore e l'ornamento), i due condividessero l'esigenza di trasmettere i valori dell'arte greca e dell'arte del Rinascimento italiano ai propri lettori e studenti. Ruskin ne tratta implicitamente, rivolgendosi alla gioventù europea:

If, after I have set before you the nature and principles of the sculpture, in Athens, Pisa, and Florence, you consider these facts, - which you will then at once recognize as such, - you will find that they absolutely justify my assertion that the state of sculpture in modern England, as compared with that of the great Ancients, is literally one of corrupt and dishonourable death, as opposed to bright and fameful life. And now, will you bear with me while I tell you finally why this is so? The cause with which you are personally concerned is your own frivolity; though essentially this is not your fault, but that of the system of your early training. But the fact remains the same, that here, in Oxford, you, a chosen body of English youth, in nowise care for the history of your country, for its present dangers, or its present duties. You still, like children of seven or eight

9 Sul progetto didattico di Ruskin, in particolare sull'importanza del disegno ma anche in riferimento all'arte greca, si rimanda agli studi di Donata Levi e Paul Tucker: Levi, Tucker 1997, 1999, 2014

10 Nella traduzione italiana di Federico Pfister per Einaudi (1943), inclusa nella raccolta Il bello nell'arte. Scritti sull'arte antica, il riferimento è alla p. 19.

Fonti, letterature, arti e paesaggi d'Europa | Sources, Literatures, Arts \& Landscapes of Europe $1 \mid \quad 92$ 
years old, are interested only in bats, balls and oars: nay, including with you the students of Germany and France, it is certain that the general body of modern European youth have their minds occupied more seriously by the sculpture and painting of the bowls of their tobacco-pipes, than by all the divinest workmanship and passionate imagination of Greece, Rome, and Medieval Christendom. («AP», 239-40)

Winckelmann, inoltre, nei Gedanken über die Nachahmung der griechischen Werke in der Malerei und Bildhauerkunst (1755), indica anche quali artisti del Rinascimento italiano avevano a suo parere seguito la via degli antichi. E sceglie, tra i tre che preferisce (Raffaello, Michelangelo e Gianlorenzo Bernini), l'opera di Raffaello, perché era stato capace di evitare gli eccessi di temperamento. ${ }^{11}$ Così anche Ruskin ha artisti prediletti, anche Ruskin teme l'elemento astratto:

All second-rate artists will tell you that the object of fine art is not resemblance, but some kind of abstraction more refined than reality. Put that out of your heads at once. The object of the great Resemblant Art is, and always has been, to resemble; and to resemble as closely as possible [...]. All the talks about abstraction belongs to periods of decadence. («AP», 282-3)

Persino l'impostazione politica del discorso sull'arte vede tra Winckelmann e Ruskin alcuni tratti in comune: entrambi attribuiscono al denaro un valore strumentale, e indicano come qualità dell'artista di successo l'originalità (Winckelmann), l'autenticità e la funzione sociale (Ruskin), a prescindere dal riconoscimento del pubblico e dei guadagni. Winckelmann sottolinea l'importan-

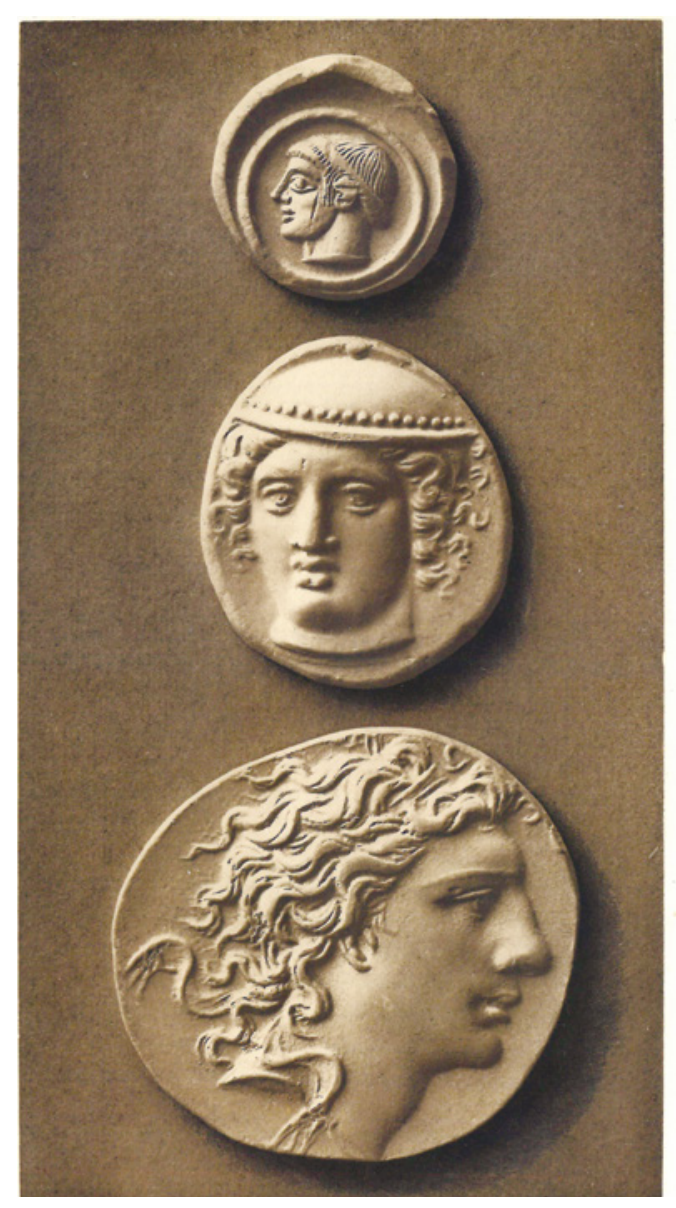

Figura 3 Esempi di monetazione dell'antica Grecia a cui Ruskin ricorre per esemplificare le fasi artistiche nel loro sviluppo tripartito dall'età arcaica attraverso l'apogeo fino al declino. Nell'opuscolo «Aratra Pentelici» la didascalia riporta: «Archaic, Central, and Declining Art of Greece. Photogravure from Greek coins» («AP», 280) za dell'autonomia economica per un artista che aspiri a

11 Nella traduzione italiana di Federico Pfister per Einaudi (1943), inclusa nella raccolta Il bello nell'arte. Scritti sull'arte antica, il riferimento è alle pp. 19 e 66.

$93 \quad$ Fonti, letterature, arti e paesaggi d'Europa | Sources, Literatures, Arts \& Landscapes of Europe 1

Fonti, etterature, arti e paesaggi d'Europa SOurces, Literatures, Arts 2 .
John Ruskin's Europe. A Collection of Cross-Cultural Essays, $85-98$ 
diventare 'originale', Ruskin sostiene che l'artista debba realizzare opere per tutti giudicando un male dei tempi il servilismo nei confronti del denaro assurto a dio. Entrambi gli studiosi sono consapevoli che non tutti i loro lettori e studenti sarebbero riusciti a seguire e a realizzare i loro insegnamenti, e anzi soltanto una netta mi- noranza era destinata a raggiungere la grandezza; dice infatti Ruskin:

Remember, the second-rate ones are a loquacious multitude, while the great come only one or two in a century; and then, silently. («AP», 282)

\section{L’influenza del pensiero di Ruskin in Germania}

Si è accennato al pensiero di Winckelmann, imprescindibile riferimento culturale per l'arte antica nell'Ottocento, per restituire un più corretto inquadramento storico alle osservazioni di Ruskin sull'arte greca: se anche quindi costituisce un interesse generalista, dove assenti risultano l'osservazione dal vero e la conoscenza diretta delle opere d'arte antica, le sue riflessioni in materia presentano aspetti di originalità. Si è pertanto ritenuto opportuno verificare se vi sia stato un qualche influsso del pensiero di Ruskin nel vivace dibattito sull'arte greca che animò la Germania nell'Ottocento. E.T. Cook e A. Wedderburn, nel volume 38 delle opere, riportano che la fama dello studioso inglese era perfino maggiore in Germania che in Inghilterra, e attribuiscono l'inizio di questa moda («fashion») all'affermazione del linguista e critico letterario Eduard Engel (1851-1938), che definì Ruskin «the Englishman's Winckelmann and Lessing in one». ${ }^{12}$ Com'è noto la scuola tedesca di antichistica, che nasce dal pensiero di Winckelmann, diviene paradigmatica a partire dagli anni Quaranta, in particolare grazie al lavoro di impronta filologica compiuto da Heirich Brunn

e da Ernst Curtius. Di diretta filiazione winckelmanniana è invece il pensiero sull'arte antica di Carl Justi, contemporaneo di Ruskin, detentore della cattedra di archeologia classica a Bonn e autore dell'opera fondamentale in tre volumi Winckelmann: sein Leben, Seine Werke und sein Zeitgenossen (1866-72). ${ }^{13}$ Pare quindi interessante constatare che una sua amica e corrispondente, che fu vicina anche a Ernst Curtius, la misconosciuta Charlotte Broicher, fu non solo tra coloro che tradussero in tedesco la selezione di opere di Ruskin, edita in quindici volumi a Lipsia per i tipi di Eugen Diederichs tra il 1900 e il 1906, curando l'edizione di due volumi di Modern Painters [fig. 4], ma al contempo fu autrice di tre volumi biografici, John Ruskin und sein Werk, editi a Lipsia e a Jena tra il 1902 e il $1907 .{ }^{14}$ Il suo interesse per Ruskin, figura vicina al pensiero tradizionale sull'arte greca di scuola tedesca (Curtius da un lato, Justi dall'altro), mi sembra confermi come l'apporto della lettura di Winckelmann nella riflessione dello storico dell'arte inglese fosse chiaramente percepibile da qualcuno che doveva conoscere bene le opere di entrambi. Lo intuì del resto lo stesso

12 Works, 38: xii e, per la citazione (tratta dalla traduzione inglese del 1902 dell'opera in tedesco), 167.

13 Justi 1866-1872. Su Carl Justi vedi Rößler 2012 e Marten, Kanz 2016.

14 I tre volumi hanno per sottotitolo: Puritaner, Künstler, Kritiker (Broicher 1902), Kunst-Kritiker und Reformer (Broicher 1907a), Sozialreformer, Professor, Prophet (Broicher 1907b). La sua opera fu recensita da Marcel Proust ne La Chronique des arts et de la curiosité del 2 gennaio 1904. Un sintetico profilo della studiosa si trova in Rößler 2012, 475, dove si apprende che a Ernst Curtius aveva anche dedicato un libretto di memorie nel 1897 (Erinnerungen on Ernst Curtius). 


\section{JOHN RUSKIN MODERNE MALER}

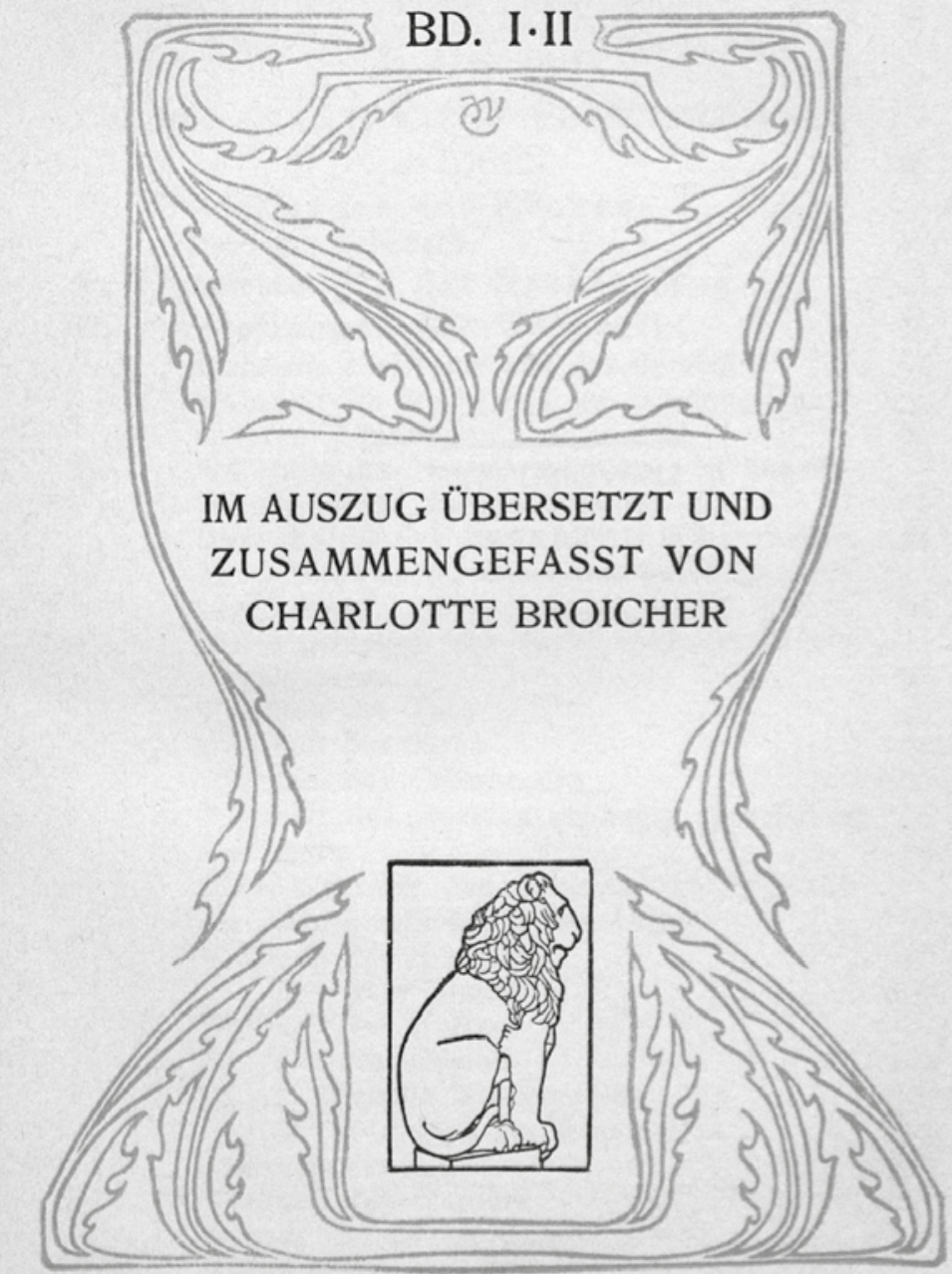

VERLEGT BEI EUGEN DIEDERICHS, LEIPZIG 1902.
Figura 4 Frontespizio dell'opera di John Ruskin, Ausgewählte Werke in vollständiger Übersetzung (Bde. 11/12). Moderne Maler (Bde. 1-2): Broicher 1902. Universitätsbibliothek Heidelberg, digital resource. https://doi.org/10.11588/diglit.3877\#०००5 
Carl Justi, anche se in una lunga lettera a Broicher del luglio 1899, di recente edita da Johannes Rößler (2012), non nasconde la sua delusione per l'assenza di pensiero sistematico in Ruskin: lo ritiene un grande maestro di stile («ein großer Stilist») ma anche un ancor più grande folle («aber ein noch größerer Narr»). ${ }^{15}$

\section{Si può parlare di pensiero originale di Ruskin sull'arte greca?}

Un altro aspetto interessante che vale la pena menzionare, in attesa di ulteriori approfondimenti, consiste nel valutare se le osservazioni sull'arte greca di Ruskin abbiano contribuito ad elaborare pensiero originale. Al proposito sembra interessante ricordare che lo stesso Charles Eliot Norton teneva da conto le opinioni di Ruskin in materia, come dimostra la prefazione già citata dell'edizione americana del 1893 a Queen of the Air, e le condivideva anche con i suoi allievi quali, ad esempio, Bernard Berenson, che esprime ammirazione per il volumetto. ${ }^{16}$ Inoltre, la riflessione sull'arte greca come espressione di ricerca di uguaglianza, vale a dire il rapporto tra apogeo artistico e sviluppo di cultura democratica, è idea condivisa da Ruskin, Norton e Berenson, secondo l'assioma che vede il Rinascimento fiorentino come corrispettivo dello splendore dell'arte della Grecia classica perché caratterizzato da un comune anelito alla pari dignità tra i cittadini. ${ }^{17}$ Per quanto ricca di ideali, la convinzione che l'emancipazione politica e la raffinazione artistica vadano di pari passo, si può spiegare come una forma di nostal- gia, di Sehnsucht, per tempi incontaminati e 'sinceri' che non esistevano più. Ė però importante sottolineare che nell'ambito di questa riflessione l'elemento ideale è talmente forte da indurre persino Ruskin ad attribuire un valore all'insegnamento dell'arte greca, nonostante si trattasse di una materia che forse i 'nordici' non potevano capire del tutto:

Gentlemen, we of the rough northern race may never, perhaps, be able to learn from the Greek his reverence for beauty; but we may at least learn his disdain of mechanism: - of all work which he felt to be monstrous and inhuman in its imprudent dexterities. («AP», 354)

A conferma dello stretto legame che nel pensiero di Ruskin intercorre sempre tra analisi della realtà contemporanea e riflessione artistica, si conclude osservando che Aratra Pentelici è costellato di preoccupazioni per la guerra franco-prussiana e termina con parole di apprensione per l'assedio di Parigi. È noto che la fine di

15 Rößler 2012, 154. Della conoscenza del Winckelmannsrede nell'opera di Walter Pater, in particolare nella stesura del noto saggio del 1867, hanno scritto da ultimi Stefano Evangelista e Katherine Harloe (2017, 66-7); vedi anche Jenkins 2018, 62-3. Evangelista e Harloe giustamente evidenziano il contributo che fornì alla formazione del pensiero di Pater e al dibattito in lingua inglese la traduzione della Storia dell'arte nell'antichità (Geschichte der Kunst des Altertums, 1764) di Winckelmann, avvenuta in forma parziale nel 1850 a opera di Giles Henry Lodge (1805-1888) con il titolo The History of the Ancient Art among the Greeks (Evangelista, Harloe 2017, 67). Forse Ruskin conosceva il volume nonostante non sia censito nella sua biblioteca (Dearden 2012); come ha dimostrato Paul Tucker (2020, 33-4) probabilmente conosceva anche i Monumenti antichi inediti (Roma 1767).

16 Mostyn-Owen 2014, 240, riporta la citazione da Berenson (One Year's Reading for Fun, 1942): «Ruskin's Queen of the Air, eloquent, magniloquent, unctuous, mad, absurd, penetrating, visionary, fantastic, all in one. What a curious mytho-poet was Ruskin. How conveniently he handles the Greek deities with the freedom of old friends whose secret histories he alone knows. Yet what sublime impudence on the part of Ruskin».

17 Scrive Ruskin: «The Greek school of sculpture is formed during, and in consequence of, the national effort to discover the nature of justice; the Tuscan, during and in consequence of, the national effort to discover the nature of justification» («AP», 228). 
quella guerra cambiò gli equilibri geopolitici europei, ridimensionando le aspirazioni della Francia e accrescendo il potere del Secondo Reich. Anche trattando di arte greca Ruskin non riusciva a tralasciare il presente; talora interpreta il pensiero degli studenti, cui fa attribuire il commento «irrelevant matter» alle questioni attuali che lui solleva. La sensibilità di Ruskin detta loro l'invito sempre attuale a interessarsi del futuro dell'Eu- ropa, a non cullarsi nella propria «frivolity», a dedicarsi alle 'arti delle pace':

I know it my duty to assert to you that the work we enter upon to-day is no trivial one, but full of solemn hope; the hope, namely, that among you there may be found men wise enough to lead the national passions towards the arts of peace, instead of the arts of war. («AP», 200)

\section{Bibliografia di John Ruskin}

I riferimenti alle opere di Ruskin rinviano al numero di volume e alle pagine di:

Cook, E.T.; Wedderburn, A. (eds) (1903-1912). The Works of John Ruskin. Library Edition, 39 vols. London: George Allen.

https://www.lancaster.ac.uk/the-ruskin/the-complete-works-of-ruskin/.

Works, 3: Modern Painters I. | 20: Lectures on Art, Aratra Pentelici, Lectures and Notes on Greek Art and Mythology (1870). | 38: Bibliography, Catalogue of Ruskin's Drawings, Addenda et Corrigenda.

\section{Bibliografia generale}

Birch, D. (1981). «Ruskin and the Science of Proserpina». Hewison, R. (ed.), New Approaches to Ruskin. London: Routledge, $142-7$.

Birch, D. (1988). Ruskin's Myths. Oxford: Clarendon Press.

Broicher, C. (1902). John Ruskin und sein Werk. Bd. 1, Puritaner, Künstler, Kritiker. Leipzig: Diederichs.

Broicher, C. (1907a). John Ruskin und sein Werk. Bd. 2, Kunst-Kritiker und Reformer. Leipzig: Diederichs.

Broicher, C. (1907b). John Ruskin und sein Werk. Bd. 3, Sozialreformer, Professor, Prophet. Leipzig: Diederichs.

Dearden, S (2012). The Library of John Ruskin. Oxford: Oxford Bibliographical Society.

Evangelista, S.; Harloe, K. (2017). «Pater's 'Winckelmann': Aesthetic Criticism and Classical Reception». Martindale, C. et al. (eds), Pater the Classicist. Classical Scholarship, Reception and Aestheticism. Oxford: Oxford University Press, 63-80. https://doi.org/10.1093/ acprof:oso/9780198723417.003.0004.

Harloe, K. (2013). Winckelmann and the Invention of Antiquity: History and Aesthetics in the Age of Altertumswissenschaft. Oxford: Oxford University Press. https://doi.org/10.1093/acprof:oso/9780199695843.001.0001.

Harloe, K. (2018). «Winckelmania: Hellenomania Between Ideal and Experience». Harloe, K. et al (eds), Hellenomania. London; New York: Routledge; Taylor \& Francis Group, 40-55. https://doi.org/10.4324/9781315277370-3

Jenkins, R. (2018). «The British Literary Reception of Greek Visual Culture in the Nineteenth and Twentieth Century». Harloe, K. et al (eds), Hellenomania. London; New York: Routledge; Taylor \& Francis Group, 56-72. https://doi.org/10.4324/9781315277370-4.

Justi, C. (1866-1872). Winckelmann und seine Zeitgenossen. 2 Bde. Leipzig: Koehler \& Amelang.

Levi, D.; Tucker, P. (1997). Ruskin didatta. Il disegno tra disciplina e diletto. Venezia: Marsilio.

Levi, D.; Tucker, P. (1999). «'A Line of Absolute Correctness'. Ruskin's Enlargements from Greek Vases and the Drawing Classes at Oxford». Birch, D. (ed.), Ruskin and the Dawn of the Modern. Oxford: Clarendon Press, 87-110.

97 Fonti, letterature, arti e paesaggi d'Europa | Sources, Literatures, Arts \& Landscapes of Europe 1 John Ruskin's Europe. A Collection of Cross-Cultural Essays, 85-98 
Levi, D.; Tucker, P. (2014). «"Drawing is a kind of language”: la didattica artistica in John Ruskin e nel dibattito inglese coevo». Annali di Critica d'Arte, 10, 215-55.

Marten, B.; Kanz, R. (Hrsgg) (2016). Carl Justi und die Kunstgeschichte. Frankfurt am Main: Vervuert.

Mostyn-Owen, W. (2014). «Bernard Berenson and Kenneth Clark». Connors, J.; Waldman, L.A. (eds), Bernard Berenson. Formation and Heritage. Villa I Tatti: The Harvard University Center for Italian Renaissance Studies, 231-47.

Rößler, J. (Hrsg.) (2012). Carl Justi. Moderne Irrtümer: Briefe und Aphorismen. Berlin: Matthes \& Seitz.

Ruskin, J. (1893). The Queen of the Air, Being a Study of the Greek Myths of Cloud and Storm. With an Introduction by C.E. Norton. New York: Maynard, Merryll \& Co. Publishers.

Tucker, P. (2020). «A 'New Clue': Ruskin's Guide to the Principal Pictures in the Academy of Fine Arts at Venice (1877), the History of Venetian Art and the Idea of the Museum». Journal of Art Historiography, 22, 1-38.

\section{Su Winckelmann}

Agazzi, E.; Slavazzi, F. (a cura di) (2019). Winckelmann, l'antichità classica e la Lombardia. Roma: Artemide.

Balestreri, I.C.R. (a cura di) (2018). Arte e cultura fra classicismo e lumi: omaggio a Winckelmann. Milano: Jaca book.

Beyer, A. et al. (Hrsgg) (2018). Winckelmann und die Schweiz. Petersberg: Michael Imhof Verlag.

Bomski, F. et al. (2017). Die Erfindung des Klassischen. Winckelmann-Lektüren in Weimar. Göttingen: Wallstein Verlag.

Bragantini, I. (a cura di) (2019). Winckelmann e l'archeologia a Napoli. Napoli: Università degli studi di Napoli l'Orientale.

Bruni, S.; Meli, M. (a cura di) (2018). La Firenze di Winckelmann. Pisa: Edizioni ETS.

Cambi, F.; Catalano, G. (a cura di) (2019). Johann Joachim Winckelmann e l'estetica della percezione. Roma: Istituto italiano di studi germanici.

Debenedetti, E. (a cura di) (2018). Johann Joachim Winckelmann (1717-1768) nel duplice anniversario. Roma: Quasar.

Décultot, É. et al. (Hrsgg) (2017). Winckelmann Moderne Antike. München: Hirmer.

Dodero, E.; Parisi Presicce, C. (a cura di) (2018). Il tesoro di antichità. Winckelmann e il Museo Capitolino nella Roma del Settecento. Roma: Gangemi.

Ferrari, S. (a cura di) (2019). La rete prosopografica di Johann Joachim Winckelmann. Bilancio e prospettive. Roma: Edizioni di storia e letteratura.

Foi, C.; Panizza, P. (a cura di) (2019). Winckelmann privato. Conseguenze di una morte inaudita. Trieste: EUT Edizioni Università di Trieste. Hase, F.-W. von (Hrsg.) (2017). Die Kunst der Griechen mit der Seele suchend. Winckelmann in seiner Zeit. Darmstadt: Verlag Philipp von Zabern.

Kunze, M. (Hrsg.) (2014). El legado de Johann Joachim Winckelmann en España. Mainz; Ruhpolding: Rutzen.

Kunze, M.; Lappo-Danilevskij, K.J. (Hrsgg) (2017). Antike und Klassizismus. Winckelmanns Erbe in Russland. Petersberg: Michael Imhof Verlag.

Walser, R. (Hrsg.) (2019). Johann Joachim Winckelmann und Bayern: eine europäische Dimension. München: Katholische Akademie in Bayern. 


\title{
La corrispondenza epistolare come rete di conoscenza, dibattito e azione Le riflessioni sulle arti e sulla tutela di Philip Webb, Giacomo Boni e John Ruskin
}

Andrea Paribeni

Università degli Studi di Urbino «Carlo Bo», Italia

Silvia Pedone

Accademia Nazionale dei Lincei Roma, Italia

\begin{abstract}
Taking as reference point the figure of John Ruskin, we intend to explore in this paper the relationship between two interesting personalities of the European artistic world at the end of the 19thcentury, Philip Webb and Giacomo Boni. Through the correspondence of these scholars held in Italian and British Archives, we consider here a variety of topics concerning the political heritage, literary and art-historical debates in modern Europe. In particular the epistolary reports and archival documentation, relating to the inspections upon monuments carried out by Boni throughout the Italian territory, reveal a sympathetic reception of the Ruskinian theories and indications concerning restoration, albeit with an application 'tempered' by the need to realize concrete and effective solutions respecting and safeguarding a monument.
\end{abstract}

Keywords Cultural Heritage Conservation. Archival documents. Anglo-Italian Cultural Relationship. Medieval Monuments in Italy. Byzantine Sculptures.

Sommario 1. Introduzione. - 2. John Ruskin, il disegno e i rapporti con Giacomo Boni. - 3. Philip Webb e il suo epistolario con Giacomo Boni.

\section{Introduzione}

Il contributo si fonda sull'analisi di corrispondenze epistolari e altri documenti, alcuni già editi, che in gran parte si conservano a Milano, presso l'Istituto Lombardo Accademia di Scienze e Lettere (ILASL), in un fondo che è stato battezzato dai suoi catalogatori 'Archivio Boni-Tea'. ${ }^{1}$ La duplicità della denominazione, che rinvia alle distinte personalità di Giacomo

1 Paribeni, Guidobaldi 2020. 
Boni ed Eva Tea, è dovuta alla articolata composizione del fondo, originato da un nucleo iniziale di documenti personali e di studio che, per disposizione testamentaria, alla morte dell'archeologo veneziano nel luglio del 1925 vennero affidati alla sua silentiaria ab epistulis, con il compito di curarne l'edizione delle parti pubblicabili; ${ }^{2}$ ad essi si aggiunsero, nel corso del tempo, moltissimi altri materiali reperiti e/o prodotti da Eva Tea al fine da un lato di approfondire la conoscenza di quel venerato maestro, con il quale la allora giovane storica dell'arte aveva condiviso solo gli ultimi anni di vita, dall'altro di dare maggiore sistematicità e spessore a quel cospicuo gruppo di inediti boniani dedicati alle risultanze degli scavi del Foro romano e del Palatino che, per una serie di sfortunate circostanze - culminate nei bombardamenti di Milano dell'agosto del 1943 che distrussero gli stabilimenti tipografici ove il volume curato dalla Tea era in bozze - tali sono rimasti fino ad oggi. L'uscita del volume, curato da Andrea Paribeni e Federico Guidobaldi, non si prefigge di pubblicare per esteso tali scritti, quanto piuttosto di fornire un catalogo sistematico dei documenti del fondo, che già Eva Tea aveva suddiviso in cartelle secondo un criterio di articolazione tematica rispecchiante i molteplici campi di studio del Maestro: archeologia, flora, conservazione e tutela dei monumenti, nonché una variegata serie di interessi che, fino ai limiti dell'estroso, toccavano i più disparati temi economici, educativi, politici e sociali [fig. 1]. ${ }^{3}$

Dal catalogo sistematico sono rimaste escluse le numerose cartelle dell'epistolario di Giacomo Boni: la de- cisione di non includere nel volume questo segmento dell'archivio è stata molto ponderata, ma alla fine nella valutazione hanno prevalso criteri di omogeneità rispetto al blocco delle cartelle tematiche. Mentre, come si è detto, queste ultime sono costituite da lotti di documenti di vario genere (elaborati e bozze di articoli, relazioni ministeriali, lettere, appunti, disegni, fotografie) affini tra loro per argomento generale che è esplicitato dal titolo della cartella stessa, quelle dell'epistolario contengono solo - o quasi solo - lettere suddivise secondo l'ordine alfabetico dei corrispondenti e quindi necessiterebbero di un approccio diverso, da condursi secondo modalità di catalogazione ed edizione più adatte a questa specifica tipologia di documenti.

Nel novero di queste lettere - per le quali è stato fornito comunque un elenco dei nominativi dei corrispondenti $^{4}$ - si trovano quelle di cui si darà conto in questa sede. Si tratta di lettere che dimostrano in quale misura il giovane ed intraprendente Boni seppe creare, e in alcuni casi mantenere nel tempo, una complessa rete di rapporti con le più rappresentative personalità artistiche del mondo anglosassone e non solo: John Ruskin (e assieme a lui anche la cugina Joan Severn) e Philip Webb, di cui si parlerà più partitamente in questa occasione, ma accanto ad essi William Morris, William Douglas Caroë e John Hebb nonché contatti indiretti con una serie di altre autorevoli figure di artisti e intellettuali come, tra le tante, Francisca Alexander, John Whalton Bunney, William Lethaby, Charles Fairfax Murray, Charles Eliot Norton, Maria Spartali Stillman. ${ }^{5}$ (A.P., S.P.)

2 Con tale titolo Boni la investì sin dal loro primo incontro al Foro avvenuto nel 1915 (Tea 1932, 2: 364). Su Eva Tea vedi da ultimo Pilutti Namer 2018; 2019, 73-80.

3 Paribeni, Guidobaldi 2020. Per precedenti presentazioni della composizione e dei contenuti dell'archivio si rimanda a Guidobaldi 2008, 2016; Paribeni 2016a.

4 Paribeni, Guidobaldi 2020, 341-5.

5 Saggi dell'epistolario con alcune di queste personalità sono stati pubblicati in passato da Eva Tea: oltre ai carteggi con Webb di cui si dirà più avanti, va ricordato quello, più contenuto quantitativamente ma di notevole interesse, con William Douglas Caroë (Milano ILASL, Archivio

Fonti, letterature, arti e paesaggi d'Europa S Sources, Literatures, Arts \& Landscapes of Europe $1 \mid$ 

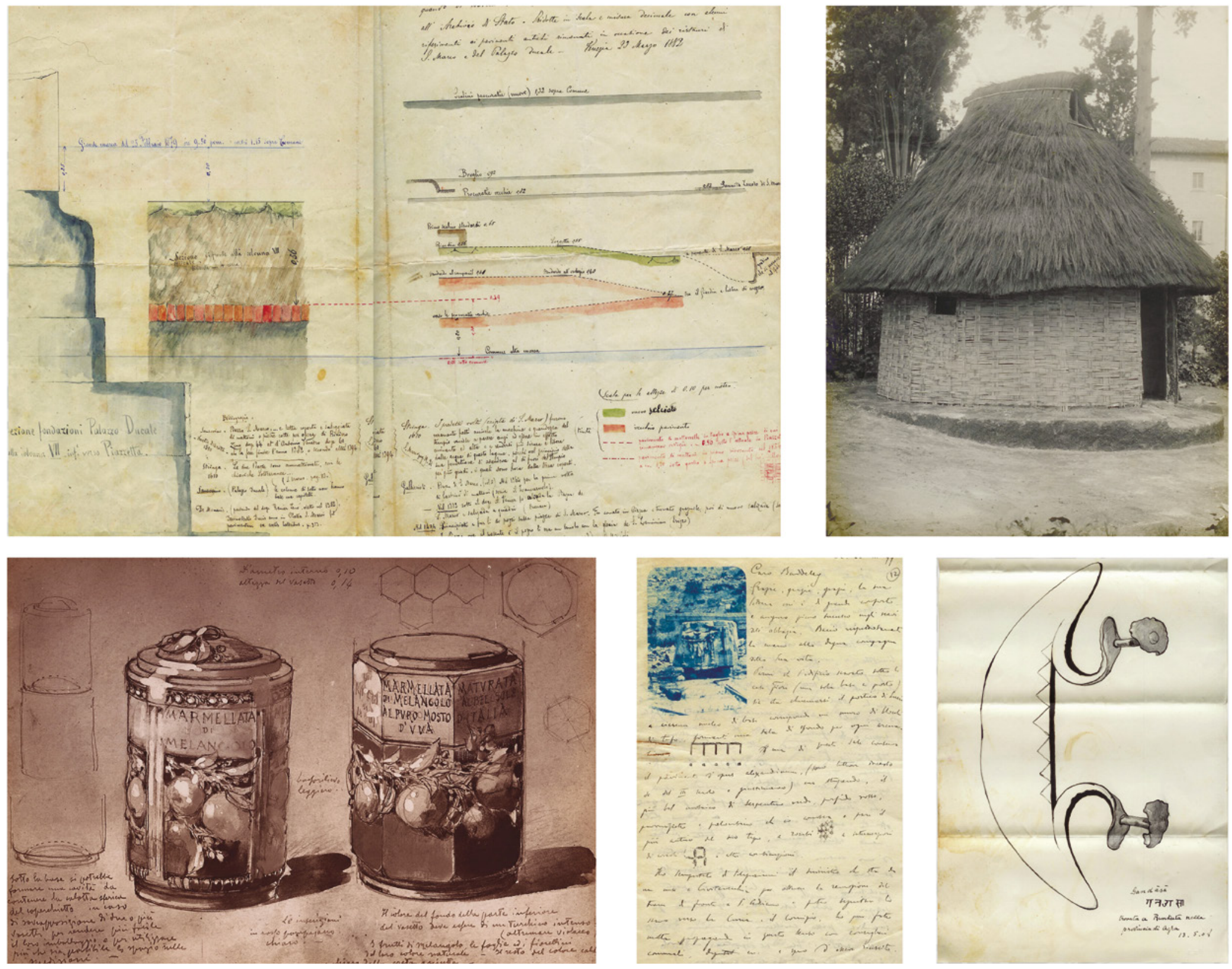

Figura 1 In senso orario: sezione stratigrafica delle fondazioni di Palazzo Ducale a Venezia (Milano ILASL, Archivio Boni-Tea, LXXIII. Calchi e disegni, doc. 2); la ricostruzione della Casa Romuli sul Palatino (Milano ILASL, Archivio Boni-Tea, XXVII/b. Casa Romuli - Riproduzioni di case, doc. 1b); disegno di gandasa (utensile agricolo) dalla località di Runkuta (Agra, India), inviato a Giacomo Boni da Eugenia Barnes (Milano ILASL, Archivio Boni-Tea, XXXIX. Epistolario B); lettera di Giacomo Boni a William St Clair Baddeley del 26 agosto 1899 (Milano ILASL, Archivio Boni-Tea, XXXIX. Epistolario B); schizzi di Boni per modelli di contenitori della marmellata di melangolo (Milano ILASL, Archivio Boni-Tea, CXLIX. Marmellata italiana, docc. 8a-b) 

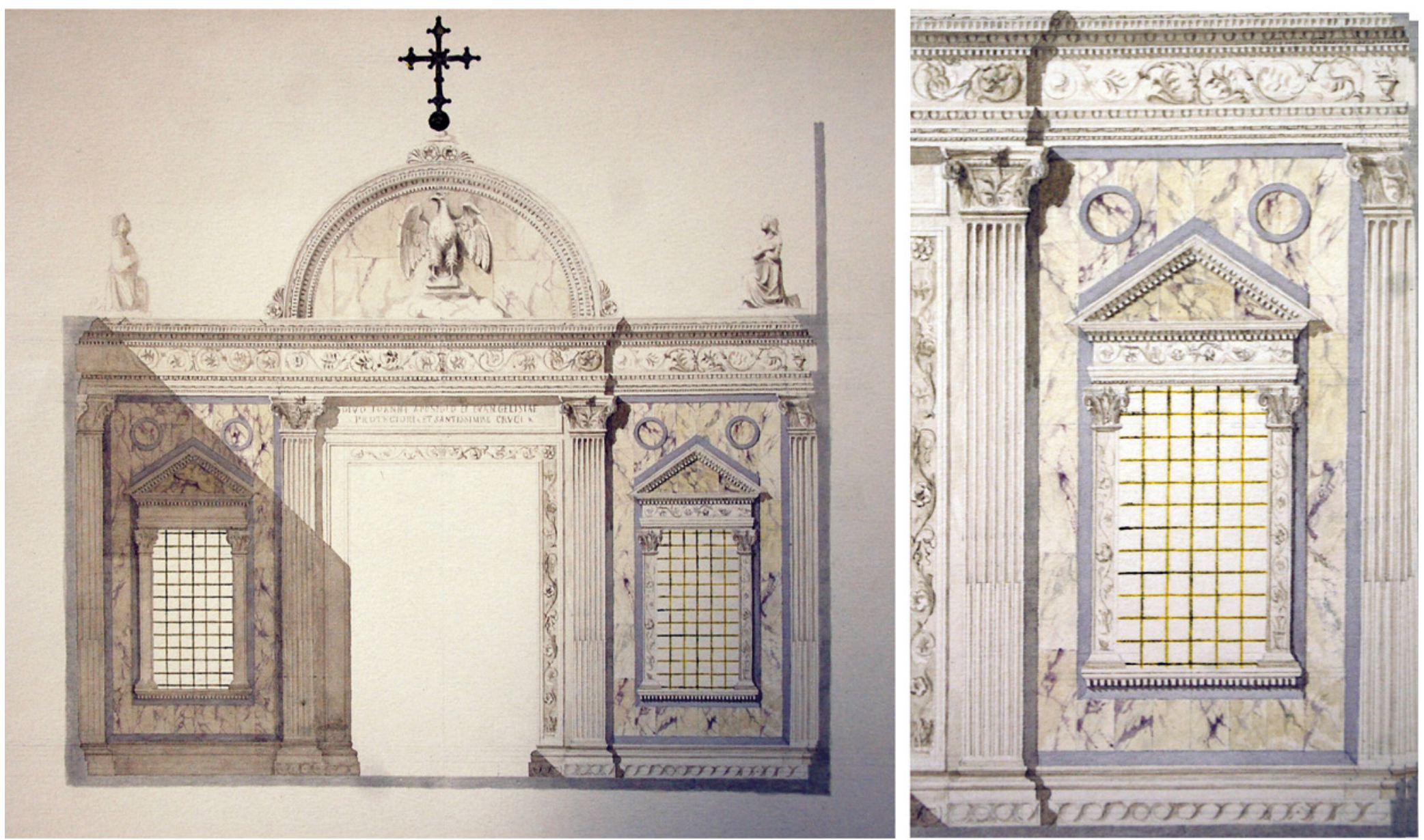

Figura 2 Giacomo Boni, disegno (intero e particolare) del portale della Scuola Grande di San Giovanni Evangelista a Venezia, realizzato nel marzo del 1883. Oxford, Ashmolean Museum, inv. WA.RS.RUD.108bis. Fotografia di Andrea Paribeni 


\section{John Ruskin, il disegno e i rapporti con Giacomo Boni}

La fitta trama di relazioni personali e interpersonali veniva costantemente alimentata attraverso un assiduo scambio epistolare che artisti, uomini di cultura, critici, studiosi ecc. erano adusi intrattenere lungo tutto il corso della propria vita. La corrispondenza - per quel che qui ci interessa valutare - permetteva, allora come oggi, quel fecondo confronto di idee, informazioni personali o risultati di carattere pratico e teorico, che costituiscono una testimonianza fondamentale per la ricostruzione degli eventi storici.

Proprio allo scambio epistolare, a far data dal 1881, sono affidati i primi contatti tra il teorico inglese e il giovane operaio-disegnatore veneziano, desideroso di legarsi al più anziano maestro non solo idealmente, abbracciando le teorie sull'arte e sul restauro promulgate da Ruskin, ma anche in termini più strettamente personali.

L'occasione dell'incontro tra Ruskin e Boni fu favorita da un più esteso 'network' di rapporti, vale a dire, da un lato, l'amicizia dello stesso Boni con il già citato pittore John Wharlton Bunney, uno dei più talentuosi allievi di Ruskin attivi a Venezia negli anni Settanta dell'Ottocento, ${ }^{6}$ e dall'altro quella con Angelo Alessandri, virtuoso disegnatore veneziano che aveva a sua volta conosciuto Ruskin a Venezia nel 1876-1877 e aveva messo il proprio talento artistico a disposizione dello studioso inglese. ${ }^{7}$ Nell'autunno del 1882 Alessandri partì da Venezia insieme a Boni per raggiungere Ruskin a Pisa con lo scopo di aiutarlo nella realizzazione di tavole, misurazioni e rilievi dei monumenti medievali della Toscana. ${ }^{8} \mathrm{Ru}-$ skin aveva avuto modo di vedere alcuni disegni di Boni che forse lo stesso Bunney gli aveva inviato e aveva così potuto apprezzarne le doti e la sensibilità artistica. Doti che Ruskin continuò a tenere in grande considerazione anche in seguito, come dimostrano i loro rapporti epistolari e personali, improntati a un vicendevole 'supporto', pur nei differenti ruoli di allievo e maestro, se non altro ideale. Boni era infatti animato dal desiderio di diffondere le idee di Ruskin e lo teneva aggiornato sulle vicende dei restauri veneziani e in seguito sulle prime esperienze romane; ${ }^{9}$ Ruskin, da parte sua, si era dimostrato disponibile ad assicurare un sostegno finanche finanziario che, in caso di necessità, potesse garantire il progresso dei lavori del giovane italiano.

Insieme alle lettere Boni inviava frequentemente a Ruskin piccoli schizzi di dettagli architettonici e rilievi scultorei, accompagnati da note esplicative e didascalie, alcuni dei quali forse riconoscibili tra i disegni di Boni oggi conservati all'Ashmolean Museum di Oxford. Nella stessa collezione si trovano inoltre le due belle tavole con la pianta e il prospetto della porta monumentale della Scuola Grande di San Giovanni Evangelista [fig. 2] e i dettagli architettonici di Ca' Dario; entrambe realizzate con dovizia di particolari e una straordinaria attenzione per le differenti qualità dei marmi. Nel marzo del 1883, le due tavole furono presentate da Ruskin alla Drawing School di Oxford, istituzione che egli contribuì fortemente a sostenere, anche finanziariamente. 
5

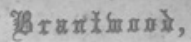

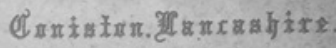

Earke doy. 1883

My dearest Boni

I got the leter with

the lovef insuption and dotart all right and keap it as a teasue, Wh hoor fous bo bury ¿read! - wueh len $h$ anverer it This hacers of the worl is erquite fur the is no kiaki wordark weth mueh - French is the af vood worth lulks is , $I$ don's wonth \& Take you from Denie, w lang as yon can save nenthin, a dircover any thirs that gon care on, - but onf nuclerstanel that th inoment yon fricd yourelf fowaless, a to succh vered and intulted,

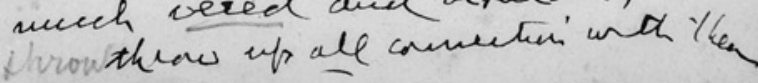

and cones at aree te ture -

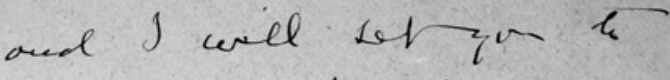

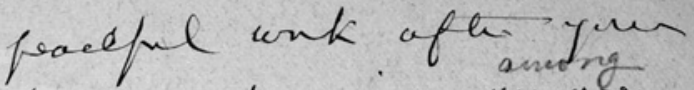
hant and unine, maty feople who will love end kavar yom.

1 phowed tome I) you dravions and ifok \& yo in nuy firt belues at Bifuel this yoas, of which you thale tow hav a cofy. Cleantuie, I send Yir all my book, te read a land a you like - yau can yw the cispolant over te th clearaian if you lean Vence vluch Lon G itlefrudu and I an er covings ifuits of is Muttion

Figura 3 Lettera di John Ruskin a Giacomo Boni del 25 marzo 1883. Milano, ILASL, Archivio Boni-Tea, LVII. Epistolario R - Ricci, Ricciardi, Rigobon, Robertson 


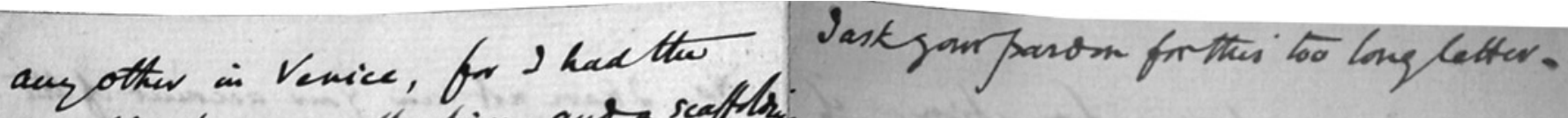
cenefit of qour attintion, aub seaftris from which 9 mbbet my ning and frigers well in to the visille, torchable work of the metioval venctians. when sîn 2 look throngh ma rogh nitk book 2 wrik to be back there, to wore allsar at the prelty brilles strey, and talk wor mi thength with $2 m$ in the suencicy - It is atlikel that I shake sor get to

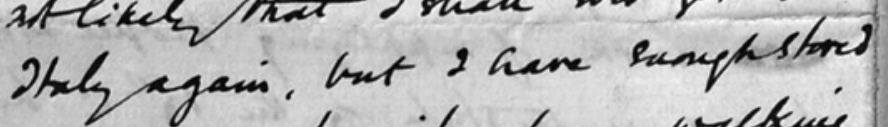
within me to siterest nuy walkuy thongth for the rot of my years.

, Raymond Buildings, Gray's Inn,

Saturtay $18^{2} \mathrm{Sef}=86$ Mydear Siguar Sovi:

Thare tentera toyplib book host, the "Correspousence resfectricy the inferisonment of M: Worts by the Prussians" 2 have nat yek heen abe to get zan the other ifformation, sither at tho flace for the tale of Sarliamentary acto, or at truesarde' for pastiamentary papurs; lut 2 hfe the athe to to adeer to which centimental reflee. tios, emes auotter mare terions So, and as suickly as fon ible. Sam truping of find a trustworthy stie. Thepeoples ate mov the worls agjent to kearch for and read of are awaking bo the fact that they care been hrribly cheated of thew the pafurs nux edp the Jame: thpe gar frisut will be whe nights aud 1 , who have sungh ow wait alitile : You mag/fromene bood, Clothuy, ant honserorf must him ( Me firffersor of huterantional

Figura 4 Lettera di Philip Webb a Giacomo Boni del 18 settembre 1886. Milano, ILASL, Archivio Boni-Tea, LXI. Epistolario U, V, W 
La propensione a una 'didattica visiva' fondata sul disegno era stata già alimentata da Ruskin negli anni Quaranta dell'Ottocento, per culminare con la pubblicazione, nel 1857, del celebre manuale The Elements of Drawing. In questa prospettiva, il disegno diventa, per chi lo esercita, strumento privilegiato di conoscenza, oltreché 'diretta' testimonianza documentaria dello stato di conservazione - e più spesso purtroppo di decadenza - del monumento. Ma la conoscenza cui Ruskin aspira è una forma di possesso specificamente visiva, che per attingere a una reale 'innocenza dell'occhio' deve progressivamente liberarsi di un sapere concettuale precostituito intorno agli oggetti della realtà e al loro modo di apparire, della dimensione che oggi diremmo più strettamente cognitiva. Di qui l'esigenza pragmatica dell'esercizio di un disegno guidato da una visione analitica, o per meglio dire 'foveale', ben lontana dalla tradizionale pratica dello sketching Tour, che si spinge spesso a concentrarsi sui dettagli, staccati, parcellizzati, fin quasi a trasformarli in una sorta di irriconoscibile 'geroglifico', come il padre dello stesso Ruskin scriveva all'amico Harrison, dotati di una speciale verità, sia pure una «verità a mo' di mosaico». ${ }^{10}$ Come è ben noto, la cosiddetta teoria dell'occhio 'innocente' avrà un certa fortuna tra gli artisti e i critici successivi, almeno fino al formalismo di Roger Fry, per poi essere severamente criticata dagli studiosi moderni - basti citare qui il lavoro ormai classico di Gombrich in Arte e illusione ${ }^{11}$ - e proprio sul piano di una dottrina 'scientifica' della percezione visiva che, in fondo, stava al cuore degli interessi di Ruskin. ${ }^{12}$ In ogni caso, tuttavia, il valore euristico del disegno come strumento di 'didattica della visione' conserverà la sua vitalità, anche oltre il pensiero ruskiniano e l'eredità dei suoi allievi. ${ }^{13}$

In questo senso, il rapporto di stima che legava Ruskin al giovane Boni, e che le 15 lettere dell'Istituto Lombardo testimoniano espressamente [fig. 3], ${ }^{14}$ poteva rappresentare una sorta di verifica delle sue stesse teorie, o almeno un loro possibile momento concretamente applicativo, dato che il lavoro sul campo del veneziano incarnava in un certo qual modo una sintesi ideale tra le istanze più teoriche della ricerca dello stesso Ruskin e le finalità pratiche dettate dalle reali necessità di conoscenza e conservazione dei monumenti artistici e della loro storia. Le lettere ribadiscono il saldo legame e la stima tra il maestro inglese e l'allievo veneziano. L'immagine che Ruskin si fece di Boni fin dagli esordi della loro conoscenza risente molto del desiderio del giovane allievo di entrare con forza nel vi-

10 Lettera di J. Ruskin a W.H. Harrison del 25 maggio 1846: Levi, Tucker 1997, 89-90.

11 Gombrich [1959] 1962, 16, 359-60.

12 Per la famosa formulazione della teoria della 'innocenza dell'occhio' di Ruskin, vedi: The Elements of Drawing (Works, 15: 27-8): «The perception of solid Form is entirely a matter of experience. We see nothing but flat colours; and it is only by a series of experiments that we find out that a stain of black or grey indicates the dark side of a solid substance, or that a faint hue indicates that the object in which it appears is far away. The whole technical power of painting depends on our recovery of what may be called the innocence of the eye; that is to say, of a sort of childish perception of these flat stains of colour, merely as such, without consciousness of what they signify, - as a blind man would see them if suddenly gifted with sight».

13 Levi, Tucker 1997.

14 Le lettere di Boni a Ruskin sono conservate a Brantwood Coniston; brani di esse sono stati pubblicati da diversi studiosi (vedi per esempio Tea 1927; 1932, vol. 1; Pilutti Namer 2013a, 2013b, 2016, questi ultimi anche per il rapporto tra Ruskin e i suoi 'discepoli' italiani). Le lettere di Ruskin a Boni, conservate presso l'archivio Boni-Tea, sono 15 e vanno dall'agosto del 1881 al luglio del 1884. Solo la prima di queste missive è contenuta nel secondo volume dedicato all'epistolario di John Ruskin all'interno dei Complete Works of John Ruskin (Works, 37: 373-4); stralci di esse anche in Pilutti Namer 2013a, 2013b, 2016. Alle missive tra Boni e Ruskin si devono anche aggiungere nel fondo milanese tre brevi lettere della cugina Joan Ruskin Severn a Giacomo Boni datate 15 aprile 1895, 1 aprile 1900 e 23 settembre 1900. 
vace dibattito sulla conservazione dei monumenti, come vedremmo più avanti. Boni rappresentò per Ruskin quella 'voce locale' che costantemente gli permetteva di essere aggiornato sulle questioni intorno al patrimonio monumentale italiano, fornendogli come già detto materiale inedito grafico e fotografico. (S.P.)

\section{Philip Webb e il suo epistolario con Giacomo Boni}

Se, nel rapporto con Ruskin, lo scambio epistolare fa da prologo al primo incontro personale, in quello con Webb esso scaturisce invece da un episodio ben preciso e altrettanto ben fissato nella memoria dei due protagonisti: lo scenario è quello delle impalcature di Palazzo Ducale, la data, il 3 aprile del 1885, è tramandata da una lettera che Webb scrisse il giorno stesso a William Morris, per ricordare la vivida impressione che gli aveva procurato l'incontro con quel «direttore dei lavori che parla e legge inglese», interessato tanto agli scritti di impronta socialista del Commonweal (l'organo di stampa della Socialist League fondata dallo stesso Morris) quanto alla poesia, gran lavoratore dotato di quella vanità necessaria a stimolare la sua naturale intelligenza. ${ }^{15}$ Sorprende da un lato la capacità introspettiva di Webb, che sa cogliere di primo acchito aspetti peculiari del carattere di Boni, assieme alla disponibilità del giovane veneziano a rischiare in proprio pur di denunciare interventi di restauro che potevano mettere a repentaglio l'integrità dei monumenti; dall'altro la sopravvalutazione del ruolo di Boni, presentato a Morris come direttore dei lavori, quando invece i suoi compiti erano molto più circoscritti, e comunque subordinati all'effettivo direttore del cantiere, Annibale Forcellini. ${ }^{16}$ Anche Ruskin, per enfasi personale o per un'errata percezione indotta da Boni - senza intenzione ma forse per effetto di quella sua vanity di cui parlava Webb - in una lettera a Charles Eliot Norton del novembre del 1882 lo definisce «master of the work on the Ducal Palace of Venice» e insignito dall'autorità governativa del potere di ispezionare qualsiasi monumento

15 «I was on the scaffolding at the Ducal Palace this morning, \& while at work I lent 'Commonweal' to the director of the works there who reads \& speaks English. He wanted permission to translate such pieces as the "Workers' share of art," which I gave him, and I promised to send him that piece and any more of the like kind of yours. He thinks the Italian workmen will be interested in it. This young fellow is well read in French German \& English poetry, and I mean to send him a volume of yours on my return, in recognition of the help he has given me in looking into the construction of buildings \&c here. He is coming to dine with me on Sat: evening when I shall have more talk with him on this and other matters. He is a rather excitable fellow working much too hard, having the necessary vanity to stimulate his natural cleverness. He is certainly not likely to let his masters be in ignorance of the mischief if they propose anything in the way of scraping to Venetian works. He has already been threatened by the engineers, who direct all works here, that they will put him on the dredging boat in the lagoons if he is not more reticent. He is poor, \& we have to put up with that, but he will get the sack soon I fancy unless he bends to what is really very ignorant authority». Tea 1940 1941, 132; Aplin 2016, 1: 288

16 In un suo scritto Annibale Forcellini illustra compiti e meriti di Boni nel restauro del Palazzo Ducale da lui diretto: «il Sig. Giacomo Boni, che venne assunto in assistenza quando era poco più che adolescente e che nel giro di pochi anni acquistò, per proprio merito unicamente, coltura sapere ed erudizioni rari a trovarsi anche in uomini d'età più provetta, si rese utile particolarmente colle sue ricerche storiche ed archeologiche e col suo fine spirito d'osservazione» (Forcellini 1887). Anche il ruolo di disegnatore svolto da Boni nel cantiere veneziano va inquadrato alla luce dei registri dei pagamenti per i lavori di restauro a Palazzo Ducale relativi agli anni 1878-1883, conservati presso l'Archivio Centrale dello Stato (ACS Min. P.I , AA.BB AA., I Versamento, 1860-1890, Busta 626, fasc. 1179/1-2), ove il suo nome ricorre soltanto in data 14 aprile 1881 , in occasione di un pagamento straordinario di £ 30, mentre con la qualifica di disegnatore figura regolarmente Luigi Vendrasco (Paribeni, Guidobaldi 2020, 39). 
pubblico, utilizzando scale e ponteggi a suo piacimento; ${ }^{17}$ un ruolo che, in quegli anni ancora lontani dall'inquadramento di Boni in seno alla Direzione Generale Antichità e Belle Arti, è difficile da immaginare. ${ }^{18}$

Boni viene ricordato da Webb nelle corrispondenze con Charles Fairfax Murray (agosto e settembre 1885), ${ }^{19}$ segno che il legame si mantenne subito dopo il ritorno dell'architetto in Inghilterra, come dimostrano del resto le lettere inviate da Boni già nel maggio di quell'anno, mentre la prima lettera di Webb di cui disponiamo [fig. 4] è del settembre del $1886 .{ }^{20}$ L'epistolario tra i due si manterrà intenso fino al 1894, per poi decrescere lentamente negli ultimissimi anni del secolo e farsi poi sporadico fino alle ultime lettere del 1907. ${ }^{21}$

Stralci dell'epistolario furono pubblicati nella voluminosa biografia di Boni scritta da Eva Tea nel 1932; successivamente sempre Eva Tea avviò sulla rivista $A n$ - nales Institutorum quella che doveva essere la pubblicazione integrale delle lettere di Boni, assieme ad alcune di Webb, arrestatasi però alle lettere risalenti al 1890..22 Nel fondo Boni-Tea dell'Istituto Lombardo è conservato il carteggio tra Eva Tea e Emery Walker, esecutore testamentario di Philip Webb, al quale la Tea si era rivolta, negli anni in cui preparava la biografia, per avere eventuali lettere di Boni; ${ }^{23}$ non è questa la sede per entrare nel merito di questa complessa e a tratti spigolosa trattativa finalizzata allo scambio e alla fruizione delle lettere Boni-Webb; ${ }^{24}$ vale però la pena di ricordare la missiva di Walker del febbraio del 1927 [figg. 5-6], nella quale il mittente annuncia alla Tea di aver ritrovato una cinquantina di lettere di Boni e di averne potuto apprezzare la proprietà linguistica - maturata da Boni grazie alle lezioni impartitegli da Elizabeth Fallon, la moglie del pittore ruskiniano John Wharlton Bun-

17 Dearden 1967; Bradley, Ousby 1987.

18 Lo stesso si può dire di William Morris, che nella sua prolusione al meeting della SPAB del 3 luglio 1889, annunciando all'uditorio la mancata partecipazione di Boni, lo presentava come colui che «now holds a position under the Italian Government which enables him to be of great service to us in stopping the unfortunate flow of restorations in that country. He could have given to us most interesting and encouraging information as to what is now going on in Italy», citato in Aplin 2016, 2: 36, n. 3). Non stupisce, pertanto che, nella biografia di Ruskin, Collingwood definisca Boni «director of the Monuments of Italy» (Collingwood 1893, 2: 205).

19 Cf. Aplin 2016, vol. 1

20 Aplin 2016, 1: 326-7

21 Aplin 2016, 4: 229, lettera di Webb a Boni del 13 maggio 1907: «At last, it seems as if we really might come together again, and see what each is like in body \& spirit - after the wear and tear of years since we looked in each other's eyes....We will hope that Whit Sunday will smile on us». E senza dubbio un fatto curioso che l'epistolario tra i due amici cessi immediatamente dopo il loro terzo incontro a ventidue anni di distanza dalla loro fatidica conoscenza sulle impalcature di Palazzo Ducale; un'ultima lettera dell'anziano e stanco Webb datata 29 luglio di quell'anno (Aplin 2016, 4: 233-4) sembra quasi sancire il distacco definitivo proprio nel ricordo di quell'ultimo breve incontro: «I would like to carry the memory of it with me into the other climate of the spirit which is steadily approaching».

22 Tea 1940-1941, 1941-1942.

23 Milano, ILASL, Archivio Boni-Tea, LXI. - Epistolario U, V, W. Le lettere vanno dal 1927 al 1932 e illustrano il tortuoso percorso attraverso il quale le lettere di Boni a Webb furono inviate tramite ambasciata ad Eva Tea, la quale le utilizzò per la biografia (Tea 1932) e in seguito per pubblicazioni specifiche (Tea 1940-1941, 1941-1942).

24 Delicata soprattutto fu la questione delle lettere di Webb a Boni, detenute dalla Tea la quale, riluttante a concederle a Walker che le richiedeva in quanto «owner of the copyright of Mr. Webb» (lettera del 30 ottobre 1931), si risolse dapprima a inviare delle copie, suscitando il disappunto di Walker («I was rather surprised that you should have sent me copies instead of the originals especially after I had entrusted you with Boni's letters for so long»: lettera di Emery Walker del 16 gennaio 1932), quindi, sempre tramite Ambasciata, a concedere in visione gli originali a patto della restituzione delle copie fatte fare a sue spese. 


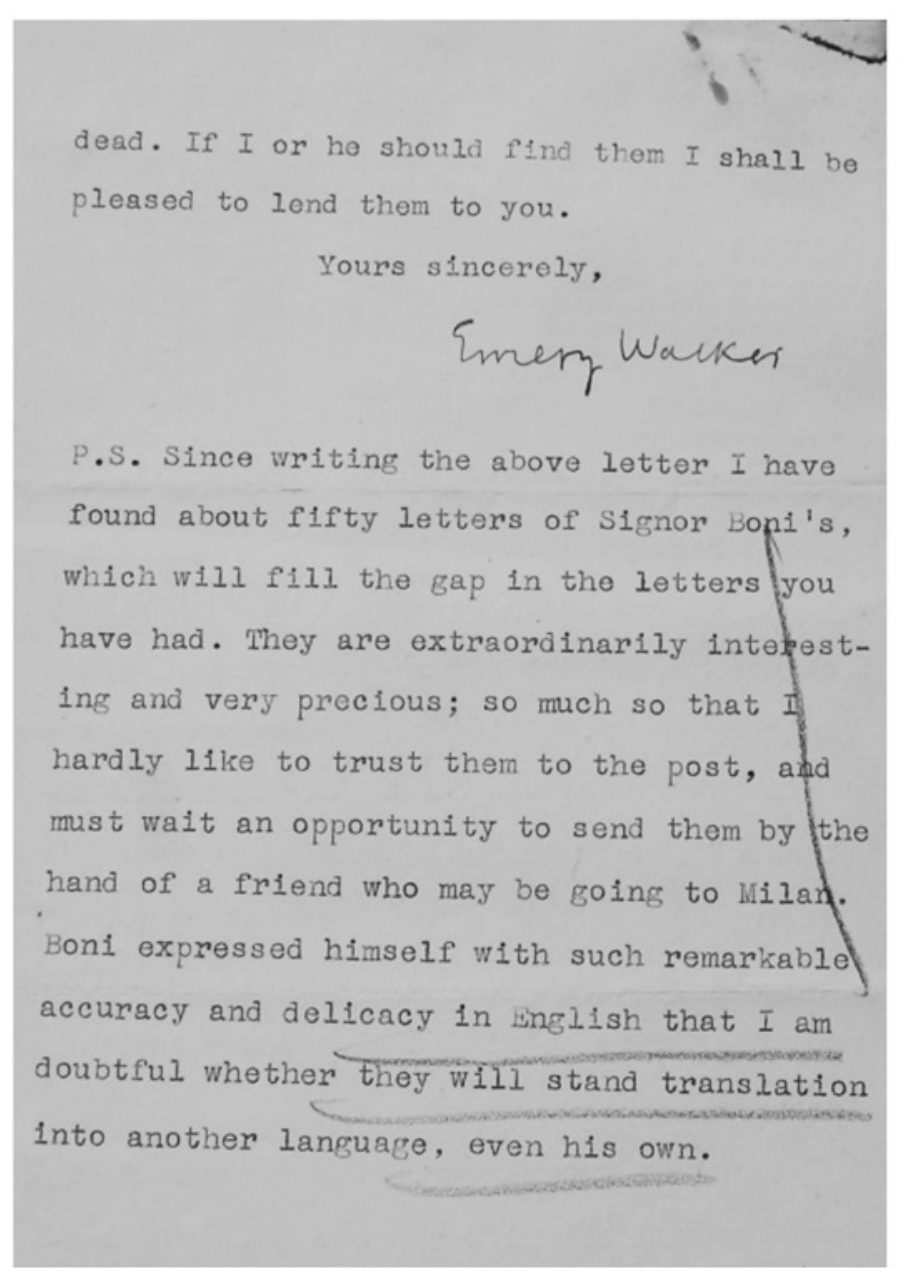

Figura 5 Lettera di Emery Walker a Eva Tea del 3 febbraio 1927. Milano, ILASL, Archivio Boni-Tea, LXI. Epistolario U, V, W

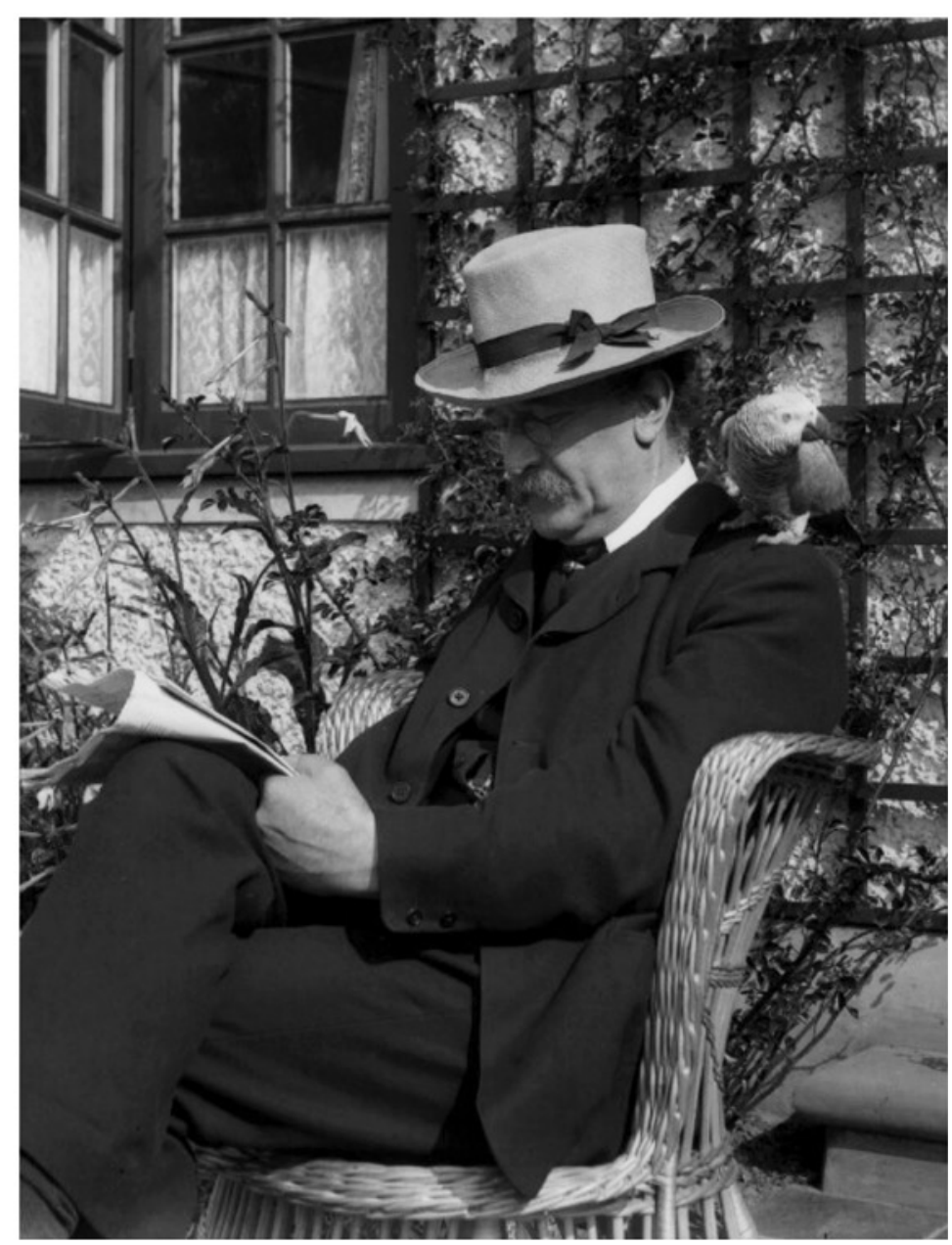

Figura 6 Ritratto fotografico di Emery Walker (1920-1933). London, National Portrait Gallery, Photographs Collection, NPG x31052 
ney - tanto da sconsigliarne una traduzione in italiano. ${ }^{25}$

Di recente le lettere di Webb a Boni sono state pubblicate nella grande opera in quattro volumi curata da John Aplin, che raccoglie l'intero corpus delle lettere dell'architetto inglese. ${ }^{26}$ Lo studioso ha editato le lettere dirette a Boni basandosi su vecchie copie fotostatiche degli originali, risalenti a circa gli anni ' 30 del secolo scorso (la realizzazione di copie non è attestata dal carteggio tra Eva Tea ed Emery Walker sopra menzionato ma potrebbe essere stata la naturale conclusione del braccio di ferro tra i due), conservate presso la Biblioteca del Courtauld Institute; Aplin però non menziona i precedenti lavori della Tea dedicati in tutto o in parte al carteggio, né sembra essere a conoscenza dell'esistenza degli originali delle lettere inviate da Webb che si trovano a Milano nel fondo Boni-Tea. Dal confronto numerico tra le copie fotostatiche del Courtauld (che non abbiamo avuto modo di esaminare autopticamente) e gli originali dell'Istituto Lombardo emerge come il lotto milanese sia largamente più completo, contando una ventina circa di lettere in più, mentre solo tre delle copie del Courtauld non trovano il loro corrispettivo nel gruppo degli originali [tab.1].

I temi trattati in questa ventennale corrispondenza sono molteplici e maturano e variano negli anni col progredire della carriera di Boni; fin tanto che il veneziano si dedicherà con costanza alle questioni della conservazione e della tutela dei monumenti, il filo diretto con Webb sarà intenso, poi, quando Boni si immergerà negli scavi del Foro lo sguardo di Webb, oramai anziano e stanco, si manterrà sempre curioso e benevolo, ma più distante e meno personalmente coinvolto. ${ }^{27}$

Non potendo ovviamente dar conto, in maniera esaustiva, del contenuto di queste numerose e lunghe lettere, segnaleremo le caratteristiche generali dell'epistolario soffermandoci su alcuni casi più particolari. Va innanzitutto detto che le lettere, costantemente dedicate allo studio e alle condizioni conservative dei monumenti italiani e, in minor parte, inglesi di epoca per lo più medievale, erano accompagnate da schizzi esplicativi inseriti nel corpo del testo e da allegati grafici - disegni e fotografie - questi ultimi soprattutto forniti da Boni come resoconto dapprima delle sue ricognizioni a Venezia e dintorni, quindi, a partire dal 1888 e con la sua nomina ad ispettore dei monumenti presso la Direzione Generale Antichità e Belle Arti, estese a tutta l'Italia e massimamente nelle regioni centro meridionali.

Negli schizzi realizzati da Webb emerge la curiosità per minuti dettagli [fig. 7] del decoro architettonico dei monumenti illustrati nelle fotografie inviate da Boni, la cui visione acuiva nell'inglese il rammarico di aver mancato di visitare tante chiese e palazzi di centri minori dell'Italia centro meridionale nel suo viaggio del 1885 e dava inoltre spunto per riflessioni di carattere storico artistico come, ad esempio, la convivenza di stilemi classici e 'barbarici' ravvisabili nello stesso manufatto: diverse lettere, ad esempio, sono dedicate alla discussione di questi temi sulla base dello scrutinio di immagini di una chiesa, indicata con il nome meno consueto e su-

25 Milano, ILASL, Archivio Boni-Tea, LXI. - Epistolario U, V, W, lettera di Emery Walker a Eva Tea, London February 3 1927: «Boni expressed himself with such remarkable accuracy and delicacy in English that I am doubtful whether they will stand translation in another language, even his own». Testimone della fase di primo apprendistato linguistico di Boni presso mrs. Bunney è il diario autografo datato al 1879 conservato presso l'Archivio Boni-Tea (Milano, ILASL, LXX.E Libretti di Appunti. Diario inglese del 1879), su cui vedi Paribeni, Guidobaldi 2020, $130-1,364$.

26 Aplin 2016.

27 Nelle sue lunghe missive Webb non si limita a registrare e commentare l'attività di tutela e le pubblicazioni del giovane amico veneziano, ma parla anche dei propri interventi di ripristino e restauro di monumenti inglesi (lettera a Boni del 28 dicembre 1890, Aplin 2016, 2: 91) e, in misura minore, della sua attività di progettazione per edilizia residenziale, in cui i dettami architettonici e decorativi dell'Arts \& Crafts si arricchivano di innovativi elementi tecnologici come, ad esempio, impianti di illuminazione elettrica (Moore 2018). 
Andrea Paribeni, Silvia Pedone

La corrispondenza epistolare come rete di conoscenza, dibattito e azione

Tabella 1 Elenco completo delle lettere di Philip Webb a Giacomo Boni.

Evidenziate in rosso le lettere conservate solo nel fondo londinese (in copia fotostatica)

e in blu quelle presenti in originale presso ILASL di Milano

\begin{tabular}{|c|c|c|c|c|c|}
\hline \multirow[t]{2}{*}{18.09 .1886} & 26.01 .1890 & 19.07.1891 & 08.01 .1893 & 05.01 .1896 & 12.09 .1905 \\
\hline & 09.02 .1890 & 16.08 .1891 & 26.02 .1893 & 24.06 .1896 & \\
\hline \multirow[t]{2}{*}{21.12 .1887} & 16.03 .1890 & 06.09 .1891 & 03.04.1893 & & 26.04.1907 \\
\hline & 30.03 .1890 & 13.09 .1891 & 27.05.1893 & 28.02 .1897 & 07.05.1907 \\
\hline 13.11 .1888 & 13.04 .1890 & 25.10 .1891 & 16.07 .1893 & 11.04 .1897 & 13.05 .1907 \\
\hline \multirow[t]{2}{*}{14.11 .1888} & 04.06 .1890 & 22.11 .1891 & 23.07.1893 & & 29.07.1907 \\
\hline & 29.06 .1890 & & 06.08 .1893 & 09.01 .1898 & \\
\hline 12.03 .1889 & 28.09 .1890 & 03.01 .1892 & 03.12 .1893 & 06.02 .1898 & $\begin{array}{l}\text { Lettera s. } \\
\text { data }\end{array}$ \\
\hline 03.06 .1889 & 19.10 .1890 & 24.01.1892 & 17.12.1893 & & \\
\hline 30.06 .1889 & 28.12 .1890 & 06.03 .1892 & & 26.08.1899 & \\
\hline 26.08 .1889 & & 27.03 .1892 & 03.01 .1894 & 18.09.1899 & \\
\hline 16.09.1889 & 18.01.1891 & 06.04 .1892 & 11.02 .1894 & & \\
\hline 17.09.1889 & 21.02 .1891 & 24.04 .1892 & 08.03 .1894 & 08.04 .1900 & \\
\hline 29.09.1889 & 29.03.1891 & 12.06 .1892 & 23.03 .1894 & 13.04.1900 & \\
\hline 06.10 .1889 & 31.03 .1891 & 03.07 .1892 & 03.06 .1894 & 15.08 .1900 & \\
\hline 27.10 .1889 & 26.04 .1891 & 17.07.1892 & 29.07.1894 & & \\
\hline 10.11 .1889 & & 21.08 .1892 & 07.10 .1894 & 01.10 .1903 & \\
\hline \multirow[t]{2}{*}{15.12 .1889} & 07.05 .1891 & 04.09 .1892 & 11.12 .1894 & & \\
\hline & 01.06 .1891 & 30.10 .1892 & & 30.01 .1904 & \\
\hline 04.01 .1890 & 21.06 .1891 & 27.11.1892 & ??.02.1895 & 11.04 .1904 & \\
\hline 12.01 .1890 & 12.07.1891 & & 26.05 .1895 & & \\
\hline
\end{tabular}

$111 \begin{aligned} & \text { Fonti, letterature, arti e paesaggi d'Europa | Sources, Literatures, Arts \& Landscapes of Europe } 1 \\ & \text { John Ruskin's Europe. A Collection of Cross-Cultural Essays, } 99-130\end{aligned}$ 


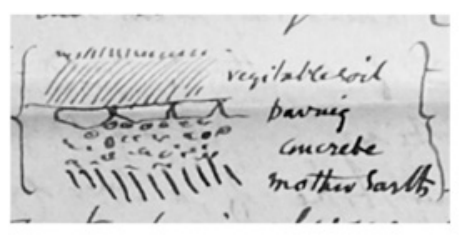

tow," is calles' "Bar", 'Cike 'barga in beantifuel tho piece of de and int planto dingrieg th the jonits like. oby last, whide is in a Silatidateg $<$ ran tie acrosit, an? as do

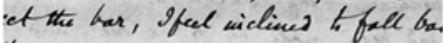
kici monar os any yeass, of it ane mileses? like the thangte of the actione fo. he acted uppa by gasses and samp t to drans of and triphtere the tie at to keep the tower anch ary from so

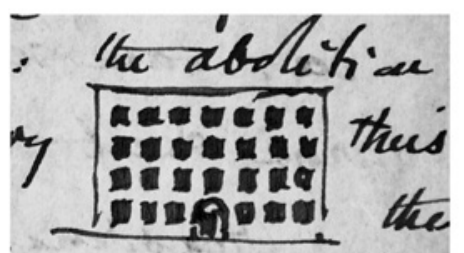
$x$ m these hell
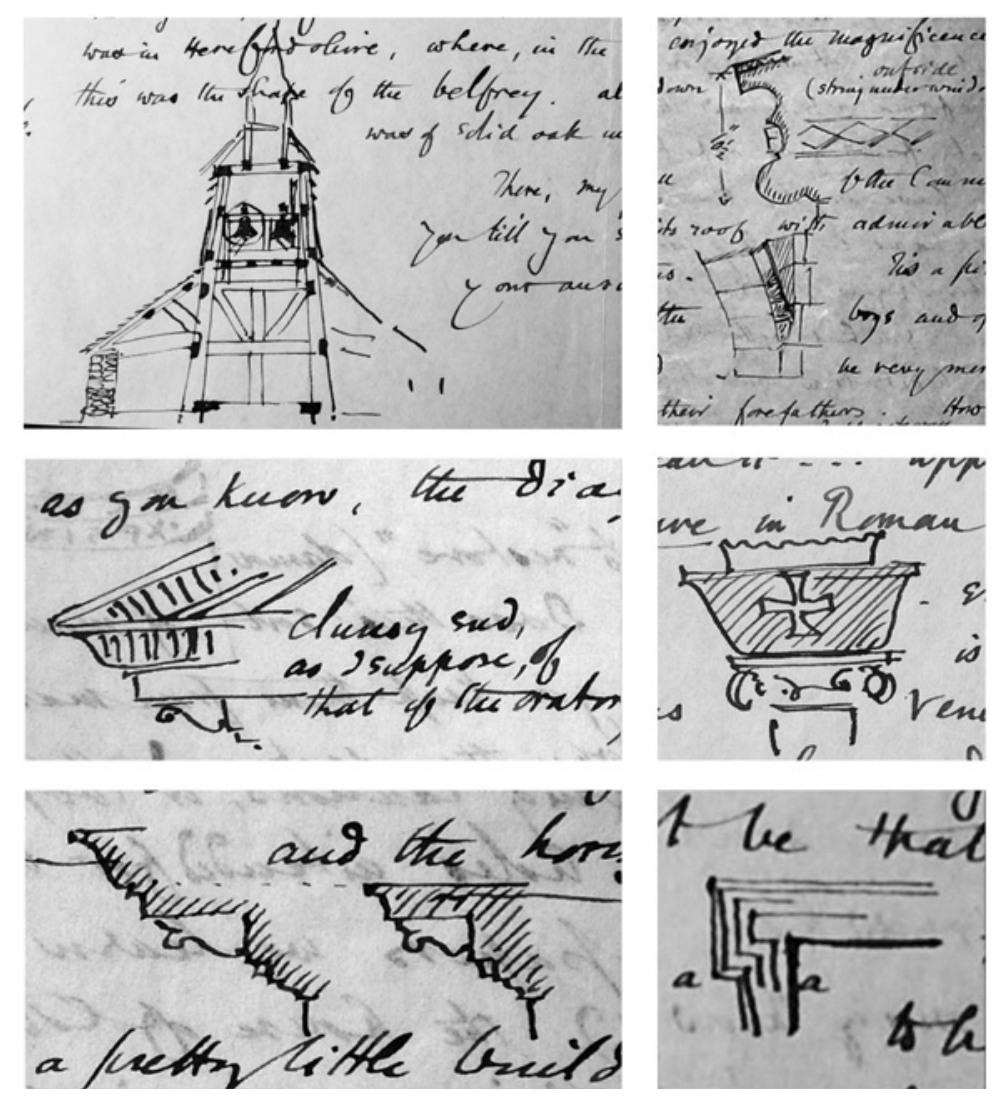

Figura 7 Philip Webb, schizzi architettonici interpolati nelle lettere inviate a Boni. Milano, ILASL, Archivio Boni-Tea, LXI. Epistolario U, V, W
The tof fo the andithane: vice fuper. be the frise casdes 19 (a) ath

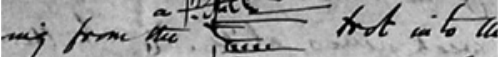
sonn as the altitit aed ratififie. Asesde so admiables derisn

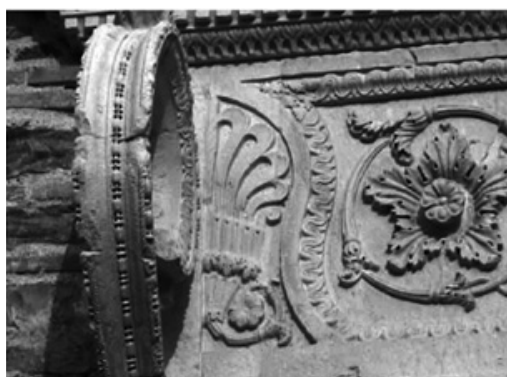

Figura 8 Lettera di Philip Webb a Giacomo Boni del 4 gennaio 1890 con dettaglio del portale della chiesa di San Salvatore a Spoleto. Milano, ILASL, Archivio Boni-Tea, LXI. Epistolario U, V, W 


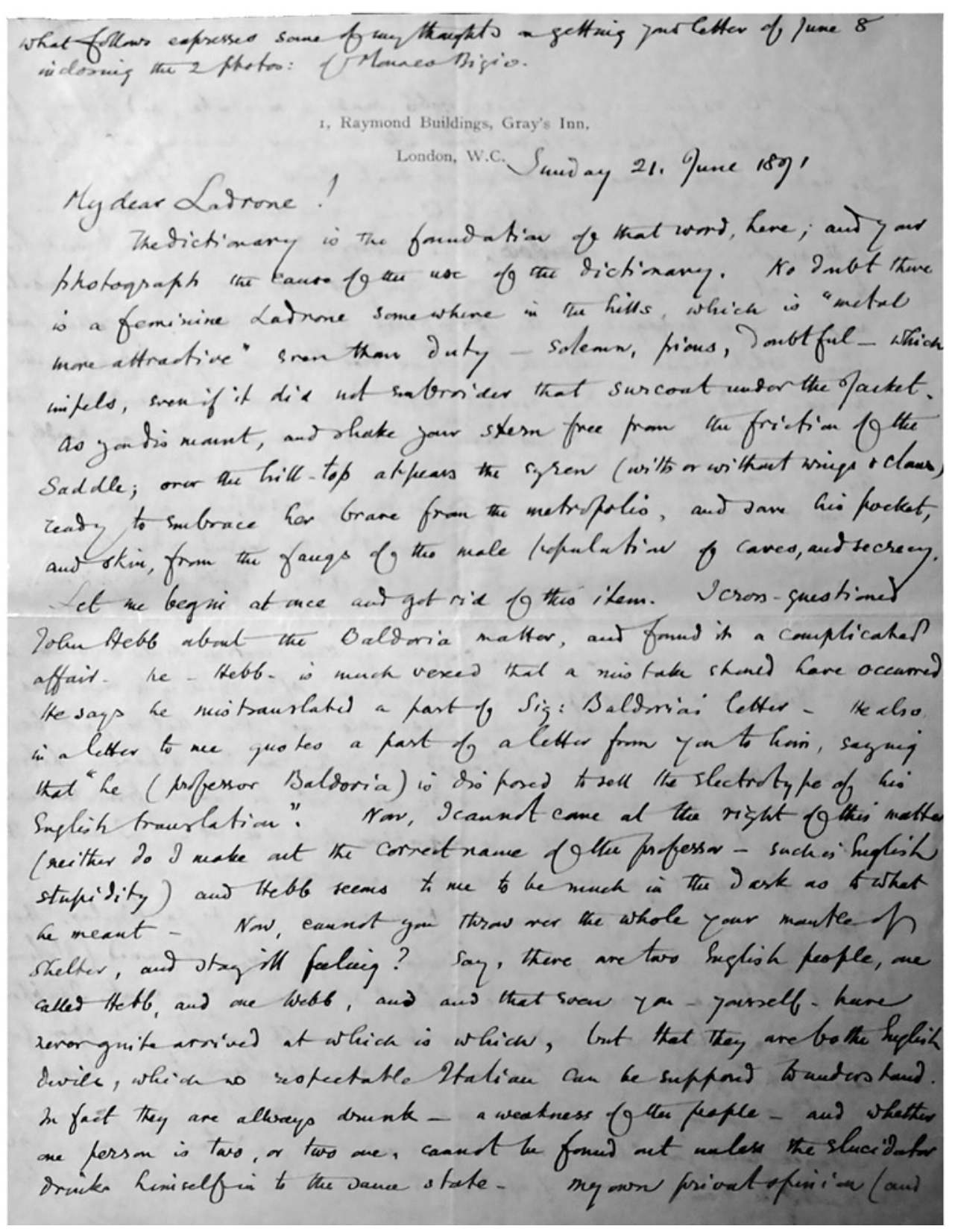

Figura 9 Lettera di Philip Webb a Giacomo Boni del 21 giugno 1891. Milano, ILASL, Archivio BoniTea, LXI. Epistolario U, V, W 


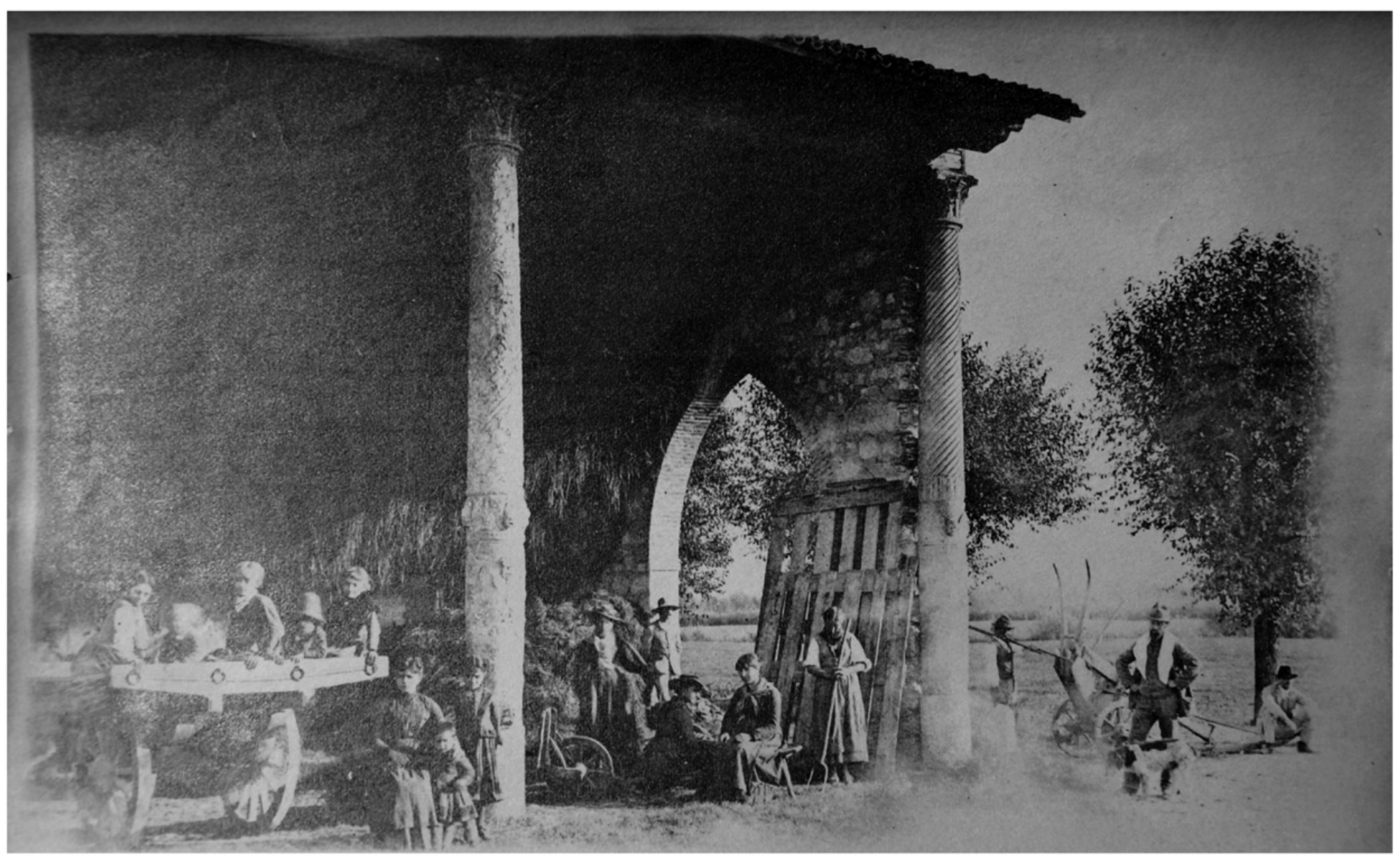

Figura 10 Stalla con colonne di epoca rinascimentale a Pontealto. Dall'album Vicenza Medioevale. Milano, ILASL, Archivio Boni-Tea, CXXXVIII. Vicenza Medioevale, doc. 1.b 


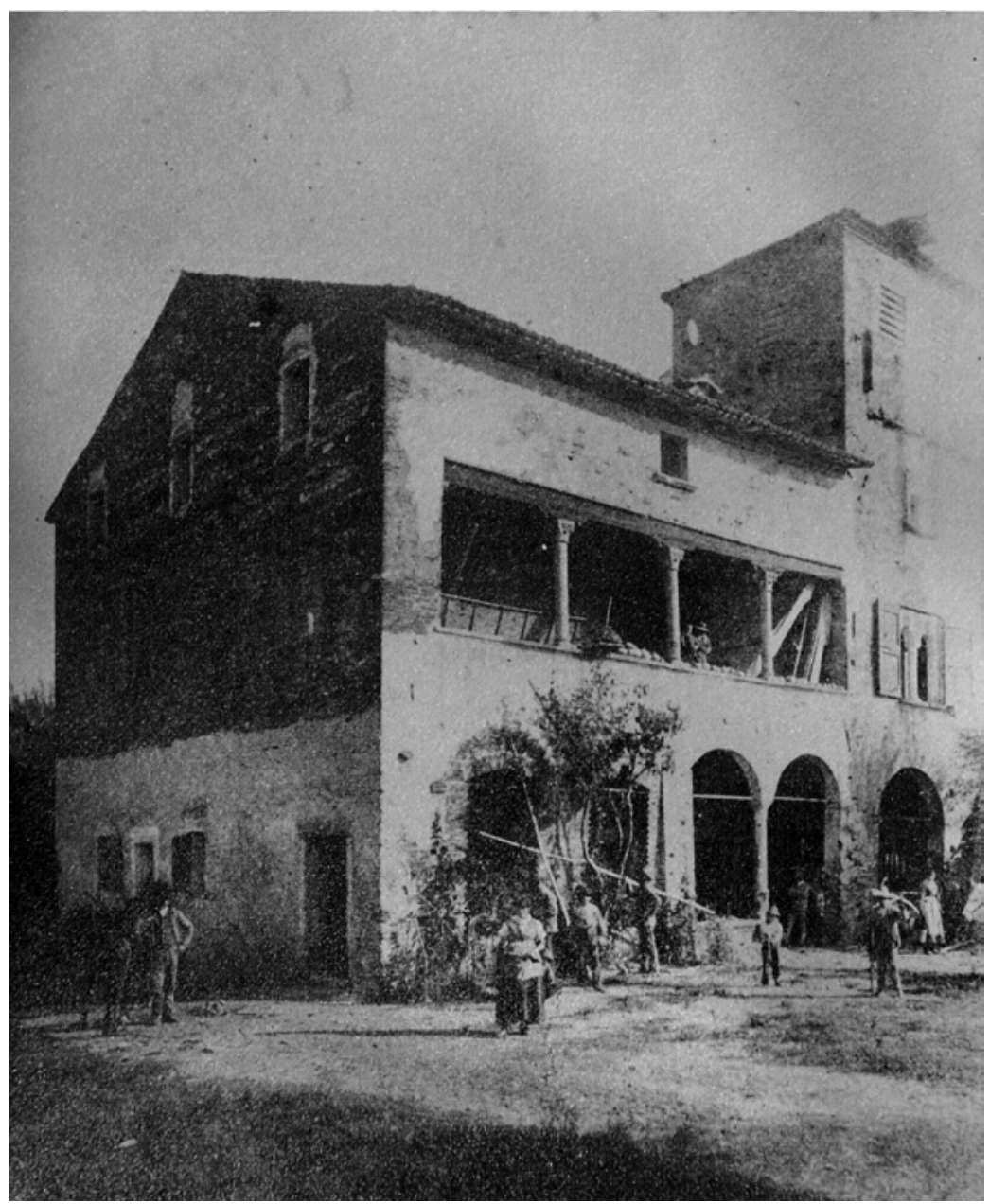

Figura 11 Casa colonica a Pontealto. Dall'album Vicenza Medioevale. Milano, ILASL, Archivio Boni-Tea
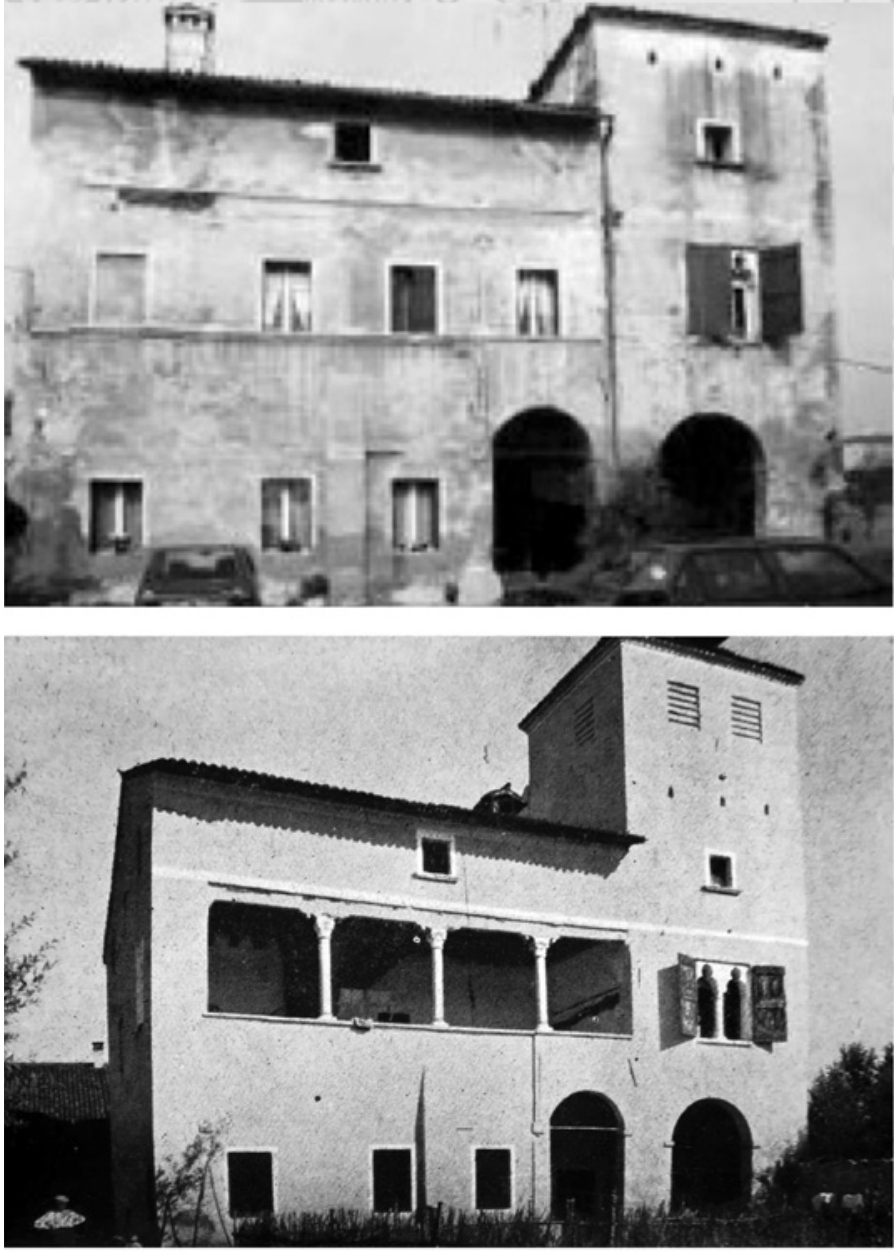

Figura 12 Località Cattane (Vicenza), Villa Loschi, Zileri Dal Verme: fasi dei cambiamenti e restauri dell'edificio dagli anni Trenta del Novecento ad oggi. Da Istituto Regionale Ville Venete 
perato di «Crocifisso Church», ma che indubitabilmente va riconosciuta nel San Salvatore di Spoleto ${ }^{28}$ per la descrizione minuziosa di particolari planimetrici e decorativi e per gli schizzi riconducibili in modo inequivocabile alle forme del portale scolpito e alla configurazione dell'abside [fig. 8]..$^{29}$

Quanto agli allegati fotografici, di essi non rimane traccia nelle lettere di Webb conservate a Milano, mentre per quanto riguarda le lettere di Boni conservate al Courtauld Institute, che non abbiamo ancora potuto esaminare de visu, la presenza fisica delle fotografie associate alle lettere è accertata solo per pochi casi, come per i due ritratti di Boni in mise da 'ladrone' allegati ad una lettera del giugno del 1891, commentati dall'amico a stretto giro di posta [fig. 9]. ${ }^{30}$

Per altre immagini di cui si parla con insistenza nell'epistolario possiamo supplire con materiali sempre presenti presso l'Archivio Boni-Tea: è questo il caso della serie di fotografie di monumenti religiosi e civili vicentini di tardo Medioevo e primo Rinascimento [fig. 10] che, nelle aspettative di Boni, avrebbero dovuto costituire un album dal titolo Vicenza Medioevale: la genesi del progetto risale alla fine del 1887 come rivela una lettera indirizzata a Webb, ${ }^{31}$ nella quale Boni descrive il suo soggiorno vicentino, nel corso del quale compì un'accurata ricognizione dei monumenti della città. Sappiamo anche che una lettera, analoga per contenuti anche se più formale data la minor dimestichezza col destinatario, Boni inviò al Royal Institute of British Architects, indirizzandola a William $\mathrm{H}$. White, forse nella speranza di ottenere dal prestigioso istituto, del quale era corrispondente, un sussidio per la pubblicazione completa dell'album. Webb in effetti contribuì con uno cheque di tredici sterline, e cercò di coinvolgere anche altri amici nel sovvenzionamento, tra cui lo stesso White, ${ }^{32}$ ma la somma non dovette risultare sufficiente, per cui l'album venne prodotto a tiratura limitatissima e senza l'apparato di note illustrative delle tavole: una copia risulta a Vicenza presso il Centro internazionale di Studi Andrea Palladio ed è stata recentemente oggetto di studio. ${ }^{33}$ Quella con-

28 Lettera di Boni a Webb del 30 dicembre 1889 (Tea 1941-1942, 168); lettera di Webb a Boni del 4 gennaio 1890 (Tea 1941-1942, 169 ; Aplin 2016, 2: 57-8). Il nome di chiesa del Crocifisso prende piede nel Cinquecento ma non ha nulla a che vedere con la croce di Alberto Sozio, come invece lascia intendere Aplin 2016, 2: 59.

29 Lettera di Webb a Boni del 4 gennaio 1890: «This door-head at Spoleto, probably built as you suggest in the Vth c., has almost perfect purity of detail and refined skill of execution. The first sign of breaking through tradition, is the semi cornice on the top of the architrave. In Byzantium, this would have been the Cornice proper. In the frieze we have the barbaric corners next to the consoles [in questo punto lo schizzo riproducente il particolare dell'architrave del portale] which is a bungle, such as a horse makes in changing from the trot into the gallop. The carving of the frieze, so soon as the artist had satisfied convention with the usual outline form of scroll, is admirably designed in detail, and cut to perfection, the tendril-like variations from the primal scroll being purely beautiful, and worthy of the descendant of the fine Greek time!». La datazione al V secolo, proposta un po' all'impronta da Boni, è stata svolta ed articolata da diversi studiosi (Russo 1992; Benazzi 2012), mentre una datazione seriore (fine VI - prima metà VII), corroborata anche dalle analisi dei materiali ceramici ed ossei rinvenuti in occasione dello smontaggio dell'architrave del portale, viene ora sostenuta da Emerick 2015-2016, 18. Vedi in generale la raccolta di saggi editi e inediti dedicati al San Salvatore di Spoleto in Bassetti, Ermini Pani, Menestò 2012.

30 Boni viene salutato da Webb con l'epiteto scherzoso my dear Ladrone in una lettera iniziata il 21 e terminata il 27 giugno 1891 , a proposito delle due fotografie allegate da Boni ad una lettera dell'8 di quello stesso mese: Aplin 2016, 2: 109.

31 Tea 1940-1941, 142

32 La lettera a William Hale White del 18 febbraio 1888 (Aplin 2016, 2: 4-5) è molto interessante per la ennesima rievocazione del fatale incontro con Boni e per la attenta descrizione delle fotografie vicentine.

33 Mattiello 2011.

Fonti, letterature, arti e paesaggi d'Europa | Sources, Literatures, Arts \& Landscapes of Europe $1 \mid \mathbf{1 1 6}$ 
servata a Milano, anche se incompleta, è comunque interessante perché da un lato sottolinea il precoce interesse di Boni per l'impiego della fotografia nello studio dei monumenti, dall'altro fornisce informazioni preziose sullo stato conservativo di strutture che, nel tempo, sono state poi alterate: si prenda ad esempio la fotografia $n$. 15 dell'album [fig. 11], corredata da una didascalia generica «casa colonica a Pontealto», ma che, a nostro parere, può identificarsi con villa Loschi, Zileri Dal Verme; la villa che compare, già con alcune alterazioni, nella monografia del Fasolo sulle ville vicentine della fine degli anni ' 20 sotto il nome di villa delle Cattane, ${ }^{34}$ è stata oggetto recentemente di un restauro che, con la riapertura della loggia e del portico, la liberazione delle bifore e il rialzamento della torre ha ripristinato l'aspetto esterno, così come testimoniato proprio dal confronto con la inedita fotografia Boni [fig. 12]. ${ }^{35}$

Il tema ricorrente nello scambio epistolare tra i due membri della Antiscrape ${ }^{36}$ era ovviamente quello delle metodologie e della prassi nel restauro architettonico. Se Webb metteva a parte l'amico veneziano di come venivano condotti i restauri nella Westminster Abbey, ${ }^{37}$ Boni dal canto suo riferiva dell'andamento di quelli condotti a Venezia, a partire da quelli di Palazzo Ducale, denunciando eccessi di zelo nel rifacimento delle sculture che avevano portato a relegare nei depositi i capitelli autentici del loggiato immortalati da Ruskin [fig. 13] per sostituirli con copie, ${ }^{38}$ ma anche smorzando i toni allarmistici di alcuni articoli che comparivano sul Times e altri giornali inglesi gridando allo sventramento e alla dissennata conduzione dei restauri in Italia. ${ }^{39}$ Questo atteggiamento di critica temperata ritorna anche in una lettera a Webb del settembre 1886 in cui la valutazione degli interventi condotti sul pavimento di San Marco, pur con qualche riserva per l'impiego di materiali nuovi nelle parti da risarcire, non è sostanzialmente negativa. ${ }^{40}$ Parole ben più severe Boni pronuncerà qualche anno più tardi, nella sua nuova veste di ispettore ministeriale, denunciando quella «elaborata ed accurata falsificazione dell'antico» che, a suo parere, la Fabbriceria di San Marco (nonostante attuasse metodi non dissimili da quelli immediatamente precedenti) perpetrava ai danni del pavimento, ma in questo caso a muovere lo sdegno del funzionario erano ragioni di conflitto tra amministrazione statale e

\section{Fasolo 1929.}

35 Per alcune fotografie delle varie fasi del complesso e una scheda informativa si rinvia a Istituto Regionale Ville Venete, VI 593. Vedi inoltre Fasolo 1929 e, in generale, sull'architettura civile gotica vicentina Barbieri 1976.

36 Era questo il nomignolo più sintetico e icastico coniato da William Morris per la Society for the Protection of Ancient Buildings, vedi in proposito Donovan 2008. Per la ricaduta dell'azione della SPAB in ambito europeo Lamberini 1998.

37 Lettera di Webb a Boni del 9 febbraio 1890 (Aplin 2016, 2: 65: «Westminster hall itself still being knocked about to make it look more mediæval than it did in Richard II days»); lettera di Webb a Boni del 24 gennaio 1892 (Aplin 2016, 2: 149, in cui i restauri vengono definiti «ridiculous 19th cent: travesties of the mediæval English building»); Lettera di Webb a Boni del 26 maggio/02 giugno 1895 (Aplin 2016, 2: 284); lettera di Webb a Boni del 9 gennaio 1898 (Aplin 2016, 2: 349).

38 Lettera di Boni a Webb .././1889, Tea 1941-1942, 163: John Ruskin, Loggia of the Ducal Palace at Venice, 1849-1850, acquerello su grafite, New York Metropolitan Museum, Rogers Fund 1908, acc. n. 08.227.39.

39 Lettera di Webb a Boni del 15 dicembre 1889, Aplin 2016, 2: 54.

40 Tea 1940-1941, 135: «Just now they think of repairing the mosaic pavement of St. Marks in a way which is neither a restoration proper (destruction of the old and relaying of a new pavement in imitation of the original) nor an absolute preservation of the old work according to archaeological views (by filling up the missing portions with a simple concrete). Now they fill up the missing portions with an imitation of the old work. This method has the advantage of saving the old work, but much of its picturesqueness is spoiled by the garish effect of the new bits of marble. These however will approach the tone of the old in a few years and to an intelligent observer it will always be possible to see which is the original». 
Figura 13 John Ruskin, Loggia of the Ducal Palace, Venice. 1849-1850. New York, Metropolitan Museum,

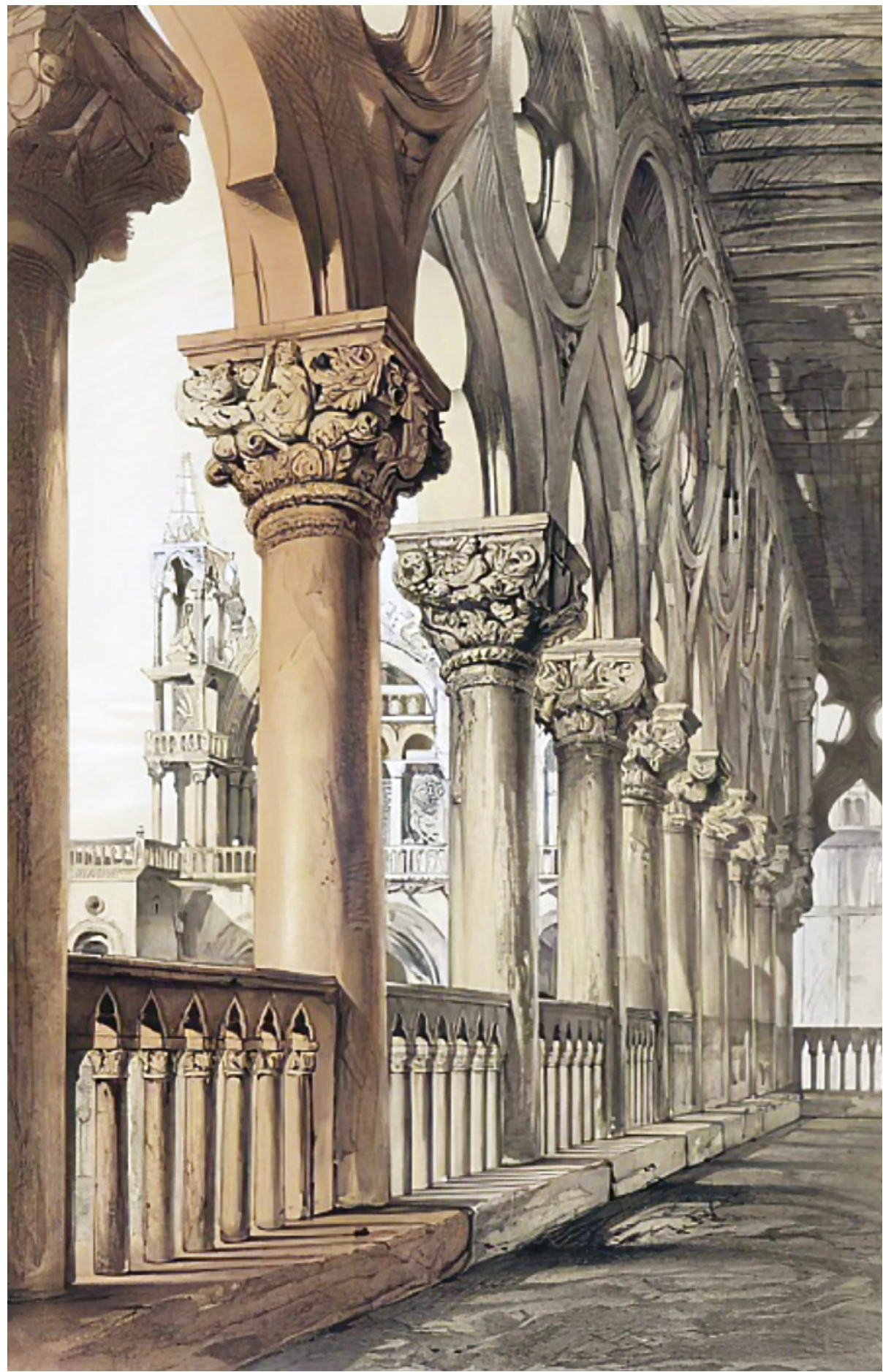




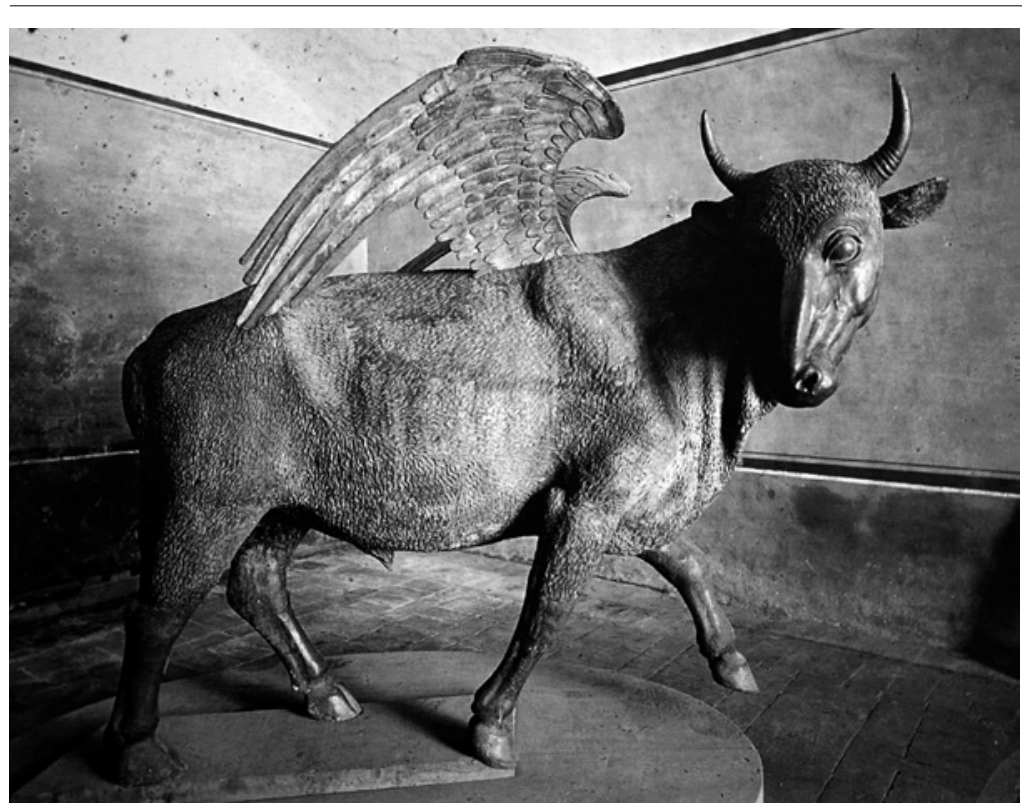

Figura 14 Toro bronzeo del duomo di Orvieto,

fotografia dopo il restauro.

Fondo Armoni Moretti ICCD D 10361

Fabbriceria circa le prerogative dell'esercizio di tutela sul monumento. ${ }^{41}$

Come dimostrano i numerosi interventi su riviste e quotidiani dell'epoca, dal Colore dei monumenti del 1883 alla raccolta Venezia imbellettata del $1887,{ }^{42}$ l'impostazione teorica di Boni in materia di restauro era tutta nel segno della condivisione del verbo ruskiniano, ovvero di sostanziale negazione di qualsiasi statuto all'atto del restauro in quanto esso, nell'imporre un intervento esterno ed estraneo a quello dei primitivi creatori dell'opera, e nell'interrompere il processo di naturale trasformazione della materia esercitato dal tempo, porta alla negazione del concetto di autenticità che, secondo una felice definizione sovente ripetuta da Boni «non costituisce il pregio principale dei monumenti, ma è condizione fondamentale di ogni pregio che essi possono avere». Su questo fondamento teorico si innesta l'azione di indirizzo e tutela negli interventi di restauro che Boni era chiamato a seguire e controllare, con scelte ed esiti che, almeno in parte, deflettevano dal pensiero ruskiniano. Lo dimostra il caso, discusso appassionatamente nelle lettere con Webb, della ricomposizione del toro bronzeo del duomo di Orvieto. Il bronzo maitanesco, «sia per difetto di fusione... sia per indebolimento degli antichi ritegni», era rovino-

41 L'argomento è trattato più diffusamente in Paribeni 2010. Va detto che le ponderate riflessioni sulle metodologie da seguire nel restauro conservativo delle pavimentazioni a commesso marmoreo troveranno la loro più lucida ed efficace applicazione negli interventi sui pavimenti in opus sectile al Foro Romano e al Palatino: vedi in proposito Lugari, Guidobaldi 2013; Guidobaldi, Bossi 2015; Guidobaldi 2020.

42 Questi e altri interventi di Boni sono raccolti ora in Franchini 2016.

119 Fonti, letterature, arti e paesaggi d'Europa | Sources, Literatures, Arts \& Landscapes of Europe 1

Fonti, letterature, arti e paesaggi d'Europa| Sources, Literatures, Arts
John Ruskin's Europe. A Collection of Cross-Cultural Essays, 99-130 
samente caduto a terra nel 1835 e i suoi frammenti erano stati in prima istanza inviati a Roma affinché sulla loro base venisse realizzato un nuovo modello. Agli inizi degli anni Ottanta prevalse invece l'idea di ricomporre il bronzo risaldando i frammenti antichi, completando «in lamina di rame a sbalzo e cesello i pezzi mancanti» e saldandoli agli antichi «mediante intelaiatura o scheletro interno di rame battuto» [fig. 14]. Le citazioni che riportiamo sono prese dal resoconto del restauro pubblicato nel 1891 da Luigi Fumi, il quale attribuisce ideazione e cura dell'intervento ad Adolfo Cozza e l'esecuzione materiale al meccanico Luigi Ravelli. ${ }^{43}$ Nella versione di Boni divulgata nelle lettere a Webb il nome e i meriti di Ravelli vengono sottolineati, mentre nessuna menzione si fa di quello di Cozza, anzi appare chiaro che Boni si attribuisce il ruolo che oggi diremmo di responsabile unico del procedimento. ${ }^{44}$ Anche nell'articolo apparso nel 1889 su Archivio Storico dell'Arte - se non scritto quanto meno ispirato da Boni - è a Ravelli che, per aver seguito le istruzioni ricevute (dal ministero quindi, implicitamente, da Boni), viene riconosciuto il merito di «un lavoro tanto lodevole da superare ogni aspettazione... senza però voler dare l'illusione che l'opera sua apparisse come antica, ma facendo anzi a bella posta distinguere il nuovo dall'originale», mentre «l'egregio architetto conte Adolfo Cozza» viene appena ricordato con un ruolo margina- le. ${ }^{45}$ Potrebbe forse essere stato questo il primo tassello del dissidio che dividerà Cozza e Boni e che esploderà, in maniera più evidente, nella querelle sui controversi scavi del Palatino del 1907 diretti da Dante Vaglieri e lo stesso Cozza, il cui esito finale fu la fusione di Foro romano e Palatino sotto l'unica direzione di Boni. ${ }^{46}$ Riflettendo sulla metodologia seguita nel restauro del bronzo orvietano emerge ancora una volta questa impostazione di fondo ruskiniana conciliata con le preminenti ragioni di salvaguardia della memoria storica e della condivisione del bene con la comunità. Sono pressappoco le stesse considerazioni espresse da Webb allorché, discutendo nel luglio del 1891 con Boni le modalità tecniche più opportune per assicurare la tenuta delle strutture murarie di una torre del XV secolo, chiudeva dicendo che «every building of intrinsically noble, or historic character, which you save to posterity, is, on your part, really socialistic, that is, for the good of the people, regardless of the individual». ${ }^{47}$

Oltre al dibattito sugli interventi di restauro, ad appassionare Webb erano le peregrinazioni che l'instancabile amico compiva per dovere istituzionale in tutta la penisola per monitorare lo stato conservativo dei monumenti e per ampliare la conoscenze del patrimonio artistico nazionale. ${ }^{48}$ Atlante alla mano, Webb seguiva Boni nei suoi spostamenti in siti a lui già noti per averli visi-

43 Fumi 1891

44 Vedi la lettera a Webb del 4 novembre 1889 (Tea 1941-1942, 158-9) e la risposta entusiasta di Webb del 10 novembre (Aplin 2016, 2: 47-50). Ovviamente il cavallo bronzeo del museo Capitolino cui Webb fa cenno - «Do you know the beautiful bronze horse in the Capitol museum at Rome, which has been so injured by time, carelessness or malice?» - è quello proveniente da vicolo delle Palme (su cui cf. Parisi Presicce 2007) e non il Marco Aurelio come intende Aplin 2016, 2: 49.

45 L'articolo su Archivio Storico dell'Arte è firmato «E.A.», sigla sotto la quale sono state cercate, di volta in volta, le personalità di Natale Baldoria, Giuseppe Coceva o lo stesso Boni; sulla questione vedi Paribeni, Guidobaldi 2020, 674, con bibliografia precedente.

46 Sul ruolo di Cozza nel restauro del toro orvietano vedi la ricostruzione dei fatti che ne dà Benocci 2002, 196, fig. 63. Sulla polemica Boni-Vaglieri che coinvolse anche Cozza, vedi Paribeni, Guidobaldi 2020, 607-9.

47 Aplin 2016, 2: 115.

48 Paribeni 2016b. 
tati nel suo viaggio italiano degli anni 1884-1885 e in altri a lui assolutamente sconosciuti. Nelle tappe di queste missioni, la cui consistenza e intensità sono confermate dalle relazioni inviate al Ministero e dai taccuini personali, Boni scovava di tanto in tanto assolute primizie, come la cassa lignea conservata nella cattedrale di Terracina, ora al Museo di Palazzo Venezia, segnalata con una fotografia a Webb nel marzo del 1889, poco prima che Natale Baldoria la pubblicasse su Archivio Storico dell'Arte; ${ }^{49}$ oppure, nell'ottobre del 1890, la cattedra lignea scovata nel santuario di Montevergine, ${ }^{50}$ la cui fotografia tanto eccita la mente di Webb, incuriosito dal carattere barbarico dell'intaglio e dalla eventuale presenza di un organico programma iconografico delle decorazioni. ${ }^{51}$ Anche se non si tratta della prima menzione in assoluto del manufatto, da attribuire probabilmente all'Itinerario da Napoli al santuario di Monte Vergine edito da Annuvola nel 1840, il sopralluogo di Boni precede comunque largamente il primo contributo scientifico del Bertaux degli ultimi anni dell'Ottocento. ${ }^{52}$

Nella curiosità manifestata da Boni e Webb verso declinazioni meno consuete dell'arte medievale uno spazio

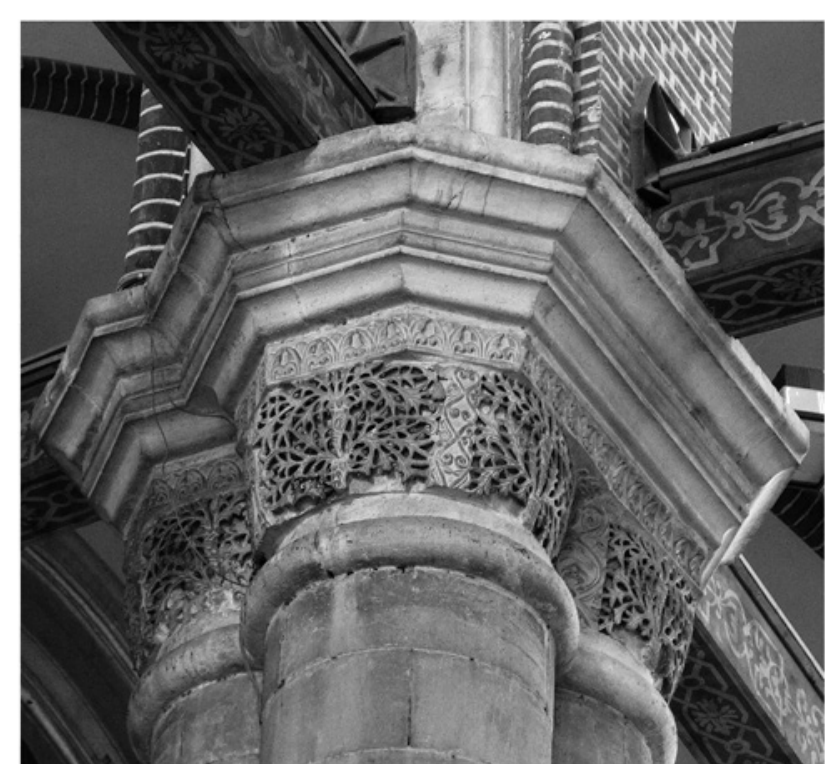

Figura 15 Venezia, basilica dei Santi Giovanni e Paolo, capitelli bizantini provenienti dal San Polieucto di Costantinopoli (Barsanti, Pilutti Namer 2009, fig. 4)

49 Lettera di Boni a Webb del 25 marzo 1889, Tea 1941-1942, 145; Baldoria 1889. Sulla cassa di Terracina vedi da ultimo Curzi 2020.

50 Lettera di Boni a Webb del 1 ottobre 1890, vedi Tea 1941-1942, 200.

51 Lettera di Webb a Boni del 19 ottobre 1890, Aplin 2016, 2: 84: «Now let me give up friendly (individual) sarcasm, and speak of the chairback. This is what I wrote in pencil when I got your photograph - "I would say, that all expression must first be barbaric. If in early (barbaric) times there is no fit expression, there will - in later life - be no expression at all" (so there is some little hope left for the Sphinx) "or only an artificial one. Without the descent into the hell of art" (touching mother earth again, after the Roman degradation) "there could have been no fresh fruitfulness, such as this splendid piece of carved human expression is. Between the 4th and the 9th centuries, there has been time enough for the grosser particles of earth to drop away, without destroying the vigour of the new youth, with the sweet smell of the dew of morning on it. It is this "quality", I take it, which makes us pass slowly, and regretfully, from the Archaic Greek expression, to that of the Pheidian time"».

52 Annuvola 1840, 12; Bertaux 1897. Sulla cattedra vedi da ultimo De Mieri 2016, con bibliografia precedente. Analoghe riflessioni sulla tempestività delle ispezioni di Boni, che battono sul tempo i primi contributi di studiosi italiani e stranieri, si potrebbero fare a proposito degli arredi liturgici abruzzesi di Santa Maria in Valle Porclaneta di cui è parola nelle lettere tra Boni e Webb nell'estate del 1891: per un approfondimento sulla questione rinviamo a Paribeni 2016b, 302-3, nota 44. Ancor prima, quando era a Venezia, Boni (lettera a Webb del 7 febbraio 1888 , Tea 19411942, 135-6) sembra precedere le prime documentazioni ufficiali del ritratto di Maometto II realizzato da Gentile Bellini (Thuasne 1888, VIII) con una sua visita alla collezione Layard a Ca' Capello («I enclose a photograph which I made of the portrait of Mehemet the Conqueror, which was once in the collection of Paolo Giovio and now belongs to Henry Layard. The fur is restored; but much about the face, the carpet and surroundings architecture is original»). Sulla frequentazione della collezione Layard da parte di artisti, studiosi e intellettuali e sulle prime documentazioni fotografiche delle opere in essa contenute vedi Riva 2020, 156-60. Sul ritratto belliniano vedi ora Rodini 2020.

121 Fonti, letterature, arti e paesaggi d'Europa | Sources, Literatures, Arts \& Landscapes of Europe 1

Fonti, letterature, artt e paesaggid Europal Sources, Literatures, Arts
John Ruskin's Europe. A Collection of Cross-Cultural Essays, $99-130$ 
aus porre at available.

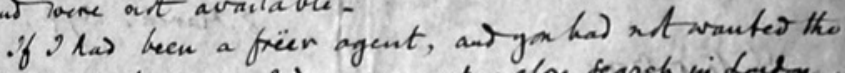
bictures at ace, 2 tare day-apas close seasch vi Labse oniqht have fous a faw from Paleotine whioh wnele

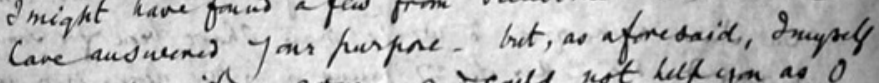

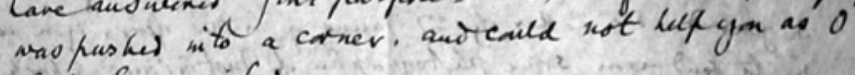
shinle have aroles.

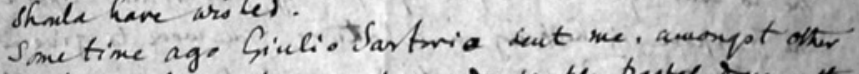

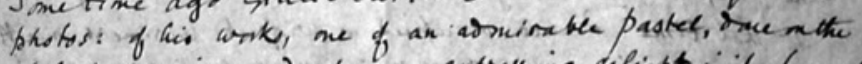
spot, of vesucio: and upa my expersing dilight nit, he sent ne sonce mone viaes fo the his tricic mantain. logrd ais true dothey, decen to me, ars so kelf ful to the

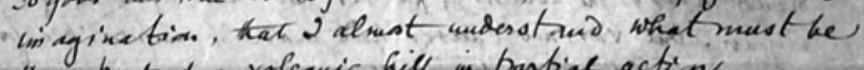
the aspect of a volcanic hill as partial action.

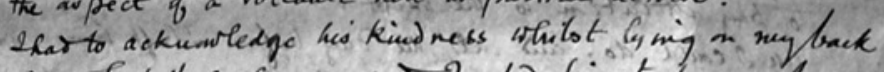

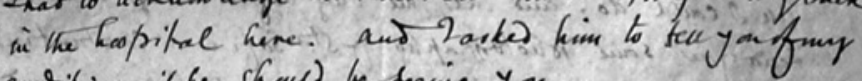
cantitin it he shail be decing y $x$ -

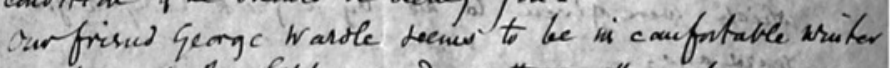

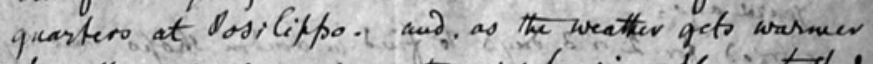

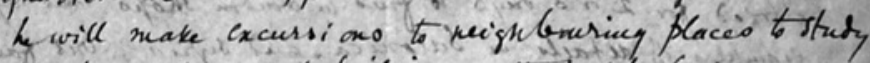
the strier of ancient builings an the prists is is reged apon.

My prisus William Lethaby - auther fo the book on Ankiketural mytto. which le dent to yox, wiske? me whan Juect wrote to yau, to ask

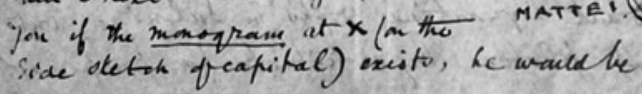

whilst les mig on ney back

(ke) fim to tea you finy

Yo be ni canfortable witer 1 the weatter gets wasmes

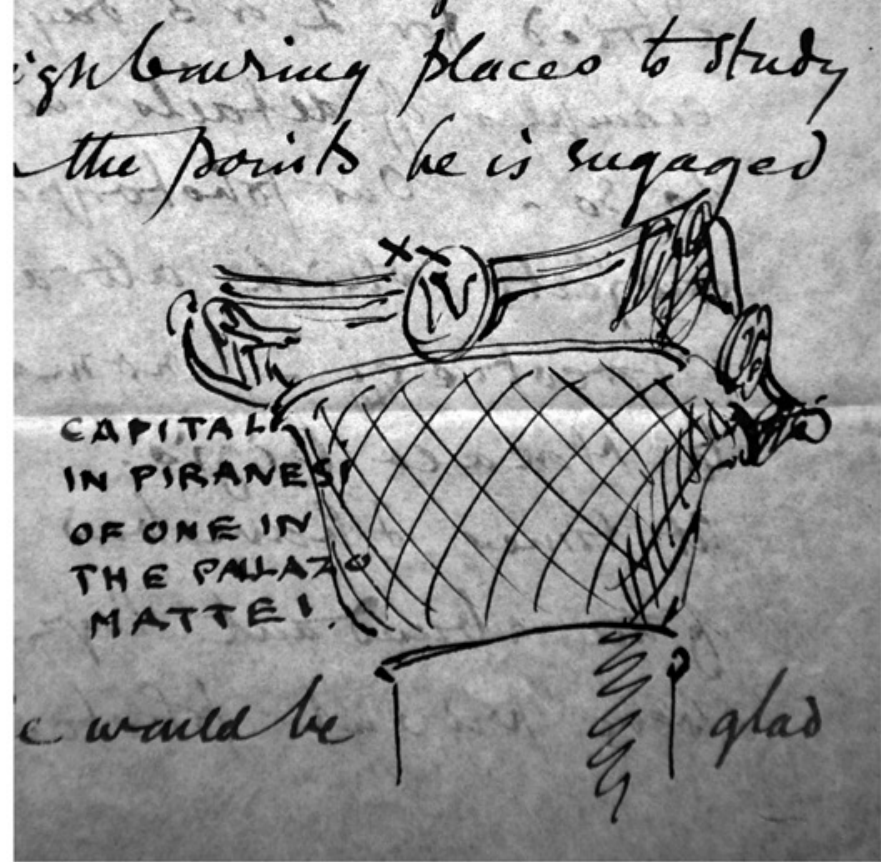

Figura 16 Lettera di Philip Webb a Giacomo Boni dell'8 marzo 1894 con schizzo del capitello in collezione Mattei. Milano, ILASL, Archivio Boni-Tea, LXI. Epistolario U, V, W 


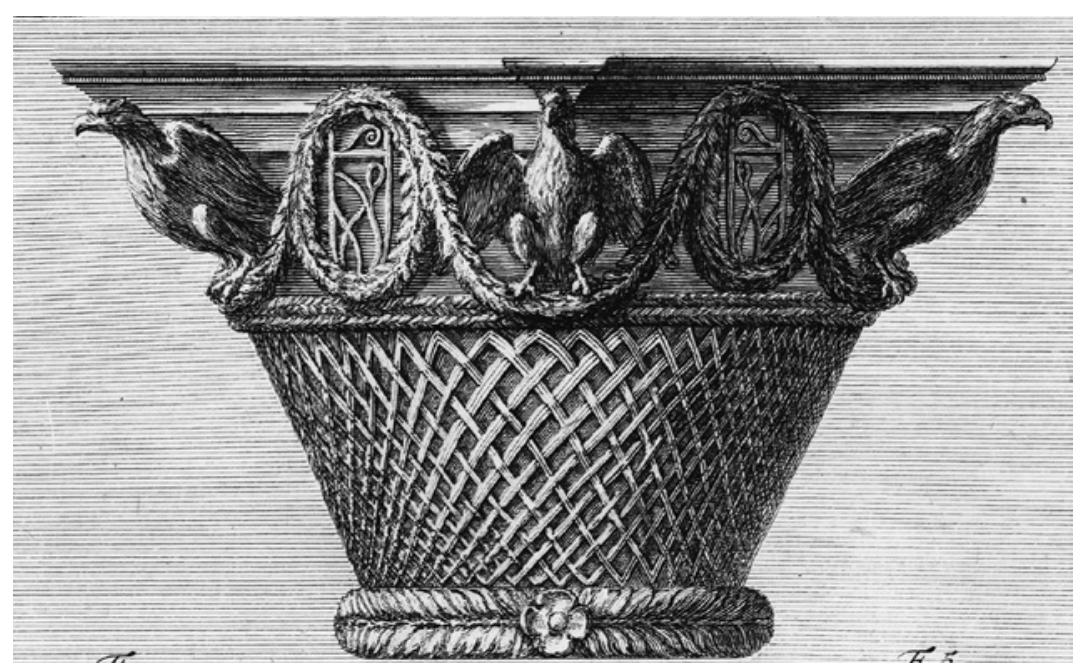

Figura 17 G.B. Piranesi, Incisione del capitello di Palazzo Mattei (Piranesi 1750, tav. 15, fig. 3) particolare lo hanno i marmi e le sculture di matrice bizantina: talvolta è la prodigiosa memoria visiva di Webb a innescare la ricerca, come nel caso dei «triple shafted "tree" capitals» da lui visti a fatica nella chiesa dei Santi Giovanni e Paolo [fig. 15] in occasione del suo soggiorno veneziano $0^{53}$ e per i quali cercava di ottenere fotografie di qualità tramite i buoni uffici di Fairfax Murray o dello stesso Boni, il quale, indotto dall'amico ad osservarli con maggior attenzione, li definiva una «sfolgorante illuminazione scaturita dalle più oscure regioni dell'arte». ${ }^{54}$ Le riflessioni di Boni e Webb su questo lotto di ca- pitelli non si spingono a suggerire per essi un contesto proto bizantino né, tanto meno, a riferirli alla allora ancora sconosciuta chiesa costantinopolitana di San Polieucto, come invece la lucida analisi di Claudia Barsanti e Myriam Pilutti Namer ha chiaramente evidenziato; ${ }^{55}$ va dato tuttavia atto ai due amici di penna di averne saputo cogliere, pur nella difficoltà di osservare sculture poste a 13 metri di altezza, le singolari e straordinarie qualità che li fanno apparentare ai tanti altri marmi provenienti dalla chiesa fatta costruire nel VI secolo da Giuliana Anicia e che, a seguito della IV Crociata, approdarono a

53 Lettera di Webb a Charles Fairfax Murray, 30 giugno 1885, Aplin 2016, 1: 298: «If you should see Alinari in Venice, suggest to him the making of goodsized photographs of the triple shafted "tree" capitals in the Ch. of S.S. John \& Paul. He might do it, if only to spite Naya. These capitals are quite beautiful and are also fresh in style».

54 Lettera di Boni a Webb, datata Epifania del 1887. «Those capitals are a mystery, their existence is to me now like a glazing shining out of the darkest region of history of art. I will not forget them» (Tea 1940-1941, 138). Sui tentativi di realizzare in proprio una fotografia dei capitelli, vedi la lettera di Boni a Webb del 13 dicembre 1886 (Tea 1940-1941, 137).

55 Barsanti, Pilutti Namer 2009.

$123 \begin{aligned} & \text { Fonti, letterature, arti e paesaggi d'Europa | Sources, Literatures, Arts \& Landscapes of Europe } 1 \\ & \text { John Ruskin's Europe. A Collection of Cross-Cultural Essays, 99-130 }\end{aligned}$ 
Andrea Paribeni, Silvia Pedone

La corrispondenza epistolare come rete di conoscenza, dibattito e azione
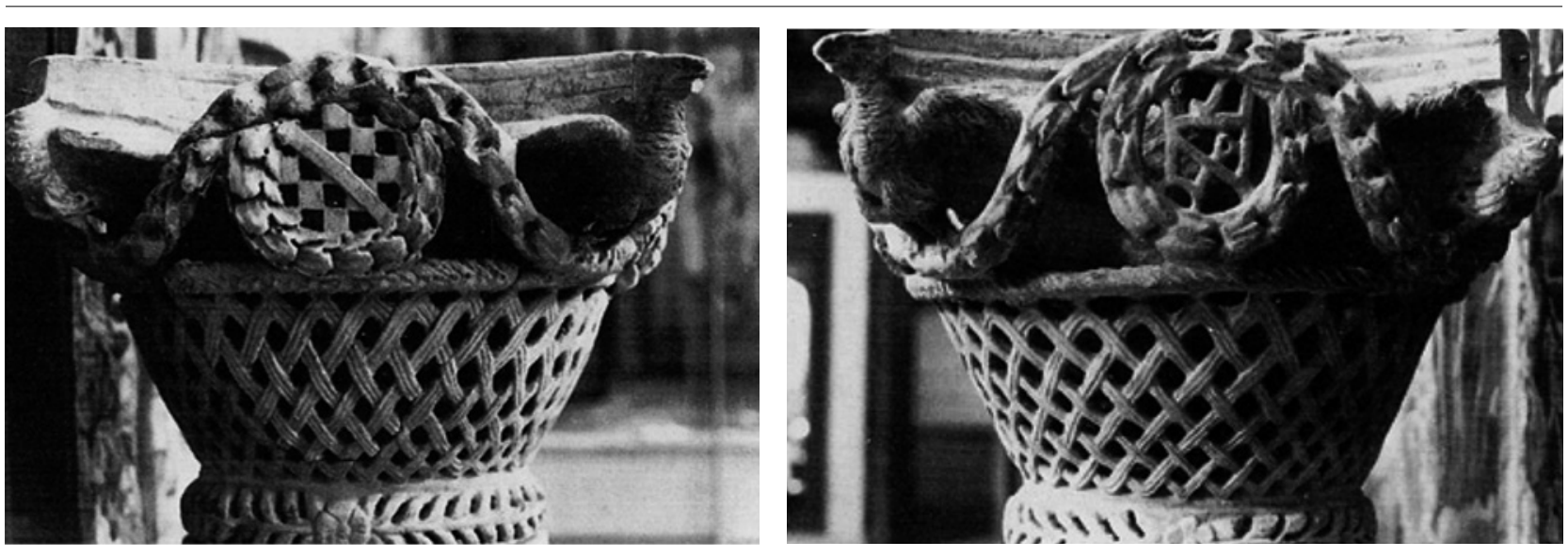

Figura 18a-b Capitelli dalla chiesa dei Santi Cosma e Damiano a Roma. Lione, Tesoro della Cattedrale (Guidobaldi 1989)
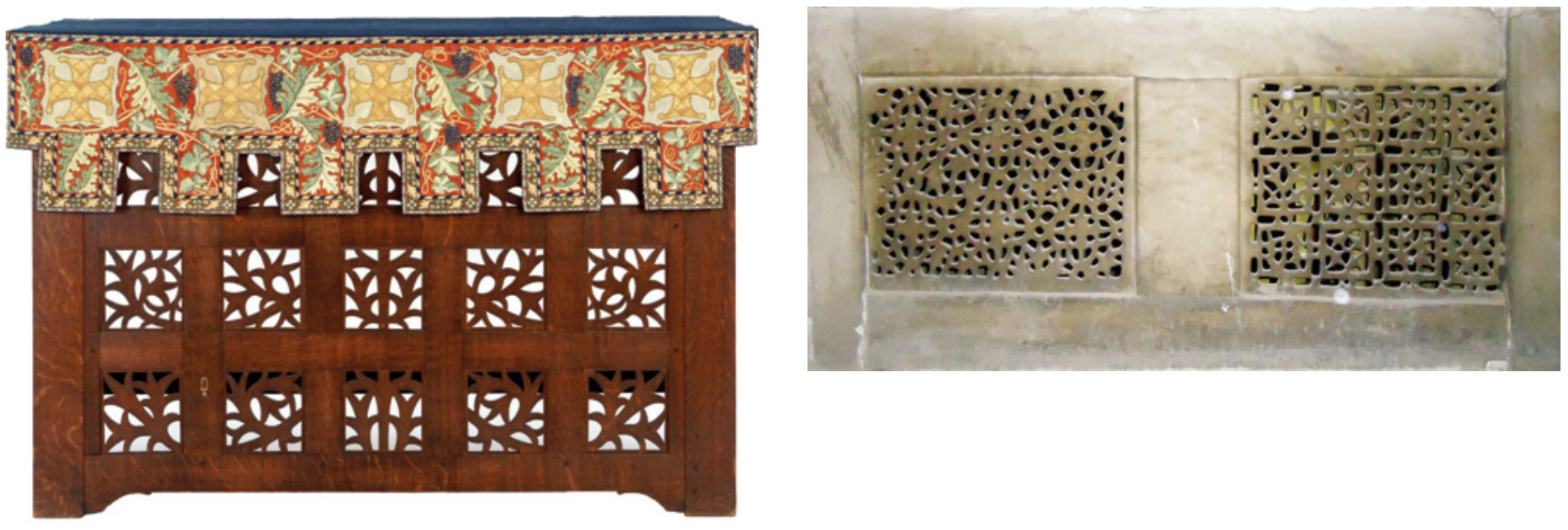

Figura 19 Altare ligneo della John Garrett \& Son su disegno di Philip Webb, 1897. Londra, Victoria \& Albert Museum (V\&A:W.4-2003)

Figura 20 Istanbul, Santa Sofia, particolare di una delle transenne giustinianee riutilizzate nella Hünkâr Mahfili (Loggia del Sultano). Fotografia Silvia Pedone 


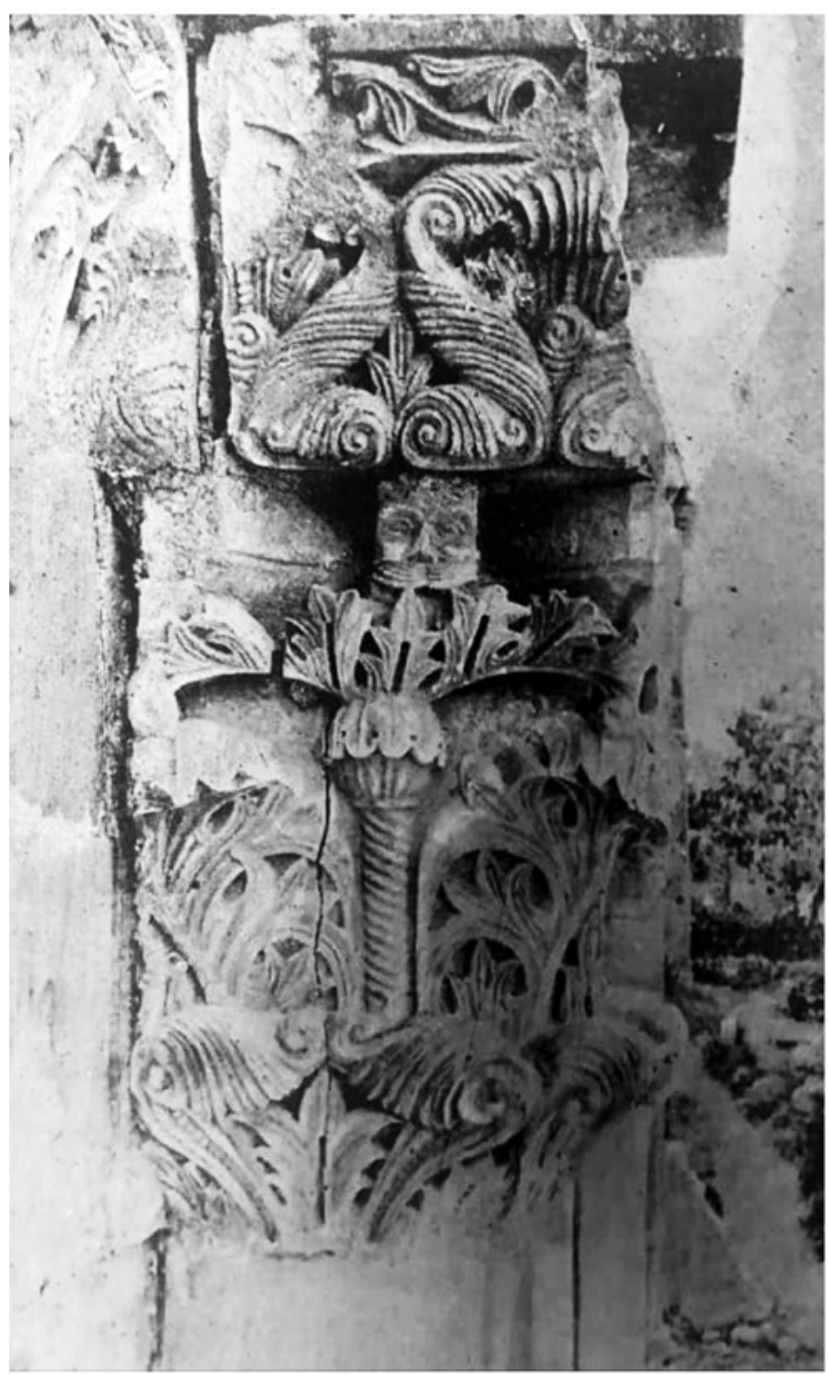

Figura 21 Abbazia di San Clemente a Casauria, capitello del portico (da Calore 1891)

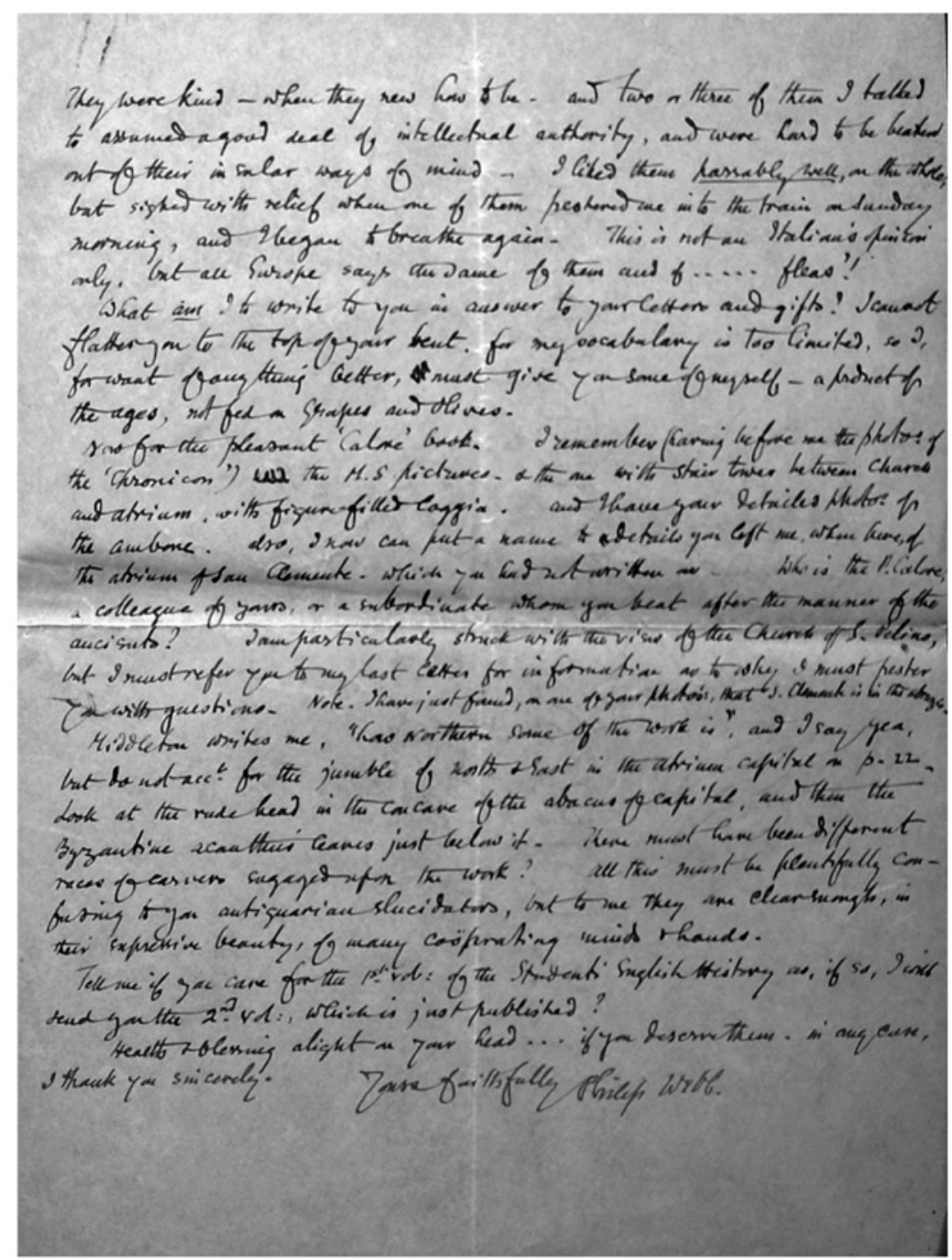

Figura 22 Lettera di Philip Webb a Giacomo Boni del 29 marzo 1891. Milano, ILASL, Archivio Boni-Tea, LXI. Epistolario U, V, W 
Venezia per ornare San Marco e altri edifici della città.. ${ }^{56}$

Qualche anno più tardi, nel 1894, la scultura bizantina di età giustinianea torna alla ribalta nelle missive scambiate tra Boni e Webb: è ancora l'inglese a muovere il primo passo su sollecitazione dell'amico William Lethaby, che aveva necessità di avere uno schizzo del monogramma di un capitello della collezione Mattei [fig.16], a lui noto dalla illustrazione che ne fornisce Piranesi [fig. 17]. ${ }^{57}$ La curiosità di Lethaby era certamente legata allo studio che questi, in sinergia con Harold Swainson, stava conducendo sulla chiesa di Santa Sofia. ${ }^{58}$ Boni tentò, senza successo, di accedere a palazzo Mattei per eseguire lo schizzo, ma se pure fosse riuscito nell'intento di entrare nel palazzo non avrebbe potuto trovare il capitello che, assieme agli altri suoi tre compagni, già dai primi anni dell'Ottocento era trasmigrato a Lione [fig. 18a-b] grazie all'acquisizione fatta dal card. Fesch, arcivescovo della città, come ha esemplarmente dimostrato Federico Guidobaldi in uno studio che ha altresì chiarito che la collocazione originaria dei pezzi era nella chiesa dei Santi Cosma e Damiano. ${ }^{59}$ Per inciso vorremmo sottolineare come lo studio analitico della Santa Sofia condotto da William Lethaby dovette avere una ricaduta sull'ambiente dell'Arts \& Crafts, nel senso di una più stretta consuetudine con stilemi e gusto decorativo tipici dell'età giustinianea: ne fa fede, tra gli altri, l'altare ligneo di- segnato da Webb ed eseguito da John Garrett \& Son nel 1897 per la Rochester and Southwark Diocesan Deaconess Institution [fig. 19], un collegio teologico femminile a Clapham Common (Londra) e ora conservato al Victoria $\&$ Albert Museum, la cui lavorazione a giorno delle foglie d'acanto richiama con grande evidenza i marmi traforati della Grande Chiesa ricostruita da Giustiniano [fig. 20]. ${ }^{60}$

Per concludere, una parte consistente dello scambio epistolare tra Webb e Boni, che qui non abbiamo il tempo di trattare estesamente, riguarda l'interesse di entrambi per i monumenti medievali dell'Italia centro-meridionale, che per il primo costituivano un campo per lo più 'ignoto' e per il secondo una occasione per occuparsi di un territorio negletto e più di ogni altro bisognoso di una ricognizione dettagliata del patrimonio artistico e architettonico al fine di assicurare una capillare azione di tutela. ${ }^{61}$

Le vivide descrizioni e le fotografie inviate da Boni dischiudevano a Webb una realtà culturale straordinariamente variegata, in cui si intrecciavano stilemi normanni, saraceni, bizantini, che suscitavano all'architetto inglese interrogativi sull'origine etnica delle popolazioni che avevano prodotto tali manufatti e sull'eventuale radicamento nel territorio di queste medesime tradizioni artistiche. La stretta convivenza di accezioni diverse suggeriva spericolate metafore enologiche circa la commistione degli stili che, grazie alla strati-

56 Su tempi e modi dell'arrivo di spolia bizantine a Venezia nel Basso Medioevo vedi ora le riflessioni critiche di Tigler 2019.

57 Lettera di Webb a Boni, 8 marzo 1894, Aplin 2016, 2: 252. Piranesi 1750, tav. 15, fig. 3: «Capitello nel Palazzo Mattei rappresentante una canestra circondata a piede da una ghirlanda di lauro. La parte di sopra è ornata di aquile, festoni e ghirlande». Informazioni su questa ricerca anche in Lethaby 1935.

58 Lethaby, Swainson 1894.

59 Guidobaldi 1989.

60 Sull'altare (V\&A:W.4-2003) e sugli altri elementi di arredo liturgico apprestati per la cappella vedi Kirk 2005, 258-61; Livingstone, Parry 2005. Per il confronto con la scultura giustinianea si prendano in esame in particolare le transenne (Barsanti 2004, 499-504) riutilizzate nella loggia del sultano realizzata tra il 1847 e il 1849 dai fratelli Gaspare e Giuseppe Fossati nell'ambito dei restauri dell'edificio, allora (e purtroppo nuovamente) moschea. Sulla recente e dolorosa chiusura dell'Ayasofya Müzesi ci permettiamo di rinviare a Pedone, Paribeni 2020.

61 L'intensa e appassionata opera di documentazione e tutela condotta da Boni in favore dei monumenti medievali dell'Italia meridionale è ben illustrata e analizzata in Giuri 2017. Vedi anche Paribeni 2016b. 
ficazione storica, aveva raggiunto esiti tutto sommato 'armonici'. ${ }^{62} \mathrm{Su}$ un piano più propriamente 'scientifico', numerosissimi sono i commenti su argomenti di dettaglio quanto su questioni più generali riguardanti il contesto monumentale del Mezzogiorno medievale: ci piace citare in chiusura la riflessione di Webb su un capitello di San Clemente a Casauria [figg. 21-22], osservato nella tavola dell'estratto del saggio di Pier Luigi Calore che
Boni gli aveva inviato, ${ }^{63}$ in cui si arriva ad ipotizzare la convivenza di due artefici diversi sullo stesso blocco di marmo, operanti a stretto contatto: un artigiano bizantino per le parti vegetali delle foglie d'acanto e un 'barbaro' le parti figurative. ${ }^{64}$ Una visione forse un po' troppo ingenua, ma certamente preconizzatrice degli accesi dibattiti sul formalismo medievale che di lì a poco avrebbero infiammato l'Europa. ${ }^{65}$ (A.P.)

\section{Bibliografia di John Ruskin}

I riferimenti alle opere di Ruskin rinviano al numero di volume e alle pagine di:

Cook, E.T.; Wedderburn, A. (eds) (1903-1912). The Works of John Ruskin. Library Edition, 39 vols. London: George Allen.

https://www.lancaster.ac.uk/the-ruskin/the-complete-works-of-ruskin/.

Works, 15: The Elements of Drawing, The Elements of Perspective, The Laws of Fésole. | 37: The Letters of John Ruskin II (1870-1889).

\section{Bibliografia generale}

Annuvola, G. (1840). Itinerario da Napoli al santuario di Monte Vergine ed a quello della Madonna dell'Arco. Napoli: Tipografia del Filatre Sebezio.

Aplin, J. (2016). The Letters of Philip Webb. Vol. 1, 1864-1887; Vol. 2, 1888-1898; Vol. 3, 1899-1902; Vol. 4, 1903-1914. London; New York: Routledge.

Baldoria, N. (1889). «La cassa di Terracina». Archivio Storico dell'Arte, 2, 242-47.

Barbieri, F. (1976). «Case e palazzi gotici». Pozza, N. (a cura di), Vicenza illustrata. Vicenza: Neri Pozza, 125-40.

Barsanti, C. (2004). «Le transenne». Guiglia Guidobaldi, A.; Barsanti, C. (a cura di), Santa Sofia di Costantinopoli. L'arredo marmoreo della Grande Chiesa giustinianea. Città del Vaticano: Pontificio Istituto di Archeologia Cristiana, 489-529.

Barsanti, C.; Pilutti Namer, M. (2009). «Da Costantinopoli a Venezia: nuove spoglie della chiesa di S. Polieucto. Nota preliminare». Nía

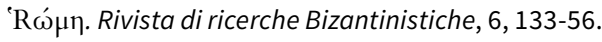

62 Lettera di Webb a Boni del 12 giugno 1892 (Aplin 2016, 2: 164): «When you pour the black wine of Bordeaux into the white wine of Orvieto, you have a spoiled mixture - untestable. But that cannot rightly be said of the mixture of Greek, Saracenic and Norman buildings in Italy, however amalgamated».

63 Calore 1891.

64 Lettera di Webb a Boni, 29 marzo 1891 (Milano, ILASL, Archivio Boni-Tea): «Look at the rude head in the concave of the abacus of capital, and then the Byzantine acanthus leaves just below it, There must have been different races of carvers engaged upon the work? All this must be plentifully confusing to you antiquarian elucidators, but to me they are clear enough, in their expressive beauty, of many cooperating minds \& hands».

65 Basti forse qui ricordare la figura e gli studi di Alois Riegl - e con lui le figure di Franz Wickhoff, Max Dvořák e Julius von Schlosser, protagonisti della Scuola viennese di storia dell'arte (Wiener Schule der Kunstgeschichte) - in cui la questione sull'arte medievale e sul formalismo divengono centrali tanto per il dibattito dell'epoca, che annovera seguaci e detrattori, quanto per la disciplina storico artistica attuale.

127 Fonti, letterature, arti e paesaggi d'Europa | Sources, Literatures, Arts \& Landscapes of Europe 1

Fonti, letterature, arti e paesaggi d'Europa| Sources, Literatures, Art 2 L
John Ruskin's Europe A Collection of Cross-Cultural Essays, $99-130$ 
Bassetti, M.; Ermini Pani, L.; Menestò, E. (a cura di) (2012). La Basilica di San Salvatore di Spoleto. T. 1, Gli studi di Mario Salmi. T. 2, Gli studi editi. T. 3, Gli studi inediti. Spoleto: Fondazione Centro italiano di studi sull'alto medioevo.

Bellini, A. (2006). «Giacomo Boni tra John Ruskin e Luca Beltrami». Lamberini, D. (a cura di), L'eredità di John Ruskin nella cultura italiana del Novecento $=$ Atti del Convegno di Studi (Firenze, 15-18 novembre 2000). Firenze: Nardini, 3-30.

Benazzi, G. (2012). «La facciata e il suo restauro (1992-2000): nuovi elementi per la conoscenza della chiesa di San Salvatore a Spoleto». Bassetti, M.; Ermini Pani, L.; Menestò, E. (a cura di), La Basilica di San Salvatore di Spoleto. T. 3, Gli studi inediti. Spoleto: Fondazione Centro italiano di studi sull'alto medioevo, 923-57.

Benocci, C. (2002). «Non modo ars sed etiam scientia: Adolfo Cozza tra arte, tecnica e progetti di architettura e di ingegneria». Tamburini, P.; Benocci, C.; Cozza Luzi, L. (a cura di), Adolfo Cozza. Ponte San Giovanni (Perugia): Quattroemme, 135-56.

Bertaux, E. (1897). «I monumenti medievali della regione del Vulture». Napoli Nobilissima, 6, supplemento.

Bradley, J.L.; Ousby, I. (eds) (1987). The Correspondance of John Ruskin and Charles Eliot Norton. Cambridge: Cambridge University Press. Bunney, S. (2007). «John W. Bunney's “big picture” of St Mark's, and the Ruskin-Bunney relationship». Ruskin Review and Bulletin, 4, $18-47$. Calore, P.L. (1891). "L'Abbazia di S. Clemente a Casauria». Archivio storico dell'arte, 4, 9-36.

Clegg, J. (2010). «John Ruskin's Correspondence with Angelo Alessandri». Hanley, K.; Sdegno, E. (eds), Ruskin, Venice and Nineteenth-Century Cultural Travel. Venezia: Cafoscarina, 69-107.

Clegg, J.; Tucker, P. (1992). Ruskin e la Toscana = Catalogo della mostra (Londra, Accademia Italiana delle Arti e delle Arti Applicate, 8 gennaio-7 febbraio 1993; Sheffield, Ruskin Craft Gallery, 20 febbraio-10 aprile 1993; Lucca, Fondazione Centro Studi sull'Arte Licia e Carlo Ludovico Ragghianti, 1 maggio-12 giugno 1993). Sheffield: Ruskin Gallery.

Collingwood, W.G. (1893). The Life and Work of John Ruskin. 2 vols. Boston; New York: Houghton, Mifflin and Company; Cambridge: The Riverside Press.

Curzi, G. (2020). «La cassa lignea di Terracina tra Riforma e Crociata». Gigliozzi, M.T.; Nuzzo, M. (a cura di), Terracina nel Medioevo. La cattedrale e la città = Atti del Convegno di Studi (Terracina 9-10 febbraio 2018). Roma: Viella, 105-12.

Dearden, J.S. (ed.) (1967). The Professor: Arthur Severn's Memoir of John Ruskin. London: Allen \& Unwin.

De Mieri, S. (2016). «Intagliatori campani, secoli XII-XIII: seggio abbaziale». Leone De Castris, P. (a cura di), Museo abbaziale di Montevergine: catalogo delle opere. Napoli: Artstudio Paparo, 44-6.

Donovan, A.E. (2008). William Morris and the Society for the Protection of Ancient Buildings. New York: Routledge.

E.A. (1889). «Restauro e ricomposizione del toro di bronzo nel duomo d'Orvieto». Archivio Storico dell'Arte, 2, 433-4.

Emerick, J.J. (2015-2016). «The Basilica of San Salvatore in Spoleto: The Structural History». Spoletium. Rivista di Storia Arte Cultura, 5253, 17-54.

Fasolo, G. (1929). Le ville del vicentino. Vicenza: Arti Grafiche delle Venezie.

Forcellini, A. (1887). «Sui ristauri delle principali facciate del Palazzo Ducale di Venezia». L'ingegneria a Venezia nell'ultimo ventennio. Venezia: Stab. Tip. P. Naratovich, 1-21.

Franchini, S.G. (2016). Quando cultura e politica salvarono Venezia: Giacomo Boni e il destino di Venezia tra Otto e Novecento. Venezia: Marsilio.

Fumi, L. (1891). Il duomo di Orvieto e i suoi restauri: monografie storiche condotte sopra i documenti. Roma: A cura del Ministero della Pubblica istruzione e dell'Opera del duomo.

Giuri, P. (2017). Giacomo Boni. Cronache sulla conservazione di un ignorato patrimonio architettonico nell'Italia meridionale. Galatina (Lecce): Mario Congedo editore, 56.

Gombrich, E. [1959] (1962). Arte e illusione. Torino: Einaudi.

Guidobaldi, F. (1989). «Origine costantinopolitana e provenienza romana di quattro capitelli del VI secolo oggi a Lione». Mélanges de l'Ecole Française de Rome. Antiquité, 101, 317-64.

Guidobaldi, F. (2008). «Le carte dell'Archivio Boni-Tea all'Istituto Lombardo di Milano. Cenni sul ritrovamento, sulla consistenza e sullo stato della pubblicazione». Fortini, P. (a cura di), Giacomo Boni e le istituzioni straniere. Apporti alla formazione delle discipline storico-archeologiche =Atti del Convegno Internazionale (Roma, Museo Nazionale Romano-Palazzo Altemps, 25 giugno 2004). Roma, 23-31. 
Guidobaldi, F. (2016). «Note dall'Archivio Boni-Tea: la progettata e mai realizzata pubblicazione di Giacomo Boni sugli scavi del Foro e del Palatino». Favaretto, I.; Pilutti Namer, M. (a cura di), Tra Roma e Venezia. La cultura dell'antico nell'Italia dell'Unità: Giacomo Boni e i contesti. Venezia: Istituto Veneto di Scienze, Lettere ed Arti, 165-81.

Guidobaldi, F. (2020). «La deperibilità dei sectilia pavimenta e un'innovativa tecnica di consolidamento sperimentata da Giacomo Boni in tre edifici del Foro romano nel 1899-1901». Esposito, D.; Montanari, V. (a cura di), Realtà dell'architettura fra materia e immagine. Per Giovanni Carbonara: studi e ricerche, vol. 1. Roma; Bristol: L'“Erma” di Bretschneider, 793-802.

Guidobaldi, F.; Bossi, S. (2015). «L'atrium Vestae nel Foro Romano: i sectilia pavimenta nel contesto strutturale del complesso». Musiva \& Sectilia, 12, (2019), 17-205.

Istituto Regionale Ville Venete, VI 593. http://irvv.regione.veneto.it/lib/pxmlServiceGate.php? fAction=XwAttachment\&fCm$\mathrm{d}=$ get\&fName=G1553PD001.PDF\&fRespMode=show\&-fId=11175.PDF.

Kirk, S. (2005). Philip Webb; Pioneer of Arts \& Crafts Architecture. Wiley Academy.

Lamberini, D. (1998). «I nobili sdegni. Le battaglie inglesi della SPAB contro i restauri nel continente e l'influsso sui proseliti europei della conservazione». Quaderni di storia dell'architettura e restauro, 20, 7-44.

Lethaby, W.R. (1935). Philip Webb and His Work. London: Oxford University Press.

Lethaby, W.R.; Swainson, H. (1894). The Church of Sancta Sophia Constantinople. A Study of Byzantine Building. London; New York: Macmillan \& Co.

Levi, P. (1899). «La riabilitazione del Foro Romano». Rivista politica e letteraria, T. 3, vol. 6, fasc. 2, 112-37.

Levi, D.; Tucker, P. (1997). Ruskin didatta: il disegno tra disciplina e diletto. Venezia: Marsilio.

Livingstone, K.; Parry, L. (eds) (2005). International Arts and Crafts. London: V\&A Publications.

Lugari, A.; Guidobaldi, F. (2013). «I rivestimenti marmorei pavimentali e parietali delle residenze di Nerone sul Palatino alla luce dei recentissimi restauri». Angelelli, C. (a cura di), Atti del XVIII Colloquio dell'Associazione Italiana per lo Studio e la Conservazione del Mosaico (Cremona, 14-17 marzo 2012). Tivoli: Scripta Manent, 613-26.

Mattiello, A. (2011). «Giacomo Boni: A Photographic Memory for the People; Documenting Architecture Through Photographic Surveys in Post-unification Italy». Caraffa, C. (ed.), Photo Archives and the Photographic Memory of Art History. Berlin; München: Deutscher Kunstverlag, 217-26.

Moore, A.H. (2018). «Designing Energy Use in a Rural Setting: A Case Study of Philip Webb at Standen». History of Retailing and Consumption, 4(1), 28-42.

Morris, W. (1893). Concerning Westminster Abbey. London: Women's Printing Society.

Paribeni, A. (2010). «Le campagne di restauro di pavimenti e mosaici nella Basilica di San Marco a Venezia alla fine dell'Ottocento: una 'elaborata ed accurata falsificazione'?». Angelelli, C. (a cura di), Atti del XV Colloquio dell'Associazione Italiana per lo Studio e la Conservazione del Mosaico (Aquileia, 4-7 febbraio 2009). Tivoli: Scripta Manent, 279-91.

Paribeni, A. (2016a). «Note dall'Archivio Boni-Tea. I materiali grafici per lo studio della Casa Romuli». Favaretto, I.; Pilutti Namer, M. (a cura di), Tra Roma e Venezia. La cultura dell'antico nell'Italia dell'Unità: Giacomo Boni e i contesti. Venezia: Istituto Veneto di Scienze, Lettere ed Arti, 183-211.

Paribeni, A. (2016b). «I monumenti del Mezzogiorno medievale nei taccuini di Giacomo Boni (1888-1898): documentazione, tutela, conservazione del patrimonio artistico nell'Italia post-unitaria». Arte Medievale, 4(6), 293-304

Paribeni, A.; Guidobaldi, F. (2020). Giacomo Boni: documenti e scritti inediti. Catalogo ragionato dell'Archivio Boni-Tea (ILASL - Istituto Lombardo Accademia di Scienze e Lettere - Milano). Tivoli: Scripta Manent.

Pedone, S.; Paribeni, A. (2020). «La Santa Sofia e il suo Museo: percorsi di lettura». Boletín de la Sociedad Española de Bizantinística, 36, 26-52.

Parisi Presicce, C. (2007). «Un cavallo di bronzo per più cavalieri. La riscoperta di un originale greco a Roma». Bullettino della Commissione Archeologica Comunale di Roma, 108, 33-54.

Pilutti Namer, M. (2013a). «Ruskin e gli allievi: Note su Giacomo Boni e la cultura della conservazione dei monumenti a Venezia a fine Ottocento». Ateneo Veneto, 3(12), 423-35.

129 Fonti, letterature, arti e paesaggi d'Europa | Sources, Literatures, Arts \& Landscapes of Europe 1 John Ruskin's Europe A A Collection of Cross-Cultural Lssays, $99-130$ 
Pilutti Namer, M. (2013b). «Mastro di Palazzo Ducale, prima che archeologo: Giacomo Boni e la Venezia dell'Ottocento». Failla, M.B.; Meyer, S.A.; Piva, C.; Ventra, S. (a cura di), La cultura del restauro. Modelli di ricezione per la museologia e la storia dell'arte = Atti del Convegno Internazionale (Roma, 18-20 aprile 2013). Roma: Campisano, 581-93.

Pilutti Namer, M. (2016). «Safeguarding Venice: Giacomo Boni and John Ruskin». Change over Time, 6(1), 24-37.

Pilutti Namer, M. (2018). «ll fondo ‘Eva Tea' al Museo di Castelvecchio: linee interpretative per una ricognizione preliminare». Verona Illustrata, 31, 147-54.

Pilutti Namer, M. (2019). Giacomo Boni. Storia, memoria, archeonomia. Roma: L'“Erma” di Bretschneider.

Piranesi, G.B. (1750). Opere varie di architetture, prospettiva, groteschi, antichità, sul gusto degli antichi Romani inventate ed incise da Giambattista Piranesi architetto veneziano. Roma.

Riva, C. (2020). «The Ca' Capello Layard and Its Art Collection: A Forgotten Anglo-venetian Treasure House of the Late Nineteenth-century». Colnaghi Studies Journal, 6, 148-69.

Rodini, E. (2020). Gentile Bellini's Portrait of Sultan Mehmed II. Lives and Afterlives of an Iconic Image. London; New York: I.B. Tauris.

Russo, E. (1992). «Su S. Salvatore di Spoleto sul tempietto del Clitunno». Acta ad Archaeologiam et Artium Historiam pertinentia, 8, 87-143.

Tea, E. (1927). «Boni e Ruskin». Il Marzocco, 14

Tea, E. (1932). Giacomo Boni nella vita del suo tempo, 2 vols. Milano: Ceschina.

Tea, E. (1940-1941). «Introduzione alla corrispondenza fra Philip Webb e Giacomo Boni». Annales Institutorum, 13, $127-48$.

Tea, E. (1941-1942). «Corrispondenza tra Philip Webb e Giacomo Boni II 1889-1890». Annales Institutorum, 14, 135-209.

Tea, E. (1959). «ll carteggio Boni-Caroë sui monumenti veneziani: 1881-1889». Archivi, 26, fasc. 1-2, 234-54.

Thuasne, L. (1888). Gentile Bellini et Sultan Mohammed II: notes sur le séjour du peintre vénitien à Constantinople (1479-1480) d'après les documents originaux en partie inédits. Paris: Leroux.

Tigler, G. (2019). «Trofei della Quarta Crociata? Punti fermi per la datazione delle facciate marmoree di San Marco». Vio, E. (a cura di), La Basilica di San Marco. Arte Storia Conservazione. Venezia: Marsilio, 131-49. 


\title{
Ruskin's Ontology of Architecture
}

Pedro Marques de Abreu

CIAUD, Faculdade de Arquitectura - Universidade de Lisboa, Portugal

\begin{abstract}
Ruskin's critique to architecture is usually understood from the subject of style, as the defence of Gothic against Classicism. If that had been the case, his writings about architecture would have lost all of their pertinacity. But that is not the case. This paper inspects the topicality of Ruskin's thinking about architecture. His observations on the subject are phenomenological observations avant la lettre: the result of his own experience, highly sensitive, and of his personal reflection upon it, deeply human. Almost a century before Heidegger, Ruskin describes the anthropological responsibility of architecture in a very similar manner to the one the German philosopher. My understanding is that Ruskin is revealing the 'dwelling' ability that pertains to architecture, and that gives it its proper identity. Without architecture's stamp on the landscape, it would not be possible for men to 'dwell' on Earth, and hence, it would not be possible for men to be rightly humans, i.e. to re-member (in Ruskin's terminology) - to accomplish that specific human trait of existence that is necessary for an authentic living, which is to be self-aware.
\end{abstract}

Keywords Architecture. Drawing. Design. Novelty. Memory. Dwelling in. Shelter. Humanity. Ruskin. Phenomenology. Intentionality.

Summary 1 Introduction. - 2 Part I: The Change of the Design Method in Architecture. - 2.1 A Matter of Style? - 2.2 Drawing's Appearance in Architectural Design. - 2.3 Advantages of Drawing in Architectural Design. - 2.4 Nuisances of Drawing in Architecture. - 2.5 Current State of Affairs. - 3 Part II: The Nature of Architecture. - 3.1 What, Then, is Architecture, According to Ruskin? - 4 Conclusion. - 4.1 From the Castle of Granson to the Greek Temple.

\section{Introduction}

"If Mr. Ruskin be right", wrote a reviewer soon after the publication of The Stones of Venice, in 1853, "all the architects, and all the architectural teaching of the last three hundred years, must have been wrong". "That is indeed precisely the fact", replied Ruskin in a later edition. "I believe the architects of the last three centuries [and he would probably agree 
in adding the ones of the next two] to have been wrong; wrong without exception; wrong totally, and from the foundation". ${ }^{1}$

Quite a bold statement! This sort of peremptoriness, the patronizing tone, drives people today to deem Ruskin's views outdated, not politically correct in the least.

Twentieth and twenty-first century theory of Architecture ${ }^{2}$ allots to Ruskin three main views on this matter: his advocacy of the gothic; ${ }^{3}$ his emphasis on ornamentation; ${ }^{4}$ and his vindication of Conservation against Restoration as relates to ancient buildings. ${ }^{5}$ Only this latter argument is considered up-to-date. Yet these perspectives match neither the whole nor the essence of what Ruskin has perceived in architecture. He was a man of extreme sensitivity towards beauty and, specifically, the beauty of architecture, a sort of sensitivity that was so correspondent to Proust, for instance (consider his introduction to the translation of the Bible of Amiens). ${ }^{6}$ Disregarding Ruskin's views would be thriftless.

Let us consider the quotation at the opening of this paper. Ruskin says, first, that all architects have been wrong "without exception". This implies that he does not see architecture as mainly a matter of personal talent, of artistic ability, otherwise some architect would have been rescued from this all-inclusive condemnation.

1 Links 1960, 9

2 Consider: Clark 1964; Di Stefano 1983; Choay 1992; Wheeler, Whiteley 1992; Lang 1999; Botton 2007.

3 "But there is a farther reason for our adopting of the pointed arch than its being the strongest form; it is also the most beautiful [...]. Not the most beautiful because is the strongest; but because its form is one of those which, as we know by its frequent occurrence in the work of Nature around us, has been appointed by the Deity to be an everlasting source of pleasure to the human mind" (Ruskin, Lectures on Architecture and Painting, I, § 8 [1853] = Works, 12: 25). Ruskin, despite all his insight, is a 19th century character, and he is not an architect. His mindset usually rests in taxonomies (a la Linnaeus); and his thought is analytical: he is not acquainted with the trial-and-error procedure (abductive) of architectural design. Thus, for him, to design a piece of architecture is to choose, among the elements of a certain language, and to compose - rather like finding the right words for a particular speech. The required unity of the work of architecture would be achieved through the correct choice of elements and correct assemblage of them - not from the beginning, as in a growing living being: something that someone familiar with the design process would have known. To imagine the possibility of a new style, specific to its time - as happened with late 19th century painters or early 20th century architects - or, even, the absence of style - as happened with the architects of the late 20th century - would be asking too much of Ruskin. For him, therefore, the main issue about aesthetics of architecture was the alternative between the two present systems of forms: the Classical or the Gothic. That is not, of course, a subject people of our time engage with. Nonetheless, his remarks about the Gothic, the reasons he presents to vindicate his pick, are quite topical. These reasons mainly have to do with the agreement with Nature. About the topicality of this argument see Abreu 2020.

4 "Ornamentation is the principal part of architecture. [...] The highest nobility of a building does not consist in its being well built, but in its being nobly sculptured and painted" ("Addenda to Lectures I and II", Edinburgh Lectures, § 57 [1854] = Works, 12: 81). Also topical are the reasons Ruskin presents in favour of ornamentation - although generally forgotten. Since the somewhat broad social refusal of the purist architecture of the 20th century, architects begin again - especially around the 1980s with the Post-Modern Movement - to give careful consideration to ornamentation. In a way, Ruskin anticipates the claim of Robert Venturi - that "less is a bore". He realizes that the order of a design should manifest itself in the details of such a design; otherwise the design will be felt as cold and incomplete. Moreover the refusal of ornamentation leads to a downgrade of the artisans involved in building, which brings about important social and economic consequences.

5 Regarding Heritage, Ruskin's thinking has been thoroughly considered since at least the Athens Chart of 1931, and it has coalesced, as one of the mainstays in the modern theories of Restauration, especially in Italy. "We have no right to touch them [the buildings of past times]. They are not ours. They belong partly to those who built them, and partly to all the generations of mankind who are to follow us. [...] Better a crutch than a lost limb" (Lamp of Memory, § XX = Works, 8: 245). These Ruskinian claims still inform the contemporary and most accepted way of relating to Heritage.

6 Writes Proust, in the "Préface”: “C'est Ruskin: si sa statue n'est pas à la porte de la cathédrale, elle est à l'entrée de notre cœur" (Proust 1904, 38).

Fonti, letterature, arti e paesaggi d'Europa | Sources, Literatures, Arts \& Landscapes of Europe $1 \mid$ 
Therefore, the issue is not of a personal or individual nature; it must be of a more substantial kind. Second, he says that all architects have been "totally" wrong, and "from the foundation". Again, identifying such profound or structural misconceptions about architecture in his contemporaries means that he has an understanding of the nature of architecture that is radically different from the one that surrounds him. Finally, he agrees that the tragic change in the understanding and practice of architecture took place about three centuries before, around the fifteenth century. What happened then? What does he really mean by all of this?
In the next pages these questions will be examined. I shall firstly try to understand what decisive change happened in the field of architecture in the fifteenth century, and how this change could affect the understanding of the nature of architecture. Then I shall review Ruskin's understanding of the nature of architecture, of its essence (because if he perceives a deficit it means that he has a more comprehensive view), presenting the reasons for his complete disagreement with the current understanding. In conclusion, I will highlight some considerations about the actuality and topicality of Ruskin's understanding of architecture.

\section{Part I: The Change of the Design Method in Architecture}

\subsection{A Matter of Style?}

Three centuries before Ruskin, we find ourselves in the heyday of the Renaissance. We all have had a general idea, since high school even, of the important changes this period brought to architecture; most people suppose that the key change was in the dominant language, from gothic to classical. Therefore one assumes that Ruskin is criticizing the use of the classical formal vocabulary, which became current during and after the Renaissance. This view would be coherent, moreover, with many other lectures and writings of Ruskin, where he defends the shapes and procedures of the gothic period, versus those used subsequently. Nonetheless it would be superficial to presume that his criticism is against classical language. Ruskin clearly confronts this misunderstanding in his 1859 lecture "Modern Manufacture and Design":

Perhaps one of the dullest and least justifiable mistakes which have yet been made about my writing, is the supposition that I have attacked or despised Greek work. I have attacked Palladian work, and modern imitation of Greek work. ${ }^{7}$ Of Greek work itself I have never spoken but with a reverence quite infinite. [M]y effort has been not less continually to make the heart of Greek work known than the heart of Gothic: [...] and my complaint of the modern architect has been, not that he followed the Greeks, but that he denied the first laws of life in theirs as in all other art. ${ }^{8}$

7 A note of the Library Edition of The Complete Works of John Ruskin, specifically indicates that: "Reference to the General Index will show how much attention Ruskin paid to Greek art".

8 John Ruskin, Modern Manufacture and Design (A Lecture delivered at Bradford, March 1st, 1859, in The Two Paths, Lecture III, § $80=$ Works, 16: 325-6). Sometimes, moreover, Ruskin shows even contempt about the subject of language or styles: "And so strongly do I feel this that I would, for my own part, at once consent to sacrifice my personal predilections in art, and to vote for the exclusion of all Gothic or Mediæval models what-

133 Fonti, letterature, arti e paesaggi d'Europa | Sources, Literatures, Arts \& Landscapes of Europe 1 
Ruskin, furthermore, occasionally even praises certain Renaissance buildings, as in the whole volume three of The Stones of Venice (1853), dedicated to the archi- tecture of that period. Still, if it is not a matter of language, what is it a matter of? I dare to say it is a matter of method.

\subsection{Drawing's Appearance in Architectural Design}

Renaissance architects discovered drawing as the fundamental tool to produce architecture; and drawing, in multiple forms, including models and Computer Aid- ed Design, has continued to be used as such until today. This apparently small change began a long series of marked ripples in the pond of the discipline.

\subsubsection{Architectural Drawing in the Middle Ages}

Before the Renaissance, drawing, or at least scale drawing, was not used to imagine and anticipate or simulate the future edifice. The Middle Ages used drawing in architecture, but mainly as a technical instrument, one that was solely used in the process of construction. They used to draw, in pavement covered with a layer of gypsum or in a whitewashed wall (although sometimes also in parchment), the stone or wood pieces that the craftsmen were to carve afterwards. These, however, were

drawings of constructive parts of the building, drawn in real size (not to scale), so the artisans could take measurements and make the pieces. ${ }^{9}$ Drawing was used neither to study an architectural idea/design nor to communicate this idea/design to the building staff - the two most essential kinds of architectural drawing that were inaugurated during the Renaissance. From the Middle Ages only a few global architectural drawings remain, but these are not drawings of invention or design. ${ }^{10}$

soever, if by this sacrifice I could obtain also the exclusion of Byzantine, Indian, Renaissance-French, and other more or less attractive but barbarous work; and thus concentrate the mind of the student wholly upon the study of natural form, and upon its treatment by the sculptors and metal workers of Greece, Ionia, Sicily, and Magna Græcia, between 500 and 350 B.C." in The Study of Architecture in Our Schools (1865) § $17=$ Works, 19: 36-7.

9 Pereira 2011, 945-1539.

10 The word 'design' in the English language, and in the field of Architecture and Art, seems to have at least four different meanings, which correspond to three different words in the Latin languages. The word 'design' translates to 'projecto' (in Portuguese), 'proyecto' (in Spanish), 'progetto' (in Italian), 'projet' (in French), meaning the process by which an object is idealized. It is the use of the word that occurs when someone speaks about 'Design Methods'. In this paper 'design' should be understood in this sense. The word 'design' also means the documents or objects by which the ideas are communicated, which represent the object prior to its building. I will use the word 'project' to translate such a notion. 'Design' also translates into 'desenho', 'diseño', 'disegno', 'dessin', which means the shape of an object from which a certain style or personality emanates (in a diverse sense from which these Latin words translate to 'drawing'). This meaning occurs when someone speaks of a "good design", or a "bad design", or the design of some architect (or designer). The fourth meaning of the word designates the discipline, that focuses on giving form, with aesthetic value, to any kind of instrument. It matches what in Italian is called 'Disegno Industriale'. In this last sense, as a discipline, I will capitalize the word: 'Design'. I will also use the same logic about the capitalization or non-capitalization of such words as Architecture: the term written with capital letter should be interpreted as the discipline; without a capital letter it should be interpreted as the object(s) produced by the discipline. 


\subsubsection{Villard de Honnecourt}

It is true that Villard de Honnecourt left a notebook with specific architectural drawings: elevations (though not sections), ground plans emphasizing the spatial and geometrical modules, analysis of the geometry of important building parts (columns, vaults, trusses...). He would have done these around the thirteenth century.

\subsubsection{Brunelleschi}

Brunelleschi is the first who is known to have used drawing to simulate constructed reality. He was so sure of the potentialities of such a tool to communicate his thinking that he didn't bother to follow the building process. He left his drawings for the façade of the Ospedale degli Innocenti in Florence to a subordinate

\subsubsection{Alberti}

Alberti theorized about this procedure and commonly used it. In his De re aedificatoria (1485), the first treatise of Architecture after the one of Vitruvius (first century B.C.), he recommends the use of scale-drawings and/or scale-models (Book II, Chapter I) inasmuch as:

[T] here you may easily and freely add, retrench, alter, renew, and in short change every Thing from one End
Still, that is more a notebook of memoranda - serving as a manual - where he took notes of the buildings he examined, in order to refer to them in the future. ${ }^{11}$ Nothing similar to the architectural drawings of Leonardo, to the recommendations of Alberti in the De re aedificatoria, or to the adventures of Brunelleschi.

and went away to visit other construction sites for which he was responsible.12 (Later, even Michelangelo did something similar when, in Rome, he sent back to Florence a scale-model, in clay, for the execution of the stairs of the Laurentian Library, whose previous plan, done when he was at Florence, had left him unsatisfied). ${ }^{13}$

to the other, till all and every one of the Parts are just as you would have them, and without Fault. ${ }^{14}$

Also,

[Y]ou will thereby have a clear and distinct Idea of the Numbers and Forms of your Columns, Capitols, Bases, Cornishes, Pediments, Incrustrations, Pavements, Statues and the like, that relates either to the Strength or Ornament. ${ }^{15}$

11 "[I]l suo Taccuino non è destinato all'esecuzione: è un insieme di idee e di forme raccolte qua e là negli edifici di cui aveva apprezzato qualche particolare o che aveva 'amato', come scrive a proposito di una finestra di Reims" (Bechmann 1988, 45).

12 Tavares 2003, 79.

13 Tavares 2012, 106.

14 Alberti 1986, 22.

15 "I therefore always highly commend the ancient custom of Builders, who not only in Draughts and Paintings, but in real Models of Wood or other Substance, examined and weighted over and over again, with the advice of Men of the best Experience, the whole Work and the Admeas- 
It is worth noticing that Alberti highlights the specificity of architectural drawing - which should be "plain and simple" - in contrast to "the design of a painter", insofar as the architect when drawing "only designs to show the real Thing itself". He even warns against the seduction of letting oneself be driven to a more artistic representation of the architectural "contrivance". ${ }^{16}$

Alberti moreover consistently declines visiting the construction site - thus, to inspect and correct the experience of space he had anticipated - and frequently complains about the builders who do not follow his scale-drawings and alter the proportions he figured. ${ }^{17}$

\subsubsection{Leonardo}

But perhaps the most articulated statement is the one of Leonardo. By the time he was painting the Last Supper, at the monastery of Santa Maria delle Grazie, Milan
This demonstrates his entire reliance on the drawing, both to communicate his ideas, and to totally conceive a piece of architecture. One may already perceive a drift in the understanding of architecture's essence, inasmuch as the focus on drawing pushes him to consider the core of architecture as "a specific eurythmy, where the agreement between a visual factor and a similar musical universal basis was pursued". ${ }^{18}$

It is no longer the experience of space that matters, but the "experience" of the drawing, of the geometrical logic of what was designed. Possibly due to this, he was considered the first modern architect.

(1495-1498), he was immersed in deep anatomical concerns. He wrote then in his notebook that if he draws a human member in 3 views (approximately the ones

urements of all its Parts, before they put themselves to the Expence or trouble. By making a Model you will have the Opportunity thoroughly to weigh and consider the Form and Situation of your Platform with respect to the Region, what Extent is to be allowed to it, the Number and Order of the Parts, how the Walls are to be made, and how strong and firm the Covering; and in a Word the all Particulars which have spoken of in the preceding Book: and there you may easily and freely add, retrench, alter, renew, and in short change every Thing from one End to the other, till all and every one of the Parts are just as you would have them, and without Fault. Add likewise, that you may then examine and compute (what is by no means to be neglected) the Particulars and Sum of your future Expence, the Size, Height; Thickness, Number, Extent, Form, Species and Quality of all the Parts, how they are to be made, and by what Artificers; because you will thereby have a clear and distinct Idea of the Numbers and Forms of your Columns, Capitels, Bafes, Cornishes, Pediments, Incrustrations, Pavements, Statues and the like, that relates either to the Strength or Ornament" (Alberti 1986, 22 = De re aedificatoria, Book II, Chapter I). Emphasis added.

16 "I must not omit to observe that the making of curious polished Models, with the delicacy of Painting, is not required from an architect that only designs to show the real Thing itself; but is rather the Part of a vain Architect, that makes it his Business by charming the Eye and striking the Fancy of the Beholder, to divert him from a rigorous Examination of the Parts which he ought to make, and to draw him into Admiration of himself. For this Reason, I would not have the Models too exactly finished, nor too delicate and neat, but plain and simple, more to be admired for the Contrivance of the Inventor, than the Hand of the Workman. Between the Design of the Painter and that of the Architect there is a Difference, that the Painter by the Exactness of his Shades, Lines and Angles, endeavours to make the Parts seem to rise from the Canvass, whereas the Architect, without any Regard to the Shades, makes his Relieves from the Design of his Platform [horizontal representation], as one that would have his Work valued, not by the apparent Perspective, but by the real Compartments founded upon Reason. In a Word, you ought to make such Models, and consider them by yourself, and with others diligently, and examine them over and over so often, that there shall not be a single Part in your whole Structure, but what you are thoroughly acquainted with, and know what Place and how much Room it is to possess, and to what Use to be applied" (Alberti 1986, 22 = De re aedificatoria, Book II, Chapter I).

17 Brandão 1964, 818.

18 Brandão 1964, 10. All the translations, unless otherwise specified, are by the Author. 
suggested by Vitruvius, in his newly found architectural treatise $)^{19}$ he can see it as if he had it in his own hand, and have a more thorough knowledge of it than if he slashed more than ten bodies. ${ }^{20}$ He produced several drawings with this. Meanwhile he began to sketch architectural drawings in his notebook. Curiously, these drawings refer to architectural structures that look very close to what Bramante was doing, around the same time (late 1470s to early 1490s) and in the same place, in the cupola of the main chapel of Santa Maria delle Grazie (Leonardo's painting too is in the monastery of Santa Maria delle Grazie): drawings of churches/tem-

\subsection{Advantages of Drawing in Architectural design}

The use of drawing, as the main instrument of design, has opened wide and delightful landscapes to architects since then: first and foremost, the pleasurable game of creation (almost like a semigod, while doodling their ideas with pencil and pen); then, the emancipation of artisanship.

The architect was [...] the idealizer of a space, was the one who had the 'idea' of the volume, its peculiarities, its secrets; he was the individual who conceived abstractly, who conceived with 'drawing'. [...] Likewise, an architect is not a manual worker, he is the one who commands the workmen. He gives his knowledge, not his manual labor. [...] As soon as the mental conception has been ples with a central ground plan and four or more chapels around, distributed according to the main axis, and with semi-spherical vaults above. Later, Bramante will repeat approximately the same layout as the module of his project for Saint Peter's in Rome (the plan was coined in a medal of 1506). It is possible to infer then, although without any solid proof, that some kind of contamination between Leonardo and Bramante took place during those overlapping years in Milan. Who gave what to whom is difficult to ascertain, but surely, during the process, drawing became the current media and space-geometry the chief concern in Architecture.

defined, the building process should follow pari passu the drawing: the 'model' of the mental image. ${ }^{21}$

Not only has the architect no longer to dirty his hands, like a common workman, but he is now able to control, on his own, bigger and more complex buildings, to command more workmen, and by this increasing the size (thus, his wage) and rate of production (decreasing the length of time for the building process). Henceforth architects will be on par with the other intellectual professions; drawing has allowed architects to climb the social ladder. ${ }^{22}$ Notwithstanding, this trend brought about several nuisances.

19 The three kinds of representation, according to Vitruvius (Book I, Chapter I, § 2, of The Ten Books on Architecture) are: Ichonografia (ground plan, representation on a horizontal plan), Orthografia (elevation, representation on a vertical plan), and Scaenographia (view from the side, in a foreshortening way, that allows to see simultaneously the front and side elevations) (Vitruvius 1998).

20 Leonardo da Vinci, Quaderni d'Anatomia, foll. 2r, 13v [in Quaderno di Anatomia B, Windsor collection], cited in Murray (1972, 124).

21 Brandão 1964, 10, 11, 14. Brandão makes other important remarks: "The most important concern of the architect is 'order', 'reason', 'ideal image', this being the converging result of spatial-mental-abstract procedures" (Brandão 1964, 13). "In short, the architect would be the thinker, not the craftsman, the philosopher who materializes his theories in spatial elements in stone. These elements portray, more or less accurately, eurythmies, symmetries, and harmonical proportions, both of the universe and of the human being" (Brandão 1964, 17).

22 Alberti writes on the Preface of the De re aedificatoria (first and second paragraphs): "But before I proceed further, it will not be improper to explain what he is that I allow to be an Architect: For it is not a Carpenter or a Joiner that I thus rank with the greatest Masters in other Scienc-

137 Fonti, letterature, arti e paesaggi d'Europa | Sources, Literatures, Arts \& Landscapes of Europe 1

John Ruskin's Europe. A Collection of Cross-Cultural Essays, $131-150$ 


\subsection{Nuisances of Drawing in Architecture}

While the architect is socially elevated, the artisan is downgraded, becoming, in the building process, something not that different from machines or animals, whose thinking is not worth considering (Ruskin calls attention to this phenomenon several times). ${ }^{23}$ Furthermore the design itself loses much richness, as it no longer includes the input of the different craftsmen in the building crew: it would be enough to compare San Lorenzo or San-

\subsubsection{The Experience of Architecture}

To the layman the judgment about the value of piece of architecture requires its full and direct experience. A person enters a building and evaluates whether or not they have a pleasurable experience from it. Such an experience develops from the input of all of the senses working together and interacting with each other. According to specialists, ${ }^{24}$ the experience of space involves twenty-one kinds of perceptive stimuli - far more than to Spirito in Florence - designs of Brunelleschi - with other medieval churches of the same city to see it: the coldness of the former is striking in comparison to the latter. Over the course of this story, what the architect feels to be the core of his discipline has changed: it has become the design, not the building. From here seeped in the splitmind effect that took over the discipline of Architecture. the usually assumed inputs of the five senses. The layman's experience of a piece of architecture is, surely, aesthetical, insofar as it depends on the sensations ('aesthesis' being at the root of 'sensation'), and also because it involves an assessment of a pleasurable effect - or, in other words, of beauty. Nevertheless, it would be inappropriate to say that the layman's common experience of a piece of architecture is an 'aesthetical' one (in the

es; the manual Operator being no more than an Instrument to the Architect Him I call an Architect, who, by sure and wonderful Art and Method, is able, both with Thought and Invention, to devise, and, with Execution, to complete all those Works, which, by means of the Movement of great Weights, and the Conjunction and Amassment of Bodies, can with the greatest Beauty, be adapted to the Uses of Mankind: And to be able to do this, he must have a thorough Insight into the noblest and most curious Sciences. Such must be the Architect" (Alberti 1986).

23 Consider Lectures on Architecture and Painting (1854) (Addenda to Lectures I and II, § $74=$ Works, 12: 97): "[F]or on the acceptance of this [principle] depends the determination whether the workman shall be a living, progressive, and happy human being, or whether he shall be a mere machine, with its valves smoothed by heart's blood instead of oil, - the most pitiable form of slave. [...] And it is with especial reference to the denial of this principle in modern and Renaissance architecture, that I speak of that architecture with a bitterness which appears to many readers extreme, while in reality, so far from exaggerating, I have not grasp enough of thought to embrace, the evils which have resulted among all the orders of European society from the introduction of the Renaissance schools of building, in turning away the eyes of the beholder from natural beauty, and reducing the workman to the level of a machine"; also, sections 15 to 16 of "The Nature of Gothic" (in The Stones of Venice, $1853=$ Works, 10: 194-5): "It is not that men are ill fed, but that they have no pleasure in the work by which they make their bread, and therefore look to wealth as the only means of pleasure. It is not that men are pained by the scorn of the upper classes, but they cannot endure their own; for they feel that the kind of labour to which they are condemned is verily a degrading one, and makes them less than men. [...] But to feel their souls withering within them, unthanked, to find their whole being sunk into an unrecognized abyss, to be counted off into a heap of mechanism numbered with its wheels, and weighed with its hammer strokes - this, nature bade not, - this, God blesses not, - this, humanity for no long time is able to endure". See also The Bible of Amiens (1884) (Chapter IV - Interpretations, § 5, last note = Works, 33: 125 ff.); The Two Paths [1858] (Lecture I, $\S 15=$ Works, 16: 268). See also Arendt 1958, especially Chs. 3 and 6.

24 VanPutte 2014, 462-3. 
usual Baumgartean sense, which implies the appreciation of an artistic accomplishment). ${ }^{25}$ They may be overwhelmed by surprise, but regardless, they will return to that place only if they have felt alright there.

When the architect was the builder as well - as happened in medieval times and still happens in vernacular

\subsubsection{The Experience of Designing}

The architect plays with forms through drawing, making models or, currently, through 3D computer drawings; he then critiques these and elects one design/idea instead of the other to be pursued and developed again, through drawing or modelling. However, the reality on which he bases his judgment and makes his choice is just a plain drawing/model. He does not engage in the full range of space perception one has of a real building. Of course it would be possible to argue that the architect, while he is drawing, is not just experiencing a drawing, that he can foresee the experience of the space he has drawn. Considering the wide range of stimuli involved in the per-

\subsubsection{Drawing as 'Abstraction'}

The use of drawing in the process of design, when one is not aware of its repercussions, brings about a reductive way of thinking about Architecture.

The phenomenological intentionality ${ }^{26}$ no longer invests the architectural object represented in the drawing. Therefore, it is not humanly possible to relate with it in a thoroughly architectural manner.

Moreover, the use of drawing to invent a space, even architecture - he could have the same experience that was described for the layman (with the twenty-one perceptive stimuli), just after the building was completed (regarding some aspects, even before). And he could immediately appreciate the result and, if necessary, correct it. Not so when the heart of the discipline is considered the design.

ception of space, such a statement is quite difficult to accept - it would require a lot of experiences of spaces, then converted to drawings or models, in order for the architect to have something as a vocabulary of drawn forms, from which he would perfectly know the whole environmental experience. Even so, the architect would be limited to applying, in his new drawings, the forms which he had experience of, the ones which belong to his vocabulary. Regarding all the forms that one architect invents - nonexperienced spatial forms - it would not be possible to claim he has a complete idea of its perceptive and existential repercussions.

when using complex drawing systems that allow the imagination of different perspectives of that space, imparts an abstraction from reality, thus a subtraction of several elements of this reality. The dimensions of the perception of space which are not possible to be represented in the Euclidean space are overlooked in the drawing. Reality is reduced to what is possible to represent by such a medium.

25 Baumgarten coined the term 'aesthetics' in his work Aesthetica published between 1750 and 1758 . He diverged from Kant and supplied the grounds a Philosophy of Art. Thereafter, 'aesthetics' has been commonly used in reference to art.

26 By phenomenological intentionality - in coarse words - I mean that the perception of every thing or being always presumes an intention the perceiver is always engaged in some kind of expectation, of which they are usually unaware. Husserl coined the term and presented it; Merleau-Ponty (1976) detailed it. 
The architect, however, when designing by drawing or modelling, tends to forget this reduction, inasmuch as it is compensated for by the continuous flux of new information, despite being closed within oneself. This leads to a kind of inebriation and estrangement. The process of design is substantially a process of dialogue between one that proposes something - the subjectcreator - and one that assesses, accepting or rejecting, what has been proposed - the subjectcritic. ${ }^{27}$ The use of drawing enables one person alone to perform, at times, both the role of creator and the one of critic, dialoguing through the drawing; one is never playing alone, which is why it is so inebriating and alienating. Besides, when reducing the elements of space, they become easier to handle and faster to process, offering themselves to endless play. Accordingly, drawing used in the architectural design process enables an immensely intense and enjoyable game of creation. Yet in this process the architect tends to forget he is only dealing with an object which offers itself to be looked at (and, beyond that, a mental object) - not an object which offers itself as dwelling place.

\subsubsection{The Criterion of Novelty}

The absentminded change of focus on the architectural perceptive elements leads to an absentminded change of criteria of assessment. The aesthetic judgment, performed during the moments of critique of the design process, is not implemented towards the full experience of the imagined architectural space, but towards the ele-
Insofar as the architect does not have the full range of space experience while completely immersed in the creative game, he tends to neglect elements of space perception that are not shown in the drawing (sound behaviour, scale etc.), or to neglect the change in the experience of certain elements present in the drawing when transported to reality (changes in the perception and appreciation of colour, light, proportions etc.). Subsequently, the architect tends to concentrate on the immediate feedback of the apparent elements (mainly on the harmonically aesthetical relationships) between the whole and the parts depicted through projective geometry. ${ }^{28}$ Yet, as stated above, throughout these periods of abstraction and absentminded drift of focus, the one who is designing does not feel he is losing something. On the contrary, the relevant elements of the foreseen architectural experience that are missing are replaced by others, which grow in importance, levelling the resulting self awareness. The final perceptive score is that the one who is designing is in total contact with reality. ments of the space that are manipulable and controllable in the drawing: (balance, order, form dynamics, harmonic proportions - in sum, aesthetic aspects of the form. ${ }^{29}$ The architect, unaware of such a change, begins to evaluate the imagined space 'plastically' - as if it were a sculpture - both because he does not receive in-

27 See Hurson 2007; Cross, Roozenburg 1992, 333; Schön 1983.

28 Regarding the concentration of the architect in the design, and namely, in the Proportion, Ruskin writes, in sections 106 to 109, in Lecture IV (1875) of the Two Paths (Works, 16: 354): "Our conclusion is - must be - that you will not amuse, nor inform, nor help anybody; you will not amuse, nor better, nor inform yourselves: you will sink into a state in which you can neither show, nor feel, nor see, anything, but that one is to two as three is to six. And in that state what should we call ourselves? Men? I think not. The right name for us would be - numerators and denominators. Vulgar Fractions. § 109. Shall we, then, abandon this theory of the soul of architecture being in proportional lines, and look whether we can find anything better to exert our fancies upon?".

29 Arnheim 1977 
formation from the drawing about the elements that are specific to the architectural experience, which are impossible to represent in the drawing (scale, light, sound behaviour etc.); and because he is not investing the represented space with the phenomenological intentionality, proper to dwelling (which would raise more natural expectations about the space). Hence the assessment does not concern the imagined space's appropriateness to dwell in, as it was supposed to.

But there is more. The architect has the notion and the actual experience of the inconsistency and controversy of an aesthetic judgment upon a plastic event. Thus even this judgment tends to drift away towards something more tangible: novelty. It is complicated to verify wheth-

\subsection{Current State of Affairs}

This - I believe - is the understanding of Architecture, which Ruskin took up arms against. Since the Renaissance, architects, through the process of design, have tended to focus on the aesthetic aspects of a space that can be represented by drawing, thus neglecting the full perceptive impact of a piece of architecture invested by dwelling intentionality.

And unfortunately, in time, this understanding has become theorized and effectuated. It would be pertinent to examine now the occurrences of such an understanding until and after Ruskin's time. I hope some brief notes can be persuasive enough.

Already in Leon Battista Alberti, the drift towards novelty and the emphasis on the artistic quality depicted in the drawing are present. He says that the architect should er or not a plastic event is beautiful, whether or not it has an aesthetic value such that grants it raison d'être; but it is easy to verify if something - even a plastic event - is new: it suffices that no one has ever done it before. Even if an architect discusses his design with other colleagues of the same profession, examining together the drawings or models (as is done traditionally in architecture schools and ateliers), because the plastic beauty of a form is a rather elusive quality, discussion will then tend to slip towards novelty: "You might not agree with me", could one perhaps say to a fellow architect, "about the balance or dynamism of my idea/design, but not about its novelty. My design is novel and that's objective - no one has ever done anything like it! I will surprise everyone". ${ }^{30}$ principally enquire in every building that which, by virtue of a well-thought artifice, profound thought or invention, is rare or admirable; and gain a habit of approving nothing but what is entirely beautiful and praise-worthy, due to the ingenuity it manifests. ${ }^{31}$

The architectural experience of the built edifice receives no mention. Boullée (1780) underlines the task of conception over that of building, in Architecture, disregarding once more the full experience of the building.

What is architecture? Shall I join Vitruvius in defining it as the art of building? Indeed no, for there is a flagrant error in this definition. Vitruvius mistakes the effect for the cause. In order to execute, it is first necessary to conceive. Our earliest ancestors built their

30 Regarding "novelty", Ruskin says in § 7 of The Poetry of Architecture [1837] = Works, 1: 8: "[I]n the wild struggle after novelty, the fantastic is mistaken for the graceful, the complicated for the imposing, superfluity of ornament for beauty, and its total absence for simplicity".

31 Alberti 1485, 142. The excerpt above was directly translated from the Latin, because the English version that was quoted throughout the paper was not faithful to the original (see De re aedificatoria, Book IX, Chapter X).

141 Fonti, letterature, arti e paesaggi d'Europa | Sources, Literatures, Arts \& Landscapes of Europe 1

John Ruskin's Europe. A Collection of Cross-Cultural Essays, 131-150 
huts only when they had a picture of them in their minds. It is this product of the mind, this process of creation, that constitutes Architecture and which can consequently be defined as the art of designing [...]. The art of construction is merely an auxiliary art. ${ }^{32}$

Accordingly, Le Corbusier, in 1923, defined architecture as "the masterly, correct and magnificent play of volumes brought together under light". ${ }^{33}$ The inappropriateness of the former definition, in what regards Architecture, becomes obvious when applied to Sculpture - because, strangely, it also applies to it although they are distinct fields.

More recently, one might recall the 1982 controversy between Christopher Alexander and Peter Eisemann,

\section{Part II: The Nature of Architecture}

\subsection{What, Then, is Architecture, According to Ruskin?}

\subsection{1 $\S 1$ Lamp of Memory}

In the first section of the Lamp of Memory, Ruskin starts by describing a very charming and smooth landscape. But then - he tells us - he is seized by a sudden chill, when it occurs to him that this exact landscape could be in the "New Continent". Immediately the experience of where the latter maintains that architecture issues forth from the world of 'ideas', as intellectual play. ${ }^{34}$ Creativity and novelty become, consequently, the main drives; the dwelling's phenomenological intentionality has been put aside.

Like the tamed beasts that regain their savagery once they have a taste of human blood (despite momentarily keeping their gentle gaze), so it happened with architects of the modern and contemporary ages: the taste of drawing in the architectural design process drew them into an unrestrained hubris which perverted Architecture. That is, I believe, the motive of Ruskin's condemnation. Nonetheless, the condemnation implies an alternative, which now ought to be inspected.

beauty and amenity vanishes, and the whole landscape becomes grey and cold, even oppressive. Only when he sees from afar the wall of Joux and the castle of Granson does the landscape regain its cheerfulness and its delightfulness. ${ }^{35}$ 


\subsection{2 § $\$ 2$ Lamp of Memory}

In continuity - and in his customary tone - he writes:

It is as the centralisation and protectress of this sacred influence, that Architecture is to be regarded by us with the most serious thought. We may live without her, and worship without her, but we cannot remember without her.

[...] there are but two strong conquerors of the forgetfulness of men, Poetry and Architecture; and the latter in some sort includes the former, and is mightier in its reality $[. . .]^{\prime \prime}{ }^{36}$

\section{What a bundle of puzzling statements!}

First: "We may live without her, and worship without her, but we cannot remember without her". Beyond being puzzling, it is also a poor claim about Architecture.
Nowadays, Memory is not that important. We can get by just fine without memory: we have books, computers, photos, digital memories...

Secondly: only Poetry and Architecture preserve Memory - so then what about History, which is usually considered the main tool in preserving the Past...?

Thirdly: Architecture includes Poetry - but is it not the other way around? Assuming 'Poetry' to be a synonym of Art (and we can presume Ruskin means exactly this, since he explains this use of the word elsewhere), ${ }^{37}$ Architecture is normally counted as one of the three visual arts...!

Finally: Architecture is mightier (than Poetry) in its reality. One could consider that the materials used in architecture endure more than those used in other arts - stone more than canvas and colour or engraving - but his point seems to be more profound.

\section{Works, 8: 224.}

37 Consider a small remark on the note of the last page of The Study of Architecture in Our Schools (1865) = Works, 19: 40fn: “[P]oetry meant, as its derivation implied - the doing. What was rightly done was done for ever, and that which was only a crude work for the time was not poetry; poetry was only that which would recreate or remake the human soul. In that sense poetical architecture was separated from all utilitarian work". Also, Modern Painters, Vol III (Part IV - "Of Many Things", Chapter I - "Of the received opinions touching the 'grand style"" = Works, 5: 28-9): "§ 12. It seems to me, and may seem to the reader, strange that we should need to ask the question, "What is poetry?" Here is a word we have been using all our lives, and, I suppose, with a very distinct idea attached to it; and when I am now called upon to give a definition of this idea, I find myself at a pause. [...] § 13. I come, after some embarrassment, to the conclusion, that poetry is "the suggestion, by the imagination, of noble grounds for the noble emotions". I mean, by the noble emotions, those four principal sacred passions - Love, Veneration, Admiration, and Joy (this latter especially, if unselfish); and their opposites - Hatred, Indignation (or Scorn), Horror, and Grief, - this last, when unselfish, becoming Compassion. These passions in their various combinations constitute what is called "poetical feeling", when they are felt on noble grounds, that is, on great and true grounds. Indignation, for instance, is a poetical feeling, if excited by serious injury; but it is not a poetical feeling if entertained on being cheated out of a small sum of money. It is very possible the manner of the cheat may have been such as to justify considerable indignation; but the feeling is nevertheless not poetical unless the grounds of it be large as well as just. In like manner, energetic admiration may be excited in certain minds by a display of fireworks, or a street of handsome shops; but the feeling is not poetical, because the grounds of it are false, and therefore ignoble. There is in reality nothing to deserve admiration either in the firing of packets of gunpowder, or in the display of the stocks of warehouses. But admiration excited by the budding of a flower is a poetical feeling, because it is impossible that this manifestation of spiritual power and vital beauty can ever be enough admired. § 14 . Farther, it is necessary to the existence of poetry that the grounds of these feelings should be furnished by the imagination. Poetical feeling, that is to say, mere noble emotion, is not poetry. It is happily inherent in all human nature deserving the name and is found often to be purest in the least sophisticated. But the power of assembling, by the help of the imagination, such images as will excite these feelings, is the power of the poet or literally of the 'Maker'”. 


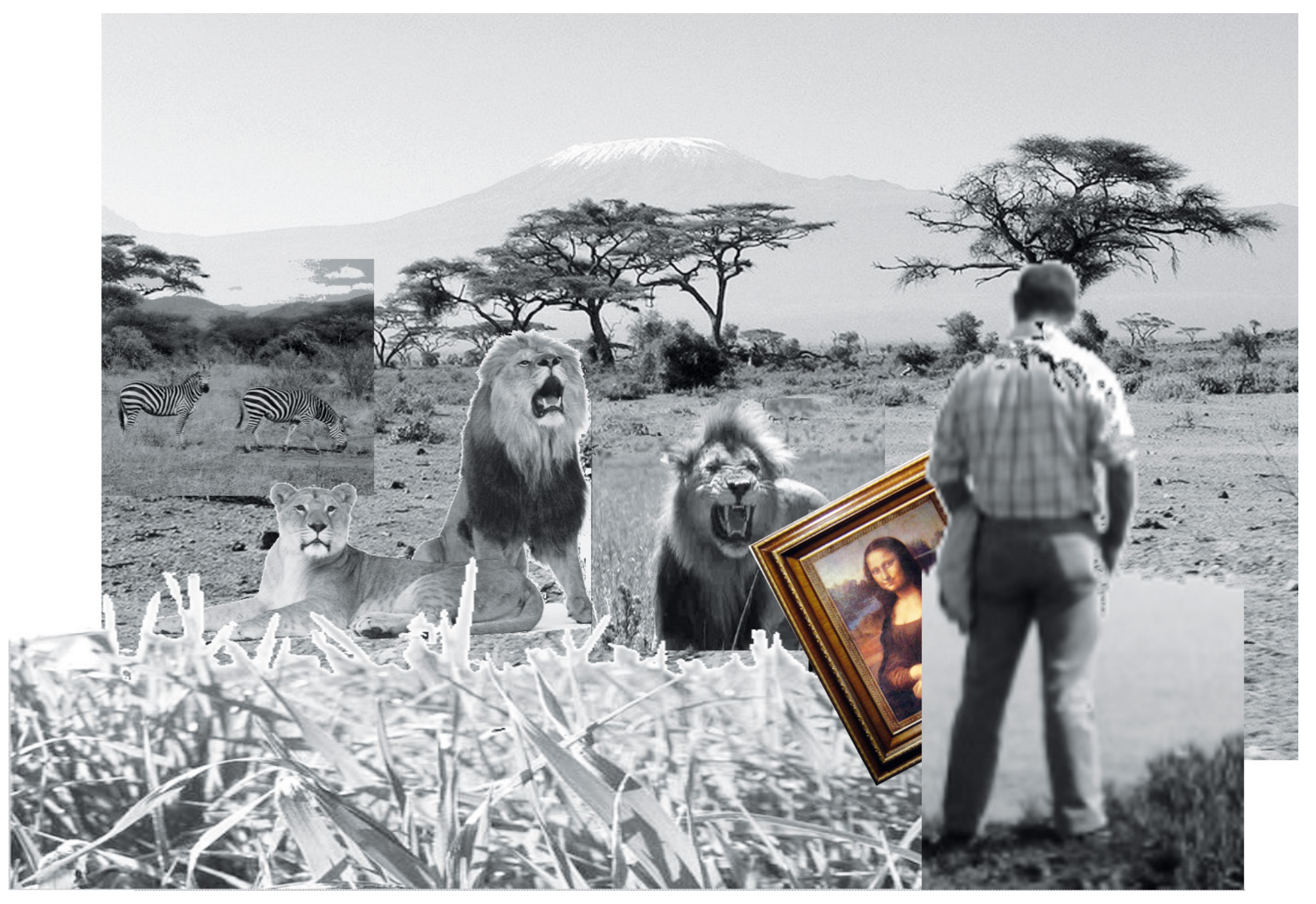

Figure 1 An interpretation of Ruskin's allegory. Fotomontage of Nuno Mesquita according to the instructions of the author 


\subsubsection{An Image}

Let us start with the last clause. Imagine one of us wins one of these foolish TV contests. The award is that your biggest wish be fulfilled. Our character is an artistic type and, therefore, he wishes to be left alone with his favourite work of art - let us say, the Monna Lisa of Leonardo (which is always so irritating to see in its overcrowded room) - to experience it calmly and thoughtfully [fig. 1]. Thus he asks to be taken to the African Savannah, to a part where there are no men, and to be left there, alone, with his favourite work of art. And so it happens: he is taken by a noisy old Land Rover, of which there are so many in Africa, to noman's land. Then the old Land Rover goes noisily away and he remains completely alone, in the wide silence of Nature. Let us imagine: he is fully absorbed, he is having an experience of intense pleasure, without any intromission, any interruption. He goes deeper and deeper into contemplation, scrutinizing every inch of the picture in perfect delight. Yet, in an instant, he hears a strange susurration - it could be the rustle of some leaves. He cannot help feeling alarmed: it could be some lizard, or a poi-

\subsubsection{Shelter?}

Architecture, not simply a shelter! A strong bunker, with its thick concrete walls, would not be a good place to enjoy a work of art. Inside its thick grey walls, without windows, one cannot help thinking about some kind of menace - the building 'speaks' of it - although being, in

\subsubsection{The Significant Other}

On the other hand, consider that this sort of event happens not only in relation to works of art. If our character was with his or her dearest, something of the kind would also take place - it would not be possible to con- sonous snake, or even some sort of predator. He had been so deeply involved in the contemplation of the painting that the murmur could have been happening for a while, without him even noticing it, and - he figures - the predator could be quite near him now. He is startled: he gets up, looks around. He sees nothing. He tries to calm himself down and get back to savouring the painting. But he cannot. Something in him has been unsettled. Despite his will, this something does not allow him to relax and take pleasure in the piece of art. He is preoccupied with his survival, and this preoccupation prevails over everything else, every other desire or volition - he is completely overwhelmed by it.

And, consider now, if the predator really comes up... The nature of this most dear piece of art would be completely forgotten, and it may even end up being used as a protective weapon - to shield himself from the beast - without any concern for damaging the painting.

Now - we may ask - what would allow our character to regain the possibility of enjoying the piece of art? Architecture, alone!

fact, safe. Only a home - not just a house - a true piece of architecture, would provide the peaceful environmental experience, which would free me from the frightening pressure of the outside and orient me in doing what I really want.

tinue with kisses and tender words if a menace hovered (though even imaginary).

Even if our character just wanted to be alone to think about his life or to meditate, under such circumstances 
that would also be impossible. And not only in the moment of the irruption of the threat; even afterwards, such a mindful state would be very hard to achieve. Concerns

\subsubsection{The Anthropological Reality of Architecture}

So this is, in my opinion, what Ruskin means by architecture being more powerful in its - I add - 'anthropological' reality than poetry/art. And this is, in my opinion, why he says that architecture "in some sort

\subsubsection{Memory}

I will not deal directly with the second puzzling statement I have mentioned above - the one concerning Poetry/Art and Architecture being the main guardians of Memory rather than History ${ }^{39}$ - but some remarks about the latter notions and their intertwining are necessary. for survival trump everything, at least in the first moment; to overcome these, solid spiritual training is required..$^{38}$

includes" art - it really does it, physically, materially: architecture offers the capsule where one can have the experience of art, and more.

38 Of course a mother with her child does it - but not in the first moment; and again one may say a mother has the "solid training" I'm talking about, having in mind the reordering of priorities being a mother signifies and all the time that, consequently, she spends with her child. One can also notice this in the Christian martyrs at Nero's colosseum, when allegedly he said: "not even dead I can take out your smile"; or, nowadays, in the Buddhist monks of Tibet, who sacrifice themselves by fire. One can accept that in these situations, a solid training occurred.

39 Concerning this issue, Ruskin's perspective is profound and deserves some study. I can only offer some brief notes about it. The clearest explanation of this difference may be found in the instruments that History and Architecture use to preserve Memory: History favours documents; Architecture, monuments. The etymology of "document" refers to the verb docere, which means to teach. The etymology of "monument" relates to the verb moneo, which means to remember, in an imperative sense. One can therefore infer that a document 'wants to be' outside the subject, so to speak, while the monument needs the subject to partake in it. Since memory is an event of the subject, in the subject, the re-happening of memory needs monuments, not documents (see Abreu 2008). Besides, memory is something of the present, not of the past - one remembers now something of the past, and so memory is always contemporary (despite having its reference point in the past). Therefore, memory requires something to reenact it, to put it into action again. That cannot be a document - or History - because it does not need the self; it exists by itself, alone, as an "impartial proof". On the other hand, an artwork demands the subject's participation - it does not exist in an objective realm; a computer, a robot, is not able to identify a work of art. A work of art always requires a human being in order to be, in order to perform its task. Only if one accepts becoming involved with a work of art, is it able to impart its message and thus to fulfil its task. Accordingly, art is an exceptional vehicle of memory. Borges speaks about it in "991 A.D." (Borges 1976). In order to be preserved, the past needs the poem, the poet, that sings of the historical event. Kierkegaard deals with it as well (in the "Eulogy on Abraham" of Fear and Trembling), saying that the hero cannot survive without the poet (Kierkegaard 1983, 15-16). Memory, in fact, being something of the present and of the self, requires something to be activated, in the subject, subjectively. Only works of art, in spite of living in a reality outside the self, possess the ability to awaken the human spirit, to partake subjectively in a human subject.

40 Le Goff 1985 
experience. ${ }^{41}$ Since Ruskin was familiar with Augustine, ${ }^{42}$ one may also reasonably presume that when Ruskin mentions "remember", he is addressing those deeper nuances of meaning in the word Memory.

"To remember" would then signify to get in touch with the inner self, to be recalled to one's humanity, ultimately to the good (the human) that one is made of. And - who does not acknowledge that?! - in order to get in touch with one's inner self, some conditions are

\subsubsection{Mental Health}

Consequently, Ruskin states, in the first page of his first lamp of architecture, "The Lamp of Sacrifice":

Architecture is the art which so disposes and adorns the edifices raised by man, for whatsoever uses, that the sight of them may contribute to his mental health, power, and pleasure. ${ }^{44}$

\section{Conclusion}

According to Ruskin it would be plausible to say, therefore, that a piece of architecture is the transformation of

\subsection{From the Castle of Granson to the Greek Temple}

Although uncommon among architects and somewhat peculiar, Ruskin's understanding of Architecture does not required: one is not able to do it not in the midst of a struggle, but rather where one senses a homely atmosphere. Says a Portuguese writer within the surprise of one of his characters: "He had never suspected how all this [he was recalling his beautiful family house] was the core of his own soul". ${ }^{43}$

Would it be possible to foresee a better purpose to Architecture, a more necessary goal and effect, in the realm of human existence...?

It is now possible to acknowledge the full weight Ruskin gives to the word 'mental', and, after the above analysis, one can accept that he speaks the truth: if the environment is not transformed, even slightly, in order to favour a total and mindful human existence, people will feel uneasy, under assault, they will not be able to freely express themselves, anxiety will grow and, in time, (if unable to overcome this anxiety) they will become depressed.

the surrounding environment as it allows for a person to be human, to feel human, and to think and act humanly.

stand alone and it is backed by quite a number of thinkers, who, more or less directly, tackle architecture. He

41 Augustine 2014-2016. Other than the Confessions, passim, refer to De Trinitate, books X, XI (especially), XII, XIV.

42 George P. Landow (1971) extensively writes on the influence of Augustine on Ruskin's allegorical method. He writes: "It is uncertain whether Ruskin knew On Christian Doctrine, though it seems likely, yet even if he did not, he had encountered similar arguments in the Confessions, which he several times mentions". (Landow 1971, 396-7) This information was kindly provided by Emma Sdegno.

43 “Corou, calou-se. Estava a pensar na sua bela casa de Azurara [...]. Nunca soubera como tudo isto era o miolo da sua própria alma!” (Régio 1993, 30). 44 Works, 8: 27. 
was, however, the first to look at architecture so profoundly. Heidegger, ${ }^{45}$ Lévinas, ${ }^{46}$ Broch, ${ }^{47}$ Eliade, ${ }^{48}$ and Gregotti ${ }^{49}$ are among those who articulated Ruskin's view. I shall discuss only one.

In several texts, Martin Heidegger addresses this subject with an understanding very much akin to that of Ruskin. In a talk to architects and engineers about the post-war dwelling crisis, Heidegger, never saying what one should understand by architecture, demonstrates that the goal of building is dwelling in. ${ }^{50}$ Then, analysing the phenomenology of dwelling, he unveils four semantic facies, which prove to be quite in alignment with Ruskin's perspective. 'Dwelling in' implies, first of all, a sense of protection. But not a random one: a sense of protection that imparts a feeling of peace. But not a general sense of peace: a feeling of peace that allows one to perceive oneself as free. And again, not any kind of freedom, but such a freedom that enables a human being to be totally in touch with their own self. Protection, peace, freedom, being oneself - all are implicitly encompassed in the poetical allegory of the first section of the Lamp of Memory discussed above; and the last feature of the four - being oneself - states essentially what is 'remembering' in Ruskin's terminology.

In a rather similar allegory to the one of Ruskin - describing the purpose and effect of a Greek Temple (a work of architecture, by antonomasia), placed alone on a cliff near the sea - Heidegger brilliantly synthesizes:

The temple, in its standing there, first gives to things their face [Gesicht], and to men their outlook on themselves. $^{51}$

Without architecture, the surrounding environment would not be able to gain a 'face'; in other words, one would not be able to figure out how to properly relate to what is around. And if the environment does not gain a 'face', men will not be able to see themselves and, consequently, to be themselves. It is as if architecture spills a sparkling and sweet perfume over things, albeit an inconspicuous one, which allows us to get in touch with things, with the other, with ourselves; a perfume or a captivating veneer, which makes things sing out: I am for you.

46 Lévinas 2011, 152-74. Lévinas's view about architecture is very synthetical, and usually neglected. He says, speaking about the home (but his observations can apply to any piece of architecture or place), it is an object among objects, yet it is a pre-condition to human existence. For, in providing a sense of welcoming, an experience of hospitality, it allows for recollection - "Recollection refers to a welcome" (Lévinas 2011, 152-6). "Recollection" can be interpreted as the specific human activity of 'reflexion' upon oneself, becoming aware of oneself, self-conscious.

47 Broch 1986a, 389-91; 1986b, 397-8.

48 Eliade 1997, 65-8; 1990, 3-116; 1987, especially "The Sacred Space”.

49 Gregotti 1996, 340-4.

50 Heidegger 2001a, $72 \mathrm{ff}$.

51 Heidegger 2001c, 42. The word 'face', in italics, does not feature in the current English translations. Nevertheless it is the term most correspondent to the German original: "Der Tempel gibt in seinem Dastehen den Dingen erst ihr Gesicht und den Menschen erst die Aussicht auf sich selbst" (Heidegger 1977, 29). 


\section{Bibliography of Works by John Ruskin}

References are to volume and page numbers in:

Cook, E.T.; Wedderburn, A. (eds) (1903-1912). The Works of John Ruskin. Library Edition, 39 vols. London: George Allen.

https://www.lancaster.ac.uk/the-ruskin/the-complete-works-of-ruskin/.

Works, 1: Early Prose Writings. | 5: Modern Painters III. | 8: The Seven Lamps of Architecture. | 10: The Stones of Venice II. | 12: Lectures on Architecture and Painting (Edinburgh, 1853), Other Papers (1844-1854). | 16: "A Joy For Ever", The Two Paths, Letters on The Oxford Museum and various addresses (1856-1860). | 19: The Cestus of Aglaia, The Queen of the Air, Other Papers and Lectures on Art and Literature. |33: The Bible of Amiens, Valle Crucis, The Art of England, The Pleasures of England.

\section{General Bibliography}

Abreu, P.M. de (2020). "Aesthetics of Well-being: Negative Effects and Strategies for Designing the Built Environment". Leal Filho, W. et al. (eds), Good Health and Well-Being. Encyclopedia of the UN Sustainable Development Goals. Cham: Springer. https://doi. org/10.1007/978-3-319-69627-0_113-1.

Abreu, P.M. de (2008). "Palazzi della Memoria”. Centofanti, M.; Mingucci, R. (a cura di), Atti del seminario internazionale di studio "Conservazione del patrimonio architettonico e urbano" (Tomar, Portogallo, 16 aprile 2008). DISEGNARECON, 2. https://disegnarecon. unibo.it/article/view/1382.

Alberti, L.B. (1485). De re aedificatoria. Libri Decem. Digitalized by Google. http://books.google.com.

Alberti, L.B. (1986). The Ten Books of Architecture - The 1755 Leoni Edition. New York: Dover Publications.

Arendt, H. (1958). The Human Condition. Chicago; London: The University of Chicago Press.

Arnheim, R. (1977). The Dynamics of Architectural Form. Berkeley: University of California Press.

Augustine of Hippo (2014-2016). Confessions. Cambridge (MA); London: Harvard University Press.

Bechmann, R. (1988). “I disegni tecnici del taccuino di Villard de Honnecourt”. Erlande-Brandeburg, A. et al. (a cura di), Villard de Honnecourt. Disegni. Milano: Jaca Book, 19-28.

Borges, J.L. (1976). “991 A.D.”. Moneda de Hierro. Buenos Aires. Emecé.

Botton, A. de (2007). Architecture of Happiness. London: Penguin Books.

Boullée, E.-L. (1976). Architecture, Essay on Art. London: Academy Editions. (Manuscript 1780, De architecture, Essai sur l'art).

Brandão, A. (1964). L.B. Alberti, Retrato de um Arquitecto Renascentista. Lisboa: Edição do Autor.

Broch, H. (1986a). "Disintegration of Values (2)". The Sleepwalkers. London: Quartet Books, 389-91.

Broch, H. (1986b). “Disintegration of Values (3)”. The Sleepwalkers. London: Quartet Books, 397-8.

Choay, F. (1992). L'Allégorie du Patrimoine. Paris: Seuil.

Clark, K. (1964). Ruskin Today. Harmondsworth: Penguin Books.

Cross, N.; Roozenburg, N. (1992). "Modelling the Design Process in Engineering and in Architecture". Journal of Engineering Design, 3(4), 325-37.

Di Stefano, R. (1983). John Ruskin, interprete dell'architettura e del restauro. Napoli: Edizione Scientifiche Italiane.

Eliade, M. (1987). The Sacred and the Profane. Sydney: Elsevier Australia.

Eliade, M. (1990). "Commenti alla legenda di Mastro Manole”. I Riti del Costruire. Milano: Jaca Book, 3-114.

Eliade, M. (1997). "Struttura e funzione dei miti". Spezzare il tetto della casa: la creatività e i suoi simboli. Milano: Jaca Book, 59-81.

Gregotti, V. (1996). "Territory and Architecture”. Nesbitt, K. (ed.), Theorizing, a New Agenda for Architecture. New York: Princeton Architectural Press, 340-4.

Heidegger, M. (1977). “Der Ursprung des Kunstwerkes”. Gesamtausgabe. 1. Abteilung, Veröffentlichte Schriften 1914-1970. Bd. 5, Holzwege. Frankfurt: Vittorio Klostermann, 1-74. 
Heidegger, M. (2001a). “Building Dwelling Thinking”. Poetry, Language and Thought. New York: Harper Collins, 141-59.

Heidegger, M. (2001b). "Poetically Man Dwells...”. Poetry, Language and Thought. New York: Harper Collins, 209-27.

Heidegger, M. (2001c). "The Origin of the Work of Art”. Poetry, Language and Thought. New York: Harper Collins, 15-86.

Hurson, T. (2007). Think better: An Innovator's Guide to Productive Thinking. New York: McGraw-Hill.

Katarxis (2004) = Katarxis 3. New Science, New Urbanism, New Architecture?. London: The Prince's Foundation for the Built Environment. Kierkegaard, S. (1983). “Eulogy on Abraham”. Kierkegaard's Writings. Vol. 5, Fear and Trembling, Repetition. Princeton (NJ): Princeton University Press, 15-23.

Landow, G.P. (1971). The Aesthetic and Critical Theories of John Ruskin. Princeton (NJ): Princeton University Press.

Lang, M.H. (1999). Designing Utopia, John Ruskin's Urban Vision for Britain and America. Montreal; New York; London: Black Rose Books. Le Corbusier (1986). Towards a New Architecture. London: Dover.

Le Goff, J. (1985). s.v. "Memoria”. Encyclopedia Einaudi. Lisboa: Imprensa Nacional Casa da Moeda, 11-50.

Lévinas, E. (2011). Totality and Infinity: An Essay on Exteriority. Pittsburgh (PA): Duquesne University Press.

Links, J.G. (1960). "Introduction” to John Ruskin, The Stones of Venice. New York: Pelican.

Merleau-Ponty, M. (1976). Phénoménologie de la Perception. Paris: Gallimard.

Murray, P. (1972). Arquitectura del Renacimiento. Madrid: Aguilar.

Pereira, P. (2011). A Fábrica Medieval, Concepção e construção na arquitectura portuguesa (1150-1550). Lisboa: Faculdade de Arquitectura da Universidade de Lisboa.

Proust, M. (1904). "Préface" to John Ruskin, La Bible d'Amiens. Paris: Société du Mercure de France.

Régio, J. (1993). A Velha Casa. Vol. 1, Uma gota de sangue. Lisboa: Círculo de Leitores.

Schön, D.A. (1983). The Reflective Practitioner: How Professionals Think in Action. New York: Basic Books.

Tavares, D. (2003). Filippo Brunelleschi. Porto: Dafne.

Tavares, D. (2012). Michelangelo. Porto: Dafne.

VanPutte, C.L. et al. (eds) (2014). Seeley's Anatomy \& Physiology. New York: McGraw-Hill.

Vitruvius, P. (1998). On Architecture. Cambridge (MA): Harvard University Press.

Wheeler, M.; Whiteley, N. (eds) (1992). The Lamp of Memory, Ruskin, Tradition and Architecture. Manchester; New York: Manchester University Press. 


\title{
Amelia Sarah Levetus (1853-1938) e il John Ruskin Club di Vienna dalla sua fondazione fino alla Prima Guerra Mondiale
}

Martina Frank

Università Ca’ Foscari Venezia, Italia

\begin{abstract}
Born in Birmingham and living in Vienna since 1893, Amelia Sarah Levetus devoted her life to facilitating the mediation between Austrian and British culture, finding a vital connection between the two countries in the thought of John Ruskin. In addition to its intense publishing activities as Vienna correspondent for The Studio magazine. An illustrated magazine Magazine of Fine and Applied Arts, Levetus was strongly committed to adult education. She was a founding member of Volksheim, the people's university, where she taught English language courses and where the John Ruskin Club was founded in 1903. This contribution traces the activity of Amelia Sarah Levetus up to the First World War and places it in the broader debate on architecture and the figurative and applied arts.
\end{abstract}

Keywords Arts and Crafts Movement. Arts and Crafts Schools. Anglo-Austrian Cultural Relationship. Magazine "The Studio". Amelia Sarah Levetus. Adult Education.

«Now the artists can breathe, and say 'We are free' [...] They had had freedom decades sooner had they had a Ruskin to fight their battle»:1 con queste parole Amelia Sarah Levetus introduce nel volume Imperial Vienna, pubblicato a New York nel 1905, gli sviluppi dell'arte viennese dell'Ottocento. ${ }^{2}$ In questo scritto, che costituisce probabilmente la prima storia culturale della città, Amelia rivela profonda conoscenza sia della storia che della realtà a lei coeva, di un ambito geografico e culturale al quale essa non appartiene per nascita ma per scelta.

1 Levetus 1905, 233.

2 Tengo a ringraziare Stephen Wildman per avermi generosamente segnalato un fascicolo sul John Ruskin Club conservato presso la Ruskin Library della Lancaster University. Per profili biografici di Amelia Levetus che contemplano anche il periodo post bellico cf. Filla 2001 e Ottenbacher 2016.

Fonti, letterature, arti e paesaggi d'Europa | Sources, Literatures, Arts \& Landscapes of Europe 1 ISSN 2724-6620 e-ISSN 2784-8507 
$E$ in verità anche il suo straordinario interesse per i fatti dell'arte non deriva da una specifica formazione disciplinare. Nata a Birmingham nel 1853 da una famiglia ebraica attiva nella comunità anche dal punto di vista religioso e con pronunciati interessi culturali e artistici, ${ }^{3}$ la formazione di Amelia è di tipo economico e pedagogico. Segue corsi a Birmingham, Cambridge, Aberdeen e St Andrews ${ }^{4}$ e due anni dopo l'arrivo a Vienna, nel 1893, si iscrive all'università di Vienna come studente straordinario di economia. Per una donna questo modo di iscrizione era ancora l'unico possibile. Al 1896 risale la sua prima pubblicazione. Si tratta di un articolo sulla rivista della Ethische Gesellschaft di Vienna, ${ }^{5}$ fondata nel 1894 dai docenti universitari Wilhelm Jerusalem e Friedrich Jodl; della Società Etica Amelia risulta peraltro anche essere segretaria. ${ }^{6}$ Collabora inoltre a un'altra rivista di nuova fondazione, Die Wage, per il cui primo numero scrive sulla condizione femminile all'università di Cambridge. ${ }^{7}$ Anche gli altri lavori pubblicistici di Amelia in questo periodo sono di impronta sociale, economica e femminista. Di vari aspetti della condizione femminile nell'impero austro-ungarico si occupano, in una prospettiva comparativa, articoli redatti per la Royal Economic Society (1897) e per riviste come Womanhood (1902) e The Englishwoman's Review (1897, 1898, 1901). ${ }^{8} \mathrm{Nel} 1897$ su invito del professore di economia Eugen Schwiedland ${ }^{9}$ è chiamata, prima donna nella storia austriaca, a tenere due conferenze universitarie sul tema delle cooperative di acquisto all'ingrosso inglesi e scozzesi. ${ }^{10}$ La notizia è talmente sensazionale da essere riportata sulla stampa quotidiana. ${ }^{11}$

A Vienna, in quegli anni Amelia frequentava anche Emily Gerard, nota anche perché Bram Stoker si ispirò ad alcuni suoi scritti per Dracula, e ancora Mark Twain e la moglie Olivia Clemens (in città dal 1897 al 1899) e altri inglesi e americani del mondo dell'economia e dell'industria. A lei si deve ad esempio, nel 1898, la mediazione tra Mark Twain e il banchiere Ludwig Kleinberg per l'ac-

3 Il padre era gioielliere e membro attivo della comunità ebraica di Birmingham. La madre Celia Moss (1819-1873) fu poeta e scrittrice, talvolta in collaborazione con la sorella Marion. La nipote Celia (1874-1936), allieva di Walter Crane alla Birmingham School of Art, diventerà una rinomata illustratrice. Per la formazione e la carriera di Celia vedi Fitzgerald 2016. Finora non è stata ritrovata una documentazione che potesse fornire indicazioni precise sui rapporti di Amelia Sarah con la sua famiglia. Non sembra tuttavia che Amelia ne condividesse le inclinazioni religiose ebraiche; nel suo certificato di morte risulta di fede anglicana.

4 Per la presenza di Levetus nel programma LLA (Ladies Literate in Arts) di St Andrews vedi Smith 2014, in particolare pp. 55, 94 , 120 e 142. Il testo contiene tuttavia alcuni sbagli e inesattezze, come, per esempio, le affermazioni secondo cui la madre di Amelia fu un'illustratrice e il padre un 'ritualbutcher'.

5 Levetus 1896

6 Swiney 1899, 268

7 Levetus 1898

8 Queste pubblicazioni valgono ad Amelia una certa notorietà internazionale nell'ambito del movimento femminista come dimostrano le ripetute citazioni dei suoi articoli. Vedi per esempio Swiney 1899, 268; La femme et le Féminisme 1900, 184.

9 Schwiedland si inserisce nell'orbita Ruskiniana: pubblica criticamente sulla cottage industry, sul lavoro a domicilio, nel quale vede un pericolo sociale ancora maggiore di quello causato dall'industria (Schwiedland 1894; Rampley 2010, 253). Questo tema è particolarmente sentito anche nel dibattito sulle arti decorative e coinvolgerà, come si vedrà, Sarah Levetus. Inoltre Schwiedland è amico fraterno di Octave Uzanne, l'editore francese che ha conosciuto in anni giovanili a Budapest e che sarà, come Levetus, un collaboratore di The Studio.

10 Qualche anno più tardi Amelia pubblica in tedesco un saggio su questi argomenti (Levetus 1900) e ancora nel 1911 seguirà un aggiornamento per la Revue d'économie politique di cui Eugen Schwiedland è membro del comitato direttivo (Levetus 1911a).

11 «Vortrag»1897, 6. 
quisto del brevetto di una «Textile-designing machine, which employed a photographic process», inventata da Jan Szczepanik, che Twain voleva introdurre negli Stati Uniti per uscire dalle sue disperate condizioni economiche. ${ }^{12}$ Szczepanik è anche l'inventore di una sorta di antenato del televisore, il telectroscopio, da cui Twain rimase profondamente colpito; ne pubblicò un articolo su The Century. ${ }^{13}$

Anche se tematiche economiche e sociali continueranno a connotare il pensiero di Amelia Levetus, al volgere del secolo i suoi interessi si orientano sempre di più verso l'arte, o meglio verso una integrazione dell'impegno e dell'analisi sociale con gli sviluppi e le tradizioni nel campo dell'arte, dell'architettura e delle arti applicate. Un buon esempio di questa integrazione è un suo articolo sulla cittadina operaia di Berndorf della famiglia Krupp che era anche un centro di produzione di posate in argentone destinate al grande pubblico. ${ }^{14}$ Nel 1902, forse in sostituzione dello storico dell'arte e traduttore di Ruskin, Wilhelm Schölermann, Amelia diviene corrispondente fisso da Vienna per la rivista The Studio. An Illustrated Magazine of Fine and Applied Arts, fondata nel 1893 a Londra da Charles Holme [fig. 1], ${ }^{15} \mathrm{ma}$ essa scrive anche per diverse altre riviste di arte e architettura austriache, tedesche, inglesi, americane, belghe, e in esse emerge di continuo il suo ruolo di mediatrice tra il mondo germanico e quello anglo sassone. La rivista The Studio ebbe un'immensa risonanza anche a
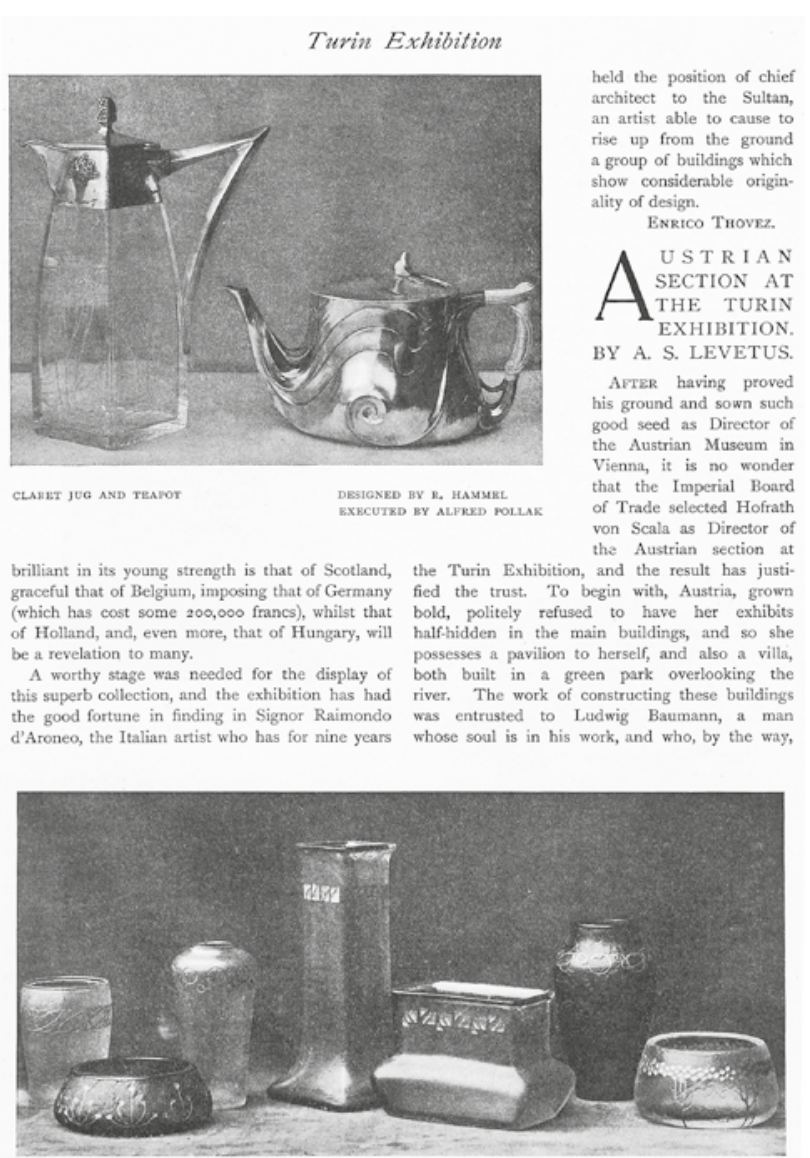

Figura 1 Prima pagina dell'articolo di Amelia Sarah Levetus sul padiglione austriaco alla Prima esposizione internazionale d'arte decorativa moderna tenutasi a Torino nel 1902, apparso nel volume $26 \mathrm{del}$ 1902 della rivista The Studio. https://digi.ub.uni-heidelberg.de/diglit/studio1902b/0059

12 Le vicende sono ampiamente documentate da lettere e da annotazioni nei diari di Mark Twain. Vedi Leary 1969, 327-33, https://daybyday. marktwainstudies.com/page/27/.

13 Twain 1898.

14 Levetus 1912.

15 Delyfer 2010.

153 \begin{tabular}{l|l} 
Fonti, letterature, arti e paesaggi d'Europa | Sources, Literatures, Arts \& Landscapes of Europe 1 \\
John Ruskin's Europe. A Collection of Cross-Cultural Essays, 151-166
\end{tabular} 
Vienna Secession Exhibition

ever-changing night; yet none can say this day is the members of the Secession, and is consequently like unto yesterday. Anidst all this tide of looked forward to with great eagerness, This time simplo lite is a fente the grains of sand, the in which is Professor Otto Wagner's model leaves of the trees, the stars of heaven, are as for the church now in process of building (the Lunatic Asylum, just ouside the city Sunrise and evening glow evoke, in all nations, of Wenna. There is so moch new in the, such a ieclings akin to worship, and the induence of the rechess

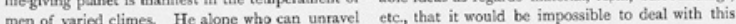
the mighty desiges of the universe and solve the and the Provessors other monumental bitidings mystery of its being, may perhaps claim to have the new General Post Offce and the project for attained the awful calmness of the Sphinx, which the Franz Joser Museum, within the timits of these

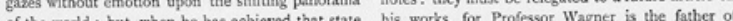
of mint a prow, for the joy of life will have departed. are many, while his pupils who have made names for G. A. QurLLHORsT. themselves are numerous, prominent among them HE TWENTY-THIRD EXHIBI- being Professor rost this exhibition represented a waiting room, TION OF THE VIENNA SECES. the bent-rood furniture having been designed SION. BY A. S. LEVETUS. Vy Marcel Kammerer (manufactured by Thonet, Tus Spring Exhibition is the only one during and Schinthal, who are the Professor's pupils
the whole year which is devoted to the rorks of and at the same time his assistants. There

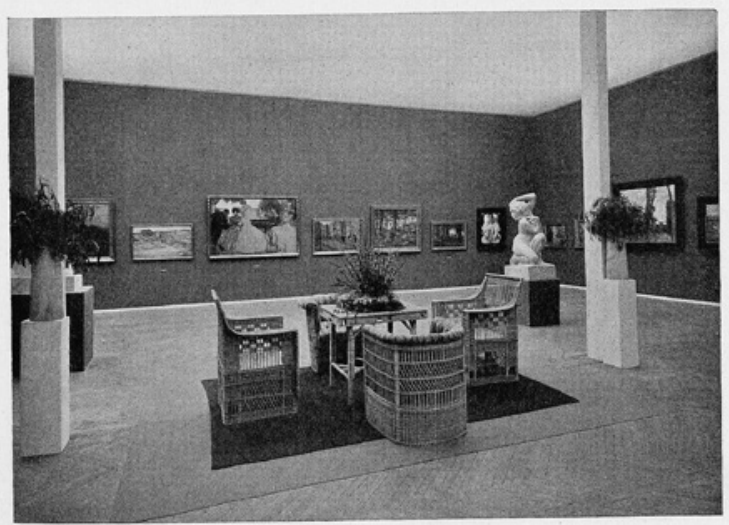

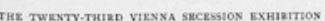

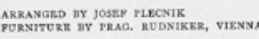

\section{A Mansion in Brussel}

BRUSSELS MANSION DE- In the decoration and furnishing of the mansion A SIGNED BY PROF. JOSEF Patists of the modern school in Vienna, such Gustav Klimt, who designed a remarkable mosaic OxE of the most notable achievements in the frieze for the dining;room, Kolo Moser, Creschkika, decoration is the mansion or "palace" recently Powolny, Leopold Forstner, and two romenertists erected in Brussels from the designs of Prof. Josef Fruu Luksch and Fruu Schleiss Simandl. The Hoffimann of Vienna for Mons. Stoclet, a wealthy gardens, which are situnted at the back of the magnate of the Belginn capital and owner of a bouse away from the road, present numerous choice collection of ancient wotks of art. Prof. interesting features in the shape of pergolns, a

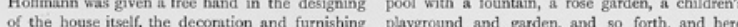
of the interior including the miscellaneous articles aguin the architect has enlisted the aid of promi of service, and also of the gardens. The mansion nent artists in the sculptural deconation of the is situated in the Avenue de Tervueren, one of the ground

main tram routes of the cily, and on that account For the carrying out of this important under. the design of the exterior lis becn kept com- taking, trined men accustomed to executing the houses it strikes a guite distinctive note. The were sent from Vienna, while the fumiuture, ss well interior of the house is of singular beauty, and, in as the table services and other applinnces, were spite of the seeming coldness of the marble walls made in the workshops of the Wiener Werkstaette and floors, a feeling of warmth is imparted to all in Vierna, the craftsmanship throughout being of the rooms by the use of appropriate colour. the highest quality. A. S. Levere

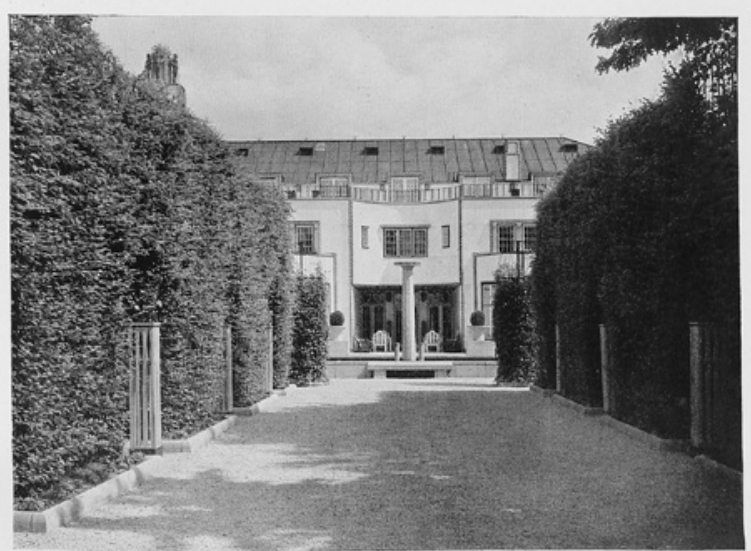

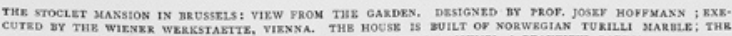

Figura 2 Prima pagina dell'articolo di Amelia Sarah Levetus sull'esposizione primaverile della Secession a Vienna del 1906, apparso nel volume 36 del 1905 della rivista The Studio. https://digi.ub.uni-heidelberg.de/diglit/international_studio26/0066
Figura 3 Presentazione di Amelia Sarah Levetus del Palais Stoclet a Bruxelles di Josef Hofmann, apparsa nel volume 61 del 1914 della rivista The Studio. https://digi.ub.uni-heidelberg.de/diglit/studio1914a/0195 
Vienna. ${ }^{16}$ Fin dal 1898 Peter Altenberg descrive in maniera assai efficace e marcatamente ironica l'attesa per l'arrivo di ogni nuovo numero nelle case viennesi:

Il quindici di ogni mese arrivava dall'Inghilterra per Jolanthe la rivista "The Studio, an illustrated Magazine of fine and applied Art". [...] Quel quindici del mese diventava un giorno di festa, o quantomeno un giorno diverso dagli altri. "Sono in Inghilterra" si diceva Jolanthe "In Inghilterra!". Un giorno qualcuno chiese al marito in uno di quei giorni: "Dov'è Jolanthe?". E lui disse semplicemente: "Ė in Inghilterra". ${ }^{17}$

Nella sua qualità di corrispondente di The Studio e di critico d'arte per una stampa internazionale Amelia documenta le scuole di arti e mestieri, le esposizioni della Secessione [fig. 2] e del Hagenbund, fa conoscere internazionalmente Josef Maria Olbrich, Josef Hoffmann [fig. 3], Koloman Moser e la Wiener Werkstätte e introduce il pubblico viennese al movimento Arts and Crafts e a artisti come Charles F.A. Voysey, Baillie Scott, Frank Brangwyn, Charles Rennie Mackintosh e i Glasgower Four. Levetus si occupa di donne artiste affermando per esempio che «a one woman show was a rare event in Vienna till Tina Blau» e presenta a più riprese lavori dell'allievo di Otto Wagner, Otto Prutscher, per citare soltanto alcuni episodi salienti. Intensa è anche la sua premura a presentare ambiti di produzione artigianale più marginali come ad esempio mobili di giunco, giocatoli, ricami e merletti, settori produttivi legati all'insegnamento nelle scuole di arti e mestieri, all'arte popolare e alla condizione lavorativa femminile, altri temi ai quali ha dedicato numerosi articoli. ${ }^{18}$

Amelia Sarah Levetus è una acuta testimone e cronista, attenta a valori etici e sociali. I suoi articoli sono generalmente in linea con quelli della rivista The Studio che promuove la diffusione a livello internazionale del movimento Arts and Crafts. Talvolta però la sua posizione sembra essere più complessa. Ne è testimonianza un suo articolo in The Studio Yearbook del 1910 nel quale descrive le ultime novità nell'ambito dell'architettura e della decorazione in Austria senza esitazioni nel citare in un'unica frase, quando ormai lo spaccatura si era consumata da tempo, Adolf Loos e diversi esponenti della scuola di Otto Wagner..$^{19}$ Non è l'unica volta che Levetus ricorda Loos, anche se nessuna delle sue opere sarà mai inclusa nei sui articoli. In un volume sulla rinascita dell'arte in Austria ${ }^{20}$ per il quale Amelia Levetus cura la parte riservata alle arti decorative, gli articoli di Loos su moda, decorazione e arredamento inglesi apparsi sul quotidiano Neue Freie Presse sono considerati come dei fattori determinanti per l'evoluzione del gusto dei viennesi, equivalenti alla leggendaria mostra di mobili inglesi presso il Museum für Kunst und Industrie nel

16 Levetus 1933; Panagl 1972; Sarmany-Parsons 1987-1988; Large 2003.

17 Altenberg 1898-1899, 14-15. Il testo si intitola Der Freund, l'amico, ma è corredato da un significativo sottotitolo: Dem Hofrath Arthur von Scala, dem idealen Vorkämpfer aristokratischer Kunst, zugeeignet (Dedicato al consigliere Arthur von Scala, il precursore ideale dell'arte aristocratica). Von Scala aveva appena inaugurato la sua nuova posizione come direttore del Museo di arte e industria con una mostra di mobili inglesi, un argomento sul quale sarà obbligo ritornare.

18 Houze 2015.

19 Levetus 1910, 219.

20 Levetus 1906, D 1

155 Fonti, letterature, arti e paesaggi d'Europa | Sources, Literatures, Arts \& Landscapes of Europe 1

Font, letterature, artie paesaggi d'Europa Sources, Literatures, Arts
John Ruskin's Europe. A Collection of Cross-Cultural Essays, 151-166 
$1897 .{ }^{21} \mathrm{Il}$ fatto che il manifesto che annuncia l'ultima delle conferenze di Loos su 'Ornament und Verbrechen' nel 1913 riporti anche l'avviso di un intervento di Levetus sulle cattedrali inglesi è soltanto un caso, ma si tratta di un tassello utile per ricomporre la posizione che Amelia Levetus aveva assunto nel mondo della cultura viennese. L'anno 1897 è quindi indicato come un momento di svolta: è l'anno di fondazione della Secessione e l'anno in cui Arthur von Scala assume la direzione del Museum für Kunst und Industrie, inaugurata appunto con la citata esposizione di mobili inglesi.

Everywhere a healthy growth in decorative art is perceptible. The Wiener Werkstätte has set a fine example, and it is a well-deserved harvest that its promoters are reaping. Starting with the ideals of Ruskin and Morris before them, they have made wonderful headway, and the development of the concern has continued without a pause. ${ }^{22}$

Il riferimento a Ruskin, che già era stato utilizzato da Levetus per descrivere il bisogno di libertà degli artisti ottocenteschi, è costante nei suoi scritti e riflette un sentire diffuso nonché una prassi di scambi personali. È risaputo che le prime traduzioni tedesche di Ruskin risalgono al 1897 (l'edizione di Strasburgo) e al 1900-1906 (quella di Lipsia); ma ricordo che già nel 1896 esisteva una traduzione ungherese di The Stones of Venice e che nella rivista Wiener Rundschau Wilhelm Schölermann aveva pubblicato nei primi mesi del 1899 articoli su e traduzioni di Ruskin. ${ }^{23}$ Tuttavia in quel clima di spiccata anglofilia che caratterizzava una buona parte della società e degli intellettuali viennesi già circolavano lavori di Ruskin in originale. Hugo von Hofmannsthal ne parla, per esempio, fin dal $1894,{ }^{24}$ e anche il letterato Peter Altenberg e il suo amico Adolf Loos non avevano certo bisogno di traduzioni. Altenberg, che, come già rilevato, aveva commentato il successo della rivista The Studio, dichiara di preferire un vaso Ruskin con naturale smalto overflow a una artificiale porcellana Meissen, ${ }^{25}$ e il suo amico Loos è ruskiniano almeno fino alla sua rottura con la Secessione quando diventa invece «il suo più assiduo nemico». ${ }^{26} \mathrm{Il}$ più convinto sostenitore di Ruskin e Morris, inizialmente in una prospettiva politica, morale e letteraria, è Joseph August Lux, che trascorse il 1897 a Londra. ${ }^{27}$ Lux divenne un attivissimo pubblicista di architettura e ricorda come nelle discussioni della cerchia di Otto Wagner Ruskin occupasse un posto di preminenza. ${ }^{28} \mathrm{Al}$ modello britannico del National Trust e al Manifesto of the Society for the Protection of Ancient Buildings di William Morris si orientavano le sue convinzioni in materia di conservazione dell'edilizia stori-

21 I rapporti di Levetus e Loos meriterebbero un approfondimento e non soltanto sulla base della ben nota anglofilia dell'architetto. Alla fine degli anni 90 entrambi sono tra gli autori della rivista Die Wage edita da Rudolf Lothar e inoltre condividevano rapporti sociali come quelli con le femministe Rosa Mayreder e Auguste Fickert. Il lascito di Loos conservato presso la Wiener Stadtbibliothek contiene diverse lettere e biglietti di Levetus indirizzati all'architetto.

22 Levetus 1912, 181

23 Schölermann 1899a, 1899b, 1899c.

24 Gilbert 1937.

25 Arlaud 2004

26 Hanisch 2018, 270-1.

27 Arlaud 2008, 2-3.

28 Hanisch 2018, 247-8. 
ca. ${ }^{29}$ Rennie Mackintosh, i cui mobili si erano visti nella capitale fin dal 1900 in numerose occasioni ${ }^{30}$ e che coltivava rapporti di amicizia con artisti e committenti viennesi, si sarebbe persino trasferito a Vienna se lo scoppio della Prima Guerra Mondiale non avesse azzerato quel progetto. ${ }^{31}$

Al ricco panorama degli scambi tra la cultura britannica e l'Europa Centrale e dell'influenza esercitata dal movimento Arts and Crafts, la cui intensità è stata messa in evidenza da studi recenti, ${ }^{32}$ appartengono anche i viaggi degli artisti. In effetti, molti artisti inglesi manifestano un interesse non tanto per Vienna quanto per diverse province della doppia monarchia. Charles Robert Ashbee, fondatore della Art Worker's Guild e della School of Handicraft a Toynbee Hall, non ha soltanto esposto alla Secessione di Vienna ma ha anche compiuto diversi viaggi a Budapest, dove ha peraltro disegnato due edifici, per visitare la colonia di artisti a Gödöllö, ispirata al modello di Ruskin e William Morris, luogo nel quale anche Amelia Levetus si recava ogni anno. Walter Crane, presidente della Arts and Crafts Exhibition Society (e maestro della nipote di Amelia Levetus, l'illustratrice Celia Levetus), oltre ad avere esposto nel 1900 e nel 1901 a Vienna le sue opere, ha percorso la Boemia, l'Ungheria e la Transilvania per scoprire e studiare l'arte popolare pre-industriale. ${ }^{33}$ Come si vedrà, si tratta di un argomento con il quale si confronterà anche Levetus.

A true feeling for art and decoration is inborn in the Austrians. Their national art is sufficient proof of this.
But the modern movement in decorative art owes its inception to outside influence - to England; its development however, comes from the Austrians themselves.

In questa frase, contenuta in un articolo del 1906 sulla rinascita dell'arte in Austria, pubblicato in The Studio, si riassume il carattere ambiguo, quasi paradossale, del pensiero di Amelia Levetus. ${ }^{34}$ Quando essa utilizza il termine di 'the Austrians', essa sembra interiorizzare una visione del mondo che sappiamo propria dell'Homo Austriacus così come la conosciamo nelle rappresentazioni dei grandi scrittori della doppia monarchia, Musil, Roth, Zweig. Quella visione accoglieva certo tutti i fermenti che avrebbero trasformato il mondo, ma il suo amato-odiato quadro di riferimento era ben saldamente l'Impero asburgico. D'altro canto Levetus è socialista, pacifista e femminista, come emerge con chiarezza sia dai suoi scritti che dal suo impegno quotidiano. Certo, si tratta di caratteri di ambiguità propri della società viennese attorno al 1900, ma Amelia Sarah Levetus non appartiene a quel mondo per nascita ma per scelta, e la sua identificazione con la Koinè Danubiana sembra essersi spinta fino ad assumerne il doppio statuto, quell'ambiguità appunto che è così tipica di molti grandi artisti e intellettuali della Finis Austriae. Levetus non esita a utilizzare il termine di arte nazionale e con questa impostazione essa tocca un nodo cruciale del rapporto tra politica, arte e artigianato. Se per Adolf Loos, riferendosi alla Kunstschau del giubileo del regno di Francesco Giuseppe 
Figura 4 Cerimonia della posa della prima pietra del Volksheim a Vienna Ottakring il 18 dicembre 1904 con Amelia Sarah Levetus al centro a destra. Fotografia di H. Schumann, Österreichische Nationalbibliothek, Bildarchiv Austria

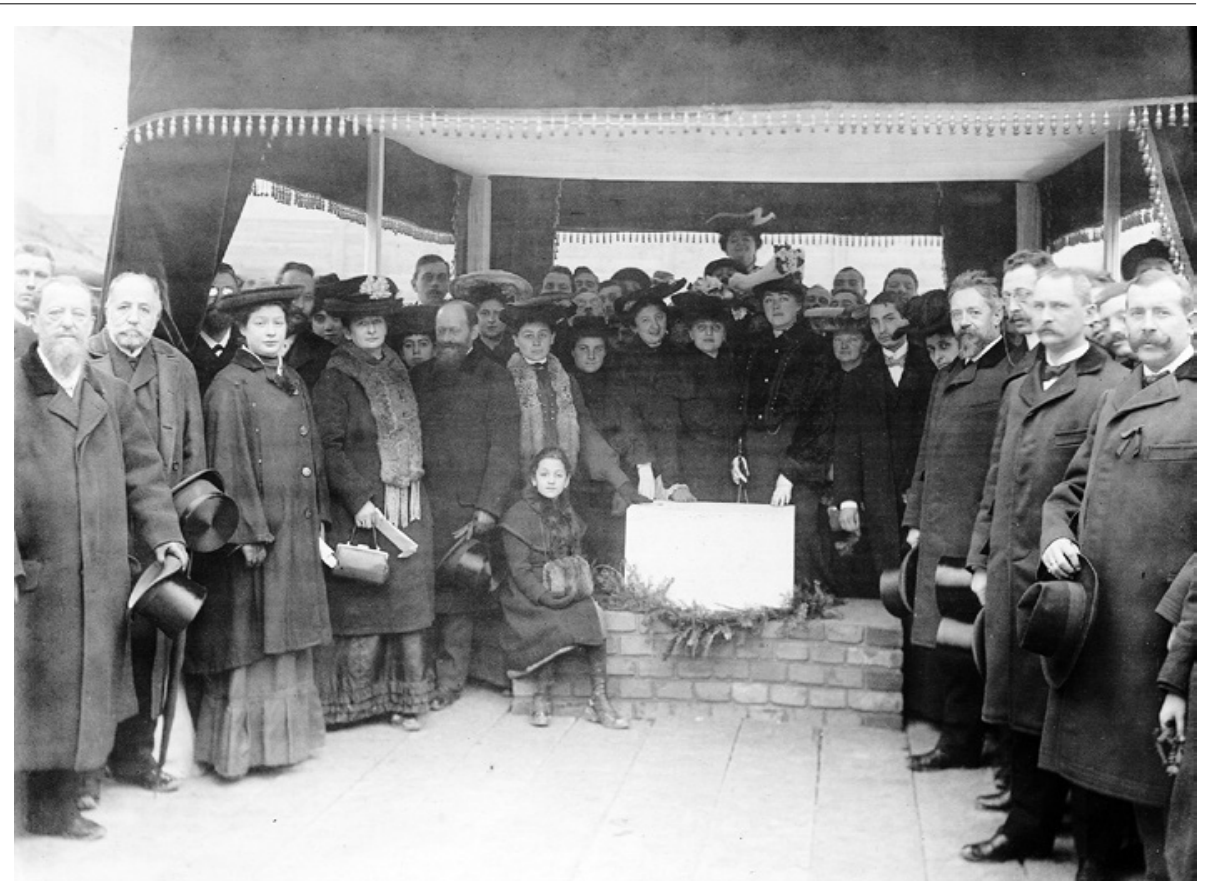

del 1908 organizzata dal gruppo di Klimt, l'appoggio del governo alle riforme dell'arte decorativa fino a fare di quest'arte il linguaggio ufficiale, equivale a una degenerazione culturale, ${ }^{35}$ Levetus, pur notando che «Austria is the only country in which the education in industrial art is organised by the State», riconosce in questa congiuntura aspetti positivi. ${ }^{36}$ Per Levetus, grazie a questa politica il progresso qualitativo delle arti decorative è accompagnato da un progresso sociale per le popolazioni rurali e in particolare per le donne..$^{37}$

Capeggiata dal viennese Museum für Kunst und Industrie, la rete delle 137 scuole statali e delle 70 scuole, private ma pubblicamente sovvenzionate, che operano nei vari settori delle arti e mestieri copre tutti i territori dell'impero. Nel clima modernista che riconosce nell'arte popolare un'importante fonte di ispirazione, questa struttura centralizzata favorisce la diffusione di un linguaggio che recepisce elementi di cultura

35 Carl Schorske ha messo in evidenza come in quel particolare momento storico al governo asburgico conveniva questa scelta rispetto all'adozione di forme ecclettiche o vernacolari che facilmente avrebbe potuto essere interpretate in chiave nazionalistica (Schorske 1981, 236).

36 Levetus 1905, 267-8.

37 Levetus 1903-1904, 325. 
popolare e rurale e che crea nuovi modelli che sono poi esportati nelle sedi periferiche. ${ }^{38}$ D'altro canto la fabbricazione di oggetti di carattere popolare per soddisfare un mercato in espansione conduce a una progressiva perdita di autenticità e originalità, con conseguente riduzione del repertorio a schemi fissi, una obiezione già avanzata, per esempio, dal critico e amico di Amelia Levetus, Ludwig Hevesi, o da Walter Crane. ${ }^{39} \mathrm{Al}$ modello produttivo statale basato sulle scuole e il lavoro domestico (Heimindustrie) si contrappongono movimenti (politicamente nazionalistici e culturalmente internazionali) di reinvenzione dell'arte popolare che promuovono modelli di unione di arte e vita chiaramente ispirati a Ruskin e Morris, ma anche a Tolstoj..$^{40} \mathrm{Ne}$ sono esempio la già nominata colonia di artisti a Gödöllő (Keserü 1988) e l'invenzione dello stile Zakopane polacco da parte di Stanisław Witkiewicz negli anni novanta dell'Ottocento. Secondo Witkiewicz gli ideali sociali di Ruskin e Morris si sono affermati a Zakopane in modo del tutto autonomo e a prescindere dalla conoscenza dell'opera di Ruskin (Crowley 1995, 9).

Gli stili vernacolari regionali sono quindi da un lato espressione dei crescenti nazionalismi ma sono anche tasselli fondanti dell'immagine che la monarchia asburgica promuove di se stessa. Oltre a confermare nella lettura dei manufatti e dei loro creatori l'adesione agli ideali di Ruskin e Morris, ${ }^{41}$ Peasant Art in Austria and Hungary, un numero speciale monografico della rivista The Studio del 1911 per il quale Amelia Levetus redige oltre all'introduzione le sezioni dedicate a Croazia, Slavonia e
Transilvania, conforta per la sua stessa impostazione editoriale quest'immagine della doppia monarchia che lo stato intende diffondere. Significativamente, il volume apre con una fitta sequenza di annunci che pubblicizzano prodotti vernacolari realizzati in lavoro a domicilio, dalle «South Slav Style Peasant Art Embroideries» alle «Hungarian Peasant Industries». Nel suo testo Levetus non manca di associarsi a quelle voci critiche su questa produzione, alle quali abbiamo appena accennato, e inizia a riconoscere nel sistema centralizzato delle scuole di mestieri il responsabile della perdita di qualità dell'artigianato rurale:

But the decay of the peasant's art is apparent in many quarters, and in its place the home industries have risen. The action taken by the Government and different societies is doing much to revive the lost arts. Schools have been organised, teachers sent from village to village to teach new methods and designs; but the schoolwork, beautifully executed as it is, loses in comparison with the naive charm expressed in the spontaneous designs and quaintness of thought. ${ }^{42}$

Non è l'unica volta che Amelia Levetus si confronta con l'arte rurale. Nei suoi corsi al Volksheim (l'università popolare appunto), sul quale torneremo tra poco, propone corsi che confrontino l'arte popolare britannica e quella austriaca e questo suo interesse si traduce anche in un'attività collezionistica che comporterà diverse donazioni, anche di oggetti inglesi e irlandesi, al Museum

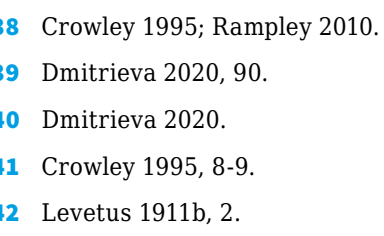


für Volkskunde (museo etnografico), fondato nel $1896 .^{43}$

Accanto alla sua attività pubblicistica tutta volta a riconoscere elementi del pensiero e delle teorie ruskiniane nelle espressioni artistiche, Amelia Levetus si dedica all'insegnamento della lingua e della cultura inglese. Nel 1901 un libero docente di storia, il socialdemocratico Ludo Hartmann, aveva fondato l'Athenaeum, un'associazione affiliata all'università che proponeva corsi formativi per un pubblico esclusivamente femminile. Uno dei suoi obiettivi era quello di migliorare la preparazione scientifica di donne intenzionate a iscriversi regolarmente all'università, le cui porte si erano loro aperte soltanto nel $1897 . .^{44}$ Levetus vi insegna (in lingua inglese e con un notevole successo di pubblico, come attestano le statistiche dei partecipanti), tenendo corsi su scrittori inglesi seicenteschi e appunto, soprattutto, su John Ruskin o Walter Pater. ${ }^{45}$

Sia Hartmann che Levetus sono tra i fondatori del Volksheim. Per molti versi questa iniziativa supera il modello inglese delle University Extensions che era stato seguito sia per i Volkstümliche Universitätskurse, inaugurati in concomitanza con l'ammissione ai corsi universitari anche alle donne, che per l'Athenaeum. I precedenti ai quali ci si orienta ora, anche per emanciparsi dalla subordinazione amministrativa dell'Università e cercare apposite sedi, sono le Universités populaires francesi e i Settlements o People's Palaces inglesi. ${ }^{46}$ Tanto è vero che in Imperial Vienna Amelia Levetus utilizza proprio il termine di People's Palace per caratterizzare il Volksheim. In un momento nel quale la parola Volk (popolo) aveva connotati essenzialmente negativi, il Volksheim promuove la diffusione di conoscenze e di ricerche scientifiche presso un pubblico popolare, includendo sia borghesi e artigiani che operai. Nella sua organizzazione e nel suo programma il Volksheim introduce significative novità che a loro volta diventeranno un modello a livello europeo. Il Volksheim non intende proporre cicli di conferenze come le università popolari francesi, né riconosce la sua missione principale nell'integrazione sociale delle classi disagiate come fanno i Settlements, né intende ripetere il modello delle scuole professionali proprio dei People's Palaces. Il suo scopo è quello di creare una struttura autonoma dedicata alla trasmissione dei saperi in un clima collaborativo e di integrazione sociale e di dotarsi per far questo delle necessarie infrastrutture, quali biblioteche e laboratori. ${ }^{47}$

L'atto di fondazione della nuova associazione ufficialmente riconosciuta nel 1901 è sottoscritto da 64 persone, la cui eterogeneità ne illustra l'ampio e trasversale sostegno: si va dai conservatori asburgici, ai Deutschliberale (liberali cattolici) fino ai socialdemocratici anticlericali. Il professore di filosofia Friedrich Jodl, con il quale Levetus aveva già collaborato nella Ethische Gesellschaft, dichiara che la democratizzazione dell'accesso alla scienza, ala letteratura, alla cultura e all'arte rappresenta un essenziale ampliamento spirituale della città, una 'geistige Stadterweiterung', parallelo a quello urbano che ha visto a partire dal 1857 l'abbattimento delle mura cittadine e il progressivo inglobamento dei sobborghi nella grande Vienna. ${ }^{48}$ Amelia

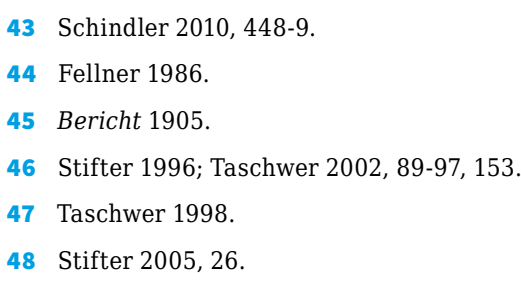




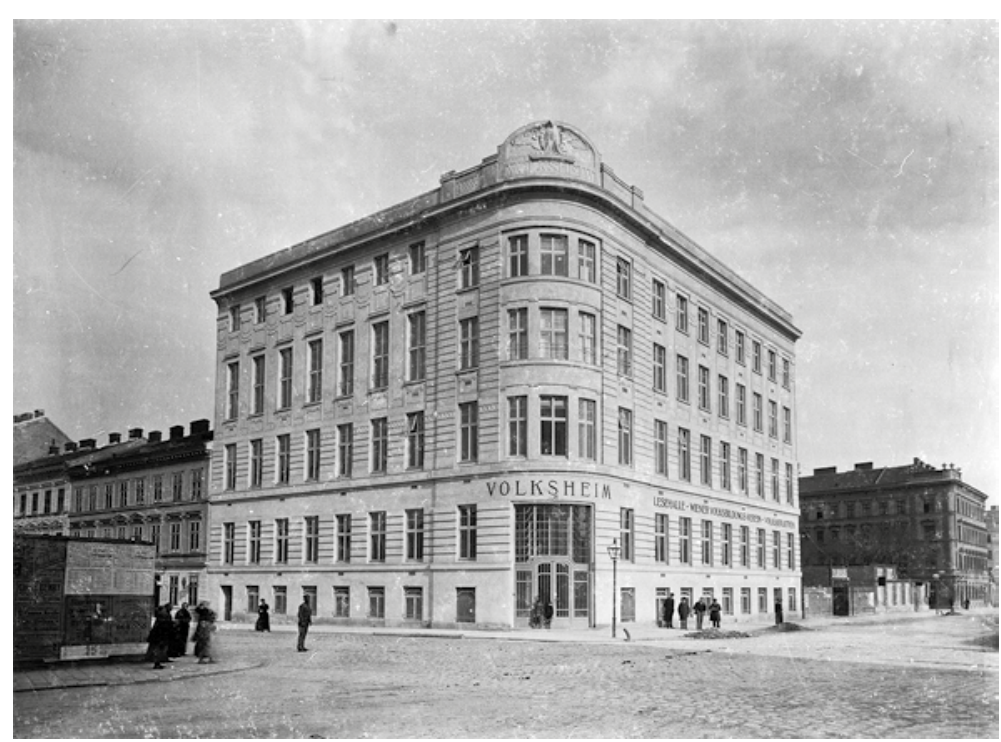

Figura 5 L'esterno del Volksheim a Vienna Ottakring in una fotografia del 1905.

Österreichische Nationalbibliothek, Bildarchiv Austria

Levetus utilizza una metafora molto simile nella sua introduzione all'arte viennese del secondo Ottocento: in quello che lei definisce il «Christmas present» dell'imperatore nel 1857, ovvero nella cessione dei suoi diritti al Stadt-Erweiterungs-Fond (fondo per l'ampliamento della città), essa riconosce la condizione adatta «to separate the clouds and bring awaking to the city». ${ }^{49}$

La missione del Volksheim era quindi la trasmissione di una visione scientifica e antimetafisica del mondo. Ciò doveva essere garantito da un rigoroso disgiungimento tra la trasmissione della conoscenza e l'ideologia. Lo scopo primario era dunque l'obiettività e la neutralità religiosa e ideologico-politica del programma educativo. ${ }^{50}$ Da parte delle autorità conservatrici, del clero cattolico e della stampa nazionale conservatrice e antisemita, la facilitazione dell'accesso all'istruzione e alla scienza per l'ampia massa della popolazione fu considerata come un atto cospirativo e un pericoloso indebolimento dell'ordine sociale esistente e quindi respinta con forza, anche perché si sospettava naturalmente che essa fosse organizzata da gruppi ebreo-massonici. L'organo del governo, la Reichspost, per esempio, scriveva che attraverso il Volksheim si volesse infettare la società con il «bacillo della peste liberale ebreo anticristiano». ${ }^{51}$

I corsi serali del Volksheim comprendono molte discipline, dalla storia alla chimica, dalla filologia alla fisica, fino all'insegnamento delle lingue. Tutti i corsi sono tenuti da professori o liberi docenti universitari di

\footnotetext{
9 Levetus 1905, 396.

0 Stifter 2020.

1 «Liberale Volksverbildung» 1900.
}

161 Fonti, letterature, arti e paesaggi d'Europa | Sources, Literatures, Arts \& Landscapes of Europe 1

John Ruskin's Europe A Collection of Cross Cultural Essays 151-16 
Figura 6 Prima pagina di un fascicolo che celebra il venticinquesimo anniversario della fondazione del John Ruskin Club. Annessi una dedica e una fotografia di Amelia Sarah Levetus e il biglietto di donazione del fascicolo alla Ruskin Library di Lancaster 
altissimo livello e, unica eccezione alla regola, non rivestendo essa alcun ruolo accademico istituzionale da Amelia Levetus, alla quale sono affidati i corsi in inglese. L'ampio sostegno morale che si era manifestato al momento della fondazione del Volksheim si tradurrà poi anche in sostegno materiale. Nel 1904 fu possibile acquistare un terreno, situato non a caso nel quartiere operaio di Ottakring, e su progetto dell'architetto Franz von Neumann fu costruita da Ludwig Faigl, un affiliato dell'associazione, una sede monumentale e funzionale [figg. 4-5]. ${ }^{52}$ I mezzi provenivano da una raccolta fondi e da ricche elargizioni, dovute, per citare solo due esempi conosciuti internazionalmente, a Karl Wittgenstein e ad Albert von Rothschild. ${ }^{53}$ Non è certo un caso, come notano Hartmann e Levetus, che l'inaugurazione della sede di questa istituzione democratica il 5 novembre 1905 coincida con la prima grande manifestazione per la rivendicazione del suffragio universale. ${ }^{54}$ Per sottolineare l'alto livello di quei corsi è utile accennare anche alle attrezzature: il laboratori di chimica e di fisica avevano dotazioni così moderne e aggiornate che persino l'Università chiese di poterle utilizzare. ${ }^{55}$

A partire dai corsi più seguiti (già nel primo anno di attività si contavano 600 iscritti) nascevano su iniziativa dei partecipanti delle 'Fachgruppen', dei club dotati di statuti propri. Quello dedicato a John Ruskin guidato da Amelia Levetus inizia la sua attività nel 1903 per «promote the teaching of that master», secondo le parole della sua fondatrice..$^{56} \mathrm{Da}$ quel che sappiamo fu questo l'uni- co club ad essere intitolato a una persona. Conoscendo la traiettoria personale di Amelia Levetus da economista a storica e critica dell'arte, è ragionevole ipotizzare che gli incontri fossero dedicati sia a Ruskin economista che a Ruskin storico e critico dell'arte. Percorrendo le notizie, purtroppo piuttosto esigue, sulle sue attività, si riscontrano in effetti sovente conferenze e seminari su vari scritti di Ruskin, ma anche su argomenti legati alle pubblicazioni di Levetus. The Seven Lamps of Architecture sono discusse nel 1904; più tardi si parlerà di The Crown of Wild Olive, e anche corsi su «I giudizi sull'arte di Ruskin» e su Modern Painters saranno tenuti più volte. ${ }^{57}$ Oggetto di studio sono inoltre William Morris e le arti decorative e popolari, affrontate in una prospettiva di comparazione tra la produzione inglese e quella austriaca. Con la fondazione e le attività del John Ruskin Club la dimensione della conoscenza e della ricezione di Ruskin e della sua cerchia acquista un significato del tutto particolare e probabilmente unico (nel panorama continentale). La cosa straordinaria non è tanto il Ruskin Club in sé e che esso parli in inglese, ma la circostanza che esso si componga anche di donne e uomini provenienti dalla classe degli artigiani, degli impiegati e degli operai.

Grazie al supporto dei partecipanti ai corsi di inglese e le attività del John Ruskin Club continuarono regolarmente anche durante la guerra. L'introduzione a un fascicolo del 1928, che celebra il venticinquesimo anniversario del club e la sua fondatrice [fig. 6] ricorda che

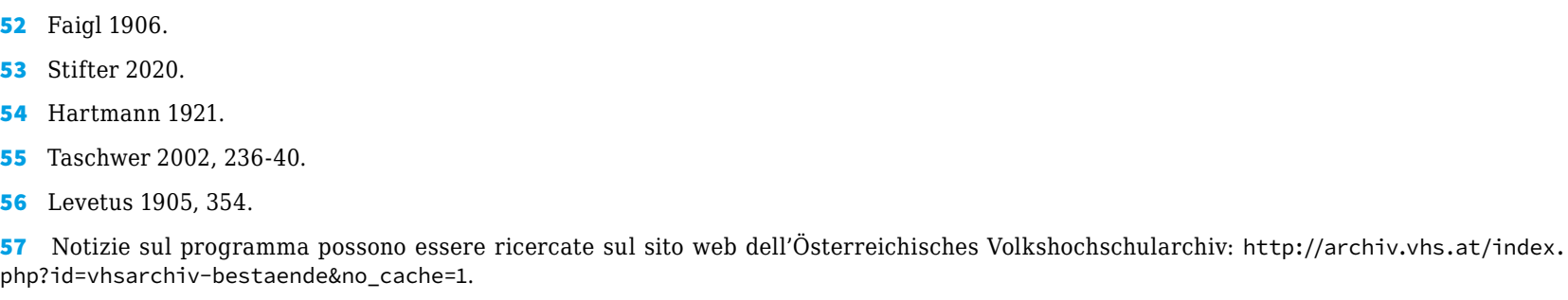

7 Notizie sul programma possono essere ricercate sul sito web dell'Österreichisches Volkshochschularchiv: http://archiv.vhs.at/index. php?id=vhsarchiv-bestaende\&no_cache=1.

163 Fonti, letterature, arti e paesaggi d'Europa | Sources, Literatures, Arts \& Landscapes of Europe 1

John Ruskin's Europe A A Collection of Cross-Cultural Essays, 151-166 
It might have been expected that the Word War would have wrought irrevocable harm to the teaching of English in an 'enemy' country - we need only to recall the position of German teaching in England during the same period -; but actually the position was quite different. ${ }^{58}$

Il periodo post bellico è inaugurato con corsi in occasione del centenario di Ruskin e negli anni accademici seguenti sono registrati insegnamenti dedicati a Unto this Last e ai giudizi sull'arte di Ruskin. «We have built together a Social State such as our Master, whose teaching we seek to follow, would have wished; in us there is peace and good will towards mankind», scrive Levetus nel ci- tato fascicolo e un contributo anonimo estende il debito nei confronti di Ruskin all'intero Volksheim:

The nobility of his mind, his high and genuine character, his lofty ideals appeal to all who have become acquainted with his writings, particularly "Unto this Last". And the group of students of the English language at the Volksheim paid the highest tribute to his principles and declared themselves his true disciples when, 25 years ago, they adopted his name for their community. For after all, it is to Ruskin that we all owe our Volksheim, which is indeed a living monument set up to him. It is a child of Ruskin's Working Men's College in London. ${ }^{59}$

\section{Bibliografia}

1903-1928 John Ruskin Club in Volksheim Vienna XVI. Wien: Vorwärts. Altenberg, P. (1898-1899). «Der Freund». Wiener Rundschau, 3, 14-15.

Arlaud, S. (2004). «Peter Altenbergs Kunst - das Ende der anglophilen Moderne in Wien?». Kokorz, G.; Mitterbauer, H. (Hrsgg), Übergänge und Verflechtungen: kulturelle Transfers in Europa. Bern: Peter Lang, 100-14.

Arlaud, S. (2008). «Les revues d'art viennoises de la fin de siècle ou comment construire une nation». Germanica, 43, 183-92.

Bericht (1905) = Verein für Abhaltung von wissenschaftlichen Lehrkursen für Frauen und Mädchen ATHENAEUM in Wien.

Billcliffe, R.; Vergo, P. (1977). «Charles Rennie Mackintosh and the Austrian Art Revival». The Burlington Magazine, 119(896), 739-46.

Crowley, D. (1995). «The Uses of Peasant Design in Austria-Hungary in the Late Nineteenth and Early Twentieth Centuries». Studies in the Decorative Arts, 2(2), 2-28.

Delyfer, C. (2010). «The Studio and the Craftsman as Artist: A Study in Periodical Poetics (1893-1900)». Cahiers victoriens et édouardiens, 71, 437-52.

Dimand, R.W. (1999). «Women Economists in the 1890s: Journals, Books and the Old Palgrave». Journal of the History of Economic Thought, 21(3), 269-88.

Dmitrieva, M. (2020). «Inventing Folk Art: Artists' Colonies in Easter Europe and their Legacy». Von Bonnsdorff, A.-M.; Ojanpera, R. (eds), European Revivals. From Dreams of a Nation to Places of Transnational Exchanges. Helsinki: Finnish National Gallery, 83-108.

Faigl, L. (1906). «Das “Deutsche Volksheim” in Wien, XVI. Bezirk». Der Bautechniker. Zentralorgan für das österreichische Bauwesen, $26(12), 1$. Fellner, G. (1986). «Athenäum. Die Geschichte einer Frauenhochschule in Wien». Zeitgeschichte, 14(3), 99-115.

Filla, W. (2001). «Miss A. S. Levetus - Kunsthistorikerin und Volksbildnerin. Porträt einer grenzüberschreitenden Pionierin». Spurensuche. Zeitschrift für Geschichte der Erwachsenenbildung und Wissenschaftspopularisierung, 12(1-4), 24-39.

FitzGerald, C. (2016). Women, Craft, and the Object: Birmingham 1880-1930 [PhD Thesis]. Warwick: University of Warwick. 
Gilbert, M.E. (1937). «Hugo von Hofmannsthal and England». German Life and Letters, 1(3), 182-93.

Hartmann, L.M. (1921). «Ein Kulturjubiläum. Zum zwanzigsten Geburtstag des Ottakringer Volksheims». Arbeiter-Zeitung, $23(111), 2$. Hertz, F.O. (1928). Race and Civilization. Trad. di A.S. Levetus. London: Kegan Paul. Trad. di: Rasse und Kultur. Leipzig: A. Kröner, 1915. Houze, R. (2015). Textiles, Fashion, and Design Reform in Austria-Hungary Before the First World War. Farnham: Ashgate.

La femme et le féminisme (1900). Paris: V. Giard \& E. Brieur.

Large, J. (2003). The Studio and the Workshops: Amelia Levetus and the British Influence on the Applied Arts in Vienna [Unpublished MA Thesis]. Budapest: Central European University.

Leary, L. (ed.) (1969). Mark Twain's Correspondence with Henry Huttleston Rogers 1893-1909. Berkeley; Los Angeles: University of California Press.

Levetus, A.S. (1896). «Sociale Hilfsarbeit». Mittheilungen der Ethischen Gesellschaft in Wien, 13, 142-5.

Levetus, A.S. (1897). «Working Women in Vienna». Economic Journal, 7, 101-9.

Levetus, A.S. (1897). «Women's Progress in Austria-Hungary». Horowitz Murray, J.; Stark, M. (eds), The Englishwoman's Review of Social and Industrial Questions: 1897. New York: Routledge. Routledge Library Editions 29 (2016).

Levetus, A.S. (1898). «Das Frauenleben auf der Universität zu Cambridge». Die Wage, 1(1), 2.

Levetus, A.S. (1900). «Gross-Einkaufs-Genossenschaften in England und Schottland». Zeitschrift für Volkswirtschaft, Sozialpolitik und Verwaltung, 9, 199-214.

Levetus, A.S. (1902). «A Women's Penitentiary. A Visit to Wiener Neudorf near Vienna». Womanhood, 8, 408-10.

Levetus, A.S. (1903-1904). «Modern Austrian Wicker Furniture». The Studio. An Illustrated Magazine of Fine and Applied Arts, 30, 323-8.

Levetus, A.S. (1905). Imperial Vienna. An Account of its History, Traditions and Arts. New York; London: John Lane.

Levetus, A.S. (1905). «The Craft Schools of Austria». The Studio, 35, 201-19.

Levetus, A.S. (1906). «Modern Decorative Art in Austria». Holme, C. (ed.), The Art-Revival in Austria. London; Paris; New York: The Studio, DI-DXII.

Levetus, A.S. (1906). «The Imperial Arts and Crafts Schools in Vienna». The Studio, 39, 323-34.

Levetus, A.S. (1909). «Glasgow Artists in Vienna: Kunstschau Exhibition». Glasgow Herald, 29 May, 11.

Levetus, A.S. (1910). «Das englische Landhaus». Innen-Dekoration. Zeitschriff für Wohnungskultur und den gesamten Innen-Ausbau, 21, 151-64.

Levetus, A.S. (1911a). «Les coopératives de gros d'Angleterre et d'Ecosse (1897-1909)». Revue d'Économie politique, 25(6), 745-64.

Levetus, A.S. (1911b). «Austria. Introduction». Holme, C. (ed.), Peasant Art in Austria and Hungary. London; Paris; New York: The Studio, 1-14.

Levetus, A.S. (1912). «Une cité ouvrière en Autriche». Revue d'Économie politique, 26(2), 193-205.

Levetus, A.S. (1933). «The European Influence of “The Studio"». The Studio, 105, 257-8.

«Liberale Volksverbildung» (1900). Reichspost. Unabhängiges Tagblatt für das christliche Volk Österreich-Ungarns, 7(50), 54.

Madden, K.K.; Seiz, J.A.; Pujol, M. (eds) (2004). A Bibliography of Female Economic Thought to 1940. New York: Routledge.

Ottenbacher, K. (2016). «Levetus Amelia Sarah». Korotin, I. (Hrsg.), Biografisches Lexikon österreichischer Frauen, Bd. 2, Wien; Köln; Weimar: Böhlau, 1972-5.

Panagl, K. (1972). Die britische Kunstzeitschrift The Studio und ihre Bedeutung für die Entwicklung des Jugendstils in Europa [Phil. Dissertation]. Wien: Universität Wien.

Rampley, M. (2010). «Design Reform in the Habsburg Empire: Technology, Aesthetics and Ideology». Journal of Design History, 23(3), 247-64.

Sarmany-Parsons, I. (1987-1988). «The Influence of the British Arts and Crafts Movement in Budapest and Vienna». Acta Historiae Artium, 33, 181-98.

Schindler, M. (2010). «"Alter Jude, Ton, glasiert”. Spuren des Jüdischen im Österreichischen Museum für Volkskunde». Johler, B.; Staudinger, B. (Hrsgg), Ist das jüdisch? Jüdische Volkskunde im historischen Kontext. Wien: Buchreihe der Österreichischen Zeitschrift für Volkskunde, 435-56.

Schölermann, W. (1899a). «Ruskin». Wiener Rundschau, 3(7), 156-8.

Schölermann, W. (1899b). «John Ruskin: Kunst und Moral». Wiener Rundschau, 3(9), 213-18.

Schölermann, W. (1899c). «John Ruskin: Kunst und Moral Il». Wiener Rundschau, 3(10), 240-2.

165 Fonti, letterature, arti e paesaggi d'Europa | Sources, Literatures, Arts \& Landscapes of Europe 1

John Ruskin's Europe. A Collection of Cross-Cultural Essays, 151-166 
Schorske, C.E. (1981). Fin-de-Siècle Vienna. New York: Vintage Books.

Schwiedland, E. (1894). Kleingewerbe und Hausindustrie in Österreich. Leipzig: Duncker\&Humblot.

Smith, E.M. (2014). To Walk upon the Grass: The Impact of the University of St Andrews' Lady Literate in Arts, $1877-1892$ [PhD Thesis]. St Andrews: University of St Andrews.

Stifter, C.H. (1996). «Knowledge, Authority and Power: The Impact of University Extension on Popular Education in Vienna 1890-1910». Hake, B.J.; Steele, T.; Tiana, A. (eds), Masters, Missionaries and Militants. Studies of Social Movements and Popular Adult Education 1890-1939. Leeds: University of Leeds, 158-90.

Stifter, C.H. (2005). «Geistige Stadterweiterung. Eine kurze Geschichte der Wiener Volkshochschulen 1887-2005». Enzyklopädie des Wiener Wissens. Bd. 3, Volksbildung. Wien: Bibliothek der Provinz Edition Seidengasse.

Stifter, C.H. (2020). «Science-Oriented Popular Education: Heterotopic Learning Venues for Scientific Knowledge in Vienna». Ash, M.G. (ed.), Science in the Metropolis: Vienna in Transnational Context, 1848-1918. New York; Oxon: Routledge.

Swiney, F. (1899). The Awakening of Women or Woman's Part in Evolution. London: Redway.

Szczerski, A. (2015). Views of Albion. The Reception of British Art and Design in Central Europe, 1890-1918. Oxford; Bern; Berlin: Peter Lang.

Taschwer, K. (1998). «People's Universities in a Former Metropolis: Interfaces Between the Social and Spatial Organization of Popular Adult Education in Vienna, 1890-1930». Hake, B.J.; Steel, T. (eds), Intellectuals, Activists and Reformers. Studies of Cultural, Social and Educational Reform Movements in Europe, 1890-1930. Leeds: University of Leeds Press, 175-202.

Taschwer, K. (2002). Wissenschaft für viele. Zur Wissenschaftsvermittlung im Rahmen der Wiener Volksbildungsbewegung um 1900. [Phil. Dissertation]. Wien: Universität Wien. https://www.academia.edu/4389849/Wissenschaft_für_viele_Zur_Wissenschafts_vermittlung_im_Rahmen_der_Wiener_Volksbildungsbewegung_um_1900_Unveröffentlichte_Dissertation_an_der_ Universität_Wien_2002

Twain, M. (1898). «The Austrian Edison keeping School again». The Century Illustrated Monthly Magazine, 56, 630-1.

«Vortrag einer jungen Dame an der Universität Wien» (1897). Neue Freie Presse, 34(11655), 2. Februar. 


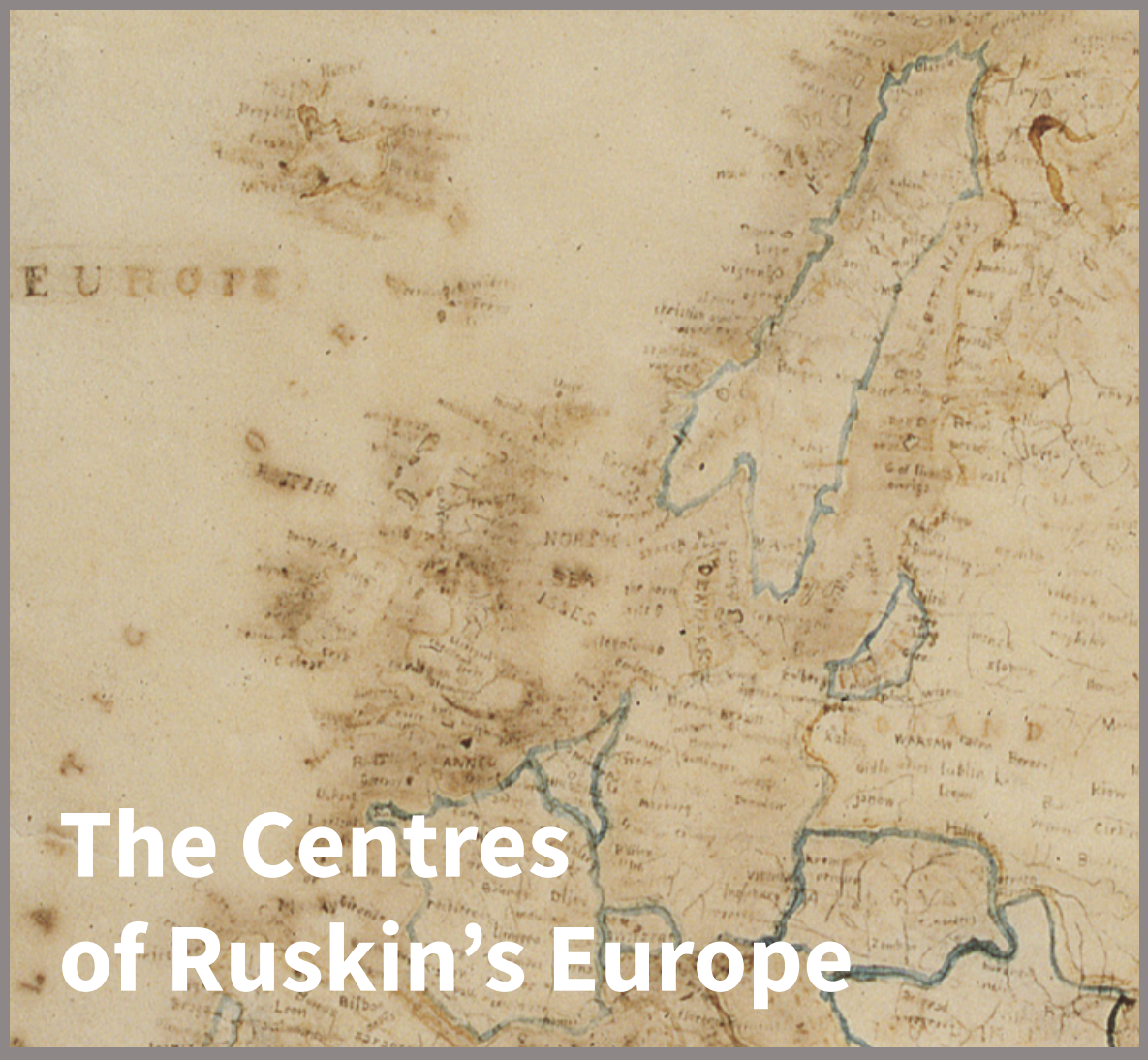




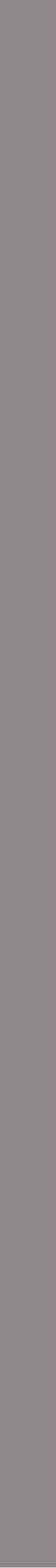




\title{
Ce qui commence à Calais : l'Europe, terrain de jeu de Ruskin
}

André Hélard

Classes préparatoires aux grandes écoles, Rennes, France

\begin{abstract}
The account, in verse, of the Ruskin family's first tour on the Continent, in 1833, opens with an evocation of Calais. This is only the first of a rather long series of passages expressing Ruskin's singular interest in Calais. What is intriguing is that Calais, in Ruskin's imaginary geography, is the place where one turns one's back on familiar England to enter continental Europe, where everything is to be discovered, the place where, in a true art of travelling, a shift of the gaze begins. At the same time, it is the starting point of what Ruskin will call, at the other end of his work, The Old Road.
\end{abstract}

Keywords Calais' experience. Disorientation. Imaginary geography. Europe awareness. Comparatism. "Grand contexte".

Sommaire 1 L'expérience de Calais. - 2 « On the Old Road ». - 3 In varietate concordia. Du regard sur l'Europe à une conscience de l'Europe.

On n'en a décidément jamais fini avec Ruskin. La place dans son œuvre de livres comme The Stones of Venice, Val d'Arno, Mornings in Florence, et même les inachevées Laws of Fésole pourrait nous amener à considérer très légitimement l'Italie comme le centre de l'Europe ruskinienne. Mais tout ruskinien connaît aussi la double affirmation, je suis tenté de dire la double provocation, de Ruskin dans Praeterita. En II, 3 (« Cumes »), il écrit :

J'ai trouvé dans mon journal du 8 mai (1841) une phrase qui me semble en contradiction avec ce que j’ai précédemment écrit des centres de ma vie et de mon œuvre : Venise et Chamonix sont mes deux maisons (ou mes deux pôles) sur cette Terre. Mais je ne connaissais alors ni Rouen ni Pise, bien que je les eusse déjà vues. Ni Genève, dont je parlais en même temps, et dont pour moi fait partie Chamonix. Quant à Venise, je la regarde de plus en plus comme une vaine tentation. ${ }^{1}$

1 Works, 35: 296. Sauf indication contraire, toutes les traductions sont faites par l'Auteur.

Fonti, letterature, arti e paesaggi d'Europa | Sources, Literatures, Arts \& Landscapes of Europe 1 ISSN 2724-6620 e-ISSN 2784-8507 
Et en II, 7 (« Macugnaga ») :

De Vérone, je ne dirai rien de plus, sinon que, même si Rouen, Genève et Pise furent pour moi des centres de ma pensée et de mon éducation, Vérone a apporté la couleur à tout ce que ces cités m'avaient appris. ${ }^{2}$

Ce que je voudrais proposer ici c'est d'oublier au moins pour un moment et Venise, et Florence et Vérone, mais aussi Rouen, Pise et Genève, et de partir à Calais, ou plus exactement de repartir de Calais. Incontestablement Calais n'est pas un centre. Ni géographiquement, vu sa situation si absolument périphérique, excentrée pour ne pas dire excentrique sur les cartes de France ou d'Europe, ni sur le plan de 'la pensée et de l'éducation' ruskiniennes. Et pourtant, la place et la fonction de Calais dans la constitution de l'Europe ruskinienne sont à la fois uniques et capitales, dépassant largement le nombre de pages, limité, qui lui sont consacrées dans les 39 volumes de la Library Edition.

\section{$1 \quad$ L'expérience de Calais}

Ce que j'appelle ainsi a été une expérience cardinale dans la vie et l'œuvre de Ruskin, et il se trouve qu'un texte de 1833 et un autre de 1885, pour ainsi dire aux deux extrémités de sa vie d'écrivain, nous disent ce qu'elle fut. Ces deux textes, plus un troisième, qui se situe fort opportunément entre les deux (1856), constituent ce que j'appellerai le 'triptyque calaisien', que je

vous propose en un premier temps de parcourir rapidement pour mesurer la place de Calais dans la vie et l'œuvre de Ruskin. Une place qui si elle est, quantitativement, sans commune mesure avec ce que lui ont inspiré d'autres lieux, à commencer bien sûr par Venise, n'en est pas moins tout à fait remarquable par son caractère puissamment déterminant.

\subsection{Premier volet du triptyque}

L'incipit du récit de 1833, en vers et en prose (alternant), du premier voyage des Ruskin sur le Continent (Account of a Tour on the Continent), se subdivise en 2 temps. Le premier évoque la traversée de la Manche :

\section{Étrange, comme l'espace qui sépare}

Une côte d'une autre côte,

Si vite, si facilement franchi,

Peut pourtant établir une si grande différence

Entre un homme et un autre homme,

Entre une race et une autre race.

Religion, langage, et même mentalité,

Le changement que l'on trouve

Est soudain et marqué. ${ }^{3}$

Ce que Ruskin privilégie d'abord c'est clairement une expérience de l'espace, avec une prise de distance avec l'espace d'origine qui introduit à la révélation de l'altérité ( " un autre homme », " une autre race »), que recouvrent les termes de différence et de changement. Si vous préfé-

2 Works, 35: 371.

3 Works, 2: 341. C'est moi qui souligne, comme dans toutes les citations à venir.

Fonti, letterature, arti e paesaggi d'Europa | Sources, Literatures, Arts \& Landscapes of Europe $1 \mid \quad \mathbf{1 7 0}$ 
rez de diversité et de variété... Car le jeune Ruskin, qui écrit cela à 13 ans et demi, met ici clairement ses pas dans ceux de Montaigne qui fit aussi son tour sur le continent, et qui dans ses Essais ( " De la vanité ») écrivait que « le voyager » propose à l'âme " la diversité d'autres vies et usances », et lui fait goûter « une perpétuelle variété de formes de notre nature ${ }^{4}{ }^{4}$

Mais Ruskin, dès le second temps de ce premier volet, dépasse cette expérience de la diversité et de la variété. L'épatante description de Calais, en prose cette fois, qui suit, donne une tout autre portée à la traversée de la Manche :

Calais est un vestibule, c'est une introduction à la France et aux Français [...]. C'est une petite France, une France en réduction. Tenez-vous sur la jetée, et regarde $z$ autour de vous. Le ciel, vraiment turquoise, est un ciel français, la mer est une mer française, l'air est un air français. [...] Et regardez les gens ; leur contenance, leur tenue, le tout ensemble est radicalement différent de tout ce que vous avez jamais pu voir en Angleterre, et pourtant les falaises d'Angleterre sont encore à l'horizon. C'est absolument extraordinaire. ${ }^{5}$

Ce qui s'exprime ici, avec un art déjà totalement ruskinien de la concentration verbale, c'est l'expérience du dépaysement. Non pas à son sens banal, pour désigner un plaisant changement de décor et d'habitudes. Mais en revenant au sens premier du verbe 'dé-payser' qui redonne au préfixe privatif toute sa force. Etre dé-paysé, comme le jeune Ruskin l'est en effet ici, c'est littéralement échan- ger un pays, le pays d'origine, pour un autre pays, avec tout ce que cela implique.

Mais s'il s'agit toujours d'espace (un vestibule, une introduction), c'est un espace qui s'ouvre tout grand au regard (regardez autour de vous, regardez les gens). Si Calais est bien, pour l'optical thinker qu'est Ruskin, le lieu du dé-paysement, c'est parce que tout ce qui y constitue le paysage, comme signe premier du pays - paysage naturel (la mer, le ciel, l'air) et paysage humain (les gens) - y est vu, dans toute la plénitude, déjà, du voir ruskinien, sous un jour radicalement différent. Et ce dé-paysement, en tant que séparation d'avec le pays/paysage d'origine, tout en désorientant le regard, le renouvelle totalement dans une sorte d'hyperactivité.

Et comme la traduction fait parfois bien les choses, le dictionnaire en ligne Reverso me propose comme traductions possibles du mot dépaysement en anglais : $e x-$ patriation, change of scenery, et disorientation et en italien, espatrio et spaesamento. En proposant même cet exemple : "Nous avons choisi le dépaysement comme condition première d'une nouvelle créativité. Abbiamo scelto lo spaesamento come condizione primaria per una nuova creatività ». ${ }^{6}$ On ne saurait mieux définir le dé-paysement dans ses diverses manifestations.

Un premier volet, donc, qui, par son caractère réflexif (tout en restant merveilleusement joyeux) dépasse de loin le simple récit de voyage. Si Ruskin était allemand, je dirais qu'il a tourné le dos à son Heimat pour pénétrer en toute jouissance dans un espace unheimlich : ce premier volet du triptyque commence d'ailleurs par le mot « étrange » et se termine par le mot « extraordinaire »...

\section{Montaigne 1962, 3: 951.}

5 Works, 2: 341-2.

6 https://www.reverso.net/translationresults.aspx?lang=IT\&direction=francese-inglese; https://www. reverso.net/translationresults. aspx?lang=IT\&direction=francese-italiano.

171 Fonti, letterature, arti e paesaggi d'Europa | Sources, Literatures, Arts \& Landscapes of Europe 1 


\subsection{Second volet de son triptyque calaisien}

C'est cette expérience fondatrice, sur laquelle Ruskin revient quelques 20 ans plus tard, dans Modern Painters IV ; là aussi, noter la position quasi inaugurale de Calais (ch. 1, «Of Turnerian Picturesque », § 2-3) dans le livre, ce qui ne va nullement de soi pour un livre dont le soustitre est « Of Mountain Beauty »:

Je n'ai pas de mots pour exprimer l'intense plaisir que j'ai toujours au moment où, après un séjour un peu prolongé en Angleterre, je me retrouve au pied de la vieille tour de l'église de Calais. [...] Je ne puis dire la moitié des plaisirs et des pensées étranges qui viennent à moi à la vue de cette vieille tour. Car elle est, en quelque sorte, l'épitomé de tout ce par quoi ce continent qui est l'Europe est intéressant. [...] Cette tour de Calais est riche d'un symbolisme infini, d'autant plus frappant que, lorsqu'elle apparaît, les sentiments qu'elle exprime sont généralement, et par contraste, à l'exact inverse de ceux qu'inspiraient les images de l'Angleterre. ${ }^{7}$

Ce second volet se subdivise lui aussi en 2 mouvements, d'une tout autre nature :

\subsection{Troisième volet du triptyque calaisien}

Ce dernier volet se trouve, comme souvent chez Ruskin, là où on ne s'attend pas à le trouver, dans un chapitre de Praeterita intitulé « Crossmount »:

Dans ma mémoire, l'infini plaisir de pouvoir flâner a. le premier mouvement est celui d'un approfondissement, mieux d'une intériorisation : désormais ce n'est plus seulement son espace extérieur (le paysage, le monde) qui est dé-paysé à et par Calais, et avec quelle radicalité (« l'exact inverse »!), c'est aussi son propre espace mental, sa façon de regarder ce monde, sa Weltanschauung : non plus seulement ciel, air ou mer, mais aussi plaisirs, pensées, images, sentiments, et surtout symbolisme ;

b. le second mouvement, encore plus intéressant, est celui d'un élargissement: Calais devient le vestibule d'un espace bien plus vaste que la France. De "France en réduction », Calais devient « l'épitomé ${ }^{8} \mathrm{du}$ Continent qui est l'Europe ». En anglais : the Continent of Europe. Ce of Europe qui serait en latin un génitif explicatif, il faut, je crois, le traduire comme tel : le Continent qui est l'Europe. Aller sur le continent, comme se contentent souvent d'écrire les voyageurs anglais, c'est seulement aller dans une autre géographie (le contraire de l'insularité britannique), mais l'appeler Europe c'est lui donner une identité propre, avec tout ce que cela comporte.

et me balancer juste au-dessus du beaupré en observant, s'il y avait la moindre houle ou une mer cassée, les plongeons de la proue dans les vagues, avec la perspective d'être le lendemain pour le breakfast à Calais, où m’attendraient les chevaux de poste, têtes

7 Works, 6: 11-12.

8 C'est-à-dire « l'abrégé d'un livre, d'une histoire ; plus particulièrement, un précis d'histoire », d'après le site du Cnrtl (Centre national de ressources lexicales et textuelles, https://www.cnrtl.fr/). 
déjà tendues droit vers le Mont Blanc, fut un de mes rares plaisirs tout à fait sans mélange. Lorsque j'acquérais un dessin de Turner il y en avait toujours un autre dont j'avais envie ; mais je ne désirais être sur aucun autre bateau que celui-là. ${ }^{9}$

C'est ici l'inscription solennelle de l'expérience du dé-paysement à Calais et par Calais à la fois dans la mémoire et dans le parcours d'une vie. Et l'on en mesure la force à ce que Ruskin l'élève, en termes de désir et de plaisir esthétique en même temps qu'existentiel, au rang et même au-dessus de l'acquisition d'un nouveau Turner. Un premier temps, dans les vagues, pour sortir de l'insularité. Un second temps pour ouvrir devant lui tout l'espace européen en ce raccourci fulgurant: "Calais où m’attendraient les chevaux de poste, têtes déjà tendues droit vers le Mont Blanc $\gg .{ }^{10}$ C'est comme un trait tiré sur la carte de l'Europe, et qui relie Calais et les Alpes. ${ }^{11} \mathrm{Et}$ ce trait dessine bien sûr une route ; et cette route, dans la vie comme dans l'œuvre de Ruskin, c'est bien sûr « the Old Road », une 'vieille route' dont Calais a donc l'extraordinaire privilège d'être en quelque sorte la tête de pont.

\section{2 «On the Old Road »}

On the Old Road... c'est le titre d'un recueil ${ }^{12}$ de différents textes où il est question de tout sauf de route, et de voyage, mais qui a imposé l'expression comme typiquement ruskinienne. En dehors de cela, Ruskin luimême ne semble guère l'avoir utilisée dans ses œuvres publiées. S'il lui arrive de l'employer, toujours avec une forte connotation de nostalgie (quand il n'y est plus) ou d'attendrissement (quand il s'y retrouve), c'est ici ou là, dans une lettre ${ }^{13}$ ou dans une page des Diaries des années 1874 et $1888 .^{14}$
Mais c'est cette expression (qu'il me semble nécessaire de conserver dans la langue originale) que les premiers biographes de Ruskin, W.G. Collingwood, au premier chef, avec son "Ruskin's Old Road », ${ }^{15}$ mais aussi E.T. Cook, qui parle de «l'old road de la plupart des voyages de Ruskin avec ses parents sur le Continent $\gg,{ }^{16}$ puis Cook à nouveau, avec A. Wedderburn, dans leurs introductions ou notes aux volumes de la Library Edition ont abondamment reprise, lorsqu'ils évoquent les voyages en Europe de leur maître et ami (par exemple

9 Works, 35: 415.

10 Works, 35: 415.

11 A vrai dire, le texte de 1833 ébauchait déjà un tel itinéraire, non seulement continental mais européen, en déclinant, tantôt en vers tantôt en prose, les étapes de ce tour : Cassel, Lille, Bruxelles, la Meuse, Aix-la-Chapelle, Cologne, St Goar, Heidelberg, la Forêt Noire, le Rhin, la Via Mala, la Splügen, Cadenabbia, Milan, le Lac Majeur, et, pour finir, déjà, Chamonix.

12 Works, 34.

13 Par exemple, sa lettre à son père, du 27 octobre 1862: "J'étais vraiment sur un nuage - comme je n'avais été depuis cinq ou six ans. Je me suis promené sur l'old, old road qui va de Genève à Chamonix, [du côté de] Bonneville » (Works, 17: lx).

14 Evans, Whitehouse 1959, vol. 3.

15 Collingwood 1903, 45-62.

16 Works, 1: 36.

$173 \begin{aligned} & \text { Fonti, letterature, arti e paesaggi d'Europa | Sources, Literatures, Arts \& Landscapes of Europe } 1 \\ & \text { John Ruskin's Europe. A Collection of Cross-Cultural Essays, 169-180 }\end{aligned}$ 
dans l'introduction aux Lectures on Architecture and Painting : $:^{17}$ "Ce volume peut être considéré comme un recueil d'œuvres de circonstance, entreprises [...] sur ce qu'il appellera plus tard 'the old road' »). ${ }^{18}$ Ainsi ontils popularisé l'Old Road chez les lecteurs de Ruskin, la parant véritablement de ce que Walter Benjamin appe-

\subsection{Ce qui se passe « on the Old Road»}

Mais ce qui m'importe ici, plus que l'origine ou l'histoire de cette dénomination, c'est bien ce qui se passe « on the Old Road », dont on ne saurait épuiser le sens et la portée en se contentant de dire que Ruskin y voyage. " On the Road », sur la route, cela m'a toujours fait penser non pas tant à Jack Kerouac qu'à Mikhail Bakhtine, et à ce que ce théoricien russe de la littérature nous dit précisément de la route dans son Esthétique et théorie du roman:

L'importance du chronotope de la route est énorme dans la littérature; rares sont les œuvres qui se passent de certaines de ses variantes, et beaucoup d'entre elles sont directement bâties sur lui, et sur les rencontres et péripéties 'en route'. ${ }^{20}$

\section{Et encore :}

Dans le roman, les rencontres se font, habituellement, 'en route'. Sur 'la grand route' se croisent les voies d'une quantité de personnes appartenant à toutes les classes, situations, religions, nationalités lait une aura. Et puis surtout, comme Ruskin l'écrit luimême dans Praeterita à propos de l'itinéraire du 'tour' de $1833,{ }^{19}$ c'était « the then only possible way », la seule route alors possible, ce qui lui suffit évidemment à lui conférer son caractère quasiment mythique.

et âges. Là peuvent naître toutes sortes de contrastes, se heurter et s'emmêler diverses destinées. ${ }^{21}$

Bakhtine évoque alors tous les héros du roman européen, dont l'histoire s'est déployée 'sur la route', avec le bric-à-brac picaresque des incidents de voyage, scènes de nuit, auberges, bagarres, rencontres édifiantes. Don Quichotte avec quoi tout commence, le Simplicius Simplicissimus de Grimmelshausen, les comédiens du Roman comique de Scarron ou le Gil Blas de Lesage, le Joseph Andrews ou le Tom Jones de Fielding, jusqu'aux Années d'Apprentissage et aux Années de Voyage de Wilhelm Meister. Ce que je propose ici c'est bien de voir Ruskin comme un de ces aventuriers 'bakhtiniens' et picaresques, lancé sur cette route qui commence à Calais. N'y a-t-il pas du Wilhelm Meister (celui des Lehrjahre) dans le jeune Ruskin, et le Ruskin vieillissant ne prend-il pas de plus en plus des allures donquichottesques ? Mais, me direz-vous, on ne peut prétendre que sur 'sa grand route' Ruskin croise, selon les mots de Bakhtine, une quantité de personnes appartenant à toutes les classes, situations, religions, nationalités et âges ?

17 Works, 12: xvii.

18 Voir encore, par exemple, Works, 16: 458; 33: xxxvii ou 35: xxx-xxxi.

19 Works, 35: 80.

20 Bakhtine 1987, 249.

21 Bakhtine 1987, 384-5. 
Bien sûr. Mais c'est que ses rencontres à lui, sur la route de l'Europe, sont d'une autre nature : elles se font par la médiation du regard (rappelons-nous le double regardez inaugural dans le premier texte de 1833 sur Calais). Tout au long de cette route, ce qu'il rencontre, lui, visuellement, optiquement, comme autant de personnages, ce sont des paysages, des monuments, des œuvres d'art, qu'il s'approprie en les dessinant immédiatement dans ses carnets et en les décrivant ou en les racontant le soir à l'auberge dans ses Journaux, qui sont le plus souvent de véritables journaux de voyage. C'est en ce sens que l'on peut tout à fait dire, en reprenant le concept de Bakhtine, que l'Old Road de John Ruskin est le lieu de toutes les rencontres, de toutes les découvertes, de toutes les expériences, de toutes les aventures. C'est en ce sens aussi que l'on peut dire, en se rappelant Leslie Stephen et son Playground of Europe (1871) - en français Le Terrain de jeu de l'Europe -, que cette Europe que l'Old Road avec ses innombrables variantes au fil

\section{2 Étapes et lieux privilégiés}

Sur cette Old Road, il y a aussi forcément des étapes, des lieux privilégiés. A l'heure des bilans, celui d'une vie et celui d'une œuvre, c'est-à-dire dans Praeterita, les titres de chapitres, Milan et Schaffhouse, le Col de la Faucille, Rome, Cumes, Fontainebleau, le Simplon, le Campo Santo, Macugnaga, La Grande Chartreuse, le Mont Velan, scandent le récit : nombre de titres de chapitres de Praeterita sont des noms de lieux et de lieux européens. Comment ne pas voir aussi dans ces lieux où des « années de voyage » permet de sillonner, est le terrain de jeu de Ruskin. Où l'œil s'en donne (si j'ose dire) à cœur joie, mobilise toute sa watchfulness et se régale de toutes les potentialités du power of seeing ruskinien.

Il faudrait, pour bien prendre toute la mesure de ce qui se passe sur ce "terrain de jeu », énumérer toutes les variantes de cette route que Ruskin et ses parents empruntent en 1833, '35, '40, '42, '47, '49 etc. Telles que Cook et Wedderburn les ont minutieusement rapportées dans la Library Edition. Il faudrait citer toutes les étapes. Faire une place à part aux visites aux cathédrales de la Terre, et une autre aux visites aux œuvres des architectes et particulièrement à l'Europe des cathédrales.

Et montrer enfin et surtout, dans le détail, comment cette 'vieille route' de l'Europe est littéralement, le lieu où s'élabore, infiniment plus que dans les musées, dans les bibliothèques ou dans son cabinet de travail, l'essentiel de l'œuvre ruskinienne, depuis Modern Painters I jusqu'à La Bible d'Amiens.

le sens se concentre et dans ces titres, dont chacun renvoie à un aspect de l'Europe, et plus largement dans les magnifiques arrêts de tel ou tel chapitre sur Abbeville ${ }^{22}$ ou sur Genève, ${ }^{23}$ ce que l'on pourrait appeler avec Proust des « réminiscences anticipées ${ }^{24}$ ou avec Pierre Bayard « un plagiat par anticipation » ${ }^{25}$ des « Géographies d'Europe » du Professeur Carlo Ossola, avec leurs 18 étapes qui promènent le lecteur d'Anderlecht à Odessa, ou de Saint-Benoît-sur-Loire à Reggio de Calabre ? ${ }^{26}$ Car c'est

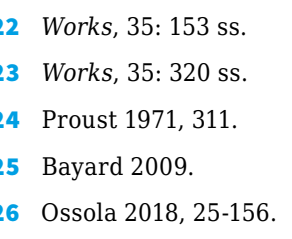


bien une géographie d'Europe, dans toute sa diversité, que dessine Ruskin, comme il en dessinait jadis la carte, reproduite sur la couverture de ce livre ?

Et cette géographie n'est pas seulement une géographie culturelle, c'est aussi une géographie affective : dans Praeterita, encore, ne nous dit-il pas que, sur cette vieille route de l'Europe, certaines étapes, certains lieux sont devenus comme ses maisons : "Où nous nous sentions chez nous [at home], c'était à Champagnole; et à Chamonix, ou à La Cloche à Dijon, ou au Cygne à Lucerne. Tous ces lieux du bon vieux temps». Il faudrait donc ici inventer un néologisme pour dire que le dé-paysement est littéralement suivi d'un re-paysement, et que ce nouveau pays, dont Calais est bien à la fois le vestibule et l'épitomé, c'est l'Europe. Ce qui commence à Calais c'est donc bien la substitution d'un nouvel être, européen, à l'être britannique, et originel, de Ruskin.

Cela ne signifie évidemment pas la disparition d'un 'être britannique', qui peut s'exprimer en termes magnifiques, comme dans ce passage de Praeterita, où Ruskin se remémore un séjour à Leamington en 1841 ou '42 :

L'automne était superbe, les blés mûrs, et [...] j'avais aux alentours, à une après-midi de marche, tout le
Warwickshire qui me touchait profondément par ce qu'il avait de tellement anglais. Les tours de Warwick bien visibles au-dessus des cimes des arbres proches ; Kenilworth, une promenade pour l'après-midi ; Stratford, à une heure de chemin au trot d'un poney; et tout autour, aussi loin que mes yeux pouvaient porter, une perfection d'Angleterre, faite non pas de collines et de vallées - cela on le trouve n'importe où, - mais de collines et d'un plat pays, à travers lequel les rivières serpentent, et où les canaux lambinent sans avoir besoin d'écluses. ${ }^{27}$

Mais il est absolument remarquable d'apprendre, dans ce même passage, que dans les jours même où il célèbre cette "perfection d'Angleterre », le jeune Ruskin est en train de lire... une Histoire de l'Europe de Sir Archibald Alison. ${ }^{28}$ Ici encore, et comme sur tant d'autres plans, Ruskin se révèle donc être un précurseur, car ce qu'il vit, entre son être britannique et son être européen, c'est quelque chose comme l'expérience des identités multiples, ou de l'identité comme feuilleté de plusieurs identités, qui ne sera pensée et analysée que bien plus tard par l'historienne française Mona Ozouf dans Composition française. ${ }^{29}$

\section{In varietate concordia. Du regard sur l'Europe à une conscience de l'Europe}

Il me reste à parler d'une dernière dimension essentielle de l'être ruskinien, moins souvent évoquée, mais qui s'accomplit pleinement sur l'Old Road. Au-delà d'un simple voyage où l'on glane des impressions, la passion de voir chez Ruskin est un véritable " usage du monde ", selon les mots d'un autre grand voyageur, Nicolas Bouvier, qui se développe, de façon très personnelle, dans la passion de comparer. Peut-être cela me vient-il de ce que

27 Works, 35: 303.

28 Auteur (entre autres) de History of Europe from the Commencement of the French Revolution in 1789 to the Restoration of the Bourbons in 1815 (Alison 1833-1843) et History of Europe from the Fall of Napoleon in 1815 to the Accession of Louis Napoleon in 1852 (Alison 1852-1859). On ne saurait mieux s'imprégner de l'esprit de l'Europe d'alors !

29 Ozouf 2009 
j'ai vécu quarante-trois ans avec une spécialiste de littérature comparée, Colette Cosnier, mais je trouve chez Ruskin un esprit et un talent de comparatiste tout à fait remarquables et ici absolument essentiels.

En disant cela, je ne suis encore pas si loin de Bakhtine ; sur la route, dit celui-ci, le héros voit « naître toutes sortes de contrastes, se heurter et s'emmêler diverses destinées $\gg{ }^{30}$ Ces contrastes, mais aussi leur indispensable pendant, les similitudes, ces destinées qui se heurtent ou s'emmêlent, on les rencontre partout dans l'œuvre de Ruskin. Car chez lui, de Modern Painters I à Praeterita, les éléments du paysage esthétique ou naturel, cathédrales humaines de Rouen ou d'Amiens ou «cathédrales de la terre » de Chamonix ou de Zermatt, font en effet plus et mieux que se succéder ou se juxtaposer, comme chez un simple collectionneur d'impressions de voyage.

L'Europe telle qu'elle est vécue par Ruskin, dans la constante mise en relation de ses aspects, devient un véritable texte, au sens premier du mot (textus, tissu ou tissage, réseau de relations), un texte qu'il ne se lasse pas de déchiffrer, de lire, d'interpréter. Un texte qui est bien ici, selon le mot fameux de Roland Barthes, « un espace de jouissance où il est possible de fouiller $\gg \cdot{ }^{31}$ Aucun passage de l'œuvre ne met mieux cela en évidence que le début de « Mountain Glory », 'La Gloire de la Montagne', le vingtième et dernier chapitre de Modern Painters IV, où se met en place une véritable Europe du paysage, ou plutôt des paysages. Paysage " résolument dépourvu de tout relief, comme la Hollande, le Lincolnshire, la Lombardie centrale [qui] m’apparaissent », écrit Ruskin, " comme une prison que je ne puis supporter longtemps $\gg \cdot{ }^{32}$ Paysage déjà un peu accidenté, où il se sent revivre, comme devant « un coteau de France, verdoyant dans la lumière du soleil avec le feuillage qui en s'élevant se découpe sur le ciel bleu », qui lui évoque aussitôt Vevey ou Côme. Avant de conclure, sur une mise en relation généralisée des types de paysages que peut offrir l'Europe, à la gloire du paysage de montagne : «Si je place le Leicestershire ou le Staffordshire juste à côté du Westmoreland, et la Lombardie ou la Champagne juste à côté du Pays de Vaud ou du Canton de Berne, je constate que l'accroissement de la somme calculable des éléments de beauté est exactement proportionnel à l'accroissement du caractère montagneux $\gg .^{33}$

Bien évidemment les cinq tomes de Modern Painters, qui ont leur point de départ dans une réflexion sur la peinture de paysage, explorent et pensent aussi, et même avant tout, une Europe de la peinture en transcendant les siècles aussi bien que les frontières, bien au-delà du projet annoncé par le fameux sous-titre de Modern Painters I. Tant et tant de pages où l'on va de Turner à Tintoret, en passant par Dürer ou Rubens, Rembrandt ou le Lorrain etc., et qui trouvent le plus splendide des couronnements dans l'extraordinaire « Two Boyhoods » de Modern Painters V, chef d'œuvre comparatiste en forme de 'Vies parallèles', ou plutôt d'enfances parallèles de Giorgione et Turner.

Mais Ruskin pense aussi une Europe des religions : « Dans les belles terres labourables d'Angleterre ou de Belgique, se développe un protestantisme ou un catholicisme orthodoxes; prospères, honorables et somnolents ; mais c'est dans les landes violettes du Highland Border, les ravins du Mont Genèvre, et sur les sommets du Tyrol que nous trouverons la foi évangélique la plus

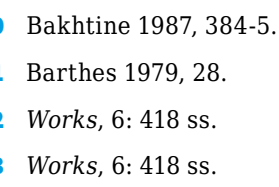


simple, et la pratique romaine la plus pure ${ }^{34}$ Et bien entendu, une Europe des cathédrales, ou de l'architecture, où de la cathédrale de Pise on passe, en l'espace d'une phrase, aux cathédrales de Caën et de Coutances, puis au gothique de Chartres et à Notre-Dame de Paris, avant de revenir à Milan et Vérone, puis en un bouquet final à « Chartres, Reims, Rouen, Amiens, Lincoln, Peterborough, Welles ou Lichfield $» .^{35}$ Et l'on se rend compte qu'au fond tout cet esprit comparatiste, qui prend ses aises dans son terrain de jeu de l'Europe, était déjà à l'œuvre dans La poésie de l'architecture, que le jeune Ruskin publia en 1838 (cinq ans après sa première escale à Calais) avec ce sous-titre : "L'architecture des nations d'Europe dans sa relation avec le paysage et le caractère national ». Et qu'il restera vivace jusque dans les œuvres les plus tardives, comme La Bible d'Amiens, où le vieux Ruskin retrouvera en des pages, superbes, qui marqueront profondément son traducteur, le jeune Marcel Proust, Venise dans Amiens !

Au-delà, Ruskin pense même une Europe du climat :

Les grandes vallées du nord des Alpes - celle du Rhin (les Grisons), de la Reuss (Canton d'Uri), du Rhône (canton du Valais), et de l'Arve (le Faucigny), - sont toutes balayées à l'heure de midi par un vent violent qui les pénètre ; une tempête quotidienne qui soulève la poussière dans le souffle de ses tourbillons, et interdit toute croissance harmonieuse aux arbres. Je n'ai été récemment ni dans la Val d’Aoste, ni dans la Valtellina, mais ni dans la Val Anzasca, la Val Formazza ou la Val d'Isella, ni dans le sud de la vallée du Saint
Gothard, il n'y a trace de l'action d'un vent mauvais comme ce vent du nord, que je suppose être, dans son essence, la forme d'été de la bise. ${ }^{36}$

Enfin, comble et summum de ce génie comparatiste de Ruskin, qui transcende même les frontières qui séparent le monde de la nature de celui de la culture : le sentiment, ou la sensation, au moment où il commence à travailler aux Stones of Venice, que « le Campanile de San Marco est en quelque sorte l'aiguille du Dru de Venise », comme il l'écrit à son père le 10 novembre $1851 .{ }^{37}$

De tout cela, qui pourrait encore être longuement illustré, je dégagerai, pour finir, quatre conclusions.

1. Du regard sur l'Europe, tout au long de l'Old Road et de ses variantes, Ruskin est bientôt passé à une conscience de l'Europe, ou une conscience européenne, et à l'Histoire qui va avec. C'est ce que disent sans équivoque les incipit des principaux paragraphes de « Mountain Glory » que je viens de citer : "Les formes d'imagination sacrée qui se manifestèrent chez les habitants de l'Europe... »; "Si je jette un large regard sur l'art de l'Europe au Moyen-Âge... »; «Un des phénomènes les plus singuliers de l'histoire de l'Europe... ». ${ }^{38}$ Autant d'exemples de la capacité de Ruskin à penser et à écrire le paysage naturel, artistique et social comme une totalité européenne, dans ses contrastes et dans ses similitudes, à quoi l'on pourrait parfaitement appliquer la devise actuelle de l’Union Européenne (UE) : In varietate concordia (unie dans la diversité). 
2. Ruskin est bien plus que l'écrivain victorien, qu'il est aussi évidemment, mais à quoi les spécialistes n'ont que trop tendance à le réduire. Il est clairement et pleinement un écrivain européen, qui demande à être lu dans un esprit proche de celui défendu par Milan Kundera dans Le Rideau. Citant Goethe qui en appelait à une « Weltliteratur», au motif que "la littérature nationale ne représente plus grand-chose aujourd'hui », Kundera y écrivait : " Il y a deux contextes élémentaires dans lesquels on peut situer une œuvre d'art : ou bien l'histoire de sa nation (appelons-le le petit contexte), ou bien l'histoire supranationale de son art (appelons-le le grand contexte) ».39 Cette route qui commence à Calais, où les Ruskin débarquèrent en 1833 pour leur premier tour sur le continent, et qui se termine à Venise en 1888, où Ruskin écrivit les derniers mots de son journal ; cette route qui transcende les frontières (en les ignorant le plus souvent), et sur laquelle une part essentielle de sa vie, de sa pensée, de son œuvre s'est déployée, inscrit bien Ruskin avec force dans le grand contexte européen, et fait de son œuvre un moment important dans le mouvement de constitution de la culture ou de l'esprit européens. L'écrivain qu'il est possède d'ailleurs une véritable et impressionnante culture littéraire européenne : à côté bien sûr de tous les 'classiques' britanniques, de Shakespeare à Keats et Carlyle en passant par Wordsworth, Byron, et Walter Scott, si cher à son père, il connaît et a lu nombre d'écrivains européens, que l'on ne cesse de croiser, souvent très fugitivement, en le lisant. Si Homère, Virgile, Dante et Rousseau ont droit à des développements plus ou moins conséquents dans tel ou tel livre, lettre, ou page des Diaries, quelques allusions, sous la forme parfois d'un simple mot, voire d'une phrase, nous laissent deviner qu'Érasme, Cervantes, Goethe, Schiller, les frères Grimm, et pour l'espace littéraire français Montaigne, Bossuet (« je prêchais aussi bien que Bossuet », se flatte-t-il dans Praeterita !), Voltaire et Diderot, Balzac, Hugo (dont il critique vertement Notre-Dame de Paris !), Lamartine, Musset (dont il cite Rolla dans Fiction, Fair and Foul), Stendhal et George Sand (dont il discute avec sa mère) sont très loin d'être pour lui des inconnus.

3. Enfin, et ceci nous ramène à l'année 2019, après avoir à Calais tourné le dos à l'Angleterre, Ruskin la replace vigoureusement dans son Europe. Le jeu même de ses phrases où le Westmoreland voisine avec la Lombardie, comme la cathédrale de Westminster avec celle de Reims, abolit en quelque sorte l'insularité britannique et, si j'ose dire en ces temps de Brexit, la réamarre à l'Europe. Et pour illustrer ce retour de l'Angleterre dans une Europe du paysage, et par-delà dans cette 'grande communauté' qu'est l'Europe de Ruskin, il faudrait encore citer les splendides pages de Praeterita, où il compare le paysage du Jura à celui des landes du Yorkshire qui, dit-il, " sont leur équivalent anglais ${ }^{40}$

4. Enfin et pour rester dans notre actualité : si Calais fut pour Ruskin le lieu où troquer son être britannique contre un être européen, en même temps que le lieu où tout commence, nous savons ce qu'est aujourd'hui Calais : le lieu, le cul- 
de-sac où tout se termine (où finit le rêve, et trop souvent la vie) pour des centaines, voire des milliers d'êtres humains que l'on appelle des « migrants » qui ont traversé l'Europe, et même le monde, avec l'Angleterre comme unique objec- tif. Troublant renversement. Face auquel on ne peut que s'interroger sur ce que nous en dirait, s'il était encore là, le Ruskin qui à partir de 1860 se construit une pensée économique et sociale, bref l'auteur de Unto this Last...

\section{Bibliographie de John Ruskin}

Les références aux œuvres de Ruskin renvoient au volume et à la page de:

Cook, E.T.; Wedderburn, A. (eds) (1903-1912). The Works of John Ruskin. Library Edition, 39 vols. London: George Allen.

https://www.lancaster.ac.uk/the-ruskin/the-complete-works-of-ruskin/.

Works, 1: Early Prose Writings. | 2: Poems. | 6: Modern Painters VI. | 10: The Stones of Venice II. | 12: Lectures on Architecture and Painting (Edinburgh, 1853), Other Papers (1844-1854). I 16: "A Joy For Ever", The Two Paths, Letters on The Oxford Museum and Various Addresses (1856-1860). | 17: Unto this Last, Munera Pulveris, Time and Tide, Other Writings on Political Economy (1860-1873). |34: The Storm-cloud of the Nineteenth Century, On the Old Road, Arrows of the Chace, Ruskiniana. | 35: Praeterita, Dilecta.

\section{Bibliographie générale}

Alison, A. (1833-1843). History of Europe from the Commencement of the French Revolution in 1789 to the Restoration of the Bourbons in 1815. 12 vols. Edinburgh: Blackwood.

Alison, A. (1852-1859). History of Europe from the Fall of Napoleon in 1815 to the Accession of Louis Napoleon in 1852. 8 vols. Edinburgh: Blackwood.

Bakhtine, M. (1987). Esthétique et théorie du roman. Paris: Gallimard.

Barthes, R. (1979). Sollers écrivain. Paris: Seuil.

Bayard, P. (2009). Le Plagiat par anticipation. Paris: Minuit.

Collingwood, W.G. (1903). Ruskin's Relics. London: Isbister and Co.

Cook, E.T. (1911). The Life of Ruskin. London: Allen.

Evans, J.; Whitehouse, J.H. (eds) (1959). The Diaries of John Ruskin. 3 vols. Oxford: Clarendon Press.

Kundera, M. (2005). Le Rideau. Paris: Gallimard.

Montaigne, M. de (1962). Essais. Paris: Gallimard.

Ossola, C. (2018). Fables d'identité. Pour retrouver l'Europe. Trad. par P. Musitelli. Paris: PUF. Trad. de: Europa ritrovata. Milano: Vita e Pensiero, 2017.

Ozouf, M. (2009). Composition française. Paris: Gallimard.

Proust, M. (1971). « Notes sur la littérature et la critique ». Contre Sainte-Beuve. Paris: Gallimard.

Stephen, L. (1871). The Playground of Europe. London: Longmans, Green \& Co. Trad. fr: Le terrain de jeu de l'Europe. Trad. par C.-É. Engel. Paris, Neuchâtel: Éditions Victor Attinger, 1934. 


\title{
Division, Juncture, System: Bridges
} and Bridge-Building in the Work of John Ruskin

Paul Tucker

Università degli Studi di Firenze, Italia

\begin{abstract}
This chapter starts by considering a recent design for a bridge named after Ruskin, whose inadequacy it attributes to disregard for Ruskin's definition of a bridge's basic function as that of safe passage over a river and misapplication of his concept of 'abstract lines' as ornament. For Ruskin, curvature determined by that function allowed bridges materially to trace such lines and so epitomize general laws governing landscape composition, natural morphology and human co-existence. Taking it as a figure of connection, threaded as a clue through his work, the chapter explores Ruskin's sense of the significance and sacredness of bridges, manifested especially in the role he assigned to an ideal Pontifex, guarantor of secure passage across national, cultural and cognitive divides.
\end{abstract}

Keywords Ruskin. Europe. Turner. Spuybroek. Bridges. Architecture. Ornament. Composition.

Summary 1 A Bridge for Ruskin? - 2 "A Bridge as Place"? - 3 A Bridge as Ornament? - 4 Bridge Passage and the 'Virtues of Architecture'. - 5 Bridge Action and Bridge Aspect. - 6 Bridges and the Laws of Design. - 7 Iron Bridges. - 8 Bridges of 'Bygone Days'. - 9 Bridges and 'The System of the World'.

The system of the world is entirely one; small things and great are alike part of one mighty whole.

(John Ruskin, Modern Painters V)

\section{$1 \quad$ A Bridge for Ruskin?}

Around fifteen years ago the Rotterdam-based architectural design office, NOX, founded and until 2010 headed by the Dutch architect Lars Spuybroek, ${ }^{1}$ won an invited competition for the design of a footbridge over the Wurm/Worm not far from Aachen, where since 1815 the river has marked the border between Holland and Germany. The competition

1 He is now Professor of Architecture at the Georgia Institute of Technology in Atlanta. 
was instigated jointly by the municipalities of Kerkrade and Herzogenrath, situated either side of the river but historically a single geographical and administrative entity within a small, semi-independent territory, the Land van Rode (or van s'-Hertogenrode). The project may no doubt be linked to the towns' active self-promotion as the binational city of Eurode. ${ }^{2}$ The bridge was apparently to have been constructed by the German engineering firm Bollinger+Grohmann. A project description on their website states that it was to have replaced a bridge built on piles, and that these would have been reused, presumably by integration into the cantilevered support of the projected $14 \mathrm{~m}$ wide circular steel floor. The floor was to have been fitted with a double curved balustrade and decorated with a pattern in mosaic comprising foliate forms in crimson and green on a pink ground. ${ }^{3}$ The bridge, however, was never actually built. ${ }^{4}$

In recent publications Spuybroek has gone about "revitalizing" ${ }^{5}$ Ruskin's conception of Gothic architecture and ornament in terms of the contemporary meth- odology and ethos of digital design, of which NOX was a pioneer. His Ruskin is a prophet of "a Gothic ontology", i.e.

a special relationship between figures and configurations, in which the figures are active parts that have a certain freedom to act, though only in relation to others and in order to form collaborative entities. This concept transcends the aesthetic opposition of structure and ornament, making the Gothic "a beauty that works," one that leads to a much broader notion of an aesthetics based on sympathy. Sympathy, in my briefest definition, is what things feel when they shape each other. ${ }^{6}$

It is not in itself surprising, then, that in his design for the Wurm/Worm bridge Spuybroek should have "used Ruskin" and even named the project after him. ${ }^{7}$ Yet neither the design itself nor the various brief texts presenting it effectively justify him in doing so.
Probably the earliest of those texts is found in the catalogue to an exhibition organized by the Zezeze Architecture Gallery in Tel Aviv in 2006. This aimed to trace "an intellectual journey starting from the writings of Ruskin, Hogarth and Worringer, through Spuybroek's inner workings, to the production of new realms of space,

2 Ehlers 2001.

3 See the project description on the website of B+G Ingenieure and Bollinger und Grohmann GMBH: https://www.bollinger-grohmann.com/ en. projects.ruskins-bridge.

4 Lars Spuybroek, personal communication.

5 Spuybroek [2011] 2016, xvi.

6 Spuybroek [2011] 2016, xvii

7 Spuybroek 2008, 259. It is called "the Ruskin Bridge" in Spuybroek 2008, "Ruskin Bridge" on http://www.nox-art-architecture.com and "Ruskins Bridge" on https://www.bollinger-grohmann.com. 


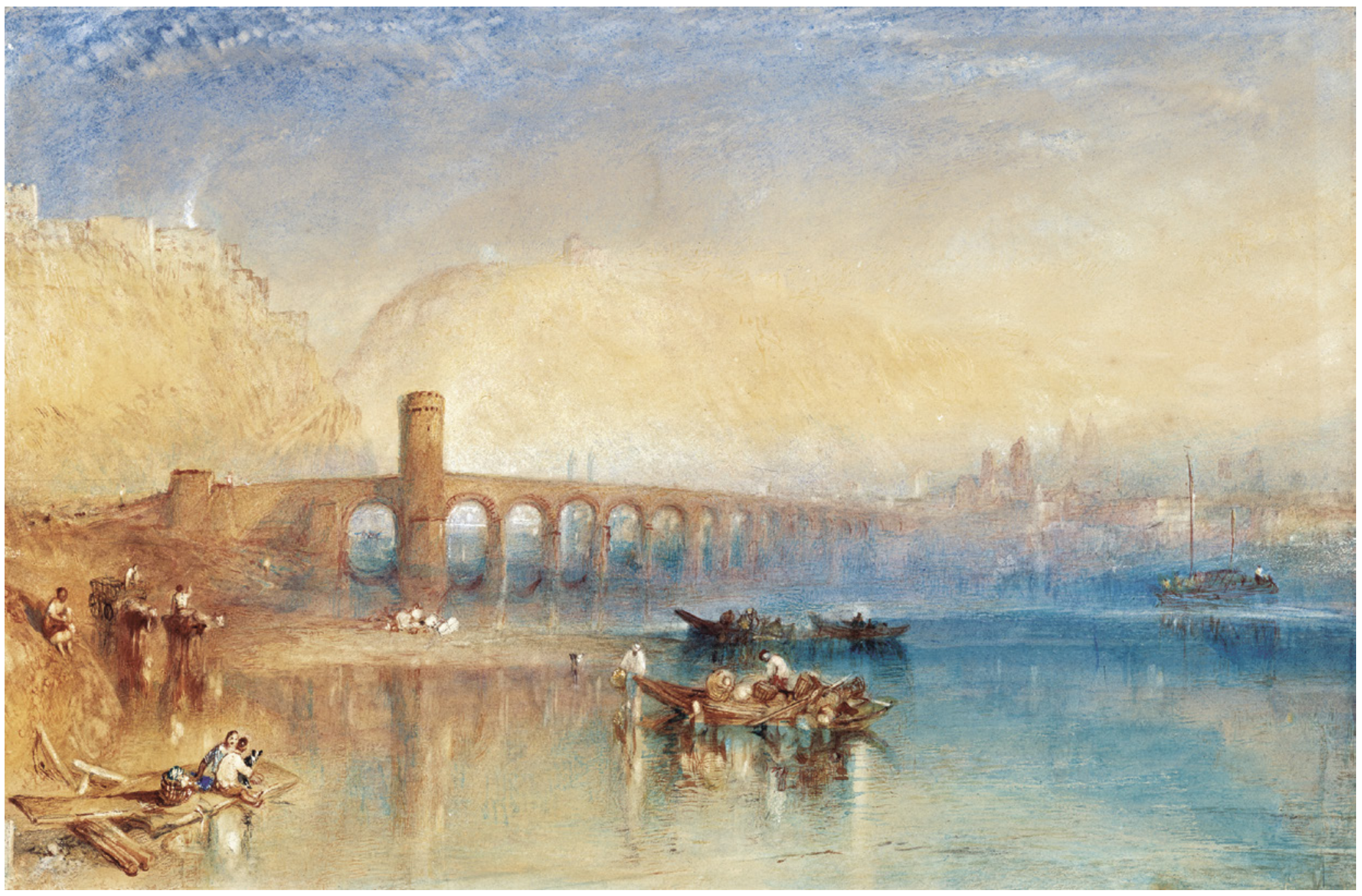

Figure 1 Joseph Mallord William Turner, Coblenz. 1842. Watercolour, $286 \times 445$ mm. Ohio, Cincinnati Art Museum (CIN6198923). Bequest of Mary Hanna. @ Cincinnati Art Museum / Bridgeman Images 
sound and movement". ${ }^{8}$ The catalogue contains a brief introduction by the exhibition's curator, Heidi Arad; selected passages by the three writers cited; extracts from an interview with Spuybroek by Ludovica Tramontin, later published in Spuybroek 2008, and annotated renders and photographs of various NOX projects, apparently provided by the architect(s).

Inarticulately and inexplicably, the note accompanying the renders of Ruskin Bridge ${ }^{9}$ quotes from The Seven Lamps of Architecture ${ }^{10}$ in support of the bridge's conceptualization as one vast ornament. The elusive chain of reasoning stems (so to speak) from the initial remark that the bridge's intended site - the Wormwildnis nature reserve - is "more like a room than a park". "Here", it is asserted,

we need a bridge that creates a middle, not a passage. A bridge that points out the center of the environment, like a chandelier in the middle of the room. So, NOX designed the first circular bridge in the world. A line as a circle, a bridge as a place. But a round bridge is not enough, that only radiates outwards, now the environment needs to relate back inward to the center. Accordingly the circle is made into a 14-meter ornament, following John Ruskin's outcry in The Seven Lamps of Architecture: "... architecture is not to imitate directly the natural arrangement, she is to place her most exuberant vegetable ornament just where Nature would have placed it". So, NOX also designed the largest ornament in the world. Here people can now sit or stand and enjoy a secret little place that has now become unique. ${ }^{11}$
The reference is to the fourth chapter of Seven Lamps, devoted to "The Lamp of Beauty" and concerned to examine the ways in which the "element of delight" resulting from "impressions of beauty" is "best engrafted upon architectural design". Moving from the assumption that "all most lovely forms and thoughts are directly taken from natural objects" and reasoning from visible "Frequency to Beauty", Ruskin poses the question of "what is or is not ornament", and in the process dismisses several traditional "forms of so called decoration in architecture" as "ugly things". These include the Greek fret, heraldic ornament, ${ }^{12}$ scrolls, ribands and "garlands and festoons of flowers". The passage (mis)quoted by Spuybroek comes from a discussion of these last and should perhaps be restored to its immediate original context (quoted elements in italics):

Closely connected with the abuse of scrolls and bands, is that of garlands and festoons of flowers as an architectural decoration, for unnatural arrangements are just as ugly as unnatural forms; and architecture, in borrowing the objects of Nature, is bound to place them, as far as may be in her power, in such associations as may befit and express their origin. She is not to imitate directly the natural arrangement; she is not to carve irregular stems of ivy up her columns to account for the leaves at the top, but she is nevertheless to place her most exuberant vegetable ornament just where Nature would have placed it, and to give some indication of that radical and connected structure which Nature would have given it. Thus the Corinth-

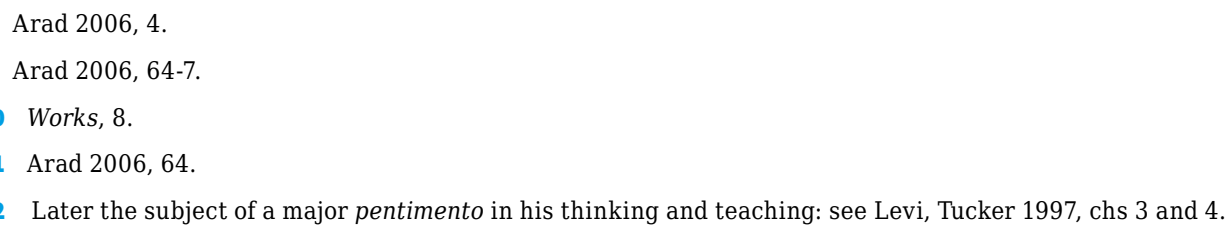


ian capital is beautiful, because it expands under the abacus just as Nature would have expanded it; and because it looks as if the leaves had one root, though that root is unseen. And the flamboyant leaf mouldings are beautiful, because they nestle and run up the hollows, and fill the angles, and clasp the shafts which natural leaves would have delighted to fill and to clasp. They are no mere cast of natural leaves: they are counted, orderly, and architectural: but they are naturally, and therefore beautifully, placed..$^{13}$

It is hard to see the relevance of the passage to the renders of Ruskin Bridge. ${ }^{14}$ Certainly, as there realized the mirrored symmetry of the bridge's foliate decoration does not "imitate directly the natural arrangement". At the same time, however, it is little more than pictographic in form and confectionary in colour, and not in the least "exuberant" in Ruskin's vitalist understanding of the term: it lacks all indication of "radical and connected structure". (It is interesting to compare it, in this regard, with the laurel in the background of Veronese's Susannah and the Elders in the Louvre which Ruskin instances and reproduces in "The Leaf Monuments" chapter in Modern Painters V and of whose "every line and leaf" he writes, "None are confused, yet none are loose; all are individual, yet none separate"). ${ }^{15}$ Last but not least, the bridge-floor can hardly correspond to the place Nature would have chosen for the living sprays supposedly evoked.

Arad 2006 fails to establish any precise connection between the design for the bridge, its somewhat garbled explication in the body of the catalogue and the appended discussion of "abstract lines" from the chapter on "The Material of Ornament" in the first volume of The Stones of Venice. ${ }^{16}$ Two excerpts from Ruskin's text are printed alongside edited images of the relevant pages from Stones, with the corresponding passages marked by underlining. The intention is evidently to highlight Ruskin's characterization of the abstract contours of natural objects - "transferred to architectural forms when it is not right or possible to render such forms distinctly imitative"17 - as universally manifesting "ever-varying curvature in the most subtle and subdued transitions" and for the most part expressing "action or force of some kind", as distinct from the circle, defined as "a line of limitation or support", and more specifically from "circular curves", which are said to be "curves of perfect rest". ${ }^{18}$

\footnotetext{
13 Works, 8: 151 (emphases added).

14 Arad 2006, 64-7.

15 Works, 7: 89-90, pl. 57.

6 Arad 2006, 72-3; Works, 9: 266-8.

7 Works, 9: 266

Works, 9: 268, 269.
} 


\section{$3 \quad$ A Bridge as Ornament?}

Indirect arguments for the assumed relevance of this characterization may be gleaned from texts by NOX/ Spuybroek published elsewhere: the project description on NOX's own website ${ }^{19}$ and the two pages devoted to the bridge in Spuybroek 2008. ${ }^{20}$ In its first half the former reiterates the substance of the note in Arad 2006, minus the quotation from Seven Lamps. After again claiming for Ruskin Bridge pre-eminence as "the first circular bridge in the world", the text continues,

John Ruskin distinguished between two types of lines, the line of limitation and the line of force. The line of limitation is the circle, the perfect form of rest, the other is the line of ornament, the line of force, wind, flow and movement. In this way we merge two concepts of the line into one single object. ${ }^{21}$

Spuybroek 2008 is more expansive. We learn that the project is "all about life" and this is the reason why the bridge floor is "covered with a huge mosaic made up of foliate curves that are pointed at one end and rounded at the other, to make the swirling forces more visible and sensible, like a turbulent vortex".22 This prompts a digression on the relation in Gothic ornament between "the pointed" ("what emerges when multiple directions cannot be reconciled and this has to be solved by a double tangency") and "the rounded" ("a sort of given [...] the basis of continuity"), itself immediately followed by the assertion,
The bridge is a huge 60 -foot-wide ornament. Imagine crossing a round bridge: you want to stay in the middle, look around at everything, talk to somebody - anything but cross over to the other side. Maybe you want to walk along the vegetal curves, looking down at the mosaic floor. All movement is concentrated in that midpoint. $^{23}$

Referring now to the "Material of Ornament" chapter from The Stones of Venice, Spuybroek confusingly rehearses Ruskin's distinction there between the abstract contours of natural objects, expressive of force, and the circular curves of limitation, support and rest as one "between lines of contour and lines of force", and implicitly avers its relevance in repeated (but unfounded) indication of consequentiality:

So we have the circle - the line of limitation, according to him - surrounded by a world of forces - violently flowing water, wind in tall trees - which end up in the circle as lines of force and action. So the powers of variation are operating at the same time as the powers of limitation. ${ }^{24}$

The concluding discussion is the most obscure and (from a Ruskinian perspective) questionable portion of the passage:

9 http://www.nox-art-architecture.com

20 Spuybroek 2008, 259-60.

21 Project description on NOX's own website (http://www.nox-art-architecture.com).

22 Spuybroek 2008, 259.

23 Spuybroek 2008, 259-60.

24 Spuybroek 2008, 260; emphases added. 
You might wonder why I'm suddenly considering a flat, mosaic ornament as a structural system, since my argument has been persistently structural. Ornament, as Ruskin says, always behaves materially, so there's no difference between, say, the wrought-iron curves that intertwine and connect to make a structural surface and the same configuration on wallpaper. As long as it configures structurally. But what it does need to do is to relate to a second materiality, that of the built system it needs to fit into. In this case, it's the system of arabesques that creates the round configuration of the circle of the bridge itself. If there were no relation between the figures and the bridge, it wouldn't work. So there's always a first-order and a second-order materiality, like with Frei Otto's analog machines, like with Semper's four elements. A materiality that informs materials. ${ }^{25}$

It is far from clear what it might mean for ornament to behave "materially". If that it acts as a structural principle, where does Ruskin say as much? Spuybroek seems rather to be invoking a conviction of his own, namely that "in the Gothic, ornament acts like structure and structure acts like ornament". ${ }^{26}$ If, on the other hand, the phrase means that realized ornament is conditioned by the nature of the materials used, this would seem to tally with Ruskin's thinking but at the same time to contradict the declared lack of difference between wrought-iron and wallpaper realizations of a particular configuration. Is Spuybroek alluding to the formal and qualitative universality of orna- ment's (in Ruskin's enumeration) primary "material”, i.e. abstract lines? Whatever the answer, it is hard to reconcile Spuybroek's assertion that in the design for the bridge it is the "system of arabesques" (a revealing choice of term) that "creates the round configuration of the bridge itself" with the impression unavoidably communicated by the renders of stencilled pattern mechanically applied to and inertly occupying an obdurately pre-existent and inassociably coloured shape and surface.

On Spuybroek's own terms, then, the bridge does not "work". Nor indeed does it on Ruskin's - and not only on those Ruskinian terms misguidedly invoked by the architect. To stay for the moment with the ornament, this is both unnaturally and "wrongly placed", in the viewer-centred architectural sense explicated in the Lectures on Architecture and Painting given at Edinburgh in 1853. ${ }^{27}$ Here the sculpted lions' heads presumed to adorn the city's Royal Institution were ridiculed not only as unmeaning (and senselessly replicated) parodies of natural form but as carved to a degree of finish inappropriate to their position "at the very top of it, just under its gutter". ${ }^{28}$ By contrast, as demonstrated by examples from Lyons and Amiens, Gothic builders reserved "their best and most delicate work" for the "foundation of the building, close to the spectator", ${ }^{29}$ while carved ornament intended by them to be seen from far below conveyed the impression of similar delicacy but was carved massively, broadly and even rudely. ${ }^{30}$

Now, the principle that actually delicate work should be placed close to the spectator implies its converse, that

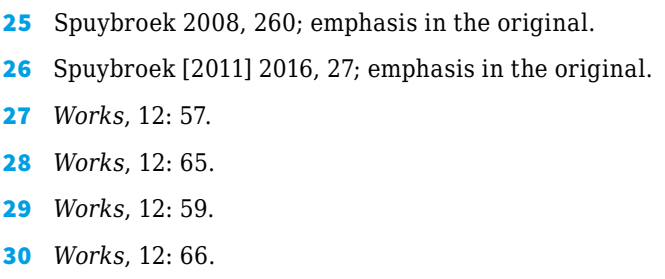


ornament placed close to the spectator should actually be delicate and not just give the impression of delicacy when seen from a distance. In the case in point, Spuybroek's overblown arabesques are not suited to their place on the bridge, if we imagine this as an object of fully situated rather than computer-mediated visual experience. The viewer would indeed be obliged to levitate to some height actually to enjoy the integral perception of "figures and bridge" simulated by some of the renders, whereas in ordinary earth-bound perception the former would appear dilated beyond capacity to comprehend and convey the vital visual link with the natural environment which the architect claims for them.

So this ornament fails to pass the test prescribed by Ruskin in the chapter from the Stones of Venice succeeding that cited by Spuybroek and which is dedicated to "The Treatment of Ornament":

The especial condition of true ornament is, that it be beautiful in its place, and nowhere else, and that it aid the effect of every portion of the building over which it has influence; that it does not, by its richness, make other parts bald, or by its delicacy, make other parts coarse. Every one of its qualities has reference to its place and use: and it is fitted for its service by what would be faults and deficiencies if it had no especial duty. ${ }^{31}$

And the criterial reference here to "use" suggests further ways in which Ruskin Bridge is unworthy of its name. For what use does or can such ornament, so realized and placed, have on a bridge? The question of course implies consideration not only of the appropriate nature and place of ornament in relation to such a structure, but, most crucially, of the use of a bridge as such. Ruskin Bridge explicitly denies its function as a bridge. Not by chance is its circular form (as its architect boasts) unprecedented, being consequent on the determination it should represent, not a means of passage but rather a "place" of rest and pause, distracting the viewer-traveller from the need or wish to "cross over to the other side". And yet, as Ruskin reminds readers of The Stones of Venice, getting "safely over the river" 32 is the minimal requirement that may be made of the bridge-builder and thereby also the very occasion and test of constructive intelligence in this elementary mode of architectural work.

\section{Bridge Passage and the "Virtues of Architecture"}

The second chapter of its first volume, dedicated to "The Virtues of Architecture", serves to rationalize and justify the structure of The Stones of Venice as a whole. It isolates and explicates two qualities of buildings which according to Ruskin are "proper subjects of law" and may (after due instruction and practice) be discerned and judged of "by a glance of the eye": their constructive "strength" and their "beauty". ${ }^{33}$ The arched bridge is instanced as an elementary example of "good construction"34 - the simplest and most economical fulfilment of function or purpose - and 


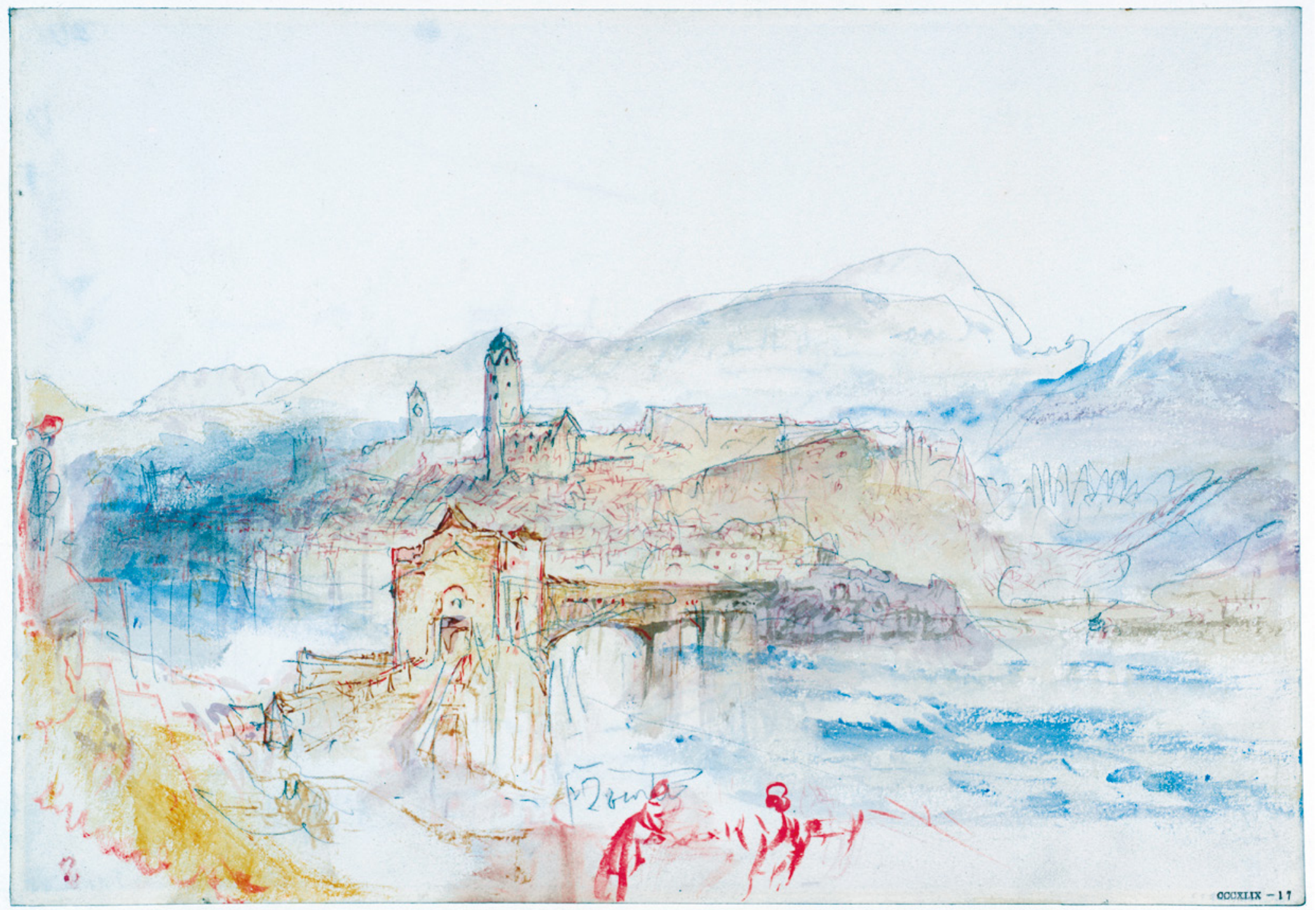

Figure 2 Joseph Mallord William Turner, Rheinfelden from the North. 1844. Graphite, watercolour and pen on paper, $229 \times 330 \mathrm{~mm}$ (support). From the Rheinfelden Sketchbook. Accepted by the nation as part of the Turner Bequest 1856. London, Tate Britain. Photo @ Tate 
Figure 3 John Henry Le Keux after John Ruskin, The Bridge of Rheinfelden. 1860. Etching. Published as PI. 83 in Modern Painters V (1860). Image scanned from 1888 edition. (C) Paul Tucker

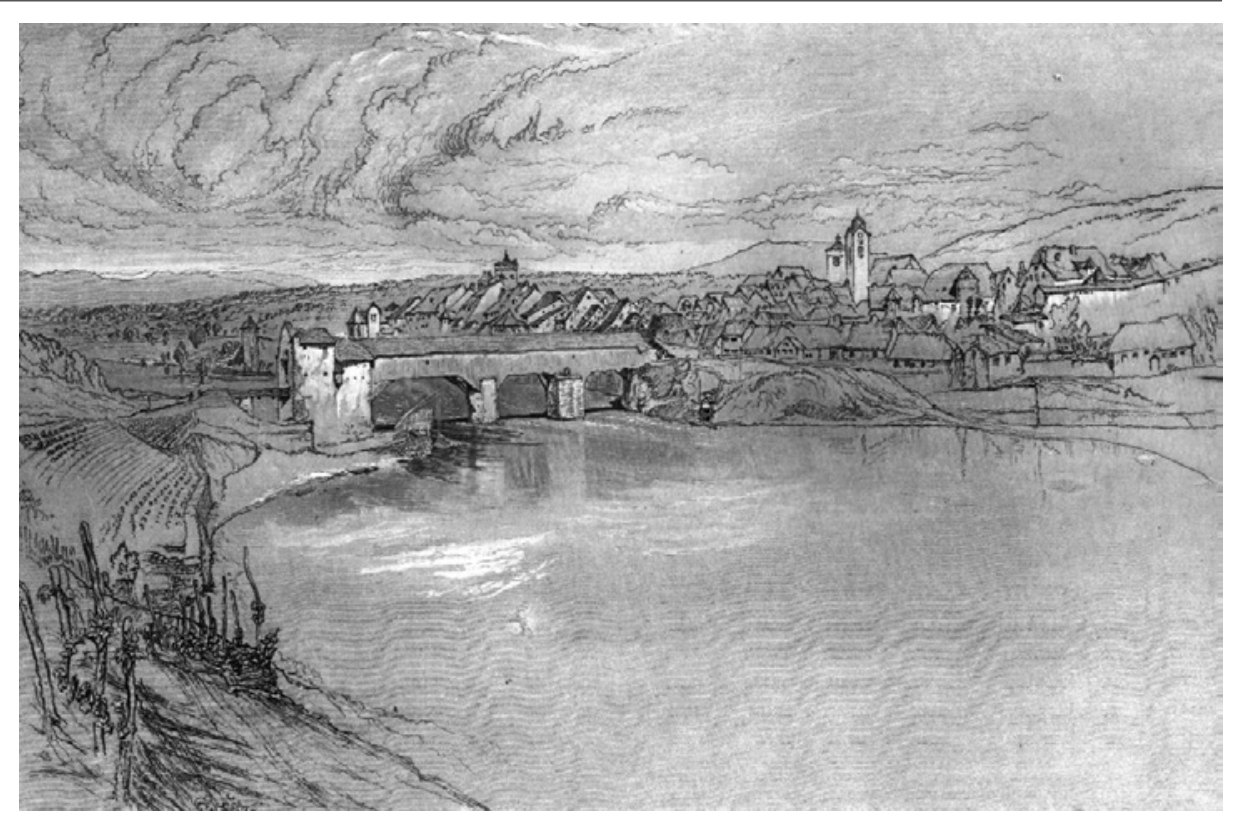

of the pleasure this affords "as the manifestation of an admirable human intelligence", ${ }^{35}$ even on the part of a "mere bridge-builder", not yet "an architect" (though in principle at least on the way to becoming one). ${ }^{36}$

For, "[s]uppose", Ruskin prompts, "we are present at the building of a bridge". What most deserves admiration is nothing very evident in the work underway, but rather the prior "choice of the curve" to be traced by the arch "and the shaping of the numbered stones, and the appointment of that number". And this for the reason "there were many things to be known and thought upon before these were decided":
The man who chose the curve and numbered the stones, had to know the times and tides of the river, and the strength of its floods, and the height and flow of them, and the soil of the banks, and the endurance of it, and the weight of the stones he had to build with, and the kind of traffic that day by day would be carried on over his bridge, - all this especially, and all the great general laws of force and weight, and their working; and in the choice of the curve and numbering of stones are expressed not only his knowledge of these, but such ingenuity and firmness as he had, in applying special means to overcome the 


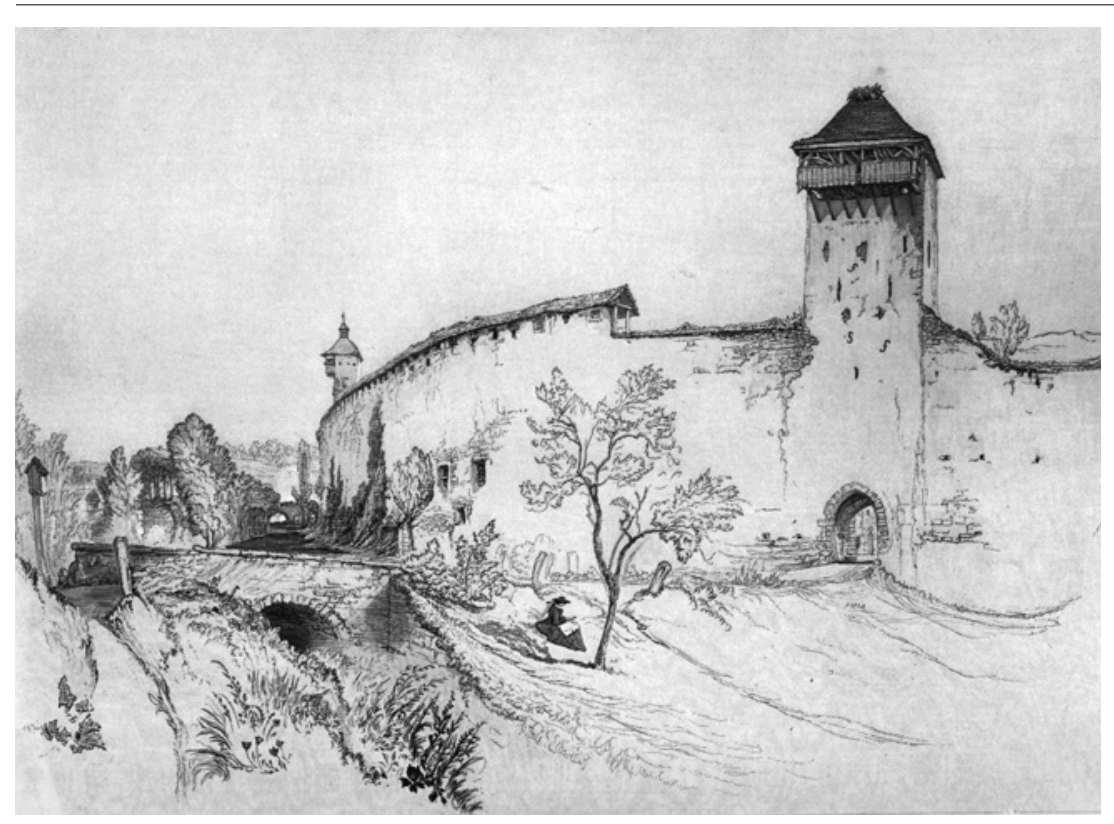

Figure 4 John Henry Le Keux after John Ruskin, Peace [the walls of Rheinfelden]. 1860. Etching. Published as Pl. 84 in Modern Painters V (1860). Image scanned from 1888 edition. (c) Paul Tucker

special difficulties about his bridge. There is no saying how much wit, how much depth of thought, how much fancy, presence of mind, courage, and fixed resolution there may have gone to the placing of a single stone of it. ${ }^{37}$

The arched bridge thus exemplifies the first virtue of architecture and the ingenuity characteristic of all art, even where this seems most practical. It does not exemplify the second virtue, calculated, should he display it, to earn the bridge-builder still higher esteem: the virtue of the bridge's beauty or decoration, manifesting, not his ingenuity but "his affections and delights". ${ }^{38}$

Yet the reference to its curve - predicated on the assumption of arched construction - suggests that, in perfect accordance with the Ruskinian understanding of ornament, the bridge might well have been so instanced. Its chosen curve might well indeed have been found to manifest precisely one of those "abstract lines" unavailingly invoked by Spuybroek, whose frequency in nature and concomitant beauty qualify them, in Ruskin's view, as 
the "first constituents of ornament". ${ }^{39}$ And this raises the possibility of a bridge itself being an ornament in quite another sense from that intended by the Dutch architect.

Aside, however, from its specific "chosen curve", the very function of a bridge fits it to enter into a broader and no less beautiful linear configuration, one integral to its landscape setting. This is well evinced by Ruskin's comments on Turner's drawing of Rheinfelden from the North (1844) [fig. 2] in his Catalogue of the Turner Sketches in the National Gallery (1857): ${ }^{40}$

A beautiful instance of serpentine continuity in composition; beginning with the red figures, the line of it winds over the bridge, back to the left in the town, up to the right by the first wall - then away to the left down into the dark shadow of the river, and returns up to the right along the mountain range, to their utmost summit. ${ }^{41}$

"Serpentine continuity" in the composition and of course - intentionally - in the landscape it depicts and in the experiential complex this images.
In Ruskin's understanding of them the "abstract lines" traceable over or across a bridge represent wide-ranging trajectories of meaning: bridges and bridge-building constitute a clue threaded through his work, of the kind he habitually looked for between individual objects or fields of interest and study, and would alert others to. Holding fast the "great connecting clue", for instance, that "[a]ll European architecture, bad and good, old and new, is derived from Greece through Rome, and coloured and perfected from the East", would allow the reader of The Stones of Venice to "string all the types of successive architectural invention upon it like so many beads". ${ }^{42}$

The architect of a bridge worthy of his name would not have neglected what Ruskin has to say or show, in writings and drawings, about the nature of bridges and their relation to rivers and landscapes, nor his understanding of the latter as expressive of inter-national histories of "moral culture" 43 - what Denis Cosgrove has called his "geographical imagination" 44 - and of the imperative need "to get safely over the river" should this, like the Wurm/Worm, represent a historically divisive

39 Works, 9: 266

40 The subject of this drawing was to be identified by Ruskin during a study-tour of Switzerland in 1858, expressly undertaken to complement his work in sorting and cataloguing the vast collection of works on paper which had been included in the so-called Turner Bequest after legal settlement of the painter's will in 1856. As Ruskin later reported in Modern Painters V (1860), "A scratched word on the back of one of them [the group of "memoranda of a bridge over the Rhine" to which the present drawing belongs], Rheinfels, which I knew could not apply to the Rheinfels near Bingen, gave me the clue to the place; - an old Swiss town [Rheinfelden], seventeen miles above Basle, celebrated in Swiss history as the main fortress defending the frontier toward the Black Forest. I went there the moment I had got Turner's sketches arranged in 1858, and drew it with the pen (or point of brush, more difficult to manage, but a better instrument) on every side on which Turner had drawn it, giving every detail with servile accuracy, so as to show the exact modifications he made as he composed his subjects" (Works, 7: 436). In the penultimate chapter of this volume, Ruskin published etchings by John Henry Le Keux after another two of Turner's memoranda and a pair of related studies by himself: one of the bridge at Rheinfelden [fig. 3] and a view of the town's walls [fig. 4], which incidentally included a small "old bridge" over their moat. Ruskin's original drawing for the former was auctioned at Christie's, London on 16 November 2006 (lot 123), while that for the latter is in the Ashmolean Museum, Oxford (WA.RS.REF.093).

41 Works, 13, 222

42 Works, 9: 34

43 Works, 17: 188

44 Cosgrove 2008, 128. 
border (at the very heart, moreover, of Ruskin's, indeed of Charlemagne's and our own fractious Europe). Such an architect would have attended to the bridge clue threaded through his work as a figure of general cohesion and coherence.

\section{$5 \quad$ Bridge Action and Bridge Aspect}

Crucially, then, the Ruskinian bridge is built and it displays in its construction the ingenuity of the builder. Indeed, in his late autobiography, Praeterita (1886-1889), Ruskin stated the view that delight at constructive cohesion in bridge-work underlay and stimulated his "early love of architecture", thanks to his inordinate fondness, as a child, of his toy bricks and in particular of an "accurately instructive" model of a two-arched bridge, "admirable in fittings of voussoir and keystone, and adjustment of the level courses of masonry with bevelled edges, into which they dovetailed, in the style of Waterloo Bridge". ${ }^{45}$

Concomitantly, as seen in the passage from The Stones of Venice quoted above, the Ruskinian bridge displays its own "strength". This however is not merely structural but 'active' in a moral sense also. Any building, like any person, as that passage explains, is expected "to act well, and do the things it was intended to do in the best way". ${ }^{46}$ In a subsequent portrait of the bridge-builder as "the village stone-mason" - occurring in a key account of Turner's 1842 watercolour of
I cannot here follow that clue in anything like the detail the topic requires, but in the remainder of this short essay will take it up at various crucial points in an attempt to sketch and elucidate Ruskin's lifelong fascination with, and general conception of bridges. the Mosel bridge at Coblen $z^{47}$ [fig. 1] in The Elements of Drawing (1857) - Ruskin traces a bridge's strength of action to its builder's capacity to understand and resolve the threat posed by the river to its safe crossing. Indeed, the type of bridge represented in Coblenz and frequently elsewhere by Turner - "with its highest and widest arch towards one side, and a train of minor arches running over the flat shore on the other ${ }^{\prime \prime 8}$ - is stated to represent "the ideal of a bridge" by virtue of its vicariously "sympathising [...] with the spirit of the river, and marking the nature of the thing it has to deal with and conquer $^{\prime 49}$ (where the mode of marking is not, as in the case of Ruskin Bridge, imitative or evocative and ornamental, but structural and contrastive). For the unequal arches answer to a universal characteristic of rivers, which

like to lean a little on one side: they cannot bear to have their channels deepest in the middle, but will always, if they can, have one bank to sun themselves upon, and another to get cool under; one shingly shore to play over, where they may be shallow, and foolish,

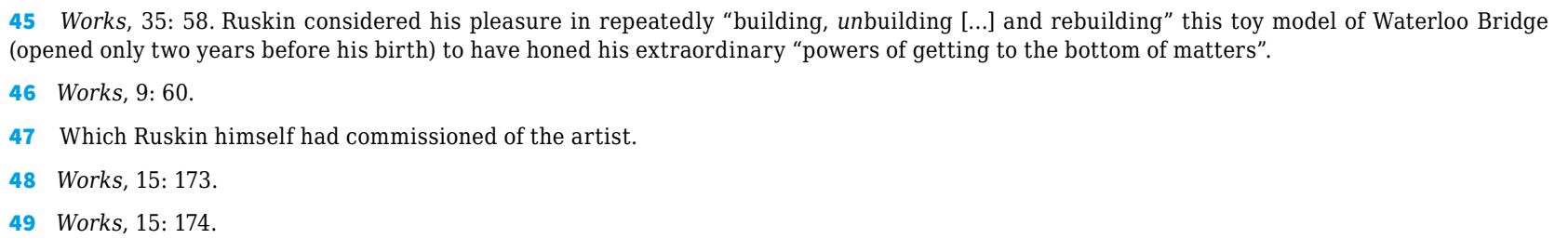


Figure 5 Jacob Philipp Hackert,

The Ponte a Mare in Pisa. 1799.

Oil on canvas, $643 \times 963 \mathrm{~mm}$.

Greifswald, Pomeranian State Museum.

(c) Wikimedia Commons

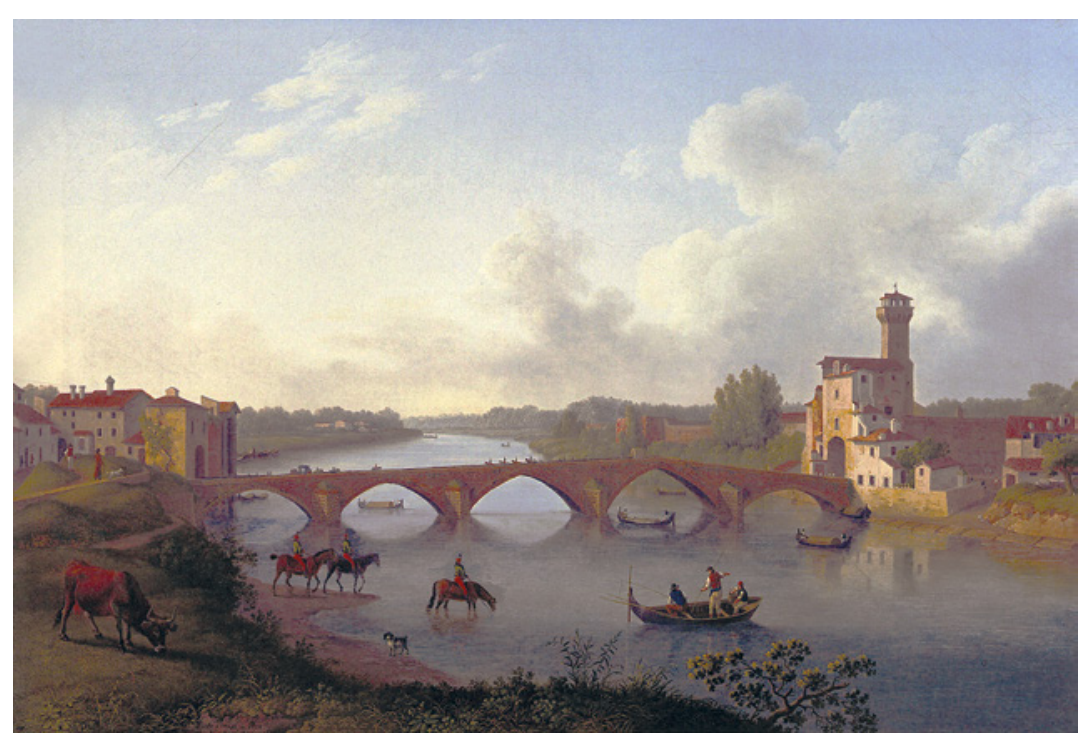

and childlike, and another steep shore, under which they can pause, and purify themselves, and get their strength of waves fully together for due occasion..$^{50}$

As a consequence, the village stone-mason typically "throws a bridge over a strong stream" by building "a great door to let the cat through, and little doors to let the kittens through":

a great arch for the great current, to give it room in flood time, and little arches for the little currents along the shallow shore. This, even without any prudential respect for the floods of the great current, he would do in simple economy of work and stone; for the smaller your arches are, the less material you want on their flanks. ${ }^{51}$

In Ruskin's thinking the bridge's active strength, its capacity for resilient endurance in strategic function, deriving from the sympathetic ingenuity of its builder, passes to its habitual users, and the bridge tends to become an index of civic endeavour and integrity and a focal point of historical destiny. And here, no doubt, is the reason for Ruskin's plan to continue the Our Fathers have Told us series, inaugurated with the publication of the first part of The Bible of Amiens in 1880, with 


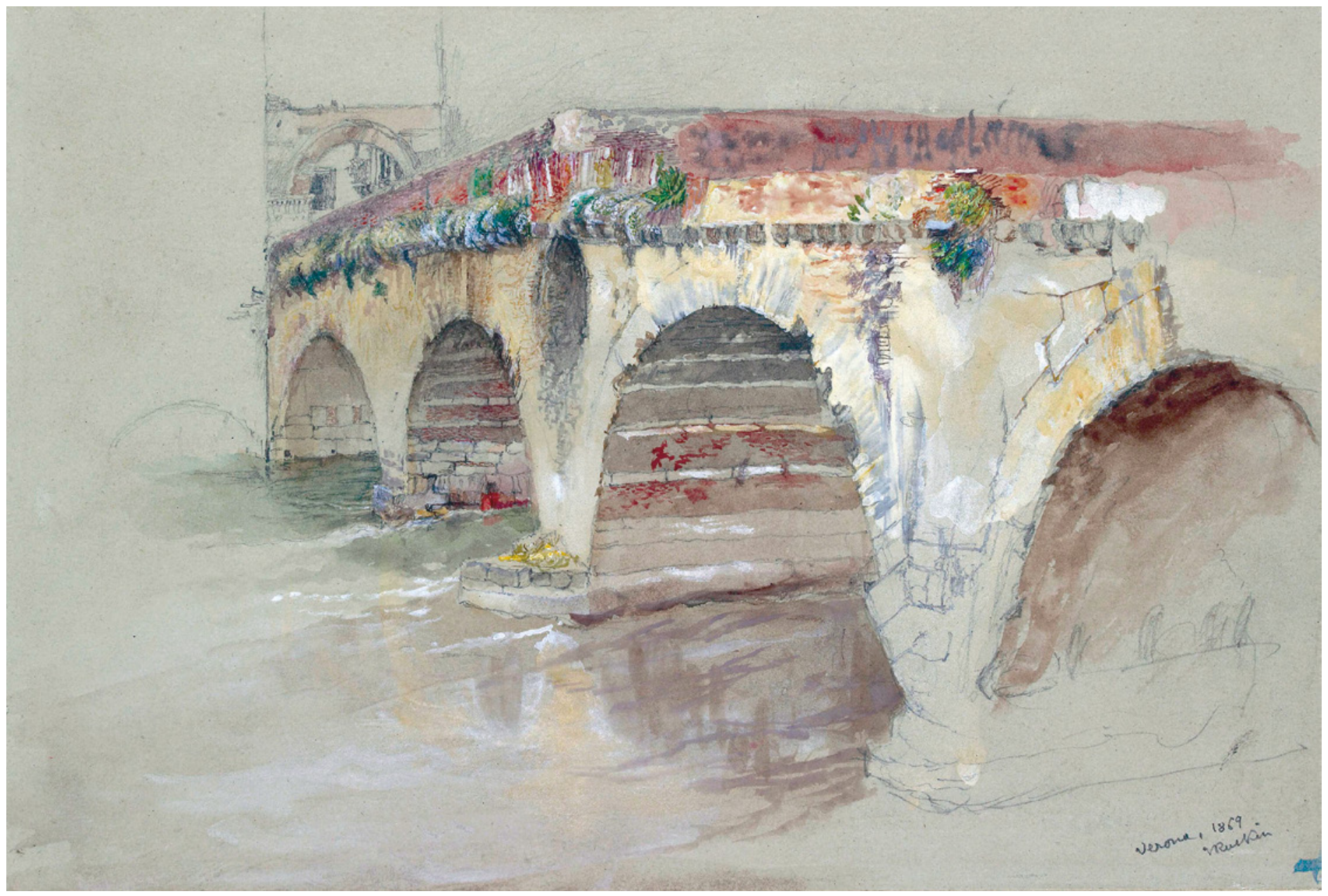

Figure 6 John Ruskin, The Ponte della Pietra, Verona. 1869. Watercolour and bodycolour over graphite on grey, wove paper, $176 \times 261 \mathrm{~mm}$. Oxford, Ashmolean Museum (WA.RS.ED.295.a). ๑ Ashmolean Museum, University of Oxford 


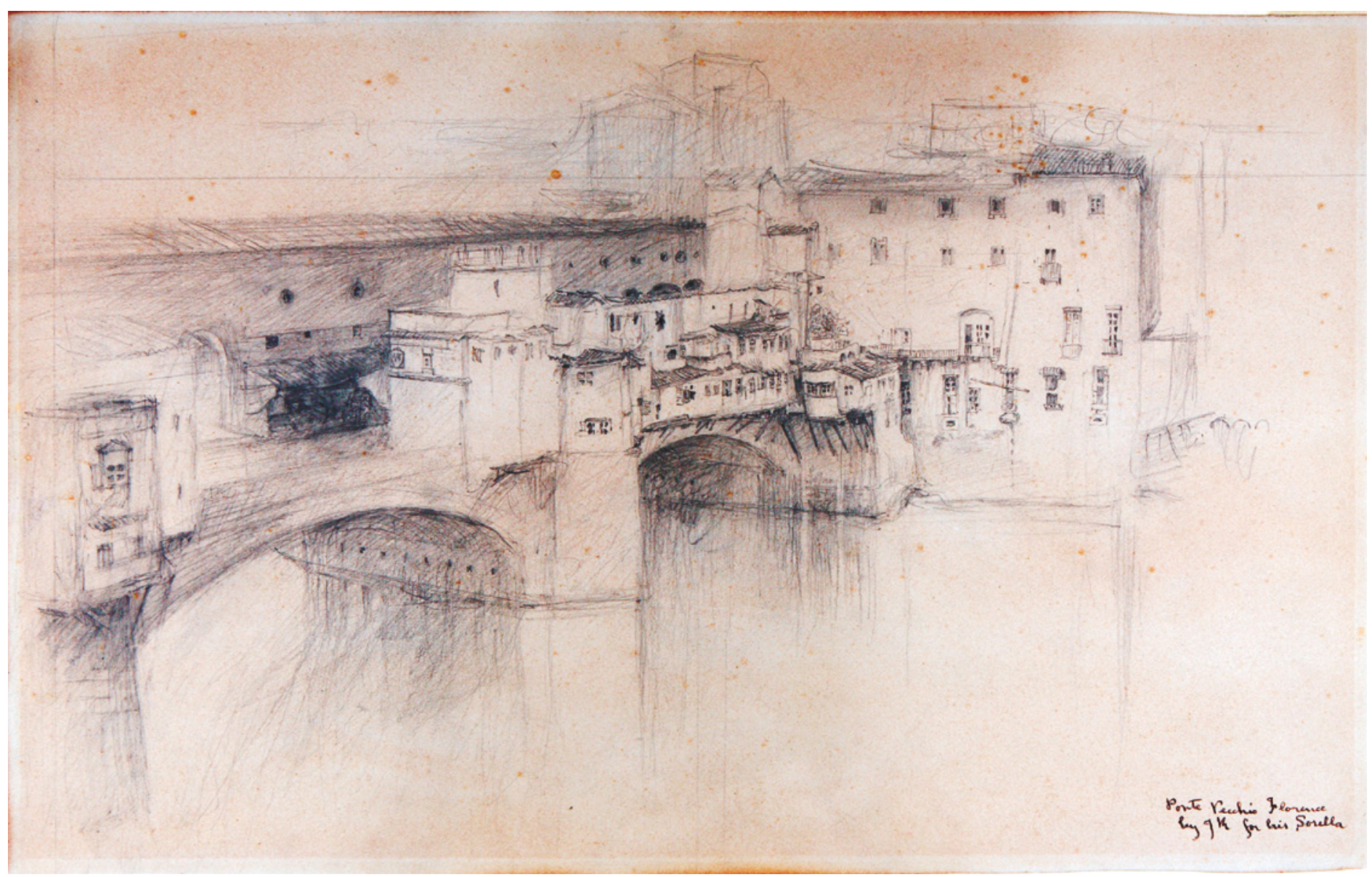

Figure 7 John Ruskin, Ponte Vecchio. 1882. Graphite on cream wove paper, $356 \times 484 \mathrm{~mm}$. Cambridge (MA) Harvard Art Museums/Fogg Museum (1957.192). Gift of Edward W. Forbes. ๑ President and Fellows of Harvard College 
volumes dedicated to, amongst others, the cities of $\mathrm{Pi}$ sa, Florence and Verona and titled with the names of the medieval bridges central or crucial to their urban configurations and natural and political histories: Ponte-a-Mare after the medieval bridge over the Arno guarding Pisa from the sea, which had collapsed in 1869 [fig. 5]; Ponte della Pietra after the Roman bridge over the Adige at Verona [fig. 6]; and Ponte Vecchio after the bridge also over the Arno but at Florence [fig. 7]. ${ }^{52}$

Here too, perhaps, is a reason for the family resemblance of many of Ruskin's late drawings of bridges, which seems to have gone little noticed, thanks perhaps to a prevailing concern to trace parallels between the development of his drawing style and his deteriorating mental state. Paul Walton, for instance, notes that after his breakdown of 1871 Ruskin's style loosened and he produced an increasing number of "excited pencil sketches" like one mistakenly described as "made at Florence in 1872" (it actually represents the Ponte pietra at Verona). ${ }^{53}$ And while recognizing that a drawing of the Ponte vecchio dating from 1882 [fig. 7] was made for the planned eponymous Our Fathers have Told us vol$u \mathrm{e}^{54}$ - to focus, like its companions, on "an architectural monument as endowed with a collective human personality" - Walton inclines to read its "quiet shadows and vaguely-sketched contours surrounding details of windows and roofs" as "private tokens of Ruskin's broken thoughts and feelings", contrasting them with the "jaunty rhythms of sharply defined forms" in a drawing of the bridge at Bremgarten in Switzerland made over two decades earlier. ${ }^{55}$ Despite the Florentine drawing's strange, soft suggestion of distance, however, it firmly renders the colossal presence of its primary object: the bridge straddling the river. And, allowing for differences in focus and emphasis, this also applies to the sketch probably made the same year and representing one end of another Florentine bridge, the Ponte Santa Trinita, which Walton adduces as evidence of the artist's "tortured sensibility" and where in his view, "instead of the voice of history, Ruskin's most desperate moods seem to be given visible form". ${ }^{56}$ Dramatic it certainly is - even alarmingly so - but equally evident is the intent, shared with the drawing of the Ponte vecchio, to exhibit massy pontal strength and reach. And the same is true of other drawings of the Ponte vecchio probably made in $1882,{ }^{57}$ as too of the "excited" 1872 sketch of the Ponte pietra cited above and other pencil and watercolour studies of that bridge made around the same time [fig. 6]. ${ }^{58}$ Moreover, though distinct in style, all recall drawings of bridges made by Ruskin in the 1860s, often in connection with

52 See Works, 33: lxv

53 Walton [1972] 1985, 110, pl. 87.

54 The drawing is probably the "pretty chiaroscuro" done on 28 October 1882 from a window of the Hotel de la Grande Bretagne on the Lungarno degli Accaiuoli, where Ruskin was staying, and which, as he wrote to his cousin Joan Severn, he thought would "make a charming plate for 'Our Fathers have told us'” (letter quoted in Ciacci et al. 2004, 191 [cat. 32]).

55 Walton [1972] 1985, 91, pl. 67; 110

56 Walton [1972] 1985, 118-19, pl. 100.

57 The Ruskin, Lancaster University, 1996 P1257, 1258 (the latter illustrated in Ciacci et al 2004, 191, cat. 32) and Christie's, London, 16 November 2006, lot 164 .

58 See also the group belonging to the City Museums, Norwich and included in Mullaly 1966, cats 63-5. 


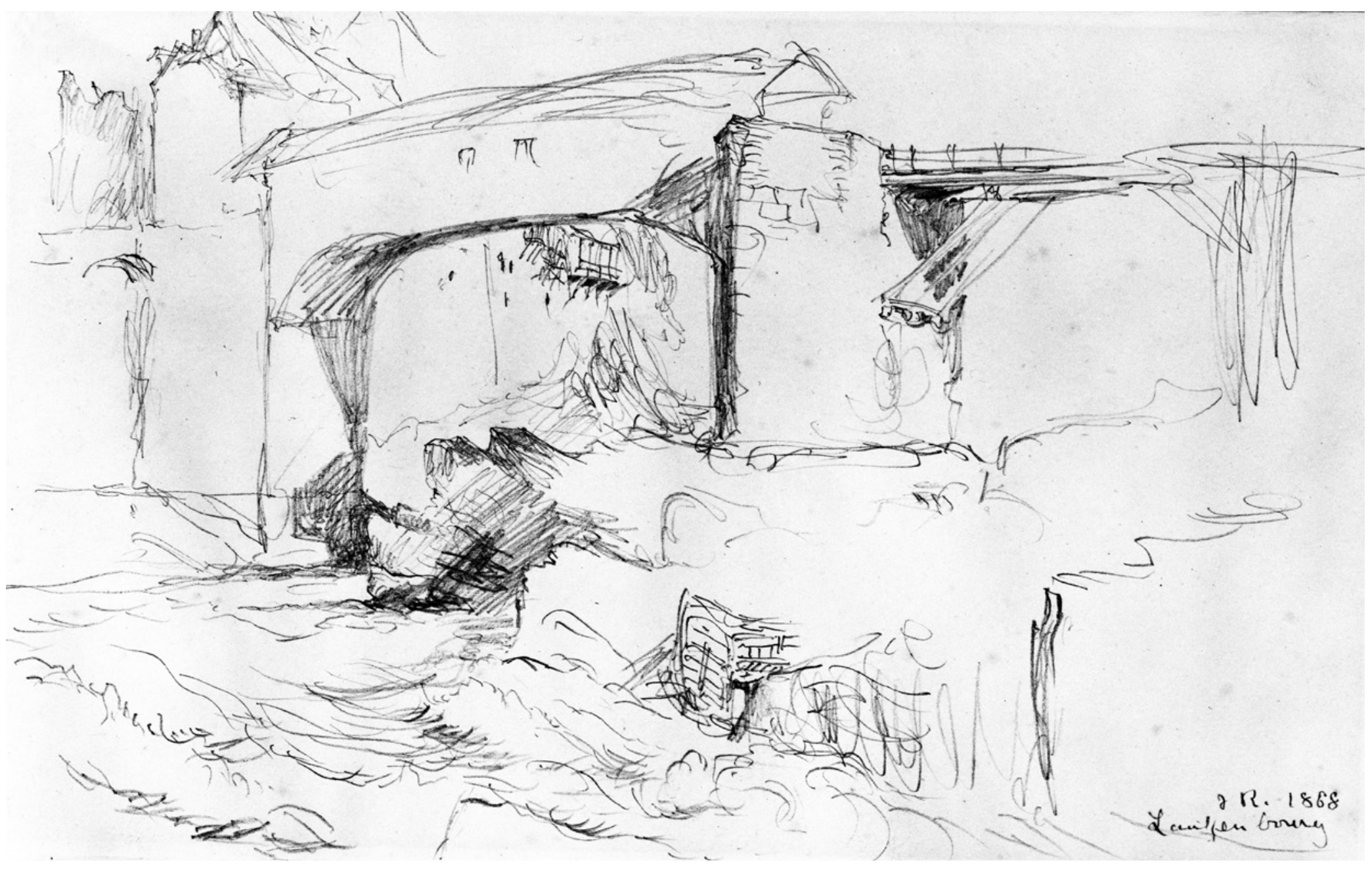

Figure 8 John Ruskin, Bridge at Lauffenbourg. 1863 [wrongly dated by Ruskin 1868]. Graphite on pale pink wove paper, $137 \times 223 \mathrm{~mm}$. Harvard Art Museums/Fogg Museum (1926.33.149). Transfer from the Fine Arts Department, Harvard University. @ President and Fellows of Harvard College 


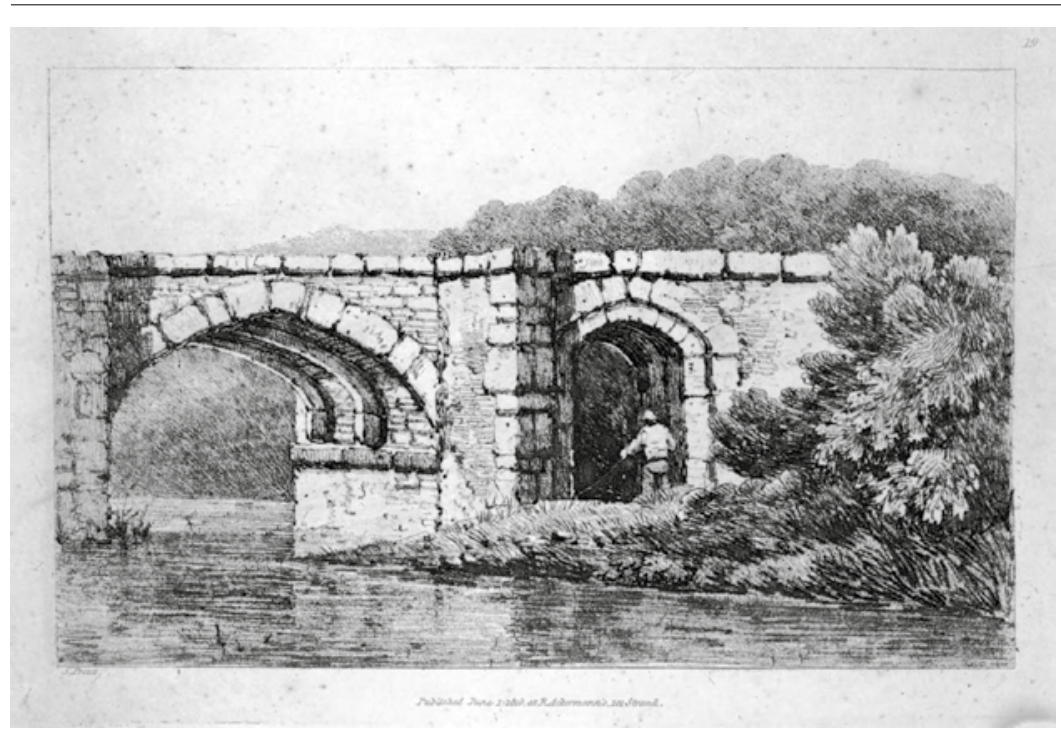

Figure 9 Samuel Prout, Two-arched Bridge, from A Series of Easy Lessons in Landscape Drawing, London: R. Ackermann, 1820. Lithograph, $210 \times 270 \mathrm{~mm}$. Image from copy held by the Fine Arts Library, Fogg Art Museum, Harvard University, digitized by Google and available from HathiTrust Digital Library (www.hathitrust.org)

his planned, but never accomplished history of Swiss towns $^{59}$ - drawings, for instance, of the bridge over the Arve at Bonneville, ${ }^{60}$ over the Rhine at Laufenburg; ${ }^{61}$ over the Reuss at Lucerne ${ }^{62}$ and to some extent the "jaunty" Bremgarten $^{63}$ too. In drawings from the 1860s through to those of the 1880s bridges stretch or stride into visual depth, varyingly tilted towards or away from the viewer on the vertical and horizontal axes so as to display their arches' tensed under-curves and - individually or in more or less taut succession - their titanic piers, knee- or waist-deep in still or swirling water.

The Ruskinian bridge, then, is strong in constructive "action". Yet like any building worth consideration as a work of architecture, it is also beautiful in decorative "aspect". This, however, as suggested earlier, is not necessarily a question of its hosting forms imitative or evocative of natural objects, but may simply entail the replication in its own functionally crucial "lines of action" of such objects' abstract contours. The bridge enjoys this quality thanks indeed to its sympathetically ingenious, hence noble ful- 


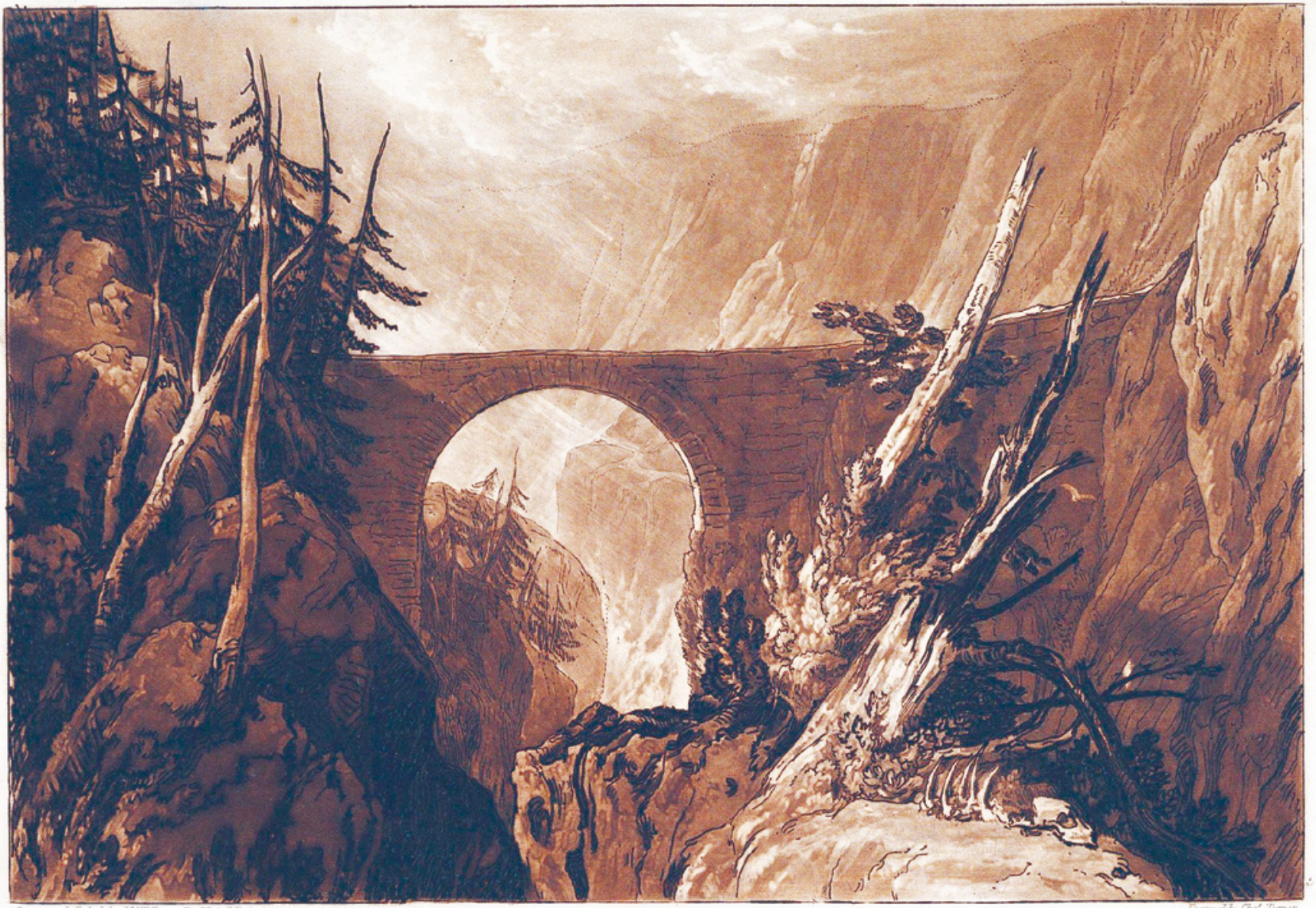

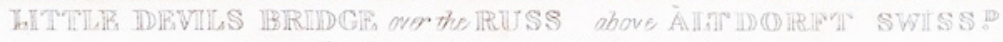

Figure 10 Joseph Mallord William Turner and Charles Turner, LITTLE DEVIL'S BRIDGE over the RUSS above ALTDORFT, SWISS , from Liber Studiorum, part IV. 1809. Etching and mezzotint. Photo of Impression in the Metropolitan Museum of Art, New York. (c) Wikimedia Commons 


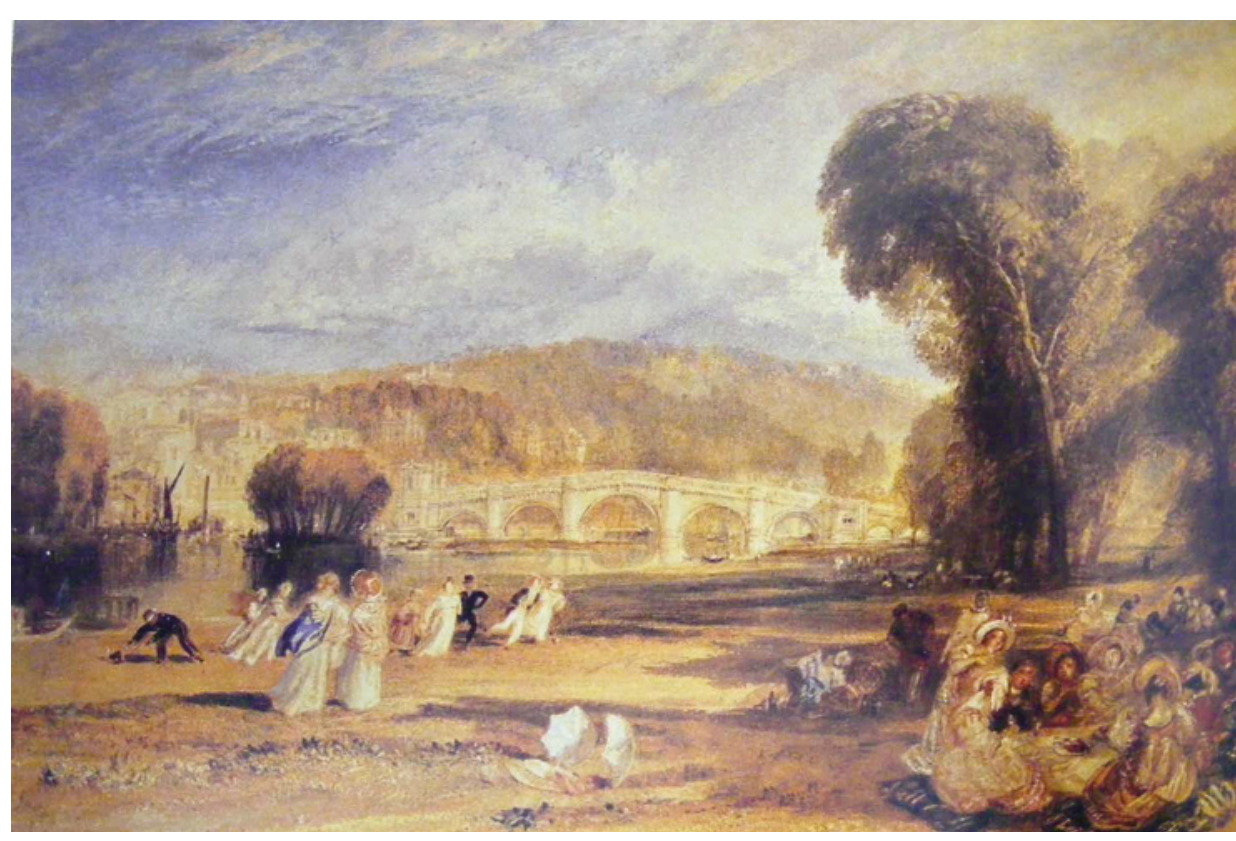

Figure 11 Joseph Mallord William Turner, Richmond Hill and Bridge. 1828-1829. Watercolour on paper, $291 \times 435 \mathrm{~mm}$. London, British Museum (1958.0712.435). Bequeathed by Robert Wylie Lloyd. (c) Wikimedia Commons filment of practical purpose. In his Edinburgh Lectures on Architecture and Painting of 1853 Ruskin stressed that "Gothic or Romanesque construction is nobler than Greek construction [Ruskin's emphasis]":

That is to say, building an arch, vault, or dome, is a nobler and more ingenious work than laying a flat stone or beam [or enormous steel disc!] over the space to be covered. It is, for instance, a nobler and more ingenious thing to build an arched bridge over a stream, than to lay two pine-trunks across from bank to bank; and, in like manner, it is a nobler and more ingenious thing to build an arch over a window, door, or room, than to lay a single flat stone over the same space. ${ }^{64}$

Thus,

all endeavours to do the thing in a grand engineer's manner, with a level roadway and equal arches, are barbarous; not only because all monotonous forms are ugly in themselves [a warning not heeded by the architect of Ruskin Bridge] but because the mind perceives at once that there has been cost uselessly thrown away for the sake of formality. ${ }^{65}$ 
By contrast, its unequal aches, motivated not by dogmatic adherence to formal symmetry, but by attentive observation of natural conditions and artful assessment of ends and means, fits the bridge to embody principles of cohesive design in natural and depicted landscape.

Ruskin conned this quality in bridges, not only in playing with his favourite toy model but in poring over and emulating the graphic work of two leading exponents of the urban and landscape variants of the Picturesque, Samuel Prout [fig. 9] and Turner [fig. 10], the first of whose watercolours to be acquired by the Ruskins, in 1839, incidentally showed Richmond Hill and Bridge ("A more wonderful or instructive piece of composition I could not have had by me", Ruskin commented in 1878 [fig. 11]). ${ }^{66}$

\section{Bridges and the Laws of Design}

In The Elements of Drawing (1857), Ruskin used Turner's depiction, in his watercolour of Coblenz [figs. 1, 12a], of the bridge over the Mosel to illustrate not only the elementary principles of bridge construction but also six of the nine Laws of Composition expounded in the extensive third part of his manual.

First and foremost, Turner's bridge exemplifies the Law of Curvature. The reader/pupil is asked to note of it that it "slopes in a gradual though very subtle curve" and is invited, taking the linear scheme of the composition provided [fig. 12b], to rule straight lines "from the base of the tower on each side to [its] ends" and thus see how their substitution for the curve damages the design. The lesson applies to "all beautiful objects whatsoever", "terminated" as these necessarily are "by delicately curved lines, except where the straight line is indispensable to their use or stability". ${ }^{67}$

Further, the dotted curves superimposed on Turner's design in Ruskin's diagram demonstrate the bridge's central role in the composition's instantiation of the Law of Radiation. This regards the beauty, not of single lines but of their union in harmonious groups, radi- ation being "the most simple and perfect" form of linear connection:

In the instance before us, the principal object being [...] the tower on the bridge, Turner has determined that his system of curvature should have its origin in the top of this tower [...] One curve joins the two towers, and is continued by the back of the figure sitting on the bank into the piece of bent timber. This is a limiting curve of great importance, and Turner has drawn a considerable part of it with the edge of the timber very carefully, and then led the eye up to the sitting girl by some white spots and indications of a ledge in the bank; then the passage to the tops of the towers cannot be missed. ${ }^{68}$

And one by one Ruskin proceeds to trace and illustrate all the curves that articulate and unify the composition.

Not only, however, does Turner's watercolour exemplify the Laws of Curvature and Radiation, but its analysis - by means of the compositional diagram already employed and of an additional enlargement [fig. 12c] of 
LETTKn m.] Ox CoLotr AND cosprostrox.

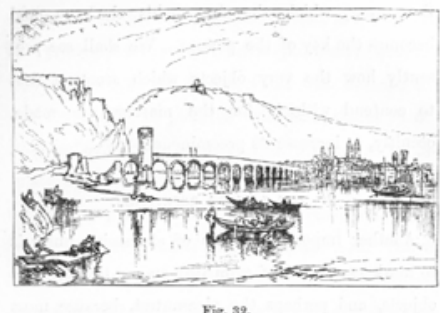

Fig. 32.

at Coblentz, the town of Coblentz on the right, Ehrenbreitstein on the left. The leading or master feature is, of course, the tower on the bridge. It is kept from being too principal by an important group on each side of it; the bosts, on the right, and Fhrenbreitstein beyond. The boats are large in mass, and more forcible in colour, but they are broken into small divisions, while the tower $\mathrm{i}$ simple, and therefore it still leads. Fhrenhreitstein is noble in its mass, but so reduced by aërial perspective of colour that it eannot contend with

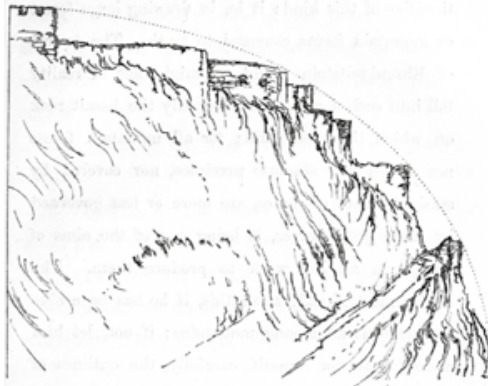

Fig. 3s.

perceive that there is a subtle cadence and harmony among them. The reason of this is, that they are all bounded by one grand curve, traced by the dotted line; out of the seven towers, four precisely touch this curve, the others only falling back from it here and there to keep the eye from discovering it too exsily.

And it is not only always possible to obtain con-

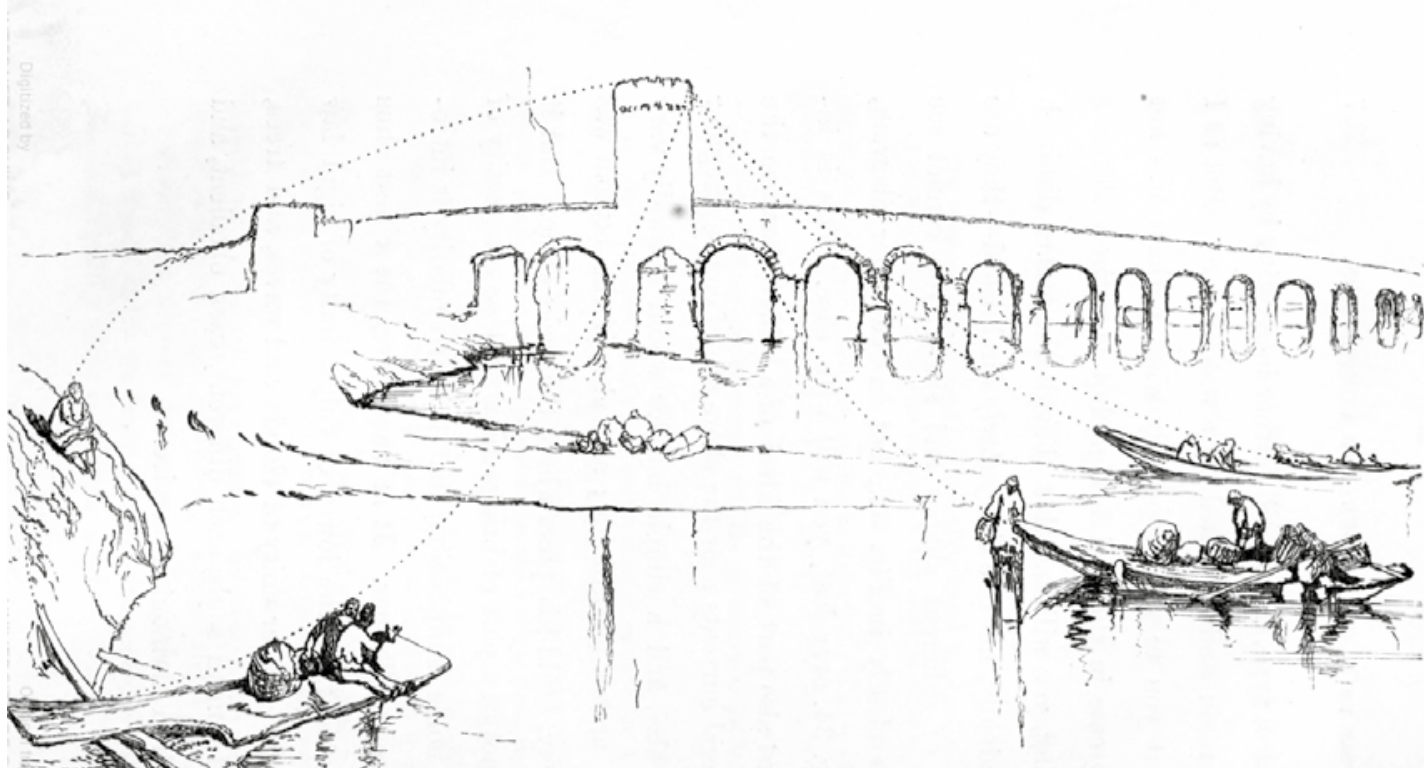

.

Fig. 34
Figure 12a John Ruskin, The Elements of Drawing. In Three Letters to Beginners, London: Smith, Elder, and Co., 1857, 253, fig. 32: woodcut by Miss Byfield after drawing by Ruskin. Image downloaded from copy at University of California digitized by Internet Archive and available from HathiTrust Digital Library (www.hathitrust.org)

Figure 12b John Ruskin, The Elements of Drawing. In Three Letters to Beginners, London: Smith, Elder, and Co., 1857, 268, fig. 34: woodcut by Miss Byfield after drawing by Ruskin. Image downloaded from copy at University of California digitized by Internet Archive and available from HathiTrust Digital Library (www.hathitrust.org)

Figure 12c John Ruskin, The Elements of Drawing. In Three Letters to Beginners, London: Smith, Elder, and Co., 1857, 271, fig. 35: woodcut by Miss Byfield after drawing by Ruskin. Image downloaded from copy at University of California digitized by Internet Archive and available from HathiTrust Digital Library (www.hathitrust.org) 
the rock of Ehrenbreitstein seen in the painting's upper left-hand corner - allows Ruskin to explain four of the remaining seven Laws also: Principality, Repetition, Continuity and Contrast. For

in every good picture, nearly all laws of design are more or less exemplified. ${ }^{69}$

And the Laws are themselves consonant with one another in so far each realizes a variant mode of Unity:

Composition means, literally and simply, putting several things together, so as to make one thing out of them; the nature and goodness of which they all have a share in producing [...] It is an exhibition, in the order given to notes, or colours, or forms, of the advantage of perfect fellowship, discipline, and contentment. ${ }^{70}$

Noble fulfilment of purpose not only fits Turner's Mosel bridge to effect the manifold unity of his composition but allows it to stand as an emblem for the very generality of the general laws by which that unity is effected. It also allows it to evoke a greater unity, extending beyond the experiential limits of the picture. For Ruskin's Laws of Composition are not limited to the realm of pictorial art, but are held to be manifest in all natural and built forms of beauty; and in his mind - as becomes clearer still in their revisioning in Modern Painters V (1860) as the unitary "Law of Help" ${ }^{71}$ - shade into those of social coexistence and harmony.
7

Such breadth of signification is one - perhaps the deepest - reason for the bitterness provoked in Ruskin by the proliferation throughout Europe of iron and tubular bridges in the "grand engineer's manner", especially if carrying the railroad. An instructive example is that of the first railway bridge at Blackfriars in London [fig. 14], designed by Joseph Cubitt. Ruskin's response to it shows the extent to which the absence of arched construction might in his view be compensated for by imaginative enhancement of function - hence the degree to which, in the arched bridge, beauty of aspect was wedded to noble fulfilment of purpose.
Only a year after its opening in 1864, in a lecture on "The Study of Architecture in our Schools" given at the Royal Institute of British Architects, the Blackfriars railway bridge was instanced by Ruskin as incontrovertible proof of "the vanity of all hope that conditions of art may be combined with the occupations of such a city" - and this despite Cubitt's "distinct attempt [...] to obtain architectural effect on a grand scale". ${ }^{72}$ The bridge's inadequacy was not, Ruskin was quick to stress, due to the materials employed:

It is not edifices, being of iron, or of glass, or thrown into new forms, demanded by new purposes, which

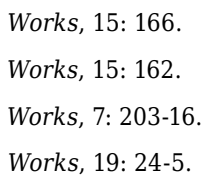




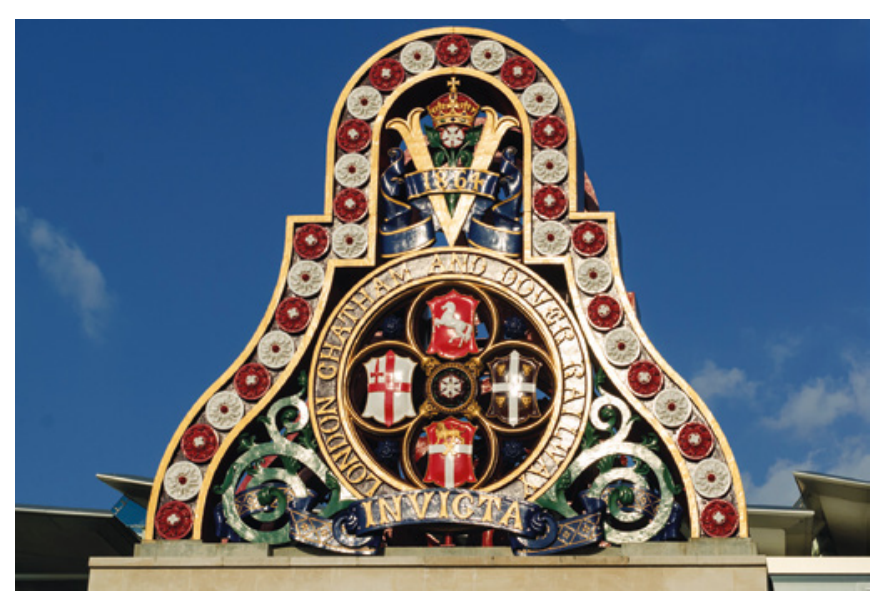

Figure 13 The badge of the London Chatham and Dover Railway, originally part of the first Blackfriars Railway Bridge (1864). Photograph by “SyndVer” (2013). @ Wikimedia Commons

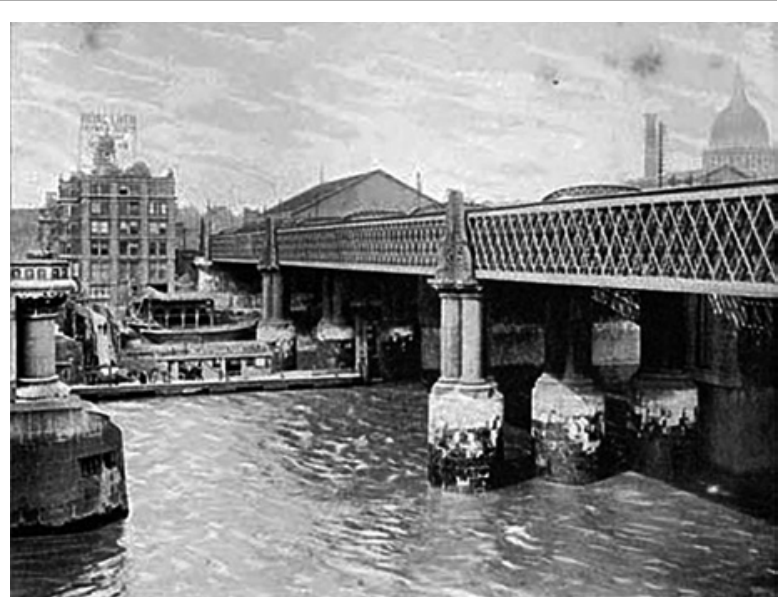

Figure 14 Joseph Dredge, Thames Bridges, from the Tower to the Source, London: Engineering, [1897], Pl. 6 (Blackfriars Railway Bridge). (c) Wikimedia Commons need hinder its being beautiful [sic]. But it is the absence of all desire of beauty, of all joy in fancy, and of all freedom in thought. ${ }^{73}$

Like the village mason's arched bridge, architectural "joy in fancy" bespoke a sort of "sympathy": delighted apprehension of a building's "main conditions of [...] structure" - in the present case the holding "a horizontal group of iron rods steadily and straight over stone piers". A Greek or Egyptian architect, Ruskin assured his audience, would have seen this clearly and

would have said to himself (or felt without saying), - It is this holding, - this grasp, - this securing tenor of a thing which might be shaken, so that it cannot be shaken, on which I have to insist. And he would have put some life into those iron tenons. As a Greek put human life into his pillars and produced the caryatid; and an Egyptian, lotus life into his pillars and produced the lily capital: so here, either of them would have put some gigantic or some angelic life into those colossal sockets. He would perhaps have put vast winged statues of bronze, folding their wings, and grasping the iron rails with their hands; or monstrous eagles, or serpents holding with claw or coil, or strong four-footed animals couchant, holding with the paw, or in fierce action, holding with teeth. ${ }^{74}$ 
At Blackfriars, by contrast, "the entire invention of the designer" seemed "to have exhausted itself in exaggerating to an enormous size a weak form of iron nut, and in conveying the information upon it, in large letters, that it belongs to the London, Chatham, and Dover Railway Company" [fig. 13]. ${ }^{75}$

Worse, in any case, than the proliferation of such bridges was their intrusion into the cherished urban and natural landscapes of Ruskin's life and mind. ${ }^{76}$ In Proserpina (1875-86), his late botanical "grammar", an extensive account of the uncomfortable and unprofitable journey from Paris to Geneva as undertaken in "latter years" by train, and as compared with the many days formerly "spent patiently and well" in covering the same distance with his parents in a pair of light twohorse carriages, reaches its climax in final sighting by the unhappy traveller, "covered with dust, and feeling as if one never should be fit for anything any more", of "the dirtied Rhone, with its new iron bridge" and of "the smoke of a new factory exactly dividing the line of the aiguilles of Chamouni". ${ }^{77}$

And not long after this, in his Guide to the Principal Pictures in the Academy of Fine Arts at Venice (1877), travellers were invited to pause in contemplation before the remains of the fourteenth-century convent and Scuola della Carità now housing the gallery - for the sake of Turner and of Ruskin himself: "for I have given Turner's lovely sketch of it to Oxford, painted as he saw it fifty years ago, with bright golden sails grouped in front of it where now is the ghastly iron bridge ${ }^{18}{ }^{78}$ designed, Ruskin points out, not proudly, by "an English engineer", ${ }^{79}$ whom he additionally blames for depriving the Venetian boatmen of ferrying work and obliging them to take instead to "begging, drinking, and bellowing for the wretched hordes at the tables d'hôte, whose ears have been rent by railroad whistles till they don't know a howl from a song"..$^{80}$

Again, the thirteenth-century fortress at Conwy in North Wales, the subject of a lovingly preserved watercolour by his father, ${ }^{81}$ token also of the castle's status as "one of four most beautiful and picturesque subjects in Europe", had been entirely disqualified as such - so he declared in an Appendix to The Art of England (1883) - since the construction by Robert Stephenson in 1848 of a tubular railway bridge across the river in front of it [fig. 15]. ${ }^{82}$

75 Works, 19: 26.

76 The Swiss tour of 1858, referred to in note no. 4 and made with a view to identifying the subjects of a series of drawings by Turner apparently of "towns along the course of the Rhine on the north of Switzerland" was specifically motivated by the "knowledge" "that these towns were peculiarly liable to be injured by modern railroad works" (Works, 7: 5; cf. Ruskin's letter to his father of 19 May 1858, quoted in Works, 5: xxix). Ruskin's own drawing of the walls and moat of Rheinfelden, included among the illustrations to Modern Painters V (pl. 84; [fig. 4] here), was published "merely to show the kind of scene which modern ambition and folly are destroying, throughout Switzerland" (Works, 7: 437n).

77 Works, 25: 454. Cf. Works, 7: 423: "Thus, the railroad bridge over the Fall of Schaffhausen, and that round the Clarens shore of the lake of Geneva, have destroyed the power of two pieces of scenery of which nothing can ever supply the place, in appeal to the higher ranks of European mind".

78 Works, 24: 172

79 Alfred Henry Neville, active in Europe since the 1830s. See Ruskin [1877] 2014, 107, 123.

80 Works, 24: 172n.

81 Works, 35: 38.

82 Works, 33: 404. Ruskin appears to have had a soft spot for modern suspension bridges, however. Thomas Telford's across the Conwy, built in 1826, goes unmentioned in the passage just cited, whereas in Praeterita childhood memories of the Menai suspension bridge - regarded with 


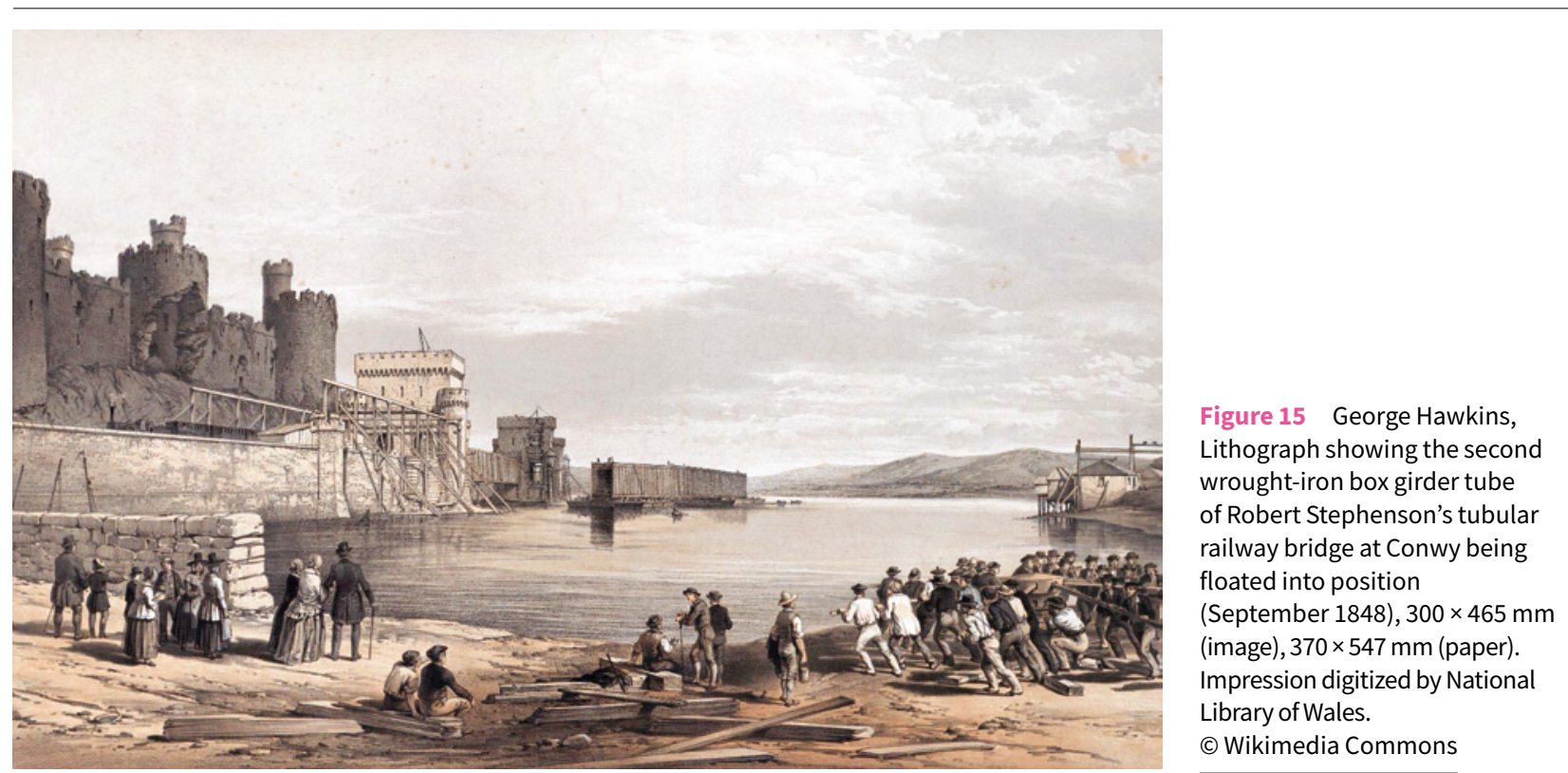

In other late writings, cultural rather than personal memory - the memory of Christian Europe - suggests the sacredness of bridges. Ruskin for instance stresses the devotional testimony preserved in their names or etymons. Writing from Assisi in 1874 he strives to impel obdurate readers of Fors Clavigera to give credence to the ecstasies of St Francis, impatiently meeting anticipated resistance with the retort:

Do you believe in Blackfriars Bridge, then; and admit that some day or other there must have been reason to call it "Black Friar's"? As surely as the bridge stands over Thames, and St. Paul's above it, these two men, Paul and Francis, had their ecstasies, in bygone days, concerning other matters than ermine tails; and still the same ecstasies, or effeminate sentiments, are possible to human creatures, believe it or not as you will. ${ }^{83}$

And in a lecture given later that same year in Oxford, as part of the "Æsthetic and Mathematic Schools of Art in Florence" series, the behaviour of the Italian "modern respectable burgess" who in Ruskin's presence had used

due admiration, he recalls, for its mechanical skill - render it preferable to Stephenson's later tubular railway bridge, the "Britannia", derogated as "the Menai tube" (Works, 35: 96).

83 Works, 28: 87

$207 \begin{aligned} & \text { Fonti, letterature, arti e paesaggi d'Europa | Sources, Literatures, Arts \& Landscapes of Europe } 1 \\ & \text { John Ruskin's Europe. A Collection of Cross-Cultural Essays, 181-212 }\end{aligned}$ 


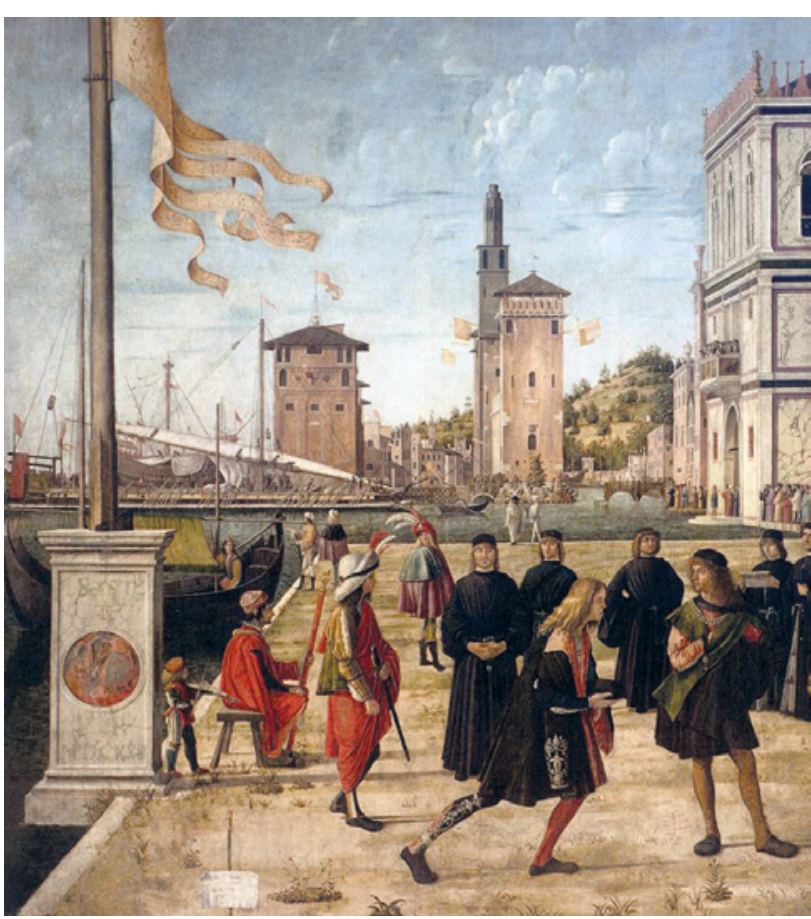

Figure 16 Vittore Carpaccio, The Return of the Ambassadors (detail), from the St Ursula series. 1490s. Tempera and oil on canvas, $2970 \times 5260 \mathrm{~mm}$ (whole). Venezia, Gallerie dell'Accademia. (c) Wikimedia Commons the face of Jacopo della Quercia's effigy of Ilaria del Carretto in the Duomo at Lucca as a hat-rest, is glossed with reference to "existing political life" in modern Italy and, incidentally (for the sake of its name), to the Ponte Santa Trinita, in Florence:

The respectable burgess, who puts his hat on the statue's face, is introducing English manufacture and liberal opinions; he is building tall chimneys close to the bridge of the Trinity, and cheap lodging-houses round the walls, and, as to the old art of the country, as fast as he can, putting his English-made hat on the face of it. That's all that it's good for now. ${ }^{84}$

Ruskin likewise recalls the medieval practice of erecting a chapel on or by a bridge. At Pisa, for example, this had been the original function of Santa Maria della Spina, as readers of Fors Clavigera were informed in an account of its personally witnessed "destruction" ${ }^{85}$ in 1872 :

It was a wonderful thing to see done. This Pisan chapel, first built in 1230, then called the Oracle, or Oratory, - "Oraculum, vel Oratorium" - of the Blessed Mary of the New Bridge, afterwards called the Seabridge (Ponte-a-Mare), ${ }^{86}$ was a shrine like that of ours on the Bridge of Wakefield; ${ }^{87}$ a boatman's praying-place: you may still see, or might, ten years since, have seen, the use of such a thing at the mouth of Boulogne Harbour, when the mackerel boats went out in a fleet at early dawn. There used to be a little

84 Works, 23: 234

85 Actually its dismantling with a view to reconstruction on a newly raised, widened and straightened Lungarno (Clegg, Tucker 1993, 79-80). 86 The Ponte Nuovo ("the New Bridge") and the Ponte a Mare [fig. 4] were two distinct bridges. The Ponte Nuovo had collapsed in the fifteenth century.

87 But see Works, 28: 533 and n. Ruskin owned two pencil drawings by Prout of the chapel on this bridge, which he placed in the Educational Series of the collection of images assembled by him in connection with his teaching at Oxford (Ashmolean Museum [WA.RS.ED.056.a/b] [http:// ruskin.ashmolean.org]). 
shrine at the end of the longest pier; and as the Bonne Espérance, or Grâce-de-Dieu or Vierge Marie, or Notre Dame des Dunes, or Reine des Anges, rose on the first surge of the open sea, their crews bared their heads, and prayed for a few seconds. So also the Pisan oarsmen looked back to their shrine, manypinnacled, standing out from the quay above the river, as they dropped down Arno under their seabridge, bound for the Isles of Greece. ${ }^{88}$

Again, users of his 1877 Guide to the Accademia in Venice were reminded that in former times, even the ruins of old bridges had been venerated. In an extended reading of Carpaccio's Return of the Ambassadors from his St Ursula series, as showing "the conditions of a state in perfect power and prosperity", Ruskin homes in on a detail in the background [fig. 16]:

Crowds on the bridges and quays, but untumultuous, close set as beds of flowers, richly decorative in their mass, and a beautiful mosaic of men, and of black, red, blue, and golden bonnets. Ruins, indeed, among the prosperity; but glorious ones; - not shells of abandoned speculation, but remnants of mighty state long ago, now restored to nature's peace; the arches of the first bridge the city had built, broken down by storm, yet what was left of them spared for memory's sake. (So stood for a little while, a few years ago, the broken Ponte-a-Mare at Pisa; ${ }^{89}$ so at Rome, for ages, stood the Ponte Rotto, till the en-

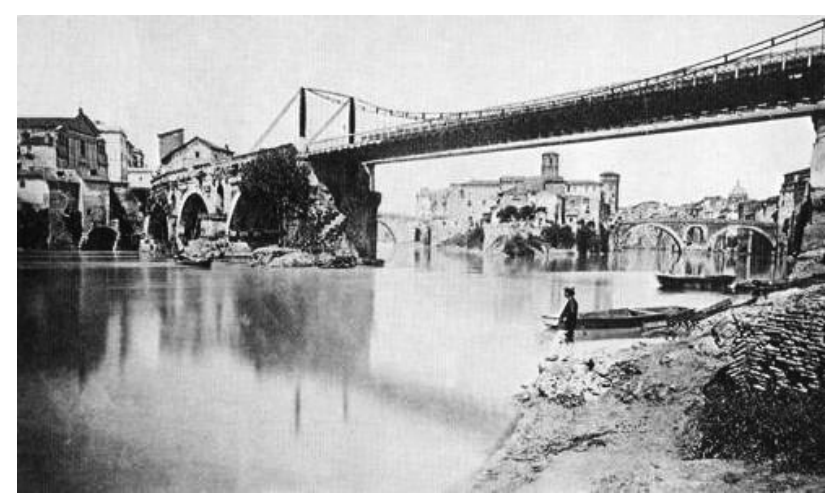

Figure 17 Fratelli D'Alessandri, Photograph of the Ponte rotto, Rome. 1875. Print in the Archivio fotografico comunale, Rome. (c) Wikimedia Commons

gineers and modern mob got at it [fig. 17], making what was in my youth the most lovely and holy scene in Rome, now a place where a swineherd could not stand without holding his nose, and which no woman can stop at). ${ }^{90}$

And similar accounts of the defilement, consequent on "modern progress", of the areas immediately surrounding, or of the scenes commanded from, bridges surviving from "bygone days", or of the bridges themselves - at Venice, Waterloo, Wakefield and Clapham (between Kirkby and Settle) - may be found in other writings of this period. ${ }^{91}$ 


\section{Bridges and "The System of the World"}

Essentially, though, for Ruskin the sacredness of bridges had to do neither with name nor adjacency of chapel, nor even with form, but (to emphasize the point one last time) with basic function (getting the traveller "safely over the river") and the infinitely expandable, but immediately comprehensible symbolism of the juncture this entailed. The arched bridge epitomized an entire architectural system and might constitute one among innumerable manifestations in natural objects and human artefacts of the "abstract lines" of beauty. More than this, however, in functional generality the bridge signalled continuous secure passage across the divides of cognitive and cultural experience and was perhaps for Ruskin a figure of the unitary "system of the world"92 which, though from ever shifting angles, he consistently envisaged and sought to explicate and to enact. For, ultimately, bridges and bridge-building came to stand for the universal security and certainty, metaphysical and moral, of which, in Ruskin's late 'Catholic' reading of society and history, one of the principal guarantors was the (according to one tradition) etymologically justified Pontifex, "bridge-builder" supreme.

In Praeterita Ruskin mocked his childish self for having been "as zealous, pugnacious, and self-sure a Protestant as you please", totally ignorant, however, of Catholic history but rather influenced by considerations such as the observation, made during the family's tours on the Continent, that

all the Catholic Cantons of Switzerland, counting Savoy also as a main point of Alpine territory, [were] idle and dirty, and all Protestant ones busy and clean - a most impressive fact to my evangelical mother, whose first duty and first luxury of life consisted in purity of person and surroundings; while she and my father alike looked on idleness as indisputably Satanic.

And he recalled in particular how his parents had

failed not, therefore, to look carefully on the map for the bridge, or gate, or vale, or ridge, which marked the separation of Protestant from the Catholic cantons; and it was rare if the first or second field and cottage, beyond the border, did not too clearly justify their exulting, though also indignant and partly sorrowful, enforcement upon me of the natural consequences of Popery. ${ }^{93}$

By contrast, the third part of Our Fathers have Told us, which Ruskin began drafting around the same time, was to be entitled Ara Coeli (after the Roman church of Santa Maria in Ara Coeli) and to "trace the foundations of the Papal power" by recounting the "transition of the Roman pontificate into the Christian Papacy". In surviving notes, transcribed and published by E.T. Cook and A. Wedderburn, ${ }^{94}$ Ruskin distinguished three types of priesthood: "natural", "Hieratic" and "Pontifical". The last of these united

the serviceable Hieratic functions with those of the Earthly Teacher, Lawgiver, and Governour, in all things pertaining to the Nation's Health, Holiness, 
and Honour. Not necessarily prophetic or oracular, but dictating constant law, and maintaining spiritual discipline $^{95}$

and was typified in western tradition by the "actively beneficent and protective functions of the Roman Pontifex Maximus". He then noted how

in the minds of all educated men the two functions of the priesthood, in divine and human service, are symbolized in their enduring names, Hieratic, from the word originally meaning Strength [...] and Pontifical - Builders of the Bridge from Earth to Heaven, builders with stones of the brook and wood of the forest, Guides of the Way, and Hospitallers of the Wayfarer. ${ }^{96}$

This interpretation of Pontifical was not in fact certain, but Ruskin, for reasons I hope to have made clear, preferred it, underpinning his gloss with a quotation from Alexander Adam as to the origin of the name of the Roman office. ${ }^{97}$ This cites the opinion of Varro in De lingua latina, who in preference to Quintus Mucius Scaevola's notion that it derived from the combination of posse (to be able) and facere (to do, make, create), opted for a derivation from facere and pons (bridge), given that the
Pontefices had been responsible for the building and afterwards the repair of Rome's first bridge, the wooden Pons Sublicius. Ruskin commends the latter interpretation in particular to the "younger reader", who would "do well", he suggests, "to learn by heart" its formulation by Varro, as reported by Adam, "attaching", he adds, "two primary ideas to it". The Pons Sublicius being, as its name recorded, a bridge built on piles (sublicae), the first of these ideas was that of "the Pontifex making safe what was dangerous, secure what was uncertain; architect not merely of wall or rock, but of foundation, amidst wave, builder of pier and arch alike"98 - where "making safe" and "secure" may in part be due to Ruskin's apparently misreading factus (from facere) as pactus (from pangere [to fasten, fix, drive or force in]), perhaps in wishful construal of the eponymous piles as manifestations of the clavus of Fors in its nail-bearing aspect. ${ }^{99}$

The second idea, quintessentially Ruskinian in its mistranslated emphasis, ${ }^{100}$ induced perhaps by inevitable comparison with the bridgeless passage of Joshua and the Israelites through the parted waters of the Jordan into the Promised Land (Joshua 3:3), was that of "'making sacred both sides of the Tiber', no more forbidding rivers to flow that they may pass into their own narrow Holy Land; but by bridge and ford now making all races known to each other, and all Lands Holy". ${ }^{101}$

95 Works, 33: 194.

96 Works, 33: 194.

97 Adam [1791] 1819, 265: "the PONTEFICES (a 'posse facere', quia illis jus erat sacra faciendi; vel potius a ponte faciendo, nam ab iis sublicius est factus primum, et restitutus sœpe, cum ideo sacra et uls et cis Tiberim fiant, Varr. L. L. iv. 15. Dionys. ii. 73, iii. 45.) were first instituted by Numa, Liv. iv. 4. Dionys. ii. 73., chosen from among the patricians". Ruskin probably read this work in his father's copy of the eighth edition, published in the year of his own birth (Dearden 2012, 6, cat. 15).

98 Works, 33: 195.

99 Works, 27: 28.

100 Ruskin seems to have interpreted sacra, not as the plural of the noun sacrum (a holy or sacred object, act or rite), but as an adjective qualifying the localities implicitly referred to in the prepositional phrase uls et cis Tiberim.

101 Works, 33: 195.

211 Fonti, letterature, arti e paesaggi d'Europa | Sources, Literatures, Arts \& Landscapes of Europe 1

Joht, letterature, artie paesaggid 'Europa Sources, Literatures, Arts
John Ruskin's Europe. A Collection of Cross-Cultural Essays, $181-212$ 


\section{Bibliography of Works by John Ruskin}

References are to volume and page numbers in:

Cook, E.T.; Wedderburn, A. (eds) (1903-1912). The Works of John Ruskin. Library Edition, 39 vols. London: George Allen.

https://www.lancaster.ac.uk/the-ruskin/the-complete-works-of-ruskin/.

Works, 3-7: Modern Painters I-V. | 8: The Seven Lamps of Architecture. | 9: The Stones of Venice I. | 12: Lectures on Architecture and Painting (Edinburgh, 1853), Other Papers (1844-1854). | 13: Turner, The Harbours of England, Catalogues and Notes. | 15: The Elements of Drawing, The Elements of Perspective, The Laws of Fésole.| 17: Unto this Last, Munera Pulveris, Time and Tide, Other Writings on Political Economy (18601873). | 19: The Cestus of Aglaia, The Queen of the Air, Other Papers and Lectures on Art and Literature. | 23: Val D'Arno, The Schools of Florence, Mornings in Florence, The Shepherd's Tower. |24: Giotto and His Works in Padua, The Cavalli Monuments Verona, Guide to the Academy, Venice, St Mark's Rest. |25: Love's Meinie, Proserpina. | 27-29: Fors Clavigera. | 33: The Bible of Amiens, Valle Crucis, The Art of England, The Pleasures of England. |35: Praeterita, Dilecta.

\section{General Bibliography}

Arad, H. (ed.) (2006). Reading Ruskin, Hogarth and Worringer. The Architecture of NOX/Lars Spuybroek=Exhibition catalogue (Tel Aviv, November 2006-January 2007). Tel Aviv: Zezeze Art Gallery. https://issuu.com/zezeze/docs/issuu/64.

Ciacci, M. et al. (a cura di) (2004). I Giardini delle regine. Il mito di Firenze nell'ambiente preraffaellita e nella cultura americana fra Ottocento e Novecento. Of Queens' Gardens. The Myth of Florence in the Pre-Raphaelite Milieu and in American Culture (19th-20th Centuries) $=$ Catalogo della mostra (Firenze, 6 aprile-31 agosto 2004). Livorno: Sillabe.

Clegg, J.; Tucker, P. (1993). Ruskin and Tuscany= Exhibition Catalogue (London, Sheffield, Lucca, January-June 1993). Sheffield; London: Collection of the Guild of St George; Lund Humphries.

Cosgrove, D. (2008). Geography and Vision. Seeing, Imagining and Representing the World. London; New York: I.B. Tauris. https://doi. org/10.5040/9780755620791.

Dearden, J.S. (2012). The Library of John Ruskin. Oxford: Oxford Bibliographical Society.

Ehlers, N. (2001). "The Utopia of the Binational City". GeoJournal, 54, 21-32.

Levi, D.; Tucker, P. (1997). Ruskin didatta. Il disegno tra disciplina e diletto. Venezia: Marsilio.

Mullaly, T. (1966). Ruskin a Verona = Catalogo della mostra (Verona, ottobre-novembre 1966). Verona: Cortella Litografia Tipografia.

Ruskin, J. [1877] (2014). Guida ai principali dipinti nell'Accademia di Belle Arti di Venezia. A cura di P. Tucker. Trad. di E. Sdegno. Milano: Electa.

Spuybroek, L. (2008). The Architecture of Continuity. Essays and Conversations. Rotterdam: V2_Publishing.

Spuybroek, L. [2011] (2016). The Sympathy of Things. Ruskin and the Ecology of Design. 2nd ed. London; Oxford; New York; Delhi; Sydney: Bloomsbury. https://doi.org/10.5040/9781474243896.

Walton, P. [1972] (1985). The Drawings of John Ruskin. [Oxford: Oxford University Press, 1972]. Reprinted New York: Hacker Art Books, Inc. 


\title{
John Ruskin and the Europe of Cathedrals
}

\author{
Claude Reichler
}

Université de Lausanne, Suisse

\begin{abstract}
On his many travels Ruskin visited religious monuments, including cathedrals. The list would be long, either by mentioning his visits in his Diaries or by describing and analyzing monuments in his works on architecture. His interest became passionate about Gothic cathedrals, and sometimes very critical for other periods. My presentation will focus on a hermeneutics of styles and mainly the Gothic style in Ruskin's work. Through this process, I will seek to understand his positions on the question of restoration, and I will once again open the file on the discussions he is initiating on Viollet-le-Duc's work: "Let us not talk about restoration, it is a lie from one end to the other", he wrote in The Seven Lamps of Architecture.
\end{abstract}

Keywords John Ruskin. Viollet-le-Duc. Religious monuments. Gothic cathedrals. Restoration.

Summary 1 The Discovery. - 2 The Truth in Gothic Architecture. - 3 "Read the Building". - 4 Conclusion

\section{The Discovery}

When the young John Ruskin explored the European continent with his parents in 1833, he was following in the footsteps of the Grand Tour undertaken by young English aristocrats in the seventeenth and eighteenth centuries. The family rented their own car and employed the services of a private courier named Salvador, who planned the itineraries, pointed out highlights, and took care of all the practical aspects of the trip. The Ruskins were also heirs to the Grand Tour because the tour on the Continent that they undertook regularly from 1833 was an essential aspect of a young man's education. It was about seeing cities, getting to know the political systems and economies of the countries travelled through, meeting important people, visiting museums and ancient monuments: in short, becoming familiar with the European civilization of which England felt a part. Moreover, on the Grand Tour, from 
the 1760s onwards, the admiration for landscapes and the visits to natural sites occupied a role that became increasingly important, until it became the core of bourgeois tourism in the Victorian era. We know how crucial this was in the young Ruskin's passion for the Alps, from his first trip onwards.

The only thing that was missing during these journeys was the learned mentor of the aristocratic tradition, who accompanied the young men to introduce them to the particularities of the countries they travelled through, to point to the masterpieces of art and architecture, to open their eyes and their souls. For John Ruskin, the role of mentor and mediator was played by the writers and scholars he read before leaving or during his travels, as well as by the draftsmen and painters whose works he admired. Among them, Samuel Prout played a prominent role, and of course, a little later, J.M.W. Turner.

If Turner was a master of landscapes, Prout, who travelled a lot by trade, had made a speciality of city paintings with their street scenes and ancient monuments. The Ruskins greatly appreciated these works, in which the attraction for the picturesque and colourful Middle Ages was marked. André Hélard recalled that the itinerary of the 1833 trip was partly suggested by the desire to see the places represented by Prout in his Sketches made in Flandres and Germany, which had just been published. ${ }^{1}$ A little later, the artist also inspired the Ruskins with his sketches of Italy and Switzerland. Churches were well represented in Prout's drawings, especially the Gothic cathedrals, whose complex and delicate architecture he liked to portray, enveloping humans in the vastness of their façades, columns and naves. From Venice to Amiens, from Rouen to Strasbourg - not to men- tion the old buildings of England, which I shall not deal with here - Ruskin's eye was first Prout's eye.

The poems written by the young Ruskin in 1833, published in 1891 under the title Account of a Tour on the Continent, testify to the influence of Prout's focus on the picturesque and on a somewhat sentimental sublime. It is not necessary to quote these verses, but rather their reinterpretation made by the elderly and sick Ruskin, who relived the sensations and visions of the past by writing his memories for Praeterita:

The vastness of scale in the Milanese palaces, and the "mount of marble, a hundred spires", of the duomo, impressed me to the full at once: and not having yet the taste to discern good Gothic from bad, the mere richness and fineness of lace-like tracery against the sky was a consummate rapture to me - with much more getting up to it and climbing among it, which the Monte Rosa seen between its pinnacles across the plain. ${ }^{2}$

This vision, where the Gothic blends with the mountain, benefitted from the help of several mediators. First of all, it was the panorama of the Alps represented by Robert Burford in the famous rotunda in Leicester Square, one or two years before the trip, in which the picturesque and the sublime merge. The quote "mount of marble" comes from Tennyson's poem The Daisy. Finally, a drawing by Prout - Piazza del Duomo - gave a beautiful graphic introduction to the young Ruskin's impressions. In Strasbourg, Ruskin had a rather negative first impression: "I was already wise enough to feel the cathedral stiff and iron-worky". ${ }^{3}$ He adds however: "But I was greatly excited and impressed [...] by finding the scene

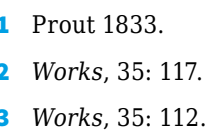


so admirably expressed by Prout in the thirty-sixth plate of his Flandres and Germany". ${ }^{4}$ In Germany, in most of his remarks, and more than anywhere else standing before the Cologne Cathedral during the 1859 trip, Ruskin displayed a virulent aggressiveness: "I find the German Gothic abominable. Cologne Cathedral an enormous failure". ${ }^{5}$ Worse still, in a letter to the painter George Richmond: "Cologne Cathedral a miserable umbug - every bit, old and new, one as bad as another". 6 The two quotations do not only testify that Ruskin experienced a se-

\section{2}

\section{The Truth in Gothic Architecture}

During his travels on the continent, Ruskin discovered all the great Gothic cathedrals and many lesser-known churches. In France and Italy, he visited the great monuments of religious architecture up to ten times, less often in Germany and Flanders. He carefully explored certain regions particularly rich in medieval architecture, such as Normandy and Picardy, the Po plain and Tuscany. As an art historian, he carried out field studies to know the buildings to which he planned to devote important essays: in Venice, Verona or Florence in Italy, in Amiens, Rouen or Chartres in France.

Everywhere, he drew to observe and better understand the architecture, before developing explanations in writing. Drawing was for him the first appropriation of an object, and also an essential mediation towards readers and listeners. His architectural books contain many drawings; he also attached sketches to the diaries and to some letters that relate observations. In his lectures, lective Europe and that he hardly liked Germany, neither for its architecture nor for its philosophers. They also show us that he had strong reservations about the achievements of the late Gothic, and that he abhorred architectural restorations and modern imitations of the Gothic in the nineteenth century. It should be recalled that work on the completion of Cologne Cathedral started again in the middle of the nineteenth century and was not completed until 1880. During his journey in 1859, Ruskin saw the undertaking being built.

he used to display his drawings. What he showed was rarely the entire monument, but the details on which he based his understanding and analysis. He did not define the architecture of a building by the structure of the whole, but by certain specific elements, in particular, for the Gothic, those that carry the ornamentation. In cathedrals, it is the arches of windows, the fillings and pinnacles, the floral decorations of capitals, the shape of buttresses, the niches of sculptures... that were subject to his most meticulous study.

Ruskin devotes a passionate attention to concrete things, and in particular to the construction materials. On church façades and in their interiors, he examines the stones and details their nuances. Proust had noted Ruskin's taste for mineral matter:

Mais jamais cependant ces pierres qu'il a tant aimées ne deviennent pour lui des exemples abstraits. Sur

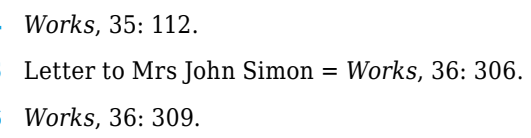


chaque pierre vous voyez la nuance de l'heure unie à la couleur des siècles. ${ }^{7}$

Each region erected its churches using the local stone that had formed in the depth of the earth over geological eras. This is for Ruskin one of the fundamental features of Gothic architecture; and this also helps to understand many of the differences between monuments. In Northern Italy, cathedrals are covered with marble; in Picardy they are built of limestone quarried in the nearby hills; in the Jura department, very old local limestone, with its yellowish colour, is used. This continuity between the earth and the building is essential in Ruskin's eyes, and led him to believe that restoration (or neo-Gothic re-creation) using new materials, such as iron, which was common in his time, was wrong, if not indeed scandalous.

In the sixth chapter of the second volume of The Stones of Venice, "On the Nature of Gothic", also published separately, Ruskin proposes six specific elements of Gothic, corresponding to six qualities of the builders. The third category is Naturalism. This category is developed by a new subdivision, which ranges artists into three classes: Purists, Naturalists in a narrow sense, and Sensualists. Ruskin bases the tripartition, as always, on criteria located at the border between aesthetics and morality: namely the rejection of evil in the works of the Purists, the rejection of good in those of the Sensualists, while artists of the second class, the class of the Naturalists, accept both good and evil:

The second, or greatest class, render all that they see in nature unhesitatingly, with a kind of divine grasp and government of the whole, sympathizing with all the good, and yet confessing, permitting, and bringing good out of the evil also. Their subject is infinite as nature.

Reading the examples given by Ruskin - all painters' names, from Giotto to Turner, from Fra' Angelico to Salvator Rosa - we understand that his tripartition reformulates the opposition between idealism and realism, and condemns certain forms of realism which Ruskin considers extreme. But the most interesting issue for us is the statement that "the Gothic builders were Naturalists", because they put the love of truth above all. This love is characterized by the concern to faithfully reproduce nature, evidently in plant ornaments, but also in narrative contents. Ruskin gives here an example that he uses in several other works: the flames of hell represented in the porch of the Saint-Maclou church in Rouen. He described it, starting with a very interesting remark:

The Gothic inventor does not leave the sign in need of interpretation. He makes the fire as like real fire as he can; and in the porch of St. Maclou at Rouen, the sculptural flames burst out of the Hades gate, and flicker up, in writhing tongues of stones, through the interstices of the niches as if the church itself were on fire. ${ }^{9}$

This statement on the explicit meaning of the sign in Gothic art, follows and concludes a comparison with a mosaic at Torcello representing the same scene in a symbolic way - "the purgatorial fire is represented in the mosaic of Torcello (Romanesque) as a red stream, longitudi-

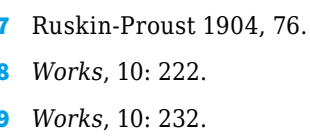


nally striped like a riband, descending out of the throne of Christ, and gradually extending itself to envelope the wicked. ${ }^{10}$ Ruskin thinks that the Gothic artist does not work with symbolism, but what we could define as with indexicality in the sense given by C.S. Peirce (1932). For him, the iconic sign in Gothic sculpture seems to be an index: the similarity between sign and object becomes a trace of the thing. Thus the sculpture of fire is a trace of the flame, the stone itself becomes fire. But he cannot do without the "as if". We should understand that Naturalism (or Realism) - truth as a trace of the thing - begins with the choice of the stone in its most concrete and geographically anchored materiality. Because the stone is at the same time sign and presence, it maintains its efficiency through the activity of the workmen, from the quarryman to the stonemason, and of the sculptor. These professions of the real are the alpha and omega of the Gothic. Unlike other artists and writers, in particular Goethe, who discovered Gothic (which he called

\section{"Read the Building"}

The porches and the chapels of the Gothic cathedrals with their numerous sculptures constitute important stages in this path to God. They both offer together the materiality of the stone and the representation of the world - the world where men live, and the other world, where they aspire to go. To the pilgrim, a sculpture represents a real fact and a statement, a geological and an eschatological reality, both as trace and as meaning. Statues belong to the monument not only as an ornament but also as a discourse. They conduct a sacred conversation with the visitors, bringing them the rustle
"German art") through his admiration for the architect of Strasbourg Cathedral, Erwin von Steinbach, Ruskin did not praise the architects of the great cathedrals; he even doubted their existence as individuals. He held no romantic belief in individual genius: his admiration goes to the anonymous craftsmen, to their simple work and the joy they find there. As we know, this is the central thesis of The Nature of Gothic.

However, Gothic Naturalism is not only about an orientation towards things, but about transforming this orientation into a spiritual path. As the central category between Purism and Sensualism, it therefore welcomes an idealism that is not epistemological, but religious. The stones of the walls and floors, the efflorescence of the capitals, the branches of the pinnacles and the lace of the roses decorating the façades, the stories carved on the porches and the light flashing from the windows, the elevation of the naves, all this material reality, unified and transformed by art, leads to God.

of thousand stories of vices and virtues, of sublime and grotesque features, of feasts and seasons, of damnation and martyrdom, of prophets and saints. In The Bible of Amiens, Ruskin devotes a long chapter to the presentation of the countless statues that populate the building, a chapter he calls "Interpretations" and to which he gives the form, sometimes critical, of a guided tour of the cathedral. There is a sense in which we can say that sculptures make cathedrals, precisely, they are a book, a Bible. Unlike Victor Hugo in Notre-Dame de Paris, Ruskin does not think that "This [the book] will kill that [the ca- 
thedral]". ${ }^{11}$ On the contrary, for him, the building comes from the book and returns to the book, in a movement of translatio that doubles and perpetuates itself in an upward spiral movement, because they both involve the same readability. He writes: "you will have to discover whether [the sculpture] is legible (and, if legible, it is nearly certain to be worth reading) ${ }^{\prime 2}{ }^{12}$ The last paragraph of The Nature of Gothic ends with the well-known formula: "Read the sculpture", of which I quote here the last sentence:

Thenceforward the criticism of the building is to be conducted precisely on the same principles as that of a book; and it must depend of the knowledge, feeling and not a little of the industry and perseverance of the reader whether, even in the case of the best work, he either perceive them to be great, or feel them to be entertaining. ${ }^{13}$

I have been tempted to relate such a sentence to the philosophy of hermeneutics, whose modern developments belong to the years in which Ruskin wrote his most important books about art and architecture. Schleiermacher was a contemporary of Ruskin. As a theory of interpretation - particularly of texts - originating in Protestant theology and appropriated in the reading of the Bible, we might think that hermeneutics has similarities with Ruskin's idea of reading churches like books. But, after having examined this hypothesis, I concluded that it has to be rejected. It seems much more relevant to think that the 'method' of interpretation promoted by Ruskin is close to that of comparatism, the science, or better the methodology appropriate to compare languages and texts, but also organs, skeletons, fossils, plants, and so on. Comparison as a way to establish the structure or to understand the functions of two or more objects that seemed similar, was a method that was theorized at the end of the eighteenth century and flourished in the Nineteenth, in the natural sciences, from anatomy to botany, and also in the historical disciplines such as philology, and studies in folklore and mythology.

Ruskin often proceeds by connecting as many objects or as many occurrences as possible, bringing the data together and comparing them, I will venture to say on the surface, as on a table or a ground plan (or as on a drawing or a painting), and not in the depth of a hidden meaning that would need to be revealed or unveiled by an interpretative approach. He thinks that, for a discerning eye, everything should be seen. Backwards and forwards, inside and outside are structured in the same way:

For the outside of a French cathedral, except for its sculpture, is always to be thought of as the wrong side of the stuff, in which you find how the threads go that produce the inside or right-side pattern. ${ }^{14}$

The metaphor of weaving is particularly appropriate for a "stuff" of stone; in the Latin languages, tissage (weaving) and texture have the same root. Throughout Chapter IV of The Bible of Amiens, on the interpretation of the Cathedral-Bible, we can find various instances that help us to better understand Ruskin's approach of connections and comparisons, whether in synchronic or in diachronic perspectives. Ruskin returns several times to 
the idea of the texture of the building, which formulates not only an image of interpretation, but also a definition of how the building was built. Bénédicte Coste, the editor of a recent French translation of The Seven Lamps of Architecture, notices that fact in her introduction: "L'édifice civil ou religieux est un texte : il se construit, il se décrira comme tel". ${ }^{15}$

Before concluding my paper, let us dwell for a while on a specific example. In a lecture on the Valley of the Somme delivered by Ruskin at the Royal Institution in January 1869, we have several occurrences of the metaphor of reading, - i.e. "In reading this architecture, you will read infallibly the faults of its builders. So we proceed to read this bit of work". ${ }^{16}$ The lecture, which begins with a remarkable description of Abbeville and the Valley of the Somme, deals with the decline of Gothic at the end of the fifteenth century, starting with the example of St. Vulfran's Church, built in a flamboyant Gothic style. From this address I shall retain two points, which I have already mentioned that find here a dazzling wording. The first concerns the primacy of matter, of this it will suffice to give one quotation:

All flamboyant architecture is essentially chalk architecture, - it is built of some light, soft, greasy stone, which you can cut like cheese, which you can drive a furrow into with your chisel an inch deep, as a ploughman furrows his field. Well, of course, with this sort of stuff, the workman goes instinctively in for deep cutting. ${ }^{17}$

The passage continues in the same humorous but seri- ous vein, to the point of explaining the proliferation of "flamboyant" shapes in the ornaments, and fanciful appearances in the sculptures, starting from the specificity of the limestone drawn from the quarries of Picardy. The demonstration that applies to Abbeville also applies, explains Ruskin, to "all late northern works" - which means to all the Northern Gothic buildings built during the fifteenth century.

The second point concerns the comparative method. Although very limited in scope and circumscribed, the lecture on St. Vulfran's and other churches of the Valley of the Somme, may be compared to Ruskin's great books. It is full of references to other Gothic buildings: Rouen, Chartres, Reims, Strasbourg, Verona and Florence, as well as English buildings, such as Chester and Newcastle Cathedrals. To confirm the use of a comparative method, it suffices to read the first paragraph of Chapter IV in The Bible of Amiens, which I have mentioned several times. One can be sure that Ruskin's important essays on architecture are like hubs, namely systems of echo and links of wonderful complexity and intensity. Ruskin's architectural drawings themselves often combine comparisons of details: windows, pinnacles, ribs, statues, façades fragments etc. Ruskin's many journeys and stays to study Gothic cathedrals call out and respond to one another in an illuminating closeness. The reader is invited to explore, through his own eyes and mind, the routes that cross through of the Europe of cathedrals that Ruskin knew and loved so well. The publishers of the Complete Works have tried to do justice to this constant linking in their General Index: $:^{18}$ anyone who consults it will notice Ruskin's prodigious comparative capacity.

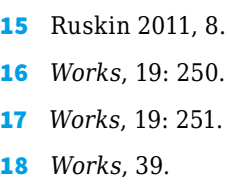




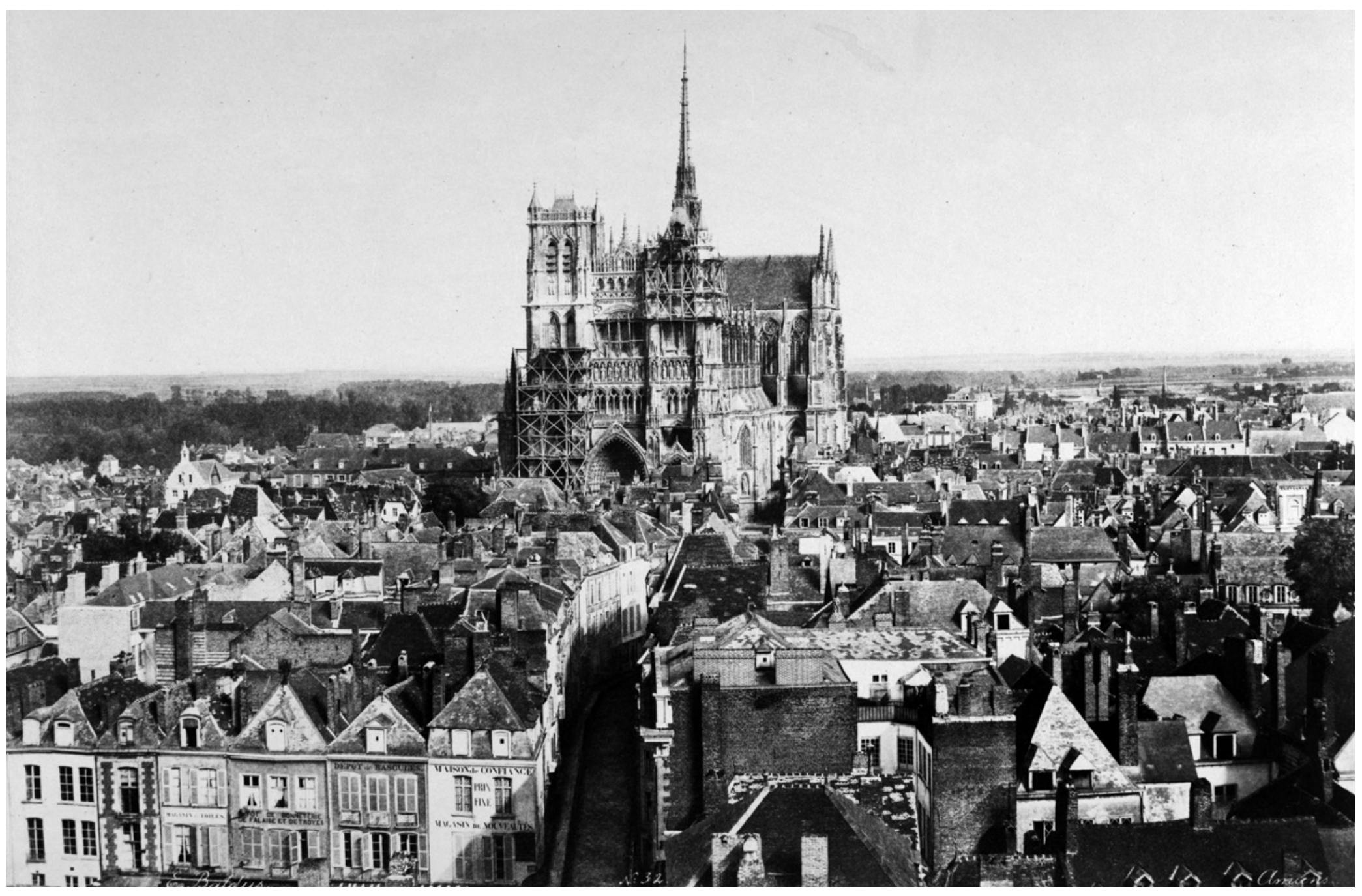

Figure 1 Édouard Baldus, Amiens, 1855. Albumen print from wet collodion negative. The Cleveland Museum of Art, Andrew R. and Martha Holden Jennings Fund 1986. CC license 


\section{Conclusion}

Unlike the comparatist scholars of his own time, who worked in historical linguistics or in folkloric or mythological history, Ruskin did not aim to reconstruct primary models. "Mother-cathedrals" from which later edifices would come, or ideal types that would provide the key to the structures of many churches that were built - both revealing national styles or moments of evolution - does not seem to be the focus of his research. It is an essential character of his thought, that its comparatism aims to specify local or historical peculiarities, to understand the singularities of such and such a building, ornament or style. The scales of the comparison can vary, of course, from the single object to the regional churches or stylistic grouping. His great joys as a researcher are realized when he discovers the perfect embodiment of a form, an absolutely unique and representative achievement. That may be part of a building, such as, in Amiens, the apse of the Cathedral which he calls, quoting Viollet-le-Duc, "The Parthenon of Gothic
Architecture", ${ }^{19}$ or a statue of the Virgin Mary that he greatly admires.

Although one cannot agree with all of Marcel Proust's remarks in his essays on Ruskin, the great French writer had such a fine and empathic understanding of Ruskin that I cannot find a better conclusion than quoting his "Preface" to the translation of The Bible of Amiens:

Il pouvait, en effet, passer d'un pays à l'autre, car la même âme qu'il avait adorée dans les pierres de Pise était celle aussi qui avait donné aux pierres de Chartres leur forme immortelle. L'unité de l'art chrétien au Moyen Âge, des bords de la Somme aux rives de l'Arno, nul ne l'a sentie comme lui, et il a réalisé dans nos cœurs, le rêve des grands papes du Moyen Âge : «l'Europe chrétienne ».20

Let us leave the great popes sleeping in their tombs, and keep the dream of a Europe unified by art and culture. 


\section{Bibliography of Works by John Ruskin}

References are to volume and page numbers in

Cook, E.T.; Wedderburn, A. (eds) (1903-1912). The Works of John Ruskin. Library Edition, 39 vols. London: George Allen.

https://www.lancaster.ac.uk/the-ruskin/the-complete-works-of-ruskin/.

Works, 10: The Stones of Venice II. | 19: The Cestus of Aglaia, The Queen of the Air, Other Papers and Lectures on Art and Literature. |33: The Bible of Amiens, Valle Crucis, The Art of England, The Pleasures of England. | 35: Praeterita, Dilecta. | 36: The Letters of John Ruskin, I (18271869). |39: General Index.

\section{General Bibliography}

Alexander, E. (1969). “Ruskin and Science”. Modern Language Review, 64(3), 508-21.

Brooks, M.W. (1989). John Ruskin and Victorian Architecture. London: Thames and Hudson.

Casteras, S.P. (ed.) (1993). Ruskin and the Victorian Eye. Phoenix: Phoenix Art Museum.

Hélard, A. (2019). "The Things to Be Seen: John Ruskin and Mr. Murray”. Devanthéry, A.; Reichler, C. (éds), Vaut le voyage ? Histoires de guides. Genève: Slatkine, 155-64.

Hugo, V. (2009). Notre-Dame de Paris. Paris: Gallimard.

Peirce, C.S. (1932). "Division of Signs". Collected Papers, vol. 2. Cambridge (MA): Harvard University Press, $227-73$.

Prout, S. (1833). Sketches Made in Flandres and Germany. London: C. Hullmandel's Lithography.

Pullen, L. (ed.) (2019). John Ruskin: The Power of Seeing = Exhibition Catalogue (The Ruskin Collection, Museum Sheffield). London: Two Temple Place.

Ruskin, J. (1983). Les pierres de Venise. Préf. de R. de la Sizeranne, trad. par M. Crémieux. Nouvelle édition avec introduction et notes de J.-C. Garcias. Paris: Hermann.

Ruskin, J. (2011). Les sept lampes de l'architecture. Éditée par B. Coste. Paris: Michel Houdiard éditeur.

Ruskin, J. (2013). Écrits sur les Alpes. Textes réunis et présentés par E. Sdegno et C. Reichler, trad. par A. Hélard. Paris: PUPS.

Ruskin-Proust (1904) = John Ruskin: La Bible d'Amiens. Traduction, notes et préface par M. Proust. Paris: Société du Mercure de France.

Ruskin-Proust (2015) = John Ruskin: La Bible d'Amiens, Sésame et les lys et autres textes. Édition établie, présentée et annotée par J. Bastianelli. Paris: Robert Laffont.

Schleiermacher, F. (1998). Hermeneutics and Criticism, and Other Writings. Cambridge: Cambridge University Press.

Sdegno, E. (2008). “Ruskin's Optical Thought: Tools for Mountain Representation”. Innocenti, L.; Marucci, F.; Villari, E. (eds), Pictures of Modernity. The Visual and the Literary, in England. Venice: Cafoscarina, 29-50.

Spurr, D. (2012). Architecture and Modern Literature. Ann Arbour: University of Michigan Press.

Walton, P.H. (1972). The Drawings of John Ruskin. Oxford: Clarendon Press.

Wheeler, M. (ed.) (1996). Time and Tide: Ruskin and Science. London: Pilkington Press.

Wheeler, M.; Whiteley, N. (eds) (1992). The Lamp of Memory: Ruskin, Tradition and Architecture. Manchester; New York: Manchester University Press. 


\title{
Il mercato antiquariale nella Venezia di Ruskin L'arte medievale in Germania
}

\begin{abstract}
Ruskin made his first trips to Venice when the city was under the Austrian domination, a long period which witnessed the dispersion of many Venetian medieval artworks. These items became of interest to a market which had to meet several requests, including high-standard commissions aimed at creating museums and evocative places. This is the case with the massive purchase by Frederick William of Prussia, in the 1840s, of some ancient medieval sculptures in Italy: among them we can find some Venetian masterpieces all bought from the same trader (Pajaro). Frederick William's brother as well, Charles, bought several Venetian artworks to replicate a Venetian cloister in the Glienicke Palace. Finally, the Church of Peace in Potsdam is adorned with a mosaic bought in Murano, once part of the demolished Saint Cyprian Church.
\end{abstract}

Keywords Art Market. John Ruskin. Gustav von Waagen. State Museum Berlin. Potsdam Friedenskirche. Klosterhof Glienicke. Venice. Medieval Art. Francesco Pajaro. Museums of Venice.

La lunga frequentazione di Venezia da parte di John Ruskin inizia nel 1835 con il suo primo viaggio in città, il primo della sua vita, ancora giovanissimo, ma che lo vede già all'opera con il disegno per fissare i luoghi e i monumenti che lo colpiscono. ${ }^{1}$ Tornerà più volte a Venezia - anche in soggiorni di lunga durata - studiandone l'architettura, la scultura e la pittura, raccogliendo appunti e rilievi in taccuini, realizzando disegni e acquerelli che utilizzerà per le sue pubblicazioni e per la didattica. ${ }^{2}$ L'opera fondamentale (aggiornata più volte) frutto di questo privilegiato rapporto con Venezia è - ovviamente - The Stones of Venice, in tre volumi corredati di splendide tavole, frutto dei suoi rilievi e disegni sul campo che restituiscono monumenti veneziani indagati nella loro struttura e organizzazione materiale, nei singoli lemmi e nelle loro componenti anche minime. Il patrimonio di disegni,

1 Hanley, Sdegno 2010; Hanley, Hull 2016.

2 Il patrimonio ruskiniano è disponibile on line: https://www.lancaster.ac.uk/the-ruskin; http://ruskin.ashmolean.org/welcome. Per i taccuini vedi: Ottani Cavina 2018, 165-87. 
di rilievi e fotografie di Ruskin trasmesso fino a noi è divenuto - al di là della lettura e dell'uso anche didattico dell'autore - preziosa fonte informativa di situazioni poi mutate e a volte compromesse. L'occhio di Ruskin privilegia in questo lavoro di decenni i palazzi medievali (pubblici e privati), alcune chiese (su tutte San Marco) e successivamente la pittura di Tintoretto.

Nell'ottobre del 1835 - dunque - Ruskin appena sedicenne visita per la prima volta Venezia con i genitori e in quello stesso anno il mosaico della chiesa smantellata del monastero muranese di San Cipriano (già sede del Seminario fino al 1817 e demolito nel giro di pochi anni) ${ }^{3}$ viene venduto all'asta. ${ }^{4}$ L'acquirente è Federico Guglielmo principe ereditario di Prussia, che aveva viaggiato in Italia nel 1828, visitando anche Venezia e Torcello. ${ }^{5}$ Il mosaico verrà destinato a una chiesa voluta dallo stesso principe a Potsdam e realizzata in stile neomedievale tra il 1844 e 1851, la Friedenskirche [fig. 1], preceduta da un chiostro e circondata da uno specchio d'acqua. La basilica si rifà a modelli romani, ben presenti a Federico Guglielmo per viaggi e studi, e ripresi anche in sue prove disegnative.$^{6}$ Lo spazio basilicale a tre navate ha un arredo liturgico in stile cosmatesco e accoglie nell'abside centrale il mosaico muranese, la cui iconografia risponde alla visione teocratica del principe prussiano nel frat- tempo diventato re: ${ }^{7}$ una Deesis con Cristo in trono tra la Vergine e il Battista, mentre ai lati si trovano i santi legati all'istituzione benedettina, Pietro e Cipriano. Il monastero si era trasferito in Murano all'inizio del XII secolo ed il mosaico, risalente probabilmente a questo secolo, ${ }^{8}$ è giunto a noi in buone condizioni. È stato recentemente restaurato, consentendo di riconoscervi un testo sostanzialmente integro e di una qualità riferibile alla produzione musiva veneziana, maturata grazie all'importante cantiere musivo marciano. ${ }^{9}$ Si trattava comunque di un monastero importante nel dogado medievale, che attirò nel tempo donazioni di acque e terreni e accolse tombe di dogi per chiudere la sua parabola come sede del Seminario vescovile e - repentinamente - essere abbandonato e venduto pezzo a pezzo. ${ }^{10}$ Questa vicenda ben esemplifica la situazione del patrimonio monumentale e artistico non solo veneziano, ma di gran parte dell'Italia del momento, dove consistenti istituzioni religiose, dopo aver perso la loro funzione per soppressioni e indemaniazioni, cambiano destinazione, vengono spogliate degli arredi o - addirittura - come San Cipriano, demolite. Una fase vividamente descritta per Venezia da Alvise Zorzi nel suo Venezia scomparsa. ${ }^{11}$

Ma oltre al mosaico muranese nel complesso della Friedenskirche trovano posto - collocate nel chiostro - nu-

4

$5 \mathrm{Z}$

6 Zuchold 2010, abb. 34.

7 Zuchold 2010; Schoenemann 2001, 319-22.

8 Polacco 1994, 1995.

9 Il restauro è stato condotto dalla dottoressa Ute Joksch (Stiftung Preussische Schlösser und Gärten Berlin-Brandenburg) nel 2017-2018. La ringrazio per la documentazione fotografica che mi ha inviato e per le informazioni.

10 Corner 1749, 3: 156-319; Zorzi [1972] 2001, 105-6, 276-7; Andreescu Treadgold 1990, 15-16; Polacco 1994, 1995; Ramelli 2000, 59-62; Moine 2013, 64-5.

11 Zorzi [1972] 2001.

Fonti, letterature, arti e paesaggi d'Europa | Sources, Literatures, Arts \& Landscapes of Europe $1 \mid 22$ John Ruskin's Europe. A Collection of Cross-Cultural Essays, 223-246 


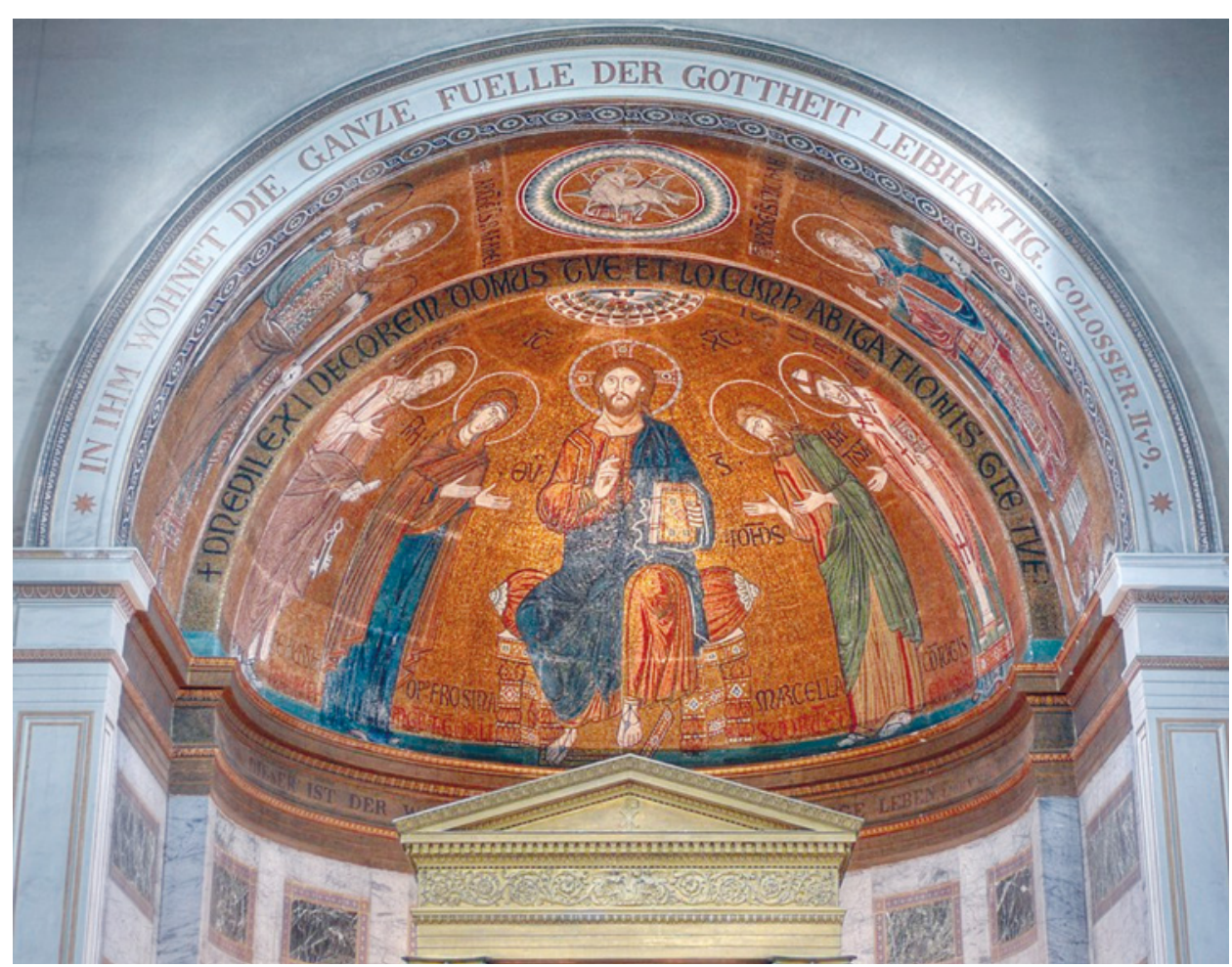

Figura 1 Potsdam Friedenskirche, abside, mosaico da San Cipriano di Murano. (c) Wikimedia Commons, Ph. Karl Heinz Meurer merose sculture di provenienza veneziana; alcune di esse all'inizio del XX secolo verranno trasmesse per volontà dell'imperatore Guglielmo II (fratello di Federico Guglielmo IV e suo successore) e destinate alla sezione dei Musei (intitolata Frühchristlich-Byzantinischen Sammlung, poi Kaiser Friedrich Museum, successivamente Museum für Spätantike und Byzantinische Kunst) ora compresa nel Bode Museum riaperto nel 2006. ${ }^{12}$ Si tratta di una formella (inv. 2922) di un pozzo (inv. 2924) e di un magnifico pluteo con pavoni (inv. 2925) ${ }^{13}$ che dobbiamo riferire ad acquisti sicuramente antecedenti al 1904, anno in cui queste opere vengono donate ai musei, forse riferibili allo stesso periodo di una consistente campagna di acquisti sulla piazza veneziana degli anni Quaranta del XIX secolo, poco dopo l'acquisto del mosaico eseguito dal console di Prussia a Venezia per conto del princi- 
pe. ${ }^{14}$ Dagli inventari e cataloghi del Museo di Berlino risulta infatti nel 1841 un consistente acquisto di sculture a Venezia dalla «Sammlung Pajaro» che rappresenta la base di partenza della sezione medievale di quel museo. ${ }^{15}$

Tra il 1840 (anno di salita al trono di Federico Guglielmo) e il 1841 infatti Gustav von Waagen - storico dell'arte impegnato nella istituzione dei Musei di stato e per le collezioni reali - viaggia in Italia ${ }^{16}$ comprando sistematicamente opere per i musei reali berlinesi o vagliando possibili acquisti, come testimonia lo stesso Wilhelm von Bode - lo studioso che ordinerà il museo che ora ha il suo nome. ${ }^{17}$ Waagen compra arte antica, ${ }^{18}$ medievale e moderna in quantità impressionanti. A Venezia acquista arte classica della collezione Grimani in mano ai commercianti veneziani Pajaro e Sanquirico. ${ }^{19}$ Un altro gruppo di sculture acquisito nel 1841 dalla raccolta Pajaro comprende pezzi veneziani (prevalentemente frammentari) riferibili all'arte altomedievale, alla stagione romanica, alcune opere d'arte bizantina disponibili, oltre a espressi d'arte rinascimentale..$^{20} \mathrm{Il}$ viaggio di Waagen (che ha come esito anche l'acquisto della Madonna
Diotallevi di Raffaello vista a Rimini ${ }^{21}$ mirava evidentemente a raccogliere una campionatura di espressioni artistiche di varie epoche e culture e - nel caso dei pezzi medievali - erano suscettibili di interesse in quel momento lastre frammentarie, capitellini, lastre di ciborio, sarcofagi, pozzi, formelle e patere che documentassero espressioni artistiche non ancora presenti nelle collezioni reali e nei costituendi musei, come esplicita Bode. ${ }^{22}$

La provenienza Pajaro e l'anno risultano senza dubbio dagli inventari del Museo di Berlino ed è interessante riscontrare come a questi pezzi medievali veneziani siano attribuiti i primi numeri inventariali, segnalando il loro arrivo precoce in una fase di istituzione della raccolta, mentre altri pezzi di provenienza veneziana risalgono ad acquisti successivi e hanno comunque numeri vicini. ${ }^{23}$

In questo primo gruppo acquisito nel 1841 (ma pervenuto nel $1849,{ }^{24}$ dopo - si noti bene - la ripresa di Venezia dagli Austriaci) rientra una lastra di ciborio (inv. 6) [fig. 2] con una iscrizione, un repertorio decorativo e modalità organizzative che consentono di collocarla nell'VIII secolo, ma è - soprattutto - strettamente confrontabile con

14 Il console è allora Cristiano Federico de Koepff (Polacco 1994, 6), un imprenditore veneziano che risulta in tale ruolo ancora nel 1844 (Manuale 1844, 163). Dal 1845 il console sarà Giacomo Treves (Manuale 1845, 165).

15 Bode 1881; Irmscher 1987; Effenberger, Severin 1992, 12.

16 Bode 1881, 70-1; Geismeier 1980, 411. Su Waagen e il suo ruolo nella formazione dei musei berlinesi si vedano anche Geismeier 1995; Meyer 2014, 183-4, 194-5.

17 Bode 1881, 70-1.

18 Heilmeyer 1995.

19 Favaretto 1984

20 Bode 1881; Irmscher 1987, 75: l'acquisto costa 13.260 franchi; secondo Irmscher un prezzo insolitamente basso: «den ungewohnlich niedrigen Preis». Waagen ebbe mano libera nella campagna d'acquisto in Italia con un finanziamento di 100.000 talleri (Geismeier 1980, 411).

21 Zavatta 2019.

22 Bode 1881, 70.

23 I pezzi inv. 21 e 22 furono acquistati nel 1882 (Volbach 1930, 39-40); inv. 23-25 nel 1888 (Volbach 1930, 42-3, 45).

24 Effenberger, Severin 1992, 12. 
altri frammenti di lastre di ciborio relative alla Basilica dei Santi Maria e Donato di Murano. ${ }^{25}$ Si tratta di frammenti di arcate riutilizzate nelle ringhiere del loggiato superiore dell'abside della basilica costruita nel XII secolo; mentre una è ancora in situ, altre (ritrovate durante i restauri ottocenteschi della basilica) sono in depositi della chiesa e del locale Museo Vetrario, già $\mathrm{Mu}-$ seo civico muranese [figg. 3a-b]. Ho già illustrato questi pezzi ricostruendo un ciborio riferibile a una fase altomedievale di quella chiesa, dismesso al momento della ricostruzione romanica, quando alcune lastre di quell'arredo, ritagliate e utilizzate a rovescio, sono state montate nella galleria absidale, mentre quella pervenuta a Berlino - pur fessurata - non mostra segni di riutilizzo. ${ }^{26}$

Dalla stessa raccolta Pajaro provengono sarcofagi interi (inv. 4 e 5), ${ }^{27}$ ma anche un pluteo bizantino in breccia verde (inv. 15). ${ }^{28}$ Sono pezzi impegnativi anche per dimensioni, ben oltre il genere prevalente nel mercato di cose veneziane (le patere e formelle anch'esse comunque presenti), ${ }^{29}$ che ci mostrano una particolare capacità dell'antiquario Pajaro di accaparrarsi pezzi di un certo interesse. Il collegamento sicuro con la chiesa muranese per lo strettissimo confronto tra la lastra di ciborio berlinese e i pezzi ancora a Murano ci consente di ipo- tizzare l'acquisizione sulla piazza di pezzi ancora di proprietà ecclesiastica, non quindi esclusivamente collegati a soppressioni e demolizioni.

Del resto Lorenzo Seguso nella sua opera Delle sponde marmoree $(1859)^{30}$ polemizza con questo stillicidio, scrivendo proprio della chiesa dei Santi Maria e Donato di Murano, quando riferisce il passaggio dalla chiesa all'antiquariato e poi alle collezioni prussiane di una scultura medievale:

Siamo inoltre giunti a sapere come il tratto di fregio su cui correva scolpita il resto dell'iscrizione poc'anzi riportata, caduto in terra non molti anni or sono, abbia servito lungo tempo di gradino di una secchera circostante al tempio e che da là, passato in un gabinetto d'antiquaria, riposi ora tranquillo in Prussia, ove noi gli auguriamo pace. ${ }^{31}$

Ma di questo rilevante acquisto per le collezioni prussiane, certificato dai cataloghi dei Musei di Berlino e dalla circostanziata notizia di Bode, non è stato possibile riscontrare ancora la documentazione negli archivi veneziani. ${ }^{32}$ Così come avvenuto per l'acquisto del mosaico nel 1835, è possibile che l'operazione sia stata seguita dal

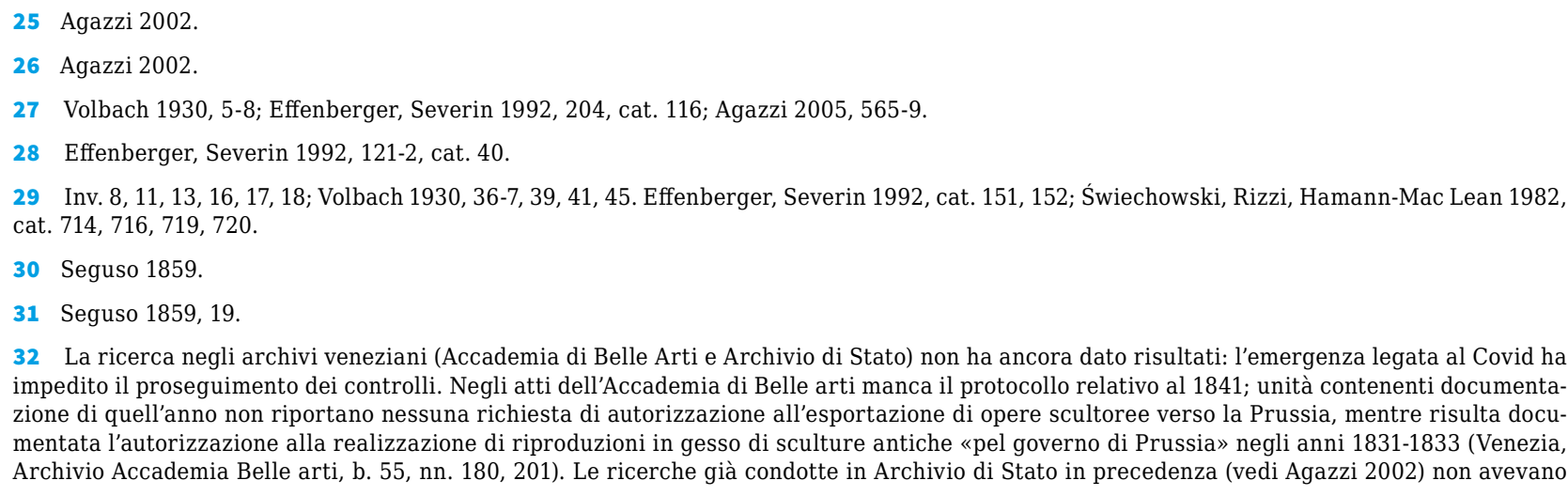
impedito il proseguimento dei controlli. Negli atti dell'Accademia di Belle arti manca il protocollo relativo al 1841; unità contenenti documentazione di quell'anno non riportano nessuna richiesta di autorizzazione all'esportazione di opere scultoree verso la Prussia, mentre risulta documentata l'autorizzazione alla realizzazione di riproduzioni in gesso di sculture antiche "pel governo di Prussia» negli anni 1831-1833 (Venezia, Archivio Accademia Belle arti, b. 55, nn. 180, 201). Le ricerche già condotte in Archivio di Stato in precedenza (vedi Agazzi 2002) non avevano 


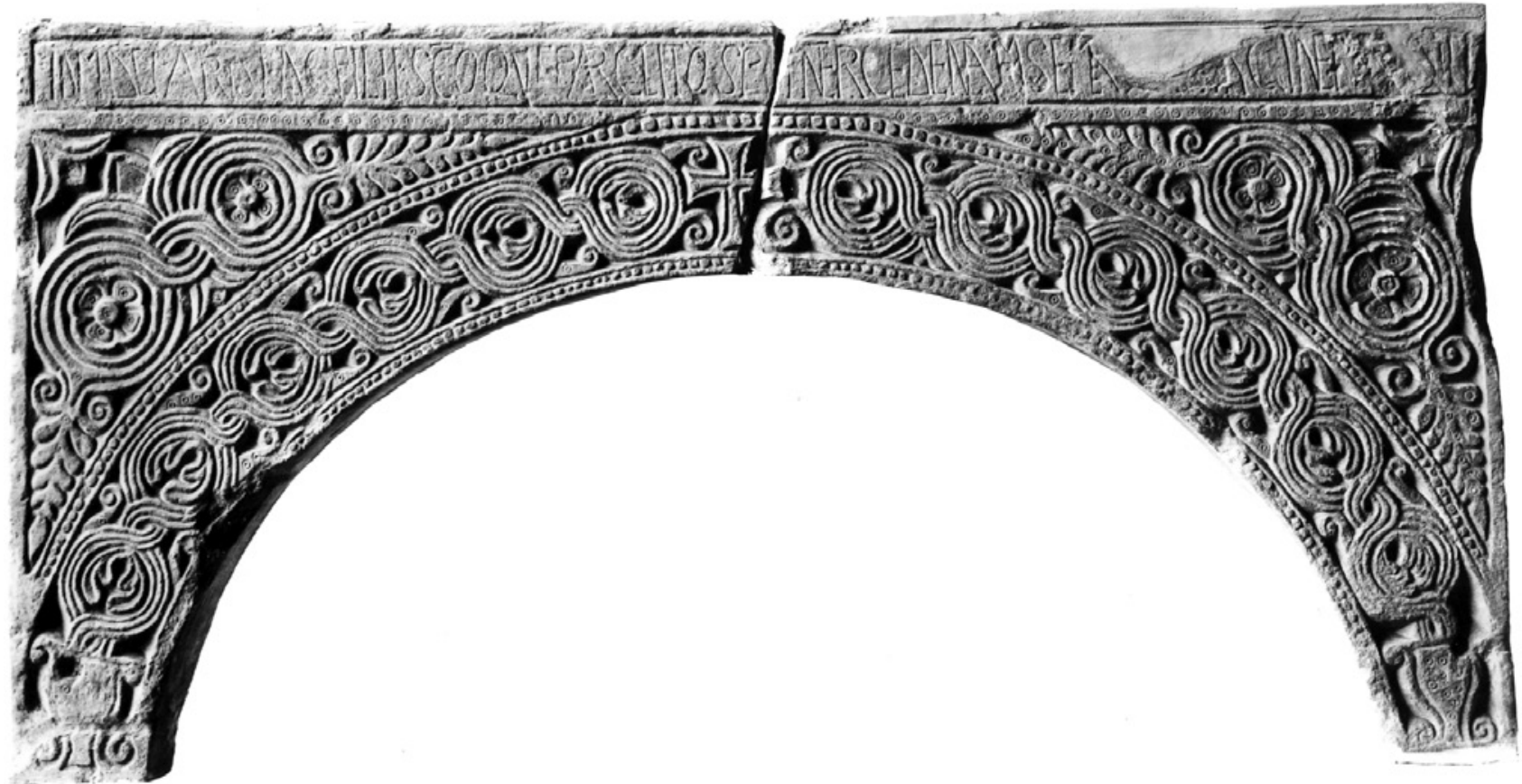

Figura 2 Berlino, Staatliche Museen, arcata di ciborio acquistata nel 1841 a Venezia. Raccolta Pajaro, inv. 6. @ Staatliche Museen zu Berlin

console di Prussia a Venezia, in quel momento ancora Federico Cristiano de Koepff. ${ }^{33}$

Di un coinvolgimento consolare si trova traccia anche in una nota del Cicogna pubblicata da Rizzi che la data al 1820: «altri oggetti d'arte furono levati dai particolari, che esistevano al di fuori sopra le loro case, per timore che il seminario voglia portar via tutto con sé. Alcuni invece per un negoziante di nome Pagliaro, e le vendette al re di Prussia e Russia col mezzo anche del console Fraiganz».34

Il riferimento alla realizzazione della raccolta del Se-

dato riscontri diretti, ma solo la conferma della vendita di materiali da parte della Fabbriceria dei Santi Maria e Donato. Auspico di poter riprendere in futuro questa ricerca sia sul fronte veneziano che su quello berlinese dove gli archivi dei musei conservano la documentazione (vedi Andreescu Treadgold 1990).

33 De Koepff fu console di Prussia fino al 1844 (Manuale 1844, 163).

34 Rizzi 1987, 11-12, 609. La schedatura dei diari di Cicogna (Archivio ricerche del Dipartimento Filosofia Beni Culturali, Università Ca' Foscari) non riporta questa citazione. Purtroppo non mi è stato possibile controllare il manoscritto originale per la chiusura della Biblioteca del Museo Correr dove i diari sono conservati. Ringrazio Isabella Colavizza per le informazioni fornitemi. 
minario per attivo impegno del Moschini confermerebbe gli anni Venti, ${ }^{35}$ ma il cenno al console di Russia Fraiganz (da leggersi come Freygang), sembra porre la circostanza negli anni Quaranta, ${ }^{36}$ momento del passaggio a Venezia di Waagen che - presa visione della disponibilità nella raccolta Pajaro - individuò delle opere da acquistare scegliendo oculatamente opere medievali che mancavano nelle collezioni reali; un procedimento che dovette sicuramente coinvolgere il console nell'espletamento della pratica di esportazione e nell'invio. ${ }^{37}$

Che gli acquisti di opere d'arte dei reali di Prussia a Venezia (e non solo) fossero seguiti dai consoli - con il coinvolgimento di Francesco Pajaro - è dimostrato anche dalla successiva vicenda dell'acquisto nel 1844 del mosaico di San Michele in Africisco a Ravenna, distaccato e smontato a pezzi dall'antiquario e trasportato a Venezia in vista della sua spedizione a Berlino. ${ }^{38}$ Danneggiato dai bombardamenti dell'assedio austriaco del 1848-1849, il mosaico viene tolto a Pajaro e consegnato a un restauratore dal console della Prussia di allora, il conte Giacomo Treves, un personaggio di rilievo sulla scena veneziana. ${ }^{39}$ Proprio grazie a questa vicenda apprendiamo qualcosa di più su Pajaro. Se è documentato come commerciante di antichità con bottega ai Tolentini, ${ }^{40}$ impegnato - come abbiamo visto - anche nella vendita della collezione Grimani ed è citato oltre che nei diari di Cicogna, anche dall'erudito Fapanni che ne registra l'attività, ${ }^{41}$ il fatto che venga incaricato dello smontaggio del mosaico ravennate dimostra il rapporto di fiducia tra il commerciante e gli acquirenti prussiani, anche tramite i rappresentanti consolari. Ma troviamo ancora maggiori informazioni in un documento del $1858^{42}$ relativo a un processo al restauratore Moro che si era occupato nel 1850 del restauro e della spedizione a Berlino del mosaico ravennate. Nel corso del procedimento giudiziario Francesco Pajaro viene chiamato a testimoniare e riepiloga la vicenda dell'acquisto, del trasporto e successivo danneggiamento dell'opera nella sua sede veneziana; constatati i danni lo stesso Pajaro suggerisce Moro come restauratore al console Treves che, affidandolo a questi, di fatto escluse il Pajaro, fino ad allora evidentemente principale referente operativo.

L'individuazione del mosaico ravennate come opera di interesse per il re di Prussia non si deve al Waagen, ma ad Alexander von Minutoli (uomo di governo e fondatore della collezione del Kunstgewerbemuseum di Berlino) che lo disegna in contesto in occasione di un sopralluogo a Ravenna (1842) [fig. 4]. ${ }^{43}$ Immediatamente Pajaro venne incaricato del trasporto:

Nel 1842 dietro incarico avuto da S.M. Il Re di Prus-
41 Fapanni, Francesco Scipione, Monumenti veneziani, Venezia: Biblioteca Nazionale Marciana, It. VII, 2289(9125), c. 187 «Nome, cognome patria dei ... reverendi antiquari, negozianti di cose antiche ... che hanno miseramente spogliato e spogliano ancora delle cose d'arte la povera città nostra [...] Pajaro, intelligente incettatore di anticaglie».

42 Andreescu Treadgold 2013, 286-7, appendice B

43 Wulff 1904, 375, abb. 1; Spadoni, Kniffitz 2007.

\section{Moschini 1842; Di Lenardo 2014}

Andreescu Treadgold 1990, 2007, 2013.

Massaro 2013.

29 Fonti, letterature, arti e paesaggi d'Europa | Sources, Literatures, Arts \& Landscapes of Europe 1 John Ruskin's Europe. A Collection of Cross-Cultural Essays, 223-246 

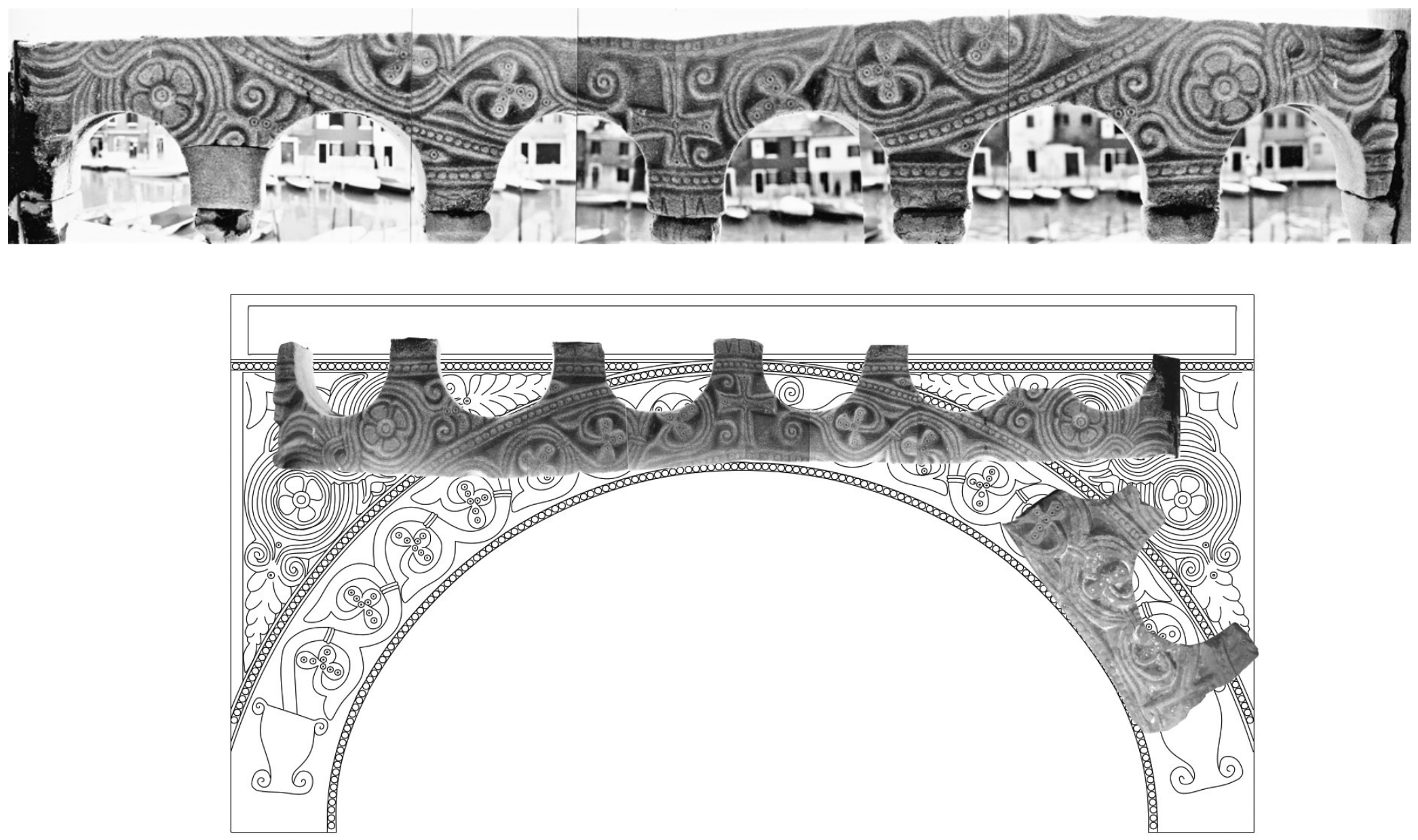

0 $1 \mathrm{~m}$

Figura 3a Murano, Basilica dei santi Maria e Donato, balaustra absidale realizzata con arcata di ciborio altomedievale. Fotografia dell'autrice, fotomontaggio

Figura 3b Ricostruzione dell'arcata di ciborio altomedievale documentata da due frammenti. Murano, Basilica Santi Maria e Donato, balaustra della loggia absidale e cappella Santa Filomena. Autore: Paolo Vedovetto 
sia mi recai a Ravenna per distaccare il mosaico che esisteva nella capella Maggiore del locale altra volta S.Michele a Ravenna, mosaico che S.M. avea comperato, e che io doveva trasportare a Venezia per ristaurarlo, loché infatti fu fatto allora per opera del mosaicista or defunto Liborio Salandri meno qualche piccola porzione di campo dorato. ${ }^{44}$

Un rilievo guida per lo smontaggio viene realizzato da Pajaro nel $1844 .{ }^{45}$ Come per il mosaico di San Cipriano anche qui l'iconografia si prestava a una lettura imperiale e l'operazione era consentita dalla situazione di privatizzazione e cambiamento d'uso. Bloccato a Venezia dalle vicende legate alla Repubblica di Manin e all'assedio austriaco, il materiale - una volta restaurato dal Moro - viene da questi spedito a Berlino nel 1850, ma alla riapertura delle casse (solo nel 1875) il mosaico risulta gravemente danneggiato e viene praticamente rifatto nel 1893, quando viene collocato nella sala bizantina del Frühchristliche und Byzantinische Museen. ${ }^{46}$ Non solo: l'affidamento al Moro determina la sottrazione di parti originali - la testa di Cristo e le teste dei due arcangeli posti ai lati - rispettivamente conservate al Victoria \& Albert Museum di Londra e al Museo di Torcello. L'identificazione delle teste come mosaici ravennati si deve a Irina Andreescu Treadgold che ha restituito un'altra vicenda di dispersione che ben rappresenta l'interesse del mercato per pezzi medievali veneziani. ${ }^{47}$

Ma al di là delle derive verso altre destinazioni di frammenti musivi, anche questo episodio documenta il flusso di materiali da Venezia a Berlino negli anni Qua-

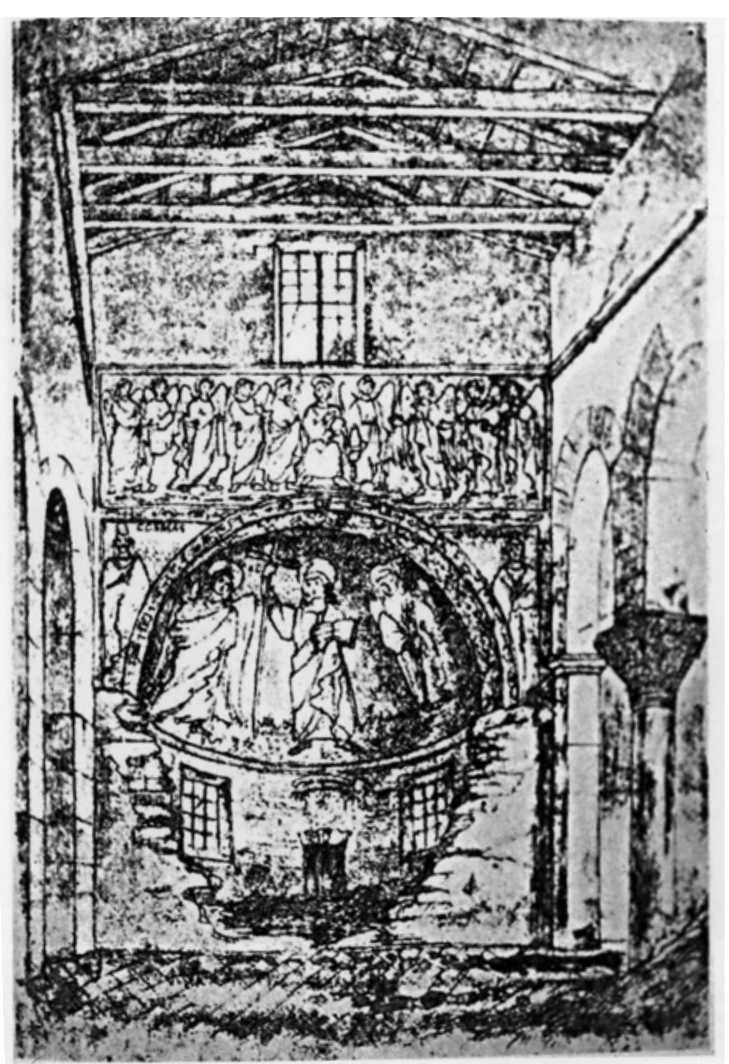

Figura 4 Ravenna, San Michele in Africisco, disegno di Alexander von Minutoli. 1842 Staatliche Museen zu Berlin, da Nehls 1991, abb. 10. (da Spadoni Kniffitz 2007)

\footnotetext{
44 Andreescu Treadgold 2013, 287.

45 Spadoni, Kniffitz 2007, fig. 14; si veda anche Nehls 1991, 166.

46 Spadoni, Kniffitz 2007.
}

47 Andreescu Treadgold 1990, 2007, 2013. Sul mercato dell'arte pittorica veneziana in questo periodo e il forte impatto delle esportazioni: Bernardello 2012-2013.

231 Fonti, letterature, arti e paesaggi d'Europa | Sources, Literatures, Arts \& Landscapes of Europe 1 
ranta per il tramite operativo di Pajaro e un interesse sistematico per l'arte bizantina e medievale, destinata prevalentemente a contesti direttamente collegati alla famiglia e alla committenza reale. Il ritardo nella consegna, la distruzione e il rifacimento nel 1875 (dopo la morte nel 1861 di Federico Gugliemo IV e quindi nella fase di interventi e donazioni da parte di Guglielmo) hanno destinato al museo anche questo compromesso brano musivo.

Ma anche il fratello di Federico Guglielmo, Carlo - come lui educato dall'architetto Karl Friedrich Schinkel - compra a Venezia e a Roma arte antica ${ }^{48}$ e medievale per il castello di Klein Glienicke: su un terreno affacciato sul lago Wannsee, acquistato nel 1824, dove già Schinkel e Peter Joseph Lenné hanno costruito e impiantato un parco, si realizzano padiglioni classicheggianti e un complesso neomedievale, il Klosterhof. ${ }^{49}$

Quest'ultimo edificio (progettato da Ferdinand von Arnim) è preceduto da una colonna con un capitello antico e un leone di san Marco, presenta un perimetrale merlato, con un muro costituito da corsi regolari in pietra alternati a più sottili in mattoni e un portale timpanato che ospita un mosaico (totalmente rifatto dopo i danni della seconda guerra mondiale [fig. 6]). Oltre il muro si apre un chiostro e lungo le pareti del portico che abbraccia tre lati sono disposti frammenti di sculture medievali, incastonate come decorazioni nel muro con incrosta- zioni marmoree ${ }^{50}$ listate da fasce orizzontali a clipei e rosette venezianeggianti. ${ }^{51} \mathrm{Nel}$ quarto lato una profonda cappella [fig. 5] accoglie numerosi rilievi e una tomba proveniente da Padova ritenuta la tomba di Pietro d'Abano, inserita in un arcosolio decorato da un fregio duecentesco a tralcio abitato e clipei nel sottarco della cappella raffrontabile con la casistica veneziana, ${ }^{52}$ mentre sulla parete di fondo sono montate altre sculture riferibili all'ambito veneziano (croce e formelle) insieme a un telamone pisano e elementi cosmateschi. ${ }^{53}$ La maggior parte delle sculture disposte lungo il portico - secondo testimonianze del tempo - proverrebbero da Venezia e in particolare dalla Certosa di Sant'Andrea soppressa a inizio Ottocento e poi demolita; ${ }^{54}$ si tratta di lastre altomedievali con decorazioni a nastro profilato evidentemente apprezzate per l'effetto decorativo, anche se spezzate. All'esterno è montata inoltre un'iscrizione muranese del 1374 che Cicogna vide ancora a Venezia nel 1856 presso un lapicida, dopo la demolizione della Scuola dei Battuti cui apparteneva. ${ }^{55}$ Anche quell'edificio muranese demolito dopo le soppressioni divenne quindi fonte di materiali per un nuovo interesse.

Sempre da Venezia doveva provenire il grande rilievo tondo con la rappresentazione di un imperatore bizantino - gemello di quello veneziano in Campiello Angaran vicino a San Pantalon -, visibile in una immagine precoce del chiostro nella parete del portico sul lato destro e

48 Goethert 1972

50 Si tratta di una decorazione parietale confrontabile con quella di Santa Sofia di Costantinopoli nel restauro allora realizzato da poco dei fratelli Fossati (Hoffmann 1999; Nelson 2004). Ringrazio Andrea Paribeni per il suggerimento.

51 Rizzi 1987, 660-1.

52 Dorigo 2003, 1: 463, 467-9.

53 Zuchold 1984 e 1993.

54 Świechowski, Rizzi, Hamman-Mac Lean 1982, 160-4; Rizzi 1987, 660.

55 Tomasin 2012. 
che venne venduto (insieme ad altre opere ora presenti in copia a Glienicke) nelle vicende successive alla prima guerra mondiale e fa ora parte della collezione dei Dumbarton Oaks. ${ }^{56}$

Nello stesso chiostro Carlo conservava (nella cappella a croce posta alla conclusione del portico sul lato sud) la propria preziosa collezione di segni imperiali: tra questi il trono di Goslar (restituito a quella cattedrale dopo la sua morte) e una croce aurea ottoniana. ${ }^{57}$ Si trattava quindi di un luogo evocativo, finalizzato a una collezione particolare. Il tondo bizantino collaborava a questa qualificazione del complesso, che aveva anche un uso privato (come risulta da una lettera della consorte del principe Carlo del 1851), ${ }^{58}$ ma caricato di riferimenti simbolici al potere con oggetti e segni imperiali, così come nella Friedenskirche eretta a Potsdam da Federico Guglielmo IV, dove l'iconografia cristiana assumeva una accezione imperiale.

In questo luogo quindi si raccolse un insieme eterogeneo di materiali medievali occidentali (soprattutto veneziani, ma non esclusivamente) e bizantini, con un gusto che rispecchia anche le scelte operate da Waagen nei primi anni Quaranta e che avrebbero determinato la nascita della raccolta poi denominata Frühchristliche und Byzantinische Kunst. Accanto all'interesse per la scultura medievale italiana (soprattutto veneziana, presenza da motivare - forse - per la disponibilità sul mercato) spicca anche l'interesse per l'arte bizantina acquistata non solo a
Venezia, ma anche in Oriente. Nel Museo berlinese giungono - come abbiamo visto - pezzi bizantini acquistati a Venezia, ma la maggior parte delle opere di quella cultura sono acquistate direttamente a Istanbul. ${ }^{59} \mathrm{Si}$ tratta di un interesse legato strettamente ad una lettura politica e teocratica, ${ }^{60}$ pienamente condivisa dal principe Carlo.

La circostanza della presenza dell'iscrizione muranese a Venezia ancora nel 1856, insieme alla cronologia della costruzione del Klosterhof (edificato negli anni Cinquanta) segnala una attività di acquisto sulla piazza veneziana anche oltre il massiccio acquisto dall'antiquario Pajaro del 1841.

Che negli anni Quaranta la casa regnante prussiana fosse particolarmente vicina e interessata alla città lagunare può essere testimoniato anche dall'apprezzamento per l'opera sulle iscrizioni veneziane di Cicogna e dalla medaglia da questi ricevuta nel 1844, circostanza riportata dallo stesso autore che pubblica la lettera accompagnatoria di Federico Guglielmo. ${ }^{61}$

Chi curava gli interessi dei reali prussiani in questa attività di acquisto collezionistico sulle piazze italiane? Sicuramente a Venezia - come abbiamo visto - dobbiamo registrare l'impegno dei consoli: probabilmente de Koepff negli anni Quaranta e poi Giacomo Treves dal 1850.

Dalle schede delle collezioni del Bode Museum (Skulpturensammlung) verifichiamo poi l'afflusso negli anni Ottanta del XIX secolo di altri materiali sculto-

56 Zorzi, Berger, Lazzarini 2019.

57 Zuchold 1984, 1993; Anderson 2019, 44.

58 Anderson 2019, 45.

59 Effenberger, Severin 1992.

60 Bullen 2003, cap. 1; Nelson 2004, 40; Ronchey 2004, 719-20.

61 Cicogna 1842, 5: 296.

233 Fonti, letterature, arti e paesaggi d'Europa | Sources, Literatures, Arts \& Landscapes of Europe 1

John Ruskin's Europe. A Collection of Cross-Cultural Essays, 223-246 
Figura 5 Klein Glienicke, Klosterhof, abside. Stiftung Preußische Schlösser und Gärten Berlin-Brandenburg/Bildarchiv.

Su gentile concessione della Stiftung Preußische Schlösser und Gärten Berlin-Brandenburg (SPSG)/Fotograf: Daniel Lindner

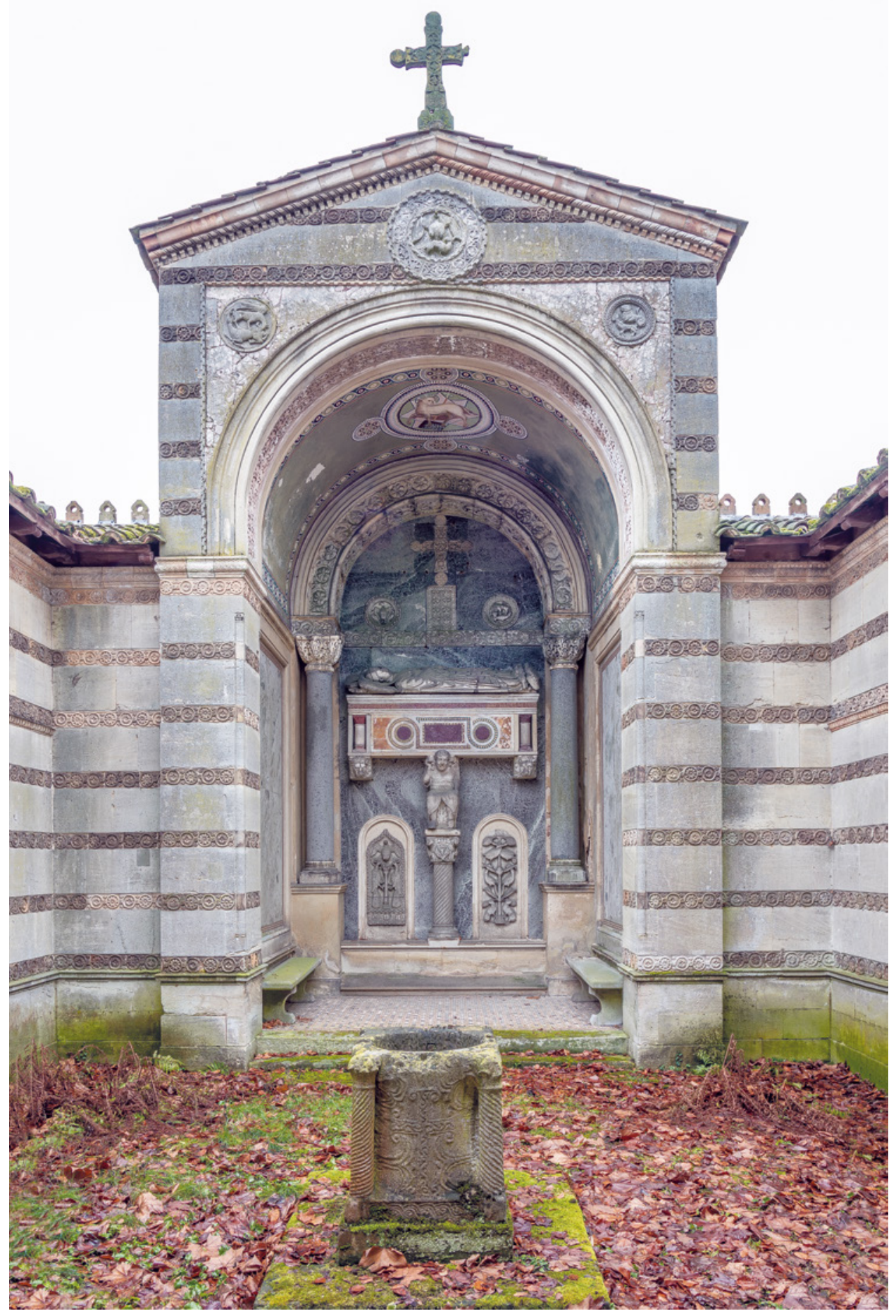




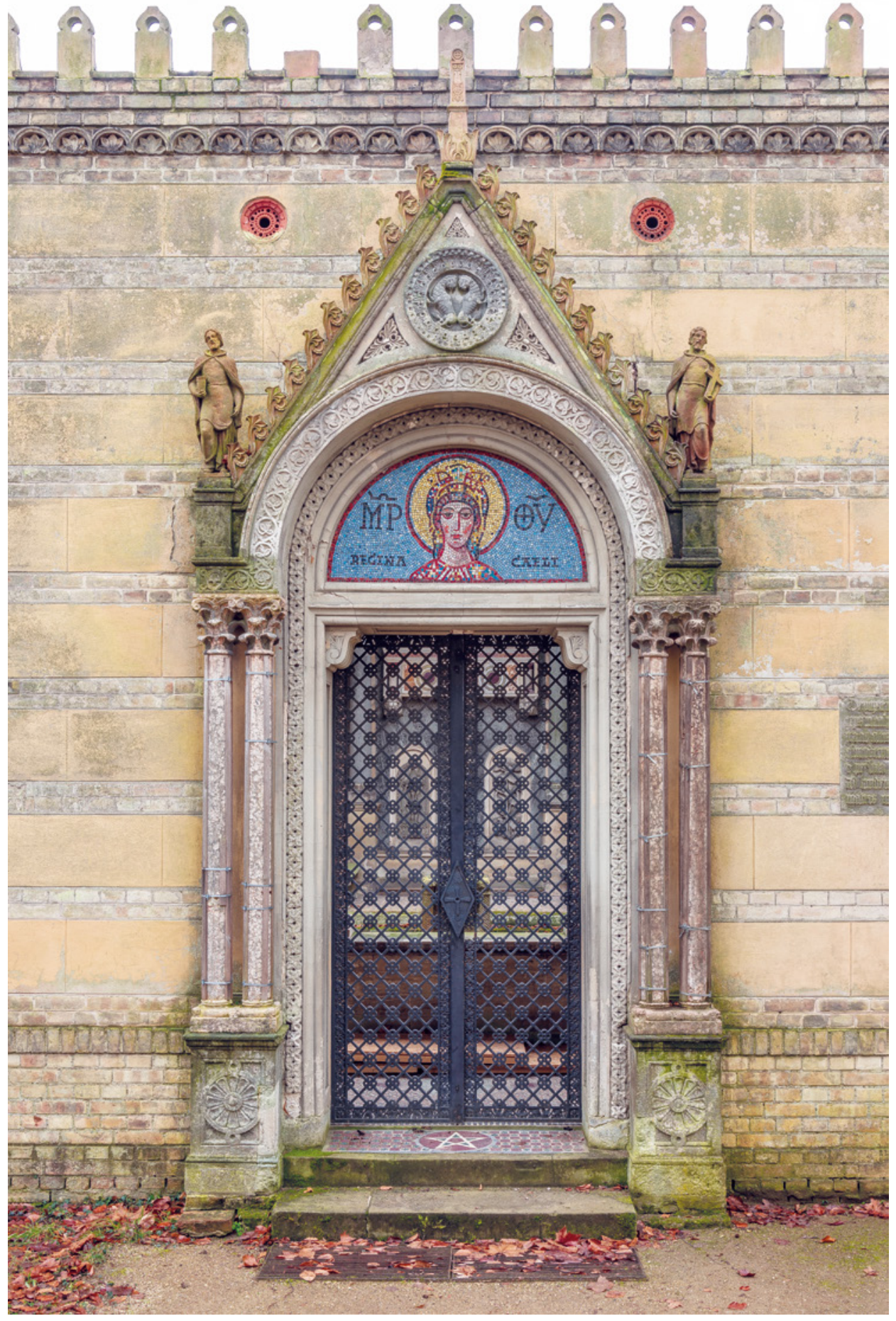

Figura 6 Klein Glienicke, Klosterhof, porta di ingresso. Stiftung Preußische Schlösser und Gärten Berlin-Brandenburg/Bildarchiv.

Su gentile concessione della Stiftung Preußische Schlösser und Gärten Berlin-Brandenburg (SPSG) / Fotograf: Daniel Lindner 
rei veneziani. Si tratta soprattutto di formelle ${ }^{62} \mathrm{ma}$ anche di un leone romanico. ${ }^{63}$ Lo stesso Bode viaggiando a Venezia negli anni Settanta fa acquisti, come il prezioso tappeto (lungo oltre 5 metri) vendutogli dalla chiesa di Burano nel 1871, ${ }^{64}$ e altre opere da antiquari con cui tiene corrispondenza. ${ }^{65}$

Un altro gruppo consistente di sculture viene consegnato ai Musei nel 1904 dall'imperatore Guglielmo: sono evidentemente sculture delle collezioni reali che entrano nel progetto di ampliamento dei musei berlinesi. ${ }^{66}$

Tra questi numerosi pezzi possiamo quindi ipotizzare più arrivi nel tempo dopo il 1841, ma forse anche altri acquisti veneziani degli anni Quaranta furono conferiti solo successivamente al Museo: un pozzo e altre formelle (come abbiamo visto) provengono infatti dal chiostro della Friedenskirche di Potsdam e facevano parte quindi del progetto neomedievale realizzato da quel decennio.

In parallelo a queste dinamiche di acquisto e disponibilità sul mercato veneziano dei frammenti di una vicenda secolare, abbattuta prima dalle indemaniazioni, poi dalle demolizioni e dispersioni, troviamo - quasi negli stessi anni - l'attenzione di Ruskin per la scultura in contesto, per i monumenti da conservare nella loro inte- grità e 'verità', difendendoli da restauri considerati dannosi. Ruskin torna a Venezia nel 1841, di nuovo nel 1845 e tra novembre 1849 e marzo 1850 per un soggiorno prolungato (di cui resta traccia consistente nei taccuini e disegni), ${ }^{67}$ viaggi che si ripeteranno nel corso della sua vita diventando sempre occasione di studi e scoperte.

I disegni acquerellati dei palazzi veneziani (come quello di Ca' Loredan del 1845) ${ }^{68}$ e quelli di San Marco (si veda quello del 1851), ${ }^{69}$ la battaglia contro i restauri del lato meridionale della basilica e tutto il lavoro confluito nell'opera The Stones of Venice, dove - per esempio - le tavole relative alla chiesa dei Santi Maria e Donato di Murano (in particolare tav. V dell'edizione 1874), ${ }^{70}$ restituiscono lo stato dell'edificio prima dei restauri: tutti questi lavori e impegni di Ruskin sono segno di tutt'altra attenzione e preoccupazione rispetto al collezionismo che immette in tutta Europa e oltremanica frammenti di rilievi, fregi, pozzi, sculture funerarie. ${ }^{71}$ Un successo che ha immancabilmente determinato la produzione di vere da pozzo, formelle e patere imitative o fraudolente. ${ }^{72}$

In parallelo a questo flusso in uscita e all'attenzione divergente di Ruskin, matura a Venezia - negli anni Cinquanta dello stesso secolo - una nuova consapevolezza.

62 Inv. 20, 22-25, Volbach 1930, 38, 39, 42-3, 45; Świechowski, Rizzi, Hamann-Mac Lean 1982, cat. 718, 721-7; Effenberger Severin 1992, cat. $150,154$.

63 Inv. 49, Volbach 1930, 53

64 Berlin: Museum für Islamische Kunst, Ident.Nr. I. 2; Cecutti 2012, 36,

65 Tüskés 2013b.

66 Volbach 1930; Effenberger Severin 1992.

67 Ottani Cavina 2018, 165-81. https://www.lancaster.ac.uk/fass/ruskin/eSoV/.

68 Ottani Cavina 2018, cat. 85, 159. http://ruskin.ashmolean.org/object/WA.RS.RUD.022.

69 Ottani Cavina 2018, cat. 65, 136.

70 Un esemplare di questa edizione, da cui si trae la figura 7 del presente testo, è conservato nel Fondo Dorigo presso la biblioteca BAUM dell'Università Ca' Foscari, Venezia.

71 Per i pozzi esportati - genere molto apprezzato - si vedano gli studi di Anna Tüskès.

72 Rizzi 1981, 55; Rizzi 1982, 87-95; Świechowski, Rizzi, Hamann-Mac Lean 1982; Tomasi 2004; Tüskés $2010,42$. 
Lorenzo Seguso nella sua opera Delle sponde marmoree (1859) polemizza con questo stillicidio. Oltre a riferire polemicamente nel passo già citato sul passaggio dalla chiesa, all'antiquariato e poi alle collezioni prussiane di una scultura medievale della chiesa dei Santi Maria e Donato di Murano, Seguso - riferendosi sicuramente a Ruskin - rimprovera ai veneziani l'incuria e ricorda ancora l'intensa attività di spoliazione negli anni Quaranta:

Dicea non ha guari un illustre oltremontano, come di tutti i popoli d'Italia, i veneziani abbiano maggior bisogno che lo straniero illustri ed asporti i loro capi d'opera prima che, smessa l'abituale indifferenza, comincino ad apprezzarne il valore. Questo severo, ma ci duole dirlo, giustissimo rinfaccio noi lo meritiamo, perché dimentichi d'ogni gloria antica, e perché vergognosamente complici a spodestarci delle più elette opere d'arte, che quando pure ci restino invendute, abbandoniamo al guasto del tempo! E fra queste vanno pure annoverate le vere da pozzo. ${ }^{73}$

Se volessimo enumerare tutte le vere passate in Inghilterra, in Prussia ed altrove dall'anno 1842 ai giorni nostri, correremmo grave rischio di stancare la pazienza del lettore. Specialmente intorno al 1847 si fù un vero affaccendarsi negli orti, nei cortili, nei magazzini più riposti. Scoperta una vera di pregio, la si levava sull'istante, il pozzo interravasi per non avere il disturbo di sostituire altra vera alla venduta. ${ }^{74}$

Eco di questo nuovo atteggiamento si trova anche nelle giunte di Zanotto all'opera di Cicognara, Diedo e Selva proprio per l'abside muranese:
L'abside singolare accennato che sofferse molti danni dal tempo, dalla imperizia di chi lo restaurò e dalla rapacità di coloro che derubarono alcuni marmi orientali e formelle, va ora ad essere condegnamente ristabilito. $^{75}$

La stessa abside è documentata nel suo aspetto ante restauro da una bella tavola di Ruskin [fig. 7]. Di quella stessa incuria per il proprio patrimonio architettonico (e quindi delle opere collegate) scrive Ruskin in un paragrafo di The Stones of Venice, dove rimprovera gli italiani per l'abbandono di tanti edifici consegnati dalle stesse Municipalità agli Austriaci, ${ }^{76}$ dimenticando - forse - che la consegna non partiva certo da un'autonoma decisione...

Quel disinteresse del resto è segnalato dallo stesso direttore delle collezioni reali berlinesi Wilhelm von Bode come per giustificare la liceità degli acquisti prussiani sul mercato italiano:

In Venedig, welches Waagen in Italien zuerst betreten hatte, fand er die Sammlung eines Herrn Pajaro käuflich, der das, was sich in den letzten Jahrzehnten in Venedig und Umgebung an Bildwerken christlicher Zeit, die Niemand achtete, dargeboten, mit dem ganzen Eifer eines italienischen Lokalforschers zusammengebracht hatte. Unter 80 meist grösseren Stücken befanden sich mehrere altchristliche Sarkophage, ein trefflicher Brunnen im Stile der Bildwerke des Dogenpalastes, die Reliefstatue eines hl. Hieronymus in der Art der Buon, zwei Schildhalter vom Hauptwerke A. Leopardi's, dem Grabmal Vendramin, mehrere Büsten

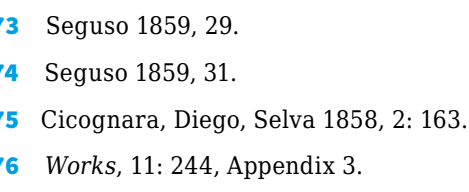




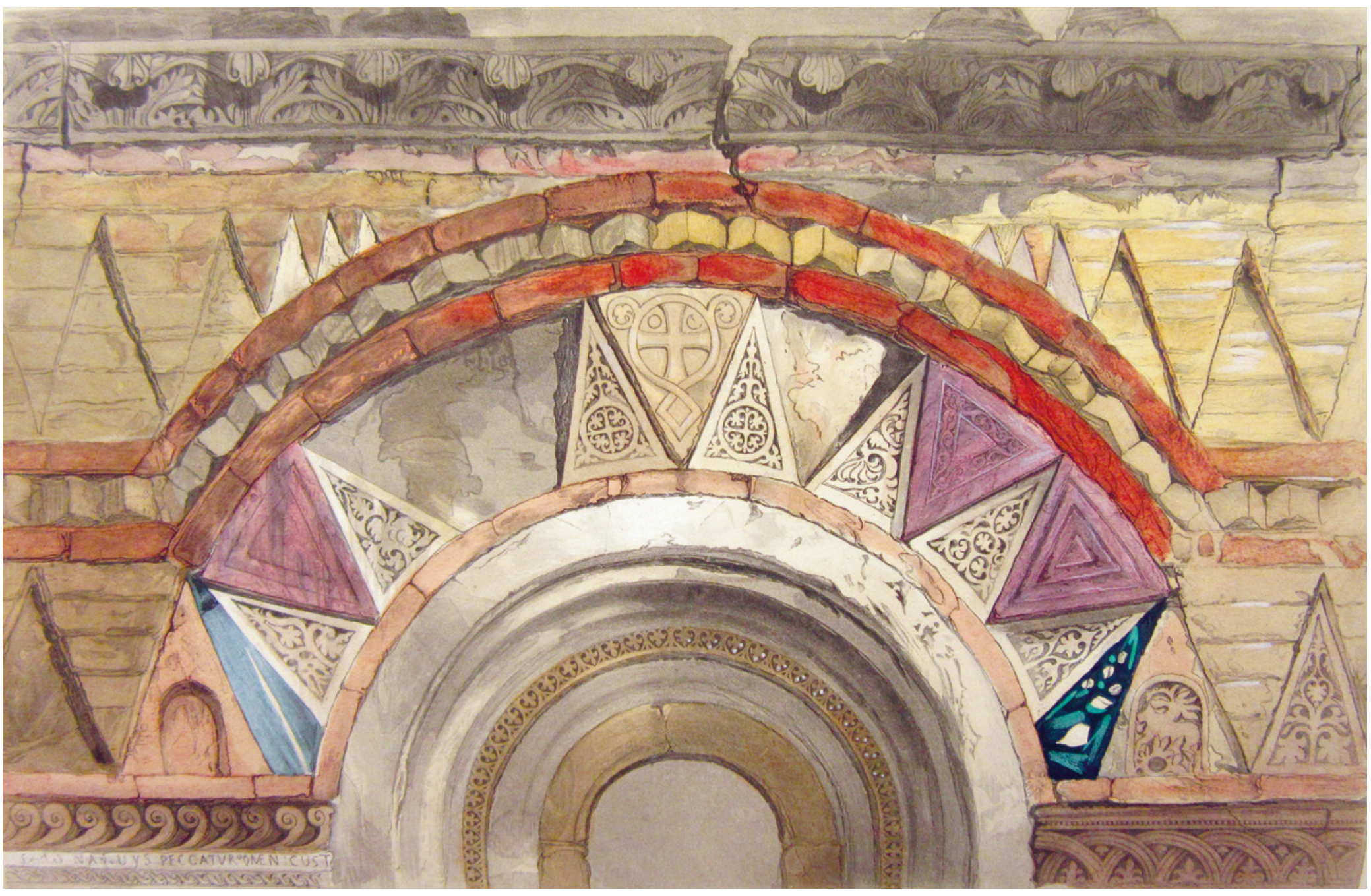

Figura 7 John Ruskin, Archivolt in the Duomo of Murano. In The Stones of Venice, vol. 2, tav. V 
von Alessandro Vittoria und eine Anzahl ornamentaler Arbeiten der verschiedensten Zeiten. ${ }^{77}$

Ma la cura per il patrimonio - in particolare quello medievale - è strettamente connessa al patriottismo: ${ }^{78} \mathrm{i}$ resti di quell'epoca sono considerati testimonianza della storia nazionale e municipale e dopo l'annessione del Veneto al Regno d'Italia, oltre ad avviare importanti restauri come quello di Palazzo Ducale per il quale è istituita una Commissione parlamentare, nascono musei dove si raccolgono le testimonianze frammentarie del passato, spesso collegate a campagne di restauro e riscoperta delle fasi antiche di monumenti, ma anche a donazioni di privati che fanno convogliare nel bene pubblico pezzi altrimenti suscettibili d'interesse per il mercato antiquario, che non cessa - ovviamente - la sua attività.

Se a Murano l'instancabile opera dell'abate Zanetti determina innanzitutto il restauro del pavimento e della basilica dei Santi Maria e Donato, con pubblicazioni e con la promozione di ricerche archeologiche, ${ }^{79}$ un altro esito di grande interesse è la raccolta dei materiali (promossa dallo stesso Zanetti) che ne sono risultati, in parte conservati nella stessa chiesa e in parte raccolti nel nuovo Museo del Municipio muranese: $:^{80}$ un museo (ora del Vetro) che negli intenti del fondatore do- veva documentare le fasi più remote dell'insediamento e degli edifici ecclesiastici della comunità muranese [fig. 8]. Analogamente le raccolte civiche della città di Venezia collocate nella nuova sede del Fondaco dei Turchi, restaurato dopo l'annessione (restauro discusso e non certo informato ai principi di Ruskin) ${ }^{81}$ a tal scopo vedevano (oltre al nucleo fondante della raccolta Correr) la disposizione al piano terra (nella corte e nel portego di facciata) di sculture medievali - in particolare vere da pozzo, bassorilievi, sarcofagi e lastre tombali - che avrebbero potuto alimentare il mercato antiquario rispondendo alla richiesta onnivora del collezionismo europeo, trovando invece lì una nuova sede, pur decontestualizzate e/o private dei nessi, essendo spesso di provenienza ignota. ${ }^{82}$ Sulla disponibilità e 'voracità' del mercato anche in questa fase e le polemiche correlate si vedano Pilutti $\mathrm{Namer}^{83} \mathrm{e}$ - in particolare - uno scritto giovanile di Giacomo Boni, il futuro archeologo in stretto rapporto con Ruskin:

Si notano alla dogana di Venezia le grosse e grandi casse, dirette all'estero, bollate col timbro del locale Regio Istituto di Belle arti, e sulle quali le guardie al confine non esercitano controllo. Contengono antiche sculture, le sponde marmoree dei nostri pezzi, stemmi, bassorilievi, balaustri, archetti a

77 Bode 1881, 71. «A Venezia, dove Waagen si era recato per la prima volta in Italia, trovò in vendita la raccolta di un signor Pajaro, che negli ultimi decenni a Venezia e nei dintorni ha raccolto immagini dei tempi cristiani che nessuno rispettava, raccolte con lo zelo di un ricercatore locale italiano. Tra i circa 80 pezzi per lo più grandi, c'erano sarcofagi paleocristiani, un'eccellente pozzo nello stile delle sculture del Palazzo Ducale, la statua in rilievo di un san Gerolamo dell'arte del Buon, due porta-scudo da un capolavoro di A. Leopardi, la tomba Vendramin, diversi busti di Alessandro Vittoria e una serie di opere ornamentali di varie epoche».

78 Si veda per gli archivi (e in particolare per l'Archivio di stato veneziano) Cavazzana Romanelli 2019.

79 Zanetti 1873, 1876, 1878, 1881.

80 Zanetti 1881; Vecchi 1995.

81 Pilutti Namer 2016.

82 Museo Civico 1899

83 Pilutti Namer 2013, 2015.

239 Fonti, letterature, arti e paesaggi d'Europa | Sources, Literatures, Arts \& Landscapes of Europe 1

John Ruskin's Europe A Collection of Cross-Cultural Essays, $223-246$ 


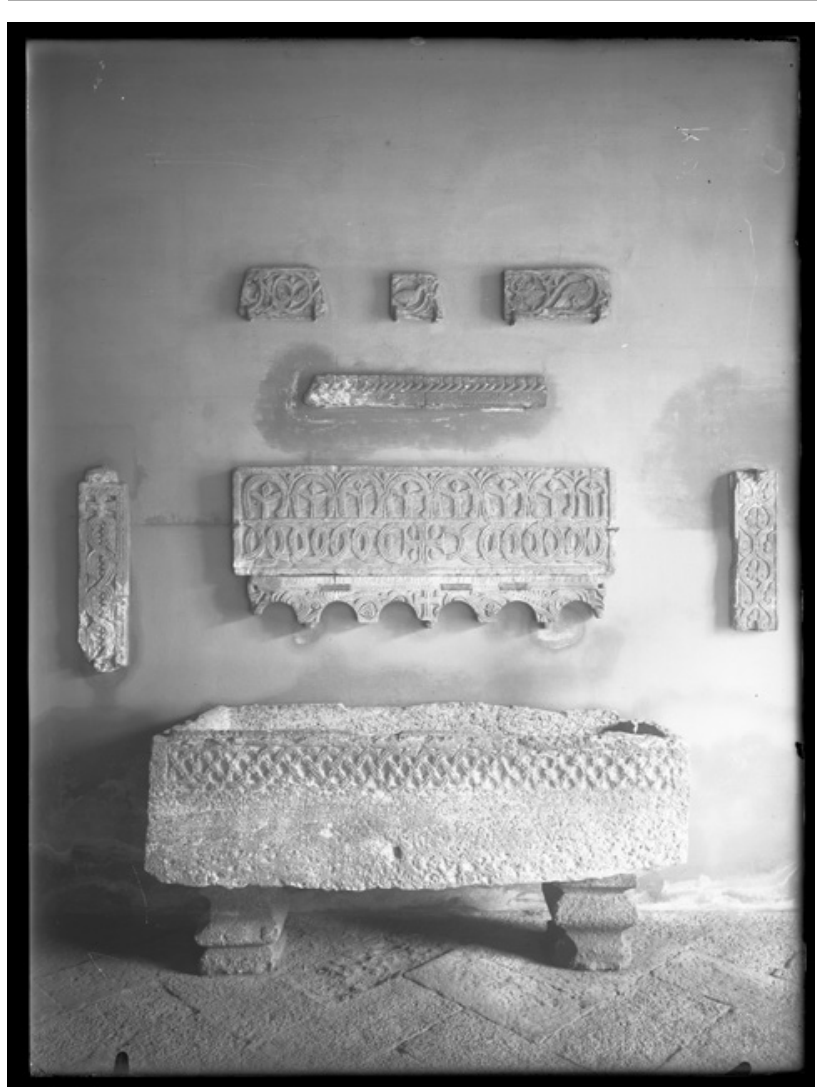

Figura 8 Murano, Museo Vetrario (già Museo Civico), allestimento dei pezzi medievali nel portico nel 1947. Venezia, Museo Correr, Archivio Fotografico, inv. M4095. 2020 @ Archivio Fotografico Fondazione Musei Civici di Venezia. Fotografia Cacco 1947 traforo, capitelli o fusti di colonna. [...] Ma non basta deplorare le giornaliere spogliazioni, commesse a danno di Venezia artistica, se non v'ha una legge che la tuteli. ${ }^{84}$

Lo stesso Ruskin alla fine del suo percorso sarà deluso, interrogandosi sulla reale possibilità di incidere sul rispetto per i monumenti. ${ }^{85}$

Ma - nonostante tutto - la dinamica di salvataggio di un patrimonio operata a inizio Ottocento dal Moschini nel creare la raccolta del Seminario ${ }^{86}$ diventa negli ultimi decenni di quel secolo prassi civica, attirando però anche gli attori del mercato di anticaglie che trovano un nuovo mercato.

Anche per iniziativa privata (come del resto era quella di Zanetti) si avviano nuove realtà museali: è il caso del Museo di Torcello. Quest'ultimo infatti nasce negli anni Settanta per l'impegno (volontario) di un privato - Nicolò Battaglini - che trova il sostegno del Prefetto di allora, Luigi Torelli (un non veneziano) ${ }^{87}$ che acquista a tal scopo un edificio e lo dona alla Provincia promuovendo l'istituzione del Museo Provinciale di Torcello che nelle intenzioni doveva documentare il territorio e la storia della Laguna nord. ${ }^{88}$

Battaglini raccoglie e convoglia materiali dalla Basilica di Santa Maria assunta e dall'isola, riceve da contadini e pescatori materiali erratici trovati in canali e barene; ${ }^{89}$ a questi primi nuclei si aggiungeranno sistematicamente materiali reperiti in scavi e restauri, ma anche doni, non

89 Il primo catalogo del Museo (Levi 1888) dà conto delle provenienze riprendendo esattamente le notizie dell'inventario stilato dal Battaglini man mano che i materiali entravano nella collezione (Agazzi 2002). 


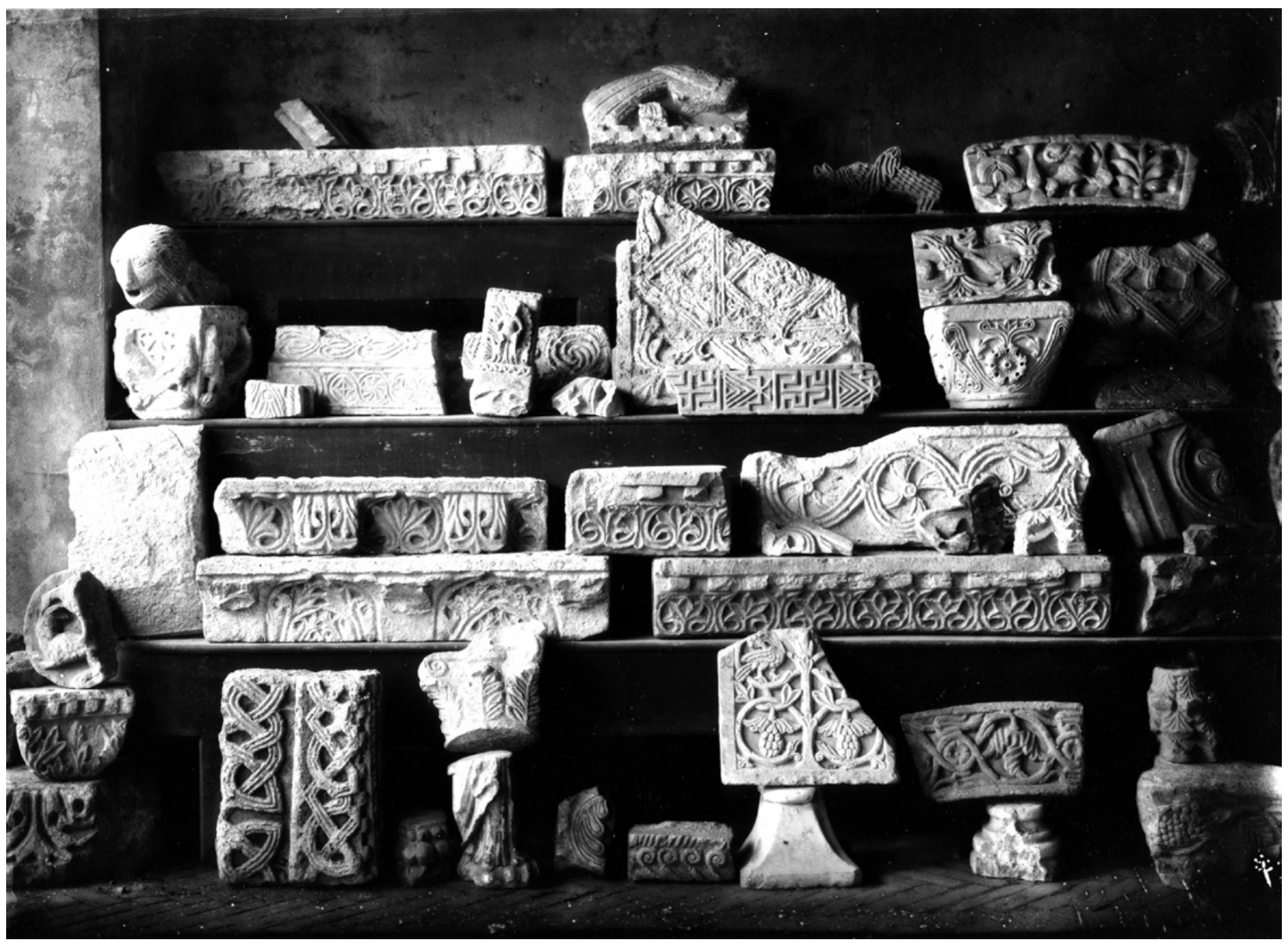

Figura 9 Torcello, Museo Provinciale, sculture medievali esposte nella loggia. Fotografia Naya Boehm 3732 
sempre di chiara pertinenza all'area. Fotografie di fine secolo illustrano l'esposizione a Torcello [fig. 9], nel portico loggiato a piano terra dei frammenti scultorei (alcuni perfettamente confrontabili con quanto arrivato in Prussia e non solo). ${ }^{90}$ Successivi ordinamenti e selezioni ai fini di rinnovate esposizioni (soprattutto nel 1930 il riordino di Callegari e uno successivo degli anni Settanta) ${ }^{91}$ hanno determinato la mancata fruizione al pubblico di una buona parte di essi, destinandoli al deposito. Se Polacco ha potuto comunque pubblicarne una parte, ${ }^{92}$ successivi lavori di catalogazione hanno consentito alcuni recuperi, ma hanno anche riscontrato ulteriori dispersioni. ${ }^{93}$ La difficoltà principale in cui versa attualmente il museo (passato ora alla Città metropolitana dopo l'abolizione delle Province) è quello dello spazio di deposito (curato e visitabile al momento, ma provvisorio). ${ }^{94} \mathrm{Nel}$ corso di 120 anni la parabola di questa istituzione sembra in discesa (se non verrà nuovamente posta al centro di un progetto che riguardi l'intero sito torcellano), così come scarsa attenzione è data ai reperti medievali del Museo di Murano. La maggior parte delle sculture raccolte negli anni Ottanta del XIX secolo per iniziativa dell'abate Zanetti è ora - pur- troppo - in deposito e al momento non accessibile. Anche la raccolta di sculture medievali dei Musei civici veneziani non gode di migliore situazione. In occasione del trasferimento dal Fondaco dei Turchi alla nuova sede nelle Procuratie di Piazza San Marco $0^{95}$ le sculture - già esposte nella grande corte e nel portico di facciata del Fondaco - ${ }^{96}$ vengono depositate al Museo archeologico nazionale ed esposte nei cortili. ${ }^{97}$ Studiate e catalogate da Polacco ${ }^{98}$ sono ora in gran parte in deposito, scomparse alla vista, oggetto di una dinamica opposta a quella che ne ha determinato la raccolta ed esposizione.

Si tratta di una rimozione fisica dettata spesso da necessità (come gli interventi di restauro alle pareti dei cortili delle procuratie), ma anche culturale, spia di un mutato peso dato a quei frammenti, relitti di una stagione che Ruskin ha contribuito a far conoscere e conservare, ma (forse) al momento non più percepiti come portatori di un valore, anche solo di testimonianza di un epoca, come intrinseca espressione di una civiltà che immetteva l'arte nei muri e nelle pietre che lo studioso inglese ha contribuito a far conoscere.

93 Oltre alla catalogazione informatizzata effettuata per la Provincia di Venezia a cura di Ennio Concina (cui ho partecipato): faccio riferimento alla schedatura dei materiali scultorei altomedievali a cura di chi scrive (con Devis Valenti, Giordana Trovabene e Myriam Pilutti Namer) per il Corpus della scultura altomedievale Diocesi di Torcello (c.d.s.).

94 Ringrazio Cecilia Casaril (Città Metropolitana di Venezia), responsabile del Museo di Torcello per le informazioni e per gli accessi al deposito.

95 Barizza 1988; Bassi, Ghirardi 2018.

96 Per la consistenza e la disposizione delle sculture: Museo Civico 1899.

97 Agazzi 1997, 117-18.

98 Polacco 1980. 


\section{Bibliografia di John Ruskin}

I riferimenti alle opere di Ruskin rinviano al numero di volume e alle pagine di:

Cook, E.T.; Wedderburn, A. (eds) (1903-1912). The Works of John Ruskin. Library Edition, 39 vols. London: George Allen.

https://www.lancaster.ac.uk/the-ruskin/the-complete-works-of-ruskin/.

Works, 3: Modern Painters I.

\section{Bibliografia generale}

Agazzi, M. (1997). «Reperti archeologici dell'area marciana: gli scavi del 1888-1889 e 1903-1905». Polacco, R. (a cura di), Storia dell'arte marciana: l'architettura = Atti del convegno internazionale di studi (Venezia, 11-14 ottobre 1994). Venezia: Marsilio, $105-22$.

Agazzi, M. (2002). «Un ciborio altomedioevale a Murano». Concina, E.; Trovabene, G.; Agazzi, M. (a cura di), Hadriatica. Attorno a Venezia e al Medioevo tra arti, storia e storiografia. Scritti in onore di Wladimiro Dorigo. Padova: Il Poligrafo, 43-53, 341-2.

Agazzi, M. (2005). «Sarcofagi altomedievali nel territorio del dogado veneziano». Quintavalle, A.C. (a cura di), Medioevo: immagini e ideologie = Atti del convegno internazionale di studi (Parma, 23-27 settembre 2002). Milano: Electa, 565-75.

Agazzi, M. (2006). «Laterizi torcellani». Trovabene, G. (a cura di), Florilegium artium. Scritti in memoria di Renato Polacco. Padova: Il Poligrafo, 23-35, 421-2.

Almanacco per le Province soggette all'Imperiale Regio Governo di Venezia per l'anno 1843. Venezia: Tip. F. Andreola.

Anderson, B. (2019). «The Prussian Tondo». Zorzi, N.; Berger, A.; Lazzarini, L. (a cura di), I tondi di Venezia e Dumbarton Oaks: arte e ideologia imperiale tra Bisanzio e Venezia. Roma: Viella, 35-49.

Andreescu Treadgold, I. (1990). «The Wall Mosaics of San Michele in Africisco, Ravenna Rediscovered». XXXVII Corso di cultura sull'arte ravennate e bizantina = Seminario internazionale di studi sul tema: "L'Italia meridionale fra Goti e Longobardi" (Ravenna, 30 marzo-4 aprile 1990). Ravenna: Edizioni del Girasole, 13-57.

Andreescu Treadgold, I. (2007). «I mosaici antichi e quelli ottocenteschi di San Michele in Africisco: lo studio Filologico». Spadoni, Kniffitz 2007, 113-41.

Andreescu Treadgold, I. (2013). «The Christ Head at the Metropolitan Museum of Art, New York, the Apse in the Bode Museum, Berlin, and Other Fake Mosaics». Entwistle, C.; James, L. (eds), New Light on Old Glass: Recent Research on Byzantine Mosaics and Glass. London: British Museum, 271-90.

Barizza, S. (1988). «Le sedi del museo: da casa Correr, al Fontego dei Turchi, alle Procuratie». Una città e il suo Museo. Venezia: Museo Correr, 291-8

Barral i Altet, X. (2006). «Ruskin deluso». Trovabene, G. (a cura di), Florilegium artium. Scritti in memoria di Renato Polacco. Padova: Il Poligrafo, 277-87.

Bassi, E.; Ghirardi, E. (2018). «La lunga strada verso casa: dal Fondaco dei Turchi a piazza San Marco, un nuovo volto per il Museo Civico Correr in seguito alla Grande Guerra». Bollettino dei Musei Civici Veneziani, 3(13), 133-8.

Battaglini, N. (1873). Il Palazzo del Consiglio di Torcello, proprietà del nob. sig. Luigig comm. Torelli... ristaurato dal cav. Nicolo Battaglini. Venezia: Tip. di Marco Visentini.

Bernardello, A. (2012-2013). «Note archivistiche sul mercato antiquario a Venezia (1815-1840). Un programma di ricerca». Atti IVSLA, t. $171,171-9$.

Bode, W. von (1881). «Die italienischen Skulpturen der Renaissance in den Königlichen Museen zu Berlin». Jahrbuch der Königlich Preussischen Kunstsammlungen, 2, 69-78.

Boni, G. (1887). «Coccodrilli archeofaghi». Venezia imbellettata. Roma, 25-8.

Bortoletto, M. (2014). «Breve storia degli scavi archeologici realizzati nell'isola di Torcello». Fozzati, L. (a cura di), Torcello scavata: patrimonio condiviso. Vol. 1, Gli scavi 1995-2012. [Venezia]: Regione del Veneto, 23-51.

243 Fonti, letterature, arti e paesaggi d'Europa S Sources, Literatures, Arts \& Landscapes of Europe 1

Fonti, letterature, arti e paesaggi d Europa Sources, Ltteratures, An 
Bullen, J.B. (2003). Byzantium Rediscovered. The Byzantine Revival in Europe and America. London; New York: Phaidon.

Callegari, A. (1930). Il Museo provinciale di Torcello. Venezia: Stamperia Zanetti.

Cavazzana Romanelli, F. (2019). «Un rimpianto lungo cent'anni. Archivi, storia, erudizione nell'Ottocento veneziano», Giorgi, A.; Moscadelli, S.; Varanini, G.M.; Vitali, S. (a cura di), Erudizione cittadina e fonti documentarie. Archivi e ricerca storica nell'Ottocento italiano (18401880). Firenze: Reti Medievali - Firenze University Press, 417-28. http://www.rm.unina.it/rmebook/index.php?mod=none_.... Cecutti, D. (2012). «Adolph Loewi e il commercio di tappeti orientali a Venezia fra Otto e Novecento». MDCCC 1800, 1, 33-42.

Cicogna, E.A. (1842). Delle iscrizioni veneziana raccolte ed illustrate, vol. 5. Venezia: G. Orlandelli.

Cicognara, L.; Diedo, A.; Selva, G.A. (1858). Le fabbriche e i monumenti cospicui di Venezia, vol. 2. Venezia: G. Antonelli.

Corner, F. (1749). Ecclesiae Torcellanae antiquis monumentis nunc etiam primum editis illustratae. Venetiis: Typis Jo. Baptistae Pasquali.

Di Lenardo, L. (a cura di) (2014). La collezione epigrafica del Seminario patriarcale di Venezia: catalogo (secoli 12-15). Venezia: Marcianum Press.

Dorigo, W. (2003). Venezia romanica: la formazione della città medioevale fino all'età gotica. Venezia: Istituto veneto di scienze, lettere ed arti; Cierre Edizioni.

Effenberger, A.; Severin, H.-G. (Hrsgg) (1992). Staatliche Museen zu Berlin. Das Museum fur Spätantike und Byzantinische Kunst. Mainz am Rhein: Philipp von Zabern Verlag.

Ferraro, G. (2019). s.v. «Torelli Luigi». Dizionario Biografico degli Italiani, vol. 96.

Geismeier, I. (1980). «Gustav Friedrich Waagen: 45 Jahre Museumsarbeit». Forschungen und Berichte, Bd. 20, $397-419$.

Geismeier, I. (1995). «Gustav Friedrich Waagen: Museumsdirektor in der Preussischen Hauptstadt». Jahrbuch der Berliner Museen, N.F. 37, 7-21.

Goethert, F.W. (1972). Katalog der Antikensammlung des Prinzen Carl von Preussen im Schloss zu Klein-Glienicke bei Potsdam. Mainz am Rhein: Verlag Philipp von Zabern.

Favaretto, I. (1984). «Una tribuna ricca di marmi. Appunti per una storia delle collezioni dei Grimani a Santa Maria Formosa». Aquileia Nostra, 55, 205-40.

Hanley, K.; Sdegno, E. (2010). Ruskin, Venice and Nineteenth-Century Cultural Travel. Venezia: Cafoscarina.

Hanley, K.A.; Hull, C.S. (eds) (2016). John Ruskin's Continental Tour 1835: The Written Records and Drawings. Cambridge: Legenda, Modern Humanities Research Association.

Heilmeyer, W.-D. (1995). «Erwerbungen klassischer Antiken durch Waagen in Italien 1841/42». Jahrbuch der Berliner Museen, 37, $39-45$.

Hoffmann, V. (1999). (a cura di). Santa Sofia ad Istanbul. Sei secoli di immagini e il lavoro di Gaspare Fossati (1847-1849) = Catalogo della mostra (Mantova, 14 novembre-31 dicembre 1999). Berna: Università di Berna.

Irmscher, J. (1987). «Zur Geschichte der Frühchristlich-byzantinischen Sammlung». Forschungen und Berichte / Staatliche Museen zu Berlin, 26, 75-80.

Lampertico, F. [1888] (2011). «Commemorazione del sen. Luigi Torelli letta al R. Istituto Veneto di Scienze Lettere ed Arti il di 12 agosto 1888». Atti del R. Istituto veneto di scienze lettere ed arti, t. 6, s. 6.

Lampertico, Fedele (2011). «Commemorazione di Luigi Torelli (1810-1887)». Marangoni, M. (a cura di), Da Palazzo Ducale a Palazzo Loredan (1843-1891). Vol. 1, Commemorazioni dei soci effettivi 1843-2010. Venezia: Istituto veneto di scienze, lettere ed arti, 617-54.

Lecomte, J. (1844). Venise, ou Coup-d'œil littéraire, artistique, historique, poétique et pittoresque, sur les monuments et les curiosités de cette cite. Paris: H. Souverain.

Levi, C.A. (1888). Catalogo degli oggetti di antichità del Museo Provinciale di Torcello, con brevi notizie dei luoghi e delle epoche di ritrovamento. Venezia: Tip. Ferrari.

Manuale (1845) = Manuale per le provincie soggette all'Imperiale regio governo di Venezia per l'anno 1845. Venezia: presso Francesco Andreola tipografo guberniale.

Massaro, M. (2013). «Giacomo Treves de' Bonfili (1788-1885): collezionista e imprenditore». Ateneo veneto, 1, 501-11.

Meyer, S.A. (2014). «Epoche, nazioni, stili (1815-1871)». Rossi Pinelli, O. (a cura di), La storia delle storie dell'arte. Torino: Einaudi, $180-238$.

Moine, C. (2013). Chiostri tra le acque: i monasteri femminili della Laguna nord di Venezia nel Basso Medioevo. Borgo S. Lorenzo: All'insegna del Giglio. 


\section{Michela Agazzi}

Museo Civico e Raccolta Correr, Elenco degli oggetti esposti (1899). Venezia: Tip. Ferrari.

Museo di Torcello: Sezione medioevale e moderna [S.l.: s.n.], stampa 1978. Venezia: Tipo-lito Armena.

Moschini, G. (1842). La Chiesa e il seminario di S.ta Maria della Salute in Venezia, descritti da Giannantonio Moschini. Venezia: G. Antonelli.

Nehls, H. (1991). «Der Altertums forscher Nicolaus Johann Heinrich Benjamin Freiherr Menu von Minutoli (1772-1846)». Forschungen und Berichte/Staatliche Museen zu Berlin, 31, 159-68

Nelson, R.S. (2004). Hagia Sophia, 1850-1950: Holy Wisdom Modern Monument. Chicago: University of Chicago Press.

Ottani Cavina, A. (a cura di) (2018). John Ruskin: le pietre di Venezia. Venezia: MUVE; Marsilio.

Pilutti Namer, M. (2013a). «Mastro di Palazzo Ducale, prima che archeologo: Giacomo Boni e la Venezia dell’Ottocento». Failla, M.B.; Meyer,

S.A.; Piva, C.; Ventra, S. (a cura di), La cultura del restauro: modelli di ricezione per la museologia e la storia dell'arte =Atti del Convegno Internazionale (Roma, Museo Nazionale Romano di Palazzo Massimo alle Terme, Università La Sapienza, 18-20 aprile 2013). Roma: Campisano, 581-593.

Pilutti Namer, M. (2013b). «Ruskin e gli allievi: note su Giacomo Boni e la cultura della conservazione dei monumenti a Venezia a fine Ottocento». Barral i Altet, X., Gottardi, M. (a cura di), La storia dell'arte a Venezia ieri e oggi: duecento anni di studi = Atti del convegno (Venezia 5-6 novembre 2012). Venezia: Ateneo Veneto, 423-35.

Pilutti Namer, M. (2015). «'Spolia’ a Venezia nell’Ottocento: Giacomo Boni e i ‘Coccodrilli archeofaghi’». Centanni, M.; Sperti, L. (a cura di), Pietre di Venezia: spolia in se, spolia in re = Atti del convegno internazionale (Venezia, 17-18 ottobre 2013). Roma: “L'Erma” di Bretschneider, 211-218.

Pilutti Namer, M. (2016). Spolia e imitazioni a Venezia nell'Ottocento: il Fondaco dei Turchi tra archeologia e cultura del restauro. Venezia: Istituto veneto di scienze lettere ed arti.

Polacco, R. (1976). Sculture paleocristiane e altomedioevali di Torcello. Treviso: Marton.

Polacco, R. (1980). Marmi e mosaici paleocristiani e altomedievali del Museo archeologico di Venezia. Roma: G. Bretschneider.

Polacco, R. (1994). «ll mosaico absidale della chiesa dei Santi Cornelio e Cipriano di Murano ora a Potsdam». Venezia Arti, 8, 5-20.

Polacco, R. (1995). «ll mosaico absidale della chiesa dei SS. Cornelio e Cipriano di Murano ora a Potsdam». Seminario Internazionale sul Tema "Ricerche di Archeologia Cristiana e Bizantina" (Ravenna, 14-19 maggio 1995). Ravenna: Edizioni del Girasole, $771-87$.

Ramelli, S. (2000). Murano medievale: urbanistica, architettura, edilizia dal 12. al 15. Secolo. Padova: Il Poligrafo.

Rizzi, A. (1981). Vere da pozzo di Venezia: i puteali pubblici di Venezia e della sua laguna. Venezia: La Stamperia di Venezia.

Rizzi, A. (1982). «Sui falsi erratici di Venezia». Ateneo veneto, n.s. 20(1/2), 309-17.

Rizzi, A. (1987). Scultura esterna a Venezia: corpus delle sculture erratiche all'aperto di Venezia e della sua laguna. Venezia: Stamperia di Venezia.

Ronchey, S. (2004). «Bisanzio continuata. Presupposti ideologici dell'attualizzazione di Bisanzio nell'età moderna». Cavallo, G. (a cura di), Le culture circostanti: la cultura bizantina. Vol. 3.1 di Lo spazio letterario del Medioevo. Roma: Salerno editrice, 691-728.

Ruskin, J. (1874). The Sea-Stories. Vol. 2 di The Stones of Venice. London: Smith, Elder and Co.

Schoenemann, H. (2001). «Architettura e urbanistica». Gert Streidt, G.; Feierabend, P. (a cura di), Prussia: arte e architettura. Colonia: Koenermann, 272-334.

Seguso, L. (1859). Delle sponde marmoree e degli antichi edifizii della Venezia marittima, disegni di Angelo e illustrazioni di Lorenzo Seguso, periodo arabo-bizantino secc. IX-XII. Venezia: Tip. del commercio.

Spadoni, C.; Kniffitz, L. (a cura di) (2007). San Michele in Africisco e l'età giustinianea a Ravenna = Atti del Convegno La Diaspora dell'Arcangelo, San Michele in Africisco e l'Età Giustinianea; giornate di studio in memoria di Giuseppe Bovini (Ravenna, 21-22 aprile 2005). Cinisello Balsamo: Silvana ed.

Świechowski, Z.; Rizzi, A.; Hamann-Mac Lean, R. (1982). Romanische Reliefs von venezianischen Fassaden: "Patere e formelle". Wiesbaden: Steiner.

Tomasi, M. (2004). «Falsi e falsari». Castelnuovo, E.; Sergi, G. (a cura di), Arti e storia nel medioevo. Vol. 4, Il Medioevo al passato e al presente. Torino: Einaudi, 871-88.

Tomasin, L. (2012). «Minima muralia: esercizio di epigrafia volgare medievale». Vox Romanica, 71, 1-12.

245 Fonti, letterature, arti e paesaggi d'Europa | Sources, Literatures, Arts \& Landscapes of Europe 1

John Ruskin's Europe A Collection of Cross Curtural Essays, 223246 
Tüskés, A. (2010). «Deux sculpture vénitiennes dans les collections du musée du Louvre». La Revue des Musées de France. Revue du Louvre, 60(4), 36-45.

Tüskés, A. (2013a). «La storiografia delle vere da pozzo veneziane». Barral i Altet, X.; Gottardi, M. (a cura di), La storia dell'arte a Venezia ieri e oggi: duecento anni di studi = Atti del convegno (Venezia 5-6 novembre 2012). Venezia: Ateneo Veneto, 265-76.

Tüskés, A. (2013b). «Mercanti veneziani e Wilhelm von Bode». Tüskés, A. (ed.), Hungary in Context: Studies on Art and Architecture. Budapest: CentrArt, 145-63.

Tüskés, A. (2017). «Three Castles - Ten Venetian Well-heads». Arte veneta, 74, 154-8.

Vecchi, M. (1995). Sculture tardo-antiche e alto-medievali di Murano. Roma: G. Bretschneider.

Volbach, W.F. (1930). Mittelalterliche Bildwerke aus Italien und Byzanz. Berlin: De Gruyter.

Allgemeine Deutsche Biographie (1896). s.v. «Waagen Gustav Friedrich», Bd. 40. Leipzig, 410-14.

Wulff, O. (1904). «Das Ravennatische Mosaik von S. Michele in Affricisco im Kaiser Friedrich-Museum». Jahrbuch der Königlich Preussischen Kunstsammlungen, 25, 374-401.

[Zanetti, V.] (1861). Dell'instituzione di un archivio comunale e di un museo nell'isola di Murano. Venezia: P. Naratovich.

Zanetti, V. (1873). La basilica dei Santi Maria e Donato di Murano. Venezia: Tip. munic. di G. Longo.

Zanetti, V. (1876). «Di una vera o sponda marmorea di un pozzo di stile arabo-bizantino esistente in Murano». Archivio Veneto, 11, 91-101.

Zanetti, V. (1878). «Le grandi lastre di marmo greco nel pavimento tessulare della basilica dei SS. Maria e Donato di Murano». Archivio Veneto, 16, 319-24.

Zanetti, V. (1881). Il museo civico vetrario di Murano. Venezia: G. Longo.

Zavatta, G. (2019). Raffaello, la Madonna Diotallevi. La vicenda storico-critica. Rimini: Agenzia NFC.

Zorzi, A. [1972] (2001). Venezia scomparsa. Milano: Mondadori.

Zorzi, N.; Berger, A.; Lazzarini, L. (a cura di) (2019). I tondi di Venezia e Dumbarton Oaks: arte e ideologia imperiale tra Bisanzio e Venezia. Roma: Viella.

Zuchold, G.-H. (1984). Byzanz in Berlin: der Klosterhof im Schlosspark Glienicke. Berlin: Presse- und Informationsamt des Landes.

Zuchold, G.-H. (1993). Der "Klosterhof" des Prinzen Karl von Preußen im Park von Schloß Glienicke in Berlin. Bd. 1, Geschichte und Bedeutung eines Bauwerkes und seiner Kunstsammlung. Berlin: Gebr. Mann.

Zuchold, G.-H. (2010). «Friedrich Wilhelm IV. und das byzantinische Gott-Königtum. Seine Kirchenentwürfe als Modell einer "Kirche der Zukunft" in Preußen». Zeitenblicke, 9(3). http://www.zeitenblicke.de/2010/3/Zuchold/index_html. 


\title{
John Ruskin and His 'Witch of Sicily', Amy Yule
}

\author{
Stephen Wildman \\ Emeritus Professor of History of Art, Lancaster University, UK
}

\begin{abstract}
John Ruskin's visit to Sicily in the spring of 1874 is largely neglected in the main biographies and generally regarded as a mere adjunct to the more significant purpose of his trip to Italy in that year, studying the work of Botticelli and other Old Masters first in Florence and at Assisi, and then in Rome. The ten-day break from study between 20 and 30 April, apparently at the casual invitation of slight acquaintances, Colonel and Mrs Henry Yule, then living in Palermo, has tended to be seen as an unimportant interlude, even though it does represent the furthest point in Europe (and therefore the world) to which he ever travelled. Recent research has uncovered a good deal more about his hosts in Palermo, and especially their daughter Amy, who begins to emerge as one of the forgotten, but not least important, figures in Ruskin's later personal life.
\end{abstract}

Keywords John Ruskin. Yule family. Travel. Sicily. Palermo.

Writing to Ruskin on 15 January 1867, Margaret Bell, Headmistress of Winnington School, Cheshire, where he was a patron and regular visitor between 1859 and 1868, referred to a "most interesting letter" received from Mrs Yule "about a year ago", promising "to send me her daughter's photograph - since which time I have heard nothing. Where is she now?". ${ }^{1}$ From this the inference has been drawn that Amy Frances Yule, only child of Colonel Henry and Mrs. Anna Maria Yule, had been a pupil at the School, and that this must have provided the connection. Four brief references to 'Amy' in letters from Ruskin to Miss Bell in May 1865 seem to corroborate this, although Van Burd, in a footnote in the Winnington Letters, cautiously points out that there was another pupil called Amy there in the early 1860s (Amy Webster). ${ }^{2}$ Ruskin may have hoped that Amy Yule would become a pupil, perhaps even offering financial support, but this can only remain speculation without further evidence.

\footnotetext{
1 Burd 1969, 599.

2 Burd 1969, 548
} 
By her own account this was not where she and Ruskin first met. We have Ruskin's word that Amy was a lively correspondent - hers were "the loveliest letters" he ever received, he told his close friend Susan Beever ${ }^{3}$ - but only two seem to survive (in the Rylands Library at Manchester University and the Houghton Library at Harvard). ${ }^{4}$ In the earlier of these, dated 26 November 1868, Amy writes from Palermo, in a frank and flirtatious way, teasing Ruskin by correcting his impression that she was nineteen:

I have so much to learn in many ways before I can carry off with dignity the grandeur of nineteen years. I am only seventeen, having been ten when I first saw you at Geneva, nearly seven years ago. ${ }^{5}$

She was indeed born in London in 1852 (and christened at Holy Trinity, Paddington, on 17 November), which fixes such a meeting at the time of the winter of 1862-63 which Ruskin spent in Switzerland.

Amy herself is even more helpful, in a remarkable aside and footnote buried within a biographical memoir of her father, perhaps not surprisingly hitherto overlooked in the Ruskin literature. Henry Yule was born in Inveresk, near Edinburgh, in 1820 - just a year younger than Ruskin - and enjoyed a successful career as an army man in India, seeing active service in the Anglo-Sikh wars of 1845-46 and 1848-49, and forming friendships with the successive governors-general Lords Dalhousie and Canning. A writer of extensive reports, as well as A Narrative of the Mission to Ava [Burma] (1858), he retired from the army in 1862, thereafter devoting himself to historical geography. Apart from co-authoring Hob- son Jobson (1886), the publication for which he is still remembered is an edition of The Travels of Marco Polo, published in 1871, and it is in a later version of this - the so-called Yule-Cordier Edition of 1903 - that Amy's extremely detailed memoir appears, dated 1902. Speaking of his return from India, she writes:

Nor did Yule [her father] find any suitable opening for employment in England, so after two or three months spent in visiting old friends, he rejoined his family in the Black Forest, where he sought occupation in renewing his knowledge of German. But it must be confessed that his mood both then and for long was neither happy nor wholesome. The winter of 1862 was spent somewhat listlessly, partly in Germany and partly at the Hotel des Bergues, Geneva, where his old acquaintance Colonel Tronchin was hospitably ready to open all doors. The picturesque figure of John Ruskin also flits across the scene at this time. But Yule was unoccupied and restless, and could neither enjoy Mr. Ruskin's criticism of his sketches nor the kindly hospitality of his Genevan hosts.

On rejoining his wife and child at Mornex in Savoy, Yule found the health of the former [his wife] seriously impaired. During his absence, the kind and able English doctor at Geneva had felt obliged to inform Mrs Yule that she was suffering from disease of the heart, and that her life might end suddenly at any moment. Unwilling to add to Yule's anxieties, she made all necessary arrangements, but did not communicate this intelligence until he had done all he wished and returned, when she broke it to him very gently. Up to

3 Ruskin to Susan Beever, Assisi, 14 April 1874; Works, 37: 94.

4 The letter at Harvard (Houghton Library, Yule papers) is dated 7 November 1871 and is chiefly of interest in confirming regular correspondence: "When I go up the mountain [Etna], I will peep over the edge of the Crater, and shall perhaps find vast accumulations of singed paper lying about with "dear Amy" on one, and "I am satisfied you never get my letters" on another".

5 Rylands Library, Manchester University, EngMS 1258/74. 
this year Mrs Yule, though not strong and often ailing, had not allowed herself to be considered an invalid, but from this date doctor's orders left her no choice in the matter. ${ }^{6}$

This last passage has implications for Ruskin's relationship with Amy, considered later; the footnote she provides here is of great interest:

I cannot let the mention of this time of lonely sickness and trial pass without recording here my deep gratitude to our dear and honoured friend, John Ruskin. As my dear mother stood on the threshold between life and death at Mornex that sad spring, he was untiring in all kindly offices of friendship. It was her old friend, Principal A.J. Scott (then eminent, now forgotten), who sent him to call. He [Ruskin] came to see us daily when possible, sometimes bringing MSS of Rossetti and of others to read aloud (and who could equal his reading?), and when she was too ill for this, or himself absent, he would send not only books and flowers to brighten the bare room of the hillside inn (then very primitive), but his own best treasures of Turner and W. Hunt, drawings and illuminated missals. It was anxious solace; and though most gratefully enjoyed, these treasures were never long retained. ${ }^{7}$

Revelations indeed: details of Ruskin's activities at Mornex are frustratingly few, nor has it been previously noted that he had taken so many of his favourite possessions to comfort him - illuminated manuscripts as well as watercolours by Turner and William Henry Hunt. The connection with Alexander (known as Sandy) Scott is also informative: "theological dissident and educationist", as he is described in the Dictionary of National Biography, he was the first Principal of Owens College (the forerunner of Manchester University) and a friend of Thomas Carlyle, F.D. Maurice and George MacDonald. He also nearly became the first editor of the Cornhill Magazine, declining the appointment at a late stage in favour of Thackeray (and thereby narrowly avoiding becoming the editor of Unto this Last). Ill health was a factor in this, and it was on a holiday on Lake Geneva that he died in January 1866, aged 60 . Teasing out these interrelationships, it is perhaps most likely that Ruskin knew him through George MacDonald, and that Scott knew from him that Ruskin could provide comfort to the Yules at Mornex in 1862.

The letter of 1869 from Amy was written from Palermo, where the Yules had pitched up in October 1864 (not 1863, as the Library Edition has it). In the years since the meeting with Ruskin at Mornex they had made a peregrination full of Ruskinian echoes: via Chambéry to Turin and Genoa, Bagni di Lucca, Venice and Verona. One can only wonder how much this itinerary might have owed to Ruskin's recommendations. From Palermo, Yule published Cathay and the Way Thither in 1866, and began work on his great edition of Marco Polo. Amy gives a description of his daily routine:

It was his custom to rise fairly early; in summer he sometimes went to bathe in the sea, or for a walk before breakfast; more usually he would write until breakfast, which he preferred to have alone. After breakfast he looked through his notebooks, and before ten o'clock was usually walking rapidly to the library where his work lay. He would work there until two or three o'clock, when he returned home, read the Times, answered letters, received or paid visits, and 
then resumed work on his book, which he often continued long after the rest of the household were sleeping. Of course his family saw but little of him under these circumstances, but when he had got a chapter of Marco into shape, or struck out some new discovery of interest, he would carry it to his wife to read. She always took great interest in his work, and he had great faith in her literary instinct as a sound as well as sympathetic critic. ${ }^{8}$

This, then, was the background for the intelligent seventeen-year-old whose letter of 1869 told of her obvious frustration, trapped between an invalid mother and a Casaubon-like father with his head buried in tales from Tartary. "You ask about my studies", she wrote, telling Ruskin of her knowledge of Italian and French, then of her instinct for - of all things - Geology, even encouraged by her mother to take "lessons from the Geologist lecturer at this University". This may have derived from being a kinswoman of Sir Roderick Murchison, twice President of the Geological Society. No wonder that Ruskin had apparently, in 1865, offered to "bring her up". The only other known letter from Amy - fleetingly cited by Van Burd in a footnote - is of January 1905, when at the age of 52 she sent a curiously belated letter of condolence to Joan Severn, Ruskin's cousin and only close relative.

That most generous offer of 1865 was infinite comfort to my mother and me at a time when comfort was sorely needed. I would not willingly say anything that should ever seem to cast reflection on my Father, but with all his fine qualities, he was not a man intended, I should think, for the parental role. Also he certain- ly had - even to the very end of his life - an almost morbid dislike to facing unwelcome facts or looking ahead. So my brave little mother had for the most part to bear her burdens alone, and I am afraid - indeed I know - that she spent many, many weary night-watches anxiously thinking out the future (the future she knew she would not see) of her undeveloped slowcoach. For 'slow-coach' I was and still am. ${ }^{9}$

So we see that no longer is Ruskin's invitation to visit Palermo something deriving from mere casual acquaintance. He was reinvigorating one of those alarmingly passionate friendships with bright young women, in this instance one whom he might once have considered a possible ward, perhaps an adopted daughter, or in the context of 1874, and her age, which he thought to be nineteen, even a possible rival in his affections to Rose La Touche, who had dismissed him from her life in the summer of 1872.

He expressed mixed feelings in a letter written to Susie Beever from Assisi on 14 April, complaining of having to break off work to go to Rome, then Naples.

My witch of Sicily expects me this day week, and she's going to take me [on] such lovely drives, and talks of 'excursions' which I see by the map are thirty miles away. I wonder if she thinks me so horribly old that it's quite proper. It will be very nice if she does, but not flattering. I know her mother can't go with her; I suppose her maid will. If she wants any other chaperone I won't go. She's really very beautiful, I believe, to some people's tastes, (I shall be horribly disappointed if she isn't, in her own dark style,) and she writes, next to Susie, the loveliest letters I ever get. ${ }^{10}$

\section{Memoir of Sir Henry Yule, 1: lx}

9 Memoir of Sir Henry Yule, 1: lx. Amy Yule to Joan Severn, 12 January 1905; Morgan Library, New York, MA 3451. At the time of Burd's reference the letter was in the collection of Helen Viljoen (Burd 1969, 549).

10 Ruskin to Susan Beever, Assisi, 14 April 1874 (Works, 37: 94). 
A very similar missive, also dated 14 April, was sent to Joan Severn, enclosing a "pretty" letter "from my Sicilian witch"11 Jeanne Clegg rightly identifies 'the witch of Sicily' as Circe of classical legend, the enchantress of whom Ruskin had written in Munera Pulveris - "no daughter of the Muses, but of the strong elements, Sun and Sea"12 - but this was not necessarily his invented nickname for Amy. That letter of 1869 had ended "Ever your affectionate little old friend, Amy" - and here one can't help but imagine a giggle and a twinkling eye - "'little old' sounds very grand, like a witch-fairy in a story book". Was she really so worldly wise, acting the enchantress and knowing exactly how to stimulate her potential rescuer's imagination? Maybe not: this was also the exact date of George MacDonald's fairy tale At the Back of the North Wind (serialized between November 1868 and November 1870), a more likely - and more innocent - source of such a soubriquet.

His companion and courier on this trip, a "delightful old German" named Klein - Frederick Crawley seems to have been left at Naples - fought for the luggage while Ruskin observed the sails of the local boats,

such jaunty little things ... just like the sprucest little thin-winged moths, with their wings all fresh-brushed and combed..$^{13}$

He had written ahead asking Amy for rooms to be arranged at the Hotel de France, apparently in the old Cala port area of the city, but it would seem that this letter was on the same boat, so there was no word from the Yules, who may not have known exactly when to expect him. First impressions of Palermo were not favourable: "A town built of large stones of the colour of mud; with an iron curled balcony to every window, and everybody's shirts - chemises - petticoats - and bedclothes hung out over them to dry". A quick visit followed to the Cathedral - also "the colour of mud" - to which he would return.

Then I asked for the 'English garden' which, when I arrived at it, down a street twice as long as the largest in Turin, I found to be about the size of Hyde Park; and that the English trees of which it consisted were chiefly palms, Indian fig, orange and lemon, the manna tree, the pepper tree, here and there a little sprig of Indian rubber and castor oil and stone pines.

This piece of sightseeing might seem an odd choice, were it not that the Giardino Inglese was the home address of the Yule family - presumably a house within the gardens..$^{14}$

Acquiring a carriage and a 'valet de place' - we hear no more of Klein in the letters - Ruskin also found (presumably in the hotel) a 'commissioner' who knew Colonel Yule. Up at half past five on Wednesday 22nd, he was annoyed to find "smoke fog over all the hills, looking exactly like the atmosphere of a summer's morning in Euston Square".

I am in a terribly bad humour, the black cold wind having actually pursued me here, - the only difference from London being the scorching and dangerous heat in the middle of the day. ${ }^{15}$ 
Henry Yule called first thing - closely followed by the British Consul, to the consternation of the hoteliers, "quite appalled at my sublimity". In 1874 this was George Dennis, diplomat and archaeologist, author of The Cities and Cemeteries of Etruria (1848) and Murray's Handbook for Travellers in Sicily (1864). It is hard to imagine Ruskin being unaware of this, and it also transpires that Dennis had the friendship and professional support of Charles Newton, Keeper of Antiquities at the British Museum, fellow student with Ruskin at Christ Church, Oxford, and brother-in-law of Arthur Severn, Joan's husband. ${ }^{16}$ It is probable that the Consul was not merely paying his respects to a distinguished visitor.

Before the day was out Ruskin had made his first excursion with Amy to Monreale, with permission to take her to Messina, if he liked ... "I don't like at all taking Amy to Messina", he told Joan, "another long tiresome twelve hours of steamer, instead of getting to my work". ${ }^{17}$ But this would be the only practical way of seeing Sicily's finest sights, Mount Etna and Taormina, given the condition and safety of Sicilian roads (banditry being rife well into the 1890s). A gloss on this is provided by an account of life in Palermo in 1881 written by Mary Paley Marshall, wife of the Cambridge economist Alfred Marshall. As she recalled,

those were the days of brigands, so one was restricted to the town for walks. It was indeed possible to go to Monreale three miles off, for armed police were stationed all the way within shouting distance, but in every other direction there was a chance that one might be briganded and a piece of ear forwarded to friends with a message that more would follow unless a large ransom were paid..$^{18}$

Amy herself, or at least her situation, Ruskin found rather troubling.

I am grieved ... by finding much that is wrong, in Amy's position, towards her father, who seems to me to deserve better of her. ... I shall give Miss Amy a lecture on behaviour to parents - in the plural. ${ }^{19}$

Of their excursion to Monreale, just a few miles south of Palermo, he says little, remarking more on the flora and fauna - "my goodness, how the lizards flashed among the rocks" - than of the great twelfth-century Cathedral with its delightful Gothic cloister and vast Byzantine mosaics. In these he noticed just two things: the blue iris growing wild on the rocks outside appearing within the decoration of the arches, and the subject of "The King presenting the temple to the Virgin - an angel sustaining it, flying forward underneath". Despite the bad humour he had confessed to Joan that

I have learned three or four inestimable truths, by coming to Sicily.

First - and not least - not to despise even the worst darkness of England - or storms of Coniston - as evils of a baser climate. That accursed wind takes them all over the earth, and the orange groves are all blight-

16 George Dennis (1814-1898), Charles Newton (1816-1894); a plaster cast of the bust of Newton by J.E. Boehm was presented to the National Portrait Gallery by Amy Yule in 1895.

17 Ruskin to Joan Severn, 22 April 1874; Clegg 1986, 125

18 Marshall 1947, 33-4.

19 Ruskin to Joan Severn, 22 April 1874; Clegg 1986, 125.

Fonti, letterature, arti e paesaggi d'Europa | Sources, Literatures, Arts \& Landscapes of Europe $1 \mid$ 
ed here, by the same storms that made me ill passing the Cenis.

Secondly, I gathered today the small blue iris, wild, on the rocks of Monreale, within half a mile of the Greek mosaics which represent it - and am now certain - matching the flower fresh gathered with the visible sea beyond Palermo - that Homer's violet-coloured sea, meant - as I have said it did, iris-coloured, and that the hitherto called 'violet' crocus of the Greeks were of this flower - the blue fleur-de-lys.

Thirdly. In the dustiest streets of Palermo, or, at least, as dusty as well could be, I saw a group of serious players at bowls - but the bowls were - oranges! I thought this a very characteristic bit of street scene.

Fourthly, I've seen Indian fig [prickly pear] in perfection as a rock plant - covering heights like Yewdale crag with masses of its blue-green leaves, fixed like painted bronze.

Fifthly, I've seen the tomb of Frederick II, and knelt at it! and am going to draw it to-morrow - God willing. ${ }^{20}$

On Thursday 23 April - St George's Day, as "Amy reminded me in time" - Ruskin kept his appointment with the Royal Tombs in Palermo Cathedral, managing a number of studies despite the distraction of a disfigured Cathedral menial and a young priest sitting behind him - an anecdote related in a later Oxford lecture (Ariadne Florentina). ${ }^{21}$ He may have begun the fine watercolour forming part of his teaching collection at Oxford and later presented to the Ashmolean Muse- um, probably finishing it on his return a few days later, on the 29 th.

He saw Amy again on St George's Day, perhaps on the drive he notes in his diary "to glorious view in evening, past Moorish bridge". ${ }^{22}$ He learned that she was a good swimmer, "and can fence, and shoot", but was shocked to learn that her mother,

of all the odd mothers ... never would let Amy read the Bible! Amy enquired timidly about it of me - just as I should of a Turk about the Koran - but she made Amy learn to swim - to fence - and the musket exercise! $!^{23}$

This may have had something to do with Mrs Yule's experience of being in Palermo when it was bombarded by the Italian fleet during a local insurrection in 1866: "cheerfully remarking that 'every bullet has its billet", Amy noted in her memoir, "she remained perfectly serene and undisturbed". ${ }^{24}$ That it was St George's Day caused Ruskin's mind to wander back to another St George, the Scuola di San Giorgio degli Schiavoni in Venice, which he left in 1872 to hasten back to England to see Rose La Touche. "If I had not let Connie \& Mrs Hilliard delay me on the Simplon", he mused to Joan, "when I left St George's Chapel to come to her, she would have married me; and all would have been well". Instead, here he was with his young witch of Sicily:

My goodness - if only R[ose] had learnt [swimming, fencing and shooting] instead of Bread from heaven,

20 Ruskin to Joan Severn, 22 April 1874; Clegg 1986, 123-24. Crossing the Mont Cenis Pass over the Alps on 4 April Ruskin had noted in his diary "pouring rain, cold, and ... black fog".

21 Ariadne Florentina (1872); Works, 22: 409-10

22 Diaries 1959, 785, entry for 26 April 1874; probably the 12th-century Ponte dell'Ammiragli.

23 Ruskin to Joan Severn, 23 April 1874; Clegg 1986, 126-7.

24 Memoir of Henry Yule 1903, 1: lix.

253 Fonti, letterature, arti e paesaggi d'Europa | Sources, Literatures, Arts \& Landscapes of Europe 1

John Ruskin's Europe A Collection of Cross-Cultural Essays, 247-258 
and would enjoy her drive with me as Amy did today; - you never saw a girl so happy!25

At five o'clock on Friday 24 April, he and Amy - possibly with the good Klein on one side and a maid on the other - took a "huge steamer" for Messina, arriving early the following morning. Ruskin slept on a sofa in his berth without undressing, so as to witness dawn and the crossing of the whirlpool Charybdis, which he found quite exciting:

With windy sea - and under any volcanic action increasing the rush of water - the place must have been so dangerous to small Greek boats as entirely to justify the myth. ... We're going on to Taormina at one o'clock ... [by train from Messina] ... but there's no fear of banditti or anything else - except just the slightest chance in the world of being swallowed up - town and all if Etna were to get suddenly hungry ... but really I've so little taste for playing at priesthood that I don't expect the fate of Korah - besides that poor little innocent Amy who forms my 'company' - may protect my wicked selfie. ${ }^{26}$

Jeanne Clegg, not unreasonably, interpreted this as a reference to Cora, often used by Ruskin as an alternative name for Persephone. However, it is spelled Korah in the letter, not Cora, and Ruskin's reference can be identified as a Biblical one, to Korah the Levite who was punished for his rebellion against Moses and challenge to the priesthood of Aaron, ${ }^{27}$ when the earth opened and swallowed him up. An obscure incident, one might think, even allowing for the Biblical familiarity inculcated by his evangelical mother, but there is another simpler explanation for this being in his mind - The Punishment of Korah figures in one of the scenes by Botticelli on the walls of the Sistine Chapel in Rome, which Ruskin had probably just looked at on his way down from Assisi, in preparation for work on his large-scale watercolour copy of Zipporah.

Sunday 26 April was a red-letter day, as he was finally to witness a scene he had imagined when a boy of ten, in 1829 writing a poem entitled Etna, presumably based on historical accounts of its eruptions:

On old Sicilia's isle a mountain roars

In sounds re-echoed from Italian shores.

[...]

Etna is quiet; but it leaves a scene

That well may fill with fear the hearts of men ${ }^{28}$

"This morning at $1 / 2$ past 4 ", the diary tells, "saw dawn on Etna: the most impressive and dreadful scene I ever yet saw in life". ${ }^{29} \mathrm{He}$ penned a long and detailed description for Joan, accompanied by a sketch. The drama of the sight seems to have shaken Ruskin out of his melancholy: "I have got out of the power of the black wind at last", and he was able to relish his visit:

Fancy; since yesterday morning at five o'clock, I have seen Charybdis - the rock of Scylla - the straits of Messina - Messina itself, now the second city in Sicily - the whole classical range of Panormus on one side - Calabria on the other - and - a line of coast unequalled in luxuriance of beauty - every crag of it

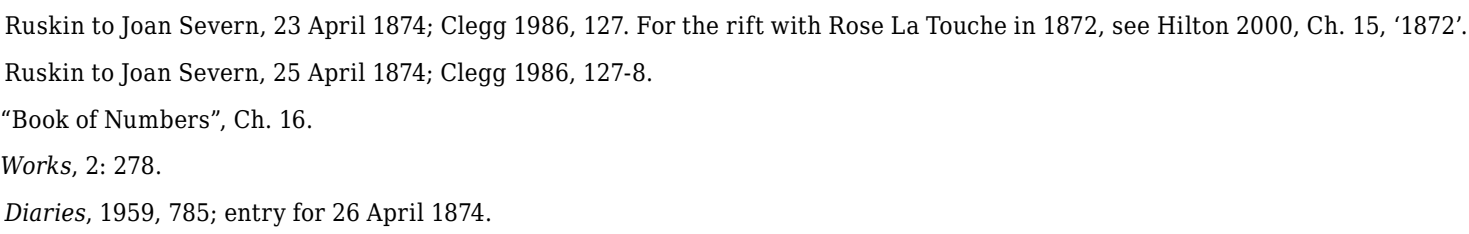


crested with Moorish or Saracenic or Norman architecture wholly new to me - a Greek theatre the most perfect in Europe, now visible on one side of the valley beneath my window - and Etna on the other. ${ }^{30}$

Ruskin's diary tells that he took Amy to see Etna on Monday 27 (having risen again at half past four), as well as going "all over [the] theatre with old cicerone". ${ }^{31}$ The next day he witnessed a memorable sunrise for the third and last time:

Where [the smoke] rose from the crater - it was in close, pure, thunderous masses of white, which took the rose of sunrise exactly as a thundercloud would - a white one, - while the rest of the mountain was still dark on the sky -: and on the opposite side, the sun rose - so as to shine exactly through one of the arches of the Greek theatre - so that on the one hand, there was Etna in full flush of sunrise - on the other, a Greek building standing up against the light - and the Apolline beams piercing it as if with Apollo's own presence - a glory as of a statue of fire beneath the arch. ${ }^{32}$

They left Taormina at 7, stopping for breakfast and lunch at Messina before continuing back at 5 in the afternoon, "poor wee Amy very sorry to go home - me think". In Messina there was just time for another "wonderful sight": at Amy's prompting they visited an apothecary's shop "that had remained unchanged since the fifteenth century ... and for that alone, I would have come to Sic- ily. I never thought to see such a thing in this world". A diagram in the letter picks out the counter, "in exquisite Florentine mosaic of Sicilian marbles; top and sides all inlaid; no duke's drawing room in Blenheim or Chatsworth can show grander marble work" and three projecting wooden presses, "exquisitely designed \& carved, holding the more precious or poisonous drugs in glass vases - Venetian - close set on tiny shelves ... the wood in places mouldered away; but no abbots chair in a Cathedral chancel could be more beautiful than each of these three presses". ${ }^{33}$ The overnight passage brought them back to Palermo early on Wednesday 29. Ruskin stayed on deck until ten o'clock, "watching the Lipari Islands, first against a sunset like Turner's Polyphemus one, and then in the moonlight". ${ }^{34}$ He got little sleep, "the moonlit sea rushing past the cabin window".

Back in Palermo, Ruskin headed back to the Cathedral, his diary recording him "hard at work on Frederick II" ${ }^{35}$ With rain setting in, he also visited the Archaeological Museum, encountering the celebrated metopes from the great Greek temples (chiefly Temple C) at Selinus (or Selinunte), dating from the seventh century B.C. That was the end of the visit. If Ruskin made any formal, or even informal, goodbyes to the Yules, or indeed to Consul Dennis, no record is known. By the evening of Thursday 30 April he was writing to Joan from on board the Campidoglio, between Ustica and the Lipari Islands, with Sicily disappearing "in a line of noble crags ${ }^{136}$ as he headed back to Naples, then on to Rome. 
Work on Botticelli was resumed over the summer of 1874, Ruskin returning to England in mid October, spending a few days at Chamonix en route. In September Rose had decided to renew contact, even to the point finally of considering marriage, but it was all too late. She was terminally ill, and after a few more or less happy encounters over the winter, he saw her for the last time in February; she died on 25 May 1875 at the age of 27.

Amy could hardly have been much in Ruskin's thoughts during this period, although there was probably correspondence following the visit. Her mother died just a month earlier than Rose, on 30 April 1875, but any feelings of sympathy would have been eclipsed by Ruskin's own devastating loss. Henry Yule returned to live in London, marrying again in 1877 Mary Wilhelmina Skipwith, the daughter of an old friend and twenty years his junior; but she died only four years later. Yule was appointed to the India Council and elected President of the Asiatic Society; he would also have become President of the Royal Geographical Society (of which he was Vice-President) but for his criticism of H.M. Stanley's vigorous activities in Africa. He died in London on 30 December 1889 and was buried at Tunbridge Wells. Musing on the trip, Ruskin had told Joan how he had been

very glad to have seen Etna, which I've to thank Amy for, for if she hadn't begged me to take her, I certainly should not have gone myself: and I have gained invaluable knowledge - To all intents \& purposes, I have been in Greece, and seen the Greek sea. ${ }^{37}$

To one whose knowledge of classical Greek culture was hitherto confined to a visit to Paestum as a youth, and hours spent in the British Museum, that was an inestimable boon, and left him quite content.

Amy Yule made no further appearance in Ruskin's life, as far as we can tell. Was he ever really serious about her? She was a lively correspondent, but having not met her since she was a girl of ten, and with Rose still in his mind, this seems unlikely. An unusually skittish letter to Susie Beever, written from Florence on 10 April, just ten days before leaving for Sicily, seems to confirm her position, as one among equals. He reported news of Rose via Joan, adding:

There's no fear of Joanie's ever thinking you write too often. Besides - you might send me a letter on the sly, you know - to the Hotel de France, Palermo. It would make my Palermo mistress so jealous, too. Please do.

Let me see - how many have I, now, after Rosie. There's first Susie - and then I think this Palermo one, Amy, who's really good as gold, too; and then there's a pretty Flora, at Woolwich [Flora Shaw, daughter of General Shaw, at 22 the same age as Amy] - and then - I felt so terribly out of my depth the other day - all in a minute - with the sister of one of my pet Oxford pupils [Lucy Drewitt, sister of Dawtrey Drewitt], a wild girl after the hounds ... Oh dear - if these good girls would but set themselves to be an example in their own drawing rooms, when they marry, of plain dressing, and charitable deed..$^{38}$

To Charles Eliot Norton, writing more seriously the day before, he reported that

I am going to Palermo to see a dear good girl, Amy Yule, who has been very lovely in her affection to me these ten years, - I don't mean like R[ose] but a quite 
simple, lovely, grateful, healthy regard, - and I want to see her[,] for everybody says she's a wonderful creature - and I know her to be so in mind. But there can never be anything to come between R[ose] \& me, now, my life is far too broken. ${ }^{39}$

So what of Amy, his "affectionate little old friend"? Whether she accompanied her father back to England after her mother's death is not known; relations with a stepmother only twelve years her senior might well have proved difficult. Her other known publications, including A Little Light on Cretan Insurrection (1879) and the co-authorship, with John Murray, of Murray's Handbook for Travellers in Greece (1884), suggest a continued life in the Mediterranean. A glimpse of her is provided in a remark by George Dennis, referring to her as "an eccentric creature ... [who] did not deign to pay a call on the British Consul to greet her father's old friend when she was staying by herself in Palermo" ${ }^{40}$

She remained Yule's only child, and would have inherited what the Dictionary of National Biography declares to have been a shade under £30,000, no small sum in 1890 . Within the next few years she moved to Tarradale House, on the Black Isle near Inverness in Scotland, which had formerly belonged to her kinsman, Sir Roderick Murchison: here she added a walled garden and a library tower. She was elected a Lady Associate of the Society of Antiquaries of Scotland in 1895; was a subscriber to the British School at Athens by 1904; a member of the Palaeontographical Society by 1906, and acted as local secretary (for Ross-shire) of the Royal Scottish Arboricultural Society. ${ }^{41}$
A lively, intelligent young woman, with an inquiring mind and an interest in geology - and gardening, as she had related in her 1865 letter - and much else besides: she must surely have attracted Ruskin intellectually, and it would seem to the disinterested observer an ideal match. But the near 34-year age difference; the continuing saga with Rose; the shocking revelation of her ignorance of the Bible: these must just have put her out of Ruskin's mind. On her part, this may not have been the case; she never married, and from the letter of 1905 to Joan Severn we learn that soon after her father's death, in or about 1891,

I wrote a long letter to Sir Henry Acland putting on record the dates and circumstances of that good deed [Ruskin's offer of 1865 to bring her up], and asking him if he thought Mr. Ruskin were well enough to care, to communicate the letter or its purport. This letter was registered, and I told him if busy not to write. No answer came, and it was not until several years after that I learnt from his son of Sir Henry's own severe illness and other troubles about that time. ${ }^{42}$

Again, alas, too late.

The Transactions of the Gaelic Society of Inverness include an obituary, following her death on 24 August 1916, aged 63.

She was a woman of uncommon gifts of mind and heart, of great force of character, and of wide and discriminating generosity. She was a patron of literature, a warm supporter of Gaelic, and a wise friend

1 Research by Dr Eric Grant into Amy Yule's life has yet to yield a photograph or portrait of her; see 'Miss Amy Yule of Tarradale House, 1852 1916', http://www. rossandcromartyheritage.org/Community/Muir-of-Ord/Folk.aspx.

42 Amy Yule to Joan Severn, 12 January 1905; Morgan Library, New York, MA 3451. I am grateful to Dr Gill Mawby for supplying a transcript. 
of education ... [it ends] As a final proof of her practical love for the advancement of learning, she bequeathed her beautiful home at Tarradale and her valuable library, together with funds for their maintenance, for the use of students who are in need of a period of rest and change. Sadly, this enlightened idea lost favour with the University of Aberdeen, and the house was sold in 2003: it has now reverted to private ownership.

\section{Abbreviations}

Diaries 1959 = Evans, J.; Whitehouse, J.H. (eds). The Diaries of John Ruskin. Vol. 3, 1874-1889. Oxford: Clarendon Press.

Memoirs of Sir Henry Yule 1903 = The Book of Ser Marco Polo the Venetian concerning the Kingdoms and Marvels of the East. Translated and edited, with Notes, by Colonel Sir Henry Yule, R.E., C.B., K.C.S.L., Corr. Inst. France. Third Edition, Revised Throughout in the Light of Recent Discoveries by Henri Cordier (of Paris) ... with a Memoir of Henry Yule by his Daughter Amy Frances Yule L.A. Scot, Ant. Scot., etc. 2 vols. London: John Murray.

\section{Bibliography of Works by John Ruskin}

References are to volume and page numbers in:

Cook, E.T.; Wedderburn, A. (eds) (1903-1912). The Works of John Ruskin. Library Edition, 39 vols. London: George Allen. https://www.lancaster.ac.uk/the-ruskin/the-complete-works-of-ruskin/.

Works, 2: Poems. |17: Unto this Last, Munera Pulveris, Time and Tide, Other Writings on Political Economy (1860-1873). |22: Lectures on Landscape, Michael Angelo \& Tintoret, The Eagle's Nest, Ariadne Florentina. | 37: The Letters of John Ruskin, II (1870-1889).

\section{General Bibliography}

Bologna, G. (2010). "Il viaggio di John Ruskin in Sicilia”. Kalós - Arte in Sicilia, 22(2), 12-15.

Bradley, J.; Ousby, I. (eds) (1987). "The Correspondence of John Ruskin and Charles Eliot Norton”. Cambridge: Cambridge University Press.

Burd, V.A. (ed.) (1969). The Winnington Letters: John Ruskin's Correspondence with Margaret Alexis Bell and the Children at Winnington Hall. London; Cambridge (MA): George Allen and Unwin; Harvard University Press.

Campisi, M.T. (2019). "Il viaggio in Sicilia di John Ruskin. Natura, Immagine, Storia”. Caccia Gherardini, S.; Pretelli, M. (a cura di), "Memories on John Ruskin: Unto This Last”, monogr. no., Restauro Archeologico, 2 vols. Vol. 1, 32-9.

Clegg, J. (1986). “Circe and Proserpina: John Ruskin to Joan Severn, Ten Days in Sicily, 1874”. Quaderni del Dipartimento di Linguistica, Università della Calabria, 2, 113-38.

Hayman, J. (1989). “John Ruskin's 'Hortus Inclusus': The Manuscript Sources and Publication History”. Huntington Library Quarterly, 52(3), 378-79. Hilton, T. (2000). John Ruskin: The Later Years. New Haven; London: Yale University Press.

“Obituary of Henry Yule”. Proceedings of the Royal Geographical Society and Monthly Record of Geography, New Monthly Series, $12(2), 111$. Marshall, M.P. (1947). What I Remember. Cambridge: Cambridge University Press.

Rhodes, D.E. (1973). Dennis of Etruria: The Life of George Dennis. London: Cecil \& Amelia Woolf.

Vitale, M.R.; Barbera, P. (2019). "Lontano dalla capitale. Il viaggio di Ruskin in Sicilia: una lettura comparata". Caccia Gherardini, S.; Pretelli, M. (a cura di), “Memories on John Ruskin: Unto This Last”, monogr. no., Restauro Archeologico, 2 vols. Vol. 1, 156-61. 


\title{
Songlines: Ruskin and the Roads of Europe
}

\section{Howard Hull}

\begin{abstract}
Ruskin's Europe can be understood as a network of roads, physical and imaginary, which interconnect in a single vital configuration. Ruskin saw this as a distinct terroir, a cultural and spatial ecosystem created over millennia. What he termed the 'Old Road' is an attempt to unify the topographical and historical discovery of physical journeying to a conceptual road of intellectual reflection and the imagination. Ruskin used, but lamented, the railways just as we use, but lament, the internet. These powerful tools come at a price, fore shortening encounter, memory and time. Ruskin fought to defend Europe's old road as one we are in danger of forgetting and which he called on us to reclaim.
\end{abstract}

Keywords Ruskin. Europe. Old Road. Roads. Travel. Tourism.

During the Cold War, 'Check Point Charlie' on Berlin's Friedrichstraße was symbolically the 'ground zero' of potential Armageddon. Across this rather makeshift set of barriers two ideologies with a willingness to risk global war confronted one another on an old road. I think it was the poet W.B. Yeats who pointed out that despite all the national borders and the military and political conflicts in the world, the roads themselves connect as if there were no borders. It is an instant source of comic delight when we see a photograph of bridge builders who, having commenced construction from two sides of a wide valley, find, as they approach the final meeting point, that their calculations are out! Roads, by their very nature, connect. The concept of the road, even the actual road itself, travels freely across boundaries. The road at Finisterre is continuous to Shanghai, even if we choose to put barriers across it.

We speak of roads in human terms. We talk of circulatory systems, of arteries, of by-passes, of networks, of nightmare roads and dream roads. We talk of one road 'feeding into' another and we even anthropomorphize roads with the feelings they induce in us. When we arrive in a city the well-planned highway on which we have been travelling

Fonti, letterature, arti e paesaggi d'Europa | Sources, Literatures, Arts \& Landscapes of Europe 1 ISSN 2724-6620 e-ISSN 2784-8507 
seems to experience something of a nervous breakdown as it disaggregates into a tangle of urban pathways. Either we brave the complexity that awaits us, or we circle the ring road until we find our way out, heading toward another city.

If we can, let us stop off in the city and find somewhere to park for a minute. Here we can explore another type of road on foot. City streets and alleyways have been shaped by centuries of human interactions. As Ruskin so eloquently teaches us, cities are like a history book and there is as much to read in the palimpsest of their roads and walkways as there is in their architecture. Less obvious, is that a large part of their history is written beyond the city limits. The city is a nodal point on a wider network, an international circulatory system of exchange, extraction and fulfillment. It is impossible to speak of the city in isolation. It gives visibility to something more fluid but omnipresent. Everywhere, the city is ultimately shaped by events arriving on the roads from somewhere else. The nature of place is in part determined by the nature of the networks that serve it. When we speak of Europe, are we speaking of its people, their possessions and places, or is Europe itself in some ways better described by the nature and character of its internal and external connections?

Our roads are teachers, structurers of behaviour, maps of the neural pathways of our culture. They are accumulated traces in the landscape of a people's self-portrait. Memorably quoted by Sir Kenneth Clark in the television series Civilization, Ruskin wrote

Great nations write their autobiographies in three manuscripts, the book of their deeds, the book of their words and the book of their art. Not one of these books can be understood unless we read the two others, but of three the only trustworthy one is the last. ${ }^{1}$
Such autobiographies are written across the surface of Europe, our roads are the desks for their manuscripts. Compare the roads of Europe to the roads of America. Europe's roads embrace the memory of many thousands of years of human culture. In America a single, soulless grid erased the tribal pathways of Native Americans, and continues to eviscerate the recent memories of a young nation.

Not that Europe is immune from the amnesia of modernity. In the Nineties Rem Koolhaas, the Dutch architect and theoretician gave this phenomenon the rather unprepossessing name of 'Hollow Core'. People in Antwerp, Brussels, Gent and the Ruhr lived and worked, he noted, in an urban landscape set between core cities and were responsible for a considerable proportion of the region's economic production and consumption. Within an hour, residents of Hollow Core could reach four international airports, eight intercity railway stations, sixteen universities, thirty-two international sport venues, and sixty-four shopping malls, while at the same time they lived in low-density neighbourhoods in a superficially green environment. Koolhaas was attempting to define the boundaries of his subject. If he was to design dwellings here where did his task start and finish?

Sitting in his study at Brantwood at half past seven in the morning on 25th February 1873, John Ruskin grappled with the same question. He was writing one of his serialized letters to the labourers of Great Britain. If he was to consider the labour - or life - of one man, what were the boundaries of his subject?

Consider, for instance, what I am doing at this very instant. It is a bitter black frost, the ground deep in snow, and more falling. I am writing comfortably in a perfectly warm room; some of my servants 
were up in the cold at half-past five to get it ready for me; others, a few days ago, were digging my coals near Durham, at the risk of their lives; an old woman brought me my watercresses through the snow for breakfast yesterday; another old woman is going two miles through it to-day to fetch me my letters at ten o'clock. [...] Somebody in the east end of London is making boots for me [...]; a washerwoman is in suds, somewhere, to get me a clean shirt for to-morrow; a fisherman is in dangerous weather somewhere, catching me some fish for Lent; and my cook will soon be making me pancakes, for it is Shrove Tuesday. ${ }^{2}$

The interconnectedness of things, the costs and consequences of actions, the displacements, gains and losses, the shifts of power and spreading of influence and energy, the interchangeable and the momentarily manifest: all these live in networks of relationships and contacts which we inhabit and which we are naturally gifted to exploit. Ruskin talks of our

innate love of mystery and unity [...] the joy that the human mind has in contemplating any kind of maze or entanglement, so long as it can discern, through its confusion, any guiding clue or connecting plan. ${ }^{3}$

Finding a language to express the nature of humanity's individual, social and cosmic inter-dependence was a European project long before Ruskin; long, indeed, before Homer. Our great megalithic structures are remnants of a sophisticated social network that was connected across huge distances, one which mapped into the cultural co-ordinates of its political economy the science of its astronomy and the rituals of its beliefs, shared from Orkney to North Africa.

The sea, or the 'whale-road' was just as important, though its routes have left their physical remains only beneath the waves and in poetry from the Seafarer to the Odyssey.

Think of Lindisfarne, or Holy Island, a remote island set in cold and stormy seas on the North East Coast of Britain. It was here, in the seventh century that Christian monks produced the illuminated Lindisfarne Gospels.

St Cuthbert's book, as Ruskin called it, is a fusion of Celtic knot-work, Nordic mythology and Christian symbolism. It was made in the middle of a thousand-year period in which Britain and Ireland were hugely popular with invaders from continental Europe. The result was a pooling of many cultural forms which the indigenous Celtic culture proved ideally adapted to interweave. Even though our ancestors seem to have selected the remotest spots on earth, these archipelagoes of islands and coastal hinterlands were at the heart of a Europe wide network of communications and trade. In an age when populations were small, vital cultures had to be international cultures.

The resulting fusion of material and spiritual interests goes to the heart of Ruskin's reading of European civilization and it is why the roads of Europe play such a key role in his unfolding consciousness. The Celtic Christian carpet pages can be read, in some senses, as a sort of map, an illustration of a network of mytho topographical relationships, somewhat after the fashion of Australian Aboriginal Songlines. The Lindisfarne carpet pages, as they are known, read both literally and symbolically. In their labyrinthine intricacy they wrest order from chaos. Like Aboriginal paintings, they seem 


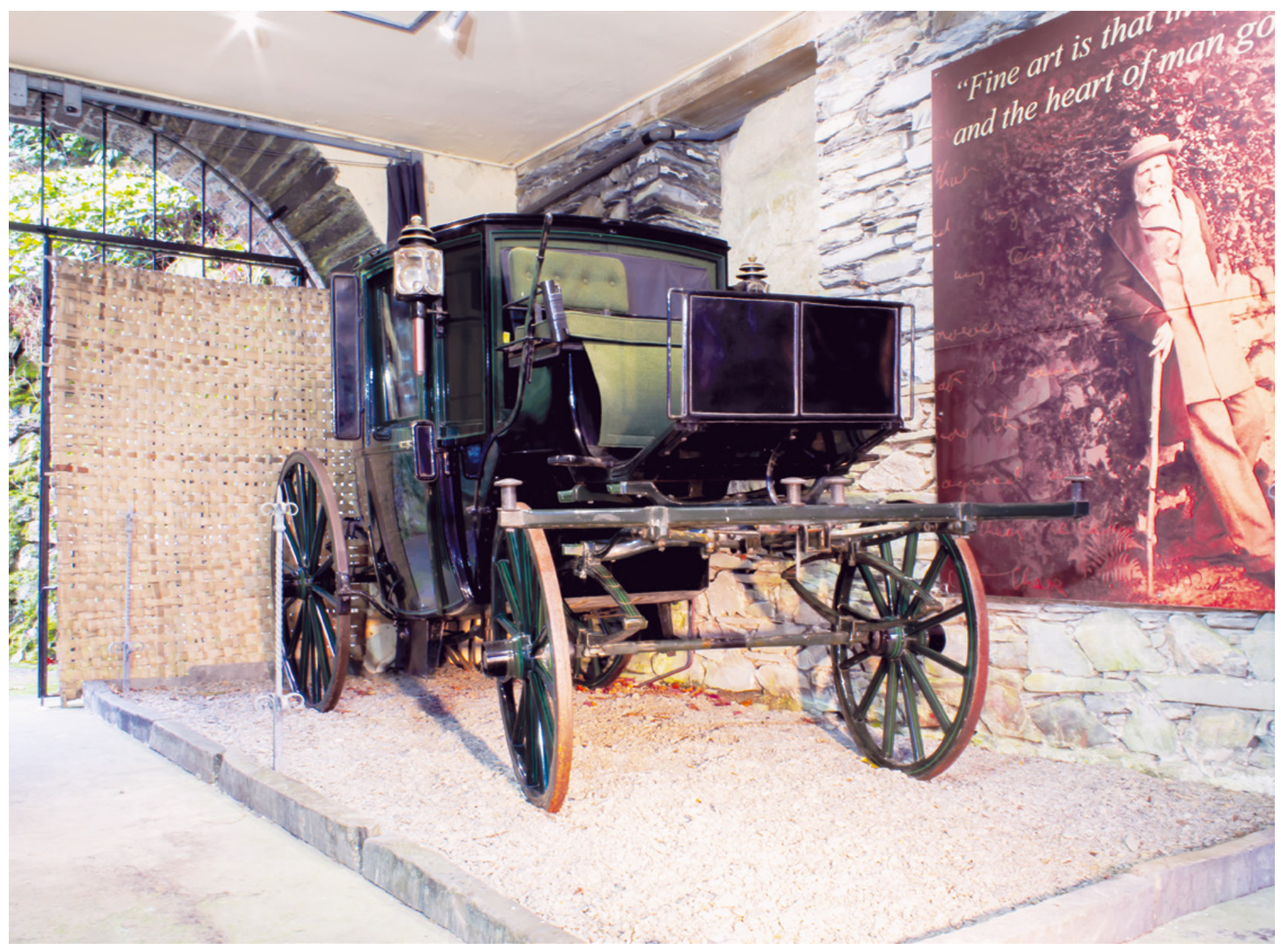

Figure 1 Double Brougham carriage built in Camberwell to Ruskin's design in 1875 for the journey between London and Brantwood. Frontal view. Whitehouse Collection, R72, Lancaster University. On display at Brantwood 
to find a language for the intangible, the language of luminous encounters on a network.

Within three centuries the early abstractions of the monks' carpet pages leapt from their vellum into the three dimensional world of the Gothic. The bedrock of the roads now literally soared into space. We are reminded in Jean Gimpel's The Cathedral Builders, that between 1100 and 1250 more stone was quarried in France than in the entire pyramid-building epoch of ancient Egypt. In the aftermath of the disintegration of the Roman Empire, a new form of Christendom was giving shape to otherwise chaotic entanglements. The roads not only carried stone and clerics, they burst alive with trade and pilgrimage. The troubadours crafted songs of the road, celebrating place in lyrical imagery, A Lunel lutz una luna luzens, bringing the variety of regional language into the mainstream of Europe's literature.

We have talked of the profound cultural legacy which gives roads character, actual and mythic, and to which we are all, as Ruskin was, heir. It is time to talk of roads of physical geography and actual kilometers. Roads of travel. Ruskin was an inveterate traveler, forever on the road.

Born in the golden era of coach travel, his earliest encounters with the landscapes of Europe were shaped by his mode of discovering them on long family carriage journeys away from his home in London. From the age of three, when he paid his first visit to the Lake District, until train travel took over the coach in Ruskin's life in the 1860s, Ruskin undertook no fewer than 15 lengthy continental tours in addition to extensive travels every year within the United Kingdom. Even in the second half of his life, despite being a regular and frequent user of the railways, coaches continued to form a vital component of most of these journeys.

The years leading up to Ruskin's birth in 1819 had seen a boom in road building in northern Europe. Pioneering engineers such as Tresaquet in France and
Macadam in Britain transformed the coaching experience. Better road surfaces allowed faster, more maneuverable coaches. The journey time of mail coaches was slashed, and carriage journeys became considerably safer and more comfortable for passengers.

A journey of 187 miles from London to Manchester, which had taken more than four days in 1754, was, by 1830 , advertised as taking 18 hours. By the 1820's the revolution in road construction meant that there were growing numbers of financially comfortable families that could contemplate recreational journeys. The Grand Tour had offered a wealthy aristocratic elite an experience of self-improving and educative cultural travel; now, popular guides to areas of outstanding natural beauty or cultural significance allowed others a version of this experience.

As sole representative of London's largest importer and retailer of sherries, with a roster of over 1,000 customers in Britain, Ruskin's father was obliged to travel extensively for business. In Great Britain and on extended trips across continental Europe, the Ruskin family travelled together, mixing business and pleasure.

As tourists, the Ruskin family's first debt was to the popular printed guides, portfolios of engravings and the poetry and reports of other travelers. However, a more exclusive opportunity was provided to the Ruskins by virtue of John James' visits to the grand houses of his customers, where they were exposed to artistic collections assembled on the Grand Tour.

The Ruskins also travelled on the coat tails of the public appetite for the Picturesque which gripped early tourism in the late eighteenth and early nineteenth century. John James and Margaret were familiar with the views or 'stations' described by authors such as Wordsworth, Linton or Thomas West, and illustrated by artists such as John 'Warwick' Smith.

Primed by his visits to the Lake District, on his thirteenth birthday Ruskin had a life-changing encoun- 
ter in the form of the gift of Samuel Roger's travel poem, Italy. Illustrated by J.M.W. Turner, Italy became the planning document for a succession of family continental tours which were to shape Ruskin's entire life. Many of Turner's views were visited, the Ruskins subsequently acquiring Turner's original watercolour study of Monks on the Road to Florence from Fiesole, a picture engraved in Italy, the site of which they visited. It was to hang in the bedroom at Brantwood until the very end of Ruskin's life.

Ruskin was keen to note his visit and compared real life to the original, stating

The little bend of wall within which they are placed is not really a part of the Franciscans' garden, but one of the turns of the road in the ascent to Fiesole.

Direct experience of the artist's subject was to be a hallmark of Ruskin's career as an art critic.

At this time Ruskin was under the influence of various drawing masters, most notably Samuel Prout and Copley Fielding from whom, in turn, he learnt topographical and atmospheric rendition and the ability to record buildings quickly and accurately. With these skills and a passionate interest in geology and architecture, Ruskin quickly fashioned his own distinctive agenda for travel and his method of work. Proud parents were happy to stop the coach for their precocious son while he got out, often drawing at some length the roadside features or views. Ruskin even records walking alongside the coach while they remained inside keeping pace with him. His famous eye for detail was thus grounded in wayside encounters taken slowly. Ruskin was able to undertake analytical and aesthetic observations concurrently. The tempo of such travel provided him with the ability to immerse himself intensely in the moment, absorbing a strong sense of place.

The slow pace of the coach on long journeys through dramatic scenery also provided an evolving succession of imagery through the window, much as the playing of a film. Descriptions in notebooks at the Beinecke, written whilst moving, as well as more famous published passages written from memory, record and exploit for dramatic effect the cumulative experience of this gradual unveiling or revelation. The influence of this style of travel also carries over into his approach as a teacher for he lays out his lessons in the shape of a journey, planning a series of experiential encounters in a carefully measured sequence. Ruskin and his teaching were also shaped by the heightened sense of anticipation such adventures provided, the sense that each discovery leads to another and that there are unimagined treasures yet to be encountered.

Being on the road thus offered Ruskin a high degree of excitement and pleasure, but it also offered something of deeper significance. When Ruskin suffered mental and physical health issues following his rejection by Adèle Domecq, and subsequently while he was a student at Oxford, the family remedy was to take him travelling. Travel linked opportunities for intellectual and sensory enlightenment with well-being and emotional healing. In his later years, following the mental breakdowns at Brantwood, Joan Severn employed the same strategy. It was a childhood experience of the healing effects of a visit to the Lake District that prompted Ruskin in 1871, while in Matlock to declare that if he could lie down in Coniston Water he would be well. The following year he bought Brantwood.

With the exception of a brief crossing of the English Channel, Ruskin's early European journeys were undertaken by horse and carriage. Although not over fond of horses and never a rider, Ruskin nonetheless loved travel by carriage. One of the more amusing and less well known pieces of Ruskin's writing is a short play or dramatic sketch which he wrote in 1836, entitled The Ascent of the St. Bernard, in which the crush of the inn in Martigny and his father's negotiations with the ostler 


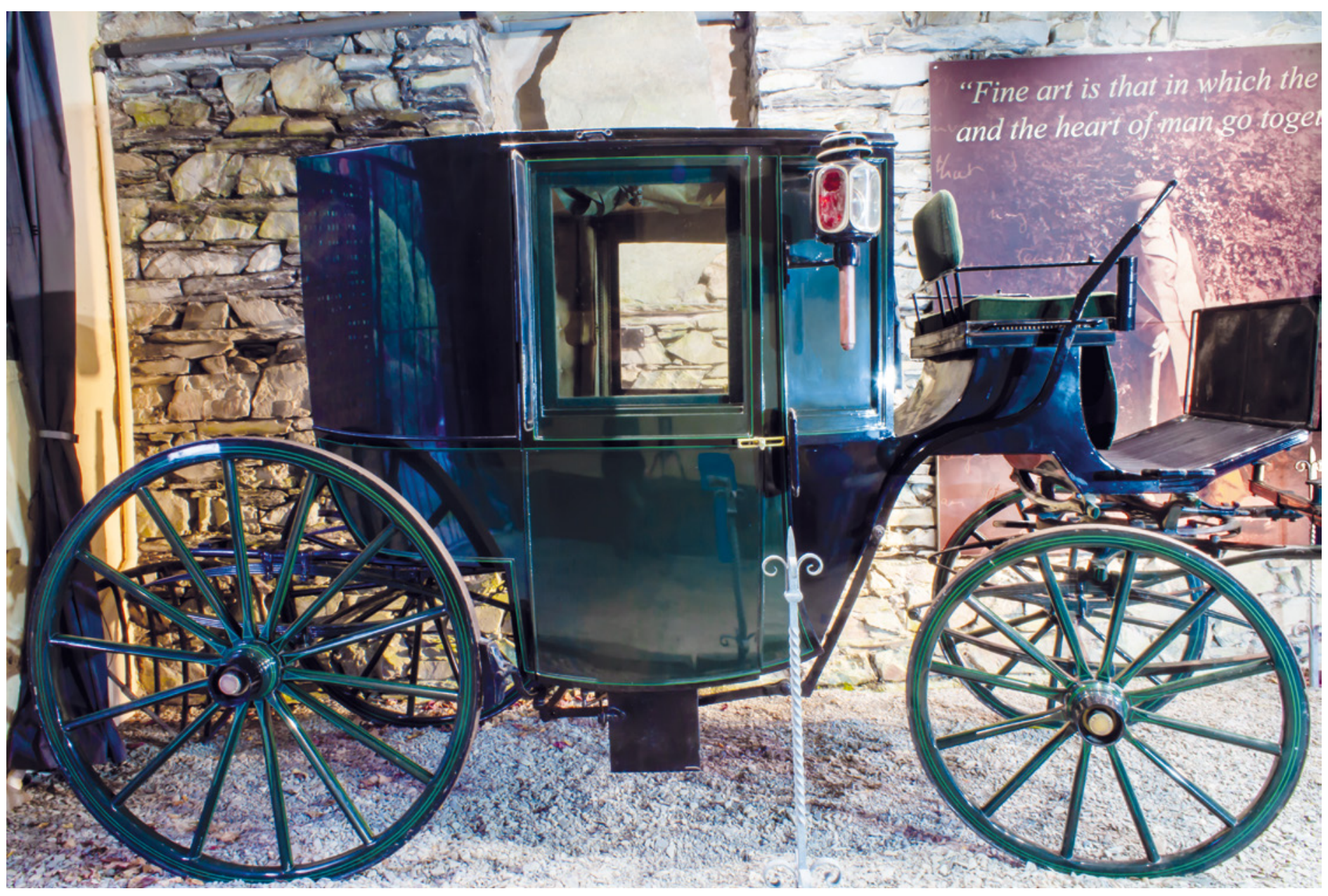

Figure 2 Double Brougham carriage built in Camberwell to Ruskin's design in 1875 for the journey between London and Brantwood. Lateral view. Whitehouse Collection, R72, Lancaster University. On display at Brantwood 
feature vividly. By the time that he was writing Praeterita Ruskin was looking back on the joys of coach travel with a nostalgia widely shared by his readers. Hugely popular in 1888 was the release of Coaching Days and Coaching Ways by W. Outram Tristram, a lively and wistful look at a past era, the illustrations of which also sold in large numbers on Ridgeways pottery.

With the expansion of the railways the old infrastructure of coaching had rapidly declined. One survivor, however, was the Coaching Inn, a predilection for stays at which Ruskin retained throughout his life. Whilst inveighing against the changes that the railways brought about, Ruskin was nonetheless a heavy user of them from the start. Not untypically, Ruskin had conflicting views: the train itself was, he wrote, an

infinitely complex anatomy of active steel, compared with which the skeleton of a living creature would seem, to the careless observer, clumsy and vile. ${ }^{4}$

But train travel reduced its passengers to "pieces of white putty that could feel pain". Instead of admiring the view

They pulled down the blinds the moment they entered the carriage, and then sprawled, and writhed, and tossed among the cushions of it, in vain contest, during the whole fifty miles, with every miserable sensation of bodily affliction that could make time intolerable. ${ }^{5}$

Just as Ruskin's enthusiasm for the technology of photography waned even as he made use of it, so the mar- vel that is the train nonetheless becomes one of his symbols of 'illth' in direct proportion to the degree to which its journey-denying ambition of speed robs its passengers of the wealth of nature through which they travel.

Ruskin's road is the old road to which he is constantly drawn back, because it is a record of both civilization and evolution. Beyond that, its many strands ultimately comprise a spiritual journey or labyrinth. The road itself is medium, metaphor and teacher, embodying the interconnectedness of his reading of European civilization, his eye for the natural world and his own inner state of being.

In 2003, Clive Wilmer and I took a Ruskin Journey by the old roads from Venice to Turin and Pamela and I, the following year, travelled on, from Turin to Paris. One late afternoon in Verona Clive and I hiked out of the city reading a long passage of Ruskin, which I have considerably shortened:

If you chance to be at Verona on a clear, warm summer's day, and to be weary - as may well happen - at the end of it, take a light carriage, and drive out at the eastern gate [...]. You will see, [...] a good road turning to the left - and [...] another turning to the left again, which, by a gradual slope, begins to ascend the hill on which the eastern walls of Verona are built. You will then presently find yourself, if it is towards evening, in the shade of those walls, [...] ascending, by a winding road, a hill [...] into the rocks of which, between you and the city walls, a steep ditch has been cut, - some thirty feet deep by sixty or eighty wide, - the defence of the city on that side being trusted to this one magnificent trench cut out of the solid rock, and to the precipice-like wall, 
above, with towers, crested with forked battlements, set along it at due intervals. It was possible to cut that rock-trench [...] without gunpowder, because the rock is a soft and crumbling limestone, on which, when you see the dusty banks of it emerge under the hedges by the roadside, you, if a member of the Royal Institution, must look with great reverence. For in that white rock there are fossil-creatures, still so like the living creatures they were once, that there it first occurred to the human brain to imagine that the buried shapes were not mockeries of life, but had indeed once lived and died. Under those white banks by the roadside was born, like a poor Italian gipsy, the Modern Science of Geology. ${ }^{6}$

With a masterly control of rhythm, Ruskin's prose ambles uphill at the pace of the carriage, the revelation of the view unfolding and its significance in history. In this one very particular place Ruskin locates us in the continuum of time and space: wherever the traveler is on this road they are part of an entity which, like Ruskin's 'veins of wealth', is the circulatory system of a single heritage in which we share.

Pamela and I spent the night in snow at the monastery at the top of the St Bernard Pass where Ruskin's dramatic sketch had placed him 180 years earlier. Not much had changed - at that point both the dogs and the monks were still there. We descended towards Chamonix and two days later to Geneva and to the strange circular underground 'city' of CERN, which houses a giant particle collider in tunnels that cut through three countries. It is where the world wide web was started.

In concert with the rest of the world, the Europe of tomorrow begins to look ever-more like this, knowable only as an abstraction, its physical necessities and cultural consciousness rendered into a great web of forces of virtual mass, looping in and out of real people, real places, real mass, powering hundreds of cities on invisible roads in a web of 'dispersed moments of concentration'. The consequences have yet to be mapped, but we know them to threaten the environment and our well-being. Ruskin warns us that the danger will be as much spiritual as it is physical. These are roads we don't yet understand, that have no obvious means of accumulating the wisdom embodied in that 'golden stain of time'. We need Europe's old road to run alongside our superhighways. Europe's Old Road is also, as William Morris described it, 'a new road on which the world should travel', because it is a self-renewing road; as Tolstoy said of Ruskin himself, it is a road 'of all times and all places'. 
Howard Hull Songlines: Ruskin and the Roads of Europe

\section{Bibliography of Works by John Ruskin}

References are to volume and page numbers in:

Cook, E.T.; Wedderburn, A. (eds) (1903-1912). The Works of John Ruskin. Library Edition, 39 vols. London: George Allen.

https://www.lancaster.ac.uk/the-ruskin/the-complete-works-of-ruskin/.

Works, 10: The Stones of Venice II. | 19: The Cestus of Aglaia, The Queen of the Air, Other Papers and Lectures on Art and Literature. |24: Giotto and His Works in Padua, The Cavalli Monuments, Verona, Guide to the Academy, Venice, St Mark's Rest. | 27: Fors Clavigera I. 


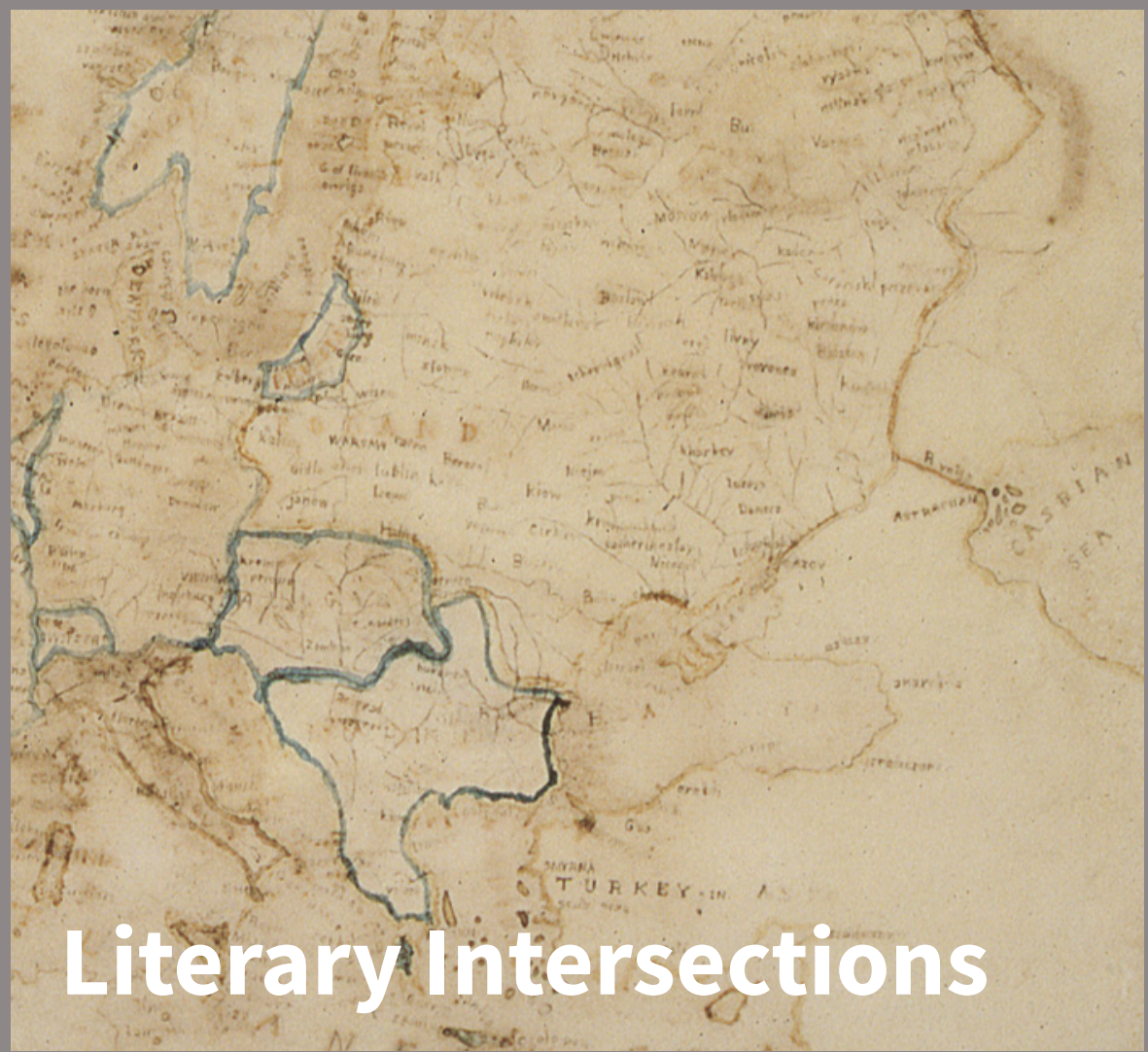




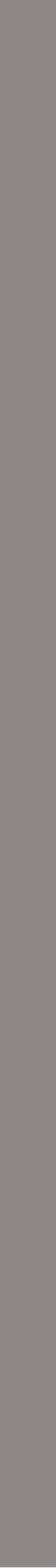




\title{
Ruskin, Dante e l'Europa romantica
}

\author{
Giuseppe Sandrini
}

Università degli Studi di Verona, Italia

\begin{abstract}
My paper aims to show how Ruskin, through Dante's work, looks for an alternative to romantic aesthetics. In Ruskin's view, Dante is Medieval Italy (his knowledge of the poet being parallel to his discovery of Italian cities such as Lucca and Verona). Gothic art is the source of Europe: so Dante is «the central man of the world» (The Stones of Venice) and the fulcrum of an aesthetic based on sharpness and allegory, the two attributes that are typical of the architecture of a gothic cathedral. Ruskin's ideas are then compared to those of Wordsworth and Coleridge, but also to the view of nature of other protagonists of the romantic age in Europe, such as Chateaubriand and Leopardi.
\end{abstract}

Keywords John Ruskin. Dante Alighieri. William Wordsworth. Samuel Taylor Coleridge. François-René de Chateaubriand. Giacomo Leopardi.

Sommario 1 Dante contro Coleridge e Milton. - 2 Il paesaggio di Matelda e un'ode di Wordsworth. - 3 Il grottesco, non il sublime. - 4 L'amore per la natura e la presenza del passato.

\section{Dante contro Coleridge e Milton}

La passione per Dante, nella vita e nell'opera di John Ruskin, nasce insieme a quella per l'Italia medievale. È durante il lungo soggiorno del 1845 che il giovane viaggiatore scopre la Commedia, come testimonia una lettera al padre del 6 maggio di quell'anno: «With this I end my day, and return home as the lamps begin to burn in the Madonna shrines, to read Dante». ${ }^{1}$ Ruskin scrive da Lucca, città che ama particolarmente, come Venezia, Verona e altre dell'Italia centro-settentrionale, preferite alla Roma classica che era stata, invece, la meta più urgente dell'Italienische Reise di Goethe. La lettura della Commedia accompagna una stagione di intuizioni e di studi sull'Italia medievale che lo porta alla stesura di The

1 Works, 4: xxx. La lettera è citata anche da Charles Eliot Norton nell'introduzione a Ruskin 1903: ix, un'antologia di note dantesche che va aggiornata con i diari e gli epistolari di Ruskin pubblicati successivamente. Come riferimento generale, cf. anche Corradini 1985. 
Stones of Venice (1851-1853): l'opera in cui «the Nature of Gothic» viene indagata nelle pietre della città lagunare, ma anche l'opera in cui si trova la celebre e risoluta definizione di Dante quale «the central man of all the world, as representing in perfect balance the imaginative, moral, and intellectual faculties, all at their highest». ${ }^{2}$

La grandezza di Dante, agli occhi di Ruskin (che legge la Commedia nella traduzione di $\mathrm{Cary}^{3}$ e nel testo originale), si basa su due qualità fondamentali che tiene sempre presenti. La prima è l'estrema nitidezza della lettera, degna del confronto con la pietra (c'è un parallelismo tra poesia e architettura: entrambe sono indispensabili per conservare la memoria di una comunità; ${ }^{4}$ in questo gli viene in aiuto l'immagine del poema di Dante introdotta nella cultura inglese dal suo amico Carlyle: «a great supernatural world-cathedral, piled-up there, stern, solemn, awful»); ${ }^{5}$ la seconda è il vertiginoso potenziale allegorico, altro attributo che avvicina la poesia a una cattedrale gotica.

Le pagine più importanti dedicate da Ruskin alla Commedia si trovano nel terzo volume dei Modern Painters (1856), ${ }^{6}$ l'opera che, iniziata per difendere l'arte di Turner, matura negli anni (attraverso la lunga interruzione che coincide con la stesura di The Stones of Venice $)^{7}$ fino a diventare una vastissima storia della civiltà europea dal punto di vista della percezione e della rappresentazione degli elementi della natura, che viene a coinvolgere così, oltre che l'arte, anche la poesia.

Nel capitolo XII della quarta parte, «Of the Pathetic Fallacy», Dante è portato come esempio di poeta che sa rendere la condizione delle anime sulle rive dell'Acheronte («Come d'autunno si levan le foglie», Inf. III 112) con «the most perfect image possible of their utter lightness, feebleness, passiveness, and scattering agony of despair, without, however, for an instant losing his own clear perception that these are souls, and those are leaves; he makes no confusion of one with the other», ${ }^{8}$ rimanendo fedele cioè, nella similitudine, alla realtà della foglia.

E il contrario, nota subito Ruskin, di quanto fa Coleridge all'inizio del suo poema Christabel (a stampa nel 1816), dove attribuisce falsamente a una foglia una vita e una volontà che non può possedere:

The one red leaf, the last of its clan, That dances as often as dance it can, hanging so light, and hanging so high on the topmost twig that looks up at the sky. ${ }^{9}$

3 La traduzione di Cary, intitolata The Vision, uscì per la prima volta in forma completa nel 1814 e venne riproposta con maggior risalto nel 1819 dopo gli apprezzamenti positivi di Coleridge e di Foscolo. Se ne conservano tre edizioni appartenute a Ruskin e da lui postillate, come riferisce Corradini $(1990,120)$, che fa riferimento a quaderni manoscritti e a libri oggi conservati alla Ruskin Library di Lancaster.

4 Si ricordi l'affermazione contenuta in The Seven Lamps of Architecture, del 1849: «there are but two strong conquerors of the forgetfulness of men, Poetry and Architecture» (Works, 8: 224).

5 Carlyle 1968, 120

6 Works, 3-7.

7 Il primo e il secondo volume di Modern Painters escono nel 1843 e nel 1846; il terzo (contenente la parte quarta dell'opera, Of Many Things) e il quarto, a stampa entrambi nel 1856, vedono dunque la luce dopo una pausa di dieci anni.

8 Works, 5: 206.

9 Ruskin cita i vv. 49-50 di Christabel, ma qui abbiamo riportato anche i due versi seguenti, dove l'immagine prosegue. Il testo è preso da Coleridge $(1996,52)$.
} 
La critica deriva dall'esigenza di verità che secondo Ruskin deve guidare anche il diletto, proprio della poesia, che si trae dall'«inganno delle passioni». Per questo $\mathrm{Ru}-$ skin, in una nota alla stessa pagina, annovera Dante, a fianco di Omero e di Shakespeare, tra i poeti di genere «creativo», contrapposti a quelli di tipo «riflessivo o percettivo» propri dell'età romantica (Wordsworth, Keats, Tennyson); la forza della Commedia, per lui, sta nella serenità di Dante, che «in his most intense moods, has entire command of himself», come aggiunge poco dopo. ${ }^{10}$

La distinzione tra «two orders of poets» ha qualche somiglianza con quella di Leopardi tra una poesia di immaginazione, propria degli antichi, e una di sentimento, tipica dei tempi moderni e venata di filosofia. Ruskin, a quanto pare, non conosceva Leopardi, ${ }^{11}$ ma poteva aver notizia della distinzione tra poesia ingenua e poesia sentimentale, per molti versi analoga, formulata da Schiller, autore che tuttavia non amava. ${ }^{12}$

La critica a Christabel implica una posizione che è di fatto antiromantica, e non solo perché Ruskin si schiera contro uno dei due fondatori del romanticismo inglese. L'argomento, infatti, è il medesimo usato appunto da Leopardi, nel Discorso di un italiano intorno alla poesia romantica (1818) contro la rosa di Byron, l'esempio di poesia moderna portato da Ludovico di Breme nel suo articolo sul Giaurro. ${ }^{13}$ Può risultare interessante confrontare certe posizioni di Ruskin con quelle del poeta italiano, ugualmente critiche verso il Romanticismo, per mettere in evidenza linee di pensiero simili all'interno del panorama europeo, anche se sviluppate in maniera del tutto indipendente.

Il Breme - riassume il Leopardi ventenne nel suo Discorso - «vuole in sostanza che il poeta avvivi checchessia tal qual è, non trasmutandolo in persona umana; e che tutto senta e viva, non però tutto il mondo sia popolato di persone: e reca per esempio certi versi del Byron dove toccando una novella Persiana degli amori della rosa e dell'usignolo, attribuisce alla rosa innamorata sospiri odoriferi». Ma, ragionando così, dimentica l'esperienza dell'infanzia: «nella immaginativa de' putti il sole e la luna appresso a poco non sono altro che un uomo e una donna, e il tuono e il vento e il giorno e la notte e l'aurora e il tempo e le stagioni e i mesi e l'ozio e la morte e infinite cose d'ogni genere non sono altro che uomini o donne». ${ }^{14}$

Quando viene a parlare del paesaggio nella letteratura medievale (capitolo XIV, «Of Mediæval Landscape»), Ruskin mette in campo subito un'altra contrapposizione, destinata anche questa a colpire il lettore inglese: «Milton's effort, in all that he tells us of his Inferno, is to make it indefinite; Dante's, to make it definite». La Commedia, dunque, è superiore al Paradise Lost per «the formality of its landscape», ${ }^{15}$ ovvero per la sua capacità di concre-

10 Works, 5: 210. L'osservazione di Ruskin si può confrontare con le riflessioni di un poeta del Novecento italiano, Umberto Saba, nelle Scorciatoie, sulla capacità di Dante di essere insieme un bambino stupito e un «uomo intero» (Saba 2001, 13-14).

11 E del resto lo Zibaldone, dove Leopardi ragiona su questo tema (pp. 734-5 dell'autografo, 8 marzo 1821), non era ancora stato pubblicato.

12 Come sappiamo da una lettera del 1879 (Works, 37: 277). Schiller discute del tema nel saggio intitolato appunto Über naive und sentimentalische Dichtung.

13 Discussioni e polemiche sul Romanticismo 1975, 1: 254-313. L'articolo del Breme era uscito nello Spettatore italiano nel 1818; nella traduzione di Pellegrino Rossi, Il Giaurro. Frammento di novella turca, era apparso a Milano nello stesso anno; Byron aveva pubblicato The Giaour. A fragment of a Turkish tale nel 1813

14 Leopardi 1989, 1: 941.

15 Works, 5: 269-70. Del resto Ruskin aveva scritto, in una nota a The Stones of Venice: «if I could only read English, and had to choose, for a library narrowed by poverty, between Cary's Dante and our own original Milton, I should choose Cary without an instant's pause» (Works, 10: 307).

$273 \begin{aligned} & \text { Fonti, letterature, arti e paesaggi d'Europa | Sources, Literatures, Arts \& Landscapes of Europe } 1 \\ & \text { John Ruskin's Europe. A Collection of Cross-Cultural Essays, 271-282 }\end{aligned}$ 
tezza, il cui esempio più lampante è l'uso dantesco dell'espressione «sovra 'l verde smalto» per indicare il colore del prato che accoglie gli «spiriti magni» nel Limbo (Inf. IV 118). Dante «knew well what enamel was» e dunque sceglie un'immagine che tiene insieme precisione tecnica e significato metafisico:

Dante means, in using this metaphor of the grass of the Inferno, to mark, that it is laid as a tempering and cooling substance over the dark, metallic, gloomy ground; but yet so hardened by the fire, that it is not any more fresh or living grass, but a smooth, silent, lifeless bed of eternal green. ${ }^{16}$

\section{Il paesaggio di Matelda e un'ode di Wordsworth}

Tanta precisione non è fine a se stessa: dietro l'opera della «natura» che qui ha «dipinto», secondo le parole di Dante, agisce lo spirito divino, proteso, attraverso la manifestazione della bellezza, alla nostra letizia.

L'altro capo dell'attenzione di Ruskin è, infatti, il significato allegorico della poesia dantesca, come risulta dalla lettura sulla quale «Of Mediæval Landscape» si sofferma più a lungo: il canto XXVIII del Purgatorio. Qui troviamo un'originale analisi della figura di Matelda, che segue fino in fondo la traccia offerta, al v. 80, dalla citazione del «salmo Delectasti» (salmo 91); l'autore di Modern Painters spiega così il rapporto tra la scena del paradiso terrestre e il sogno del canto precedente, in cui Lia e Rachele sono apparse come prefigurazioni di Matelda e di Beatrice:

Leah gathers the flowers to decorate herself, and delights in Her Own Labour. Rachel sits silent, contem-
Il verde senza vita del Limbo viene confrontato, subito dopo, con l'«erba» e i «fior» della valletta dei principi (Purg. VII 73-81), dove Ruskin sottolinea la «mediæval accuracy» nell'elencare i colori, chiamati con i nomi dei pigmenti usati dai miniatori, e propone anche una chiosa pittorico-minerale all'espressione «fresco smeraldo»:

It is evident that the "emerald" here means the emerald green of the illuminators; for a fresh emerald is no brighter than one which is not fresh, and Dante was not one to throw away his words thus.

plating herself, and delights in Her Own Image. These are the types of the Unglorified Active and Contemplative powers of Man. But Beatrice and Matilda are the same powers, Glorified. And how are they Glorified? Leah took delight in her own labour; but Matilda - "in operibus manuum Tuarum" - in God's labour: Rachel in the sight of her own face; Beatrice in the sight of God's face. ${ }^{17}$

Matelda rappresenta la felicità che si prova nel vivere, sulla terra, lavorando alla scoperta delle opere di Dio («in operibus manuum Tuarum exultabo», dice il salmo): una felicità perfetta, «the life of the terrestrial paradise, being a true foretaste of heaven, and beginning in earth, as heaven's vestibule». La figura di Matelda si identifica quindi con l'«ideal landscape» descritto da Dante, che rovescia l'immagine iniziale della «selva oscura» nella 
«divina foresta», quasi una «new nature, converting pathless ways into happy ones».18 La scena di Matelda che raccoglie fiori pare a Ruskin la più importante non solo della Commedia ma di tutta la poesia europea, perché

it contains the first great confession of the discovery by the human race (I mean as a matter of experience, not of revelation), that their happiness was not in themselves, and that their labour was not to have their own service as its chief end. It embodies in a few syllables the sealing difference between the Greek and the mediæval, in that the former sought the flower and herb for his own uses, the latter for God's honour. ${ }^{19}$

Qui Dante incarna («embodies»), scolpisce in poche sillabe il sigillo del suo mondo, l'esperienza di una felicità che si può aprire solo in un'altra dimensione. Si capisce, allora, perché Harold Bloom ha definito Ruskin "one of the first, if not indeed the first, "myth" or "archetypal" critic», indicando la sua implicita teoria della letteratura nel principio per cui «all great poetry whatsoever is allegorical» $\mathrm{e}$ "that what is allegorizes is a fundamental myth of universal man, his fall from Paradise and his quest for a revelation that would restore him to Paradise». ${ }^{20}$ Questo mito centrale, di cui Bloom rintraccia l'origine in una «experience of paradisal intimations within a wholly natural context» (con riferimento all'ode di Wordsworth Intimations of Immortality from Recollections of Early Childhood), è un'eredità del romanticismo, ma declinata secondo l'idea tipicamente ruskiniana dell'epoca gotica come infanzia universale dell'umanità, capace di immaginazione e di «perpetual wonder». ${ }^{21}$

Wordsworth è l'altro fondatore del romanticismo inglese, che Ruskin giudica superiore a Coleridge, se non come poeta, sicuramente come uomo ${ }^{22}$ (la stessa valutazione di Dante si regge, ricordiamo, soprattutto sulla sua qualità di «central man»). Val la pena di leggere un punto chiave dell'ode Intimations of Immortality from Recollections of Early Childhood, che esprime insieme l'importanza decisiva di un singolo elemento della natura, individuato dalla sua familiarità con la vita del poeta, e il suo rimandare con insistenza a un'esperienza altra, perduta. Sono i vv. 51-57 dell'ode, a stampa nel 1807:

- But there's a tree, of many, one,

A single field which I have look'd upon,

Both of them speak of something that is gone:

The pansy at my feet

Doth the same tale repeat:

Whither is fled the visionary gleam?

Where is it now, the glory and the dream? ${ }^{23}$

Ruskin è per certi versi il fedele discepolo di Wordsworth; da un'altra sua poesia, The Excursion, prende non a caso l'epigrafe che sta in capo ai volumi di Modern Paint-

18 Works, 5: 275

19 Works, 5: 280. I vv. 40-63 di Purg. XXVIII sono riportati da Ruskin, qualche pagina prima, non nella consueta traduzione di Cary, ma in quella più recente di Cayley (stampata tra il 1851 e il 1855 e apprezzata da Rossetti), che si differenzia perché conserva la terza rima del poema dantesco.

20 Bloom [1965] 1987, xvi.

21 Bloom [1965] 1987, xxiv, xviii. Bloom riprende un passo di The Stones of Venice (Works, 11: 66).

22 «Wordsworth has a grand, consistent,perfectly disciplined, all grasping, intellect [...] Coleridge may be the greater poet, but surely it admits of no question which is the greatest man», scrive il giovane Ruskin al suo «college tutor», il reverendo W.L. Brown, in una lettera del 1843 (Works, 4: 392).

23 Wordsworth 1997, 136.

\begin{tabular}{l|l}
275 & $\begin{array}{l}\text { Fonti, letterature, arti e paesaggi d'Europa | Sources, Literatures, Arts \& Landscapes of Europe } 1 \\
\text { John Ruskin's Europe. A Collection of Cross-Cultural Essays, 271-282 }\end{array}$
\end{tabular} 
ers, nella quale si dichiara servitore «of Nature and of Truth». Ma la sua posizione ricorda, di nuovo, quella del giovane Leopardi del Discorso di un italiano intorno alla poesia romantica, che guarda però non al mondo cristiano medievale, bensì all'antichità greca e alla«sterminata operazione della fantasia», ripercorsa da ognuno di noi nel tempo dell'infanzia, che ha dato vita alle credenze mitologiche. Di «perpetual wonder», e insieme di fatale rimpianto, è piena anche la pagina del Discorso in cui Leopardi rievoca quella condizione:

Quando il tuono e il vento e il sole e gli astri e gli animali e le piante e le mura de' nostri alberghi, ogni cosa ci appariva o amica o nemica nostra, indifferente nessuna, insensata nessuna; quando ciascun oggetto che vedevamo ci pareva che in certo modo accennando, quasi mostrasse di volerci favellare; quando in nessun luogo soli, interrogavamo le immagini e le pareti e gli alberi e i fiori e le nuvole [...] quando la maraviglia tanto grata a noi che spessissimo desideriamo di poter credere per poterci maravigliare, continuamente ci possedeva. ${ }^{24}$

Leopardi, lontano da una visione spiritualistica del mondo, orienterà la sua poetica verso il recupero dell'esperienza nella dimensione affettiva del ricordo. Scrive nello Zibaldone (p. 4418, 30 novembre 1828):

All'uomo sensibile e immaginoso, che viva, come io sono vissuto gran tempo, sentendo di continuo ed immaginando, il mondo e gli oggetti sono in certo modo doppi. Egli vedrà cogli occhi una torre, una campagna; udrà cogli orecchi un suono d'una campana; e nel tempo stesso coll'immaginazione vedrà un'altra torre, un'altra campagna, udrà un altro suono.

L'intuizione di Wordsworth («But there's a tree, of many, one / A single field which I have look'd upon») sembra più vicina al pensiero di uno scrittore del Novecento italiano, Cesare Pavese, che afferma nel suo saggio «Del mito, del simbolo e d'altro»:

Ora, carattere, non dico della poesia, ma della fiaba mitica è la consacrazione dei luoghi unici, legati a un fatto a una gesta a un evento. [...] Così a ciascuno i luoghi dell'infanzia alla memoria; in essi accaddero cose che li han fatti unici e li trascelgono sul resto del mondo con questo suggello mitico. ${ }^{25}$

Ruskin, da parte sua, crede che «the greatest thing a human soul ever does in this world is to see something, and tell what it saw in a plain way». ${ }^{26}$ Vorrebbe contrapporre alla «Pathetic Fallacy» l'aderenza alla semplice realtà delle cose: il che - nota Bloom - rappresenta una critica del romanticismo dal suo interno. È questo il dilemma estetico che lo spinge a sostenere tanto il paesaggio già quasi impressionista di Turner quanto la pittura medievalista dei Preraffaelliti. Tuttavia Ruskin non mostra interesse per il tema romantico del poeta esule e solitario, caro al suo amico Dante Gabriel Rossetti; non legge Dante romanticizzandolo, ${ }^{27}$ ma cercando di risalire alla sorgenti della sua estetica.

24 Leopardi 1989, 1: 919.

25 Pavese [1945] 1974, 139.

26 Works, 5: 333.

27 Ruskin, comunque, non dà a questo termine un significato opposto a "classico". Scrive infatti in The art of England, Lecture I, 1883: «I use the word "romantic" always in a noble sense; meaning the habit of regarding the external and real world as a singer of Romaunts would have regarded it in the Middle Ages, and as Scott, Burns, Byron, and Tennyson have regarded it in our own times» (Works, 33: 269). 
Del resto già in The Stones of Venice la rivendicazione di Dante quale «the central man of all the world» avveniva all'interno di un capitolo intitolato «Grotesque Renaissance», dunque sotto una precisa categoria estetica che sarà il caso di indagare per chiarire meglio il pensiero di Ruskin e tentare di offrire un'integrazione alla lettura di Bloom. Il «noble grotesque» di cui Dante è il campione contraddistingue infatti l'arte di un'epoca,

\section{Il grottesco, non il sublime}

Proprio la valutazione altamente positiva del grottesco segna un altro distinguo di Ruskin rispetto alle concezioni estetiche del romanticismo inglese. Significativo, ancora, il confronto con Coleridge, che notava

Dante's occasional fault of becoming grotesque from being too graphic without imagination; as in his Lucifer compared to Milton's Satan. Indeed he is sometimes horrible rather than terrible- falling into the $\mu 1 \sigma \eta$ tòv instead of the $\delta \varepsilon ı v o ̀ v$ of Longinus; in other words, many of his images excite bodily disgust, and not moral fear. ${ }^{29}$

Il richiamo all'antico autore del Sublime dà la chiave della preferenza accordata a Milton: il grottesco per il poeta romantico è un difetto (anche se aggiunge che «the faults of great authors are generally excellencies carried to an excess»), causato dalla qualità «troppo grafica» di certe figure di Dante, che sono sentite come «prive di immaginazione» rispetto alla sublimità di Paradise Lost. quella gotica, non ancora del tutto sottoposta al dominio della ragione, come ribadisce questo principio generale:

wherever the human mind is healthy and vigorous in all its proportions, great in imagination and emotion no less than in intellect, and not overborne by an undue or hardened pre-eminence of the mere reasoning faculties, there the grotesque will exist in full energy. ${ }^{28}$

Abbiamo riportato sopra il pensiero dell'autore di Modern Painters, che preferisce Dante a Milton proprio per la sua capacità di definizione. Ora è il caso di andare a ripescare, tra le chiose dantesche dei diari di Ruskin, un appunto (datato Ginevra, 1 maggio 1849) a proposito di Inf. XIII 91-92: «Note how graphic the description of the speaking of the trunk by puffs». ${ }^{30}$ Qui l'aggettivo "graphic», che serve a sottolineare con entusiasmo il vigore dell'immaginazione dantesca («Allor soffiò il tronco forte, e poi / si convertì quel vento in cotal voce»), contiene probabilmente un'implicita risposta a Coleridge, per cui il «too graphic» era il segno di un grottesco deteriore.

D’altra parte la stessa considerazione dell'idea di sublime pone Ruskin fuori dalla linea miltoniana. Già nel 1843 il giovane autore del primo libro di Modern Painters lanciava un attacco frontale alla teoria di Burke, che aveva fatto scuola nella cultura britannica dei decenni precedenti, affermando che «sublimity is found wherever anything elevates the mind». Sublime non è dunque la paura della morte o il brivido di chi sente mi-

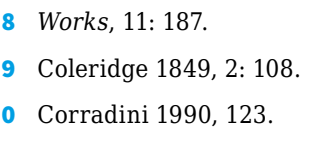


nacciata la propria sopravvivenza, ma la contemplazione e la coscienza del proprio destino: «There is no sublimity in the agony of terror». ${ }^{31}$

L'estetica di Ruskin cerca di uscire dalla soggettività della percezione grazie a una fede intatta nell'apparenza delle cose, cui corrisponde il recupero, attraverso la facoltà della "penetrative imagination», ${ }^{32}$ di un legame con la verità, in senso trascendente. Da qui prende luce anche il concetto di grottesco, che il libro III di Modern Painters riprende da The Stones of Venice, articolandolo in tre tipologie, la terza delle quali, la più nobile, viene definita «Art arising from the confusion of the imagination by the presence of truths which it cannot wholly grasp ${ }^{3} .^{33}$

Siamo, di nuovo, all'idea della conoscenza imperfetta come fonte insieme di felicità e di ispirazione, tema che invita a confrontare ancora Ruskin con Leopardi: si pensi al pensiero dello Zibaldone (p. 100, 8 gennaio 1820) sui poeti antichi che «descrivendo con pochi colpi, e mostrando poche parti dell'oggetto, lasciavano l'immaginazione errare nel vago e indeterminato di quelle idee fanciullesche, che nascono dall'ignoranza dell'intiero». Resta aperta, e difficile da sanare,la contraddizione tra definito e vago: "vagueness is not the sign of imagination, but of its absence», ribadisce Ruskin criticando Milton. ${ }^{34}$ Ma è questo il terreno dove possono incontrarsi l'anonimo artigiano gotico, intento a scolpire i particolari di una cattedrale, e il pittore di paesaggio dell'Ottocen- to, smarrito in quell'«oscurità di cuore ${ }^{35}$ che, secondo Modern Painters, è il carattere più profondo della modernità. Ruskin pensa innanzitutto a Turner, il cui pennello insegue i profili mutevoli delle nuvole; non dimentichiamo che la settima parte del suo libro si intitola «Of Cloud Beauty» e che anche lui potrebbe dire, come L'Étranger del primo dei poemetti in prosa di Baudelaire (raccolti in volume solo dopo la sua morte, nel 1869, e oggi noti col titolo Le Spleen de Paris), «J'aime les nuages... les nuages qui passent... là-bas... là-bas... les merveilleux nuages!». ${ }^{36}$

Un esempio di «true grotesque», tratto dall'arte medievale italiana, è il grifone che «carries on his back one of the main pillars of the porch of the cathedral of Verona». Ruskin scrive che

the Lombard workman did really see a griffin in his imagination, and carved it from the life, meaning to declare to all ages that he had verily seen with his immortal eyes such a griffin as that. ${ }^{37}$

Il grifone fa da pietra angolare della chiesa perché la sua doppia natura, «its unity of lion and eagle», suggerisce, per analogia, le due nature, umana e divina, di Cristo: non a caso Ruskin rimanda in nota al canto XXIX del Purgatorio. ${ }^{38}$ La visione di Dante nel paradiso terrestre, dove è appunto un grifone a tirare il carro della Chiesa, è condivisa dal semplice «workman» del duomo di Vero-

31 Works, 3: 128-30.

32 Ruskin definisce questa forma più alta e intuitiva di immaginazione nel secondo volume (contenente la parte terza) di Modern Painters, dove il confronto tra la descrizione delle fiamme in Milton e Dante (Purg. XXVI 4-8) è a tutto vantaggio di quest'ultimo (Works, 4: 249-50)

33 Works, 5: 130.

34 Works, 5: 271.

35 Per la «darkness of heart» dell'artista moderno, dovuta a «our want of faith», vedi il capitolo «Of Modern Landscape» (Works, 5: 322).

36 Baudelaire 1975-1976, 1: 277.

37 Works, 5: 140-1.

38 Works, 5: 147. 
na, le cui pietre si accendono, come dirà The Bible of Amiens, di un "glow of mythic fantasy». ${ }^{39}$

Alison Milbank nota che le due nature del grifone si manifestano a Dante riflesse negli occhi di Beatrice (Purg. XXXI 121-123) perché il mistero dell'incarnazione, simboleggiato dalla «doppia fiera», non ci può apparire che, paolinamente, «per speculum». Ruskin è vicino al Victor Hugo della prefazione al Cromwell, secondo il quale il realismo grottesco di Dante è indivisibile dal suo essere poeta cristiano, dalla sua visione dell'uomo come creatura duplice, composta di una parte mortale e di una immortale; e bisogna riconoscergli il merito di aver tentato di costruire un'estetica non idealistica, «truly modern but also truly Dantesque». ${ }^{40}$

\section{L'amore per la natura e la presenza del passato}

Nella sua autobiografia Praeterita il vecchio Ruskin, rievocando un viaggio nello Yorkshire compiuto quando aveva diciotto anni, scrive: «I felt, for the last time, the pure childish love of nature which Wordsworth so idly takes for an intimation of immortality»; e aggiunge: «In myself, it has always been quite exclusively confined to wild, that is to say, wholly natural places».43 $\mathrm{Di}$ questo sentimento della natura selvaggia, esclusivo della prima giovinezza e cantato nell'ode di Wordsworth, discute già il capitolo di Modern Painters intitolato «The Mo-
Sarebbe qui via d'uscita dalla «Pathetic Fallacy»; senonché, come dice Bloom, lo stesso Ruskin è diventato poi «the major Romantic myth-maker of the Victorian era». ${ }^{41}$ In questa ambivalenza c'è non solo la «aesthetic tragedy», ma anche il dramma personale di uno scrittore che nella seconda parte della sua vita parla a un vasto pubblico in veste di profeta di una nuova società, ma è insieme costantemente rivolto verso il proprio infelice cammino di uomo sulla terra. Il che determina una svolta anche nel suo modo di guardare a Dante: Sesame and Lilies (1865) propone la definizione della Commedia come «a love-poem to his dead lady; a song of praise for her watch over his soul».42 ral of Landscape». Qui però Ruskin nota che in lui tale piacere «was never independent of associated thought»: la sua contemplazione del paesaggio era sempre associata a un libro letto o a una storia ascoltata.$^{44} \mathrm{Il}$ fatto è che

the charm of romantic association can be felt only by the modern European child. It rises eminently out of the contrast of the beautiful past with the frightful and monotonous present; and it depends for its force on the existence of ruins and traditions, on the re-

\footnotetext{
39 Works, 33: 121.

40 Milbank 2011, 153.

41 Bloom [1965] 1987, XXVII.

42 Works, 18: 116. Il tema sarà sviluppato, in particolare, da Borges nei Saggi danteschi: «Io sospetto che Dante edificò il miglior libro che la letteratura abbia mai prodotto per interpolarvi alcuni incontri con l'irrecuperabile Beatrice» (Borges 1984-1985, 2: 1307).

43 Works, 35: 218-19.

44 Works, 5: 365. Viene in mente di nuovo Pavese, L'adolescenza: «Nessun ragazzo, nessun uomo ammira un paesaggio prima che l'arte, la poesia - una semplice parola anche - gli abbiano aperto gli occhi» (Pavese [1945] 1974, 150).
} 
mains of architecture, the traces of battle-fields, and the precursorship of eventful history. The instinct to which it appeals can hardly be felt in America, and every day that either beautifies our present architecture and dress, or overthrows a stone of mediæval monument, contributes to weaken it in Europe. ${ }^{45}$

Questa consapevolezza spiega perché, secondo il Ruskin di The Seven Lamps of Architecture (1849), la natura esercita un fascino maggiore quando mostra i segni del lavoro umano: il paesaggio di Champagnole, nel Giura francese (descritto all'inizio del sesto capitolo del libro, «The Lamp of Memory») lo attrae proprio perché comprende edifici come il castello di Joux, che corona uno spalto ripido e boscoso. «It is a spot which has all the solemnity, with none of the savageness, of the Alps», scrive Ruskin a proposito del fiume Ain, che scorre tra alte rocce sormontate da un bosco di conifere; sulla cima del dirupo, l'osservatore è sorpreso non da un senso di sublime, ma da «the sudden blankness and chill which were cast upon it when he endeavoured, in order more strictly to arrive at the sources of its impressiveness, to imagine it, for a moment, a scene in some aboriginal forest of the New Continent». ${ }^{46}$

C'è forse, qui, un implicito distinguo rispetto a chi aveva esaltato proprio le selvagge foreste dell'America settentrionale come una perfetta immagine della natura cristiana, recante nella sua solitudine l'impronta del Creatore: si pensi a Chateaubriand (autore che Ruskin non nomina mai, ma del quale certamente poteva aver notizia) e alla sua Atala, nata come inserto romanzesco del Génie du Christianisme. Nella visione di Chateaubriand, il Cristianesimo ha il merito di aver liberato la natura di ogni residuo di presenza mitologica: «puisque c'est cette religion, qui, chassant de petites divinités des bois et des ondes, lui a permis de peindre les déserts dans toute leur majesté». ${ }^{47}$

Si tratta di una posizione esattamente opposta a quella di Leopardi (lettore precoce e attento di Chateaubriand) che, nella canzone Alla Primavera o delle favole antiche, scritta nel 1822, rimpiange accoratamente la natura «abitata» degli antichi. Val la pena di ricordare, a questo proposito, le pp. 63-4 dello Zibaldone:

Che bel tempo era quello nel quale ogni cosa era viva secondo l'immaginazione umana e viva umanamente cioè abitata o formata di esseri uguali a noi, quando nei boschi desertissimi si giudicava per certo che abitassero le belle Amadriadi e i fauni e i silvani e Pane ec. ed entrandoci e vedendoci tutto solitudine pur credevi tutto abitato e così de' fonti abitati dalle Naiadi ec. e stringendoti un albero al seno te lo sentivi quasi palpitare tra le mani credendolo un uomo o donna come Ciparisso ec. e così de' fiori ec. come appunto i fanciulli.

Ruskin sta oltre l'ideale alternativa tra Leopardi e Chateaubriand, due contrastanti campioni della sensibilità europea del primo Ottocento; e non gli basta nemmeno lo spiritualismo romantico di Wordsworth. La presenza per lui più necessaria e vitale è quella del passato, di un passato che - ragiona all'inizio della quinta parte di Modern Painters - si possa avvertire vivo nel presente: cosa inconcepibile nell'ordinata e monotona Inghilterra, ma ancora attuale in certe città della vecchia Europa, dove «we feel the ancient world to be a real thing, and one with the new». E qui interviene il suo talento di viaggia- 
tore, capace di tenere insieme la lettura della Commedia e la ricognizione dei monumenti legati in qualche modo alla poesia dantesca, per esempio l'arca di Cangrande:

At Verona we look out of Can Grande's window to his tomb; and if he does not stand beside us, we feel only that he is in the grave instead of the chamber, - not that he is old, but that he might have been beside us last night. ${ }^{48}$

È un atteggiamento di ascolto che, come sappiamo, troverà un erede pochi decenni dopo. Nel 1906 Proust, introducendo la sua traduzione di Sesame and Lilies, partirà anche da Dante per certe emblematiche considerazioni sull'opportunità di riconoscere nel mondo di oggi «la place inviolable du Passé: - du Passé familièrement surgi au milieu du présent». Nella riflessione di
Proust, l'epifania del passato può avvenire attraverso la parola dei grandi poeti come attraverso uno scorcio di città italiana, prima fra tutte la Venezia figlia della Grecia e dell'Oriente:

Que de fois, dans la Divine Comédie, dans Shakespeare, j'ai eu cette impression d'avoir devant moi, inséré dans l'heure présente, actuel, un peu du passé, cette impression de rêve qu'on ressent à Venise sur la Piazzetta, devant ses deux colonnes de granit gris et rose qui portent sur leurs chapiteaux grecs, l'une le Lion de Saint-Marc, l'autre saint Théodore foulant aux pieds le crocodile, - belles étrangères venues d'Orient sur la mer qu'elles regardent au loin et qui vient mourir à leurs pieds, et qui [...] continuent à attarder au milieu de nous leurs jours du XII siècle qu'elles intercalent dans notre aujourd'hui. ${ }^{49}$ 


\section{Bibliografia di John Ruskin}

I riferimenti alle opere di Ruskin rinviano al numero di volume e alle pagine di:

Cook, E.T.; Wedderburn, A. (eds) (1903-1912). The Works of John Ruskin. Library Edition, 39 vols. London: George Allen.

https://www.lancaster.ac.uk/the-ruskin/the-complete-works-of-ruskin/.

Works, 3-7: Modern Painters I-V. | 8: The Seven Lamps of Architecture. | 9-11: The Stones of Venice I-III. | 18: Sesame and Lilies, The Ethics of Dust, The Crown of Wild, Letters on Public Affairs (1859-1866). |33: The Bible of Amiens, Valle Crucis, The Art of England, The Pleasures of England. |35: Praeterita, Dilecta. |37: The Letters of John Ruskin, II (1870-1889).

\section{Bibliografia generale}

Baudelaire, C. (1975-1976). Euvres complètes. Texte établi, présenté et annoté par C. Pichois. 2 vols. Paris: Gallimard.

Bloom, H. [1965] (1987). «Introduction». The Literary Criticism of John Ruskin. Selected, edited, and with an introduction by H. Bloom. New York: Da Capo.

Borges, J.L. (1984-1985). Tutte le opere. A cura di D. Porzio. 2 voll. Milano: Mondadori.

Carlyle, T. (1968). On Heroes, Hero-worship, and the Heroic in History. London: Oxford University Press.

Chateaubriand, F.-A.R. de (1802). Génie du christianisme, ou Beautés de la religion chrétienne. Nouvelle édition. 4 vols. Paris: Migneret. Coleridge, S.T. (1849). Notes and Lectures upon Shakespeare and some of the old Poets. Edited by H.N. Coleridge. 2 vols. London: Pickering. Coleridge, S.T. (1996). I poemi demoniaci. A cura di M. Pagnini. Firenze: Giunti.

Corradini, C. (1985). «"Lecturae Dantis” di John Ruskin». Studi danteschi, 57, 271-302. Poi in Corradini, C. (1989). Saggio su John Ruskin: il messaggio nello stile. Firenze: Olschki, 59-89.

Corradini, C. (1990). «Note dantesche inedite dai “Diari” di John Ruskin». Studi danteschi, 62, 117-51.

Discussionie polemiche sul Romanticismo (1975). A cura di E. Bellorini, ristampa a cura di Anco Marzio Mutterle. 2 voll. Roma-Bari: Laterza.

Leopardi, G. (1989). Tutte le opere. A cura di W. Binni, con la collaborazione di E. Ghidetti. 2 voll. Firenze: Sansoni.

Milbank, A. (2011). «Dante, Ruskin and Rossetti: Grotesque Realisme». Havely, N. (ed.), Dante in the Nineteenth Century. Reception, Canonicity, Popularization. Bern: Peter Lang, 139-58.

Pavese, C. [1945] (1974). Feria d'agosto. Torino: Einaudi.

Proust, M. (1987). «Préface du traducteur. Sur la lecture». Ruskin, J., Sésame et les Lys. Édition établie par A. Compagnon. Bruxelles: Complexe.

Ruskin, J. (1903). Comments of John Ruskin on the "Divina Commedia". Compiled by G.P. Huntington. Boston; New York: Houghton, Mifflin \& C.

Saba, U. (2001). Tutte le prose. A cura di A. Stara, con un saggio introduttivo di M. Lavagetto. Milano: Mondadori.

Wordsworth, W. (1997). Poems. Poesie (1798-1807). A cura di A. Righetti. Milano: Mursia. 


\title{
The Stones of Venice: Lady Augusta Gregory and John Ruskin
}

\begin{abstract}
Lady Augusta Gregory was among those Victorian Anglo-Irish genteel women who were deeply influenced by Ruskin's views on the political and artistic history of Venice. She stayed at Ca' Cappello during he visits to Venice, Sir Austen Henry Layard and Lady Enid Layard's beautiful palace on the Grand Canal. She often walked the streets of the Mediterranean city in search of architectural details that Ruskin mentioned in The Stones of Venice. The chapter considers the significance of these Ruskin-inspired sojourns on the formation of Lady Gregory's aesthetic sensibilities at the turn of the twentieth century and reveals the true subject matter of one of her Venetian sketches, now held at the National Library of Ireland.
\end{abstract}

Keywords Ruskin. Lady Gregory. Lady Layard. Sketching. Travel writing.

Summary 1 Introduction. - 2 Ruskin and The Stones of Venice. - 3 Lady Gregory and the Stones of Venice. -4 Conclusion.

\section{Introduction}

John Ruskin's three-volume The Stones of Venice ${ }^{1}$ may have been only one of several travel books written about the Apennine Peninsula during the Victorian period, but it was certainly the one that exerted the most influence on the writers of the Irish Literary Revival between 1880 and 1930. My recent study, Lady Gregory and Irish National Theatre: Art, Drama, Politics, ${ }^{2}$ explores the seminal influence of Ruskin's social thought in Ireland during the Revival period, as expressed in the volumes of The Stones of Venice and in later works such as Time and Tide and Fors Clavigera. ${ }^{3}$ Lady Augusta Gregory herself was a dedicated Ruskinian who disseminated Ruskin's thoughts in various ways through the work of

\footnotetext{
1 Works, 9-11.

2 Remport 2018.

3 Works, 17; 27-29.
} 
the Co-operative Movement, the Home Industries Movement and the Abbey Theatre, known at the time as the Irish National Theatre. ${ }^{4}$ Dramatist, director and designer, Lady Gregory of Roxborough in the west of Ireland county of Galway, became a powerful player in the Irish literary scene. She overcame countless struggles when managing the Abbey Theatre in Dublin and fought doggedly during and after the years of the First World War for a public space in the city to house the impressive art collection of her nephew Hugh Lane.

Lady Augusta was the wife of Sir William Gregory of Coole Park, descendant of a long-line of Gregorys in British colonial service, including the famous East-India Company. She is mostly remembered as a friend of William Butler Yeats, Noble Prize-winning poet and playwright of Dublin/Sligo and of George Bernard Shaw, Oscar and Noble Prize-winning playwright from Dublin. Lady Gregory's achievements, however, should be appreciated in their own rights, acknowledging her immense knowledge of literature and art, and her love of Ireland. It was this love of Ireland, in fact, that rocked the boat of her friendship with Lady Enid Layard, who used to welcome her to Ca' Cappello from the early 1880s until the late 1900s. Sir Henry and Lady Layard showed Lady Gregory the best of what Venetian life had to offer, including visits to the Academy, the Ducal Palace, St. Mark's Cathedral, and the Rossini Theatre. Lady Layard reveals in her diaries that when walking around Venice, she and her husband would carry around with them a Ruskin book or booklet, reading out passages from the much-valued publications (Layard, September $22,1882)$. When Sir William and Lady Gregory arrived at Venice for the first time as a married couple in Octo- ber 1881, their first excursion on Sir Henry's gondola was out to the Stabilimento, followed by visits to the Academy (to view the new exhibition), to St. Mark's (to see the Pala d'Oro), to the Seminario (to view pictures and sculptures), and to the Lido (for the Layards' customary afternoon/evening stroll). Lady Gregory had much to learn because Sir Henry and Sir William were fervent art lovers, paintings in particular, and were associated with both the National Gallery and the British $\mathrm{Mu}$ seum in London. These two men were boundless wells of information on the history of European art, including that of Italy. Sir Henry and Sir William were also involved with the Arundel Society that published John Ruskin's explanatory notes on Giotto's frescoes in the Arena Chapel in Padua.

Lady Gregory herself had read Ruskin's The Stones of Venice when she was still a young, single woman, living with her family in County Galway's Roxborough House. She wrote in her autobiography that when she was reading Ruskin's book - that she had found in the library of a nearby landed estate of Castle Taylor - she believed it was "without much prospect of ever seeing Venice itself". ${ }^{5}$ Augusta's confessions about reading the book are particularly interesting because of the context in which they are given: her comments on The Stones of Venice are immediately followed by the story of Sir William Gregory's marriage proposal, indicating perhaps an early instance of Sir William's marked influence on the aesthetic education of the soon-to-be Lady Gregory. All the greater was her delight when she finally saw Venice herself and met Sir Henry on his gondola. ${ }^{6}$ In her memoirs, Lady Gregory dedicated a whole chapter to Sir Henry, recounting the instance of Ruskin's visit to Ca' Cappello to 
examine the illustrious Layard Collection that included a Carpaccio, a Luini, and works by Giovanni Bellini. After the death of her husband in 1894, Lady Layard took to making copies of these paintings with a view to including them in a book celebrating the collection. Lady Layard was very fond of drawing and sketching - learning

\section{Ruskin and The Stones of Venice}

Ruskin wrote The Stones of Venice following two visits to Venice between 1849 and 1852. Observing a traveller's descent from the Alps to the Po Valley and then onto the Adriatic, Ruskin describes the arrival of river pebbles into the Veneto region as follows:

When the eye falls casually on a map of Europe, there is no feature by which it is more likely to be arrested than the strange sweeping loop formed by the junction of the Alps and Apennines, and enclosing the great basin of Lombardy. [...] The character of the Lombardic plains is most strikingly expressed by the ancient walls of its cities, composed for the most part of large rounded Alpine pebbles alternating with narrow courses of brick; [...] The finer dust among which these pebbles are dispersed is taken up by the rivers, fed into continual strength by the Alpine snow, so that, however pure their waters may be when they issue from the lakes at the foot of the great chain, they reach the Adriatic; the sediment which they bear is at once thrown down as they enter the sea, forming a vast belt of low land along the eastern coast of Italy. The powerful stream of the Po of course builds forward the fastest; on each side of it, north and south, some of the trade from Ruskin himself - and Lady Gregory soon followed her friend in documenting the streets of Venice. Leaving Ca' Cappello for long walks around the city, she studied Venetian architecture with a keen eye - her sketches are testimonies of her love of Venetian art and her knowledge of Ruskin's The Stones of Venice.

there is a tract of marsh, fed by more feeble streams, and less liable to rapid change than the delta of the central river. In one of these tracts is built RAVENNA, and in the other VENICE. ${ }^{7}$

These astute observations about the journey of the mountain pebble from the French-Italian Alps to Veneto would have described Ruskin's own descent from the east of France to Northern Italy, travelling from Chamonix through Milan to Venice, as he usually did. Whether in company or on his own, Ruskin tended to rest in Chamonix in the French Alps to take in the beauty of the mountains and the warm welcome of Chamonix's inhabitants. ${ }^{8}$

Ruskin's remarks about the traveller's first views of Venice are no less poetical. Writing under the spell of English romanticism that coloured Lord Byron's views of the city, Ruskin describes the arrival of the visitor into the winding lagoons as follows:

And at last, when its walls were reached, and the outmost of its untrodden streets was entered, not through towered gate or guarded rampart, but as a deep inlet between two rocks of coral in the Indian Sea; when first upon the traveller's sight opened the long rang-

7 Works, 10: 10-11.

8 Hilton 2002, 33; Sdegno 2015, 33.

285 Fonti, letterature, arti e paesaggi d'Europa | Sources, Literatures, Arts \& Landscapes of Europe 1 John Ruskin's Europe. A Collection of Cross-Cultural Essays, 283-296 
es of columned palaces, - each with its black boat moored at the portal [...]; when first, at the extremity of the bright vista, the shadowy Rialto threw its colossal curve slowly forth from behind the palace of the Camerlenghi; that strange curve, so delicate, so adamantine, strong as a mountain cavern, graceful as a bow just bent; when first, before its moonlike circumference was all risen, the gondolier's cry, "Ah! Stali", struck sharp upon the ear, and the prow turned aside under the mighty cornices that half met over the narrow canal, where the plash of the water followed close and loud [...]; and when at last that boat darted forth upon the breadth of silver sea, across which the front of the Ducal Palace, flushed with its sanguine veins, looks to the snowy dome of Our Lady of Salvation, it was no marvel that the mind should be so deeply entranced by the visionary charm of a scene so beautiful and so strange, as to forget the darker truths of its history and its being. ${ }^{9}$

Besides illustrating Ruskin's beautiful prose style, the above passage is interesting from the point of view of Lady Gregory's own account of her arrival in Venice decades later, an arrival that was recorded in her diary and later published in her memoirs in the 1930s. She writes in her memoirs:

"Sir Henry Layard met us with his gondola and welcomed us to Ca Capello" was the first of many a like entry. And these visits stand out in my memory with their days of delight, the sight of the passing boats, the rafts from the mountain forests, the sound of the water plashing against the wall at night, the cry of "Stali" as a gondolier turned the sharp corner from the narrow to the great canal. ${ }^{10}$

Of course, the gondolier's cries were familiar to all visitors, not just to Lady Gregory and Ruskin. Ruskin himself wrote a short piece about the gondoliers rowing along the narrow canals entitled "The Gondolier's Cry", which is attached to the second volume of The Stones of Venice. ${ }^{11}$ There is, however, a distinct Ruskinian strain in Lady Gregory's lines as they run into an extended description of Ruskin' visit to Ca' Cappello in October 1888. Her images of "passing boats", "rafts from the mountain forests" and "sound of the water splashing against the wall at night" when hearing the gondolier's warnings, ${ }^{12}$ resonate strongly with Ruskin's descriptions of Venice of the "colossal curve" of the Grand Canal, the "gondolier's cry" along the waterways, where "under the mighty cornices that half met over the narrow canal [...] the plash of the water followed close and loud, ringing along the marble by the boat's side". ${ }^{13}$ This echo of Ruskin's style in Gregory's writing carries considerable significance: in the memoirs that she wrote much later in life, she expressed her desire to be remembered not just for her Irish friends and associates but also for whom she called her "Athenaeum Friends": Sir Frederic Burton, Sir Alfred Lyall, Alexander William Kinglake, Henry James, Robert Browning, and Sir Henry Layard. Significantly, to different degrees, each of these painters and writers responded to Ruskin's narrative accounts of Venice.

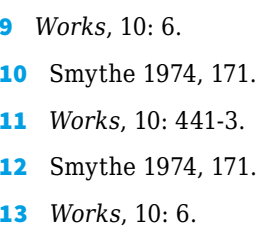


Lady Gregory describes Sir Henry as effectively the British ambassador to Venice "so great was his position" due to his highly esteemed work in the fields of art and archaeology. ${ }^{14}$ He was, as Gregory writes, "free to exercise in his spacious palace the wide and liberal hospitality" that he had learned during his sojourns in the Middle East. ${ }^{15} \mathrm{Ca}^{\prime}$ Cappello on the Grand Canal may have been a spacious building and may have been welcoming to visitors but was not one of the palaces that Ruskin studied in detail in The Stones of Venice. British hospitality was, of course, on a shoe-string during Ruskin's visits to Venice in 1849-1850 and 1851-1852. Tim Hilton remarks that when the Ruskins arrived in Venice, Rawdon Brown was "practically the only English person there: the others, like most of the Venetian aristocracy, had left the city before the [Austrian] siege began" in 1849. ${ }^{16}$ During that siege, many palaces received considerable damage both in their structure and in their ornamentation and after the siege many of the façades were vanishing under the brisk reconstruction effort of the Austrian authorities. ${ }^{17}$ Robert Hewison notes that through sketches, watercolours and daguerreotypes, Ruskin tried to record whatever he could of the famous buildings. ${ }^{18}$ Ruskin's motivations in making the journey to Venice, recording the buildings and writing The Stones of Venice were, of course, manifold but some of them are worth reiterating here. He intended to record and canonise the city's architecture from the Byzantine through the Gothic to the Renaissance; he made the case that Byzantine and Goth- ic architecture were superior to that of the Renaissance; and, further to this, he wanted to demonstrate "how the rise and fall of the Venetian builder's art depended on the moral or immoral temper of the State". ${ }^{19}$

When it comes to the discussion of Byzantine architecture, Ruskin studies a number of palaces, including the Fondaco dei Turchi, the Rio-Foscari House, the Madonetta House, Ca' Farsetti, and Ca' Loredan. The Fondaco dei Turchi was in a dire condition when Ruskin saw it. Only the middle section of the building survived the ravages of historical battles (both Napoleonic and Hapsburg). Nonetheless, Ruskin saw in it a real beauty, observing in detail the system of its arches, the ornamentation of its capitals, and the colouring of its waterfront façade. The Rio-Foscari House, the Terraced House (Palazzo Mengaldo), $\mathrm{Ca}^{\prime}$ Farsetti and $\mathrm{Ca}^{\prime}$ Loredan receive similar sympathetic treatment, Ruskin seeing them as "indicative of Byzantine workmanship". ${ }^{20}$ This is high praise from the art critic who considers Byzantine architecture, steeped in medieval Christian religiosity, more valuable than Greek architecture, known for its use of human proportions and exhibiting an ancient pagan spirit. Ruskin compares Byzantine embellishment to Greek ornamentation, and draws the conclusion that the former is superior in style because of its closer affinity to the natural world. Ruskin writes that Byzantine shafts in Venice show a "greater love of nature" than the most ornamented of Corinthian columns because no two sides of a Byzantine capital are alike. He argues that this il-

\footnotetext{
14 Smythe 1974, 171.

15 Smythe 1974, 172

16 Hilton 2002, 141.

17 Hewison 1978, 12.

8 Hewison 1978, 12.

9 Works, 9: 14.

Works, 10: 152.
} 
lustrates how the motifs of these capitals take their inspiration directly from nature, filled with a wide variety of leaves and flowers. ${ }^{21}$ Ruskin continues these observations with a reflection on Byzantine colouring, drawing connections between the colouring of Venetian shafts and façades and the "bright and pure colour which, in a modified form, was afterwards the root of all the triumph of the Venetian schools of painting"..22 Similar to the case of sculptural details on Venetian buildings, Ruskin finds divine ordinance in Byzantine colouring. When compared to the darker hues of a Renaissance oil painting, the natural Venetian Byzantine palette is once again found more desirable: Ruskin perceives in it a strong association with the sphere of the Divine. He remarks that "the more faithful and earnest the religion of the painter, the more pure and prevalent is the system of his colour". ${ }^{23}$

Ruskin continues in much the same vein when he studies Gothic architecture later on in the second volume of The Stones of Venice: he notices once again the arrangement of stories, the relation of the central to the lateral arches on waterfront façades, and the embellishment of structural elements such as doors, windows, columns and balconies. He claims that one can notice a "loss of unity of conception which regulated Byzantine composition". ${ }^{24}$ Byzantine architecture, claims Ruskin, is "centralised in its ornamentation as much as in its proportions". ${ }^{25}$ He further examines the use of twisted shafts, decorative chamfers, intricate traceries, intrigu- ing spires, and the recurrent use of fleur-de-lis motif in Gothic architecture. ${ }^{26}$ He notes that balconies are often used in order to enhance the architectural splendour of a building, writing that "the Gothic palaces owe half of their picturesque effect" to the decorative design of their balconies. ${ }^{27}$ By way of illustrating the point, he selects a number of Venetian palazzos for examination, including the Ca' Falier, Marco Querini's Palace and the Ca' Sagredo.

Since the balconies of these palaces are decorated both with eye-catching balustrades and stunning windows, this examination offers the art critic a further chance to elucidate his categorisation of Venetian windows, comparing them to those of the late Gothic style found in Verona. ${ }^{28}$ Lady Gregory later takes a note of these balconies with their laced windows and decorative balustrades, recording some of them in her sketchbooks during the 1890s. She follows Ruskin in noting the Gothic archways and windows of the Ducal Palace, the building that is one of the main focuses of Ruskin's attention in his appraisal of Venetian architecture both in the first and the second volume of The Stones of Venice, alongside St. Mark's Cathedral. As for the Doge's Palace, Ruskin examines its structural elements and its long history dating back to the early medieval times. Additionally, he suggests a new way of understanding its historical significance, one that has at its heart a spiritual/theological interpretation of beauty. As he often did before and 
would do repeatedly after the publication of the work, he connects natural light with the realm of the Divine, claiming that the way in which natural light falls on the Palazzo Ducale through the holes of its traceries and other sculpted elements resembles the way in which God's light shines down on humankind and the world. Ruskin is persistent in saying that Medieval Gothic was first and foremost an ecclesiastical style and, as such, should be appreciated for its devotional aspects above all else. At the end of the second volume of The Stones of Venice, after an extended analysis of the Ducal Palace, he returns to where he started the volume, only this time adding a new, devotional layer to his description of Venice and the Alps. About his walk around the Ducal Palace looking over to the white-capped mountains of the Alps, Ruskin writes that

God had done a greater work in breathing into the narrowness of dust the mighty spirits by whom its haughty walls had been raised, and its burning legends written, than in lifting the rocks of granite higher than the clouds of heaven, and veiling them with their various mantle of purple flower and shadowy pine. ${ }^{29}$

\section{$3 \quad$ Lady Gregory and the Stones of Venice}

Sir William Gregory and his wife took a different route to that of Ruskin, arriving at Venice via the Brenner Pass from Munich through Austria (Gregory, October 26, 1881). On their way to Venice, the Gregorys stopped to wonder at the Gothic architecture in Verona, visiting the town's medieval cathedral, Sant'Anastasia, and its Dominican church, San Zeno (Gregory, October 27, 1881). Soon after the Gregory's arrival in Venice, Sir Henry Layard invited them to a gondola ride in the direction of St. Mark's Cathedral, despite the gondoliers' ongoing strike against large steamboats threatening to take their livelihood (Layard, October 31, 1881). A few days later, Sir William and Lady Gregory returned to the cathedral to view the Pala d'Oro, the golden and enamelled altar screen inside the old Byzantine church (Layard, November 1, 1881). Lady Gregory marvelled at the ornamentation of the cathedral and enjoyed the evening Vespers during which the golden Byzantine altar piece was uncovered for the worshippers (Layard, November 1, 1881). Lady Augusta saw for herself the shafts, the capitals, the

structure wonderfully described by Ruskin. On their way back home, the Layards and Lady Gregory went to the Lido, to land at Santa Maria Elisabetta and walk around until darkness fell on land and sea. Enid Layard records the evening and its colours in a truly Ruskinian manner: "We could not take a long walk as it got late \& we came home the distant mountains tipped with snow stood out clear on one side the Dolomites \& the Alps were towards Trieste $\&$ on the other the hills above Bologna. The sun set was lovely \& the water shot with all colours, blue, pink \& green \& gold" (Layard, November 1, 1881).

Sir William and Lady Gregory were to enjoy the beauty of the Italian countryside later; first there was much religious art to discover in the local churches. Gentile and Giovanni Bellini were among Sir Henry Layard's favourite artists, whose tableaux formed an important part of the Layard Collection that decorated the walls of Ca' Cappello. The Bellini brothers were Renaissance artists. Ruskin admired their paintings because of their devotional quality. Ruskin asserted that while the work 
of other Italian artists could be studied easily in a museum or a gallery, "Tintoret and Bellini can be judged of only in Venice". ${ }^{30}$ Agreeing with Ruskin on this matter, Sir Henry and Sir William lead numerous art tours around Venice in order to locate a beautiful Bellini tableau or altarpiece. By the side of both men, Lady Gregory visited the most famous historical locations of the city. She visited the Basilica dei Santi Giovanni e Paolo, site of the funeral service and burial of a long list of Venetian doges, where she learned about Venetian history and studied Giovanni Bellini's polyptych of San Vincent Ferrer (1464-1468) (Gregory, November 4, 1881). There she learned that Santi Giovanni e Paolo was the church where Gentile and Giovanni Bellini had been buried in the early sixteenth century. Lady Gregory went to see Bellini's triptych at the Basilica di Santa Maria Gloriosa dei Frari near Ca' Cappello, and she saw a Bellini altarpiece (Madonna and the Child, 1505) in San Zaccaria, alongside further paintings at the Galleria dell'Accademia on the Grand Canal (Gregory, November 1-2, 1881). Bellini's several Sacre Conversazioni along with his and Andrea Previtalis's Allegories were exhibited at the Accademia alongside Paris Bordon's The Fisherman Presenting the Ring to Doge Gradenigo (1534) and Vittore Carpaccio's St. Ursula Cycle (1490s) (Gregory, October 30, 1881). Lady Gregory tentatively attributes one other painting to Bellini, one that she entitles The Last Supper, located in the church of San Salvatore. She raises questions regarding this attribution and today the biblical tableau is, in fact, attributed to Bellini's assistant, Vittore Carpaccio. She takes a further note of the Bellini at the Ducal Palace, as well Carpaccio's St. George and the Dragon in the Scuola di San Giorgio degli Schiavoni, of which Ruskin wrote several times during his long career as art critic, from St. Mark's Rest to Fors Clavigera $^{31}$ (Gregory, November 4, 1881). A year later, in preparation for the Gregorys' next visit to Venice, the Layards returned to the Scuola to admire the St. George series that had been carefully studied by Ruskin in St. Mark's Rest: "We took with us Ruskin's little book on them \& amused ourselves vastly reading it on the spot" (Layard, September 22, 1882).

Ruskin's good friend, Rawdon Brown, assisted Sir Henry in assembling the Venetian collection of paintings at Ca' Cappello. Sir Henry's collection was renowned among both local and international cognoscen$t i$; so much so, argues art historian Cecilia Riva, that "[t] he collection was considered one of the most beautiful in the whole Veneto". ${ }^{32}$ Riva adds that following the death of Lady Enid Layard in the early 1910s, Gino Fogolari, who was the Director of the The Royal Galleries of the Academy of Venice, "made a plea for transforming Ca' Cappello Layard into a branch of the National Gallery and keeping the paintings within Italy". ${ }^{33}$ The Layard collection had great art historical value and abounded in North Italian religious artworks, including Gaudenzio Ferrari, Bartolomeo Montagna, Francesco Bonsignori, Andrea Previtali, and Bernardino Luini. Sir Henry owned Carpaccio's The Departure of Ceyx, a painting linked to the St. Ursula Cycle at the Accademia, and Paris Bordon's Christ Baptizing St. John Martyr, also linked to the Accademia, in addition to Gentile Bellini's The Sultan Mehmet II (1480) and Giovanni Bellini's Virgin and the Child (1490s), as well as a tableau from Giovanni Bellini's work-

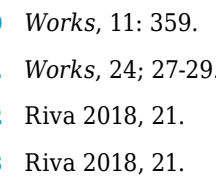


shop, Adoration of the Kings (1475-1480). ${ }^{34}$ Sir Henry and Lady Layard were immensely proud of their collection, one that also contained artefacts from the Middle East and artworks from Spain, two locations where Sir Henry had spent a considerable amount of time as archaeologist and as a British ambassador. Lady Layard records in her diary that during Lady Gregory latest visit to $\mathrm{Ca}^{\prime}$ Cappello in May 1896, she decided to make copies of the paintings in the Layard Collection with a view to publishing these in an illustrated catalogue for the wider public to read (Layard, May 4, 1896). Lady Layard sat down daily to this task, giving Lady Gregory time to discover the lagoons of Venice for herself. There is a significant change here from previous visits, in that in the 1880s Lady Gregory used to walk about Venice accompanying her husband and Sir Henry to a place of great art historical significance. A decade later, she walks about the streets of Venice as a cognoscente of the local art world who had taken many lessons at the history of the Veneto region and of Northern Italy. On her return home to Ca' Cappello, Lady Gregory recognizes her friend's dedication to her new art project, encouraging Lady Gregory herself to make sketches of Venice. So she decides to walk about, making visual records of some of the buildings and monuments in the district in which Ca' Cappello is located, San Polo, as well as further afield towards St. Mark's Cathedral in the Sestiere di San Marco.

One of the buildings that she records is Ca' Cappello itself, the house in which she is staying, and one of the people she sketches is her host, Lady Enid Layard. Lady Gregory's sketch of her friend, now held at the National Library of Ireland, is of enormous significance for a number of reasons: first, in the perfection of the lines and the exactness of proportions, it is testament to $\mathrm{Au}$ gusta Gregory's skill as an artist; second, it documents Enid Layard as an artist in her own right, exhibiting not just her ability to draw but also her knowledge of Renaissance art; and third, it showcases Augusta Gregory's knowledge of the oft-used pictorial compositional techniques of 'painting-within-the-painting'. Lady Gregory's sketch depicts a female figure seated comfortably on a mount on the left, a figure who faces a painted tableau that is hanging on the wall, and which takes up much of the middle section of the sketch: ${ }^{35}$ Lady Gregory's drawing has not yet been identified: Melita Cataldi has suggested that the female figure on the left of the scene was Lady Gregory herself and thus the drawing was a form of self-portrait; ${ }^{36}$ the National Library of Ireland catalogue makes no identification as to the subject matter of Lady Gregory's sketch. ${ }^{37}$ Cataldi's claim of the sketch being a self-portrait can be refuted by both Lady Gregory's sketch itself and by Lady Layard's diary entry from 13 May 1896. Lady Gregory herself wrote on the sketch next to the drawing figure of the woman: "the green drawing room - May 13 - " and Lady Layard's diary entry for 13 May 1896 reads: "copying the Holy Family by Bissolo". Since Lady Layard destroyed her own drawing later that day, Lady Gregory's sketch is the only existing evidence of her friend working on the Bissolo on 13 May 1896. Beside this, the sketch is significant for another reason in relation to Gregory criticism: as mentioned earlier, it is an early piece of evidence of Lady Gregory's use of a well-known compositional device, the 'painting-within-the painting', that was used by artists from

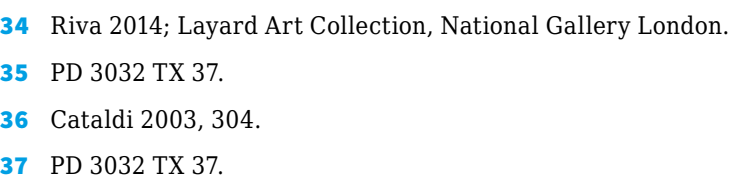


the Renaissance onwards. Lady Gregory had knowledge of this devise from Spanish sources: the paintings of Diego Velázquez which were exhibited at the Prado in Madrid. Sir William and Lady Gregory visited the Layards in Madrid during Sir Henry's term as British ambassador there and visited the museum to view the famous Spanish masters, including Diego Velázquez (Gregory, April 16, 1887). Sir William and Lady Gregory also owned a Velázquez, Christ in the House of Mary and Martha (1618), which Sir William bequeathed to the National Gallery in London in $1892 .{ }^{38}$ Later as dramatist of the Abbey Theatre in Dublin, Lady Gregory would make use of this pictorial devise of the 'painting-within-the-painting' that she had seen employed in many European paintings, connecting her Irish plays to a wider European artistic tradition, part of which she had sampled during her art tours of the Apennine and the Hibernian Peninsulas. Lady Gregory's short rural Irish plays - the likes of The Image (1909) and Hyacinth Halvey (1906) - will pay homage to this European pictorial tradition that she had studied for long hours in the galleries and museums of Rome, Venice, Bologna, Florence, Verona and Venice, making her plays unique in the context of the Irish Literary Revival from the 1890s to the 1920s. ${ }^{39}$

Ca' Cappello was decorated by the paintings of the Italian masters, carving out a special place for the Layard Collection in Venice. As good friend of Enid Layard, Augusta Gregory continues to return to Venice after the death of Sir William in 1892 and the death of Sir Henry in 1894. Many of her earlier forms of entertain- ment continue, with visits to the Picture Gallery of the Academy, St. Mark's Cathedral and the Ducal Palace, but she begins to discover new ways of amusing herself. She takes long rides on Enid's gondola, going to the Giudecca, the Lido and the island of Murano. She also takes long walks around the seemingly floating streets, sketching the old, medieval architectural features around her. By this time, she had acquired the 1887 edition of Ruskin's Examples of the Architecture of Venice from Venice, which reiterated once again the exquisiteness of Venetian architecture. ${ }^{40}$ While strolling on the streets of Venice, she completes a number of sketches, all of which illustrate Ruskin's artistic influence on her now well-developed aesthetic sensibility. One of the locations she draws is the Basilica di Santa Maria Gloriosa dei Frari, where she had seen the Pesaro Triptych by Giovanni Bellini, alongside works by Titian, Veneziano, and Vivarini. ${ }^{41}$ Lady Gregory chooses to make a drawing of a side entrance to the Franciscan church, an entrance ornamented with Bartolomeo Bon's relief of Virgin and St. Francis. Bon's rather impressive Gothic traceries embellished also the Ducal Palace and the Ca' d'Oro on the Grand Canal. On the other side of town, she sketches the Calle del Paradiso near the Ponte del Paradiso. ${ }^{42}$ Stephen Kite draws attention to Ruskin's continuous interest in tabernacles, gables, and traceries, and in what he calls "Venetian affection for triangular motifs at different scales". ${ }^{43}$ Kite sees this demonstrated in Ruskin's description of the buildings of Murano in the second volume of The Stones of Venice and in "the gables of certain

38 Remport 2011, 50-1.

39 Remport 2018, 121-56.

40 Sotheby and Co. 1972, 54.

41 PD 3032 TX 49

42 PD 3032 TX 49

Kite 2012, 154

Fonti, letterature, arti e paesaggi d'Europa | Sources, Literatures, Arts \& Landscapes of Europe $1 \mid 292$ 
streets, such as the famous Calle del Paradiso". ${ }^{44}$ Situated en route to St. Mark's Cathedral, the Calle and Arco del Paradiso are remnants of the glorious days of Venice, with the crests of the Foscari and Mocenigo families exhibited under the protective arms of the 'Madonna of Mercy'. Lady Gregory's attention to Gothic detail in these sketches is stunning, exposing her careful attention to architectural detail influenced by John Ruskin's work as travel guide and art historian.

Two further sketches reveal her interest in Venetian Gothic architecture. Near the Ponte del Paradiso, there are a number of houses that exhibit exquisite Medieval features, two of which she drew on one of her excursions around the city. ${ }^{45}$ Lady Gregory's sketch of the Gothic window with the pointed arch, and with the curtain casually falling on the window-sill, dated 19 June 1896, is reminiscent of Ruskin's drawing of the windows of $\mathrm{Ca}^{\prime}$ Falier, one of the more prominent sketches of Ruskin's in the second volume of The Stones of Venice. ${ }^{46}$ Lady Gregory makes a little drawing next to this Gothic window: that of a domestic balcony with a traced balustrade, straight clean side shafts and a round arch to complete the architectural composition. This drawing brings to mind Ruskin's argument about the coexistence and correlation between ecclesiastic and domestic architecture during the period of Venetian Gothic. ${ }^{47}$ Ruskin persistently and fervently argues in the second volume of The Stones of Venice that, in part, the beauty of Venetian architecture lies in the fact that the same Gothic features that ornament religious buildings decorate non-ecclesiastical constructions, such as noblemen's palaces or common houses. Discussing St. Mark's Cathedral, the art critic elucidates the nature of domestic architecture in Northern Italy:

at the time when the best of them were built, every man's house was a kind of temple; a figure of the Madonna, or of Christ, almost always occupied a niche over the principal door, and the Old Testament histories were curiously interpolated amidst the grotesques of the brackets and the gables. ${ }^{48}$

Once again, Ruskin's religious attitude saturates his art historical writings, drawing attention to what he perceived as the close connection between the builders, the building and the sphere of the Divine.

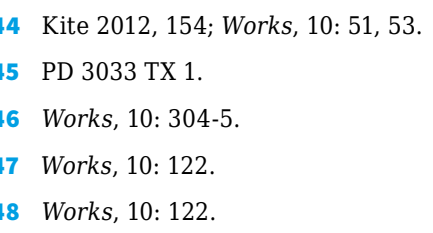


Figure 1 John Ruskin, The Four Venetian Flower Orders, from The Stones of Venice, vol. III, tav. X. London: Smith, Elder and Co., 65, Cornhill, 1853. Courtesy of the Marciana Library, Venice

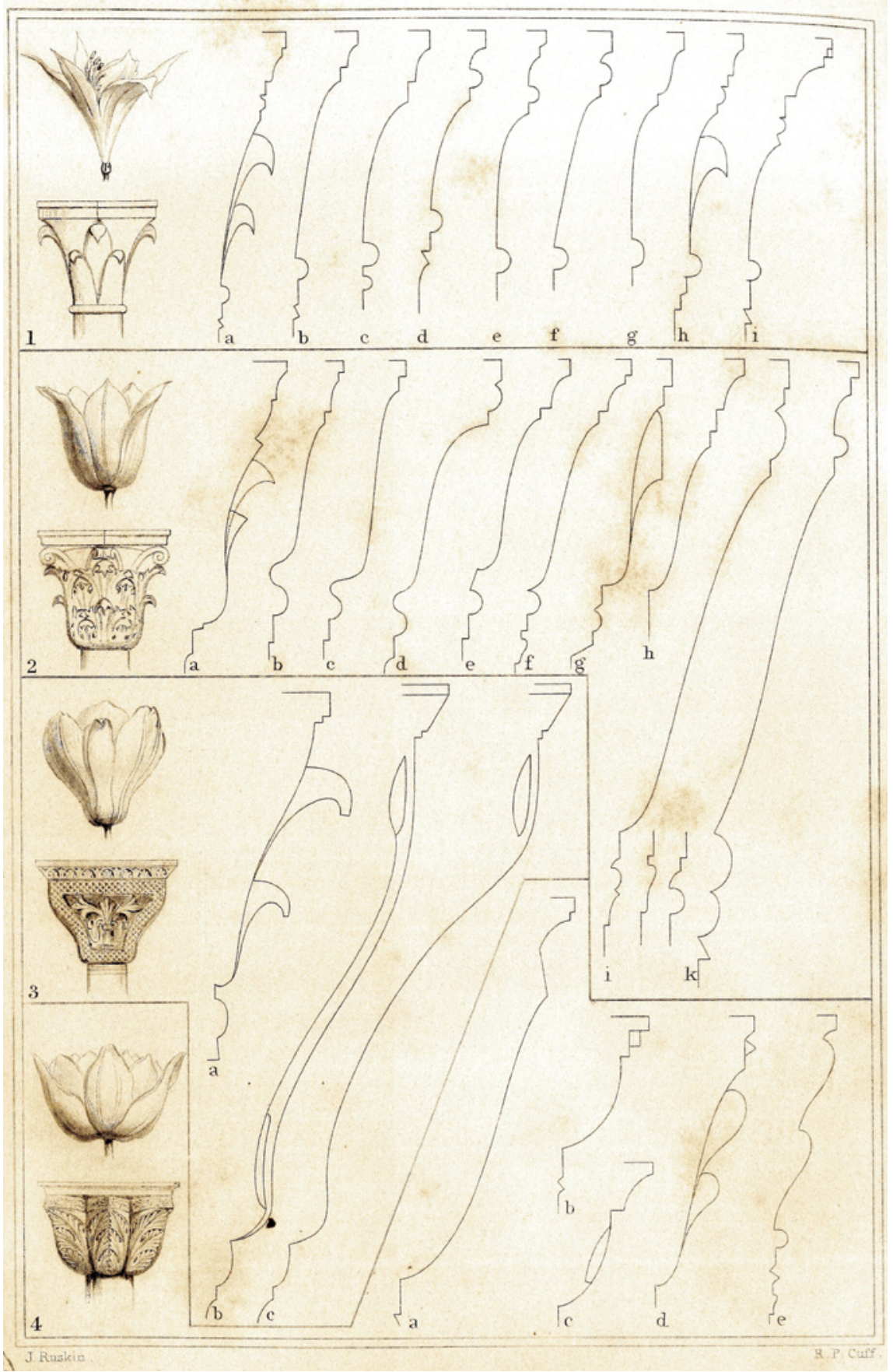




\section{Conclusion}

Lady Gregory's Ruskin-inspired art tours need to be understood in the context of the whole of her cultural experience in Venice during the 1880s and 1890s. While her early diaries from the period are clear indications of her education in Venetian art and architecture, her later diaries are true revelations of her mounting interest in the multifaceted cultural and political discussions carried out at $\mathrm{Ca}^{\prime}$ Cappello during those two decades. While at Venice, she attends several cultural programmes such as the performance of Lord Byron in Venezia at the Marionette Theatre (Layard, October 24, 1885), Verdi's famous and scandalous Rigoletto at the Teatro Rossini (Gregory, November 5, 1881; Layard, November 5, 1881), and I due Foscari, also at the Teatro Rossini (Layard, November 1, 1887). She visits the Palazzo Contarini del Bovolo to see its beautiful spiral staircase (October 28, 1881); the Palazzo Correr that housed Teodoro Correr's fabulous Venetian collection (Layard, September 27, 1882); the Palazzo Barbaro that was renovated by Daniel and Ariana Sargent Curtis to showcase its Baroque splendour (Layard, May 14, 1896); and the Palazzo Labia to view Venetian artist Giovanni Battista Tiepolo's famous frescoes from the late 1740s (Layard, June 9, 1896). Because Lady Gregory's diaries of the period either consist of extensive descriptions of paintings (especially those from the 1880s) or wide-raging accounts of local Venetian gossip (especially those from the 1890s), Irish literary critics and Gregory biographers have been led to believe that her diaries from the period are of little importance, insignificant to her later work for the Irish Literary Revival that would bloom from the 1900s to the 1920s. The exact opposite is the case: Lady Gregory's visits to Venice are of immense significance for her later work as founder, dramatist and designer of the Abbey Theatre in Dublin and for her involvement in the artistic and cultural circles of early-twentieth-century Ireland. Her visits to Venice and her many Ruskin-inspired tours of the beautiful city shaped her artistic and aesthetic sensibilities, as evidenced in her sketchbooks from the period. More important still, her experience of fin-de-siècle Venice would leave a deep imprint on her life and work in Ireland, providing a further instance of Ruskin's wide-ranging influence on the period of the Irish Literary Revival beyond that of the more obvious cases: William Butler Yeats, Oscar Wilde and George Bernard Shaw. 


\section{Bibliography of Works by John Ruskin}

References are to volume and page numbers in:

Cook, E.T.; Wedderburn, A. (eds) (1903-1912). The Works of John Ruskin. Library Edition, 39 vols. London: George Allen.

https://www.lancaster.ac.uk/the-ruskin/the-complete-works-of-ruskin/.

Works, 9-11: The Stones of Venice I-III. | 17: Unto this Last, Munera Pulveris, Time and Tide, Other Writings on Political Economy (1860-1873). |

24: Giotto and His Works in Padua, The Cavalli Monuments, Verona, Guide to the Academy, Venice, St Mark's Rest.| 27-29: Fors Clavigera I-III.

\section{General Bibliography}

\section{Primary Sources}

Emory University Atlanta (GA), Robert W. Woodruff Library, Gregory Family Papers, Special Collections and Archives Division.

Gregory, Lady Isabella Augusta. Holograph Diary, 1880-1882. Gregory Family Papers, Special Collections and Archives Division. Atlanta: Robert W. Woodruff Library.

Gregory, Lady Isabella Augusta. Typewritten Diaries, 1882-1892. Lady Augusta Gregory Papers, Albert A. and Henry W. Berg Collection, New York Public Library.

Gregory, Lady Isabella Augusta. Sketchbooks, Prints and Drawings Department, National Library Ireland. PD 3032 TX - 3033 TX.

Layard Art Collection. https://www.nationalgallery.org.uk/about-us/history/collectors-and-benefactors/austen-henry-layard.

Layard, Lady Mary Enid Evelyn. Add. MS 46153-46170: 1869-1912. British Library. https://www.browningguide.org/lady-layards-journal/.

Pethica, J. (ed.) (1996). Lady Gregory’s Diaries, 1892-1902. Gerrards Cross: Colin Smythe.

Smythe, C. (ed.) (1974). Seventy Years, Being the Autobiography of Lady Gregory. Gerrards Cross: Colin Smythe.

Sotheby and Co. (ed.) (1972). Catalogue of Printed Books Formerly in the Library at Coole, the Property of the Lady Gregory Estate. Sotheby Auction Catalogue for 20 and 21 March 1972. London: Sotheby and Co.

\section{Secondary Sources}

Cataldi, M. (2003). “Lady Gregory’s Sketchbooks”. Deandrea, P.; Tchernikova, V. (eds), Roots and Beginnings. Venice: Cafoscarina, $303-16$. Hewison, R. (1978). Ruskin and Venice. London: Thames and Hudson.

Hilton, T. (2002). John Ruskin. New Haven; London: Yale University Press.

Kite, S. (2012). Building Ruskin's Italy: Watching Architecture. Farnham: Routledge; Ashgate.

Remport, E. (2011). “I Usually First See a Play as a Picture': Lady Gregory and the Visual Arts”. Irish University Review, 41(2), 42-58.

Remport, E. (2018). Lady Gregory and Irish National Theatre: Art, Drama, Politics. Basingstoke: Palgrave Macmillan.

Riva, C. (2014). "La collezione Layard nel catalogo 1896". Predella, 35, 53-78.

Riva, C. (2018). "An Art World Insider: Austen Henry Layard and the Nineteenth-Century European Art Trade". Journal for Art Market Studies, 2. https://doi.org/10.23690/jams.v2i2.28.

Sdegno, E. (2015). “The Alps”. O’Gorman, F. (ed.), The Cambridge Companion to John Ruskin. Cambridge: Cambridge University Press, $32-48$. 


\title{
From Ruskin's Amiens to Proust's Venice Reflections on the Diapered Screen
}

\author{
Emily Eells
}

CREA, Université Paris Nanterre, France

\begin{abstract}
The paper focuses on the first of Ruskin's two translations into French, La Bible d'Amiens (published in 1904, followed by Sésame et les lys in 1906), and on how Proust reshaped Ruskin's work in his lengthy introduction and copious footnotes. Proust chose to translate The Bible of Amiens in order to acquaint a French readership with Ruskin's only full-length study of France and French Gothic architecture, and also because Ruskin considered the work to be representative of his entire 'system'. This paper examines how Proust appropriates Ruskin's text, making it his own through the addition of his invasive critical apparatus. The fact that Proust started to work on La Bible d'Amiens when he was in Venice in 1900 casts an Italian hue on his version, which emphasizes the parallels Ruskin drew between the Venice of Picardy and the Queen of the Adriatic.
\end{abstract}

Keywords John Ruskin. Marcel Proust. The Bible of Amiens. La Bible d'Amiens. French Gothic architecture.

Summary 1. Translating Ruskin's Stones of Amiens. - 2. Reflections of Ruskin's Italy in Proust's Novel.

...those blessed days when with a few other disciples "in spirit and in truth" of the Master we went about in Venice in a gondola, listening to his predication at the water's edge, landing at each of the temples that seemed to rise from the sea, proffering the object of his description and image of his very thought, imparting such light to his books, as to-day sheds on them their immortal lustre. ${ }^{1}$

The spirit and cadence of the epigraph could be ascribed to Ruskin, though in fact the lines were written originally in French, by Marcel Proust, as a footnote to his translation of The

1 Ruskin-Proust 1904, 245-6: “...ces jours bénis où, avec quelques autres disciples 'en esprit et en vérité' du mâ̂tre, nous allions en gondole dans Venise, écoutant sa prédication au bord des eaux, et abordant à chacun des temples qui semblaient surgir de la mer pour nous offrir l'objet de ses descriptions et l'image même de sa pensée, pour donner la vie à ses livres dont brille aujourd'hui sur eux l'immortel reflet". This footnote by Proust is from the translation of Nordlinger 1955, 61 . 
Bible of Amiens. The possible confusion about their authorship illustrates the formative role Ruskin played in Proust's writing and how he not only translated Ruskin texts but also transposed his texts into his own.

Proust discovered Ruskin in November 1893 when the first translated extracts of his work were published in the Bulletin de l'Union pour l'action morale. One of them ends with Ruskin's bidding in St. Mark's Rest:

Qu'aucun vrai disciple de moi ne soit « Ruskinien» pour toujours ; qu'il suive, non pas moi, mais l'impulsion de sa propre âme et la direction de son Créateur.

No true disciple of mine will ever be a 'Ruskinian'! - he will follow, not me, but the instincts of his own soul, and the guidance of its Creator. ${ }^{2}$

Proust appears to rewrite that quotation in the preface to his translation of Sesame and Lilies:

Et c'est là, en effet, un des grands et merveilleux caractères des beaux livres (et qui nous fera comprendre le rôle à la fois essentiel et limité que la lecture peut jouer dans notre vie spirituelle) que pour l'auteur ils pourraient s'appeler "Conclusions" et pour le lecteur "Incitations". Nous sentons très bien que notre sagesse commence où celle de l'auteur finit, et nous voudrions qu'il nous donnât des réponses, quand tout ce qu'il peut faire est de nous donner des désirs.

And there, indeed, is one of the great and marvellous features of beautiful books (and one which will make us understand the role, at once essential and limit- ed, that reading can play in our spiritual life) which for the author could be called "Conclusions" and for the reader "Incitements". We are fully aware that our wisdom begins where that of the author ends, and we would like to have him give us answers, when all he can do is give us desires. ${ }^{3}$

Reading Ruskin instilled in Proust the desire to write, and this excerpt from his preface to Sésame et les lys (1906) shows how he reworked and overwrote Ruskin's texts.

Proust translated and heavily annotated two volumes of Ruskin's works: The Bible of Amiens (the French version was published in 1904), followed two years later by Sesame and Lilies. Before embarking on his À la recherche du temps perdu, he also penned two pastiches of Ruskin's style as a means of ridding himself of its influence. This paper will focus on his first translation and how he rewrote Ruskin's text by adding a preface of nearly a hundred pages and numerous footnotes such as the one cited above. A disciple of Ruskin's, Proust used his work as a framework on which to construct his œuvre. This study will examine how Proust's voice harmonized with the voice of the Master while revealing how he struck chords of dissonance in a vain attempt to silence the Ruskinian resonance in his work.

The subtitle of this paper - "reflections on a diapered screen" - echoes Ruskin's use of the term: according to the Shorter Oxford English Dictionary, it was originally the name of a textile "woven with patterns showing up by opposite reflections from its surface, and consisting of lines crossing diamond-wise, with the spaces filled up with parallel lines, leaves, dots etc.". ${ }^{4}$ The word is also used to refer to the ground pattern in a painting or on a 


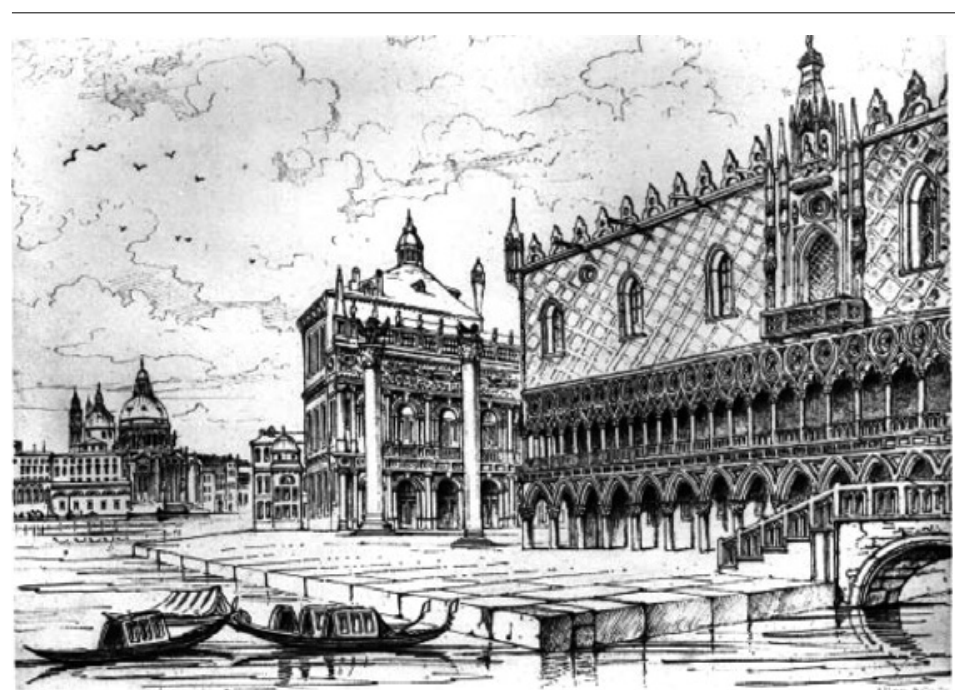

Figure 1 John Ruskin, The Ducal Palace, Venice, 1835. In Works, 35: PI. 8, facing 182. Scanned image and text by George P. Landow.

http://www.victorianweb.org/painting/ruskin/ drawings/14.html wall, for example when Ruskin describes the façade of the Ducal palace in Venice and its "the diaper pattern of the red and white marbles represented as a bold panelling in relief". ${ }^{5}$ That quotation comes from the passage in Praeterita in which Ruskin relates his first visit to Venice and the drawing he did of the Doge's Palace when he was seventeen, where the diaper pattern is evident [fig. 1]. Proust, who claimed to know Praeterita by heart, would have noted Ruskin's use of the term "diaper". Equally, he would have picked up the two instances when Ruskin used the word in his description of the Byzantine art and mosaics of the Basilica of St Mark's in Venice:

Hence arose the universal and admirable system of the diapered or chequered background of early orna- mental art. They are completely developed in the Thirteenth century, and extend through the whole of the fourteenth, gradually yielding to landscape and other pictorial backgrounds [...]. The whole space of the brick wall was considered as a background; it was covered with stucco, and painted in fresco, with diaper patterns. ${ }^{6}$

Venetian diapering provides a suggestive image of how, on the one hand, Proust constructed a kind of mottled screen through which we read Ruskin's Bible of Amiens today and how, on the other hand, Proust made Ruskin's works into a kind of patterned screen onto which he projected his multi-volume novel $\dot{A}$ la recherche du temps perdu (In Search of Lost Time). He first uses the term dia- 
pré in its opening volume, when his young narrator reflects on his childhood reading:

Dans l'espèce d'écran diapré d'états différents que, tandis que je lisais, déployait simultanément ma conscience, et qui allaient des aspirations les plus profondément cachées en moi-même jusqu'à la vision tout extérieure de l'horizon que j'avais, au bout du jardin, sous les yeux, ce qu'il y avait d'abord en moi de plus intime, la poignée sans cesse en mouvement qui gouvernait le reste, c'était ma croyance en la richesse philosophique, en la beauté du livre que je lisais, et mon désir de me les approprier, quel que fût ce livre.

In the sort of screen dappled with different states of mind which my consciousness would unfold at the same time that I was reading, and which ranged from aspirations hidden most deeply in myself to the completely exterior vision of the horizon that I had, at the bottom of the garden, before my eyes, what was first in me, innermost, the constantly moving handle that controlled the rest, was my belief in the philosophical richness and beauty of the book I was reading, and my desire to appropriate them for myself, whatever that book might be. ${ }^{7}$

Proust explains here how reading sets in motion a dynamic between the depth of the self and the outside world, and in this passage the diapered screen seems to be placed both at the back of the reader's mind's eye and on the horizon in front of his lifted eyes. The reader's desire to appropriate the philosophic wealth and beauty of the book he is reading simulates Proust's own experience of reading Ruskin. In a working version of this passage, Proust makes the link with Ruskin explicit by spelling out the book title The Bible of Amiens. In the same rough draft, he associates the book the young narrator is reading with other Ruskinian allusions - its "paysage montagneux d'eaux vives" (the mountain landscape with its flowing streams), the "grappes de fleurs" (the clusters of flowers) and the "collines boisées" (the piny hills) - which all figure in Ruskin's evocation of the Jura at the beginning of "The Lamp of Memory", the penultimate chapter of The Seven Lamps of Architecture. ${ }^{8}$ The formative experience of reading is thus imprinted with Ruskin's texts, testifying to the fundamental importance they had on Proust.

\section{Translating Ruskin's Stones of Amiens}

The Bible of Amiens is an important, late contribution to Ruskin's work on the Gothic architecture of Northern France, an area which, "dull as it seems to most travelers", was to him "a perpetual Paradise". ${ }^{9}$ Proust managed to persuade the Mercure de France to publish his

French translation of the volume, stressing in a letter to its editor the importance of acquainting a French readership with what Ruskin had to say about their country and culture:

7 Proust 1913-1927, 1: 83. The quoted translation is from Lydia Davis (Proust 2002, 86)

8 The manuscript passages from Cahier $29 \mathrm{f}^{\circ} 82 \mathrm{r}^{\circ}$ are transcribed in Proust 1913-1927, 1: 753. Proust borrows from Robert de La Sizeranne's French translation of Ruskin in La Sizeranne 1909, 6-8.

9 Works, 6: 419. 
Je prétends que si l'on ne devait traduire qu'un Ruskin, c'est celui-là ne fût-il pas le plus beau qui devrait être publié. Parce que c'est le seul qui soit sur la France, à la fois sur l'Histoire de France, sur une ville de France et sur le Gothique français. [...] Enfin vous savez que Ruskin le considérait comme tout à fait représentatif de son système.

I believe that if only one Ruskin volume were to be translated, it should be this one, even if it is not the most beautiful. Because it's the only one about France, that is about French history, a French town and French Gothic. [...] Finally, you know that Ruskin considered it as perfectly representative of his system. ${ }^{10}$

By 'system', Proust is probably referring to the conclusion Ruskin reaches at the end of The Bible of Amiens: "the Life, and Gospel, and Power of [the history of Christianity], are all written in the mighty works of its true believers [and] the simplest, completest and most authoritative in its lessons to the active mind of North Europe, is this on the foundation stones of Amiens". ${ }^{11}$ As the editors of the Library Edition explain, Ruskin's aim was to revisit "some passages of early Christian history, in order to illustrate the spirit which lit the Lamps of Christian Architecture". ${ }^{12}$

Proust read The Bible of Amiens in Venice in the spring of 1900, and even inscribed the name of his hotel and his room number in his copy. ${ }^{13}$ He probably started on the project of translating it during that stay in Venice which would explain why the critical apparatus he added to the translation has an Italian hue. Notably when he annotates Ruskin's third chapter entitled "The Lion-Tamer" about St Jerome, by adding a long passage from $S t$ Mark's Rest describing the Carpaccio frescoes depicting the life of the patron saint of translation. He echoes Ruskin's following instructions:

let your boatman take you across to San Giorgio Maggiore; there you can moor your gondola under the steps in the shade, and read in peace, looking up at the pillars when you like ${ }^{14}$

in his advice about where to read The Bible of Amiens:

Le meilleur endroit pour lire ce chapitre est l'église San Giorgio dei Schiavoni à Venise. On prend une gondola et dans un calme canal, un peu avant d'arriver à l'infini frémissant et miroitant de la lagune on aborde à cet "Autel des Esclaves" où on peut voir (quand le soleil les éclaire) les peintures que Carpaccio a consacrées à saint Jérôme.

The best place to read this chapter is the church of San Giorgio dei Schiavoni in Venice. You should take a gondola and, in a tranquil canal, a little before reaching the sparkling, shimmering expanse of lagoon, you will come to the "Shrine of the Slaves" where you will be able to see (when the sun illuminates them) Carpaccio's paintings related to St Jerome..$^{15}$
10

12

Proust's copy of The Bible of Amiens in the 1897 edition published by George Allen (London) is held in the Département des Estampes of the Bibliothèque nationale de France. Proust wrote "Hôtel de l'Europe, 61" on the flyleaf of his copy.

4 Works, 24: 208

Ruskin-Proust 1904, 219. 
Figure 2 Notre-Dame d'Amiens. Photograph of a detail of the southern portal. Amiens. @ Wikimedia Commons

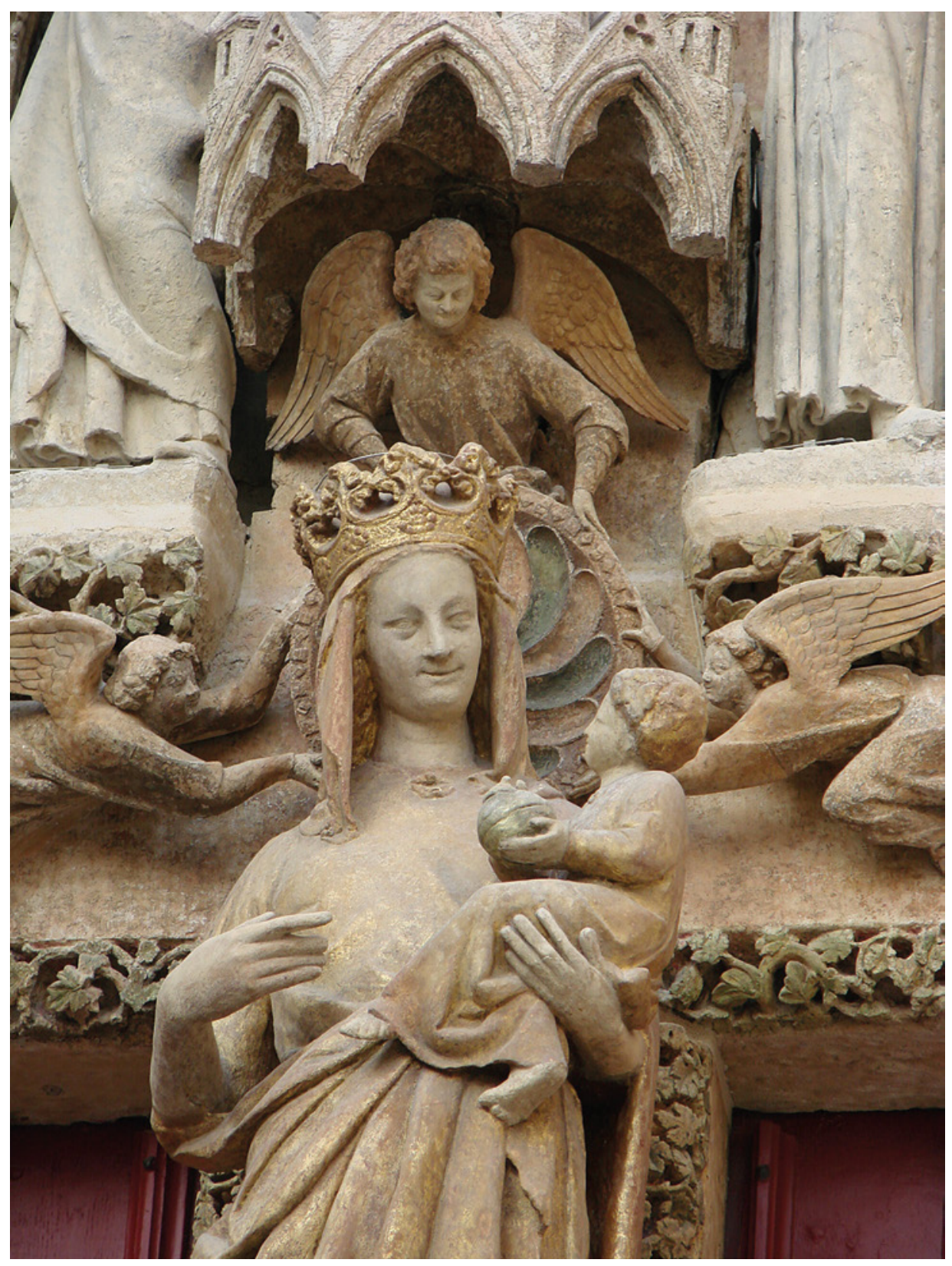


Ruskin presents Amiens in relation to Venice, playing on its nicknames 'the Venice of France' and 'the Venice of Picardy' and, as Proust notes, illuminating the stones of northern France with a "un reflet magique d'Italie" (a magical reflection of Italy. ${ }^{16}$ At the beginning of the volume, Ruskin wonders what distinguishes the light and air in Amiens:

Why should this fountain of rainbows leap up suddenly here by Somme, and a little Frankish maid write herself the sister of Venice, and the servant of Carthage and Tyre? ${ }^{17}$

He further questions how the "Frankish maid" is related to her Venetian and classical counterparts. Ruskin focuses on the statue of the Virgin in the southern portal, "the pretty French Madonna" who is a native of Amiens, quarried from the local stone [fig. 2]. He uses the French word for a maidservant to characterize her as a "little white-capped Amienoise soubrette". ${ }^{18}$ A symbol of French Christianity, she supplanted Joan of Arc and represented "a merrier faith for France", 19 though headed, according to Ruskin, towards the guillotine of the French Revolution. She typifies the Nurse-Madonna, or Madonna in decadence, traditionally represented by Raphael, but exemplified as "a good French type" here. At this point in his translation, Proust in- tervenes in a footnote to add this quotation from Modern Painters:

the crowned Queen-Virgin of Perugino sank into a simple Italian mother in Raphael's Madonna of the Chair. ${ }^{20}$

Ruskin had taken issue with Raphael who no longer considered art to be at the service of religion but used a religious subject as an excuse for his paintings, which he expressed in this chiasmus:

In early times art was employed for the display of religious facts; now, religious facts were employed for the display of art. ${ }^{21}$

According to Ruskin, this was emphatically not a "healthy change". ${ }^{22}$ Proust's footnote pointing to this assessment of Raphael exemplifies how he accentuated the Italian component of Ruskin's volume on Amiens, producing a multi-facetted text reflecting in various directions.

In addition to cross-referencing Ruskin's works, Proust also compiled a kind of encyclopedia of British culture so that his readers would understand Ruskin's references. He annotated his own copy with a question-mark next to the name 'Greatheart' indicating that it needed to be identified, ${ }^{23}$ and added a footnote spec-

16 Ruskin-Proust 1904, 62.

7 Works, 33: 26-7

8 Works, 33: 27

9 Works, 33: 128.

0 Works, 5: 78. Translated by Proust in footnote 2: Ruskin-Proust 1904, 323-4.

Works, 5: 77, emphasis in original.

Works, 5: 78

The annotation is made in Proust's copy, page 31 (see fn. 12). 
ifying that he was a character from Bunyan's The Pilgrim's Progress. ${ }^{24}$ Similarly, he annotated Ruskin's reference to Thomas More:

Décapité en 1535, sur l'ordre de Henri VIII, pour avoir refusé de prêter le serment de suprématie.

Beheaded in 1535, under Henry VIII's orders, because he had refused to swear supremacy to him. ${ }^{25}$

Proust was clearly targeting a French audience, not only filling in the gaps in their knowledge but also making them feel at home with the translation by punctuating it with references to their culture. He is so invasive that his notes often outweigh Ruskin's original text, as is the case on page 320 of the translation. Footnote one about the sculpted calendar is a lengthy extract from Mâle's L'Art religieux du XIIlème siècle en France (French Religious Art of the Thirteenth Century). It is one of Proust's numerous "translator's notes" which frequently quote Mâle, thus framing Ruskin's text with a French critical apparatus. Mâle's emphasis on the humanity of the sculptures at Amiens resonate with Ruskin's own values:

Tout cela est simple, grave, tout près de l'humanité. Il n'y a rien là des Grâces un peu fades des fresques antiques : nul amour vendangeur, nul génie ailé qui moissonne. Ce ne sont pas les charmantes déesses florentines de Botticelli qui dansent à la fête de la Prima- vera. C'est l'homme tout seul, luttant avec la nature ; et [l'œuvre est] si pleine de vie, qu'elle a gardé, après cinq siècles, toute sa puissance d'émouvoir.

All of that is so simple, earnest, and close to humanity. There is nothing of the somewhat insipid Graces of ancient frescoes: no Cupid gathering grapes, no winged genius harvesting. Neither are there Botticelli's charming Florentine goddesses dancing to celebrate Spring Awakening. It is man alone, fighting against nature, and the art work is so full of life that after five centuries, it has kept all its emotive power. ${ }^{26}$

The footnotes on the opposite page of Proust's translation ${ }^{27}$ pursue the idea that the sculptures reflect their location, focusing on the sculpted calendar of the months of the year coupled with the signs of the Zodiac above them. Proust again cites Mâle to make comparisons between the sculptures of Amiens cathedral and those of other French cathedrals, creating a kind of temporal calendar which maps their locations. In note three, he comments that in the month of March, "il n'est plus permis de rester au coin du feu" (sitting at home by the fire is no longer allowed), so the workers are depicted in the vineyards. The variation in climate between the regions accounts for the different stages in the agricultural cycle: in Chartres, the vine is being pruned, whereas in Amiens, the peasant is digging [fig. 3]. Proust cites Mâle's indication that there were vineyards in Amiens in the Middle Ages, even though they are not there anymore. ${ }^{28}$

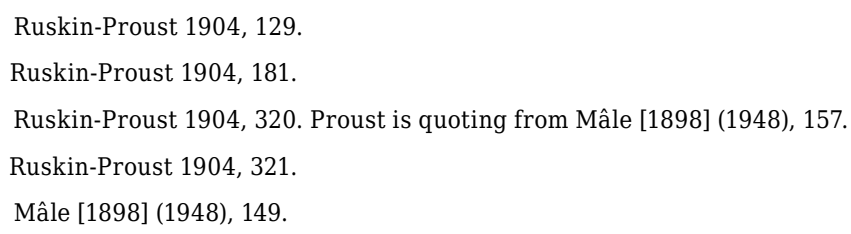




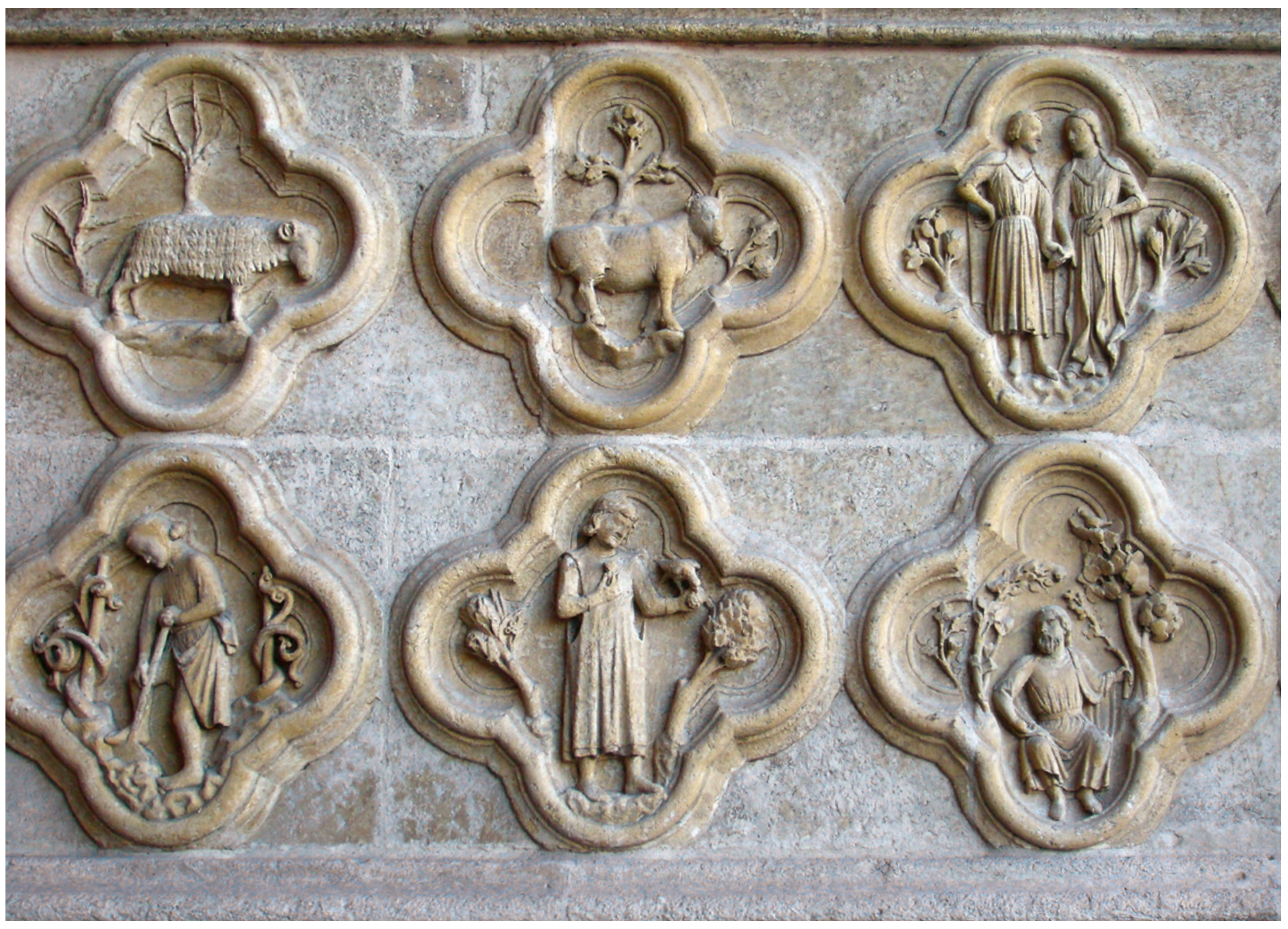

Figure 3 Notre-Dame d'Amiens Cathedral, Northern Porch. Detail of Zodiac signs and months of the year. From left to right: Aries, Taurus, Gemini (above); March, April, May (below). @ Wikimedia Commons 
In their edition of Ruskin's text, Cook and Wedderburn borrow that note from Proust ${ }^{29}$ which, as well as several other references they make to Mâle, intensifies its French dimension.

Proust also refers to French painters, thus making the peritext he adds to Ruskin's original a kind of diapered screen decorated with French motifs. He recounts his Ruskinian pilgrimage to Amiens, revealing how the effects of the journey and the weather on the day he visited the cathedral modified his view of the cathedral. In this way, he seems to be following the precept Ruskin defines in Modern Painters when he writes:

If a painter has inventive power he is to treat his subject in a totally different way; giving not the actual facts of it, but the impression it made on his mind [...]. Now, observe, this impression on the mind never results from the mere piece of scenery which can be included within the limits of the picture. It depends on the temper into which the mind has been brought, both by all the landscape round, and by what has been seen previously in the course of the day. ${ }^{30}$

Proust's sensitivity to how the light played on the cathedral's façade partakes in Ruskin's own preoccupations though he illustrates his point with reference to an artistic movement unknown to Ruskin, namely French Impressionism. Proust focuses on how the aspect of the cathedral changes at different times of day, invoking the notion of the colour of time which he equates with Monet's series of views of Rouen cathedral. This passage from Proust's introduction to his translation of The Bi- ble of Amiens also pinpoints what Ruskin sees as an almost organic relationship between the cathedral and its natural setting, as Proust noted:

Ruskin ne séparait pas la beauté des cathédrales du charme de ces pays où elles surgirent.

Ruskin does not separate the beauty of cathedrals from the charm of the countries where they loom. ${ }^{31}$

Proust's remark on the light reflecting on the stones reads as a poetic definition of Monet's series depicting the façade of Rouen cathedral, where

Sur chaque pierre vous voyez la nuance de l'heure unie à la couleur des siècles.

On every stone you can see the shade of the hour blended together with the colour of the centuries. ${ }^{32}$

Ruskin's work inspires Proust to write this passage of poetry in prose:

Quand vous voyez pour la première fois la façade occidentale d'Amiens, bleue dans le brouillard, éblouissante au matin, ayant absorbé le soleil et grassement dorée l'après-midi, rose et déjà fraîchement nocturne au couchant, à n'importe laquelle de ces heures que ses cloches sonnent dans le ciel, et que Claude Monet a fixées dans des toiles sublimes où se découvre la vie de cette chose que les hommes ont faite, mais que la nature a reprise en l'immergeant en elle, une ca-

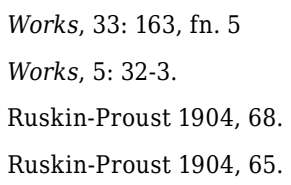


thédrale, et dont la vie comme celle de la terre en sa double révolution se déroule dans les siècles, et d'autre part se renouvelle et s'achève chaque jour, - alors, la dégageant des changeantes couleurs dont la nature l'enveloppe, vous ressentez devant cette façade une impression confuse mais forte.

When you see the western façade of Amiens for the first time, blue in the mist, dazzling in the morning, drenched and lusciously gilded in the afternoon sun, rosy and already coolly nocturnal at sunset, at whatever hours its bells chime in the sky, and which Claude Monet has immortalized in his sublime canvasses, where this man-made creation reveals its life but which nature has reclaimed by immersing it in her, a cathedral, whose life, like that of the earth with its double revolution - unfolding over the centuries while renewing and completing itself every day -, then, releasing it from the changing colours in which nature envelops it, you will experience in front of this façade a confused but strong impression. ${ }^{33}$

While the comparison he made with Monet was in keeping with Ruskin's artistic sensitivities, the analogy Proust drew between Ruskin and Gustave Moreau is strikingly heterogeneous given Moreau's mystic, oneiric, mythological qualities. Proust first drew the parallel in his article published in the Gazette des Beaux-Arts on 1 April 1900 and then repeats it in his preface to the translation of The Bible of Amiens:

Comme "Les Muses quittant Apollon leur père pour aller éclairer le monde", une à une les idées de Ruskin avaient quitté la tête divine qui les avait portées et, incarnées en livres vivants, étaient allées enseigner les peuples.

Like "The Muses leaving Apollo their father to go and enlighten the world", one by one Ruskin's ideas had left the sublime head which had borne them and, embodied as living books, had gone forth to instruct the people. $^{34}$

The comparison is highlighted by its prominent position at the beginning of Proust's article, next to a photograph of Ruskin. At first sight, it is an incongruous juxtaposition: on the one hand, we have the portrait of the dour, earnest sage of Coniston and on the other, the languorous, homoerotic figure of Apollo [fig. 4]. Proust's image of the diffusion of Ruskin's weighty tomes as the diaphanous female muses surrounding Apollo is equally incongruous.

Proust himself questioned the pertinence of the comparison between Ruskin and Moreau, though he justified it on the grounds that both attached importance to symbolism, Moreau in his practice of art for art's sake, Ruskin as a devotee of the religion of beauty:

Il n'y a certes pas lieu de comparer Ruskin à Gustave Moreau, mais on peut dire qu'une tendance naturelle, développée par la fréquentation des Primitifs, les avait conduits tous deux à proscrire en art l'expression des sentiments violents, et, en tant qu'elle s'était appliquée à l'étude des symboles, à quelque fétichisme dans l'adoration des symboles eux-mêmes, fétichisme peu dangereux d'ailleurs pour ces esprits si attachés au fond au sentiment symbolisé qu'ils pouvaient passer d'un symbole à l'autre, sans d'être arrêtés par les diversités de pure surface. 


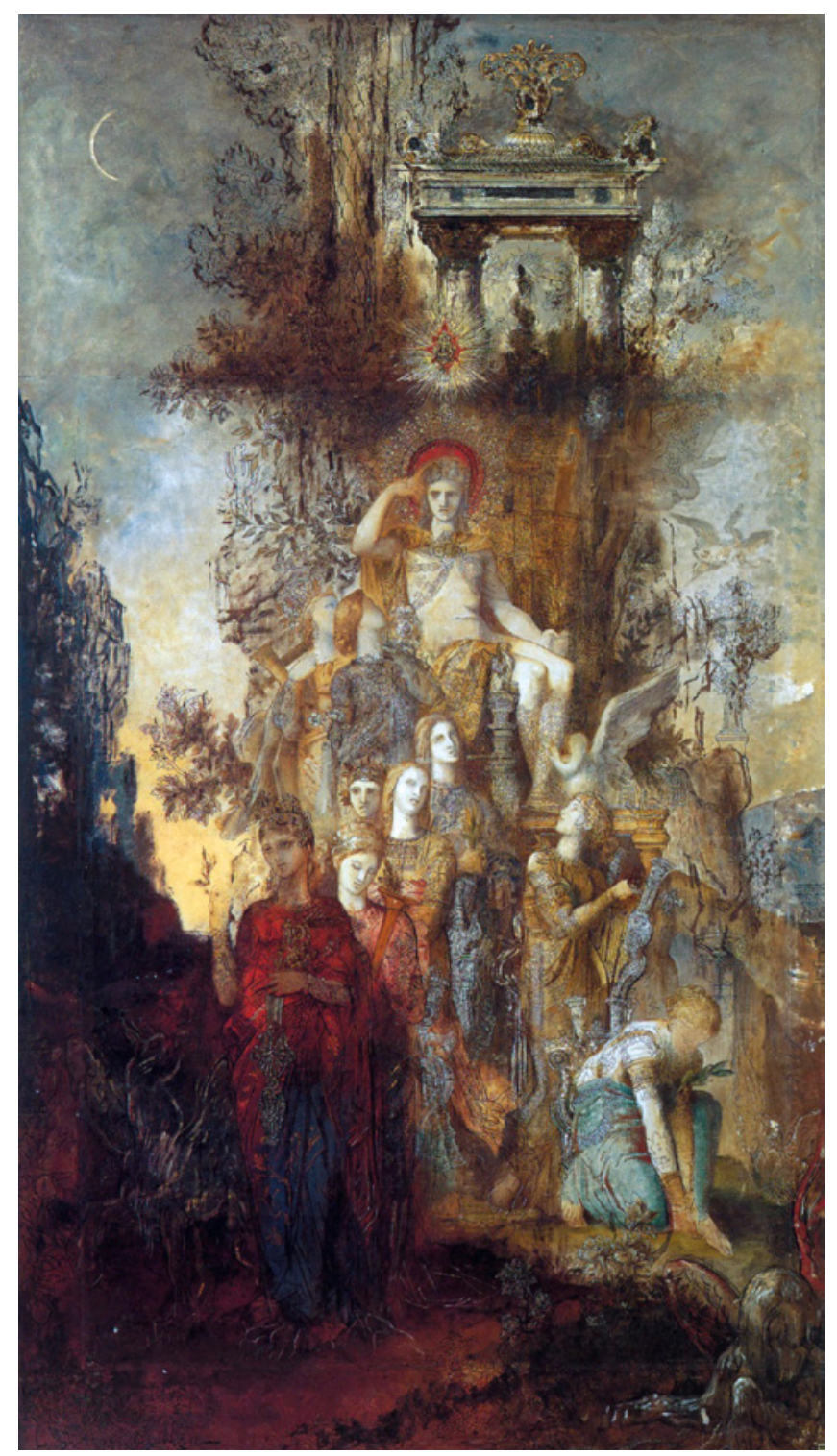

Figure 4 Gustave Moreau, Les Muses quittant Apollon, leur père, pour aller éclairer le monde. $1868.292 \times 152 \mathrm{~cm}$. Paris, Musée Gustave Moreau

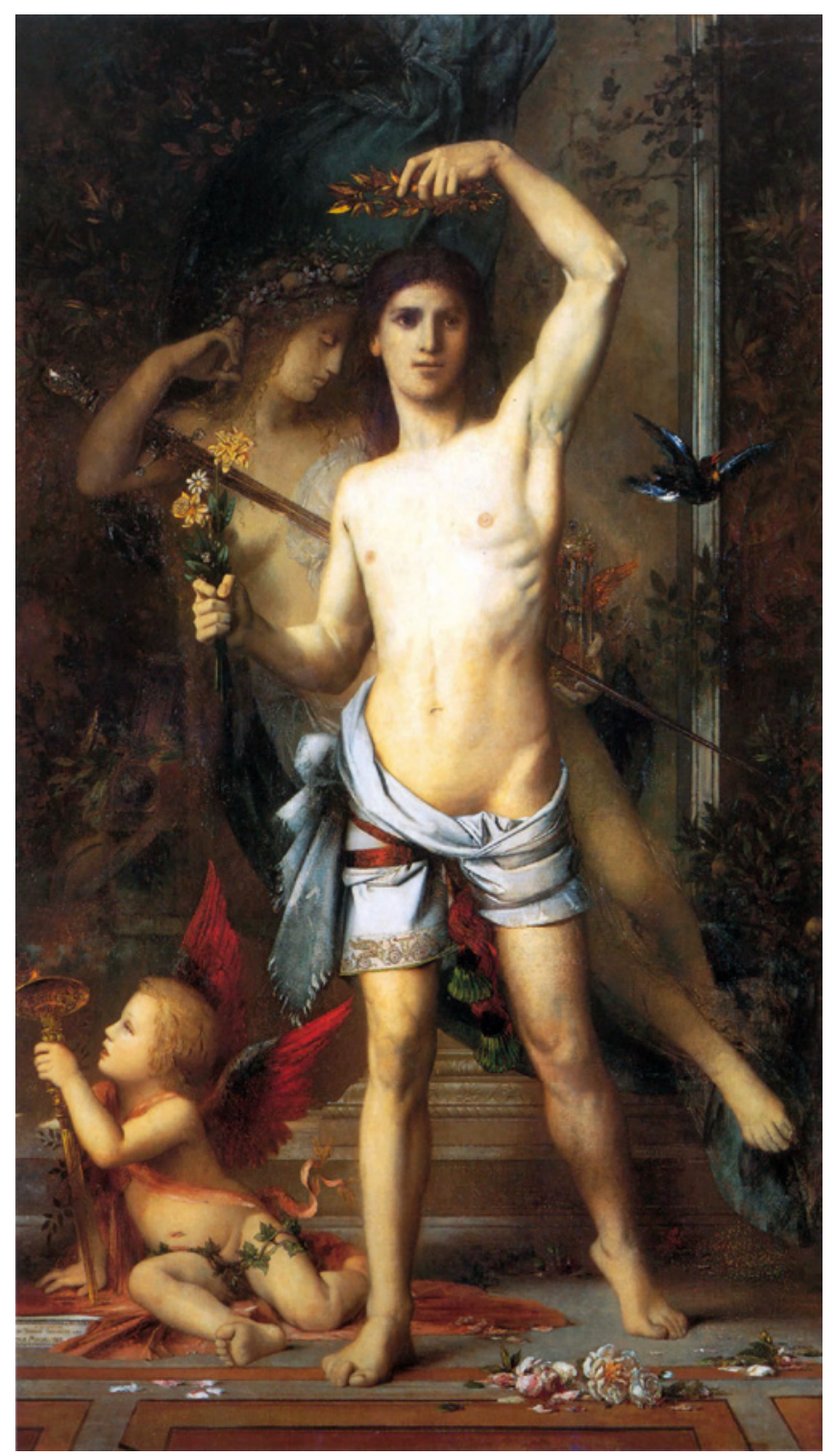

Figure 5 Gustave Moreau, Le jeune homme et la Mort. 1865. $215.9 \times 123.2 \mathrm{~cm}$. Cambridge (MA), Fogg Art Museum 
There are no grounds for comparing Ruskin to Gustave Moreau, but one may say that a natural tendency, developed through familiarity with the Primitives, had led both to proscribe the expression of violent feelings in art, and, in as much as this was applied to the study of symbols, to proscribe a certain fetishism in the worship of symbols themselves, not a very dangerous fetishism for minds so attached in reality to the feeling symbolized that they could pass from one symbol to another without being hindered by superficial diversity. ${ }^{35}$

Proust draws on Moreau's symbolism elsewhere in the preface when he makes The Young Man and Death [fig. 5] into an image of Ruskin's senile insanity:

à l'extrême vieillesse, la pensée déserta la tête de Ruskin, comme cet oiseau mystérieux qui dans une toile célèbre de Gustave Moreau n'attend pas l'arrivée de la mort pour fuir la maison.

in very old age, thought deserted Ruskin's head, like the mysterious bird in a famous canvas by Gustave Moreau which does not wait for the arrival of death to flee from the house. ${ }^{36}$

Picturing Ruskin as Moreau's ephebic young man standing at the door of the kingdom of death and crowning himself with Apollo's laurel leaves would indeed be surprising but Proust's attention here is focused on the bird taking flight which he reads as a symbol of Ruskin's mental state.

Proust had also associated Moreau with Ruskin's work in the preparatory notes of the preface to his translation.

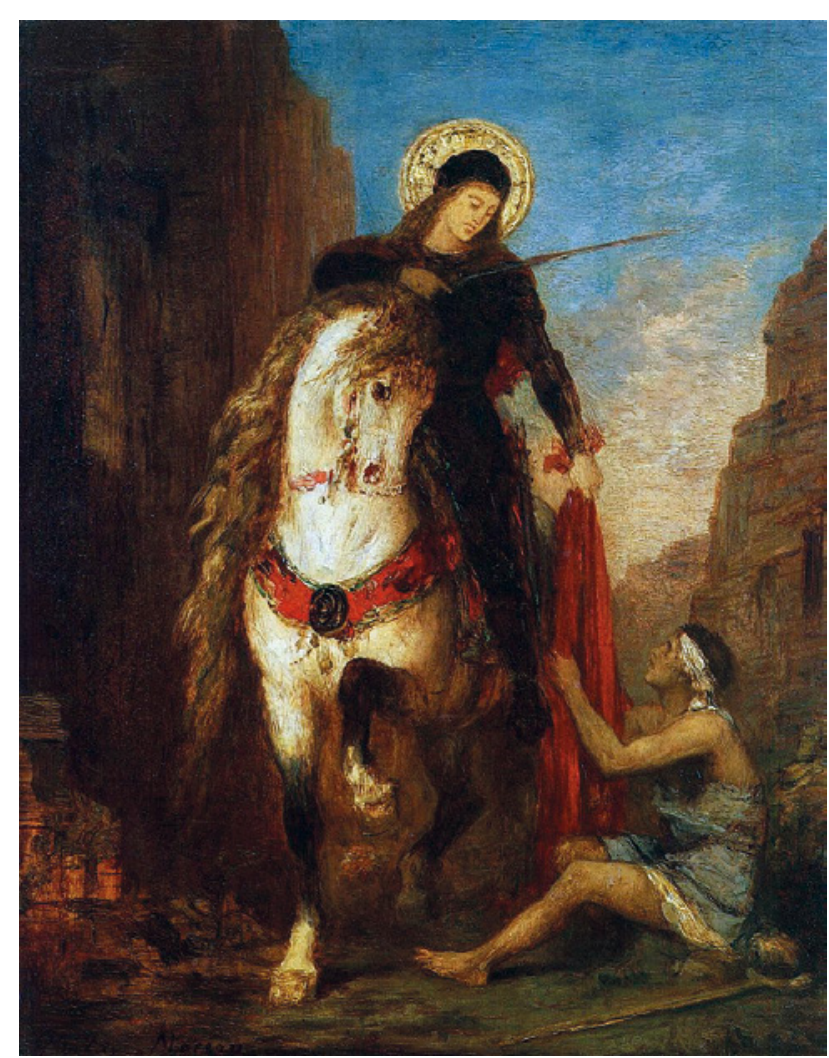

Figure 6 Gustave Moreau, Saint Martin partageant son manteau. 1882. Private Collection. (c) Wikimedia Commons 
Figure 7 Paul César Helleu (1859-1927), Intérieur de l'église abbatiale de Saint-Denis. $1891 \mathrm{ca}$. Oil on canvas, $194 \times 155 \mathrm{~cm}$. Boston (MA), The Isabella Stewart Gardner Museum. The Athenaeum. https://www.gardnermuseum.org/ experience/collection/10732

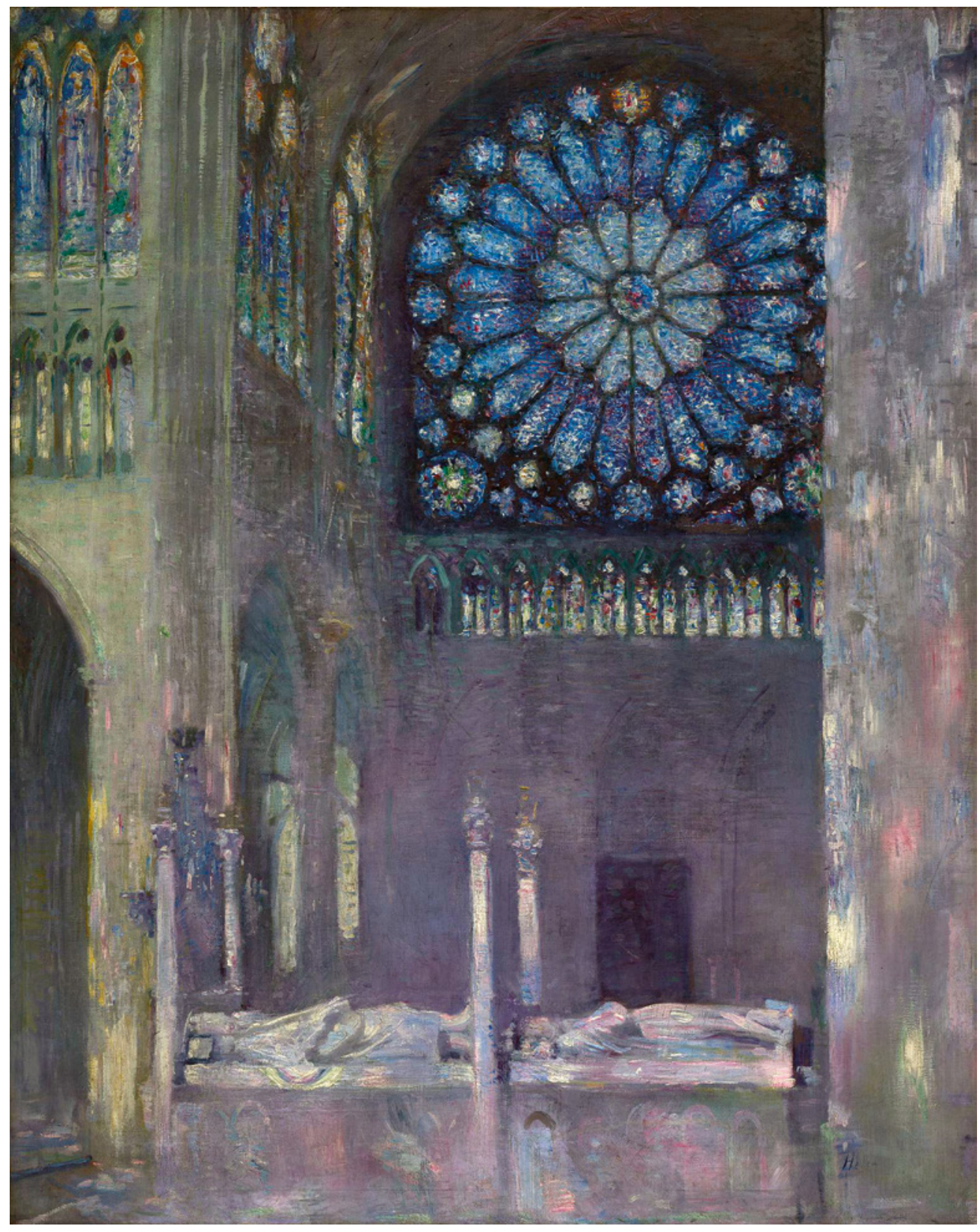


Commenting on the passage in which Ruskin relates how St Martin gave a cloak to naked man in Amiens [fig. 6], he wrote that we should go to St. Acheul church to find:

le souvenir de St. Martin auquel Ruskin a consacré des pages admirables que semble illustrer le charmant St Martin de Gustave Moreau.

a reminder of St. Martin about whom Ruskin wrote some admirable pages which Gustave Moreau's charming $S t$ Martin seem to illustrate. ${ }^{37}$

Proust's use of the verb illustrer (to illustrate), though deleted from the final version, reveals how he drew on French culture to illuminate Ruskin's text, in the sense of "to shed light on".

Proust's reference to Paul Helleu (1859-1927), another French artist outside the corpus studied by Ruskin, again indicates how he wanted to break from the master's influence. Helleu is named in a footnote praising his depictions of the interior of cathedrals ${ }^{38}$ in which he excelled in capturing the light of the stain-glass windows [fig. 7]. He was one of the models of Proust's fictitious painter Elstir, so his footnote not only recasts Ruskin's text as a work belonging to the aesthetics of the French fin-de-siècle but also foreshadows his own work.

Proust attempted in vain to break from Ruskin and tried to purge himself of his influence by parodying his style in his pastiches. He wrote a three-page pastiche of Modern Painters as a dedication to Jean Sardou, in the gift-copy of his translation of Sésame et les lys. In that unpublished pastiche is titled Extrait de Ruskin (Extract from Ruskin), ${ }^{39}$ Proust compares the portrait of the ded- icatee bathed in the Parisian evening light to paintings by Turner with the reflection of the sunset on the Salute in Venice. He imitates Ruskin's hyperbolic style and the condescending tone with which he addresses philistine English travelers:

Le plus remarquable Turner que je connaisse représente M. Jean Sardou sortant de l'Odéon dans la lumière oblique d'un de ces glorieux couchants qu'excelle à reproduire le peintre ; comme le jeune savant est placé devant l'entrée des artistes, le naïf spectateur anglais s'imagine que l'uniforme qu'il porte est un costume de théâtre, bien que cela ne soit pas. En réalité il n'est que la matérialisation, en moelleuses apparences de velours cerise, de ces rayons écarlates qui empourprent la Salute dans la Venise voisine et dans Didon à Carthage.

The most remarkable Turner I know depicts Mr. Jean Sardou leaving the Odeon theatre in the slanting sunrays of one of those glorious sunsets which the English painter excels at reproducing; as the young scholar is positioned in front of the stage door, the naïve English spectator believes that the uniform he is wearing is a theatre costume, though it is not. In fact, it is merely the materialization in soft fabric looking like soft cherry-coloured velvet, of the scarlet rays which bathe in purple the Salute in the Venice nearby and in Dido at Carthage.

Through this play of light rebounding between Italy and France, Proust echoes the associations Ruskin establishes between the two countries. As Proust had noted in the

\footnotetext{
37 Proust 1971, 755. For Ruskin's description of St Martin, see Works, 33: 41.

38 Ruskin-Proust 1904, 32.

39 This copy sold at Sotheby's in 2018. See http://www.sothebys.com/en/auctions/ecatalogue/2018/livres-et-manuscrits-pf1803/lot.154. html?locale=en
}

$311 \quad \begin{aligned} & \text { Fonti, letterature, arti e paesaggi d'Europa| Sources, Literatures, Arts \& Landscapes of Europe } 1 \\ & \text { John Ruskin's Europe. A Collection of Cross-Cultural Essays, 297-316 }\end{aligned}$ 
preface to his translation of The Bible of Amiens, Ruskin's thought moved seamlessly from one country to another, recognizing the unity of Christian art across Europe and highlighting how the architectural treasures of northern France cohere with the "bijoux éblouissants"

\section{2}

\section{Reflections of Ruskin's Italy in Proust's Novel}

Proust's reading of Ruskin is imprinted in his novel and can even be felt in his choice of titles, as Proust's biographer George Painter suggests:

Ruskin had shown himself aware of Time Regained: it was a book whose very title, Praeterita, might be literally translated as "Things Past", or "Temps Perdu". ${ }^{2}$

Jérôme Bastianelli has made a similar association between Ruskin's title The Two Paths and Proust's paired titles Du côté de chez Swann and Le Côté de Guermantes:

Selon l'explication qu'il en donne dans la préface, le titre, qui n'est pas sans rappeler "les deux côtés" de Combray, vient de l'objectif que se donne l'auteur : permettre au lecteur de choisir "entre deux types d'études, l'une menant à l'épanouissement, l'autre à l'asphyxie de ses facultés", et d'identifier "l'instant précis de la vie où la voie bifurque, un embranchement menant au mont des Oliviers, et l'autre à la vallée de la mer Morte". (dazzling jewels) of Italy. ${ }^{40}$ Ruskin's achievement, according to Proust, was to have made the dream of the great popes of the Middle Ages a reality: through his appreciation of the arts, he succeeded in creating a "Christian Europe". ${ }^{41}$

According to the explanation Ruskin gives in the preface, the title, which anticipates the two "paths" in Combray, comes from the objective the author sets: to offer the reader the choice "between two modes of study, which involve ultimately the development, or deadening, of every power he possesses" and to identify "the hour and the point of life when the way divides itself, one way leading to the Olive mountains - one to the vale of the Salt Sea". ${ }^{43}$

Proust's novel follows Ruskin in tracing the opposing the path of enlightening aesthetics and that of a sybaritic social life.

Recognizing his indebtedness to Ruskin, Proust clearly wanted to mark his departure from him, even degrading him in his novel by associating him with the password to a male brothel. ${ }^{44}$ One of Proust's characters also derides him by dubbing him "Lord John Ruskin" before calling him a "sombre raseur" (a deadly bore). ${ }^{45}$ The only other two explicit references to Ruskin are more neutral, namely when he is called upon to motivate the young nar-

40 Ruskin-Proust 1904, 61.

41 Ruskin-Proust 1904, 61

42 Painter 1983, 265.

43 Bastinelli 2017, 714. Quotations from The Two Paths (Works, 16: 253-4).

44 Proust 1913-1927, 4: 411

45 Proust 1913-1927, 2: 99. 


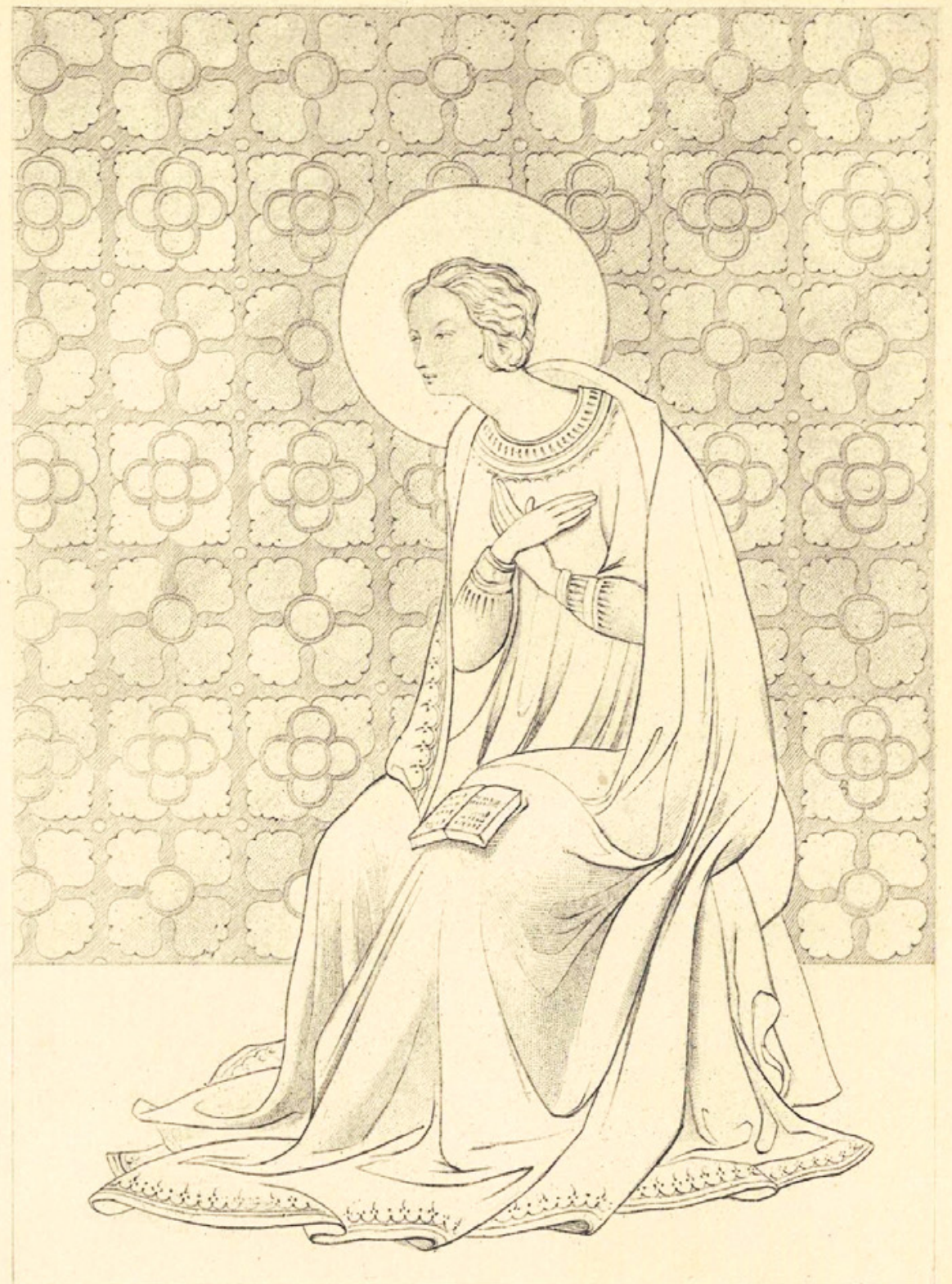

Figure 8 John Ruskin, Ancilla Domini, frontispiece of Works, 7. Drawing from the painting by Fra Angelico. http://www.victorianweb.org/painting/ruskin/ drawings/33.jpg 
rator's trip to northern France, ${ }^{46}$ and when the narrator recalls going to the Baptistery of St Mark's to work on Ruskin during his stay in Venice. ${ }^{47}$

Even though he is not named more than four times in the several thousand pages of Proust's text, Ruskin's influence permeates it. One example echoing Ruskin's use of the word 'diaper' is the image of Florence at Easter conjured up by the narrator:

le rêve [...] du printemps le plus diapré [...], celui qui couvrait déjà de lys et d'anémones les champs de Fiesole et éblouissait Florence de fonds d'or pareils à ceux de l'Angelico.

[the dream of] the most dappled spring [...] the spring which was already covering the fields of Fiesole with lilies and anemones and dazzling Florence with golden grounds like those of Fra Angelico. ${ }^{48}$

Proust's use of the word 'diapré' here resonates with Ruskinian overtones as he probably had in mind the diapered screen behind Fra Angelico's Madonna, which he knew thanks to Ruskin's drawing of it, reproduced as the frontispiece in Modern Painters volume five [fig. 8]. ${ }^{49}$

If Proust's text reflects images from Ruskin in this way, it refracts his prose in the sense that it modifies its sphere of reference. An eloquent example is when Proust adapts Ruskin's passage in The Stones of Venice on how aspects of geology can be related to art and makes it into an image of memory. He first translates the following passage from Ruskin in a footnote to his translation of The Bible of Amiens:

The colours of marble are mingled for us just as if on a prepared palette. They are all shades and hues [...], some being united and even, some broken, mixed, and interrupted, in order to supply, as far as possible, the want of the painter's power of breaking and mingling the colour with the brush. But there is more in the colours than the delicacy of adaptation. There is history in them. By the manner in which they are arranged in every piece of marble, they record the means by which that marble has been produced, and the successive changes through which it has passed. And in all their veins and zones, and flame-like stainings, or broken and disconnected lines, they write various legends, never untrue, of the former political state of the mountain kingdom to which they belonged, of its infirmities and fortitudes, convulsions and consolidations, from the beginning of time..$^{50}$

He then highlights the significance of Ruskin's point when he works that quotation into the conclusion of the first part of his novel, Combray. He constructs his key passage on memory on an intertext which is Ruskinian

46 Proust 1913-1927, 2: 9.

47 Proust 1913-1927, 4: 224

48 Proust 1913-1927, 1: 379. Davis' translation from Proust 2002, 390

49 Proust knew the volume on Florence, published in the Villes d'art célèbres series in 1906, where the word 'diapré' is also used (Gebhart 1906, 6). The description of the Florentine hills include the anemones - though not the lilies - which flower in Proust's mind's eye: "Vers la fin d'avril, la colline apparait diapree, rayonnante ; les amandiers et les pêchers font pleuvoir sur les champs leur neige blanche ou rose ; les anemones, les iris, les œillets, les boutons d'or, les pervenches foisonnent le long des sentiers" (Towards the end of April, the hill has a mottled, radiant look: showers of white and pink snowflakes fall from the almond and peach trees; the pathways abound with anemones, irises, carnation, buttercups and periwinkles).

50 Works, 11: 38. The footnote is in Ruskin-Proust 1904, 251.

Fonti, letterature, arti e paesaggi d'Europa | Sources, Literatures, Arts \& Landscapes of Europe 1 | 314 
both in essence and in the repetition of such terms as "marble", "veins" and "colours":

Tous ces souvenirs ajoutés les uns aux autres ne formaient plus qu'une masse, mais non sans qu'on ne pût distinguer entre eux - entre les plus anciens, et ceux plus récents, nés d'un parfum, puis ceux qui n'étaient que les souvenirs d'une autre personne de qui je les avais appris - sinon des fissures, des failles véritables, du moins ces veinures, ces bigarrures de coloration, qui dans certaines roches, dans certains marbres, révèlent des différences d'origine, d'âge, de "formation".

All these memories added to one another now formed a single mass, but one could still distinguish between them - between the oldest, and those that were more recent, born of a perfume, and then those that were only memories belonging to another person from whom I had learned them - if not fissures, if not true faults, at least that veining, that variegation of colouring, which, in certain rocks, in certain marbles, reveal differences in origin, in age, in "formation". ${ }^{51}$

Ruskin might not be named here, but Proust has clearly rewritten him in his French text. It illustrates how he built his cathedral of a novel using coloured and veined Ruskinian stones, making his prose radiate with reflections from Ruskin, like the gilt diapered screen in Fra Angelico's Annunciation. 


\section{Bibliography of Works by John Ruskin}

References are to volume and page numbers in:

Cook, E.T.; Wedderburn, A. (eds) (1903-1912). The Works of John Ruskin. Library Edition, 39 vols. London: George Allen.

https://www.lancaster.ac.uk/the-ruskin/the-complete-works-of-ruskin/.

Works, 3-7: Modern Painters I-V. | 9-11: The Stones of Venice I-III. | 16: "A Joy For Ever", The Two Paths, Letters on The Oxford Museum and various addresses (1856-1860). | 24: Giotto and His Works in Padua, The Cavalli Monuments, Verona, Guide to the Academy, Venice, St Mark's Rest. |33: The Bible of Amiens, Valle Crucis, The Art of England, The Pleasures of England. | 35: Praeterita, Dilecta.

\section{General Bibliography}

Bastianelli, J. (2017). Dictionnaire Proust-Ruskin. Paris: Classiques Garnier. Gebhart, É. (1906). Les Villes d'art célèbres: Florence. Paris: Henri Laurens.

La Sizeranne, R. de (1909). Pages choisies. Paris: Hachette.

Mâle, É. [1898] (1948). L’Art religieux du XIIlè siècle en France. Paris : Armand Colin.

Nordlinger, M. (1955). Marcel Proust and His Time. London: Wildenstein Gallery.

Painter, G.D. (1983). Marcel Proust. Harmondsworth: Penguin.

Proust, M. [1913-1927] (1987-1989). À la recherche du temps perdu. 4 vols. Edité par J.-Y. Tadié. Paris: Gallimard.

Proust, M. (1971). Contre Sainte-Beuve, précédé de Pastiches et mélanges et suivi d’Essais et articles. Edité par P. Clarac et Y. Sandre. Paris: Gallimard.

Proust, M. (1976). Correspondance, vol. 3. Edité par P. Kolb. Paris: Plon.

Proust, M. (2002). The Way by Swann's. Transl. by Lydia Davis. London: Allen Lane.

Ruskin-Proust (1904) = John Ruskin: La Bible d'Amiens. Transl. by M. Proust. Paris: Mercure de France.

SOED (1973) = Shorter Oxford English Dictionary. 2 vols. Oxford: Clarendon Press. 


\title{
Edited by Ruskin: Francesca Alexander's Roadside Songs of Tuscany
}

\author{
Emma Sdegno
}

Università Ca' Foscari Venezia, Italia

\begin{abstract}
In 1907 Cook and Wedderburn published the volume XXXII of the Library Edition, grouping together a series of texts "Edited and Arranged by John Ruskin": Studies of Peasant Life: The Story of Ida, Roadside Songs of Tuscany, Christ's Folk in the Apennine, Ulric the Farm Servant. Since most of the works were authored by Francesca Alexander the volume is in effect a tribute to this American artist. In this paper I outline the history of the editing of Francesca Alexander's Roadside Songs of Tuscany, from the manuscript "Francesca's Book" to the published edition issued in parts between 1884 and 1885. I argue that Ruskin's interest in the project bears a special relationship to the publication of the Fioretti di San Francesco in mid-nineteenth-century French and English versions, and to the ideological context that generated those publications. Ruskin's declared aim of conveying to the English mind "some sympathetic conception of the reality of the sweet soul of Catholic Italy" was generated within this context, and his idea of publishing Francesca's manuscript in a heavily edited and thoroughly new form justifies comparison with continental research into Medieval literature in the last decades of the Nineteenth century.
\end{abstract}

Keywords Francesca Alexander. Roadside Songs of Tuscany. The Story of Ida. Italian Folk poetry. Fioretti di San Francesco. Cardinal Manning. Frédéric Ozanam.

Summary 1 On the Old Road. -2 Fanny Alexander and the Peasants of the Abetone. -3 "Francesca's Book". - 4 The Little Flowers of Francesca. - 5 A "Unique" and "Very Sumptuous" Book. 6 "The Main Lessons".

On the Old Road

John Ruskin's editorial works are utterly neglected, in part because of for their ancillary nature, but also because they belong to his last years, which are still little studied and somewhat undervalued. The guidebooks to Venice and Florence of the late 1870s and 1880s - St Mark's Rest, The Guide to the Principal Pictures in the Academy Venice and Mornings in Florence - and even Fors Clavigera (1871-1884) and the Oxford lectures of the same pe- 
riod - are provocative in that they partially contradict the aesthetic and theological assumptions of his earlier works. Their complex, non-linear argumentation is challenging, and the abundance of rhetorical tropes carries a host of half-submerged references which hardly ever come to the surface. Ruskin's multilayered discourse does not aim at definitive answers, but at opening up a vital space for meditation. After the wreckage of the Franco-Prussian war, a conflict that affected him painfully and magnified his personal emotional wreckage, his lifelong concern for Europe, its culture and its heritage, takes on a sense of desperate urgency, and of mystical reliance. It was in this context that Ruskin conceived of his edition of Roadside Songs of Tuscany, a work he considered highly and that contemporary reviewers defined as "unique" and "very sumptuous". In exploring its genesis I shall recall his penultimate European journey, and some readings that may well have played an important part in shaping the work as it eventually appeared.

On 5 August 1882, five months after suffering his third and most severe attack of mental illness to date, Ruskin set off, on his doctor's recommendation, on what was to be his last visit to Tuscany. He was accompanied by his valet and by the young artist who was to be his first biographer, W.G. Collingwood, who recorded aspects of the journey in three chapters of his Ruskin's Relics. The tour was meant to consolidate Ruskin's recovery and was equally divided, in terms of the time spent, between France (Champagne, Burgundy and the Jura) and Tuscany (Pisa, Lucca and Florence). The French itinerary took him "on the old road", as Ruskin called it, ${ }^{1}$ along the beaten track of places he had visited with his parents and which would be recollected in his autobiography Praeterita (1885-1889). ${ }^{2}$ The Continental old road was engraved in the story of his personal life, and (partly) coincided with the myths, legends, and histories of Europe's past. In his late work Ruskin considered this legacy from a new focus, one in which the old family routes are interlaced with the 'Road of Our Fathers'. As early as the 1860s - as Cook and Wedderburn suggest - Ruskin had begun planning a series comprising "Studies in Christian History and Architecture" to be entitled Our Fathers Have Told Us. "The work", announced Ruskin, "will consist of ten parts, each taking up some local division of Christian history, and gathering, towards their close, into united illustration of the power of the Church in the Thirteenth Century". ${ }^{3}$ The Bible of Amiens, published in 1880, is the first and only volume of the series to be completed. A study "descriptive of the early Frank power, and of its final skill", it is a close reading of a portion of the map of Europe that Ruskin had explored repeatedly in the course of his working life, a portion coinciding with a "highly restricted geographical area delimited by his personal experience", whose "imaginative and rhetorical geography" could however "vary considerably", as Denis Cosgrove has pointed out. ${ }^{4}$ After the Bible of Amiens Ruskin's researches were directed further back in time, towards a closer study of the earlier history of Christianity. The visit he made on 2 September 1882 to the Benedictine monastery of Cîteaux, the cradle of the Cistercian reformation, was to be a climactic experience with respect

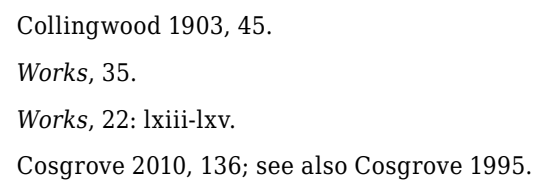


to this. ${ }^{5}$ Ruskin never accomplished his ambitious project, and perhaps he did not intend to. In his strained mental condition, he could not aim at writing such a comprehensive work, but he needed to clarify the outlines of that history as a framework for his researches on art, religion, and modern life. This is testified to by the large quantities of notes he left, and which are partly published in volume XXXIII of the Library Edition. ${ }^{6}$ Fragments of Ara Coeli and Valle Crucis, the books which were to deal respectively with "the foundations of the Papal power", and with "the monastic architecture of England and Wales", surface through the 18821884 letters of Fors Clavigera, and through his notes to Roadside Songs of Tuscany.

\section{Fanny Alexander and the Peasants of the Abetone}

The story of its editing begins on 5 October 1885, when Ruskin and Collingwood arrived in Florence and were introduced by the former's American friend and artist, H.R. Newman, to the Alexanders, a family of Massachusetts expatriates who had settled in Tuscany in 1853. Francis, a Boston portrait painter, and Lucia Gray Swett, a wealthy woman of aristocratic connections, were part of that large circle of Anglo-American artists living in Florence in the late nineteenth century, and with respect to whom their daughter Fanny must have been quite eccentric. Born in Boston in 1837, Esther Frances, known as "Fanny", spoke Italian as her second mother tongue. She was particularly and unusually connected with the poorest among the local people, and cultivated her drawing skills in composing precious missal-like sheets of drawings of flowers and folk songs, with the care, the devotion and the restraint of an amanuensis [fig. 1].
The Alexanders habitually spent their summer holidays in the Apennines at Abetone, where Fanny established an extraordinarily close, sympathetic relationship with the peasant women of the village. A deeply pious Lutheran Evangelical, Fanny was fascinated by the religious beliefs, traditions, and legends that were transmitted mainly through singing among the contadini. In these mountains" as Van Brooks puts it "everyone sang, the farmers, the shepherds and the charcoal-burners, who, as they watched their fires at night, kept one another company by singing together and improvising verses". ${ }^{7}$ Musically gifted herself, Fanny started recording the contadini songs and their tunes in her manuscript. ${ }^{8}$ This careful work was intended to be both documentary and artistic, and had philanthropic purposes, as Fanny aimed to sell the manuscript to some American patron and redistribute the money among her poor Abetone friends. ${ }^{9}$ Her method was similar to that of Lady Augusta Greg-

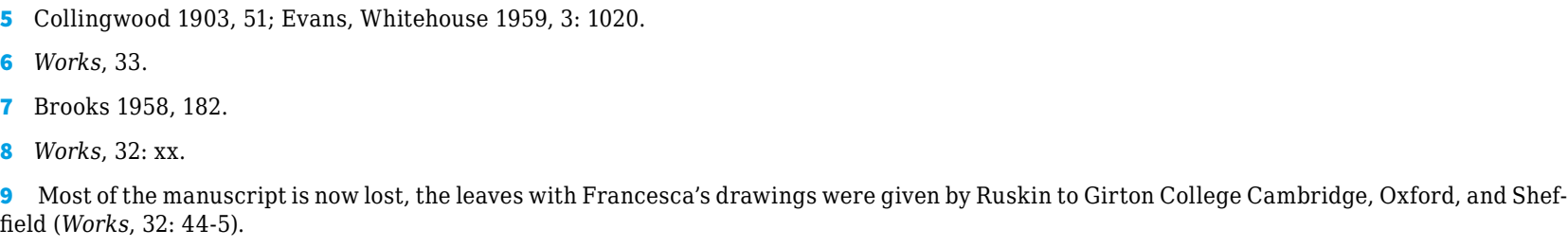


ory, who collected orally transmitted folk tales among the Irish peasantry. ${ }^{10}$ Fanny's interests were, however, more closely intertwined with a bond of solidarity, as local people saw her as a "miracle-worker", who "nursed the invalids [...], sent scrufulous children to the seaside, bought mattresses, dresses and shoes for them and paid their rent when it was overdue". ${ }^{11}$

A well-known source of Fanny's knowledge of Tuscan oral culture was Beatrice Bernardi di Pian degli Ontani, an illiterate improvisatrice from whose viva voce Fanny transcribed and translated most of the Tuscan songs, rispetti, and stornelli. A woman in her sixties when Fanny met her, Beatrice was a celebrity in the Florence salot$t i$, although she continued to live a peasant life of hardships to the end. In Roadside Songs Beatrice is given a leading place: her portrait opens the collection and about ten pages are devoted to first-hand details of her biography. Another major source was Edwige Gualtieri, Fanny's affectionate, pious, and musical housemaid, whose fame was to be wholly due to Ruskin's edition of Roadside Songs of Tuscany.

\section{“Francesca's Book"}

On 9 October 1882, in hyperbolic terms that recall his descriptions of some revelatory moments in his life such as his encounter with Tintoretto in the Scuola di San Rocco in 1845 - Ruskin wrote to Mrs Alexander saying that their meeting had marked a turning point in his life:
Fanny's meant her collection to be an elegiac monument to a territory and its people, to peasant life, to universal feelings, to orally preserved old music. Her drawings and the poems she transcribed and translated were generated by an intense relationship with the place. Neither a true local nor a complete foreigner, but a combination of both, Fanny's transcultural stance enriches the image of her Tuscany with an insider's understanding, combined with the wonder of an outsider. Her work partakes of that literary interest in folk traditions and songs that had emerged in Italy in the 1840s, when Niccolò Tommaseo, Giuseppe Tigri, Antonio D'Ancona, Giambattista Giuliani and others had started gathering and publishing a substantial corpus of materials and establishing metrical forms and rhymes, variants, and theories as to their origins and paths of transmission. ${ }^{12}$ In addition, the British expatriate Ouida had set her novel A Village Commune (1881) in the Abetone, giving in the appendix a documented account of the place and of Beatrice di Pian degli Ontani. ${ }^{13}$ All of these works are acknowledged in Roadside Songs of Tuscany.

I've taken a new pen - it is all I can! - I wish I could learn an entirely new writing from some pretty hem of an angel's robe, to tell you with what happy and reverent admiration I saw your daughter's drawings yesterday; - reverent, not only of a quite heavenly gift of genius in a kind I had never before seen, - but

10 George 2003, 227. This is the only published article on Roadside Songs of Tuscany, it focuses on Alexander's translations into Italian, but refers to an unrecorded edition of 1888

11 Brooks 1958, 182.

12 Tommaseo 1841; Tigri 1869; D’Ancona 1878; Giuliani 1879.

13 Ouida 1881. 
also of the entirely sweet and loving spirit which animated and sanctified the work, and the serenity which it expressed in the surest faiths and best purposes of life. ${ }^{14}$

He proposed buying the manuscript, which had proved to be closely related "to [his] work in England", he would pay the sum that the family had asked for it (600 guineas) and place it in St George's Museum. ${ }^{15}$ His idea was to exhibit the manuscript at Sheffield for the benefit of the Companions of the Guild of St George and of local peasants. To this purpose, he wished Fanny to write "by way of introduction to it - such brief sketches as she may find easy of arrangement of the real people whose portraits are given". The main object of the sketches would be "the conveying to the mind of our English paesantry (not to say princes) some sympathetic conception of the reality of the sweet soul of Catholic Italy". ${ }^{16}$

The meeting marked a turning point for Fanny too. The news of Ruskin's visit and of his interest in her manuscript spread rapidly throughout Florence, and she became a celebrity overnight. In December she wrote to a friend that she felt "temporarily on the list of distinguished people", that her house had been invaded by "the strangest variety of people [...] of every possible nationality", asking to see her work in a frenzy of Ruskin emulation. ${ }^{17}$
When Ruskin returned to England in mid November 1882 he was in a state of high enthusiasm over his new treasure, and quickly began disseminating references to Fanny's work in his lectures. On 1 March 1883, in the first of his Oxford lectures devoted to "Realistic schools of painting", he presented Francesca Alexander, along with three other young artists - Giacomo Boni, Angelo Alessandri, and Lilias Trotter - claiming that Francesca's drawings "carry with them certain evidences of the force of the religious feelings on the imagination". ${ }^{18}$ It must have been at this time that he began referring to Fanny in his public writings as "Francesca". ${ }^{19}$

Ruskin introduced Francesca's work on several occasions, arousing considerable interest in his new friend and her work. On 6 June, at Prince of Wales Terrace, Kensington he delivered a private lecture on "Francesca's Book" before two hundred attendees which included Matthew Arnold, Henry Lowell, Lord Leighton, the Burne-Joneses; Cardinal Manning had also received an invitation..$^{20}$ Several newspapers reported the event, all claiming to disclose the identity of the mysterious Francesca. All of them variously noticed that the lecturer was "in capital health and spirits", that the second part was all devoted to "Francesca's Book", a work "written and illustrated by a Miss Alexander", whose original pen-and-ink drawings were shown. ${ }^{21}$ The Tablet, a Lon-

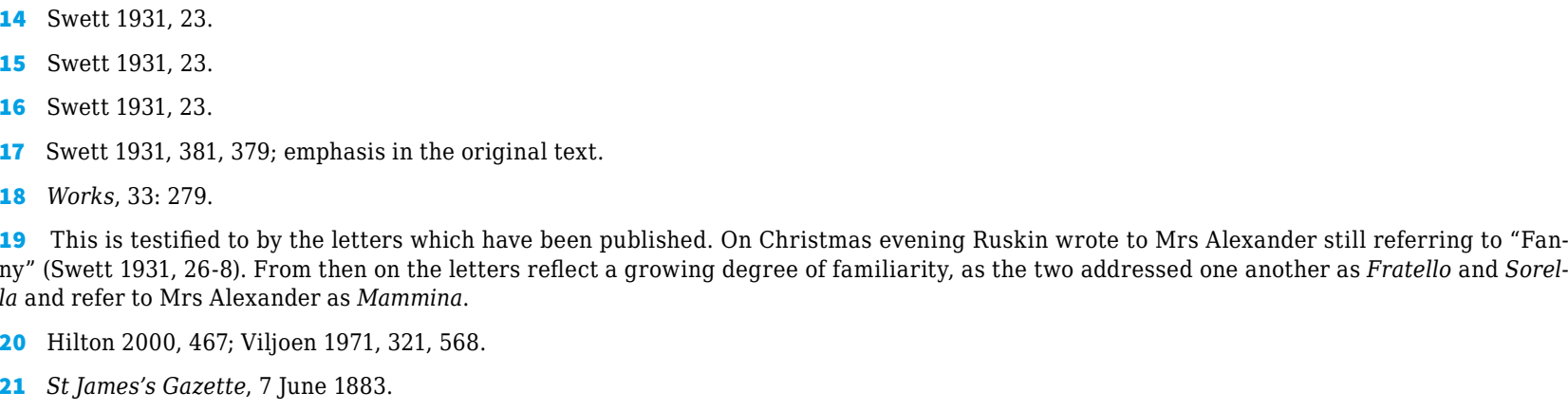


don Catholic newspaper, specified that the venue was the home of Mrs Bishop, a Catholic lady, ${ }^{22}$ and the Western Daily Press, reporting the news the following week, announced that the forthcoming publication of the "series of drawings [...] with some descriptive verses", ${ }^{23}$ had been anticipated by Francesca Alexander's The Story of Ida: Epitaph on an Etrurian Tomb. Finally, a lengthier review in the Spectator of 19 June reported the lecture in greater detail, saying that Ruskin had mentioned some correctable flaws in Francesca's rendering of the human figure, but expressed his unconditional praise of the strength and delicacy of her flower drawings, which compared only to those of Leonardo da Vinci's. The association with flowers then had led him to see the folk legends that Francesca had learnt from Beatrice degli Ontani as "the sparks which have kindled her imagination and given life to her skill", sparks that must have reminded Francesca "in her innocent freshness, of the Fioret$t i$ which, six centuries ago, gathered round the memory of St. Francis". ${ }^{24}$

This often-quoted reference to the Fioretti is interesting and deserves some attention. When the first two issues of Roadside Songs were published in August 1884, Ruskin wrote to Francesca again comparing the work to the Fioretti of St. Francis, and this time made closer reference to the book's purpose and to some additional notes he had inserted. "I am very, very happy" he said, "about the form the book is taking-the little supplementary bits, enable me to fit it all together into what will be the loveliest thing ever seen, and to more good than the fioretti di San Francesco" ${ }^{25}$

\section{The Little Flowers of Francesca}

The association with the Fioretti might imply more than a general evocative allusion. The connection had been first made at the beginning of June 1883 by Cardinal Henry E. Manning, in his letter of thanks for his copy of The Story of Ida. Concluding her introduction to Francesca Alexander's The Hidden Servants, Anna Fuller reports the Cardinal's words:
It is simply beautiful, like the Fioretti di San Francesco. Such flowers can grow in one soil alone. They can be found only in the garden of Faith, over which the world of light hangs visibly, and is more intensely seen by the poor and the pure in heart than by the rich, or the learned, or the men of culture. ${ }^{26}$

22 Tablet, 9 June 1883, 886.

23 Ruskin's own report of the lecture is given in a letter to Francesca dated 10 June, "I lectured on your book last Tuesday to all my best London friends and made them ever so happy [...] I simply said to the London people that I was not worthy to have such a book to show them. [this is not reported by any reviewer]. But it is nice that you like so much what I've said about the two religions" (Swett 1931, 32-3).

24 Works, 32: 535-8.

25 Works, 32: 77; Swett 1923, 77

26 Alexander 1903, ix; Works, 32: xxiii. Anna Fuller's extract too is given in the Library Edition with no further details. Cook and Wedderburn play down the relationship between Ruskin and Cardinal Manning, whose friendship with Ruskin helped fuel suspicions that he was about to con-

Fonti, letterature, arti e paesaggi d'Europa | Sources, Literatures, Arts \& Landscapes of Europe 1 | $\mathbf{3 2 2}$ 
Writing to Mrs Alexander on 22 June, Ruskin referred to a letter by Manning he had forwarded to Francesca. ${ }^{27}$ Cook and Wedderburn laconically inform us that Ruskin "saw something of Cardinal Manning in his later years" and that "some of the Cardinal's letters were accompanied by gifts of books such as the Fioretti of St Francis", ${ }^{28}$ but no reference to the gift occurs in the Library edition, ${ }^{29}$ nor are we informed that it was Cardinal Manning who first translated and published the Fioretti into English in 1864 under the title of Little Flowers of St Francis. Manning's reference to the Fioretti in connection with The Story of Ida echoes his own preface to the translation, where he defined the stories of the poor saints collected in the anonymous florilegium, as "admirable poems in prose" which

may justly be compared to flowers which give evidence of the season which has brought them forth, but do not reveal the name of the gardener who planted them. Every page of this little book breathes of the faith and the simplicity of the Middle Ages. [...]. Indeed, no one author could have composed this book. Compiled from a variety of sources, it is as if it were the work of a whole century. ${ }^{30}$

In his preface Manning also pointed out that the Fioretti were not to be considered as "superficial trivial sketches, only intended to familiarise the public mind with the austere virtues of the cloister"; rather, the stories, in "their great simplicity", were "full of strong doctrine, and fitted for men deeply versed in theology", and provided a typological reading of episodes in the lives of St Louis, of St Clare, and of St Francis, acknowledging the distinguished French scholar, Professor Ozanam as his source. Manning's edition was, in fact, greatly indebted to Frédéric Ozanam (1813-1853), a distinguished Catholic scholar who had translated a selection of the Fioret$t i$ into French. This constituted a part of his wide literary-historical source study, Poètes Franciscains en Italie au treizième siècle (1852), corresponding to Chapter VII, entitled "Les Petits fleurs de saint François". Historical and literary studies merged with social engagement in Ozanam, who was also the founder of the Society of St Vincent de Paul. This twofold commitment emerges in his studies of early Franciscan poetry, where the poetical and religious value of poverty are foregrounded. Poverty is also seen as a stylistic cypher by Ozanam, who praises the Fioretti as true poetry and sees prose as the fittest form for telling the epic of the poor. ${ }^{31}$ It is no surprise then to discover that, among the altarpieces encountered in his rides through the Umbrian villag-

vert to Catholicism, something he had to officially deny in 1887.

27 Swett 1931, 339.

28 Works, 36: xxxvi.

29 We are informed that the Fioretti was a late discovery of Ruskin's, and that in Praeterita (Work, 35: 523) Ruskin acknowledged that his American friend Charles Eliot Norton had introduced him to the Fioretti, and two letters to Norton of 1874 and 1879 where the work is mentioned, are reported with no details of the edition (Works, 37: 279). In Mornings in Florence (1875-1877) Ruskin had quoted and translated a lengthy passage from an edition that Cook and Wedderburn (Works, 23: 356), overlooking a date discrepancy, wrongly identify with Leopoldo Amoni's (Roma: Biblioteca Apostolica Vaticana 1889).

30 Manning 1884, v.

31 "Mais le livre des Petits fleurs de saint François est écrit en prose, et il a ce point commun avec tant de poèmes du moyen age écrits d'abord en vers pour le grand plaisir des grands, mais qui ont fini par trouver en prose une forme plus populaire et durable. [...] et on peut ajouter que les pompes de la poésie eussent mal convenu à l'épopée des pauvres" (Ozanam 1852, 203-4).

$323 \quad \begin{aligned} & \text { Fonti, letterature, arti e paesaggi d'Europa | Sources, Literatures, Arts \& Landscapes of Europe } 1 \\ & \text { John Ruskin's Europe. A Collection of Cross-Cultural Essays, 317-334 }\end{aligned}$ 
es, he sought the one in honour of St Zita. ${ }^{32}$ To this maid servant and patron saint of Lucca, Ruskin gives a leading role in Roadside Songs, placing Francesca's drawings and the "Ballad of Saint Zita" in the first two issues followed by a lengthy note on her hagiography. Such correspondences encourage us to believe that Ruskin had some knowledge of Ozanam's work and thought. As a Medievalist scholar, endowed with a particularly keen sensitivity to the language of art, and actively engaged in charitable works, he certainly had the traits that would have attracted Ruskin's interest.

Frédéric Ozanam's work had been introduced into England by Kathleen O'Meara in her biography of $1876 . .^{33}$ Two years later a second edition of the book appeared with a fifteen-page preface by Cardinal Manning, ${ }^{34}$ in which Manning made an outright political statement that was in effect a call to European Christians and to the clergy to carry out their duties. The Cardinal presented Ozanam as "one of the most brilliant of the brilliant band" of nineteenth-century French Catholic writers who had left "an indelible mark upon the country". ${ }^{35}$ His key contribution lay in his proposal for a future republic Commonwealth of Europe, an idea that originated in a 'fascination' with medieval culture combined with modern socio-polit- ical theories. Ozanam's major source of inspiration was Alexis De Tocqueville's Democracy in America, Manning argues, quoting extensively from the English 1865 edition of that work, ${ }^{36}$ which proposes an egalitarian model that responds to the urgent social issues thrown up by the "violent shock of opulence and poverty which is making the ground tremble under our feet". ${ }^{37}$ The questions that Cardinal Manning raised in his Preface were of momentous importance to Ruskin, and we can imagine their emerging in the substantially unrecorded exchanges between the two friends in the early 1880s.

That Manning might have been the vehicle by which Ruskin came to know Ozanam by way of O'Meara's biography seems therefore to be quite plausible, and further echoes in Roadside Songs seem to support this hypothesis. In introducing the Fioretti, O'Meara reports a veiled reference by Ozanam to his wife Adèle - whom he calls his "Beatrice" - and her "delicate hand" in translating the "little flowers". Interestingly, in reporting these words, O'Meara expands the flower metaphor implied in the Fioretti and defines them as the "fragrant little flowers that grew in the lowly spots along the road". ${ }^{38}$ It is tempting to imagine that Roadside Songs of Tuscany might have been inspired by O'Meara's image. This is a reference which

32 Ozanam 1852, 10.

33 An Irish Catholic expatriate to Paris, Kathleen O'Meara (1839-1888) wrote novels under the pseudonym Grace Ramsay, and in her own name wrote some biographies and lives of saints; she was the Paris correspondent of The Tablet.

34 O'Meara 1878. The book went through seven editions by Catholic and Christian publishers in the United States between 1878 and 1915

35 O’Meara 1878, vi, viii.

36 O’Meara 1878, xi-xvi.

37 O'Meara 1878, xv.

38 "They are anonymous 'it being the effort of mysticism to be forgotten of men before God', remarks the gleaner who presents them to us; and he adds, 'Here I pass the pen to a had more delicate than mine'. Whilst he was plunged in the arid researches of the archives, this hand 'more delicate than his' was culling the fragrant little flowers that grew in the lowly spots along the road, and forming them into a bouquet whose perfume refreshed him in many an hour of weariness and pain. Perhaps the fact of their being translated by her whom he styled his Beatrice may have added another charm to those exquisite idylls in Ozanam's eyes, and account in a measure for the fascination which they possessed for him to the least" (O’Meara 1878, 289; emphasis added). 


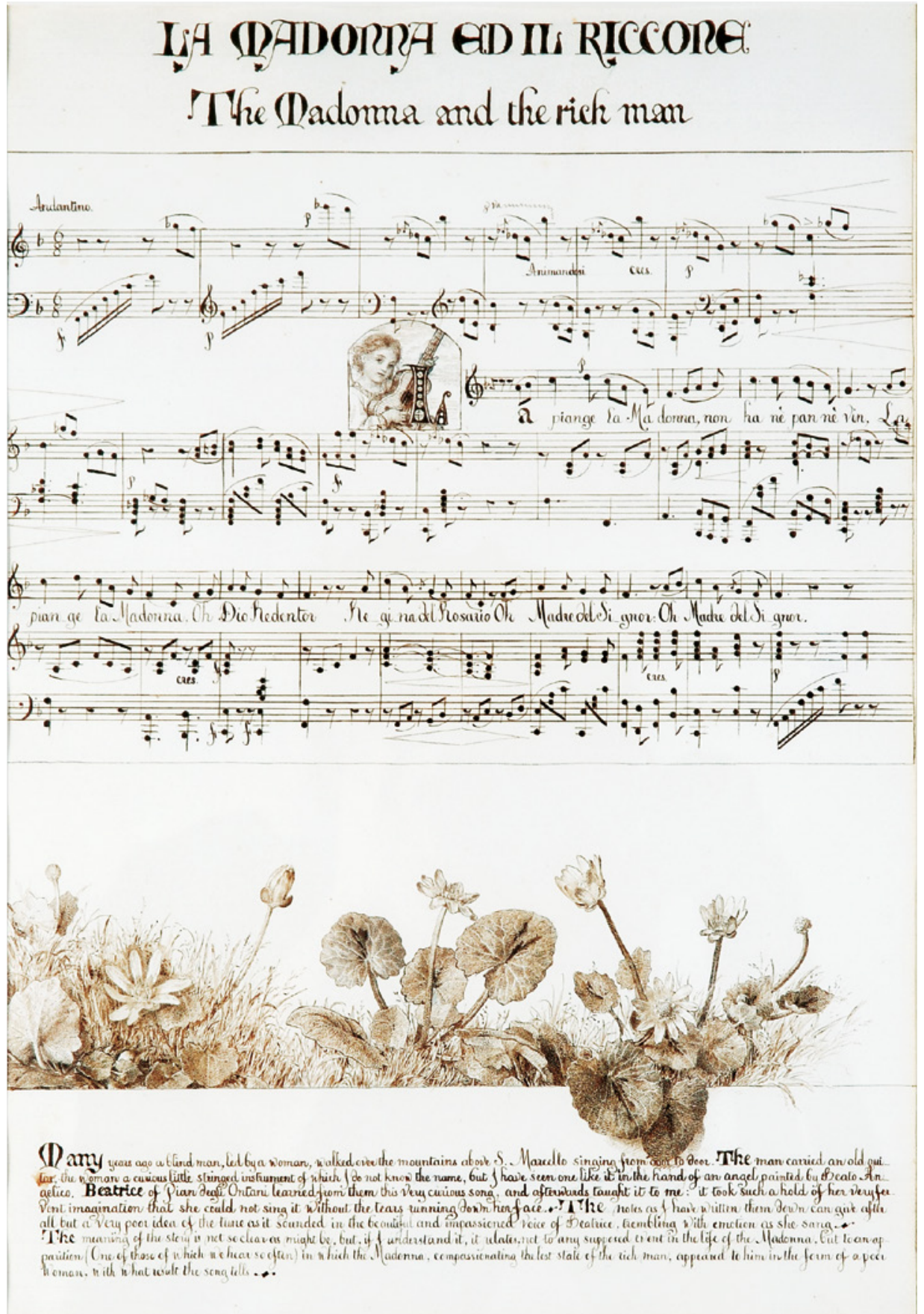

Figure 1 Esther Frances "Francesca" Alexander, La Madonna e il Riccone, title page to The Madonna and the Rich Man. Collection of the Guild of St George, Museums Sheffield

The meaning of the story is not so clear as might be; but, if I understand it, it relates, not to any supposed event in the life of the Madonna, but to an apparition (one of those of which we hear so often) in which the Madonna, compassionating the lost state of the rich man, appeared to him in the form of a poor woman; with what result the song tells. From Francesca Alexander's MS. book (Works, 32: 108) 
would have been particularly appropriate to Francesca, whose "delicate hand" had not only transcribed and translated the poems, but had also illustrated them with her extraordinary flower drawings. The chain of connections and correspondences may be read as constituting a multi-layered flower-and-song association that determined Ruskin's choice of the final title of the book. As we know the titles of Ruskin's late works are outcomes of half-obscure, densely personal, highly evocative processes.

\section{$5 \quad$ A "Unique" and "Very Sumptuous" Book}

Sometime around 10 May, Ruskin received "Francesca's Book" from Florence. ${ }^{40}$ On the 13th he shared with her his 'bewilderment' at its beauty and preciousness, trusting that she would "soon know how precious it [would] become to uncountable multitudes". He hinted at the need to change the form of the manuscript and, announcing the imminent publication of The Story of Ida, he said that once Ida began to become known he would make "this book" known at Oxford..$^{41}$

The Story of Ida is the first of the works by Francesca that Ruskin published and the one least heavily edited. When Ruskin first saw the manuscript, he was struck by the association between the fragile young Italian girl and Rose La Touche, the young woman he passionately and devastatingly loved, who had died in 1875. But what also struck him was the ecumenical potential of the story, as Francesca reported:
The excitement and inadequacy that Ruskin expresses in his letters on Roadside Songs after the summer 1883, when he had conceived the full sense of the project, can be thus related to the complexity of the endeavour and the 'holiness' of the Fioretti model. On 1 October from Kenniwe Castle, Galloway, he wrote to Mrs Alexander: "I've got type settled, and my own notions a little - but I'm a profane creature to have a charge of such a thing". ${ }^{39}$
He said a good deal about my little story of Ida, which he had just read, and quite took my breath away by proposing to take it away and have it printed. He said it would be a very useful religious book [...] especially from the absence of all sectarian feeling in it, and he seemed much pleased at the strong friendship and religious sympathy between Ida and myself, belonging as we did to two different and usually opposing churches. And in connection with this, he spoke with much sadness of the enmity between different Christian sects, saying that he had known good Christians, in all of them (which is my own experience). ${ }^{42}$

The need to bridge the fracture between Protestant and Catholic Churches and overcome what he saw as one of the greatest cultural barriers dividing Europe from England (and dividing England itself), was a strong concern of late Ruskin, and the potential he found in the work of

40 He had received Francesca's manuscript from a "Quaker woman" who visited Francesca in Florence in Spring 1883 (Swett 1931 , 384 ). Ruskin's letter is dated "Herne Hill. Sunday 13 May 1883" (Swett 1931, 28-9).

41 Swett 1931, 28-9.

42 Swett 1931, 376. 
Francesca, a pious American Evangelical woman who collected the religious poems of Catholic contadini, became gradually clearer. At this stage, the idea of keeping the manuscript at St George's Museum had given way to the prospect of a - possibly imminent - publication of the work. In the meantime, he had received from Francesca the "short biographical sketches", which were to accompany the drawings.

Although not completely defined as yet, the idea of a serial publication was also taking shape. On 24 October 1883 Fanny wrote to her friend Lucy Woodbridge: "As nearly as I understand, some part of the book of the Roadside Songs is to be printed in numbers, but I do not know how much, nor when it is to appear".43

The ten issues of Roadside Songs of Tuscany appeared between April 1884 and August 1885. Ruskin worked intensely on one issue at a time, gaining the attention of the public step by step. Each of the thin issues was composite, consisting of 25-30 pages of heterogeneous materials: a number of folk songs, two drawings and the prose sketches of the peasants by Francesca, and some Editor's notes. By December 1884, four issues had come out, meeting with puzzled reviews in the newspapers, which experienced difficulty in framing it. A lengthy piece in The Evening News and Star of that November foresaw that "when completed" the work would be "probably unique in the world of art and letters" ${ }^{44}$ When the whole book appeared, in September 1885, in the shape of a 340-page folio hardback volume, it was greeted as a "very sumptuous book" whose socio-historical interest to the British public was, according to the reviewer, jeopardized by the hardly accessible format - said the reviewer [fig. 4]. ${ }^{45}$
In the course of editing Ruskin had completely rearranged Francesca's manuscript, selecting from among the drawings and folk songs and changing their order, so as to place first the Ballad of Santa Zita, instead of the two religious hymns that opened the manuscript, and close the book with a version of the legend of St Christopher he had expressly asked Francesca to transpose into prose to make the story clearer. The central section included two long religious songs - The Madonna and the Rich Man [fig. 2] and The Madonna and the Gipsy - and Francesca's drawing of Christ and the Woman of Samaria accompanied by a translator's note. These texts formed the backbone of the collection, what we might call its Christian framework, and were built around Francesca's drawings. Ruskin assigned great importance to the people who had sat for the drawings, the "originals" - as Francesca called them - of the Madonna, the Samaritan, St Christopher and the Gipsy. He saw a resonance in their lives with the episodes and legends from the Gospel of which the songs speak. He thought of their stories as new Fioretti, stories of poor, everyday saints, survivals of that monastic European spirit he had been on the track of for some years. This is suggested by the Editor's preface to the first issue, where he informs the reader that Francesca had chosen her models because they shared some "circumstances and habitual tone of mind" with the figures of the saints they represented. ${ }^{46}$

Originally intended just to complement the drawings, the sketches of the peasants' lives in fact constitute the larger part of the complete work: 136 of 340 pages, about two thirds of the whole book. Their prominence is ensured by an index of twenty-one names that opens the

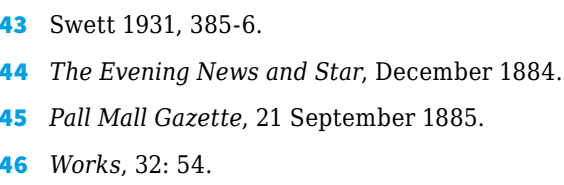


Figure 2 Esther Frances "Francesca" Alexander, Non ho né pan né vin cosa ti posso dar? $381 \times 277 \mathrm{~mm}$ In The Madonna and the Rich Man (Works, 32: 104). Collection of the Guild of S George, Museums Sheffield

"The pretty young girl who sits for the Madonna is named Emilia; but I must not tell the name of her family, nor where she lives, for fear that it might be heard of [...]. I have not much to tel about Emilia (or, as we usually call her La Madonnina); she is very beautiful, and has sat for all the Madonnas in the book. [...] but the girl sitting in the

wooden chair, whose face does not appear, deserves that I should tell a little about her. [...] Afterwards her face appears several times in the course of the book, [...] poor Paolina [... I must tell the story".

Francesca Alexander (Works, 32, 110)

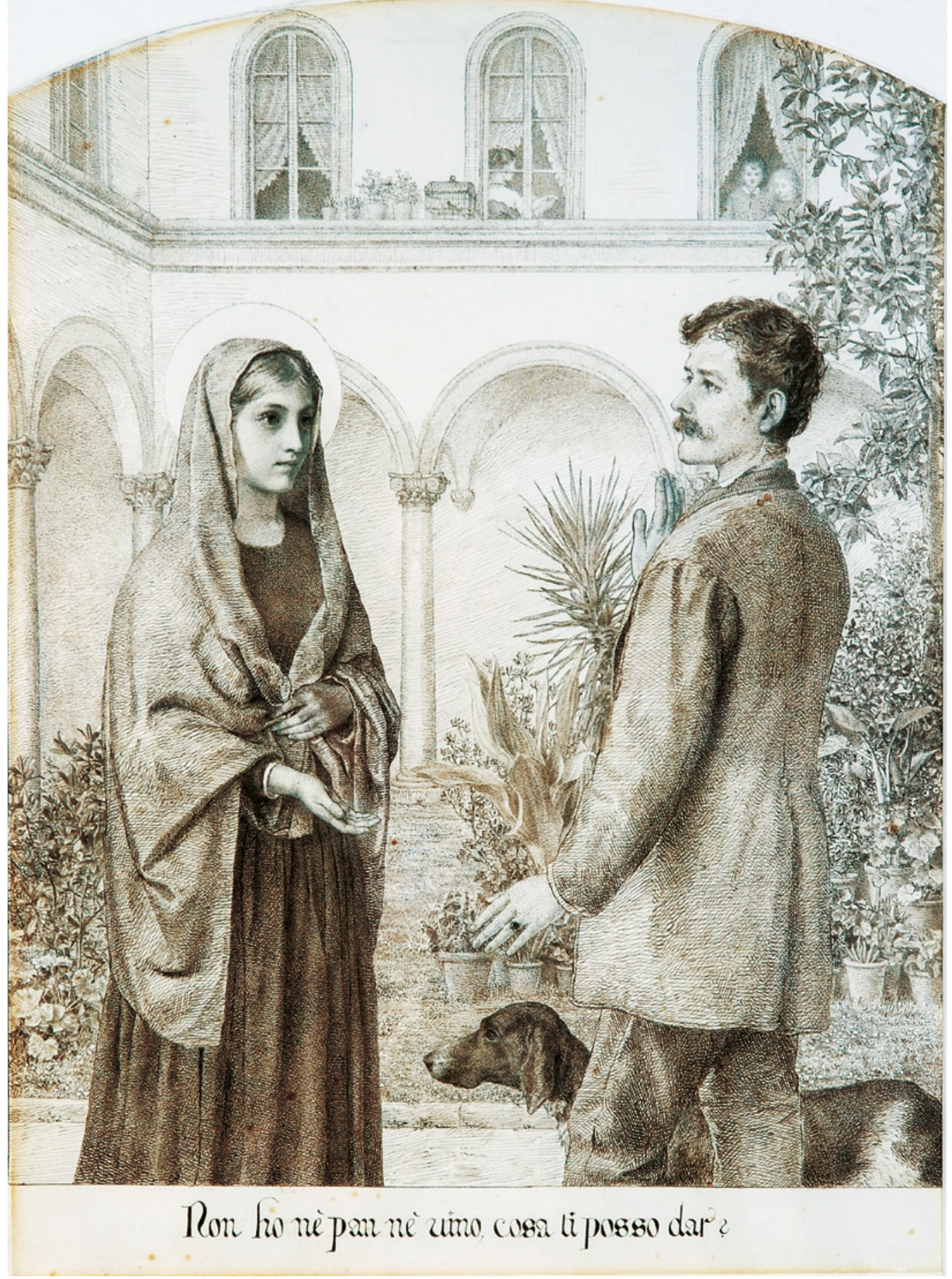



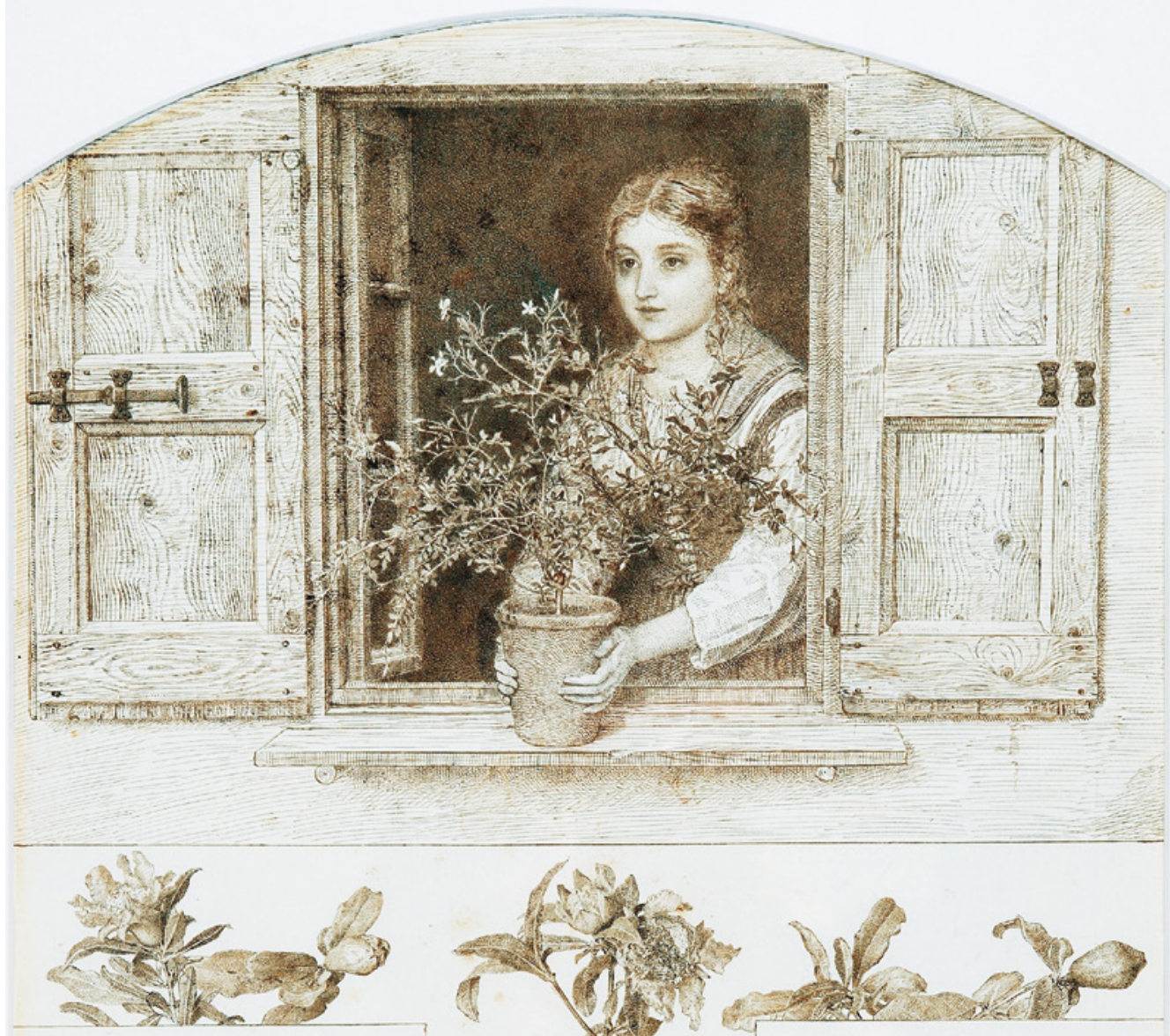

\section{P19. here on the plain a little house f see,}

- Andinthat house my lady lives herself: Beside the door a green pomegranite tree. - A jessamine bloomingon thewindow Comelove and set thy jessamine in the Sing, f can hear thecat thy window there. Come love and set thyjessamine in the sun Bing, f will answer when the song is done.

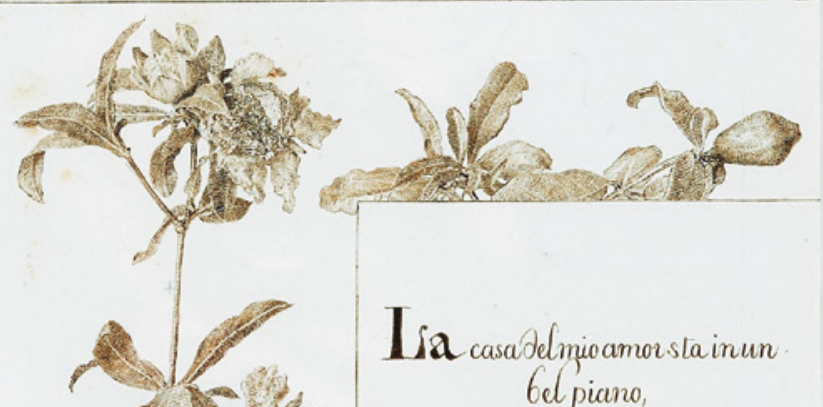

$$
\text { Gel piano, }
$$

Rimpeltoallamia par ungiardino. - Appiédelleuscioc úun bel melagrano - Allafinestracihaun gelsumino Pigliaquel gelsumin, meltilo alfíesco; Piglia quel gelsumin meltilo al sole Cantapursu, che li rispondo amore. Cantapursu,che ti-ispondo a questo.
Figure 3 Esther Frances "Francesca" Alexander, The Jessamine Window. $389 \times$ $284 \mathrm{~mm}$. Works, 32: 142. Collection of the Guild of St George, Museums Sheffield

"Paolina's portrait is given [...] as the girl setting a jessamine at her window [...] And though I think all these faces are pretty, not one of them gives even a faint idea of her beauty, which I found it entirely beyond my power to represent". Francesca Alexander (Works, 32: 115)

"The Jessamine Window, with its pretty lesson in window-gardening, is given to Sheffield". John Ruskin (Works, 32: 158 fn) 
volume, in which are listed the "Persons whose characters are sketched, or some account given of passages in their lives, in illustration of the songs of Tuscany". ${ }^{47}$ The sketches are given "in Francesca's own colloquial, or frankly epistolary, terms, as the best interpretation of the legends revived for us by her, in these breathing images of existent human souls". ${ }^{48}$

In Roadside Songs the association between a saint and her/his "original" turns out to be far from systematic; sometimes it is only hinted at, a mere suggestion, and sometimes it is abandoned in favour of another character who in the picture appears dimly and at a distance [fig. 2]. Francesca tells us in a confident and assertive narrative voice about Gigia, Lucia Santi, Geminiano Amidei, Emilia, Paolina, their brothers, mothers, sisters-in-law, neighbours, donkeys: in effect recreating a whole community. "However", she writes at one point, "I am not writing a history of Cutigliano, but of Assunta, who lived in one of its steep narrow streets, just flights of low steps, but with beautiful gardens between the old houses, and roses and jessamines hanging over their walls". ${ }^{49}$ Ruskin the teacher and mentor who educates Francesca's drawing skills, gives her also the status of narrator, encouraging her to write a wealth true of stories which was to overflow into the subsequent collection of stories published serially as Christ's Folk in the Apennine (1887-1889).

Such prose sketches occur in issue after issue of Roadside Songs. This creates the effect of a community of people with whom British readers become familiar gradu- ally, as with characters in Victorian serial novels. They also interlace with the group of texts of rispetti - shorter songs composed of hendecasyllabic lines, with an abababcc or ababccdd rhyme scheme - and stornelli, short, proverbial, three-line songs each focusing on a flower (e.g. "Flower of the Pea", "Flower of the Maize"). Francesca's drawing - "sincere and true as the sunshine; industrious, [..]; modest and unselfish, as ever was good servant's work for a beloved Master" - seize and render those correspondences with "candour and lack of ostentation" [fig. 3]. ${ }^{50}$ Interestingly, the ballads and songs that are reported and translated all treat of encounters between strangers: the Madonna and the rich man, the Madonna and the gipsy, as well as the drawing of Christ and the woman of Samaria. Moreover, all the figures involved in these encounters are women, as models of benevolence and acceptance, and to womanhood, in the collection, Ruskin attributes a "guiding power". ${ }^{51}$

The editorial intervention is massive. Ruskin organizes the work in such a way as to give prominence to the Christian frame and to the peasants' portraits and lives, adding substantial notes to orient the texts and make the discourse relevant to contemporary Britain within the European context. The tone and approach are that of Fors Clavigera, the monumental epistolary work he addressed to the workmen and labourers of Britain. After an almost continuous run of more than fifteen years, Ruskin ended the Fors Clavigera series in December 1884, a date midway through the serial publication of Roadside Songs, which addresses a similar audience,

47 Works, 32: 50. The emphasis on the peasants' lives stands out in the 1885 volume edition, while the Library Edition prints the sketches in smaller fonts.

48 Works, 32: 88

49 Works, 32: 147

50 Works, 32: 52. Her drawing reflected a discipline of the hand responding to criteria of "rightness" that Ruskin had advocated from the 1860s, see Levi, Tucker 2011

51 Works, 32: 224 
and casts a similar critical, unsettling glance on European events and British insularity, particularly in the Editor's "Notes on Santa Zita", "On the Priest's Office", "On the Gipsy Character", "On St Christopher". In the Preface Ruskin associates Tuscan folksongs with ancient Greek epic verse, in consonance with Tommaseo, Tigri, and Ouida, but referring to Émile Boutmy's Philosophie de l'Architecture en Grèce and his stylistic description of the homeric sentence as "explicit and undisturbed narrative or statement of emotion". ${ }^{52}$ In the "Note on the Life of Santa Zita" he reconstructs the story of the patron saint of Lucca through a close comparison between the 1615 version of the poem in ottava rima by Guaspari di Bartolomeo Casentino's Vita e morte della beata Sita, which Francesca had translated, and the account given in Alfonso Villegas's monumental work, Il Sagro Leggendario della vita di Gesù Cristo, di Maria Vergine, e de' Santi (1757). Ruskin's enquiries had revealed that the thirteenth-century maiden servant was recognized in the time of Dante as "a very notable creature and one of practical power throughout Europe"..$^{53}$ The long "Note on the Gipsy Character" is a tough attack against British intolerance, where Ruskin points at the "much happier wisdom" of the Italian peasantry to show "how deeply and cruelly the scorn of the Gipsy race had infixed itself in the minds of the prosperous middle classes of our own island, at the beginning of the century", referring to the 1797 entry in the Encyclopæedia Britannica. ${ }^{54}$

Published in September 1884, few months before he bid farewell to the readers of Fors Clavigera, the "Notes on the Priest's Office" have a pivotal role within the collection: "I leave the reader", says Ruskin, "for a while to

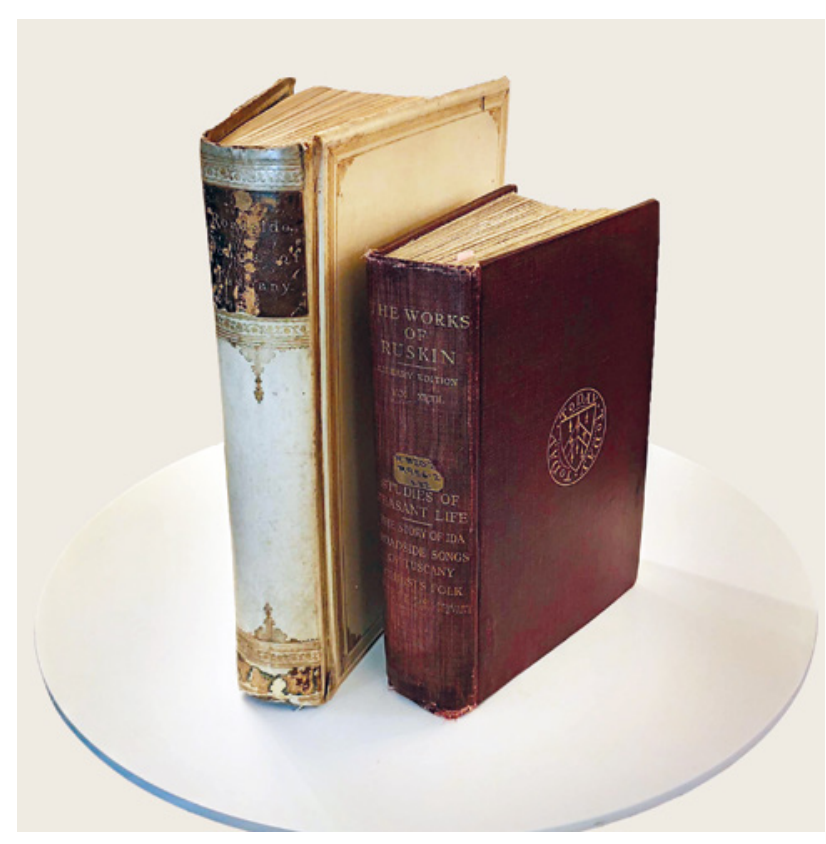

Figure 4 Francesca Alexander, Roadside Songs of Tuscany, London, George Allen, 1885. E.T. Cook and A. Wedderburn, The Works of John Ruskin, Library Edition, vol. 32, Studies of Peasant Life

his own reflections on the people he will make acquaintance with in Francesca's stories, and on the circumstances which have made them what they are". ${ }^{55}$ These "Notes" constitute a dense theological digression on political and religious institutions in Europe, which seems at odds with the context of Tuscan peasantry, and only can be understood if related to his research into Monasticism and Early Christianity. The focus is on the clergy, whose irrele- 
vance in contemporary life, says Ruskin, is confirmed by their absence from the dramatis personae in "higher imaginative" literature, as well as by their appearing as a desacralized body. He then provocatively maintains the bishops to be "responsible for all evils in Europe", and quotes from Pope Gregory I's letter to Emperor Maurice in Milman's History of Latin Christianity. The issue alluded to actually constituted an iceberg in the history of Christian controversies: it dates back to 587-88 AD when John IV of Constantinople, supported by Emperor Maurice, attributed to himself the title of ecumenical patriarch, and Gregory the Great responded by introducing the title of Servus Servorum Dei, declaring the pope's humility before God. The reference is left implicit, with no footnotes to clarify it, a flash in the theological background of a discourse that revolves around the theme of religious consecration, ruling power, and wealth. While stating that the evil of Europe is in having lost the vow of poverty, Ruskin maintains that the beneficial effects of religious men are still at work "in secret channels", and that their "modest and constant virtues" are "at the root of what yet remains vital and happy among European races, as Francesca's book will display". ${ }^{56} \mathrm{He}$ reminds us that in the Bible and in Homer political and religious bodies were "consecrated offices", while among the Romans the figure of the Pontifex maximus was "simply one of the functions to which any citizen was eligible, as to that of consul or dictator". ${ }^{57}$
Here Ruskin refers the reader to forthcoming, but in fact never published, chapters in Ara Coeli on the life of St Gregory, and in Valle Crucis on St Benedict, works that "may be both read in connection with the Tuscan songs". ${ }^{58}$ Finally he considers the significance of anointment and of the vows of poverty taken by monks and priests, saying that while in monks the vow is part of their desire to worship God in seclusion, ${ }^{59}$ in Priesthood "magnificence" was "entirely needful" - as Tintoretto's Circumcision of Christ in the Scuola di San Rocco ("the most beautiful existing symbol of all priesthood") showed.$^{60}$ He recalls here that Gregory I "civilized the Saxons and Lombards" by "the use made of music, metal work and painting" and blames the attacks of "typically modern protestants" against the "use of splendour" by the Church, and concludes that all ancient cultures were "born in equal simplicity", citing Gibbon's portrait of Caliph Omar as a model of frugality and munificence: "Wherever he halted, the company without distinction was invited to partake of his homely fare, and the repast was consecrated by the prayer and exhortation of the Commander of the Faithful". ${ }^{61}$

As in Fors Clavigera, Ruskin's discourse here follows a path that is not linear, but one which moves "disjointedly through constantly shifting, idiosyncratic viewpoints". ${ }^{62}$ Read in the context of the spiritual search on which he was engaged at this time, however, we may grasp a sense of the theological questions and social concerns it raises.

56 Works, 32: 118

57 Works, 32: 119. Italics in the original.

58 Works, 32: 119. Italics in the original.

59 Works, 32: 120

60 Works, 32: 121.

61 Works, 33: 121-3.

Stoddart 1998, 47 


\section{6 "The Main Lessons"}

Coinciding with the birth of the interest in peasantry from the $1870 \mathrm{~s}^{63}$ Roadside Songs of Tuscany was perceived as a composite work whose ethnographic interest was soon acknowledged. ${ }^{64}$ In assembling its texts and images, Ruskin performed a complex act of cultural mediation by means of multiple processes of translation.

Roadside Songs was intended to intersect with British culture and be relevant to it within the European context. Ruskin conducted his reader through his historical and philological searches, connecting the Tuscan peasantry to the sources of Western civilization. His method is analogical and associative, his statements aiming not to be normative but suggestive and reformative. Initially Ruskin had meant to provide teaching through Francesca's stories of the contadini, but later gave up this attempt for reasons of which he wrote in a final "Note on the Vision of St Christopher":
I had partly hoped, in closing this series of pictures of the hearts of the Italian peasantry, to indicate the main lessons they seemed to bear for us all. But I am abashed before their strength and innocence, and able to draw only this one conclusion of deep practical import, - that the only service we can rightly render them is to love them. ${ }^{65}$

The teacher and scholarly editor thus withdraws from the work, letting the book close with two sections of letters from Francesca that compose a mosaic of stories told by her maid Edwige, about women and children, on family life and mutual help, poverty and charity. The volume ends with the evening prayer that, Francesca assures in a note, all peasants sing to their children. It is an appropriate ending for a book which was not intended to be a monument of an idealised view of rural life, but a memorial of living Tuscan peasants that aimed to rekindle, in modern Britain, that mysticism of everyday life which, in his old age, Ruskin saw as the core of Europe's legacy.

\section{Bibliography of Works by John Ruskin}

References are to volume and page numbers in:

Cook, E.T.; Wedderburn, A. (eds) (1903-1912). The Works of John Ruskin. Library Edition, 39 vols. London: George Allen. https://www.lancaster.ac.uk/the-ruskin/the-complete-works-of-ruskin/.

Citations indicate volume number and pages only, as it follows: Works, [vol.]: [p.].

Works, 22: Lectures on Landscape, Michael Angelo \& Tintoret, The Eagle's Nest, Ariadne Florentina. |23: Val D'Arno, The Schools of Florence, Mornings in Florence, The Shepherd's Tower.|32: Studies of Peasant Life. |33: The Bible of Amiens, Valle Crucis, The Art of England, The Pleasures of England. |35: Praeterita, Dilecta. |36-37: The Letters of John Ruskin I-II.

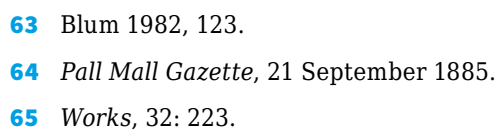




\section{General Bibliography}

Alexander, F. (1884-1885). Roadside Songs of Tuscany, 10 issues. London: George Allen.

Alexander, F. (1885). Roadside Songs of Tuscany. London: George Allen.

Alexander, F. (1887-1889). Christ's Folk in the Apennine. London: George Allen.

Alexander, F. (1897). Tuscan Songs. Cambridge (MA): Riverside Press.

Alexander, F. (1903). The Hidden Servants and Other Very Old Stories Told Over. With a preface by A. Fuller. London: David Nutt.

Blum, J. (1982). "Fiction and the European Peasantry: The Realist Novel as a Historical Source". Proceedings of the American Philosophical Society, 126(2), 122-39.

Boutmy, É. (1870). Philosophie de l'Architecture en Grèce. Paris: Ballière.

Brooks, W. Van (1958). The Dream of Arcadia. American Writers and Artists in Italy 1760-1915. London: Dent.

Clegg, J.; Tucker, P. (1993). Ruskin and Tuscany. London: Lund Humphries.

Collingwood, W.G. (1903). Ruskin's Relics. London: Isbister and Co.

Cosgrove, D. (1995). “Mappa Mundi, Anima Mundi: Imaginative Mapping and Environmental Representation”. Wheeler, M. (ed.), Ruskin and Environment. The Storm Cloud of the Nineteenth Century. Manchester: Manchester University Press, 76-101.

Cosgrove, D. (2010). "Ruskin's European Visions". Geography and Vision. Seeing, Imagining and Representing the World. London, I.B. Tauris, $135-51$.

D’Ancona, A. (1878). La poesia popolare italiana. Livorno: ed. F. Vigo.

Evans, J.; Whitehouse, J.H. (eds) (1959). The Diaries of John Ruskin. 3 vols. Oxford: Clarendon Press.

George, J.-A. (2003). “Translating Tuscany: Francesca Alexander's Roadside Songs (1888)”. Forum for Modern Language Studies, 39(2), 227-38. https://doi.org/10.1093/fmls/39.2.227.

Giuliani, G. (1879). Sul vivente linguaggio della Toscana. Firenze, Lettere.

Hilton, T. (2000). John Ruskin, The Later Years. Yale: Yale University Press.

Levi, D.; Tucker, P. (2011). “The Hand as Servant': John Ruskin, Professor of the Manual Arts”. Predella, 29, 1-27.

Manning, H.E. (1884). The Little Flowers of St. Francis of Assisi. Translated from Italian and ed. by. London: Burns and Lambert.

Moorman, J.R.H. (1940). The Sources for the Life of S. Francis of Assisi. Manchester: Manchester University Press.

O'Meara, K. (1876). Frederic Ozanam, Professor At the Sorbonne: His Life and Works. Edinburgh: Edmonston and Douglas.

O'Meara, K. (1878). Frederic Ozanam, Professor At the Sorbonne: His Life and Works. With a Preface of his Eminence Cardinal Manning. London: Kegan Paul.

Ouida [Louise de la Ramée] (1881). A Village Commune. London: Chatto and Windus.

Ozanam, F. (1852). Poètes Franciscains en Italie au treizième siècle avec un choix de petits fleurs de Saint Francois traduits de l'italien, suivis de recherches nouvelles sur les sources poétiques de la divine comédie, septième édition. Lyon: Librairies Catholique Emmanuel Vitte.

Ozanam, F. (1919). Franciscan Poets in Italy of the Thirteenth Century. Translated and Annotated by A.E. Nellen and N.C. Craig. London: David Nutt.

Ruskin, J. (2018). Looking at Tintoretto with John Ruskin. Edited by E. Sdegno. Venezia: Marsilio.

Stoddart, J. (1998). Ruskin's Culture Wars. "Fors Clavigera" and the Crisis of Victorian Liberalism. Charlottesville: University of Virginia Press. Swett, L.G. (1931). John Ruskin's Letters to Francesca and Memoirs of the Alexanders. Boston: Lothrop, Lee \& Shepard.

Tommaseo, N. (1841). Canti popolari toscani corsi illirici greci. 4 voll. Venezia 1841.

Tigri, G. (1869). Canti Popolari Toscani. Firenze: Arnaldo Forni editore.

Viljoen, H.G. (ed.) (1971). The Brantwood Diary of John Ruskin. Yale: Yale University Press. 


\title{
Ruskin's Islamic Orient and the Formation of a European Ideal
}

\begin{abstract}
The Ruskinian attitude towards the non-European, and in particular, the Islamic 'other', may at first seem definitive. This position being made evident from Ruskin's descriptions of the lugubrious nature of Islam's sacred scripture, its peoples, arts and Weltanschauung. This paper argues, contrary to this bien pensant view, that Ruskin's oeuvre intimates an ongoing, lasting and unfinished discussion with the Islamic Orient, from the earliest drafts of The Stones of Venice to later discourses on morality, history and religion. Whilst the sympathies for the refinement and delicacy of Islamic art and its influences upon the Venetian Gothic are well documented in the literature, Ruskin's engagement with Islam (both positive and negative) has yet to be fully explored. This paper endeavours to warp and weft the strands of these ideas into a sustained discussion of Ruskin's ideals in his oeuvre.
\end{abstract}

Keywords Ruskin. Islam. Europe. Orient. Aesthetics. Gothic.

Summary 1 Introduction. - 2 Ruskin's Parlance with Islam and Islamic Art. - 3 Ruskin's Aesthetic Ideals and Islamic Art. - 4 The Autochthonous Gothic and the Islamic Presence. - 5 Remnants of the Islamic "Lava Stream" in Ruskin and the "Eastern Question".

\section{Introduction}

The medieval retelling of the ancient Parzival drama in Wolfram von Eschenbach's hands stands as a propitious moment in European literature, an epic whose unconstrained desire to explore the summum bonum allegorises the Holy Grail as an enchantment with the Christ figure. Contended to deliver religion not as mere theology but as sacrament (Scruton 2020), Wolfram's tale, curiously, necessitates this quest as a meeting with the world of Islam. The drama begins with Parzival's father, Gahmuret, seeking adventure and fortune in the East, accepting a commission at the court of the Caliph in Baghdad. This leads him ultimately to the African kingdom of Zazamanc and the beautiful Moorish (Muslim) Queen, Belacane. There he fights and defends the city from invaders winning the battle 
as well as the hand of the queen, with whom he has a son, Feirefiz. ${ }^{1}$

The perturbations within Gahmuret, however, lead him back to Europe where he marries again and fathers a second son, Parzival. Later as a young knight, Parzival will find himself in search of the Holy Grail and chances upon, unbeknownst to him, his elder stepbrother in a duel. Realising the Moor with whom he has drawn swords is his brother, the narrative leads ultimately to their reconciliation and Feirefiz embracing Parzival (and Christianity) with the latter becoming the new Grail King. Later in Wagner's adaption, Parsifal, his final opera, illusions to the world of the East (and Islam) are not without consequence, such as in Act II wherein the Guardians of the Grail are set upon the 'northern mountain of Gothic Spain' and must contend with the perfidious Klingsor whose adjoining realm nestles yonder "on the southern slope of the same mountain range, facing Moorish Spain". 2

Parsifal ultimately destroys the heathen kingdom, not before making a sign of the Cross over its ruins. It seems in the Wagnerian mien; redemption is delivered at the cost of an annihilation of that which was; atonement in what has perished. In Wolfram's earlier rendition, however, vindication of the other does not come without mutual reclamation. ${ }^{3}$ The rapprochement with the Islamic world as foundational to the birth of modern European literature is not without irony, and haunts the pages of many a thinker and their ruminations on what it means to inhabit ' $\mathrm{Eu}$ rope' today. In the elision between these retellings, there exists a further concomitant attitude to how cultures and ideas move, rest and meet across time, namely, between 'consilience' of world views as well as the domination of 'influence' wherein one quells the other.

The seeming antipathy between these views has consequences and is also convenient to us in order to assess John Ruskins' views on Islam which, as shall be argued, are far from insignificant to his thought. In this regard, and with an analogy to Percival, Ruskin neither fully entertains Wolfram's attitude by which the other is conciliated in the moment of acceptance nor Wagner's consummate submission to the will of the other. We may argue, initially at least however, that Ruskin's position is more 'Wolframian' in his sensibility towards the natural outgrowth of the Gothic from Islamic precedents than Wagner's ahistorical reading which shares little appetite for cultural consilience. The intention of the present meditation is then to consider Ruskin's ideas on Islam, art and Gothic in order to evaluate those claims, which are for the most part unfinished, and their place and conception of the 'great community' that is Europe.

\section{Ruskin's Parlance with Islam and Islamic Art}

In an account of Ruskin's life, the Orient, recognised as principally, and yet not exclusively, as Islamic in mien, can be said to have played an important and long-stand-

ing role. The few works dedicated to this area of Ruskin reveal their significance to his architectural thoughts, and also to his ideas on geography, nations and their

1 The name Feirefiz is derived from the Old French vais and fiz meaning 'colourful son' and whose complexion is described by Wolfram as that of a "Magpie" (Gray 1974).

2 Bassett 2008, 109.

3 Bell 2013, 263. 
histories as well as their connection to art and beauty. ${ }^{4}$ The mention of Islam, Muslims and the Orient is therefore more than a passing dalliance in his writing and in the development of his ideas. Part of this interest can be read as cohabiting with the variety of his intellectual interests, and much like them, which had enchanted his thoughts early in life, were later reviewed with a certain distance and even disapproval, and yet never truly banished from the rich intellectual imagination at his disposal. Therefore, any definitive remarks concerning Ruskin's praise or opprobrium for Islam and its sensibilities in art are premature if not occasioned upon his larger intellectual projects.

Ruskin's Victorian England and its ideas of Islam at this time can be read as a longer tradition of fascination with oriental culture. ${ }^{5}$ The popularity of the Great Exhibition of 1851 - in the form of Thomas Moore's Oriental romance Lalla Rookh, Owen Jones' exposition and lauding of Islamic architecture as optimised in the Alhambra, as well as the eroticising and licentious novella of Emily Barlow (The Lustful Turk) and the popularity of harem art etc. - all engage with an exoticised oriental preponderance. In this milieu, and within the closer intellectual orbit of Ruskin, were the fastidious works of individuals such as Robert Hay, Edward Lanes and Owen Jones and the 'Hay's Group', which forged a new intellectual interest in Islamic aesthetics and design. Their burgeoning school is what Crinson defines as a 'New Orientalism', which showed a new sense of urgency and intellectual enquiry had infected the study of Islamic architecture - a kind of parallel to the oriental renaissance of the later eighteenth and early nineteenth centuries when western scholarly knowledge of oriental languages and texts flourished. What had until then largely been either a textual study [...] now became concerned [...] with the reading of artifacts, especially architecture, and a deeper consideration of their worth for contemporary production. ${ }^{6}$

This does not preclude, however, Ruskin's disdain for the popularising of Arabian fancy and the laudation in the Hay's Group more generally and yet specifically of the Alhambra and its ornamentation, found in Owen Jones' work The Grammar of Ornament. ${ }^{7}$ As a younger man, Ruskin mentions in Praeterita, an early ambition of his was, if fate had joined him with his father's business partner in Spain, to write a history of the early Caliphs of Islam. ${ }^{8}$ Moreover, we know of his at least passing interest in the founder of the religion, Muhammad, from early letters. Having read Thomas Carlyle's lectures On Heroes, Hero-Worship, and The Heroic in History (1841), in which Carlyle identifies Muhammad "Hero as Prophet", Ruskin would remark on this depiction in a letter to his father: "read some of Carlyle's lectures. Bombast, I think; altogether approves of Mahomet, and talks like a girl of his 'black eyes'” (Atwood 2013, 247). These remarks made whilst he was still only twenty-two are perhaps expected, and yet not only would his views on Carlyle change

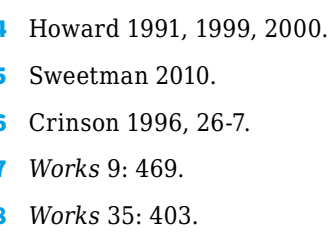


(referring to him as his "Master" and "Papa" in later correspondence), ${ }^{9}$ but also (as Atwood has shown) his ideas of the 'hero' to express a vision not too dissimilar to that of Carlyle (though not a coterminous sympathy with Muhammad). Where this early interest arose is unsure, and yet its fomenting may be recognised from literature he had read as an undergraduate, famously the Arabian Nights. In a later autobiographical reflection, Ruskin would express his views on his Protestant upbringing with reference to the work writing:

If he [the reader] will look back to what I have told of the chapter-learning, he will not find it spoken of as immediately delightful or resultful. For any effect it had on my own character hitherto, I might as well have learned the Koran in Arabic. The effect up to this time had been merely literary and imaginative, forming my taste, and securing my belief in the supernatural - or quasi-belief - gradating into the kind of credit I gave the Arabian Nights. ${ }^{10}$

Of his panoply of acquaintances, many had connections with the Muslim world. Charles Augustus Howell, for example, served as his secretary from 1865 to 1868 and had lived in Morocco, in which his chosen lifestyle in North Africa was akin to a "sheik of an Arab tribe".11 David Urquhart, a Scottish member of Parliament and Turkish Diplomat serving on a trade mission, worked closely with the court of Sultan Mahmud II, especially Koca Mustafa Reşid Pasha. Urquhart's affection for Turkish life led to a number of influential publications such as Turkey and its Resources (1833) and The Pillars of Hercules (1850), and became famed for introducing Turkish Baths in Victorian London. E.T. Cook and A. Wedderburn mention Ruskin and Urquhart at one time saw much of each other and that on the latter's encouragement, Ruskin would build a house for himself (Avcioglu 2011, 244). Max Müller, Fellow of Christ Church, and editor of the influential fifty volume Sacred Books of the East (1879-1910) was Oxford's first Professor of Comparative Philology and known to Ruskin. Ruskin also held correspondence with the prominent dramatist, Thomas Henry Hall Caine, author of Mahomet, a fouract historical sympathetic work based on the life of $\mathrm{Mu}-$ hammad, and who subsequently visited Ruskin at Coniston. ${ }^{12}$ Moreover, he knew and admired artists who had gone to the East, such as John Frederick Lewis, who had sojourned a decade in Muslim lands, producing over 600 works. ${ }^{13}$ Amongst the Pre-Raphaelite Brotherhood, William Holman Hunt travelled to the Holy Land, via Egypt as well as Sir Frederick Leighton. And whilst these examples serve not any conclusive proposition of Ruskin's own interests, for fear of an associative fallacy, such relationships serve to render the intellectual world of his operations as potentially not unfamiliar with the world of Islam. 


\section{Ruskin's Aesthetic Ideals and Islamic Art}

The fact of being amongst the first art theorists since the Renaissance to recognise, as a prototypically Christian and European expression of art, that the Gothic foments within the unlikely fulcrum of Islam, is a significant intellectual contribution. Its relevance in Ruskin's ideas of Islam oscillated between erecting strictly dialectical positions in which Europe stands on one side, to a distant appreciation of Islam's achievements. In either case, there is depth and complexity wrought in his ideas, which can be seen to oppugn their appearance often considered in the broader literature. For Ruskin, goodly work is a condition of beauty, serving its own reward by honestly attending to the natural materials, and nature more generally, in the craftsman's ward as well as the world which surrounds him. Beauty then ushers forth an embodying of truth tethered not only in the craftsman's hands but also the age of a people and the sensibilities of their conditions. ${ }^{14}$ Ideals for art and architecture, which for Ruskin were forged by a lifelong study and admiration of the Gothic, meld a philosophy of moral work and a poetics of life. In drawing on his knowledge of architectural history, Ruskin's claims for an Islamic influence on the Venetian Gothic, in the first volume of Stones of Venice, and historically locates this from around the 1180s. He claims that

the Doric and the Corinthian orders are the roots, the one of all Romanesque, massy-capitalled buildings - Norman, Lombard, Byzantine, and what else you can name of the kind; and the Corinthian of all Gothic, Early English, French, German, and Tuscan.
Now observe: those old Greeks gave the shaft; Rome gave the arch; the Arabs pointed and foliated the arch. The shaft and arch, the framework and strength of architecture, are from the race of Japheth: the spirituality and sanctity of it from Ismael, Abraham, and Shem. ${ }^{15}$

Expressing the connective-tissue between the East and West, Ruskin draws on the analogy of a "lava stream" to illustrate the outgrowth of the Arab (read Islamic) influence into the architectural vision and world view of European art:

The Arab, therefore, lay under no disadvantage in colouring, and he had all the noble elements of constructive and proportional beauty at his command: he might not imitate the sea-shell, but he could build the dome. The imitation of radiance by the variegated voussoir, the expression of the sweep of the desert by the barred red lines upon the wall, the starred in shedding of light through his vaulted roof, and all the endless fantasy of abstract line, were still in the power of his ardent and fantastic spirit. Much he achieved; and yet, in the effort of his overtaxed invention, restrained from its proper food, he made his architecture a glittering vacillation of undisciplined enchantment, and left the lustre of its edifices to wither like a startling dream, whose beauty we may indeed feel, and whose instruction we may receive, but must smile at its inconsistency. ${ }^{16}$

\section{Landsdown 2019.}

15 Works, 9: 34.

16 Works, 9: 282. See Appendix 22 in the first volume of the Stones for a further exposition on this topic, headed "Arabian Ornamentation" (Works, 9: 469). 
A little later, Ruskin will use the image of a "lava stream" to indicate where the marks and warmth of Islamic influences were handed to other forms of building: ${ }^{17}$

The Arab banished all imagination of creature form from his temples, and proclaimed from their minarets, "There is no god but God." Opposite in their character and mission, alike in their magnificence of energy, they came from the North and from the South, the glacier torrent and the lava stream: they met and contended over the wreck of the Roman empire; and the very centre of the struggle, the point of pause of both, the dead water of the opposite eddies, charged with embayed fragments of the Roman wreck, is VENICE. ${ }^{18}$

In light of these examples, Crinson argues, when Ruskin "wrote The Stones of Venice [his views] of Islamic culture was a positive one that could emphasize both its deep seriousness and its vibrancy". ${ }^{19}$ Furthermore, that there is a positive reception in the manner of the interpolation of world views with Islam with that of Europe is evidently a "fusion" or at least an "infusion of aesthet- ic modalities". ${ }^{20}$ Ruskin will further build on the typos of Islamic influences with regard to the Ducal Palace as the great meeting of the varying influences on Venice, claiming between approximately the Ninth to the Eleventh centuries Venice had adapted almost entirely the appearance of Mamluk architecture. ${ }^{21}$ This is illustrated by Ruskin in the evolution and the fluidity, for example, of pointed arch progression.

Later, the evolution of Ruskin's ideas shows how geography, morality and sentiment are influential causes in the development of art and help distinguish farther the ties between Islamic and Christian aesthetics. For example, in the third volume of the Stones he develops a theory of the five climes with each having a particular affinity to art and topography. Here Ruskin's definition of the 'Northern' and the 'Southern Savage' are useful as a means to discern respective artistic capabilities of peoples. Whilst the Northern peoples are defined by reason and the careful imitation of nature in figuration, the Southern sensibility is towards imagination and pathos. Whilst not used consistently throughout his work, especially in the first volume of the Stones, pastiches are given liberal borrowings between Byzantine

17 Analogy to the volcanic is not always positive such as in a discussion of Turner's Vesuvius (Works, 22: 13). In the Seven Lamps, and appropriately in the discussion on the "Lamp of Life", Ruskin discloses another reading of the lava stream and one in which, analogously to a nation's providence and destiny is "first bright and fierce, then languid and covered, at last advancing only by the tumbling over and over of its frozen blocks. And that last condition is a sad one to look upon [...] in Architecture more than in any other; for it, being especially dependent, as we have just said, on the warmth of the true life, is also peculiarly sensible of the hemlock cold of the false" (Works, 8: 193).

18 Works, 9: 38 (capitals in original). These thoughts resonate with a robust earlier rendition in Gibbons, which Ruskin was familiar with, namely, that "The creed of Mohammed is free from suspicion or ambiguity; and the Koran is a glorious testimony to the unity of God. The prophet of Mecca rejected the worship of idols and men, of stars and planets, on the rational principle that whatever rises must set, that whatever is born must die, that whatever is corruptible must decay and perish" (Gibbons 1993, 5: 339).

19 Crinson 1996, 53.

20 Crinson 1996, 53. In the case of Venice, this is evident further in the ways its nomenclature mirrors eastern traditions. For example, the Italian fondaco is derived from the Arabic funduq (trading post) (Howard 2002,36) and that the city itself was described by the prominent art historian Giuseppe Fiocco as a great suq (Arabic for marketplace) (Howard 1991, 59).

21 Works, 9: 41. 
and the Arab (Islamic) as defining the southern enunciation of art. ${ }^{22}$ This view is further accentuated in the fifth volume of Modern Painters $(1860)^{23}$ where he dissects the globe based on five distinct climes of which the 'sand-lands' (Muslim lands) harbour the qualities of intelligence necessary for religious art to flourish. In both accounts, the emphasis on Islamic art as 'serious' and 'sensuous' is evident. ${ }^{24}$

Ruskin would write in The Poetry of Architecture ${ }^{25}$ that the national character of a people and the offerings of beauty which they present to the world are the products of clime, terrain, temperament and morals. Such ideas, which Ruskin later develops, are certainly not unique to him, since they reoccur in nineteenth century aesthetic ruminations and have strong pre-modern antecedents. ${ }^{26}$ Of further interest is the apparent contradiction in Ruskin which pairs this aesthetic with the moral values of a people begging the question: "how could the Muslims produce beauty having been infidels, in the view of Ruskin?". ${ }^{27}$ In The Two Paths, for example, his reaction to the popularising of oriental art by Owen Jones' Grammar of Ornament ${ }^{28}$ as well as the fifth volume of Modern Painters seems to point to an answer namely, that fidelity to nature and the study of natural form marks European distinction, in whose wake, the Islamic mien opted for the fantastical, natural conventionalism and ludibrium of the imagination..$^{29}$ The problem is that disobedience to natural form is, as a consequence, corollary to laxity in the moral tenor of a people, since nature, for Ruskin, is itself a moral pedagogue. ${ }^{30}$ The natural conventionalism of Islamic art (embodied in the geometric abstraction of its ornamentation) would lead to it embracing 'mere pleasure' and persuasion to the fancies of the fantastical (thus Ruskin's critique of the

22 The close proximity of these respective visions of architecture are most commonly used by Ruskin to define Byzantine art as 'contemplative', 'mystic', 'mythical', and 'symbolic'; it had 'constancy', 'want to freedom', 'petrification', 'formalism', and 'monotony'. By contrast, Islamic was described by words such as 'exquisite', 'ardent', and 'fantastic'; it had 'excitement', 'enchantment' and 'evanescence' (Crinson 1996, 50).

23 Works, 7.

24 Crinson 1996, 49. Ruskin's sentiments towards the development of an aesthetic by virtue by a topographical medium is recorded amongst his earliest cognitions on art in fact, whilst still a first-year undergraduate at Oxford.

25 Works, 1: 11-73.

26 A case in point would be Fergusson's A History of Architecture in All Countries (1865), in whose introduction a theory of race and architecture is developed.

27 Howard 1999

28 In particular, see Lecture I, The Deteriorative Power of Conventional Art Over Nations (Works, 16: 259-92).

29 This is not unusual and serves the normative critique of the 'Muslim Mind' as one possessed by sensuality. See an early distinction between the different qualities of mind in Ruskin's distinction between 'aesthesis' and 'theoria' in Modern Painters (Works, 4: 42). One may also take the further example of his comparison of a Persian manuscript with a Turner drawing, taken from the third lecture in the Queen of the Air, in which he notes: "at this moment there is open beside me as I write, a page of Persian manuscript, wrought with wreathed azure and gold, and soft green, and violet, and ruby and scarlet, into one field of pure resplendence. It is wrought to delight the eyes only; and it does delight them; and the man who did it assuredly had eyes in his head; but not much more. It is not didactic art, but its author was happy: and it will do the good, and the harm, that mere pleasure can do" (Works, 19: 394).

30 Wilmer 2015

341 Fonti, letterature, arti e paesaggi d'Europa | Sources, Literatures, Arts \& Landscapes of Europe 1

Fonti, etterature, artie paesaggi d'Europa Sources, Lteratures, Arts
John Ruskin's Europe. A Collection of Cross-Cultural Essays, 335-350 
Alhambra as based on similar lines). ${ }^{31}$ Such arguments reveal Ruskin's contradictory thoughts on the matter; and yet, to conclude this as his abjuration of Islamic art and aesthetics would be premature.

\section{The Autochthonous Gothic and the Islamic Presence}

Ruskin's writings are seldom consistent and often open for appraisal, so too must it be with his views on Islamic art. Due to the centrality of the Gothic in his oeuvre, we see a necessary arch back to his conceptions of beauty and truth, rendering Islamic art as partial progenitor of a style and descendant of those ideas in the Gothic. This rapprochement is of central importance since it may be argued, as it shall be here, that at least some Islamic ideals which give birth to the great Gothic styles are conceived by terms acceptable to Ruskin's own thought. This may better be surmised as not an associative fallacy but rather an elective affinity to those ideas, praised in Ruskin, and found on the common ground of architectural practice and thought. As such, these ideals are themselves to be found, in one fair way, within Ruskin's own discourses on the Gothic. Whilst this may seem platitudeness, due to the aforementioned lauding of Gothic origins, they presume also a rethinking of the place of Islam within Ruskin's oeuvre. It is, building upon the work of Deborah Howard and others, to argue that there is a complexity to which one must turn in handling Ruskin's views and cannot be limited to the advance of political ideals and intellectual expectations anticipated in his latter oeuvre. It is for this reason that one can measure this parlance, pentimento like, in the articulation of his own rendering of the most noble aesthetic ideas to be found in The Seven Lamps of Architecture and the Nature of Gothic. ${ }^{32}$ Again, this is not sur- prising due to the abiogenesis of the Gothic, and yet the connections one may make with Islamic architecture make the less inconspicuous case for a larger treatment of Islam in his thinking.

In the Seven Lamps, for example, and in the lamp of "Sacrifice" particularly, the dedication of craft to a supreme being, reflects equally in man's love and obedience to that being. Islamic architecture is defined by Ruskin as an oblation to God, however misinformed it may be in his eyes, nonetheless summoning a deity to which these labours are directed. Similarly, the lamp of "Truth" inheres the dedication to an honesty towards materials and structure and that of "Power" namely, inspiring awe in reaching the sublimity of nature, as allegorised in stone. One may allude to the references in Ruskin's treatment of the Ducal Palace as an appropriate example of this. Here, the grandeur and splendour of the building sits as a mirage of power and truth and can be found in Ruskin's lauding of examples of dressed stone from Mamluk (Cairo) buildings. Again, the lamp of "Beauty" explicates that the beautiful resonates in due understanding of nature and natural forms, which in itself is the lauding of the Divine, in the labours of nature. Speaking of the balcony at Campo San Benedetto (Venice), for example, we may allude to Ruskin's use of the Cinquecento arabesque in this period as illustrative of this lamp. He continues:

31 Due to this inherent climatic pathos, the seriousness of art leads to a inescapable cruelty of a people and "cruel nations" for Ruskin were products of "an inheritance of ignorance and cruelty, belonging to men as spots to the tiger or hues to the snake" (Works, 16: 307).

32 Works, 8 and 10 
It is but the arresting upon the stonework of a stem or two of the living flowers, which are rarely wanting in the window above (and which, by the by, the French and Italian peasantry often trellis with exquisite taste about their casements). This arabesque, relieved as it is in darkness from the white stone by the stain of time, is surely both beautiful and pure; and as long as the renaissance ornament remained in such forms it may be beheld with unreserved admiration. ${ }^{33}$

In the exhortation of the lamp of "Life", we may point to Ruskin's description of Basilica di San Marco, as a point of reference of this principle and within which he points out the arabesque as amongst its most lovely features,

Those columns of the principal entrance are among the loveliest in Italy; cylindrical, and decorated with a rich arabesque of sculptured foliage, which at the base extends nearly all round them, up to the black pilaster in which they are lightly engaged. ${ }^{34}$

In the lamp of "Memory" and his discussion of the domicile and its rightful permanence in the built environment, he criticises modern tenements and their restrictions and compares them alternatively to the natural

tents of the Arab or the Gipsy by their less healthy openness to the air of heaven, and less happy choice of their spot of earth; by their sacrifice of liberty without the gain of rest, and of stability without the luxury of change. ${ }^{35}$

From the Nature of Gothic, we may further draw out similar themes relating back to Ruskin's high demands on architecture as found in the workings of the Southern Savage mind of the Muslim. Of these, "Savageness", for example, offers the primal and unrestrained mien which in Ruskin's works features the subtilty of soul and gentility of labouring which renders the capacities of life fecund for the character of our built world. We may add further that for Ruskin savageness captures an aptitude of thought inclined to the "south" and to be found in the works and thoughts of Muslim nations namely, their untamed and approximate closeness to nature as evident. ${ }^{36}$ He starts with and appropriates the "southern savage gothic" as the mise en scène for the Gothic. Identifying this savage with the "necessity for invention, its purposeful unfinished quality and its revulsion to mere imitation as three defining features". ${ }^{37}$ The art of "Changefulness" in the design of craftsmen can be elicited from his choice of "Arabian Windows" taken from the Campo Santa Maria Mater Domini, where Ruskin takes account of it being "one of the richest fragments in the city: and a beautiful example of the fantastic arches which I believe to have been borrowed from the Arabs". ${ }^{38}$ Curious is how the element of changefulness is exacted in the very medium of this transitional moment in the Venetian Gothic. In describing its façade, Ruskin writes "two of the circular ornaments at the points of the arches are larg-

\footnotetext{
3 Works, 8: 75.

4 Works, 8: 202

Works, 8: 227

Connelly 2015.

Works, 17: 196-7.

Works, 11: 320.
}

343 \begin{tabular}{l|l} 
Fonti, letterature, arti e paesaggi d'Europa | Sources, Literatures, Arts \& Landscapes of Europe 1 \\
John Ruskin's Europe. A Collection of Cross-Cultural Essays, 335-350
\end{tabular} 
er than the rest; that the lateral windows are broader than the three intermediate ones; and that, of the lateral windows themselves, the one on the right is broader than that on the left". ${ }^{39}$ And that this curious lack of regimentation in the exterior is a hallmark of the "Arabic period" since he further argues:

the same thing takes place - one of the lateral openings is larger than all the rest; and I have not as yet been able to discover the reason for such an arrangement, as these groups of windows appear to have always lighted one room only. ${ }^{40}$

As for the principle of Naturalism, Ruskin opens a concomitant discussion on capitals and the construction of the bell on columns. This he does through observing the relative labours of truncation in capital formation by positing stability and refined proportions, when met with the shaft, by starting the masons cut further down with a deeper incision to harness their gradation. This middle ground between the over and under cutting which may be acute in both concave or convex and concludes that

the actual form of the capitals of the balustrades of St. Mark's: it is the root of all the Byzantine Arab capitals, and of all the most beautiful capitals in the world, whose function is to express lightness. ${ }^{41}$
"Grotesque" is the attenuation of the imagination to "delight in fantastic and ludicrous [...] images" ${ }^{\prime 2}$ as a mark of the Gothic. It reveals a certain freedom and flight from the conventions of normative refined ornament upon dressed stone exteriors whose potential for an expressive commune with the work of the craftsmen, is reminiscent of the fantastical in Islamic building. ${ }^{43}$ "Rigidity", at the other end, is an activity wrought in the dynamics of the buildings whose "peculiar energy [...] gives tension to movement, and stiffness to resistance, which makes the fiercest lightning forked rather than curved". ${ }^{44}$ Whilst making a clear distinction between the Northern and Southern tribes which accentuate the relative traditions of rigidity in architecture, the place given to Gothic stands as a hallmark for its temper of character which resists the authority of the horde and the individual against the presence of time. This Ruskin speaks in contrast to the "languid submission" of the south. ${ }^{45} \mathrm{Fi}$ nally, as for "Redundance" it is the "uncalculating bestowal of the wealth of its labours" such that in the accumulation of architectural facts a tapestry of labour is emergent, pentimento like, for the eyes of the observer. ${ }^{46}$ As Ruskin argues:

And although, by careful study of the school, it is possible to arrive at a condition of taste which shall be better contented by a few perfect lines than by

40 Works, 11: 320. In this regard the discussion of the horseshoe round, or as Ruskin names it the 'Arabic and Moorish arch' is relevant to its appearance as modulation its ability to be modulated as thus found in the 'Early English and French' Gothics (IX. 161).

41 Works, 11: 139

42 Works, 10: 239.

43 Crinson 1996.

44 Works, 10: 239

45 Works, 10: 241.

46 Works, 10: 243.

Fonti, letterature, arti e paesaggi d'Europa | Sources, Literatures, Arts \& Landscapes of Europe $1 \mid \quad 34$ 
a whole facade covered with fretwork, the building which only satisfies such a taste is not to be considered the best. ${ }^{47}$

Therefore, in the managing of the eye and articulating of its sharpness,

we cannot say, therefore, that a building is either Gothic or not Gothic in form, any more than we can in spirit. We can only say that it is more or less Gothic, in proportion to the number of Gothic forms which it unites. ${ }^{48}$

Herein lies the rub namely, that the Gothic is itself the product of a consilient tradition which is defined in Rus- kin through, or in opposition to, its providence(s). The examples shown to illustrate this point offer only a propaedeutic account of how these ideas bear presence for Ruskin. As Ogden argues, such complexity in the articulation of the Gothic is perhaps a consequence of his broader familiarity with Islam that as a thinker he is "unfamiliar to Victorian and post-colonial scholars alike, a Ruskin who both defines "Gothic" in racially chauvinistic terms as well as, paradoxically, invokes Arab architectural influences as an endless illustration of the best of Venetian Gothic". ${ }^{49}$ Again, this is not surprising, given the place of Islamic architecture in the development of the Gothic for Ruskin and serves to ensure that perhaps there is a sustained and unfinished quality to his ideas on the matter.

\section{Remnants of the Islamic 'Lava Stream' in Ruskin and the 'Eastern Question'}

As with many matters in his works, it is clear how and exactly why Ruskin's views on Islam and Muslims changed over time. The need to differentiate from the Southern Savage, the vituperative remarks on the Indian Mutiny (1856), Ruskin's comments in The Two Paths as well as the inaugural address as Slade Professor (1870) all famously exhort the imperial project and thus exculpate England's yoke over lesser nations and are perhaps not the final word on the matter. Critiques by post-colonial scholars such as Edward Said, ${ }^{50}$ which would paint Ruskin as a muse for the colonial project, are set, it seems, to a premature tone; perhaps this is only a partial corrective in designating him 'colonialist'. At the other end, to give an example, one must question the extent to which a national project, or European vision, within Ruskin is itself tenable. As Stoddart has recently done convincingly, analysing his thoughts on such claims, concludes that "Ruskin is perhaps the sharpest critic of his own deployment of nationality as a corrective to the problems of modern social formations". ${ }^{51}$ Subsequently, later in life there is a partial and potentially significant desire to cogitate upon the East, as in November 1876, when Ruskin writes in Fors Clavigera of his intentions to pen a work on the "Eastern Question" and in particular on the religion, history and politics of the Islamic Orient:

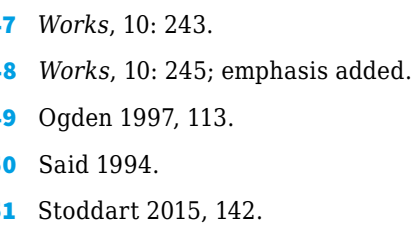


I want to write a long note in Byzantine empire - Commanders of the Faithful, - Gran Turks - and the 'Eastern Question'. But can't: and perhaps the reader will be thankful'.52

Left as a musing, as his earlier intentions to pen the history of the first Muslim Caliphs, what would have been made at this juncture in his career as an arch back to the Orient, is left to historical conjuncture. Specifically, from the 1870s, the advent of Ottoman reforms, British involvement in Egypt and the dissolution of the East Indian Company may all have been grist for Ruskin's mill. Equally, such events may have changed and called into question his own reticence with the European industrial world, slavery, religion and the state of the Northern Savages condition. There seems to be no place for anything in Ruskin, especially the later works, which would however encourage a movement towards the place of a wholly congenial relation to Islam. His retreat into the North, figuratively and temporally, cleft upon the hilltops and mountainous storms, is where he felt most comfortable in the end, in the retreat from convictions of his youth and ultimately the retreat of his reason. However, it is of note that references to Islam do not cease in his later works and correspondence. Writing in St Mark's Rest (1879), for example, he argues

A marvellous thing - the Protestant mind! Don't think I speak as a Roman Catholic, good reader: I am a mere wandering Arab, if that will less alarm you, seeking but my cup of cold water in the desert; and I speak only as an Arab, or an Indian, - with faint hope of ever seeing the ghost of Laughing Water. ${ }^{53}$

As Francis O'Gorman has shown, Ruskin's religious proclivities serve as no "easy labels for his theological position[s]" during this period.$^{54}$ Later Islamic references are equally evident such as in his daily musings. For example, one such appears in a letter to Mrs. Arthur Severn Llangollen on October 15, 1883:

Just came in from the most delicious walk I ever had in England or Wales. Never saw anything like the beauty of the valley between wavy hills of pasture gilded with Fern like an Arabian book - romance or Koran - broidery of gold on silk. No heath! - all grass, crag, fern and divinest woods and fields below, and Valle Crucis with its Cross and Abbey and lateral brook. Birds everywhere - and I've seen two water ouzels! Off at 12 for Oxford! ${ }^{55}$

Commenting on these references offers, if anything, the dexterity of a fecund mind whose resources were drawn from a great well of diverse ideas. It may also, and not without the presence of a Southern Savage, the noble Arab, show the dwelling of serious art and its lasting impressions on Ruskin. In the latter published oeuvre (The Bible of Amiens, published between 1880 and 1885),

52 Works, 38: 738. The time of this writing was an especially tumultuous period in his life with Rose La Touche dying the previous year and he himself resigning the Slade Professorship just three years later. What these thoughts would have culminated in is unclear though using his writings on colonial Britain we may not be surprised to hear more of the same though with greater intensity of spirit. It is also important to note that Ruskin's cogitations on related matters may have been the basis for his Eastern Question. For example, whether it may have been similar in candor to his series of letters entitled The Italian Question (Works, 36: 319) is uncertain. For a nuanced view on his ideas of empire see Faulkner 2000.

53 Works, 24: 277-8; emphasis added. The reference to "Laughing Water" is from Longfellow's The Song of Hiawatha.

54 O'Gorman 2015, 152

55 Works, 37: 468-9; emphasis added 
which was intended to draw further on religious history and architecture and stands as an important addition to the articulation of the thinkers' thoughts, Ruskin writes, with reference to the Southern Savage and his simplicity:

The influence of Egypt vanishes soon after the fourth century, while that of Arabia, powerful from the beginning, rises in the sixth into an empire whose end we have not seen. And you may most rightly conceive the religious principle which is the base of that empire, by remembering, that while the Jews forfeited their prophetic power by taking up the profession of usury over the whole earth, the Arabs returned to the simplicity of prophecy in its beginning by the well of Hagar, and are not opponents to Christianity; but only to the faults or follies of Christians. They keep still their faith in the one God who spoke to Abraham their father; and are His children in that simplicity, far more truly than the nominal Christians who lived, and live, only to dispute in vociferous council, or in frantic schism, the relations of the Father, the Son, and the Holy Ghost. ${ }^{56}$

This chastising of the Protestant traditions of his time, to which he himself was a product and devoted for much of his life, seems apt at a time in which he is weary from Carlyle's demise and walking into another mental breakdown the following year. ${ }^{57}$ His religion becomes that of
Saint Ursula, his Gothic that of the North, his land that of Scotland's gelid hills. In disregarding so much he becomes a martyr of the redemption he himself sought in art. However, and curiously, the Arts and Crafts Movement, much of which he inspired, did not resist in the same way and rather, unlike Ruskin himself, would tread about the southern soils of the imagination, opening the door, left ajar by Ruskin, to the world of Islam and the Orient. This influence of Islamic art has yet to be written into the broader history of the movement, though it is evident that a sustained and reverent relation existed. We may draw on as examples of William Morris and his passion for Persian design and love of oriental literature, the Pre-Raphaelite visual obsession with the Islamic world; the typographer Emery Walker's North African, Islamic art collection; the calligrapher Edward Johnston's student, John C. Tarr, who lived in North Africa and greatly admired Islamic artistic culture; the rise of poly-chromaticism in Neo-Gothic design; Edwin Landseer Lutyens Mughal-inspired gardens etc. The alacrity with which these ideas were taken hold a decisive turn in the wake of Ruskin's legacy. As Ogden has shown,

on the latter half of the 19th century [...] Particularly in what the Victorians called the Near East and over territory historically occupied by Arabs (especially Egypt), English architects and builders partially re-

56 Works, 33: 95-6; emphasis added.

57 As was his custom throughout his life, and especially during the latter period, Ruskin wanted to donate precious manuscripts, paintings, geological materials and miscellany to charitable causes as well as friends. He is recorded to have donated at least (of which we know) of his Arabic Quran manuscripts. One such given to the Whiteland's College on Christmas Day (1880) having been described as 'very interesting' and bound in "silken satchel with a gold cord, every page being profusely ornamented in flower scrolls and gold" (Works, 30: 339). The second was also an illuminated manuscript addressed to Rev. J.P. Faunthorpe on December 6, 1881 stating: "The other [book for donation] is - I don't know what, for I can't read it, and don't know even its right way upwards! So I am ashamed to have it among my books any more, but I think with its pretty silken cover, binding and all, it is just the thing to show your girls what sort of a thing a Book should be!" (Works, 37: 381). For Ruskin's inscription in the latter see Works, 38: 426. This of course does not elide those remarks from Ruskin which he makes, of the supercilious and invective variety during this same period. In a letter to Charles Eliot Norton, for example, on February 10, 1863 Ruskin addresses the extremities of battle found in the enemy Civil War in which Norton had been working for the Loyal Publication Society, and compares them with the founder of Islam, writing "Mahomet was mild, Christian-like and rational, in comparison" (Works, 36: 432).

$347 \quad$ Fonti, letterature, arti e paesaggi d'Europa | Sources, Literatures, Arts \& Landscapes of Europe 1 
shaped Imperial Britain into an Orientalized Italy. Just as in medieval Venice, Arab styles proliferated. ${ }^{58}$

It is due to his compendious interests, to which we ought to give a broad concession in judging Ruskin and his ideas of Islam and the making of European ideals. It seems that one must and in so doing, we do not close the chapter on Islam in his thought but rather necessitate the need for further nuance. As Ruskin writes of the intellectual labours of thought in Arrows of the Chace (1880),

in the building of a large book, there are always places where an indulged diffuseness weakens the fancy, and prolonged strain subdues the energy: when we have time to say all we wish, we usually wish to say more than enough; and there are few subjects we can have the pride of exhausting, without wearying the listener. ${ }^{59}$
It is a fitting epithet for Ruskin's own oeuvre (and life), that ceaseless fecundity of ideas to animate and stir his listeners did also dissipate his own energy. As for those "listeners" of Ruskin, our present account evidently says little enough to "weary the listener" on the matter, and yet offers the importance of drawing out these ideas further in evaluating his work. As with Ruskin's other and many discourses battling for attention, we cannot settle on Islam's place in that panoply as taking much room, yet more perhaps than it is often accorded. Concluding his thoughts then on Islam, amidst paradox and polemic, we may mediate that they are neither a paean, as in its place in the development of an entirely wholly European expression, nor do they abnegate its place therein. Ruskin's thoughts are a concatenation wherein the Northern Savage ultimately succeeds where he and his mind would eventually find rest. 


\section{Bibliography of Works by John Ruskin}

References are to volume and page numbers in:

Cook, E.T.; Wedderburn, A. (eds) (1903-1912). The Works of John Ruskin. Library Edition, 39 vols. London: George Allen.

https://www.lancaster.ac.uk/the-ruskin/the-complete-works-of-ruskin/.

Works, 1: Early Prose Writings. |3-7: Modern Painters I-V. | 8: The Seven Lamps of Architecture. | 9-11: The Stones of Venice I-III. | 16: "A Joy For Ever", The Two Paths, Letters on The Oxford Museum and various addresses (1856-1860). | 17: Unto this Last, Munera Pulveris, Time and Tide, Other Writings on Political Economy (1860-1873). | 19: The Cestus of Aglaia, The Queen of the Air, Other Papers and Lectures on Art and Literature. |22: Lectures on Landscape, Michael Angelo \& Tintoret, The Eagle's Nest, Ariadne Florentina. |24: Giotto and His Works in Padua, The Cavalli Monuments, Verona, Guide to the Academy, Venice, St Mark's Rest. | 30: The Guild and Museum of St. George. |33: The Bible of Amiens, Valle Crucis, The Art of England, The Pleasures of England. | 34: The Storm-cloud of the Nineteenth Century, On the Old Road, Arrows of the chace, Ruskiniana. |35: Praeterita, Dilecta. |36-37: The Letters of John Ruskin I-II. | 38: Bibliography, Catalogue of Ruskin's Drawings, Addenda et Corrigenda.

\section{General Bibliography}

Allen, V. (1997). Hall Caine: Portrait of a Victorian Romance. Sheffield: Sheffield Academic Press. Avcioglu, N. (2011). Turquerie and the Politics of Representation, 1728-1876. London: Routledge.

Atwood, S. (2013). “'Leading Human Souls to What is Best'. Carlyle, Ruskin, and Hero-Worship”. Sorensen, D.R.; Kinser, E.B. (eds), On Heroes, Hero-Worship, and the Heroic in History: Thomas Carlyle. New Haven: Yale University Press, 247-59.

Bassett, P. (2008). Wagner's Parsifal: The Journey of a Soul. Kent Town: Wakefield Press.

Bell, H.R. (2013). Wagner's Parsifal: An Appreciation in the Light of His Theological Journey. Eugene (OR): Cascade Books.

Connelly, S.F. (2015). "John Ruskin and the Savage Gothic”. Journal of Art Historiography, 12(12), 1-16.

Crinson, M. (1996). Empire Building: Orientalism and Victorian Architecture. London: Routledge.

Faulkner, P. (2000). "Ruskin and the British Empire". Journal of the William Morris Society, 14(1), 54-66.

Fergusson, J. (1865). A History of Architecture in All Countries. Oxford: Oxford University Press.

Gibbons, E. (1993). The Decline and Fall of the Roman Empire. 6 vols. London: Everyman's Library.

Gray, C.Jr. (1974). "The Symbolic Role of Wolfram's Feirefiz". The Journal of English and Germanic Philology, 73(3), 363-74.

Howard, D. (1991). "Venice and Islam in the Middle Ages: Some Observations on the Question of Architectural Influence". Architectural History, 34, 59-74.

Howard, D. (1999). "Ruskin and the East". Architectural Heritage Society of Scotland, 10(1), 37-53.

Howard, D. (2000). Venice \& the East: The Impact of the Islamic World on Venetian Architecture 1100-1500. New Haven, Yale University Press.

Howard, D. (2002). The Architectural History of Venice. New Haven. Yale University Press.

Jones, O. (2008). The Grammar of Ornament. London: A \& C Black Publishers.

Lansdown, R (ed.) (2019). John Ruskin: Selected Prose. Oxford: Oxford University Press.

O'Gorman, F. (2015). "Religion”. The Cambridge Companion to John Ruskin. Cambridge: Cambridge University Press, $144-56$.

Ogden, D. (1997). “The Architecture of Empire: 'Oriental' Gothic and the Problem of British Identity in Ruskin's Venice”. Victorian Literature and Culture, 25(1), 109-20.

Said, E. (1994). Culture and Imperialism. London: Vintage.

Scruton, R. (2020). Wagner's Parsifal: The Music of Redemption. London: Allen Lane. 
Mujadad Zaman Ruskin's Islamic Orient and the Formation of a European Ideal

Stoddart, J. (2015). "Nation and Class". O’Gorman, F (ed.), The Cambridge Companion to John Ruskin. Cambridge: Cambridge University Press, 130-43.

Sweetman, J. (2010). The Oriental Obsession. Cambridge: Cambridge University Press.

Wilmer, C. (2015). “Creativity”. O'Gorman, F. (ed.), The Cambridge Companion to John Ruskin. Cambridge: Cambridge University Press, $230-46$. 


\title{
John Ruskin and Kenji Miyazawa An Idea of Nomin-Geijutsu (Peasant Art) and its European Legacy
}

Yasuo Kawabata

Japan Women's University Tokyo, Japan

\begin{abstract}
My paper concerns an aspect of Ruskin's influence on modern Japan, focusing on the life and work of Kenji Miyazawa (1896-1933), poet and author of children's stories, who organised Rasu Chijin Kyōkai (Rasu Farmers Association) in the poverty-stricken farming communities of northern Japan, Hanamaki, Iwate prefecture, in 1926. The association was arguably influenced by Ruskin's Guild of St George, as well as by his commitment to the Working Men's College. Ruskin's work had been introduced into Japan during the Taishō Era (1912-1926), with quite a few works translated into Japanese, including Unto This Last and Modern Painters.
\end{abstract}

Keywords Kenji Miyazawa. Nōmin-Geijutsu (Peasant Art). Rasu Chijin Kyōkai (Rasu Farmers Association). Taishō Era. Modern Japan. John Ruskin. Guild of St George. Working Men's College.

Summary 1 The Foundation of Rasu Chijin Kyōkai (Rasu Farmers Association). - 2 The Draft of Nömin-geijutsu Gairon Köyō (An Outline Survey of Peasant Art). - 3 The Problematics of the Japanese Term "Geijutsu". - 4 How Miyazawa Inherited Ruskinian Legacy.

\section{The Foundation of Rasu Chijin Kyōkai (Rasu Farmers Association)}

The title of my paper might be considered, I fear, out of place of the general theme of "A Great Community: John Ruskin's Europe". In a way it is, as I focus on a Japanese poet, but I intend to show you some aspect of Ruskin and the European Legacy from my own circuitous route. I will start by giving a short biography of my protagonist, Kenji Miyazawa (18961933), who was one of the most popular poets and fantasy writers in Modern Japan, though his present fame is in stark contrast to his obscurity while he lived. 
Figure 1 Unknown photographer, Portrait of Kenji Miyazawa (1896-1933). Late 1920s. Kamakura Museum of Literature archives. (c) Wikimedia Commons

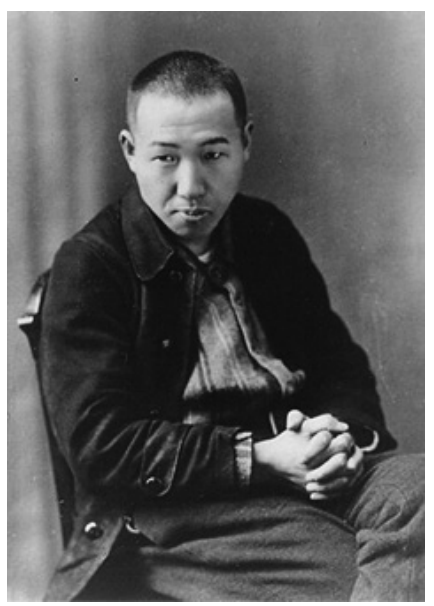

Born in 1896 in the small town of Hanamaki in Iwate Prefecture, the north-eastern part of mainland Japan (Tōhoku-chihō), a district once called "the Tibet of Japan" for its long, cold winters and grudging soil, he spent most of his life in his native countryside. He published only two books in his lifetime: a collection of children's tales entitled Chumon no Oi Ryori-ten (The Restaurant of Many Orders), and the first section of his most famous work of poetry, Haru to Shura (Spring and Ashura). He left voluminous manuscripts of children's tales, fairy tales and poems behind, including his masterpiece Ginga Tetsudō no Yoru (Night on the Milky Way Railway), most of which were published posthumously. Besides literary works, his many-sided activities included a high-school teacher, an agricultural engineer, an activist of the Nichiren Buddhist sect, an Esperantist, as well as a garden designer, a typical example of which is the "Tearful Eye" flower bed designed by Miyazawa in 1927, and he was a social reformer, advocating Nomin-Geijutsu (peasant art) to create a new kind of farming community. My paper mainly concerns the latter facet of Miyazawa as a social reformer, though we should not forget that it is closely connected with other activities. After graduating from Hanamaki Nōrin-Gakkō (Hanamaki Agricultural and Forestry School), Miyaza- wa became a teacher at Hanamaki Nō-Gakkō (Hanamaki Agricultural School) in 1921. Taking the job, he developed close relationships with the farming community. He taught a new class called "The Art of Farming" through which he established friendly connections with the local boys who came as students. Subjects of the class he was in charge of were algebra, English, chemistry, agricultural production, agronomy, soil fertility and meteorology. He was also given an experimental paddy, a small field for rice cultivation.

Though he enjoyed teaching at the school, Miyazawa left the position in 1926 to establish Rasu Chijin Kyōkai (Rasu Farmers Association). He declared then his intention of becoming a paesant himself, which was regarded by his parents and local inhabitants as one of his eccentricities, as he was the eldest son of the wealthiest family in the town, and this change of profession was very unexpected. His actions proved, however, that he was in earnest. At a riverside cottage owned by his family in Shimoneko, where he was staying at the time, Miyazawa gathered together a group of young people from farming families in the community and lectured to them on agronomy and other subjects. The association also engaged in plays, music, and other cultural activities. Nearby were the fields he cultivated during this period of his life. 


\section{The Draft of Nōmin-geijutsu Gairon Kōyō (An Outline Survey of Peasant Art)}

It was probably shortly before he began his new life that Miyazawa wrote the final draft of "Nōmin-geijutsu Gairon Kōyō" (An Outline Survey of Peasant Art), in which he envisioned peasant art as it might be in the future, contrasting his ideal with the present state of general unhappiness of ordinary peasants. It was, as Mallory Fromm, scholar of Miyazawa, explains: "a statement of his mature inner vision written in a sort of shorthand that reflects the urgency and excitement of the labours he was shortly to commence". ${ }^{1}$ Hence the general obscurities of the text.

The name "Rasu" of Rasu Chijin Kyōkai is such an unfamiliar term in Japanese that a number of explanations have been proposed as to its meaning. Perhaps it came from English "rustic", or just the reverse of "(a)sura", a demon god of Indian or Buddhist mythology. One of the tenable etymologies is that it might be derived from the name "Ruskin", as a critic Itsuo Onda first suggested. ${ }^{2}$ Although Miyazawa does not actually mention Ruskin in the text, Katsumi Itō, his pupil and a member of the association, recalled later that Miyazawa stressed the importance of Ruskin in the class. ${ }^{3}$ This reference seems plausible as we can find definite connections between Miyazawa and Ruskin through William Morris, whose name Miyazawa does mention several times in his "memo" for "A Revival of Peasant Art". The association was arguably influenced by Ruskin's Guild of St George, as well by his earlier commitment to the Working Men's College.

As a matter of fact, in the early decades of the twentieth century, Ruskin and Morris were, along with Tolstoj, Kropotkin, Edward Carpenter, highly valued by Japanese intellectuals as the most important Western thinkers in the modern era. During the first quarter of the century alone, over twenty works by Ruskin were translated into Japanese, including Modern Painters, Sesame and Lilies, Unto This Last, to mention but a few.

As for Morris, the first Japanese version of News from Nowhere, entitled Risō-kyō (An Ideal Place), was published in abridged form in 1904 by Heiminsha, the Society of Commoners (translated by Toshihiko Sakai, one of the Japanese pioneer socialists), which was followed by Morris's principal lectures on art and society: "Decorative (Lesser) Art", "Art of People", "Beauty of Life", "Useful Works vs. Useless Toils", "Art, Wealth, Riches", "Art and Socialism", and so on. It is not too much to say that Japanese versions of Ruskin and Morris flooded in during the period.

In "The Revival of Peasant Art", Miyazawa wrote:

Our ancestors, though poor, lived quite happily.

They possessed both Art and religion.

Today we have only work and existence.

Religion, now enervated, has been replaced by modern science, yet science is cold and dark.

Art has departed our midst and become wretchedly decadent.

Those we now call people of religion and Art monopolize and sell Truth, Goodness, and Beauty.

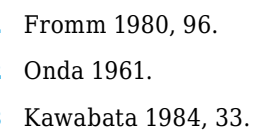


We lack both the strength and the necessity to purchase.

We must now walk a true, new path and create our own Beauty!

Burn away grey labour with Art! ${ }^{4}$

In the first two lines, we can notice a kind of Medievalism in Miyazawa's mind. He added some comments for the last line ("Burn away grey labour with Art!") in his memo: "The revival of Art must be the revival of joy in labour. / Morris, 'Art is man's expression of his joy in Labour'". ${ }^{5}$ Morris was probably brought to Miyazawa's attention by Hisao Honma, critic and scholar of
English Literature, who published Seikatsu no Geijutsu-ka (Making Life Art), a biography of Morris. ${ }^{6}$ However, reading Miyazawa's summary of Morris's concept of an ideal community where art is revived, there is no doubt that he read News from Nowhere as well as some of Morris's lectures in Japanese translation.

\section{The Problematics of the Japanese Term 'Geijutsu'}

It should be noted here that there is a certain difference in nuances between the word 'art' and the Japanese term 'geijutsu', though they are generally considered to be equivalent words, the latter being a translation word (hon'yaku-go) like 'minshu-shugi' (democracy), 'bunka' (culture), 'jiyū' (liberty), 'ren'ai' (love), 'bi' (beauty), or even the pronouns of 'kare' (he), 'kanojo' (she), and 'karera' (they). ${ }^{7}$ A Japanese encyclopaedia defines 'geijutsu' as "a general term given to one of the particularly human activities to create original value". ${ }^{8}$ When the Japanese people hear the word 'geijutsu', they usually associate it with distinctive forms of 'art' apart from our ordinary life. From the start, the word 'geijutsu' has gained a rather exclusive, high-class, quality: 'the Art' or 'the fine arts' in its most modern sense of the word, denoting special skill or works of painting, en- graving, or sculpture. It is due to the particular ideology of the people in the early Meiji era (in the late nineteenth century), who tried to introduce the concept of 'art' in such a sense into Japan, discarding instead older, more general, connotations it had. In short, 'geijutsu' was conceived as something remote from the daily life of common Japanese people including, of course, peasantry, that is over the half of the Japanese population in the 1920s. No one in pre-modern Japan certainly would have used the word with such an implication.

On the other hand, as we have just seen, 'art' had become increasingly alien word for working class people in Britain after the Industrial Revolution. Ruskin and Morris are significant in criticizing the situation in which 'art' was monopolized by the well-to-do. When Morris, inspired by Ruskin, insisted on the importance

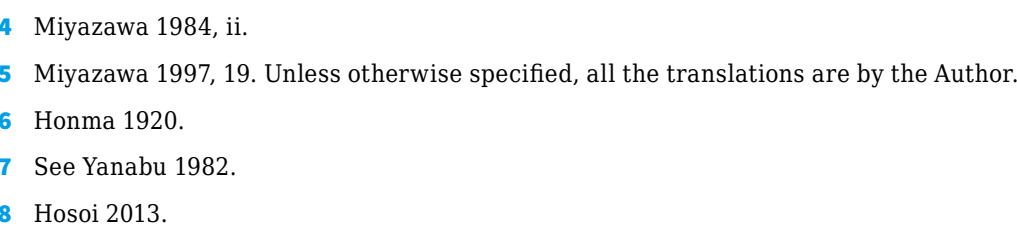


of "lesser arts" or "decorative arts", he tried to stop such a monopoly. He declares, "I do not want art for a few, any more than education for a few, or freedom for a few". ${ }^{9}$ In the same lecture, Morris points out that the love of luxury and show has ruined the arts since the Renaissance. Therefore, he says we must return to the traditional yeoman's house and the humble village church for the best of English decorative art, which is "never coarse, though often rude enough, sweet, natural, and unaffected, an art of peasants rather than of merchant princes of courtiers".${ }^{10}$ He goes on to say:

A peasant art, I say, and it clung fast to the life of the people, and still lived among the cottagers and yeomen in many parts of the country while [...] stupid pomp had extinguished all nature and freedom, and art was become [...] the mere expression of that successful and exultant rascality which in the flesh no long time afterwards went down into the pit for ever. ${ }^{11}$
It is precisely this emphasis on peasant crafts that determined the whole direction of the later Arts and Crafts movement. It was a call for a return to functional simplicity in "the arts of life", and Morris certainly believed that this would follow from substituting production for use for production for profit. ${ }^{12}$ In other lecture on "Art and Labour" (1884), he proposes to take the word 'art' in a wider sense:

[B]y art, I do not mean only pictures and sculpture, nor only these and architecture, that is beautiful building properly ornamented; these are only a portion of art, which comprises, as I understand the word a great deal more; beauty produced by the labour of man both mental and bodily, the expression for the interest man takes in the life of man upon the earth with all its surroundings, in other words the human pleasure of life is what I mean by art..$^{13}$

\footnotetext{
9 Morris 1914, 26.

10 Morris 1914, 18.

11 Morris 1914, 18.

12 Henderson 1967, 200.

13 Morris 1965, 94-5.
} 


\section{How Miyazawa Inherited Ruskinian Legacy}

In Miyazawa's project for Rasu Chijin Kyōkai, we can discern definite influences of Ruskin and Morris. There are similarities between Miyazawa and the people engaged in the Arts and Crafts movement. Miyazawa, too, did not want art for the few. "What does the word 'artist' mean to us?", he asks in "Creators of Peasant Art", a section of An Outline Survey of Peasant Art:

Professional artists will one day cease to exist.

Let everyone adopt an artist-like sensibility.

Ceaselessly express yourself along the lines of your individual genius.

We are, each of us, artists at one time or another. ${ }^{14}$

Indeed, he found frequent occasions when the ordinary life could be transformed into art, as seen in his poem

entitled Dai-san Geijutsu (The Third Art):

When I was furrowing the field

I found a little man with grey hair

standing behind me.

"What d'you intend to sow?" he asked me.

"I will sow seeds of red turnip", answered I.

"Never furrow like that, then", he said,

and he showed me the way himself, furrowing the plot obliquely.

I stood still, moved, entirely enchanted.

The sun shone and the wind blew,

the shadows of the two on the sand,

the river glittering over there.

I was almost in ecstasy, thinking, "what kind of Indian-ink drawings or flavour of a sculptor's chisel could

[surpass this!"15

The sole qualification for membership of Rasu Chijin Kyōkai was a connection, even if only tenuous, with farming or 'soil' (hence the word 'chijin' or 'men of the earth'). There were no fees or dues. Everything needed by the organization was supplied by Miyazawa. About twenty young men joined. The majority were ex-pupils of Miyazawa from Hanamaki High School of Agriculture, and students who had attended his lecture series at Iwate Na- 
tional High School. A few members were from Morioka, the biggest city in the area. The association's activities were at first only a few, but gradually they increased. A small 'orchestra' of six or seven youths was formed by Miyazawa, who sold some of his books to purchase the instruments. He held record concerts and story readings at the association on Saturday afternoons and evenings for whoever came, usually the local children.

Its activities were short-lived - only two years - as his physical condition declined during the period, and he died of a lung disease in 1933 at the age of $37 . \mathrm{Nev}$ ertheless, his experiments of Nomin-geijutsu, as well as his theory expressed in "The Outline Survey", were very unique and valuable, and still deserve close attention.

The years from 1910 through to the late 1920s, which include the Taishō period (1912-1925) and cover the period Miyazawa worked, were among the most crucial periods in modern Japanese history. Since the 1860s, when it opened its country to the world after two and half centuries of its isolation policy, the Meiji restoration saw a drastic modernization throughout the country. The modernization sometimes took the shape of haphazard adoption of Western mode of industrialization, with the negative results of urbanization, destruction of the traditional way of life, decline of crafts as well as environmental pollution.

In this period there was a trend among Japanese intellectuals to introduce and translate the ideas of Western thinkers who were critical of industrialization in favour of the pre-modern mode of community. One significant aspect of the reception of Ruskin into Japan was linked to this trend. It can be said that Ruskin was invoked in the context as a thinker of Counter-Industrialization, with its emphasis on the values of the medieval guild system in which the working people were not "broken into small fragments and crumbs of life".${ }^{16}$ Miyazawa's work can be contextualized in the general movement of Nomin-geijutsu or "peasant art" and one of his great contributions was that he presented the Ruskinian idea of art and society to us in his own way as a vital European legacy still relevant for Japan today. 


\section{Bibliography of Works by John Ruskin}

References are to volume and page numbers in:

Cook, E.T.; Wedderburn, A. (eds) (1903-1912). The Works of John Ruskin. Library Edition, 39 vols. London: George Allen.

https://www.lancaster.ac.uk/the-ruskin/the-complete-works-of-ruskin/.

Works, 10: The Stones of Venice II.

\section{General Bibliography}

Fromm, M.B. (1980). The Ideals of Miyazawa Kenji [PhD dissertation]. London: University of London.

Fromm, M.B. (1984). Miyazawa Kenji no Risō. Translated by Y. Kawabata. Tokyo: Shōbun-sha.

Henderson, P. (1967). William Morris: His Life, Work and Friends. London: Thames \& Hudson.

Honma, H. (1920). Seikatsu no Geijutsu-ka (Making Life Art). Tokyo: Santoku-sha.

Hosoi, Y. (2013). "Geijutsu". Heibonsha Dai-hyakka Jiten (Heibonsha Encyclopedia). Tokyo: Heibonsha, Electronic Dictionary.

Kawabata, Y. (1984). “Minshu-geijutsu Gakkō no Shisō: Rasukin, Morisu, Kenji” (The Idea of Art School for People: Ruskin, Morris, and Kenji). Shin Nihon Bungaku (New Japanese Literature), 442: 21-35.

Keene, D. (1987). Poetry, Drama, Criticism. Vol. 2 of Dawn to the West: Japanese Literature in the Modern Era. 2 vols. New York: Henry Holt. Miyazawa, K. (1984). "Nōmin-geijutsu Gairon Kōyō" (The Outline Survey of Peasant Art). Fromm 1984, i-x.

Miyazawa, K. (1995). "Dai-san Geijutsu" (The Third Art). Shin Kōhon Miyazawa Kenji zenshū (New Variorum Edition of the Complete Works of Kenji Miyazawa). Vol. 5, Text. Tokyo: Chikuma Shobo, 30-1.

Miyazawa, K. (1997). "Nōmin-geijutsu no Kōryū" (The Revival of Peasant Art). Shin Kōhon Miyazawa Kenji Zenshū. Vol. 13, Text. Tokyo: Chikuma Shobo, 17-20.

Morris, W. (1914). "The Lesser Arts". Morris, M. (ed.), The Collected Works of William Morris, vol. 22. London: Longmans, 3-27.

Morris, W. (1965). “Art and Labour”. LeMire, E.D. (ed.), The Unpublished Lectures of William Morris. Detroit: Wayne State University Press, 94-118.

Onda, I. (1961). “'Rasu' Toiugo no yurai: Kenji to Rasukin” (The Origin of the Word “Rasu”: Kenji and Ruskin). Yojigen (Fourth Dimension), 6, 2727-34.

Yanabu, A. (1982). Hon'yaku-go Seiritsu-jijō (How the Words for Translation Were Coined). Tokyo: Iwanami Shoten. 


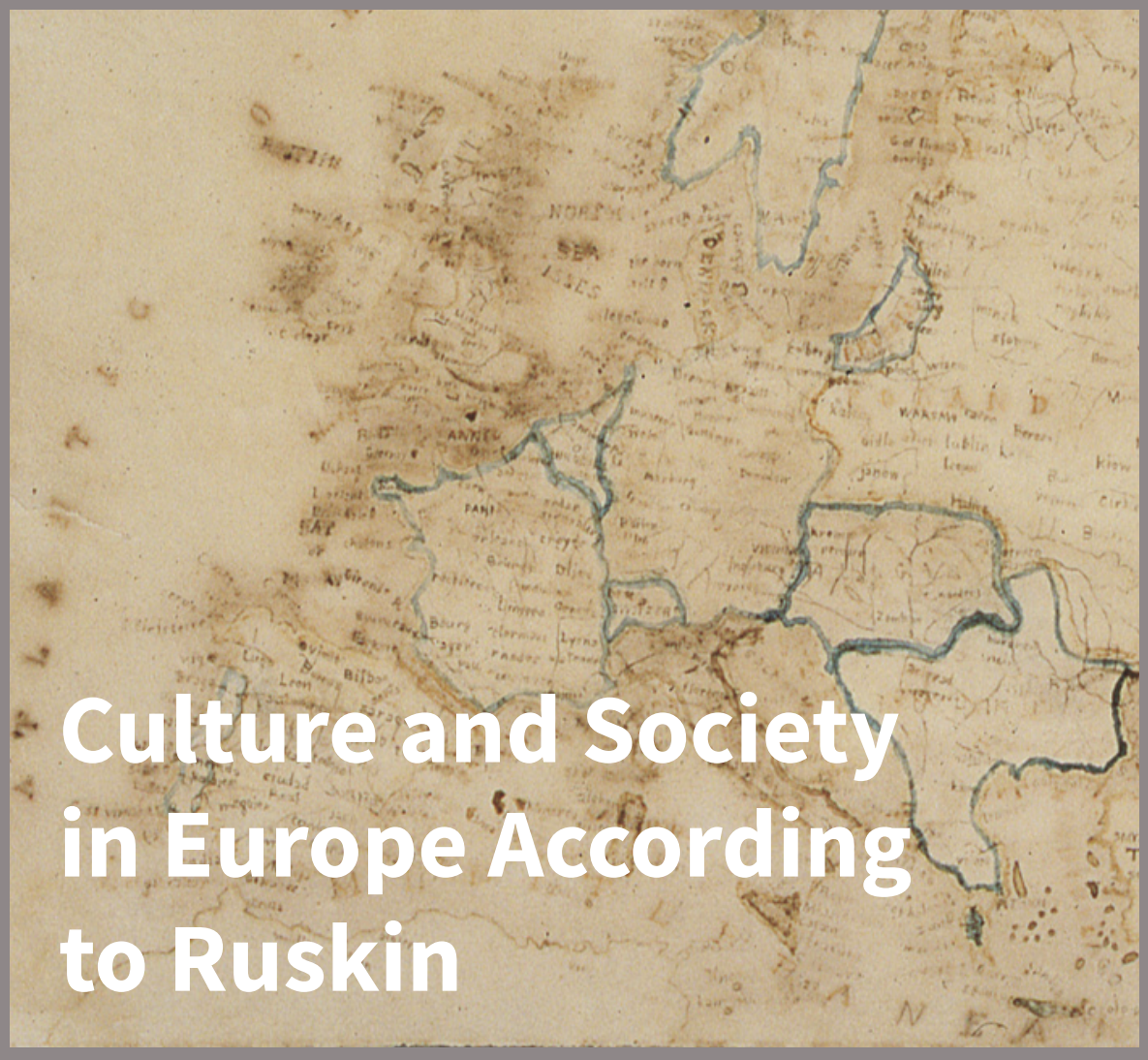




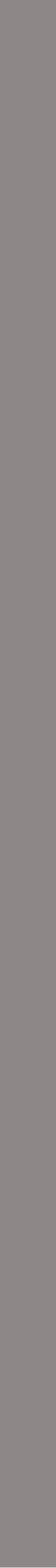




\title{
Tra nostalgia preindustriale, ghildismo e rinascita nazionale Il pensiero sociale di Ruskin nel dibattito culturale italiano
}

Laura Cerasi

Università Ca' Foscari Venezia, Italia

\begin{abstract}
Ruskin's social criticism, which in Unto This Last (1862) harshly condemned the effects of industrialism by mythologizing medieval age and craftmanship, had a wide influence on social reformers of various political orientations. While his work as an art critic was promptly received in the Italian cultural debate, his social criticism found little audience, at least until the turn of the century, and anyway not in the sphere of economic and sociological culture. In this contribution I examine how the circulation of Ruskin's social thought in the Italian cultural debate between the 19th and 20th centuries was inscribed in the renewed interest in the social function of art, advocated in the Florentine literary journal II Marzocco with particular reference to the work of L. Tolstoj by young intellectuals such as U. Ojetti, A. Orvieto, and E. Corradini, as well as established critics as A. Conti.
\end{abstract}

Keywords Nationalism. National heritage. Transcultural history. John Ruskin. Unto this Last. Il Marzocco. Lev Tolstoj's reception in Italy.

Sommario 1 Importanza di un necrologio. - 2 Arte e rinascita, nazione e rigenerazione. - 3 Antiindustrialismo e ghildismo. - 4 Difese d'arte: convergenze parallele.

\section{Importanza di un necrologio}

Come sarà giudicata l'opera dell'Old man of Coniston da coloro che soli si sentono capaci di giudicare il bene e il male?

Può apparire singolare che uno dei più tempestivi necrologi italiani di Ruskin, il primo in una rivista d'arte e letteratura, facesse leva in chiave latamente anti-nietzchiana non sul valore di critico d'arte ma sullo spessore etico del grande vittoriano. Tuttavia Angelo Conti, il Doctor Mysticus delle pagine dell'Emporium, il modello dello spirituale Daniele Glauro del

1 Conti 1900. 
Fuoco dannunziano, il teorico della contemplazione estatica del bello necessaria per cogliere l'attimo rivelatore dell'essenza platonica dell'opera d'arte ${ }^{2}$ - tratteggiava il suo ritratto di Ruskin non richiamando alcuno fra i suoi scritti d'arte e architettura, ma ricordando invece soprattutto la sua figura di filantropo e di riformatore sociale. La scelta, in se stessa, non era estemporanea: già John Hobson aveva impostato la sua fondamentale interpretazione del pensiero sociale di Ruskin in base all'assunto che fosse inestricabile dalla sua radice letteraria e artistica. ${ }^{3}$ Piuttosto, fin dal titolo del suo contributo ( $\mathrm{La}$ religione dell'amore), Conti alludeva, volendosene differenziare, a quello che allora era il principale veicolo di conoscenza di Ruskin in Italia, La Religion de la beauté del critico francese Robert de La Sizeranne. ${ }^{4}$ Secondo il critico romano, «la parte più pura e più profonda della sua eredità» era l'insegnamento impartito a «due intere generazioni» non solo ad «amare l'arte e la natura», ma anche a «sentire la nobiltà e la serietà della vita». A suffragio, Conti componeva un collage di citazioni dove con varie accentuazioni lo scrittore di Brantwood dichiarava l'inscindibilità di sentimento estetico - anzi, anche del sentimento vitale tout court - e coscienza sociale, e sottolineando «quelle parole che io già citai in un mio articolo: essere cioè mille volte meglio che i marmi del Partenone cadano in polvere e si scolorino le gote dipinte della Gioconda che veder diventare pallide le gote delle donne reali e lacrimosi per il freddo e la fame gli occhi dei bambini nati per la gioia». ${ }^{5}$ Conti faceva però un passo ulteriore, e ricordando che «il Ruskin ha vissuto per oltre dieci anni fra gli operai, spendendo per la loro educazione e per il loro aiuto i quattro o cinque milioni ereditati dal padre», situava il valore dell'insegnamento ruskiniano nella sua declinazione pratica:

Certamente le cose che Ruskin ha scritte avrebbero avuto la metà del loro valore se egli non le avesse fatte risplendere con l'esempio. Il popolo non crede e non ha creduto mai all'amore di chi non sa rinunziare a molte fortune per alleviare le altrui miserie, non crede a chi predica la carità e la compassione stando a casa sua tra i tappeti, i profumi, i conviti e le avventure. ${ }^{6}$

Angelo Conti faceva parte della piccola pattuglia di conoscitori diretti dell'opera di Ruskin, il cui accesso ai testi dello scrittore di Brantwood era precedente alla mediazione di La Sizeranne, principale via d'accesso all'opera ruskiniana per un pubblico più vasto. ${ }^{7}$ La subordinazione dello scrittore d'arte e architettura al riformatore sociale rifletteva perciò una consapevole scelta interpretativa, che andava inscritta nella riconfigurazione dell'orientamento critico di Conti allora in corso. In quel torno d'anni, il critico romano stava maturando il superamento dell'estetismo decadente e dannunziano, indirizzandosi verso l'affermazione della necessità di un fondamento etico e sociale del fatto artistico e letterario: il richiamo, riportato in apertura, al giudizio di «coloro

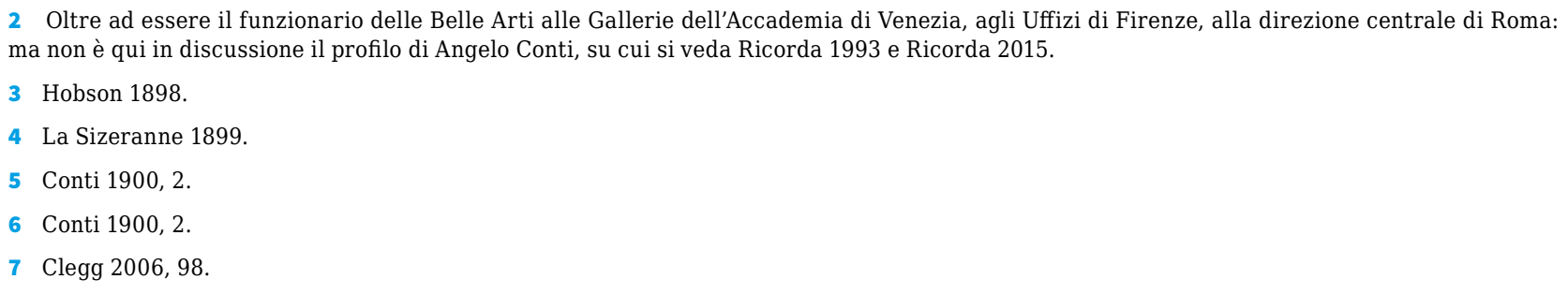


che si sentono capaci di giudicare il bene e il male» andava in questa direzione.

Non era un caso che questa valorizzazione in chiave etico-sociale del profilo complessivo di Ruskin, imperniato sulla sua visione della vita economica e sulla sua azione riformatrice, comparisse in un foglio d'arte e letteratura come il Marzocco, e non in una delle riviste di economia politica e di scienze sociali, nelle quali, infatti, non è stata trovata traccia di discussione di Unto this Last. ${ }^{8}$ Le scienze economiche andavano infatti perfezionando il loro profilo scientifico, che si definiva proprio attraverso l'abbandono delle concezioni etiche ed eclettiche dell'economia e l'adozione dell'ortodossia marginalista sulle orme di Walras e Pareto, aumentando contestualmente il proprio peso nelle istituzioni accademiche. ${ }^{9}$ Il Marzocco, invece, stava maturando come Conti una ridefinizione dei propri riferimenti culturali, allontanandosi dall'estetismo dannunziano delle origini e abbracciando, soprattutto attraverso l'inclinazione tolstoiana di uno dei due fratelli Orvieto, Angiolo, una declinazione etica e sociale della concezione dell'arte e della letteratura, che negli anni si sarebbe orientata soprattutto in un'azione in difesa del patrimonio artistico fiorentino e nazionale. ${ }^{10}$
L'importanza del Marzocco nella penetrazione e discussione delle opere di Ruskin in Italia è stata opportunamente rilevata. ${ }^{11}$ Ad un esame ravvicinato dei fitti dibattiti che vi si intrecciavano, tuttavia, emerge come l'interesse per Ruskin si inserisse in un momento cruciale del riorientamento culturale della rivista, avviato da una discussione sulla funzione sociale dell'arte, su cui non è stata ancora richiamata l'attenzione. Proprio a partire dal necrologio a firma di Angelo Conti, e nel breve volgere di pochi numeri, nel foglio fiorentino le diverse posizioni via via maturate precipitavano in nette opposizioni, fino alla rottura traumatica culminata con l'allontanamento di Enrico Corradini dalla direzione. Ripercorrere brevemente alcuni passaggi della discussione che si è snodata fra autunno 1899 e primavera 1900 permette di restituire Il Marzocco al ruolo rivestito nella cultura italiana a cavallo dei due secoli: un ruolo di rilievo, non riducibile al solo valore prolettico di successive e iconiche stagioni culturali cui è stato confinato a seguito della liquidazione postuma comminata da protagonisti del calibro di Croce e Prezzolini. ${ }^{12}$ E insieme, consente di recuperare alcuni rilievi del tessuto di riferimenti e implicazioni culturali che ha filtrato l'interesse per le opere di Ruskin a cavallo dei due secoli, improntandone la ricezione.

8 Guerzoni 2006, 143.

Augello 1992; Augello, Bianchini, Guidi 1996; Michelini, Guidi 2001; Augello, Guidi 2019.

10 Cerasi 1990. Il Marzocco nasceva nel febbraio 1896 interamente finanziato dai fratelli Angiolo e Adolfo Orvieto. Angiolo aveva retto la direzione del foglio in una prima fase, seguito da Enrico Corradini e poi dal fratello Adolfo.

11 Clegg 2006; Bertoni 2006; Del Puppo 2006.

12 Cerasi 2003.

363 Fonti, letterature, arti e paesaggi d'Europa | Sources, Literatures, Arts \& Landscapes of Europe 1

Fohn, Ruskin's Europe. A Collection of Cross-Cultural Essays, $361-374$
Jort 


\section{Arte e rinascita, nazione e rigenerazione}

Se qualcuno qui ha tirato in ballo l'arte aristocratica, la bellezza pura e l'arte per l'arte, molto probabilmente aveva intenzione di servirsene come arma contro la sciatteria, la volgarità, contro coloro che scrivono senza sapere la grammatica e non hanno ingegno sufficiente per farne a meno, contro coloro che esercitano la scrittura come un mestieraccio facile e alquanto rimunerativo [...] In quanto alla bellezza pura, all'arte aristocratica e all'arte per l'arte, non ho alcun rimorso personale. Non le ho mai nominate e mi fanno l'effetto del fumo negli occhi. ${ }^{13}$

Con la brutalità che gli stava diventando caratteristica, Corradini liquidava il dannunzianesimo estetizzante delle origini - che pure non era affatto episodico, D'Annunzio avendo tenuto a battesimo la rivista ${ }^{14}$ - accentuandone il carattere strumentale. In questo, Corradini coglieva il tratto militante che aveva marcato la rivista, nata con il proposito dichiarato di avviare una battaglia culturale palingenetica in nome della «bellezza» e di una versione dell'Arte per l'Arte tendenzialmente simbolista. ${ }^{15} \mathrm{Di}$ cui colpisce non tanto l'ovvio collocarsi nel solco della reazio- ne antipositivista, ${ }^{16}$ quanto i toni aggressivi e agonistici impiegati per «respingere i moderni barbari» dal «chiuso campo dell'arte», ${ }^{17}$ e l'intento pedagogico ritenuto necessario per la formazione di lettori e lettrici avvertiti: «risuscitata l'arte, bisognava fare il pubblico. Bisognava mettersi tra la moltitudine dei beoti, maschi e femmine, ed educarla un tantino». ${ }^{18}$ L'obiettivo della rinascita artistica e letteraria si inquadrava infatti entro il più vasto ma inscindibile obiettivo della rigenerazione nazionale, che ne era cornice e insieme giustificazione ultima. ${ }^{19}$ L'emersione della nazione italiana come spazio culturale e politico entro cui dimensionare i propositi palingenetici si era profilata nella rivista fin dai primi mesi, innescata dalle considerazioni di Ugo Ojetti sulla mancanza di una letteratura nazionale,$^{20} \mathrm{e}$ accelerata dall'intervento periodizzante di Mario Morasso, che avrebbe portato alla ribalta la generazione dei Nati dopo il '70 come interpreti della Terza reazione letteraria, incaricata di portare a compimento il superamento sia dell'agonizzante positivismo, che del "cosmopolitismo nordico" da cui erano segnati «spiritualismo, decadentismo, misticismo, simbolismo»: «La terza reazione si incarna nelle pure fonti eterne e solen-

13 Corradini 1899.

14 Ed essendosi i giovani redattori impegnati per difenderlo dalle accuse di plagio («La questione dannunziana» 1896; «Dell’impresa dei beoti» 1896).

15 Garoglio 1896, 1896a; Ojetti 1897; Tumiati 1897.

16 Numerosi gli interventi contro il metodo storico, la ricerca positiva, lo scientismo, il metodo sperimentale, l'erudizione, la critica lombrosiana e 'psicopatologica' e i loro corollari politici ed ideologici; contro l'umanitarismo, contro l'arte 'democratica', contro la generale decadenza culturale e l'imbarbarimento del gusto («Per Noi», 1896; «Per la letteratura e per l'arte» 1896; Zuccoli 1896; soprattutto, Zuccoli 1897).

17 «Prologo» 1896.

18 Zuccoli 1897.

19 Cerasi 2012.

20 Ojetti 1896. La discussione avrebbe avuto un'eco duratura, poi ripresa in articoli sulla Nuova Antologia (Gnoli 1897). Sul primo Ojetti si vedano De Lorenzi 2004 e Nezzo 2016, 13-37. Va osservato che Ojetti aveva esordito con una serie di interviste agli scrittori italiani (Ojetti 1895), e che il tema della letteratura nazionale sarebbe riemerso in età matura, ponendosi retoricamente l'interrogativo se In Italia, l'arte ha da essere italiana? (Ojetti 1941). 
ni dell'arte nazionale, nella semplicità, nella forza, nell'anima e nella terra natale». Alla nuova generazione si imponeva «il dovere superbo, genitura del fato di Roma, il dovere di dare alla patria sentimento di sé [...], di ringagliardire lo spirito nazionale, di ricostruire moralmente la razza in una organica unità etnica». ${ }^{21}$ Dietro il pungolo dell'aggressiva enfasi morassiana, ${ }^{22}$ la discussione culminava in una vasta Inchiesta sulla politica dei letterati, da cui emergeva un orientamento incline all'impegno degli scrittori nella formazione dello spirito pubblico. ${ }^{23}$ Era uno dei segni, che andavano moltiplicandosi nella cultura italiana, della chiusura di un periodo, e della definizione di un nuovo sistema di coordinate culturali proprio dell'età dei nazionalismi. ${ }^{24}$

Quale nesso poteva sussistere fra questi aggressivi e brutali prodromi di un arrembante patriottismo culturale, e il profilo cosmopolita del visionario di Brantwood? Il terreno comune era l'assunzione della necessità di una pur indeterminata finalizzazione cui indirizzare la produzione letteraria ed artistica:

La letteratura è vanità di vanità e non è scusabile se non quando è messa al servizio di qualcosa che la trascende, la domina e la purifica [...] L'arte per l'Arte è sterile e vuota, così come la virtù per la virtù. L'arte e la letteratura sono oggi un ninnolo vano appunto perché dimenticano ciò. ${ }^{25}$
Si trattava di un terreno programmaticamente rivendicato:

L'artista quindi non si deve rinchiudere in una torre d'avorio [...] Sosterremo concordi la profonda e sostanziale relazione dell'Arte con la Vita e quindi con la religione e quindi con la morale, con l'educazione e con la politica, con la con la scienza e con le condizioni ed i problemi sociali tutti quanti. ${ }^{26}$

Ma che non veniva assunto secondo accenti del tutto condivisi. Enrico Corradini, sulla scia della morassiana 'egoarchia' ${ }^{27}$ lo declinava in termini di un barresiano determinismo vitalistico: occorreva arrendersi alla «necessità della natura umana», come il padre Dante aveva realizzato nella «sublimità incommensurabile» del V Canto dell'Inferno. "Nell'affermazione di una legge di natura che non si può vincere l'uomo si redime, perché l'uomo è tanto più libero quanto più obbedisce alla sola natura che è in lui». ${ }^{28}$

Non era un caso che Corradini desse forma al suo determinismo attivistico in occasione di una discussione su Resurrezione di Tolstoj. Seguendo una suggestione di Ferdinand Brunetière, Angelo Conti annunciava l'incipiente «vero rinascimento della poesia», che sarebbe prodotto «dalla apparizione di una letteratura religiosa e sociale, la quale si proporrà non di abbrutire ma

23 Tutte le risposte all'Inchiesta sulla politica del letterati sono state pubblicate nei nrr. 19, 20, 21 e 22, del Marzocco, fra il giugno e il luglio del 1897.

24 Mangoni 1985.

25 Neal 1899.

26 «Ai lettori» 1900 .

27 Corradini 1898; Morasso 1898.

28 Corradini 1900. 
di rendere migliori gli uomini. E il primo libro nuovo io credo sia quella meravigliosa Risurrezione di Tolstoi»», ${ }^{29}$ che traduceva in forma narrativa le nette formulazioni sulla moralità dell'opera letteraria formulate in Che $\mathrm{Co}$ sa è l'arte?. ${ }^{30}$ «Io vorrei che, dopo aver letto Resurrezione tutti i miei compagni di lavoro si proponessero di fare una guerra spietata a tutto ciò che è pura letteratura, pura arte e pura bellezza». ${ }^{31}$ Angiolo Orvieto, a seguire, dichiarava il suo incondizionato entusiasmo per l'ultimo romanzo dello scrittore di Jàsnaja Poljana, paragonandolo alla Divina Commedia per l'identità dell'ispirazione morale, l'indicazione del cammino dall'errore al giusto, dalla menzogna alla verità:

E parso a molti e anche a me lo confesso, che in Leone Tolstoi il filosofo, il cristiano fervente avessero diminuito l'artista e anzi dovessero finire con l'ucciderlo. [...] Lo dicono, l'ho detto anch'io, ma non è vero: e se non ci fosse già per dimostrarlo la Divina Commedia (scusate se è poco) ci sarebbe ora anche questa Resurrezione, che con la Commedia ha parecchi punti in contatto. ${ }^{32}$

Il paragone con Dante era per Corradini insostenibile. Quelli di Tolstoj erano «prediche», un «modello del nuovo genere letterario ultramorale», ma non avevano nulla a che spartire con il genio di Dante, che era tale perché capace di accettare gli aspetti controversi del- la natura umana: «la morale dei sermocinatori bisogna che taccia e accetti le leggi della vita come sono». ${ }^{33} \mathrm{La}$ discussione si accendeva in un botta e risposta, che rivelava il profilarsi inconciliabile di un'antropologia crudamente realistica e deterministica da una parte, ed etico pedagogica dall'altra ( Est modus in rebus caro Corradini, e io non vedo perché l'uomo debba con tanto accanimento coltivare, esasperare, inviperire ciò che tu chiami "la sola natura che è in lui" ed alla quale quanto più l'uomo obbedisce, tanto più tu lo proclami libero»); ${ }^{34}$ mentre Corradini rigettava il «facile entusiasmo» di Orvieto richiamandogli il carattere di finzione della letteratura: "Che la Maslova dimostra come si possa amare più il prossimo di noi stessi, lo so; ma vorrei che me lo dimostrasse un po' meno la letteratura e un po' più la natura umana»; ${ }^{35}$ al che Orvieto ribatteva essere la natura umana perfettibile, e perciò oggetto dell'azione di «educatore convinto e fervido» del romanziere russo. ${ }^{36}$

Il solco che si era scavato con la discussione su Tolstoj si approfondiva intorno al necrologio di Ruskin, tanto da indurre Corradini, in capo a due settimane, a lasciare la direzione della rivista. L'apprezzamento rivolto da Conti al filantropo e riformatore sociale, creatore della Guild of St. George, fondata nel 1871 con il lascito dell'eredità paterna, risultava particolarmente urticante per Corradini. In nome dell'accettazione piena della natura umana, andava rigettato anche l'assistenzialismo cari-

29 Conti 1899

30 Tolstoj 1899.

31 Conti 1990a.

32 Orvieto 1900.

33 Corradini 1900.

4 Orvieto 1900a.

5 Corradini 1900a

Orvieto 1900c. 
tatevole. I bisognosi non esistono, l'altruismo è astratto e innaturale, la vera realtà è la forza.

Al solito il divario è tra il porre a fondamento la viltà e la quietudine, e il porre la forza e la volontà [...]. Ad un certo punto tu gridi: non sentite la voce del popolo? Altro se la sentiamo. Anche a chi non vuole si fa sentire. Ma non è una voce cristiana che annunzia pietà: è una voce feroce che annunzia la guerra. ${ }^{37}$

Al di là della chiara indicazione della direzione politica che andava assumendo l'elaborazione corradiniana, merita qui rilevare come il Ruskin di Angelo Conti, da cui Corradini si sentiva oltraggiato e che era stato assunto a occasione ultima per il consumarsi di una divisione che maturava da tempo, era un Ruskin francescano, medievalista, anti-industriale, spirituale il cui profilo, di fatto, si sovrapponeva a quello di Tolstoj. In altri termini, se in al- cuni protagonisti del dibattito, come lo stesso Conti o Domenico Tumiati, la conoscenza di Ruskin era diretta, ${ }^{38}$ la sua diffusione era invece agganciata alla crescente attenzione che a cavallo dei due secoli veniva riservata allo spiritualismo tolstojano: le cui opere, è bene ricordarlo, apparivano in traduzione francese quasi in contemporanea all'edizione russa, e conoscevano perciò immediata circolazione anche fra i lettori colti italiani. ${ }^{39} \mathrm{Ed}$ era dall'ombra dell'eremita di Jàsnaja Poljana che i richiami al pensiero sociale del visionario di Brantwood sarebbero rimasti in parte oscurati. In questo senso, il profilo culturale di Ruskin perdeva un po' della sua poliedricità, per assimilarsi alla potente figura dell'aristocratico contadino russo: ancora alla fine del decennio, l'influente anglo-fiorentina Vernon Lee - che pure ne apprezzava profondamente l'opera $-{ }^{40}$ riproponeva la linea di discendenza tra Carlyle, Tolstoj e Ruskin, nel segno dell'opposizione 'profetica' alla modernità industriale e finanziaria occidentale. ${ }^{41}$

\section{Anti-industrialismo e ghildismo}

Non stupisce, dunque, che come è stato più volte notato la prima traduzione italiana del vigoroso atto d'accusa dei costi sociali del trionfante capitalismo liberale lanciato in Unto This Last comparisse quasi quarant'anni dopo l'edizione originale,$^{42}$ quando anche in Italia analoghi costi sociali cominciavano ad essere percepiti e patiti. Soprattutto, non stupisce che la traduzione fosse proposta in forma di compendio riassuntivo delle rapsodiche quanto radicali proposizioni ruskiniane di critica dell'economia politica, ${ }^{43}$ atto a comparire nelle biblioteche popolari promosse dall'associazionismo socialista insieme alla simpatetica presentazione di Ruskin e il suo

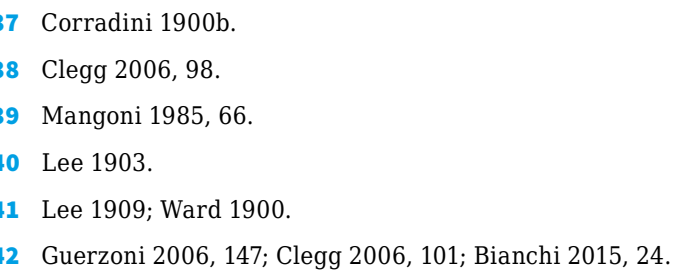

Si tratta del compendio di Unto This Last ad opera di Thomas Barclay, tradotto da Ernestina D'Errico e pubblicato con il titolo I diritti del lavoro presso Mongini nel 1900 (Ruskin 1900).

367 Fonti, letterature, arti e paesaggi d'Europa | Sources, Literatures, Arts \& Landscapes of Europe 1

Fonti, letterature, arti e paesaggid'Europa Sources, Literatures, Arts
John Ruskin's Europe. A Collection of Cross-Cultural Essays, $361-374$ 
apostolato sociale pubblicata a stretto giro: ${ }^{44}$ in tal modo situandosi nella linea di ricezione da parte del socialismo umanitario, allora in Italia nella sua massima espansione, degli argomenti di denuncia del capitalismo industriale e dell'economia politica liberale provenienti da autori di orientamento conservatore, inaugurata dall'apprezzamento degli strali di Carlyle da parte del giovane Marx. ${ }^{45}$

Forse più significativo, in ottica transculturale, il fatto che la prima traduzione integrale di Unto This Last fosse stata affidata dall'editore Voghera al giovane Giovanni Amendola, allora vivamente animato da interesse per lo spiritualismo contemporaneo e impegnato nella Biblioteca filosofica; e che Amendola ne sottolineasse la tempestività, osservando che «il tema principale di questo libro - e cioè i rapporti dell'economia con l'etica - è diventato oggi di moda, il che prova che il mistico e sognante apostolo anglo-sassone aveva il dono di un'intuizione sostanzialmente buona», ${ }^{46}$ testimoniando della migrazione della ricezione della critica sociale ruskiniana presso l'area del vocianesimo e delle istanze di riforma morale allora in affermazione; ciò che trovava conferma in un piccolo studio introduttivo, che segnalava come una delle ragioni del perdurante interesse per l'opera di Ruskin fosse l'esigenza della «rieducazione civile ed estetica del popolo nostro». ${ }^{47}$

Era un'esigenza di rigenerazione morale che richiamava il rilancio del pensiero sociale di Ruskin, in particolare del suo ruralismo anti-industrialista, che andava allora sviluppandosi nelle discussioni del londinese
New Age Circle e nelle colonne della rivista The New Age, animati da Arthur Penty e Alfred Orage. ${ }^{48}$ Nel 1906 Penty, nel suo influente The Restoration of the Gild System, dichiarava il suo debito con Ruskin, precisando che la sua opera intendeva essere una illustrazione concreta di quanto il pensatore di Brantwood aveva solo accennato:

Readers of the following pages will probably be aware that the idea of restoring the Gild system as a solution of the problems presented by modern industrialism is to be found in the writings of John Ruskin, who put forward the proposition many years ago. Unfortunately, however, as Ruskin failed to formulate any practical scheme showing how the Gilds could be re-established in society, the proposal has never been seriously considered by social reformers. Collectivism may be said to have stepped into the breach by offering a plausible theory for the reconstruction of society on a co-operative basis, and Ruskin's suggestion was incontinently relegated to the region of impractical dreams. My reason for reviving the idea is that while I am persuaded that Collectivism is incapable of solving the social problem, the conviction is forced upon me that our only hope lies in some such direction as that foreshadowed by Ruskin, and in the following chapters I hope to show that it is not impossible to discover practical ways and means of re-establishing the Gilds in our midst. ${ }^{49}$

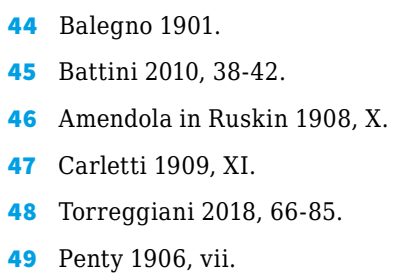


L'interesse di ricerca per questi temi si è di recente avviato anche in Italia, ricostruendo il quadro ampio di una tradizione pluralista di pensiero su rappresentanza degli interessi e corpi intermedi, trasversale alle diverse famiglie politiche liberali e conservatrici: gli idealisti inglesi, il ghildismo corporativo fabiano di George D.H. Cole, il New Age Circle di Penty e Orage, il distributismo di Gilbert K. Chesterton, i cattolici inglesi, i progetti planisti degli anni Trenta. ${ }^{50} \mathrm{Si}$ tratta di un tessuto culturale, mai maggioritario ma importante, in cui le relazioni si annodano in luoghi d'incontro - il Balliol College di Oxford, le riviste, da New Age appunto a The Eye-Witness, e si riconoscono in maestri e ispiratori comuni - John Ruskin, William Morris, il cardinale Manning. L'ispirata critica ruskiniana all'economia liberale e liberista, tiepidamente recepita nel momento della pubblicazione di Unto This Last, sarebbe stata via via ripresa dalle correnti del ghildismo corporativo primonovecentesco passando attraverso l'influenza dell'anti-macchinismo di Ruskin su Morris e il movimento Arts and Crafts. La riappropriazione della dimensione estetica del gesto creatore artigiano si riverberava nel movente etico, religioso e in subordine politico dei dibattiti del New Age Circle: il cui obiettivo era la rigenerazione della società, il ritrovamento di un'armonia dell'uomo con se stesso, di un 'buon vivere' che nella sua rettitu- dine riconcili l'essere umano con la natura e la società. Si trattava di una rivoluzione «political, economic, and, we would add, moral», come sosteneva Orage nel 1907. ${ }^{51}$ Non era un caso, in quest'ottica, che i protagonisti si muovessero nel campo dell'arte e dell'architettura, come Arthur Penty, dove l'estetica del vivere ne comporta anche la rigenerazione morale. Nella bellezza è contenuta una dimensione etica la cui valenza politica puntava ad una nuova dimensione comunitaria.

Incorporato, per così dire, in questo filone di critica anti-industrialista, utopistica, organicista e corporativa, il pensiero sociale di Ruskin trovava un canale di penetrazione che superava i confini delle isole britanniche. Per apprezzare la circolazione in Italia del pensiero sociale ruskiniano merita perciò guardare alla sua penetrazione indiretta, attraverso William Morris soprattutto, che aveva un suo riverbero nella presenza e nel network culturale degli anglo-fiorentini. ${ }^{52}$ Ancora Vernon Lee, nel suo Enchanted Woods, aveva contribuito ad popolarizzare una versione colta del canone del pittoresco, come rivelazione della «divinity of places» della sostanza spirituale conservata nei luoghi: ${ }^{53}$ un genius loci della toscanità antimoderna, modellato nell'armoniosa corrispondenza fra natura e cultura, fra paesaggio, arte ed architettura che emanava dalle colline toscane ed era racchiuso fin nelle decorazioni più umili. ${ }^{54}$

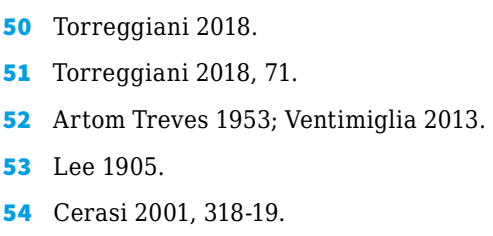




\section{Difese d'arte: convergenze parallele}

La linea che muove da William Morris e Arts and Crafts può riuscire utile a mettere a fuoco un tratto originale della ricezione di Ruskin nel dibattito culturale italiano del primo Novecento, che lo disincagliava dall'assimilazione al campo tolstojano, e definiva un suo spazio sulla base dell'intreccio fra critica sociale, rigenerazione morale e sentimento estetico che attraversa in modo inscindibile l'opera ruskiniana

Per tornare al necrologio di Angelo Conti, merita rilevare come l'ammirazione per l'opera filantropica di Ruskin, a partire dalla costituzione della Guild of Saint George, fosse sentita come un incitamento all'azione, che faceva leva sul valore etico dell'arte secondo la prospettiva ruskiniana. Proprio a Firenze, lanciata dalle pagine del Marzocco si costituiva in quei mesi una sorta di emanazione di Arts and Crafts, una Società per l'arte pubblica, ispirata ad un esempio belga, ${ }^{55}$ con l'intento di «diffondere nel pubblico la convinzione che l'arte è uno dei principali mezzi di incivilimento e di benessere materiale, ed una delle più importanti questioni sociali», nella persuasione che «il dovere della presente generazione è quello di ravvivare nell'anima nostra la tradizione di bellezza che è tradizione italiana». ${ }^{56}$ ricevendo una certa attenzione sul piano nazionale. L'esigenza di disciplinare e rivestire di decoro artistico le manifestazioni della modernizzazione urbana e di «tutto ciò che il progresso ha acquistato di utile alla vita pubblica contemporanea», che riceveva una certa attenzione sul piano nazionale, ${ }^{57}$ nasceva nel seno dell'élite politica e culturale fiorentina, venendo promossa dal sindaco Torrigiani, che aveva po- chi mesi prima appoggiato anche un'iniziativa 'conservativa' del patrimonio artistico come l'Associazione per difesa di Firenze antica. Presieduto dal principe Tommaso Corsini, il sodalizio si era costituito per intervenire presso la stampa italiana e straniera e assicurare la «tutela del carattere e del patrimonio storico ed artistico di Firenze», puntando a conciliare le esigenze della modernizzazione con quelle dell'arte:

La nostra Associazione non è, come da taluni si vorrebbe far credere, un centro di opposizione ad ogni lavoro di risanamento che l'igiene e i bisogni di una città moderna esigono. [...]. Essa si propone soltanto di conciliare, ancor più di quello che non si sia fatto fino ad oggi, le ragioni dell'Arte e della Storia con quelle dell'edilizia; si studia di salvare dalla distruzione, per quanto è possibile, quegli edifici dell'antica Firenze che ricordano i tempi più gloriosi della sua storia e che tanto contribuiscono a dare ad alcuni quartieri quel carattere che amano gli artisti e che i forestieri ci dicono essere la più potente attrattiva della nostra città. Tuttavia ciò noi crediamo conciliabile, nella maggior parte dei casi, con quei lavori di risanamento e di abbellimento che una città progredita e civile può richiedere. ${ }^{58}$

Tutela del patrimonio e disciplinamento della modernizzazione erano due aspetti correlati. Le battaglie culturali a favore della 'rinascenza italica' fondate sulla tradizione artistica si traducevano, soprattutto al passaggio dei due secoli, in impegno per la conservazione e la tute- 
la del patrimonio artistico, che andava acquistando un rilievo crescente:

I segni di questo salutare risveglio si vedono ormai da ogni parte. La Società per l'Arte Pubblica si propaga assai rapidamente: associazioni per la tutela e la preservazione degli antichi monumenti sorgono e fioriscono in quasi tutte le nostre città; si fanno sempre più gradite e frequenti le mostre d'arte antica; proposte e progetti per rintracciare e mettere alla luce antichi dipinti, coperti dal bianco, dalle antiche chiese e dai palagi pubblici, deturpati o negletti, si seguono l'una all'altra. ${ }^{59}$

Non si trattava di un fatto locale. Le ricerche, che si sono moltiplicate in anni recenti, sulle origini del movimento per la tutela del patrimonio culturale e naturale hanno posto in evidenza l'importanza del tessuto di iniziative, dibattiti, pubblicazioni, associazioni e riviste che sul piano nazionale ha accompagnato i primi provvedimenti legislativi, e dato l'impronta alle politiche di conservazione del secolo scorso, che affondano le proprie radici nella cornice di costruzione della nazione e nella formazione dell'identità nazionale. ${ }^{60}$ Sul piano locale, il dibattito sulle 'difese d'arte' prendeva forma in una fitta trama associativa delle classi colte fiorentine che facevano leva su una rappresentazione di fiorentinità non (o non soltanto) localistica per condurre una battaglia culturale nel segno dell'antimodernità, che costituisse di fatto un indirizzo di sviluppo per la città imperniato su artigianato e turismo. ${ }^{61}$

Qui torniamo allora, circolarmente, allo scontro in occasione della morte di Ruskin con cui abbiamo aperto queste riflessioni, e alla rilevanza del Marzocco non solo nella storia della cultura italiana nel passaggio fra Otto e Novecento, ma nel dispositivo di ricezione di Ruskin che si sta cercando di ricostruire. Se per Enrico Corradini, dopo l'allontanamento dalla direzione della rivista, si apriva la strada dell'attivismo politico, che l'avrebbe portato a fondare con Il Regno il primo foglio del nazionalismo italiano, il drappello di ruskiniani rimasti nel Marzocco individuava nelle 'difese d'arte' l'ambito d'elezione dell'impegno del letterato: alcuni di loro, come Angiolo Orvieto, avrebbero intensificato la propria attività al centro del network associativo cittadino; Ojetti guadagnava presto una posizione di primo piano nella critica d'arte; altri ancora, come Giovanni Rosadi, deputato del 'bel San Giovanni' avrebbero promosso lungo tutto il decennio i primi passi della legislazione conservativa, tenendo a battesimo la prima legge di protezione dei monumenti del $1908 .^{62}$

La divergenza, tuttavia, non sarebbe durata a lungo: allo scoppio della guerra mondiale, ruskiniani e antiruskiniani si sarebbero trovati a militare nello stesso versante del fronte patriottico-nazionale, accesamente interventisti: Ojetti responsabile dell'Ufficio di tutela dei monumenti, Orvieto alla mobilitazione civile, Corradini con l'Idea nazionale in prima linea nella battaglia bellicista. La convergenza nel fronte patriottico-interventista degli estimatori del visionario di Brantwood - in forza peraltro di quello stesso impegno per la rinascita nazionale che era stato affidato alla militanza per la 'bellezza' e all'impegno per le 'difese d'arte' - segnala, se non altro, la profondità delle trasformazioni culturali e politiche intervenute tra il periodo mediovittoriano e il primo decennio del Novecento, e l'intrinseca ambiguità contenuta nell'emergere della dimensione nazional-patriottica. 


\section{Fonti}

«Ai lettori» (1900). Il Marzocco, V, 1, 7 gennaio.

«Dell'impresa dei beoti» (1896). II Marzocco, I, 10, 5 aprile.

«La questione dannunziana». (1896). Il Marzocco, I, 3, 16 febbraio.

«Per la letteratura e per l'arte» (1896). Il Marzocco, I, 37, 11 ottobre.

«Per Noi» (1896). Il Marzocco, I, 5, 1 marzo.

«Prologo» (1896). Il Marzocco, I, 1, 2 febbraio.

Balegno, E. (1901). Ruskin e il suo apostolato sociale. Milano: La Poligrafica.

Carletti, R. (1909). Il pensiero di Ruskin e sua influenza sui contemporanei. Torino: Paravia.

Chiappelli, A. (1900). «ll risveglio degli studi sull'arte in Italia». Il Marzocco, V, 23 dicembre.

Chilovi, D. (1903). «Le Società di abbellimento e le esposizioni per l'arte pubblica». Nuova Antologia, fasc. 16 febbraio, 643-57.

Conti, A. (1899). «Le tendenze dell'arte II». II Marzocco IV, 47, 24 dicembre.

Conti, A. (1900). «La religione dell'amore (John Ruskin)». Il Marzocco, V, 4, 28 gennaio.

Conti, A. (1900a). «"Secol si rinnova"». Il Marzocco, V, 1, 7 gennaio.

Conti, A. (1900b). «Difendiamo Firenze». Il Marzocco, V, 33, 18 agosto.

Corradini, E. (1898). «Uomini e idee del domani». Il Marzocco, III, 14, 8 maggio.

Corradini, E. (1899). «Intorno alle Tragedie dell'anima». Il Marzocco, IV, 33, 17 settembre.

Corradini, E. (1900). «Francesca e Katucha». Il Marzocco, V, 6, 11 febbraio.

Corradini, E. (1900a). «Ama il prossimo tuo...». Il Marzocco, V, 12, 25 marzo.

Corradini, E. (1900b). «Lettera aperta ad Angelo Conti». Il Marzocco, V, 5, 4 febbraio.

Corsini, T. (1898). «Lettera aperta al sindaco Torrigiani». Bollettino dell'Associazione per la difesa di Firenze antica, 1(1), 15 dicembre.

Gargàno, G.S. (1898a). «Un congresso importante». Il Marzocco, III, 30, 28 agosto.

Gargàno, G.S. (1898b). «Sul primo Congresso dell'Arte pubblica». Il Marzocco, III, 42, 27 novembre.

Gargàno, G.S. (1898c). «La Società per l'Arte Pubblica». Il Marzocco, III, 45, 18 dicembre.

Gargàno, G.S. (1899a). «Per l'Arte pubblica». Nuova Antologia, fasc. 1 giugno.

Gargàno, G.S. (1899b). «L'Oeuvre Nationale Belge». Il Marzocco, IV, 29, 4 settembre.

Garoglio, D. (1896). «Ancora per l'arte aristocratica». Il Marzocco, I, 19, 7 giugno.

Garoglio, D. (1896a). «Sopra le forze di Björnson». Il Marzocco, I, 38, 18 ottobre.

Gnoli, D. (1897). «Nazionalità e arte». Nuova Antologia, vol. 32, s. 4, t. 67, 16 febbraio.

Hobson, J.A. (1898). John Ruskin Social Reformer. Boston: Dana Estes \& Company.

La Sizeranne, R. de (1899). Ruskin et la Religion de la Beauté. Paris: Hachette.

Lee, V. [Violet Paget] (1905). The Enchanted Woods and Other Essays on the Genius of Places. London; New York: John Lane.

Lee, V. [Violet Paget] (1909). Gospels of Anarchy and Other Contemporary Studies. New York; London: T. Fisher Unwin.

Morasso, M. (1897a). «Ai nati dopo il '70. La terza reazione letteraria». II Marzocco, II, 1, 2 febbraio.

Morasso, M. (1897b). «La politica dei letterati, I, II pregiudizio dell'astensione». Il Marzocco, II, 13, 2 maggio.

Morasso, M. (1898). Uomini e idee del domani. L'egoarchia. Torino: Bocca.

Neal, T. [Angelo Cecconi] (1899). «Arte e Accademia». II Marzocco, IV, 44, 3 dicembre.

Ojetti, U. (1895). Alla scoperta dei letterati. Torino: Bocca.

Ojetti, U. (1896). «La Grande illusione». Il Marzocco, I, 8, 22 marzo.

Ojetti, U. (1897). «Individualismo e Arte». II Marzocco, II, 4, 28 febbraio.

Ojetti, U. (1941). In Italia, l'arte ha da essere italiana? Milano: Mondadori.

Orvieto, A. (1900). «Resurrezione». Il Marzocco, V, 4, 19 gennaio.

Orvieto, A. (1900a). «Giasone e Nekludov». Il Marzocco, V, 7, 18 marzo.

Orvieto, A. (1900b). «E poi, basta». Il Marzocco, V, 13, 1 aprile. 


\section{Laura Cerasi}

Penty, A.J. (1906). The Restoration of the Gild System. London: Swan Sonnenschein and Co.

Ruskin, J. (1900). I diritti del Lavoro. Riassunti da Thomas Barclay. Roma: Mongini.

Ruskin, J. (1908). Le fonti della ricchezza (Unto this last). A cura di G. Amendola. Roma: Voghera.

Tolstoj, L. (1899). Che cosa è l'arte? Milano: Treves.

Tolstoj, L. (1900). Resurrezione. Milano: Treves.

Tumiati, D. (1897). «Un libro idealista e il neo-simbolismo». Il Marzocco, II, 38, 24 ottobre.

Ward, M.A. (1900). Prophets of the Nineteenth Century. Carlyle, Ruskin, Tolstoi. London: Gay and Bird.

Zuccoli, L. (1896). «Indietro!». Il Marzocco, I, 24, 12 luglio.

Zuccoli, L. (1897). «Riepilogo IV». Il Marzocco, II, 33, 19 settembre.

Zuccoli, L. (1897a) «Riepilogo I, II, III». II Marzocco, II, 29 agosto, 5 settembre, 12 settembre.

\section{Bibliografia}

Artom Treves, G. (1953). Anglo-fiorentini di cento anni fa. Firenze: Sansoni.

Augello, M.M. (1992). «La nascita di una professione accademica: Gli economisti italiani post-unitari (1860-1900)». Quaderni di storia dell'economia politica, 10(3), 3-39.

Augello, M.M.; Bianchini, M.; Guidi, M.E.L. (a cura di) (1996). Le riviste di economia in Italia. Dai giornali scientifico-letterari ai periodici specialistici (1700-1900). Milano: FrancoAngeli.

Augello, M.M.; Guidi, M.E.L. (a cura di) (2019). Economisti e scienza economica nell'Italia liberale (1848-1922). Una storia istituzionale. Milano: FrancoAngeli.

Balzani, R. (2003). Per le antichità e belle arti. La legge n. 364 del 20 giugno 1909 e l'Italia giolittiana. Bologna: il Mulino.

Battini, M. (2010). Il socialismo degli imbecilli. Propaganda, falsificazione, persecuzione degli ebrei. Torino: Bollati Boringhieri.

Bertoni, C. (2006). «Croce e il ruskinismo italiano. I rapporti con Conti e “Il Marzocco"». Lamberini, D. (a cura di), L'eredità di John Ruskin nella cultura italiana del Novecento. Firenze: Nardini editore, 31-64.

Bianchi, B. (2015). «Arte, lavoro, domesticità. Il pensiero di John Ruskin interpretato dalle donne e dagli uomini del suo tempo (18601930)». DEP. Deportate, esuli, profughe, 27, 23-47.

Cerasi, L. (1990). «Burocrazia, strutture comunali, beni culturali nell'immagine del "Marzocco"». Studi Storici, 4, 843-65.

Cerasi, L. (2000). Gli Ateniesi d'Italia. Associazioni di cultura a Firenze in età giolittiana. Milano: FrancoAngeli.

Cerasi, L. (2001). «Fiorentinità. Percorsi di un'ideologia identitaria fra Otto e Novecento». Studi Novecenteschi, 62, $311-43$.

Cerasi, L. (2003). «Per una pedagogia della tradizione. Appunti sul nazionalismo del primo "Marzocco"». Cercles. Revista d'Història Cultural, 6, 53-73.

Cerasi, L. (2012). Pedagogie e antipedagogie della nazione. Istituzioni e politiche culturali nel Novecento italiano. Brescia: La Scuola.

Clegg, J. (2006). «La presenza di John Ruskin in Italia cento anni fa». Lamberini, D. (a cura di), L'eredità di John Ruskin nella cultura italiana del Novecento. Firenze: Nardini editore, 95-108.

De Lorenzi, G. (2004). Ugo Ojetti critico d'arte. Dal "Marzocco" a "Dedalo". Firenze: Le Lettere.

Del Puppo, A. (2006). «Aspetti della ricezione di Ruskin nella stagione delle riviste». Lamberini, D. (a cura di), L'eredità di John Ruskin nella cultura italiana del Novecento. Firenze: Nardini editore, 119-35.

Del Puppo, A. (2012). Modernità e nazione. Temi di ideologia visiva nell'arte italiana del primo Novecento. Macerata: Quodlibet.

Gori, A. (2014). Tra patria e campanile. Ritualità civili e culture politiche a Firenze in età giolittiana. Milano: FrancoAngeli.

Guerzoni, G. (2006). «La ricezione italiana del Social and Economic Criticism di John Ruskin 1850-1950». Lamberini, D. (a cura di), L'eredità di John Ruskin nella cultura italiana del Novecento. Firenze: Nardini editore, 136-55.

Mangoni, L. (1985). Una crisi fine secolo. La cultura italiana e la Francia fra otto e Novecento. Torino: Einaudi.

Michelini, L.; Guidi, M.E.L. (a cura di) (2001). Marginalismo e socialismo nell'Italia liberale. Annali della Fondazione Giangiacomo Feltrinelli. Milano: Feltrinelli.

$373 \quad$ Fonti, letterature, arti e paesaggi d'Europa | Sources, Literatures, Arts \& Landscapes of Europe 1 John Ruskin's Europe A A Collection of Cross-Cultural Essays, $361-374$ 
Nezzo, M. (2016). Ugo Ojetti. Critica, azione, ideologia. Dalle Biennali d'arte antica al Premio Cremona. Padova: Il Poligrafo.

Papi, F. (2008). Cultura e tutela nell'Italia unita 1865-1902. Todi: Tau.

Pellegrino, A. (2012). La città più artigiana d'Italia. Firenze 1861-1929. Milano: FrancoAngeli.

Piccioni, L. (2014). Il volto amato della patria. Il primo movimento per la protezione della natura in Italia 1880-1934. Trento: Temi.

Ragusa, A. (2011). Alle origini dello Stato contemporaneo. Politiche di gestione dei beni culturali e ambiente tra Ottocento e Novecento. Milano: FrancoAngeli.

Ricorda, R. (1993). Dalla parte di Ariele. Angelo Conti nella cultura di fine secolo. Roma: Bulzoni.

Ricorda, R. (2015). «ll 'fuoco giorgionesco': da Angelo Conti a D’Annunzio». Archivio d'Annunzio, 2, 129-41. http://doi.org/10.14277/2421-292X/AdA-2-15-9.

Torreggiani, V. (2018). Stato e culture corporative nel Regno Unito. Milano: Giuffré.

Troilo, S. (2005). La patria e la memoria. Tutela e patrimonio culturale nell'Italia unita. Milano: Mondadori.

Ventimiglia, G.M. (2013). «Il ruolo della cultura inglese nella definizione del restauro come disciplina». Tomaselli, F. (a cura di), Restauro anno zero. Roma: Aracne, 284-360. 


\title{
Ruskin in Translation: Versions of Unto this Last in a Few Europeans Languages Toward a Reception History of John Ruskin's Social Thought
}

Jean-Yves Tizot

Université Grenoble Alpes, France

\begin{abstract}
The article proposes to review the reception and transmission of John Ruskin's social thought as expressed in Unto this Last (1862), translated and edited in different European languages. The text itself offers little room for variation, so that interpretations are voiced mainly in the editors' introductions or afterwords, when they exist. This study finds that very few foreign versions do justice to Ruskin's radicalism in his anti-capitalist and anti-industrial critique of modern society, his rejection of 'progress' and his refutation of political economy, while most take advantage of the occasion to express their own political and social views, often in blatant betrayal of Ruskin's ideas.
\end{abstract}

Keywords Anti-capitalism. Anti-industrialism. Anti-machinism. Christian socialism. Degrowth. Democratic Liberal. Interpretation. Liberalism. Liberal Italy. Marx. Marxism. Political economy. Radicalism. Ruskin. Socialism. Social reform. Social change. Translation. Version. Unto this Last.

Summary 1 Introduction. - 2 The Dutch Versions. -3 The German Versions. - 4 The Italian Versions. -5 The French Versions. -6 The Spanish Versions. -7 Conclusion.

\section{Introduction}

Unto this Last (hereafter UTL) is generally recognised as the book which most clearly marks Ruskin's evolution from a mainly arts-centred thinker (though not without views on society) to a critic of modern society in general (including its art and culture). The book was initially a series of separate articles which appeared in the Cornhill Magazine in 1860, but the first essays met with such an outraged response from readers that the editor, William Thackeray, though a friend of Ruskin's, resolved (under pressure from the owner George Smith) to cut the series short, and to limit to four the number of articles the magazine would 
publish. The scandal was caused by Ruskin's open criticism of both "the modern soi-disant science of political economy" ${ }^{\prime 1}$ and the exploitative attitude of employers towards their workers, which the dominant economic doctrine of the time could be used to justify - although it did not overtly encourage it - through the argument of the priority of self-interest in economic relations.

The first essay of the series, "The Roots of Honour", was probably the most shocking and offensive for ordinary believers in the orthodox economic doctrine of the day. It contains what is probably the strongest formulation of Ruskin's rejection of the claims to scientificity of a doctrine he placed squarely in the same category of "popular creeds" such as "alchemy, astrology, witchcraft". ${ }^{2}$ More pointedly, Ruskin attacks in his first essay the fundamental premise of political economy, previously popularised by Adam Smith, that self-interest is the principal motive of actions and exchanges of economic importance. The attack, in spite of the occasional jolliness and pleasantness of tone, is radical and epistemological in nature. It is possibly one of the earliest critiques of the much-debated theory of so-called "instrumentalism". ${ }^{3}$ The essay is not entirely eristic however, and Ruskin also proposes his own view that gain or profit should be secondary in all economic activities, as they are (or should be) in medicine or architecture for example: economic relationships are grounded, overtly or covertly, in moral considerations, and are ultimately expressions of a moral code. Ruskin took stock of the results of the economy of his time and concluded that the moral code on which it was based was harmful for the majority and therefore wrong.

The second essay ("The Veins of Wealth") develops further the idea that service to others is the true meaning of economic relations, and therefore that honour (or honesty) should be the central value in exchanges of all kinds. In the absence of honest foundations for the economy, says Ruskin, the wealth of some entails the exploitation of others: "the art of making yourself rich, in the ordinary mercantile economist's sense, is therefore equally and necessarily the art of keeping your neighbour poor". ${ }^{4}$ In "Qui Judicatis Terram", the third essay of the series, Ruskin prolongs the argument of honourable economic relationships and links it to the question of moral assessment. The deep and innate sense of fairness and justice ingrained in human beings always serves as the existential background and moral index for economic interactions. Unfair compensation for work is thus the sign of a relationship in which employers abuse their privileged position of power to steal (value) from their workers, and both categories are (more or less clearly) aware of that fact. The discourse of political economy is produced to justify this relationship or to conceal it when it cannot be justified. When Thackeray decided to interrupt the series of articles, he nevertheless allowed Ruskin, by way of apology, to write a fourth essay that might be longer than the previous ones. In "Ad Valorem" Ruskin redefines terms which he believed political economists had misunderstood or misused. "Value" features prominently among these abused notions, and

$1 \operatorname{UTL}$ (=Works, 17), 25 .

2 Works, 17: 25.

3 Instrumentalism, as explained for example by the US American philosopher John Dewey, means that theories are valued not for the realism of their assumptions but for their "predictive capacity". In economics, this epistemological doctrine has been endorsed by most mainstream authors in recent decades, and for example by Milton Friedman and Friedrich von Hayek. Ruskin criticises the very fact that the two aspects can be divorced for one another as symptomatic of the loss of perspective of modern political economy.

4 Works, 17: 44

Fonti, letterature, arti e paesaggi d'Europa | Sources, Literatures, Arts \& Landscapes of Europe $1 \mid \mathbf{3 7 6}$ 
Ruskin's analysis leads him to conclude that value is intrinsic to things and objects, and that it consists essentially in their life-enhancing or life-supporting capacity. Ruskin summarised this idea in his famous aphorism "There is no Wealth but Life". This sentence is followed by words that illustrate the moral imperative informing all of Ruskin's investigation of just distribution and criticism of political economy:

Life, including all its powers of love, of joy, and of admiration. That country is the richest which nourishes the greatest number of noble and happy human beings; that man is richest who, having perfected the functions of his own life to the utmost, has also the widest helpful influence, both personal, and by means of his possessions, over the lives of others. ${ }^{5}$

Powerful and shocking as these criticisms and alternative views on political economy might have appeared at the time, the preface written by Ruskin for the book in which the four articles were collected in 1862 must have outraged many a reader beyond anything else the volume contained. But it also contributed not a little to Ruskin's reputation as a "social reformer", anti-capitalist and Communist, among other designations. There Ruskin forewarned readers that the conclusion he would like them to reach after reading the book was no less than the necessity of four broad lines of social reform resulting in

a. a compulsory public training and education system;

b. linked to the latter, a public system "for the production and sale of every necessary of life, and for the exercise of every useful art"; c. a secondary training and placement system to deal with the problems of unemployment and qualification; and

d. "Lastly, - that for the old and destitute, comfort and home should be provided; which provision, when misfortune had been by the working of such a system sifted from guilt, would be honourable instead of disgraceful to the receiver". ${ }^{7}$

Remarkably, this preface, which probably offers one of the clearest summaries of Ruskin's perspective on social and economic issues, is almost never mentioned, or very laterally, in the foreign versions' introductions or editorial notes - just as in many discussions of the meaning of UTL in English-language versions. Certainly, its message does not sit well with most of the ideological prisms through which UTL, as an expression of Ruskin's social views, is usually read and translated. This relative obliteration of the preface in foreign versions is in fact surprising, since UTL is usually chosen among Ruskin's social theory works for its clarity of purpose and expression. One could be led to suspect that the content of the preface is perhaps too clear.

On the whole, although it was cut short by the decision of the director of the Cornhill to terminate the series after the fourth essay, UTL is probably the more elaborate and complete expression of Ruskin's rejection of the "philosophy" which the business and employing classes (and their social dependents) used as a justification of the dominant order, the doctrine of economic liberalism (or political economy). When he began his attack, Ruskin was coming out of a long spiritual crisis, and entering a new phase in his intellectual life:

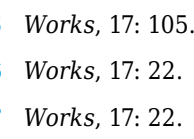



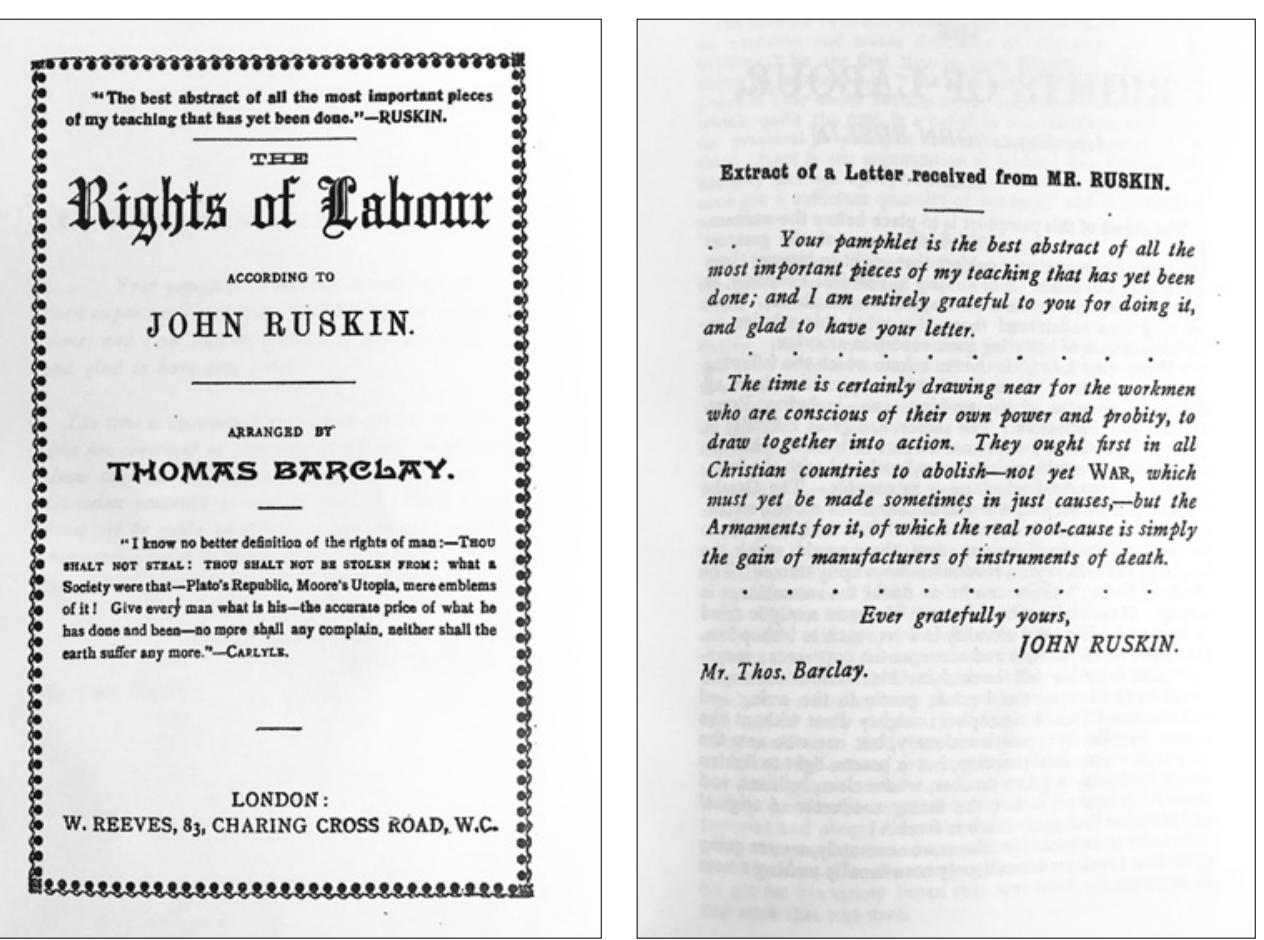

Putting his zeal at the service of this world, he set himself the mission of showing, by every possible means, criticism, satire, invective and sarcasm, that modern society was fundamentally evil, that the freedom on which it purported to rest, more formal than real, served to hide greed. ${ }^{8}$

The book was clearly perceived as a danger by the propo- nents of free trade and the defenders of a separation between economics and ethics. As Anthony notes, "[t]he reaction was also significant in its hostility, as though his critics saw more clearly than his friends had done, the truly radical nature of the attack that he directed". ${ }^{9}$ A leading article in that bastion of free-trade, the Manchester Examiner, expressed in October 1860 the fear that if Ruskin was not crushed "his wild words will touch the 
springs of action in some hearts, and ere we are aware a moral floodgate may fly open and drown us all". ${ }^{10}$

But UTL also garnered the sympathetic attention and enthusiasm of many social reformers, radicals and socialists (who must have looked beyond Ruskin's professed anti-socialism). In a small pamphlet published by a certain Thomas Barclay around 1890 entitled The Rights of Labour according to John Ruskin [fig. 1a]. the author offers in his introduction an interesting (if somewhat sycophantic) characterisation of Ruskin as a social and political prophet: "one of the greatest thinkers of any age" - no less - "Ruskin has been aptly termed the 'Modern Plato'" ${ }^{11}$ Ruskin's views, according to Barclay, can enlighten the working class and help it in its struggle toward emancipation. The subject of the pamphlet, Barclay writes in the introduction, is "Political Economy, in other words the relation of Capital and Labour", and Ruskin's teachings in this respect are essential, because:

[u]ntil working men understand thoroughly what this relation is, all hope is vain of bettering their condition as a class [...] Unto this Last is the book from which the following extracts are taken. It met with bitter opposition from all the usual enemies of the working man, including Press, Priests and Professors. The author had great difficulty in getting it published; a fact not to be wondered at when we consider its revolutionary character, combined with the logic, grace, and vigour of which he is so capable. ${ }^{12}$
There are other reasons, beyond the kind of reverence for both message and style displayed by Barclay in his pamphlet, for the relative success of UTL, at home and abroad, to do with the form and structure of the collection of essays. Jeanne Clegg in her study of Ruskin's "presence" in Italy at the beginning of the Twentieth century, remarks that "the predilection for UTL, with the consequent exclusion of Munera Pulveris and Fors Clavigera" was largely due to "its methodical organisation" (four essays written in numbered sections) as well as "its abstract approach". ${ }^{13}$ That predilection had a purpose:

The desire of extracting from Ruskin's writings a set of distinct, concise and coherent ideas is an almost constant aspect of their reception and transmission in the first half of the Twentieth century, in England as well as in Italy. ${ }^{14}$

This is still true, as the present article will try to show, not only for these two countries, but in the wider international context of the various translations and the different 'versions' suggested by the editors. There are versions of UTL in Japanese, Chinese and I found a reference to one in Malayalam, the main language of the Indian state of Kerala. The ambit of the present investigation is limited to Europe - only a small part of Europe really - but this is a beginning and there is scope for yet more interesting discoveries. The reasons for UTL's worldwide success as a favoured aspect of Ruskin's social ideas cannot be discussed further here at any great 
length. However, it cannot suffice of course to explain it by motives of form or structure, or, as Barclay put it, the "logic, grace, and vigour" of Ruskin's prose. Essentially, UTL's appeal has to do with what Barclay calls its "revolutionary character".

Barclay's booklet displays on page 2 an extract from an undated letter [fig. 1b] from Ruskin to the author which gives another intimation of Ruskin's views in matters of relations between Capital and Labour. Ruskin first kindly describes the pamphlet as "the best abstract of all the most important pieces of my teaching that has yet been done", and further assigns to workers "first of all in all Christian countries" the historical task of abolishing not War itself, but the means of waging war, that is "the Armanents for it, of which the real root-cause is the gain of manufacturers of instruments of death". ${ }^{15}$ For Ruskin, war between "Christian countries" - his approximation for Europe - was fostered by and for the profit of capitalists, as is made clear in many more precise passages in Munera Pulveris, Ruskin's second series of economic essays, notably, but subsequently also in Fors Clavigera. As P.D. Anthony remarked in his study of Ruskin's "social theory"16 "it was Ruskin, not Marx nor Engels" who wrote the following, one of the harshest and most direct condemnations of European capitalists:

Occult Theft - Theft which hides itself even from itself, and is legal, respectable, and cowardly, - corrupts the body and soul of man... And the guilty Thieves of Europe, the real sources of all deadly war in it, are the Capitalists - that is to say people who by percentages on the labour of them; instead of by fair wages of their own. The real war in Europe, of which this fighting in Paris is the inauguration, is between these and the workman, such as they have made him. ${ }^{17}$

Ruskin's anticapitalism originally stemmed from his visceral opposition to industrialism as a mode of social organisation and its associated cultural expressions. In effect, the twin streaks of anti-capitalism and anti-industrialism run unbroken through all of Ruskin's later social commentary writings, but their early roots are in his art criticism, where they also continued to develop in the later phase. Clive Wilmer in his introduction to the Penguin Classics edition of Ruskin's works - tellingly entitled UTL and others writings - outlines the trajectory of a thinker apparently interested only in art initially, but whose commentary and critique becomes increasingly loaded with social preoccupations, and whose aesthetic ideas are ever more linked to or even based on ethical concerns, until this evolution reached a tipping point:

With The Political Economy of Art the balance of Ruskin's work is turned on its head. He began as a critic of art who incidentally commented on the state of society. In the Manchester lectures he was transformed into a social critic who illustrates his arguments with observations about art. [...] That is not to say that Ruskin criticized his age merely because its ugliness offended him. His condemnation of modern culture implied a condemnation of more deeply rooted social failures. What he sought to expose was a society statistically rich that could find no employment for its workers, lamented over-production as a cause of poverty, accepted the notion of planned obsolescence, encouraged an arms race as a source of eco- 
nomic growth, allowed extremes of poverty and starvation to co-exist with ostentatious luxury, professed Christianity but saw poverty as a law of nature not to be tampered with, and expected the majority of its people to rest content in conditions of squalor and brutal ugliness. ${ }^{18}$

In UTL, however, Ruskin's anti-industrialism extends chiefly to the relationship between workers and employers, which he finds finally to be dishonourable and exploitative. Ruskin does not deal extensively in UTL with the questions of inferior standards of work and production, the stultifying and dehumanising routines which operatives are subjected to in manufactures, or the disciplinary implications of factory work, all of which and more are developed elsewhere. The anti-industrial element in UTL is not yet anti-machinist, ${ }^{19}$ and is therefore mostly a function of his anti-capitalist outlook.

The question of the interpretation of Ruskin's critique of capitalism and modern society as expressed in UTL is potentially contentious given the number and considerable variety of the contenders, as the review of some foreign examples will illustrate. Beyond that variety, his intellectual legacy falls broadly into two rather distinct categories. On the one hand there are those who, like the so-called "Organic Radicals" ${ }^{20}$ and various degrowth movements, along with other social critics (J.-C. Michéa in France and Eugene McCarraher in the USA for example $)^{21}$ believe that the critique which Ruskin formulat- ed more than 150 years ago is still valid nowadays, and possibly more so given the state of permanent crisis of globalised capitalism and its catastrophic consequences on so many levels. In this view, Ruskin's "social theory" is incompatible with the acceptance of capitalism and industrialism. The implication is that, in general, Ruskin's ideas have not been carried out in practice, and that "welfare" reforms and the subsequent welfare forms of capitalism are not descended from Ruskin's damaging critique of any form of capitalism or industrial organisation of society - as the four points of the preface as well as the radical critique of liberalism contained in UTL clearly show.

On the other hand, the more commonly held or at least publicly expressed view of Ruskin's social thought stands in stark contrast with that radical interpretation. This is perhaps only an apparent paradox, since this more consensual view proposes a version of Ruskin's ideas which is more readily acceptable by the general public as well as many Ruskin scholars. Perhaps fittingly, it behoved C. Wilmer, the former Master of the Guild of Saint George, to formulate (in 1984, long before he became Master) in his introduction to the Penguin Classics edition of Ruskin writings, what is probably the most widely circulated version of this orthodox thesis. Wilmer concludes that "Ruskin's influence is incalculable", in Britain (and beyond), through the permeation of his ideas into early twentieth-century Liberal "social reforms", post-war Labour social security

\section{Wilmer 1997, 18.}

19 In the sense that machines, according to Ruskin, should not in general replace human work or labour, but make them feasible, or their difficulty acceptable. Ruskin was against 'machinism' understood as the tendency to systematically replace work and labour by machine operations, which results in lower levels of qualification and less interesting work (for the majority of the workforce), and of course is motivated by the search for lower levels of "cost of labour". Ruskin's view is probably unique in articulating such a deep critique of the technological drive of modern societies at the time, and his voice remains remarkably isolated in the present context of almost complete domination of applied techno-science.

20 For a brief presentation see for example https://orgrad.wordpress.com/our-principles/, and on Ruskin as precursor of contemporary environmental awareness https://orgrad.wordpress.com/a-z-of-thinkers/john-ruskin/.

21 McCarraher 2019a; 2019b; 2019c; Michéa 2013.

381 Fonti, letterature, arti e paesaggi d'Europa Sources, Literatures, Arts \& Landscapes of Europe 1

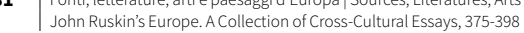


and nationalisations and, later, consensus-politics welfarism. ${ }^{22}$ To take up but one of these "examples", the so-called social reforms of the Liberal governments, it is well-known, were directly inspired from very different quarters, namely Bismarck's Germany, which Lloyd George visited for closer inspection before adopting and adapting for Britain several socio-economic adjustment measures, among which state pensions in 1908.

In terms of Ruskin's own thought and action, this view is very debatable. For one thing, the "incalculable influence" thesis only takes into account one side of Ruskin's ambivalence towards the problem of injustice, when in fact Ruskin represents "a rare embodiment of that contradiction between material improvement and root and branch opposition to the prevailing economic system". ${ }^{23}$ But more fundamentally because "[t]he 'practical' side of Ruskin is often banal and always ineffective", ${ }^{24}$ and is no match, or equal counterpart, for his ethical criticism. In deciding whether Ruskin's more important legacy is that of a unique fundamental opponent of capitalist industrialism or that of a rather unimaginative benevolent reformer, his limited, often amateurish and more or less charitable endeavours, including those of the Guild of St George, cannot easily be considered to represent a valid counterargument to his sweeping epistemological and moral attack against political economy, especially as expressed in UTL. By contrast to the failure of Nineteenth century radical criticisms of the machinery issue to address the "social problems" as fundamentally economic in nature - "a failure which acted to protect political economy from the criticisms of its methodology and its doctrines and industrialisation", according to Maxine Berg - ${ }^{25}$ "Ruskin goes beyond radical criticism of social questions by maintaining a most resolute intellectual criticism of political economy". ${ }^{26}$

That the "revolutionary character" of Ruskin's proposals was never incarnated in practical policy terms has not prevented the more benign view of Ruskin as a "social reformer" and central inspirator of today's society to enjoy a considerable degree of success, at least ever since J.A. Hobson's John Ruskin. Social reformer (1898). In wider terms, Ruskin's influence is often confused with the influence that various interpretations of some of his ideas may conceivably have had. The National Health Service, the provision of free libraries and museums, a number of "socialist" policies, Philip Snowden or M.K. Gandhi might be said to have been influenced by Ruskin, but fundamentally these policies and people differed from Ruskin in intention. The ultimate target of Ruskin's criticism of modern society is industrialism and capitalism because of what they did to human work, and that he wished undone. ${ }^{27}$ Ruskin denounced and opposed the employment relationship of capitalism and the work practices of industrialism. There is of course formidable evidence that employment relationships have globally deteriorated and work practices become more tiring, stressful or de-humanising over the past period of so-called "neo-liberalism". But writing before the early stages of the latest phase in the evolution of capitalism ("neo-liberalism"), P.D. Anthony already observed the

22 Wilmer 1997, 30.

23 Anthony 1983, 123.

24 Anthony 1983, 123

25 Berg 1980, 296.

26 Anthony 1983, 123.

27 For further developments on this aspect of Ruskin's influence see for example Anthony 1977 and Anthony 1983.

Fonti, letterature, arti e paesaggi d'Europa | Sources, Literatures, Arts \& Landscapes of Europe $1 \mid$ 
documented failure of welfare capitalism to improve dramatically the condition of the working man:

Explanations of this fundamental failure range from attacks on the sectionalism and economism of the trade unions, on the corruption of the leaders of the Labour Party, on the bureaucratic tendencies of all labour institutions, on the overpowering hegemonic control of capitalism, on the influence of the communication media over the working class, on 'consumerism', on social conditioning of the proletariat, on socialisation. The most likely explanation for the failure of the proletarian apocalypse lies in none of these; it is that the condition of the working man's life has, in many cases, not improved but has deteriorated, that its deterioration has been made acceptable to him by financial reward and by limitations of its duration, by the better provision of 'fireside humanity'.

But that is not sufficient, says Ruskin. We cannot set out to atrophy human qualities in men's working lives and expect them to flourish at night and at the week-ends. ${ }^{28}$

The view that Ruskin's ideas have been carried out in practice was both extended and deepened in the past years by the Financial Times journalist and author Andrew Hill, who started referring to Ruskin in reasoned defence of top management pay levels in his column around the turn of the new millennium. ${ }^{29}$ The 2013 Pallas Athene edition of UTL has a short introduction by Hill preceded by a shorter foreword by the Master of Guild - an interesting indication of a certain consensus. In it Hill bemoans the fact that, for too long, "the left annexed Ruskin's ideas on social reform", then quotes approvingly Wilmer's verdict from the Penguin Classics edition that "no political label quite fits Ruskin's politics", to reveal, with a great sense of continuity and relevance, his interpretation of UTL:

In fact, looked at as a kind of moral for capitalists, Unto this Last - which reflects Ruskin's devotion to order and hierarchy in society, not to equality as such - offers much that the moderate right should appreciate. ${ }^{30}$

This idea made more progress in 2019 with Hill propagating the view, in a book appropriately published the year of Ruskin's bicentenary, Ruskinland, that Ruskin's legacy can be traced "almost everywhere" (the book is suggestively subtitled How John Ruskin shapes our world). If Ruskin's shadow is everywhere in today's society because he contributed so much to its shaping, he could not be too radically opposed to the general direction of its evolution: quid est demonstrandum. Given its sociological bases, the consensus across the centre of British political ideology between left-of-centre welfarism and the "moderate right" on the meaning of Ruskin's contribution is a solid one. Conversely, the radical interpretation is fragilised by is marginality. Their opposition points to Ruskin's own ambivalence, and, beyond, to the importance of Ruskin's voice for the present day, and, perhaps, for the future.

Even though the rift between the radical and the reformist approaches is cardinal, interpretations of UTL are not always, or exclusively, determined by political ideology. What follows confirms the (obvious) assumption that each editor (sometimes also in the capacity of translator) of UTL in a foreign language is given an opportunity to appropriate the meaning of the book and,

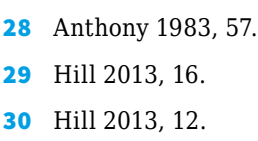


through it, the whole of Ruskin's "social theory", by bending and twisting the interpretation of that theory to fit particular goals. Various attempts at appropriation of
UTL have come from such improbable intellectual landscapes as a suspicious branch of yoga or the Vatican-approved "economic theory" of the Focolari, among others.

\section{The Dutch Versions}

2.1

I have found two translations of UTL in Dutch, only of them with an introduction. The first Dutch version [fig. 2], published in 1901, is entitled Dezen Laatsten Ook ("These Last Too"). The translator was Paul Horrix, a free-thinker, pacifist, internationalist and also a psychic. Horrix was the private assistant of a certain Dr Pieter Eijkman, a diagnostic radiographer, naturopath, hygienist, anthropologist, and, like Horrix, an internationalist pacifist. The pair became famous a few years after the translation of UTL came out, in 1905, when they proposed a plan to build the "World Capital of Internationalism" among deserted dunes near The Hague, with a view to propagating the idea of an international union of states in favour of world peace, in the wake of the 1899 Hague Peace Conference.

\section{2}

The second Dutch version is purely a translation, without editorial apparatus. The translator was a certain Hugenholtz-Zeeven, who also translated William Morris's series of lectures Art and Society [fig. 3a]. It is entitled De Laatstgekomenen ('The Last Arrived' or 'The Last Come'), which is also close to the original and refers directly to the parable of the workers in the vineyard. Typically, it also dates from 1901, and belongs in the international wave of translations that appeared in the wake of Ruskin's death. Interestingly, it is the last issue in a collection called the "International Library" ('Internationale Bibliotheek'), which seemed to specialise in "socialist" (in the broad sense) authors such as Marx, Kautsky,
The very short (one page) introduction does not really help in determining what kind of Horrix's interpretation of UTL was, and why he might have wanted to propagate Ruskin's ideas. As verbose and vague as it is short, the introduction explains that Ruskin was "preoccupied" by "so-called socialism" (Horrix even uses the word "fear). There is no way to know whether Horrix understood what Ruskin meant exactly by socialism (i.e. mainly skill- and category-based workers' mobilisation), or if he was in favour of a radical change of economic system, or indeed shared Ruskin's radical anti-capitalist outlook. There is the possibility, based on a gross misunderstanding, that Horrix wanted to be associated with Ruskin because of his apparent "anti-socialism".

Bebel, Bellamy, Morris, Blatchford, the Barnetts, among others, but also in "progressive" authors like J.S. Mill or Henry George, or even Christian reformers as diverse as Tolstoy and William Booth [fig. 3b]. Given this background, it is all the more regrettable that the translator or editor did not write an introduction as they seemed to situate Ruskin within a group of radical critics of capitalism and sometimes "anti-systemic" thinkers.

There do not seem to be more recent versions of UTL in Dutch or Flemish but of course further research will be needed to ascertain that. 
It seems that there have been four or five versions of UTL in German so far. One of them, however, is a rare book which I have not been able to see at this time, entitled

\section{1}

Diesem Letzten (To This Last), with an introduction by Christine $\mathrm{Ax}$, is the latest German version published to date (it came out in 2017). The text itself, however, is a reprint of the first translation of UTL ever published in German, the work of a certain Anna von Przychowski. This is the German version mentioned in the Library Edition's Bibliographical Note (BN). Typically, the Przychowski translation came out in 1902, in the wake of Ruskin's death. The Library Edition's Bibliographical Note indicates that "[a]n introduction by the editor (Wilhelm Schölermann) occupies pp. 5-8", and that it has "an index (not translated from Mr. Wedderburn's), pp. 182-196". This original edition is very difficult to find and I have not seen it. A special 2019 bicentenary edition (of the 2017 version) was produced with a different cover picture, and on page ii an Otto Eckmann Jugendstil frontispiece from the original 1902 front cover, an ornate box containing the word "Ruskin" in Eckmann's signature typeset. I have only been able to obtain a copy of the introduction by Wilhelm Schölermann ("Zur Einführung") on the internet, but have not been able to verify its authenticity as yet. The introduction asserts the importance of Ruskin's social and political thought (as expressed in UTL), and welcomes the publication of the first German translation in the fifth volume of Ruskin's selected works. Schölermann seems to think that Ruskin advocated the rediscovery of the personal dimension of social relations, and the necessary harmony of the latter - an approach which is supposed to proceed from Ruskin's knowledge of art, which gave him
Die Adern des Reichtums ("the Veins of Wealth" which is the title of the second essay in UTL). The other two are more easily available and better known.

a special insight into the laws of organisation. This is rather perceptive, but the resulting social and political interpretation falls far short of Ruskin's own ambition to change the organisation of society deeply and durably so as to make exploitation, injustice and dishonesty as residual as possible. Schölermann also salutes Ruskin's effort to redefine the 'real' meaning of value as the life-enhancing power of things and processes, as opposed to their prices as commodities.

The 24-page introduction by C. Ax is entitled "Nur Leben ist Reichtum", literally "Only life is Wealth" - a paraphrase of "There is no wealth but life", one of the most famous sentences in UTL, from the fourth essay, "Ad Valorem", §77). The back cover tells us that Ax is an economist, philosopher, science teacher and author who researches and writes on questions of sustainable development with a particular emphasis on local economies, manual work and degrowth. She teaches and researches at the Sustainable Europe Research Institute (SERI) of Vienna, Austria. In 2013 she co-authored Wachstums Wahn ("Growth Madness") with F. Hinterberger, also from the SERI. According to Ax, Ruskin figures prominently amongst the socalled "precursors" of degrowth, and he was also an early champion of a "basic unconditional income", and a promoter of "social entrepreneurship" avant la lettre. In her view, environmentally aware economists, environment activists and critics of economic growth share Ruskin's fate of disparagement and defamation by the established authorities, especially when they are accused of being 


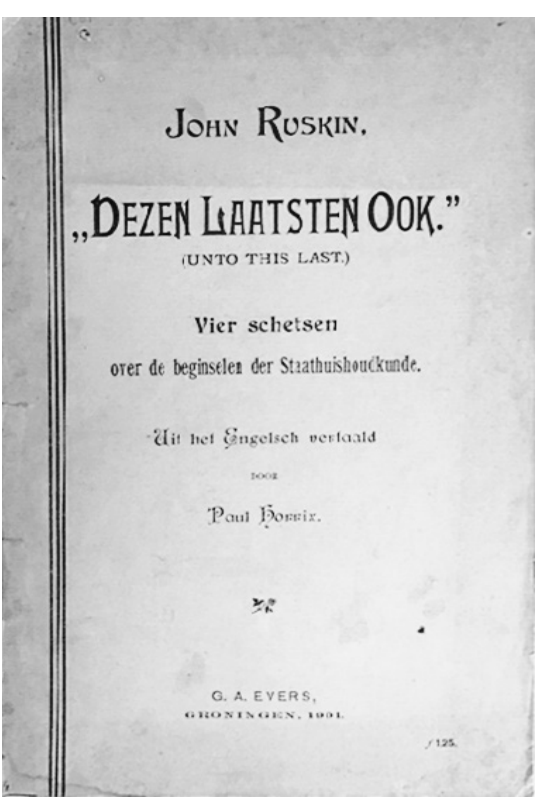

Figure 2 Horrix, Paul (ed. and transl.). John Ruskin, "Dezen Laatsten Ook" (Unto this Last). Vier schetsen over de beginselen der Staathuishoudkunde. Groningen: G.A. Evers, 1901. Front cover. Free of rights
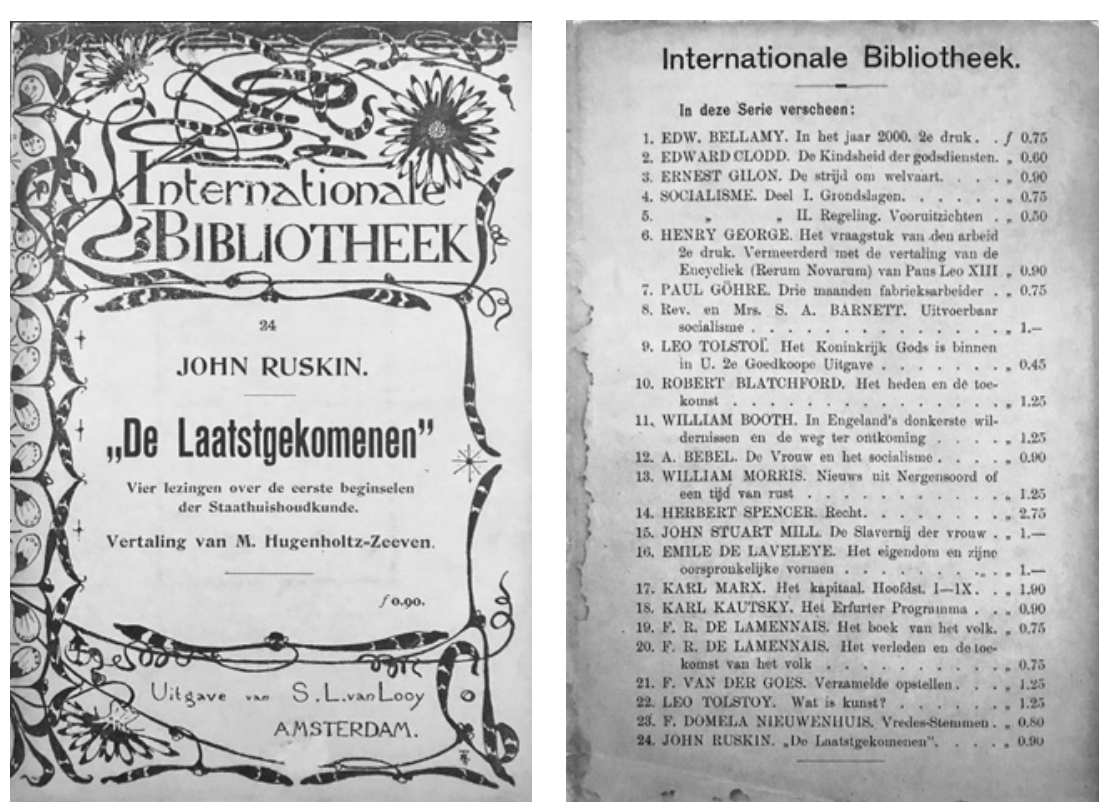

Figures 3a-b Hugenholtz-Zeeven, M. (transl.). John Ruskin, "De Laatstgekomenen". Vier lezingen over de eerste beginselen der Staathuishoudkunde. Amsterdam: S. L. van Looy, 1901. Front cover. Free of rights "enemies of Progress". In Ruskin she sees an early and powerful critic of what Lewis Mumford called the "Megamachine" (Mumford, The Myth of the Machine, 1967-1970), the techno-scientist system that subjects individuals in advanced societies to the yoke of technology.

More generally, Ax seems to endorse Ruskin's critique of the capitalist economy and of its theoretical justification, the doctrine of economic liberalism. However, the final paragraphs of Ax's introduction expose an interesting interpretative twist, in total contradiction with Ruskin's no- tion of work: building on the link between Ruskin and his most famous follower, William Morris, Ax extrapolates that "neither was an enemy of machines". While Morris was at best ambivalent in his attitude to machinery and the necessity and morality of the use of machines, Ruskin was in fact probably as close as it is possible to be to an anti-machinist, on several levels. As P.D. Anthony put it rather convincingly in his John Ruskin's Labour, for Ruskin "[m]echanical work is to be avoided, because its performance entails the loss of control of the workman in his operation. If avoidance is impossible, the simplest forms of 
machinery must be used first". ${ }^{31}$ Ax nevertheless contemplates the possibility that the increased computerisation of social relations, first and foremost of which work relations - so-called "digitalisation" - might succeed where the Arts \& Crafts movement, the workers' movements and Communism have failed, that is to say in the re-appropriation of work and leisure. Ruskin, of course, was a consistent enemy of (so-called) progress, a foremost opponent to capitalism and industrialism, and a radical critic

\section{2}

The second recent German version of UTL bears the same title as the first, Diesem Letzten, and actually first came out in 2011, before the version just reviewed. There was a second edition the same year, and a third in 2015, perhaps a sign of commercial success. The translator and editor is Uwe David, who wrote an introduction as well as an afterword. David relied heavily on the Przychoski translation but modernised or adapted some of the vocabulary "for today's readership", that is often into more casual language. The editorial production is of rather elaborate and well-researched, complete with original footnotes and the translator's own footnotes (with their own reference endnotes in the last pages).

As for the interpretation suggested by the introduction and afterword, this is a very surprising version of UTL. One the one hand, the reading of Ruskin's UTL suggested by David in his introduction is not very original in itself, and in fact rather benign. Unsurprisingly, David posits the relevance of Ruskin's critical mes- of the domination of the technological and scientist mentality and practice, not an occasional detractor of their "excesses". "Progressive" thinkers of all hues who, unlike Ruskin, do not fundamentally question the existence and perpetuation of capitalism, might frequently find it difficult to reconcile their fundamental belief in "progress" (of the kind that can take place within "reasonable" capitalism) with what Anthony calls Ruskin's "resolute intellectual criticism of political economy". ${ }^{32}$

sage to "the problems of our time", ${ }^{33}$ but Ruskin in his view was neither pro-capitalist, nor communist, but a critic of contemporary society with a message of personal moral reform. On the other hand, readers might promptly suspect that the aim of David's enterprise in publishing a new German version of UTL was not essentially about expressing Ruskin's "message" in more contemporary German than Anna von Przychowski's translation. The longer afterword (14 pages) is where readers really find out about David's real motivations, even though the second page of the book is an early giveaway: it displays a portrait of "Shri Mataji Nirmala Devi" (1923-2011), who is thanked "for her inspiration and her support". Shri Mataji was the founder of a new form of "yoga" after she experienced (in 1970) a "spiritual awakening", during which she felt that she had found the way to bring about "en-masse Realisation" (through "Sahaja Yoga" or "spontaneous yoga"), which could then enable mankind to "formulate collective and sustainable answers to the challenges of our 
times", ${ }^{34}$ a change called for and "announced by all the Great Scriptures". According to David, Ruskin was obviously a "Great Awakened", and the sacrifice of his for- mer comfortable life of fame and success for the pursuit of a moral mission is evidence for David that Ruskin was himself nothing less than a "Guru".

\section{The Italian Versions}

Probably because of Ruskin's prolonged and intense relation with the country, Italy has produced many versions of UTL, probably the most of any single country. They are examined here (more or less) by order of appearance. Guido Guerzoni in his study of "The Italian reception of Ruskin's economic and social criticism 1850-1950" remarks in his opening words that his contribution might with reason have been entitled "The very partial and tardy Italian reception of John Ruskin's economic and social thought" ${ }^{35}$ The conclusion of partiality of reception is of course universally valid, given the polemical nature of Ruskin's ideas, while that of tardiness is probably true for most countries outside the UK, where Ruskin's social ideas were known as soon as they were published. Guerzoni further notes that in spite of extensive research he was not able to find "any significant trace in any form whatsoever of his economic and social thought during the period between Italian Unity [1870] and the date of his death". ${ }^{36}$ Again, the finding applies as a general rule to all the countries reviewed here. Guerzoni mentions ${ }^{37}$ "the text presented as the first Italian edition of UTL", I diritti del lavoro, published by Luigi Mongini in Rome in 1900. Mongini (like the second Dutch translation reviewed above) placed Ruskin in the same catalogue as eminent socialists (Marx, Engels, Lassalle etc.), but his UTL was in fact a translation of Thomas Barclay's The Rights of Labour according to John Ruskin, by Ernestina D'Errico. It does in effect present a version of UTL, but the 24-page editing work, as far as I can gather, is Barclay's, not Mongini's (the cover indicates "riassunti da Tommaso Barclay", "summarised by Thomas Barclay").

probably a reprint of the same translation, from 1936, but which mentions only Francesco Chimenti as translator. ${ }^{38}$ This one has a one-page foreword about Ruskin, in which Chimenti notes that Ruskin "introduced the moral element of justice" in his study of "social sciences and

34 Translated from the site French Sahaja Yoga website: https://sahajayoga.fr/.

35 Guerzoni 2006, 136.

36 Guerzoni 2006, 143.

37 Guerzoni 2006, 147.

38 I am very grateful to Jeanne Clegg for providing me with a copy of this text, which is not easily available. 
political economy". In the same vein, he comments that in UTL Ruskin "proclaims human dignity in society and condemns any principle or action that considers Man as a subpart of a machine".

The short introduction is followed by a longer "Note out of place, as a reminder of how much England owes us", three

\section{2}

The second Italian version dates from 1908 and is entitled Le fonti della ricchezza (literally "The Sources of Wealth", close to the title of the second essay in UTL) and is the work of the famous anti-Fascist politician Giovanni Amendola. ${ }^{40}$ This is another example of an early-twentieth century translation, and it is typical of the "liberal" interpretation which prevailed at the time. Amendola (1882-1926) was initially a journalist and professor of philosophy at the University of Pisa, and later in politics was a member of the Radical Party for two decades and later for a few years of the Democratic Liberal Party. He was always a determined opponent of the great Liberal leader and several times Prime Minister Giolitti, whose parliamentary opportunism ("transformism"), clientelism and corrupt practises he did not condone.

In the short but dense introduction (8 pages) to his 1908 translation, Amendola makes it clear that he sees Ruskin as a sort of "primitive Christian", an "economic apos-

\section{3}

Amendola's translation has been republished without his introduction in recent years (between 2003 and 2014) pages of rambling remarks on the greatness of Roman, and therefore Italian, civilisation, as well as an attack on Eden in the wake of the Abyssinia crisis. As Jeanne Clegg observed, "this preface [...] is neither the first nor the last of the Fascist uses of Ruskin, which were in fact not limited to Italy only" (here a note refers to the founder of the "Ruskin Societies" J.H. Whitehouse). ${ }^{39}$ tle". Although Amendola had been a Socialist Party member as an adolescent and belonged to the left wing of the Liberal movement, he was no socialist, and a Marxist even less. In this introduction he wished Ruskin's positive influence might have extended to the Italian socialists, whom he saw, along with the economic liberals, as proponents of "the same economic exaggerations against which Ruskin fought his battle".

Amendola's version of Ruskin's UTL and of Ruskin himself set the tone and the vision that would dominate after him, perhaps to this day still, in Italy. His political fame and his tragic end at the hands of the Fascists have certainly contributed to add respectability to his interpretation of Ruskin as a Liberal Christian "social reformer". This situation has done nothing, conversely, to clarify Ruskin's attitude to capitalism in general and his singular notion of (non-Marxist) Communism - two aspects of Ruskin's thought that Amendola did not see, or did not want to discuss. 
Figure 4 Villani, Felice (ed. and transl.). I diritti del lavoro (Unto this Last). Bari: Laterza e Figli, 1946. Front cover. Free of rights

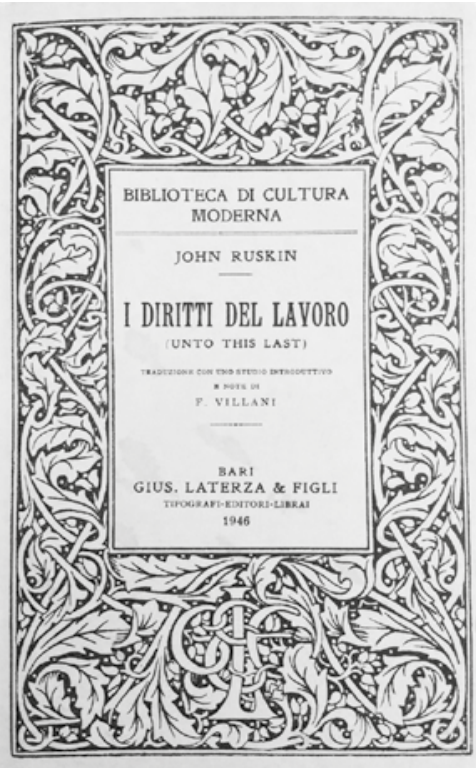

good translation since the employer of the workers in the vineyard wanted to pay the last workers first...), and $A$ quest'ultimo ("Unto this Last"), both with the erroneous subtitle "Four Essays in Christian Socialism" (the original subtitle being "Four essays on the first principles of political economy"...). These recent reprints have no

\section{4}

The next Italian version is I Diritti del lavoro (1946) by Felice Villani, translator and editor [fig. 4]. The title, which translates as "The Rights of Labour" was probably borrowed from the Mongini edition mentioned earlier, and so indirectly from Thomas Barclay's pamphlet. Interestingly, Villani's book is dedicated "to the memory editorial apparatus, only a few words on the back cover simply stating that "Ruskin's warning [...] portended the values of the anti-globalisation movement and the factors behind the crisis of the world economy with tragic lucidity". The overall impression is one of opportunistic substandard publishing work.

of Giovanni Amendola, who in Italy first understood and divulgated this work of Ruskin's" ${ }^{41}$

Villani wrote a long "Introductory Study" ("Studio introduttivo") - 82 pages in all - which is very dense, as well very erudite and well written, and makes for one of the 
most interesting introductory pieces for UTL. In it Villani competently places Ruskin's work and its evolution in its historical context of British liberalism and its discontents. For Villani, Ruskin the art critic and the avuncular moralist is "the minor Ruskin", or "not the best Ruskin". The "best Ruskin" for Villani is the Ruskin of the so-called "economic writings", UTL and Munera Pulveris, because they still speak to us today of problems and issues of our contemporary world..$^{42}$

Villani places Ruskin'S thought between individualism (the necessity of individual moral reform) and socialism (a moral economic life for a democratic and just community). Villani, very much like Amendola, belonged to the liberal democratic Christian left. Both believed that the evangelical message should serve as the underlying moral doctrine of that democratic liberal left-wing, hence the attraction of some of Ruskin's Gospel-inspired views. Villani offers thus a very articulate interpretation of $U T L$, and in a way it is probably the classic middle-of-

\section{5}

Cominciando dagli ultimi is the most recent translation of UTL into Italian, published by Edizioni San Paolo in a collection called "Classics of Christian Thought". The translation was the work of Riccardo Ferrigato and is introduced by Luigino Bruni, an economist working in the Christian social tradition inherited from the social doctrine of the Church, who is currently involved in the "Economy of Communion project", which seeks to promote internationally the community-based business model initially put in place by Silvia "Chiara" Lubich in the "Focolari" movement (officially the "Work of Mary"). the-road, consensual, "liberal" view of Ruskin's politics, which still dominates to this day. Villani comes back to the definition of the balance between liberalism and socialism several times, and offers a good summary of this view: for him the ideal economy would be "a social economy, not a socialist economy; a liberal economy, not a laissez-faire economy" ("Economia sociale, non socialistica; economia liberale, non liberistica")..$^{43}$ That this approach can be viewed by some as "left-wing" or "socialist" in any way is perhaps an indication of the state of contemporary political ideology rather than a faithful representation of Ruskin's own ideas. According to Guerzoni, however, Villani “placed Ruskin's economic and social criticism in its true historical context, withdrawing it, who knows for how long, from the rapacious hands of all those who wanted to appropriate its spoils and brandish them like a banner". ${ }^{44}$ That is well said, but Villani cannot escape the simple fact and common rule that an interpretation is an interpretation.
Bruni sees Ruskin as a critic of Nineteenth-century capitalism and its "theory", but adds that Ruskin "today would be even more critical of our capitalism and our economic theory, which have exacerbated the vices which Ruskin identified and stigmatised in his generation". Bruni places Ruskin "on the side of the utopian socialists (he attempted, as is known, to build, like Robert Owen, an ideal community of labourers), of Karl Marx, of John Hobson, and, in Italy, of Arturo Labriola, Achille Loria, Ugo Rabbeno, so many theoreticians of cooperation and many social Christians". In this long

\footnotetext{
2 Villani 1946, 6.

3 Villani 1946, 61

Guerzoni 2006, 150-1.
} 
list of comrades and companions, Ruskin stands out, however, because of certain "remarkable notes of modernity and originality" which are especially visible in UTL according to Bruni, who reviews two of these points in more detail.45

The first one is that Ruskin refutes and refuses the view that self-interest is the first and only motivation of all economic behaviours, of work in particular, and Bruni put a special emphasis on the importance of what Ruskin called "social affections". The second point underlined by Bruni is Ruskin's singular definition of wealth. Bruni does not mention or comment on Ruskin's fundamental definition of wealth, which is anything and everything that contributes towards the maintenance and increase of life, a conception expressed in his famous aphorism "There is no Wealth but Life" ("Ad Valorem", § 77). Bruni understands, perhaps hastily, that Ruskin viewed economic inequality (whether of income or wealth is not clear) as necessary to the maintenance of the motivation to work in a "market economy", because in the absence of a "wealth incentive", workers would tend not to want to work. Besides the fact, known to most economists, that income is not the same thing as wealth (in many respects), Ruskin probably thought otherwise, since his emphasis, as remarked earlier, is principally on moral motivations and "social affec- tions". More generally, Ruskin's economic thought is set against an ideal horizon which is not "the market economy", or even less capitalism, but some form of social organisation radically different from, and in many ways opposed, to both.

In his introduction Bruni refers readers to Giuseppe Toniolo and "a large number of the Catholic economists from the Nineteenth century to the present day". Toniolo (a Catholic Saint since 2011) is an interesting figure of the right-wing Italian Catholic Movement. He founded the Catholic Union for Social Studies in 1889, and in 1894 elaborated the "Programme of Catholics Against Socialism" (also known as the "Milan Programme", after the 1894 Milan congress of the Union), in the wake of Rerum Novarum. It is possible to consider that the ideas once propagated by Saint Toniolo live on in various forms, in particular in the so-called "Economy of Communion" (EoC). Like Toniolo's own views, the EoC is an economically liberal, business-oriented model, and the evil of capitalism is seen to reside mainly in finance. This kind of 'criticism' of capitalism (capitalism produces poverty and exclusion...) might make recruiting Ruskin ("reddest of red"!) into a charismatic Catholic social reform movement either very difficult, or simply counter-productive, if anyone with a notion of what Ruskin was about is watching.

\section{$5 \quad$ The French Versions}

There are two French versions, typically published more than a century apart. They seem to be some of the most radical of all interpretations, even including the Brit- ish ones. But even today Ruskin in France remains little known, or only superficially as a "reactionary" or "Christian socialist". In an article devoted to Ruskin's influence 
in France, Bénédicte Coste ${ }^{46}$ noted that the first translations of Ruskin into French, in the early 1890s, were in fact fragments dealing with moral and social themes, not aesthetics (this was the work of "social" Catholics in bulletins of their society L'union pour l'action morale), but also that the near-exclusivity of the reception of Ruskin as an art critic in France, in particular in the wake of R. de la Sizeranne's Ruskin et la religion de la beauté (1897), has resulted historically in the almost total oblit-

\section{1}

The first French version of UTL came out in 1902 under its English title, with the subtitle "Il n'y a de richesse que la vie" ("There is no wealth but life") [figs. 5-6]. The title page repeats the English "Unto this Last" and has, in brackets, a translation of the phrase: "Même à ce dernier..." ("Even to this last..."). The translation itself was the work of Emile Peltier, a Catholic abbot as well as a fervent internationalist Esperantist, and socially involved on the "social" side of French Catholicism.

The substantial introduction (33 pages) is signed "H.-J. Brunhes". This is not one name, but two names in one: the introduction was the result of a collaboration between Henriette and Jean Brunhes, a couple of prominent "social Catholics". Not surprisingly, the introduction extols Ruskin's vigorous indictment of the "pseudo-science of political economy", and insists that it reflected Ruskin's "religious beliefs", and accorded with the new social doctrine of the Church. Peltier and the Brunheses were sympathisers of the same social movement, that of the progressive Catholic bourgeoisie who criticised the global role of science "in the service of gold", "to give and confirm to the voracious appetites of the rich their titles of legitimacy". ${ }^{47}$ eration of the other aspect of Ruskin's thought. The scarcity of French translations of UTL, or indeed of any of Ruskin's economic and social writings to the present day (a situation which Coste calls "the strange case of John Ruskin, or how French translations have 'forgotten' his political and social thought") is both a sign and a consequence of this blinkered reception which began even before Ruskin's death.

Jean Brunhes (1869-1930), born to an educated Catholic middle-class family, was an eminent geographer, one of the founders of modern human geography. Politically he was on the left-wing of social Catholicism in France and an early-days member of Marc Sangnier's movement "Le Sillon". Jean Brunhes's wife, Henriette (1872-1914), was also a devoted Social Catholic, and was one of the founders of the "Ligue sociale des acheteurs", a consumers' cooperative on the model of what was being done in the USA at the time. This was not their first encounter with Ruskin, since together they had already published Ruskin et la Bible, pour servir à l'histoire d'une pensée ("Ruskin and the Bible, towards an intellectual history") in 1901. They were no political revolutionaries, socialists, communists or anarchists, and they seemed to hope for a form of capitalism civilised by the application of interpretations of the Gospel such as Ruskin's own, but they placed Ruskin among their favourite protesters "against the new tyranny of our modern societies", together with Carlyle, between the German archbishop Ketteler and the "very first theorists of socialism and anarchism". Their 


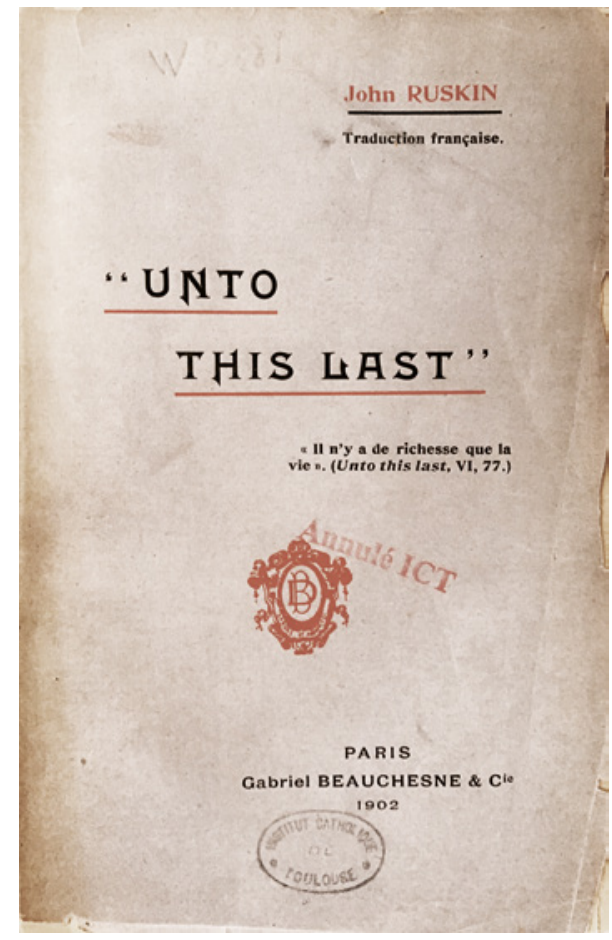

Figure 5 Brunhes, Henriette and Jean (éds); Peltier, Emile (transl.). Unto this Last. Il n'y a de richesse que la vie. Paris: Gabriel Beauchesne et Cie éditeurs, 1902. Front cover. Free of rights

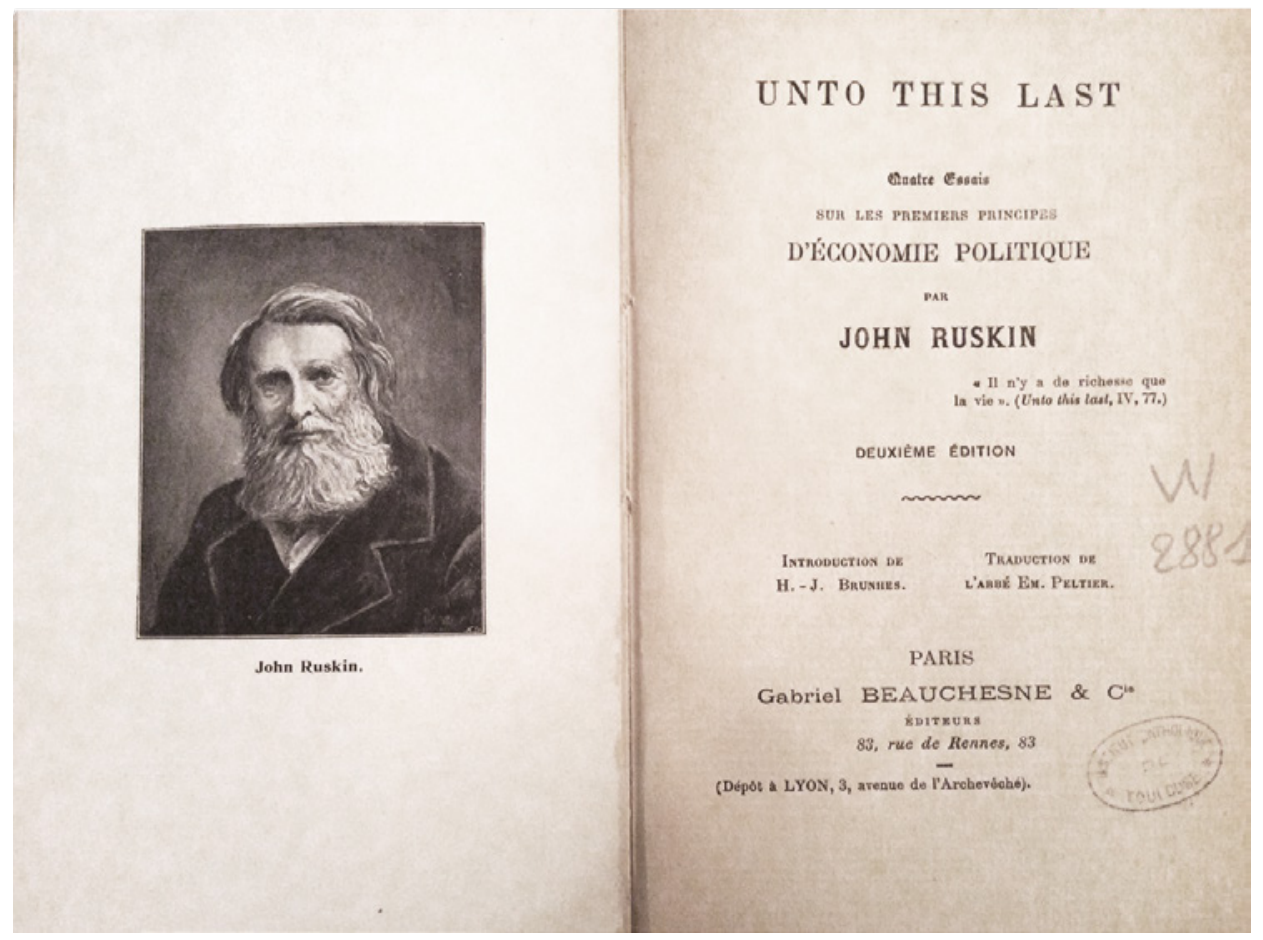

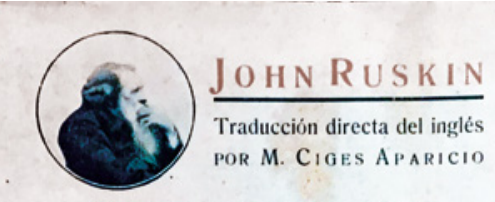

-UNTO THIS LAST HASTA ESTE OLLIMO

\section{Estidios Sociales}

Cuatro estudios sobre

Economia politica
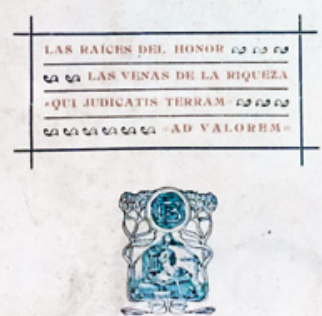$$
\text { MaDriD }
$$

Figure 6 Brunhes, Henriette and Jean (éds); Peltier Emile (transl.). Unto this Last. Il n'y a de richesse que la vie. Paris: Gabriel Beauchesne et Cie éditeurs, 1902 Title page. Free of rights

Figure 7 Ciges Aparicio, M. (ed.). Unto this Last (Hasta este último). Libreria de Fernando Fé, Madrid, c. 1910. Free of rights 
interpretation of Ruskin's ideas in UTL extends beyond political economy, and like Ruskin himself, they criticise Western civilisation for erring and losing itself into capitalist and industrial barbarity. ${ }^{48}$ For them, UTL is proof that Ruskin was a precursor of their struggle for more justice and, above all, more dignity:

"UTL was a manifesto and it remains a document: in this double capacity, it belongs not only in the history of literature, but in the history of ideas; it deserves, in truth, to take its rightful place in the history of economic doctrines". 49

\section{2}

The most recent French version (2012) is by Pierre Thiesset and Quentin Thomasset, both editors and translators, and is entitled Il n'y a de richesse que la vie ("There is no Wealth but Life"). Thiesset and Thomasset are two former students of journalism at odds with the current media world and its race for fast news, e-mail alerts and news flashes, the instantaneousness of the social media, and so on. Its legal status is that of a not-for-profit voluntary sector organisation. There is a short "editor's note" (4 pages) with significant references: William Morris (for socialism and sobriety), Gandhi (frugality and austerity) and Nicholas Georgescu-Roegen (for degrowth) are seen as heirs to Ruskin's ideas. There is no mention of the Brunheses' interpretation, even if the notes from their edition have been kept for
The Brunheses' interpretation of UTL, though not squarely anti-capitalist, managed to capture some of the radicalism of Ruskin's critique of capitalist social relations, which is more than can be said of many other versions. Theirs remains a deeply religious interpretation of Ruskin's social ideas and they believe that "the Old and New Testaments not only are omnipresent in Ruskin thought, but they are also the deep cause of its progress", ${ }^{50}$ a view not necessarily shared by all readers of Ruskin nowadays.

this text. The editor's note states a critique of capitalist productivism, industrialism, consumerism collectively described as an "impasse". ${ }^{51}$ The suggested reading however is clearly pro-degrowth - both translators-editors are members of the French "décroissance" movement. Thiesset is a regular contributor to the monthly review La décroissance and wrote a section on Ruskin in a book devoted to fifty presumed "precursors of degrowth", ${ }^{52}$ where Ruskin is described as "a major author against industrial civilisation, who struggled to defend a world infused with beauty, faith, honour and harmony and not based on the relentless development of productive forces". ${ }^{53}$

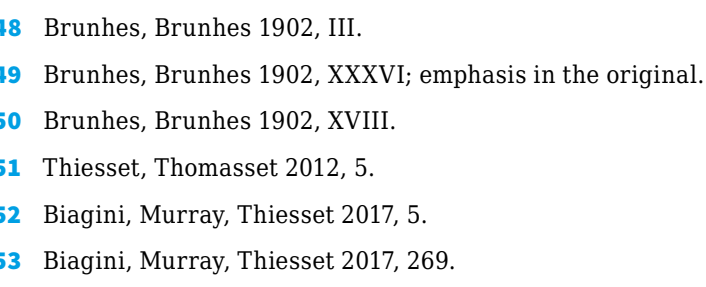




\section{The Spanish Versions}

A Spanish version entitled Unto this last (Hasta este último), a "direct translation form the English by Mr Ciges Aparicio", came out around 1908-10 [fig. 7]. It does not have an introduction (but has its own index). Another version was published under the title $A$ este último in 2002. The text was translated by Dolores Mármol and Paulino Fajardo, and the latter is the author of a long introductory study (86 pages), a text adapted from his doctoral thesis research entitled "Business management and social justice". Fajardo is a lawyer and partner at the international consultancy Davies Arnold Cooper and a specialist of civil liability. On UTL, his outlook is clearly that of the "benevolent" strand of human resources management which has flourished in recent years, with a rather benign indictment of the excesses of globalised capitalism and a call for more "solidarity", but all within a gentler form of capitalism including more of the celebrated "corporate social responsibility". Fajardo's interpretation of Ruskin, a combination of "the use of affection by the employer as a management tool" and "making economic growth, progress and wealth compatible with his parameters of social justice", regrettably extends misunderstanding into nonsense.

\section{Conclusion}

In spite of their diversity, the different versions of Ruskin in foreign translations reviewed here all share a common feature: none of them refer to the politically radical implications of the four points presented by Ruskin in his preface. Ruskin placed them at the beginning of UTL, because there were, as it were, its conclusions, the practical consequences of the ideas contained in the four essays. Somehow the various commentators managed to overlook them, along with a few others of Ruskin's central beliefs.

Only a few of the foreign versions take up Ruskin's critique of machinism and industrialism (including urbanism), either as dehumanising for workers or simply as a cultural and practical impasse (mostly the French versions and Ax's German version, but ambiguously so, and Chimenti in a few words). Similarly, Ruskin is rarely portrayed as an enemy of Progress. Nor can Ruskin's original critique of political economy easily be digested by contemporary political ideologies based on the liberal views of justice, equality and progress, because Rus-

kin refused them altogether. Among the few versions reviewed here, only the recent French interpretation does justice to Ruskin's multiple criticisms of capitalist social relations by noting that globally they amount to a wholesale rejection of that mode of accumulation and its attendant modes of life.

Most versions import their own religious, political, ideological or personal agendas with varying degrees of relevance into the discussion of UTL, and of Ruskin's general character as a "social reformer". Indeed the assumption serving as an ideological centre of gravity for all these versions is that Ruskin's message, being supposedly essentially moral, is primarily a capital-compatible reformist vision, if not a programme. Any serial and serious reading of Ruskin's deliberately "social" writings - but the rest will serve too - will show how far wrong that interpretation obviously is.

Unsurprisingly, this view reflects the one prevailing today, in Ruskin's own country, that his ideas are compatible with the left-and-right-of-centre "social demo- 
cratic" consensus, or now perhaps a post-neo-liberal consensus, with Wilmer in the moderate left position and Hill on the other side of a centre that is, in effect, not a dividing line but a common political and ideological ground. The famous mot of uncertain attribution which says that "it is easier to imagine the end of the world than the end of capitalism" certainly applies to most of the editors of foreign versions of UTL, even though Ruskin certainly tried his best to give imagination more power. If the treatment of this work after his death is any indication of the incomprehension Ruskin had to face in his lifetime concerning "the most serviceable things [he had] ever written", little wonder then that, pursuing this effort and meeting with more of the same reception, he felt increasingly disheartened about the prospect of social change without violence.

\section{Bibliography of Works by John Ruskin}

References are to volume and page numbers in:

Cook, E.T.; Wedderburn, A. (eds) (1903-1912). The Works of John Ruskin. Library Edition, 39 vols. London: George Allen.

https://www.lancaster.ac.uk/the-ruskin/the-complete-works-of-ruskin/.

Citations indicate volume number and pages only, as it follows: Works, [vol.]: [p.].

Works, 17: Unto this Last, Munera Pulveris, Time and Tide, Other Writings on Political Economy (1860-1873). | 27: Fors Clavigera I.

\section{English and European languages versions of John Ruskin's Unto this Last (1862)}

Amendola, G. (a cura di) (1908). Le fonti della ricchezza (Unto this Last). Roma: Enrico Voghera.

Ax, C. (Hrsg.) (2019). Diesem Letzten. Übersetzung von A. von Przychowski. 2nd ed. Frankfurt am Main: Westhafen Verlag.

Barclay, T. (ed.) (2017). The Rights of Labour According to John

Ruskin. Dehli: Facsimile Publishers.

Brunhes, H.; Brunhes, J. (éds) (1902). Unto this Last. Il n'y a de richesse que la vie. Trad. par E. Peltier. Paris: Gabriel Beauchesne et Cie éditeurs.

Bruni, L. (a cura di) (2014). Cominciando dagli ultimi. Trad. di R. Ferrigato. Cinisello Balsamo: Edizioni San Paolo.

Chimenti, F. (a cura di) (1936). A Quest'Ultimo. Principii Fondamentali di Economia Politica. Bari: Pasini e Figli.

Ciges Aparicio, M. (ed.) (s.d.). Unto this Last (Hasta este último). Cuatro ensayos sobre los primeros principios de economía política. Madrid: Libreria de Fernando Fé.

D’Errico, E. (a cura di) (1900). I diritti del lavoro. Roma: Luigi Mongini editore.

Fajardo, P. (ed.) (2002). A este ultimo. Cuatro ensayos sobre los principios básicos de la economía política. Trad. de P. Fajardo, D. Mármol. Salobreña (Granada): Alhulia.

Hill, A. (ed.) (2013). John Ruskin: Unto this Last. Foreword by Clive Wilmer. 2nd ed. London: Pallas Athene.

Horrix, P. (ed.) (1901). Dezen Laatsten Ook (Unto this Last). Vier schetsen over de beginselen der Staathuishoudkunde. Groningen: G.A. Evers. Hugenholtz-Zeeven, M. (ed.) (1901). De Laatstgekomenen. Vier lezigen over de eerste beginselen der Staathuishoudkunde.

Ruskin, J. [1862] (2014). Fino all'ultimo. Unto this Last. Quattro saggi di socialismo cristiano. Cercenasco: Marco Valerio.

Thiesset, P.; Thomasset, Q. (éds) (2012). Il n’y a de richesse que la vie. Vierzon: Éditions Le pas de côté.

David, U. (ed.) (2015). Diesem Letzten. Vier Aufsätze über die wichtigsten Prinzipien des Volkswirtschaft. 3rd ed. Norderstedt: Books on Demand. Villani, F. (a cura di) (1946). I diritti del lavoro (Unto this Last). Bari: Laterza e Figli.

Von Przychowski, A. (Hrsg.) (1902). Diesem Letzten. Vier Abhandlungnen über die ersten Grundsätze der Volkswirtschaft. Einführung von W. Schölermann. Leipzig: Verlag Eugen Diederichs.

Wilmer, C. (ed.) (1997). John Ruskin: Unto this Last and Other Writings. 2nd ed. London: Penguin Books.

397 Fonti, letterature, arti e paesaggi d'Europa S Sources, Literatures, Arts \& Landscapes of Europe 1

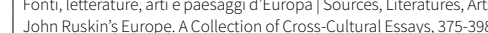




\section{General Bibliography}

Anthony, P.D. (1977). The Ideology of Work. London: Tavistock Publications.

Anthony, P.D. (1983). John Ruskin's Labour. A Study of Ruskin's Social Theory. Cambridge: Cambridge University Press.

Berg, M. (1980). The Machinery Question and the Making of Political Economy. Cambridge: Cambridge University Press.

Berliner Freie Studentenschaft (Hrsg.) (1916). John Ruskin. Die Adern des Reichtums. Jena: Eugen Diederichs.

Biagini, C.; Murray, D.; Thiesset, P. (2017). Aux origines de la décroissance. Cinquante penseurs. Paris; Vierzon; Montréal: L'échappée; Le pas de côté; Ecosociété.

Brunhes, H.; Brunhes, J. (1900). Ruskin et la Bible. Pour servir à l'histoire d'une pensée. Paris: Perrin.

Clegg, J. (2006). “La presenza di Ruskin in Italia cento anni fa”. Lamberini, D. (a cura di), L'eredita di John Ruskin nella cultura italiana del Novecento. Firenze: Nardini, 95-108.

Coste, B. (2013). “'No disciple of mine': une étape de la réception française de John Ruskin”. Dick, J.K.; Schwerter, S. (éds), Traduire: transmettre ou trahir? Réflexions sur la traduction en sciences humaines. Paris, Éditions de la F-MSH, 131-45.

Guerzoni, G. (2006). "La ricezione italiana del Social and Economic Criticism di John Ruskin, 1850-1950”. Lamberini, D. (a cura di), L'eredità di John Ruskin nella cultura italiana del Novecento. Firenze: Nardini, 136-55.

Hill, A. (2019). Ruskinland. How John Ruskin Shapes Our World. London: Pallas Athene.

Hobson, J.A. [1898] (1904). John Ruskin. Social Reformer. London: James Nisbet \& Co.

Jaudel, P. (1973). La pensée sociale de John Ruskin. Paris: Librairie Marcel Didier.

McCarraher, E. (2019a). Ruskin Was Right. Prophets of Ecological Disaster. https://www.commonwealmagazine.org/ruskin-was-right.

McCarraher, E. (2019b). Comrade Ruskin. How a Victorian Visionary can Save Communism from Marx. https://www.plough.com/en/ topics/justice/comrade-ruskin.

McCarraher, E. (2019c). The Enchantments of Mammon. How Capitalism Became the Religion of Modernity. Harvard: Harvard University Press. Michéa, J.-C. (2013). Les mystères de la gauche. De l'idéal des Lumières au triomphe du capitalisme absolu. Paris: Flammarion. 


\title{
The Apostle of Beauty: Some Turn-of-the-Century Perceptions of Ruskin in Central and Eastern Europe
}

\section{Stuart Eagles}

Independent Scholar

\begin{abstract}
Ruskin's writings were widely available in a range of European languages by the early years of the Twentieth century. We focus here on the Czech lands (mainly Bohemia), Hungary and Poland, with some comparative references to Russia. In these nations, Ruskin was found in partial and complete translations of individual works, anthologies of selected passages, critical studies, journal articles and in the debates these publications helped to stimulate. Ruskin was also read both in the original English, and widely in French translation. But it was not until Ruskin's ideas began to circulate in these countries' native languages that Ruskin's literary merit and philosophical insights could be seriously engaged with.
\end{abstract}

Keywords Ruskin's reception. Translation. Hungary. Czech. Poland. Russia. Leo Tolstoj. Robert de la Sizeranne.

Summary 1 Ruskin's Reputation. - 2 Ruskin, the Apostle of Beauty. -3 Interpreters and Interpretations. -4 Women Interpreters.

Ruskin did not generally approve of his work being translated. When, in 1896, the committee of the Welsh National Eisteddfod asked his permission to include translations from his poems in their concert programme, W.G. Collingwood replied that Ruskin

has always felt extremely indisposed towards translations from his works, and it would perhaps be hardly fair to persons to whom he has refused permission to translate into French and German, if he were now to sanction translations into Welsh. ${ }^{1}$

Despite or regardless of this, Ruskin's writings were widely available in a range of European languages by the early years of the Twentieth century. We will focus here on the Czech 
lands (mainly Bohemia), Hungary and Poland, with some comparative references to Russia. ${ }^{2}$ In these diverse nations, Ruskin was found in partial and complete translations of individual works, anthologies of selected passages, critical studies, journal articles and in the debates these publications helped to stimulate. Ruskin was also read both in the original English, and widely in French translation. But it was not until Ruskin's ideas began to circulate in these countries' native languages that Ruskin's literary merit and philosophical insights could be seriously engaged with.

The peak of Ruskin's appeal to translators and critics, and the height of his reception, was reached in these countries, as in many other parts of the world, at the turn of the Nineteenth into the Twentieth century. Ruskin was discussed with increasing frequency and interest from the late 1890s, but his death at the start of 1900 triggered an explosion of obituaries and critical retrospectives in a period hungry for fresh ideas. The dislocating effects of urbanisation, rapid industrial growth, the mechanisation of agricultural and industrial production, the specialisation of labour, and the mushrooming of city squalor, all combined with the ambiguous and uncertain potential of technological invention, social experimentation, and cultural innovation, to contribute to a fevered atmosphere at once threatening and exciting, full of possibilities, good and bad.

The space available here is barely sufficient to provide more than the briefest selective sketch of some of the lessons derived from Ruskin by a few of his most fascinating interpreters. Ruskin's influence was felt in the fine, applied and industrial arts. He was admired for his role in inspiring and supporting the Pre-Raphaelites, and for providing the theoretical basis for the practical achievements of William Morris and the Arts \& Crafts movement. A wide variety of individuals and groups cited aspects of his thinking and called on his authority in cultural debates about the purpose and value of artistic movements and national styles. The vital connecting element was Ruskin's insistence on the vital importance of beauty in nature and art. This is not to deny that he had his detractors and opponents. Nor is it to suggest that Ruskin's legacy was uncomplicated or uncontested. For many conservatives, his perceived celebration of man's harmony with nature in the Middle Ages, and his emphasis on traditional methods and standards of art production, validated their resistance to modern orthodoxies, and privileged the special work of the hands above all else. Many modernists opposed to Ruskin saw this is as essentially stifling of creative innovation, truth to nature being apparently antithetical to abstraction. But for modernists who saw value in Ruskin's ideas - and there were many - his rejection of industrial capitalism, opposition to exploitative labour relations and belief that art was for the everyday and for all, licenced a range of initiatives to make art more accessible. Inevitably there were differences of interpretation from individual to individual and group to group within these countries, as well as between them. Describing Ruskin's little-known translational history, and the affinities and differences in his reception in central and eastern Europe, helps to map Ruskin's global reach and to understand his influence in a way that underlines the inspirational or recuperative value of his insights to a diverse and significant range of thinkers and practitioners all too commonly ignored in the English-speaking west.

2 For a broad survey of the reception of British art and design in the Czech lands, Hungary and Poland, as well as Germany and Austria, see Szczerski 2015. For surveys of Ruskin's influence on the Czech Arts \& Crafts, see Vybíral 2004; for his reception in Hungary, see Péteri 2005; and for a brief survey of his influence in Poland, see Ulita 2007. For a contextualised and detailed account of Ruskin's influence in Russia see Polonsky 1998, Eagles 2011, Eagles 2016 
Conspicuous among Ruskin's champions was Leo Tolstoj who declared in 1898 that "John Ruskin is one of the most remarkable men not only of England and of our generation, but of all countries and times". ${ }^{3}$ For him, Ruskin was "a philosopher, political economist, and Christian moralist". In addition to publishing his own short selection of paraphrased Ruskinian aphorisms, Tolstoj also caused one disciple in particular, Lev Pavlovich Nikiforov (1848-1917), a Socialist Revolutionary, to translate three books about Ruskin (two from French), twelve books by Ruskin, and to produce four books of selected passages as well as his own short biography of him. ${ }^{4}$ But Tolstoj's interpretation of Ruskin, and the extent of Nikiforov's copiousness, were exceptional. In Russia, as in Bohemia, Hungary and Poland, the dedication was less intense and the focus of attention was largely on Ruskin's aesthetic theory, in particular his claims for the vital moral importance of natural and artistic beauty.

\section{Ruskin's Reputation}

The master of religious painting, and a leading member of the Mir Iskusstva (World of Art) movement in Russia, Mikhail Nesterov (1862-1942), recalled in his memoirs, "I and my generation were raised on the views and concepts of art of Ruskin and theorists like him". ${ }^{5}$ In 1900, the journal Magyar Iparmüvészet (Hungarian Applied Arts) commented in an obituary of Ruskin that many Hungarians were unsure whether he had been "a polar explorer or a Russian novelist", ${ }^{6}$ but by 1904 the same journal declared that "Ruskin has a very good name in Hungary today". ${ }^{7}$ This latter article speculated that whilst it was possible that Ruskin's influence would prove to be a passing fad, it might yet cause "the whole Hungarian nation" to "settle on a new culture

in which Ruskin's teaching is vivid and thriving". ${ }^{8}$ For the Hungarian artist, Aladár Körösfői-Kriesch (18631920), writing in the same year, Ruskin's influence was so pervasive that "We are all his disciples, whether we have read a line of him well or not". ${ }^{9}$ The Czech journalist, essayist and author, Gustav Jaroš (1867-1948), writing under his pseudonym Gamma in the influential journal, Volné Sméry (Free Directions) in 1900, remarked:

How thick must be the Chinese wall between England and Bohemia if the first accounts of a man of such importance as John Ruskin are only timidly starting to reach us in a few fragmentary passages

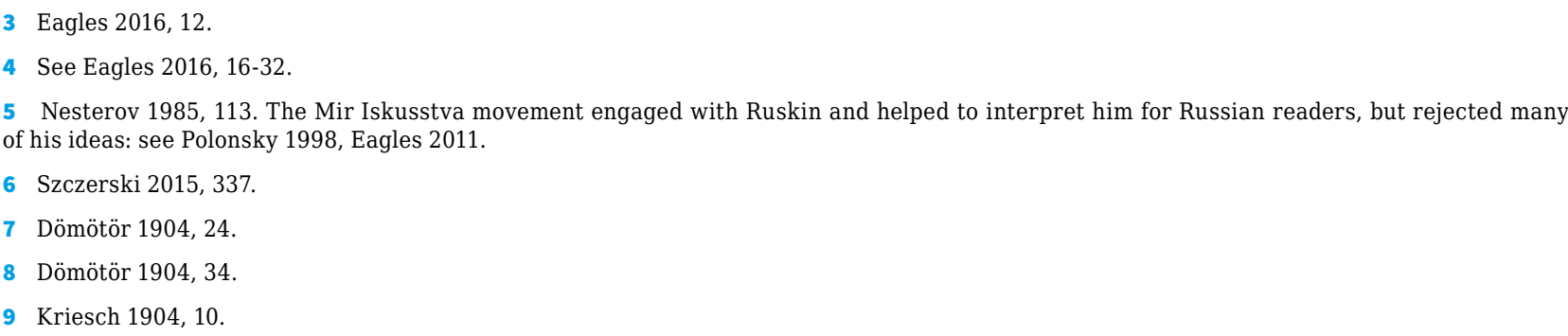


after his great, famous and profound work has been ongoing for fifty years. ${ }^{10}$

The lawyer, journalist and critic of society and economics, Stanislaw Koszutski (1872-1930), wrote in 1900 that until recently Ruskin was almost completely unknown in Poland, but that it was to be hoped that with the recent slew of "obituaries, dissertations and articles", he would become known in "all the splendour of his beauty and strength". Ruskin was, he added, "one of the greatest writers of the Nineteenth century". ${ }^{11}$

Translations appeared unsystematically, out of sequence, often out of context, and coverage was not consistent across national borders. Of Ruskin's major works, only Sesame and Lilies appeared in its entirety in all four languages: translated into Russian twice, first by Nikiforov in 1900 (the first of his 11-volume Sobranie Sochinenii Dzhona Reskina (The Collected Works of John Ruskin), and second, by Olga Soloveva, the following year; in Polish, also in 1900, by Ruskin's most prolific Polish translator, Wojciech Szukiewicz (18671944); in Czech, in 1901, by F.X. Šalda; and in Hungarian, in 1911, by Klára Farkas. ${ }^{12}$ The Crown of Wild Olive appeared in Russian, Polish, and Czech, all in 1900; Unto this Last in Russian in 1900, Hungarian in 1904, and Czech in 1910; Ethics of the Dust in Russian and Polish, both in 1901; The Queen of the Air in Polish in 1901, and Czech in 1903. Lectures on Art was translated into Russian three times (twice in 1900, and again in 1907) and appeared in Czech in 1901; Mornings in Florence appeared in Russian in 1902, and Czech in 1919; The Political Economy of Art in Russian in 1902 and in Czech in 1925; while The Two Paths appeared before the Second World War only in Czech (in 1909). The Eagle's Nest and The Laws of Fesole were also translated into Russian in 1903 and 1904 respectively.

The Seven Lamps of Architecture did not make it into any of these languages in its entirety in this period, but a critical summary and analysis was published in Polish in 1902 by the influential architect and conservator, Jan Sas Zubrzycki (1860-1935). Ruskin's multi-volume works were scarcely attempted in their entirety by translators, but circulated in numerous extracted passages that appeared in anthologies, critical studies, and journal articles, though Frondes Argrestes (being a selection from Modern Painters) and a 363-page selection from Fors Clavigera made standalone publications in Russian in 1902 and 1905 respectively, and remarkably all three volumes of The Stones of Venice appeared in Hungarian between 1896 and 1898, published by the Magyar Tudományos Akademia (Hungarian Academy of Arts and Sciences).

10 Jaroš 1900, 89. The article was headed with a drawing, apparently of Ruskin, by the artist, Jan Priesler (1872-1918), for a long time editor of the journal, and later an art professor.

11 Koszutski 1900, 1-2.

12 It also appeared in Romanian in 1914, translated by Constantin Antoniade (1880-1954), a hugely important Romanian jurist, diplomat, writer, philosopher, and historian of the Renaissance: Comori si gradini: trei conferinte (Treasures and Gardens: Three Lectures), Bucharest: Universale. 


\section{Ruskin, the Apostle of Beauty}

Common to many - but by no means all - translators and critics of Ruskin in turn-of-the-century Russia, Poland, Hungary, and the Czech lands was their interpretation of him as an apostle. A Christian teacher leading his disciples through the blighted landscape of the present, Ruskin shone the light from his lamps of Beauty and Truth to point the way to a future capable of recuperating the sympathy and reverence for nature common in pre-Renaissance Europe when God's gifts were apparently properly valued and head, heart and hand were harmonized in a whole human soul.

Ruskin's legacy was to a large extent framed in central and eastern Europe by the influential French art critic and historian, Robert de la Sizeranne (1866-1932), and especially by his magisterial study, Ruskin et la religion de la beauté, which appeared in Paris in 1897. It was translated into Russian twice, both in 1900, once by Nikiforov, and once by T. Bogdanovich. A year earlier an essay on Ruskin had appeared under the title "Religiya krasoty" (The Religion of Beauty) in the Russian journal Russkoe bogatstvo (Russian Treasure) by the essayist, critic, and theosophist, Adelaida Gertsyk (1874-1925), later a notable and highly original Decadent poet. ${ }^{13}$ A Polish translation of Sizeranne's work was published in Lviv in 1898-1899, as John Ruskin i kult piękna by the eminent writer and literary critic, Anto- ni Potocki (1867-1939). It appeared just before Potocki established himself as the pre-eminent ambassador of Polish culture in France, having established the Koło Polskie Artystyczno-Literackie (Polish Artistic and Literary Association) in Paris in 1897. Sizeranne's study was cited in Polish obituaries of Ruskin, such as that which appeared in the journal, Architekt (Architect) by Jan Zawiejski (1854-1922), the historicist architect.

A short study by Lev A. Bogdanovich, Dzhon Reskin: 'Apostol religii krasoty' (ocherk) (John Ruskin, 'Apostle of the Religion of Beauty' [An Essay]) was published in Moscow by I.A. Mamontov just after Ruskin's death in January 1900. In his account, Bogdanovich firmly establishes Ruskin as the "father of the aesthetic" and the "apostle of the religion of beauty", ${ }^{14}$ varying the phrase to "the father of British aesthetics" ${ }^{15}$ as he characterises Brantwood as a site of pilgrimage to which disciples expectantly turned up with their "Kodaks" determined to take away a memento of their visit. ${ }^{16}$ He hails "the apostolic spirit of John Ruskin" ${ }^{17}$ and, deploying a significant metaphor, he compares Ruskin to a "Great Anglo-Saxon physician"18 determined to "use his powerful means to cure his brothers". ${ }^{19}$ Ruskin is the "eternal advocate of real virtues" whose writings are "monographs about the medicinal qualities and properties of beauty". ${ }^{20}$

13 Gertsyk produced the Russian translation of Mornings in Florence.

14 Bogdanovich 1900, 3.

15 Bogdanovich 1900, 6.

16 Bogdanovich 1900, 5.

17 Bogdanovich 1900, 22

18 Bogdanovich 1900, 7.

9 Bogdanovich 1900, 8.

o Bogdanovich 1900, 9. 
In June 1900, the translator and literary critic, Zinaida Vengerova (1867-1941), wrote a lengthy obituary of Ruskin in the journal Vestnik Evropy (The Herald of Europe), though she expressed an anxiety felt by other modernists, in Russia and elsewhere, that Ruskin's message had the potential to stifle creativity in its perceived emphasis on the social utility of art, but her enthusiasm for him was always strong. Her essay was later collected under the title, "Dzhon Reskin, Apostol krasoty" (John Ruskin, Apostle of Beauty). In 1903 she succinctly encapsulated the admiration of many sympathetic modernists by declaring that "[Ruskin] caused a revolution in art, he managed to convince us that art is not a luxury but a necessity, that beauty is a necessary element of life". ${ }^{21}$ In Hungary, in 1904, Körösföi-Kriesch compared Ruskin to 'the apostles' for revealing the harmony and beauty of nature by insisting that the artist should show a mirror to nature and thus reflect their own soul. ${ }^{22}$ Two years earlier, Frigyes Spiegel (1866-1933), the architect and craftsman, writing in the journal Magyar Iparmüvészet (Hungarian Applied Arts), called Ruskin the "free-spirited apostle" who taught that "mechanical industry could be counter-balanced only by the revival of the handicrafts". ${ }^{23}$

The Pole, Stanislaw Koszutski concluded his essay on
Ruskin with the assessment that the "words and deeds of this apostle [...] have received a wide hearing at home and abroad": "Everywhere, his words echo" with the message that "the most beautiful reward of life is life itself; the joy of creation is the reward which God himself destined for us". ${ }^{24}$ The Czech teacher, translator and cultural historian Otakar Josek (1854-1926) wrote an article on Ruskin in the journal, Osvěta (Enlightenment) entitled "Apostol krásy a altruismu" (Apostle of Beauty and Altruism), and Miloš Seifert (1887-1941), a school teacher, naturalist, writer, and translator, long interested in Ruskin, entitled his 1937 study, John Ruskin, apostol pravdy a krásy. Myslenky a dilo (John Ruskin, Apostle of Truth and Beauty. Ideas and Work); as a young man, in 1910, he had translated Unto this Last. The founder of modern Czech cultural criticism, František Xaver Šalda (1867-1937), whose translation of Sesame and Lilies we have already noted, called Ruskin, in a short piece entitled "Johna Ruskina vyklady o umĕni” (John Ruskin's Interpretations of Art), published in the influential journal, Volné Sméry (Free Directions), "the apostle of beauty" in everyday life who crucially recognised its joy. ${ }^{25} \mathrm{He}$ credited Ruskin with influencing his increasing conviction that intuition was the most important element in artistic creation.

\footnotetext{
21 Vengerova 1903, 173

22 Kriesch 1904, 9.

3 Spiegel 1902, 97.

Koszutski 1900, 7.

5 Šalda 1902, 132
} 
Framing Ruskin as the Apostle of Beauty nevertheless neither strictly limited nor gave any special coherence to how Ruskin was understood. A more detailed look at a few of the Hungarian, Polish and Czech interpreters and interpretations of Ruskin reveal further continuities and variations in Ruskin's reception in the region.

Körösföi-Kriesch's account of Ruskin, given in his 1904 lecture "Ruskin művészi hitvallása" (Ruskin's Artistic Creed), was a sensitive account which demonstrated a widely-read appreciation of Ruskin, and an understanding of Ruskin's style that unarguably influenced his own mode of expression. He argues that although "most people do not even know it" it was Ruskin's medievalism that provided the impulse for "the whole modern art movement" as he saw it. ${ }^{26}$ Ruskin's central contribution was as "a prophet, a seer" who established "new and deep connections between things". ${ }^{27}$ The analysis begins with Modern Painters and Ruskin's reverence for nature and its truthful representation in the artwork of Turner. Körösföi-Kriesch traces Ruskin's interest in nature to his geological studies at Oxford, his friendship with Henry Acland, and the European travels he undertook with his parents, connections which inspired him to see the world simultaneously through the eyes of the scientist and the artist. In Italy, Körösföi-Kriesch argued, Ruskin was able to study the great Christian medieval artists and discovered "the joy of the eyes" in the artistic "worship of nature". ${ }^{28}$ The Renaissance destroyed the rhythm of life and undermined the harmonious relations between man, nature and society, a rupture which had led to the people of Europe "not looking any more for God" but instead revelling unthinkingly in splendour. ${ }^{29}$ It was time once again to embrace the soul of the artist. Seven Lamps and Stones "found a strong echo in England and across the continent as well", both among "the people and especially the majority of artists" ${ }^{30}$ Körösföi-Kriesch shared in Ruskin's admiration for the art of the Middle Ages, produced in a Christian community, and he endorsed Ruskin's religious reverence for nature. Ruskin's argument that "everyone has the right to and need for art as much as air and daily bread" ${ }^{31}$ attracted him keenly: Ruskin, he believed, was the first to argue that "moral and artistic truths stem from one and the same source and that the latter cannot exist without the first". ${ }^{32}$ For Körösföi-Kriesch, Ruskin's argument was organic and holistic, binding the arts with every aspect of life.

Ruskin was presented as a visionary who pioneered and gave expression to a noble aesthetic theory which Morris gave practical form. Their shared sense of the vital importance of preserving ancient buildings, their promotion of handicrafts, and their concern for the welfare of the working man were exemplary and should be followed in Hungary. Unto this Last, quoted extensively,

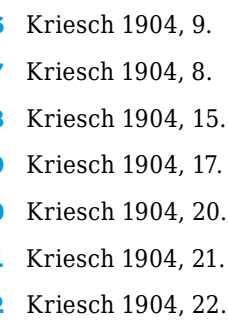


is described as a "modern gospel" in which Ruskin argued for "a common life based on love alone"..$^{33}$ In this text, Ruskin had shown "what is truly valuable in human life, vital and lasting" for "noble and happy" souls. ${ }^{34}$ There was perhaps more wishful thinking than truth in his assertion that although Ruskin was attacked for his economic theories, "it never for a moment discouraged" him. ${ }^{35}$ Ruskin's attack on the factory was "violent" because work there destroyed the soul. ${ }^{36}$ Ruskin's practical legacy was vested in the Oxford Museum, established with his friend Acland, and represented "every branch of human knowledge, the manifestation of the creative spirit in all directions", ${ }^{37}$ and built in the gothic style which expressed his ideas, though "perhaps not quite so clearly and eloquently as his works". ${ }^{38}$ Ruskin had stirred the soul (literally, he says, "boiled") and had led the way to a new art and a new life. ${ }^{39}$ Ruskin, together with the Pre-Raphaelites, had "handed the arts back to life" and "extended their boundaries in every direction". ${ }^{40}$

Together with Sándor Nagy (1869-1950) KörösfőiKriesch believed that the Ruskinian-Morrisian ideal was being lived in the Transylvanian villages where folk art and crafts were still being practiced in an unspoilt region of what they apparently called "Ruskinian Lands". ${ }^{41}$ From 1901, in Gödöllő, twenty miles from
Budapest, Körösföi-Kriesch lived a Tolstoyan life in an artists' colony to which friends and colleagues followed him. It was a creative utopia consciously informed by the ideas of Ruskin and Morris, and encompassing work in stained glass, leather, furniture-making, textile-weaving, interior design, and book illustration. The textile, carpet and tapestry workshop, which Körösföi-Kriesch personally directed, achieved success and renown, and it endured for twenty years. There are instructive comparisons to be drawn between Körösföi-Kriesch and the Polish art theoretician, painter, and architect, Stanisław Witkiewicz (1851-1915), who also considered that he had realised the Ruskin-Morris dream in soutern Poland at Zakopane, where he developed the "styl zakopiańsk", admirably detailed in an essay by Marta Wiszniowska, and not requiring amplification here.

We have already mentioned Miloš Seifert's translation of Unto this Last and his study of Ruskin published in 1937. He provides another example of Ruskinian ideas being put into practice. He became the father of the Czech Woodcrafting Movement. He was, in common with many of Ruskin's most ardent disciples around the world, a vegetarian, a pacifist, a campaigner for nature conservation, and an ecologist. Immediately prior to publishing his major study of Ruskin in 1937 he wrote a mono-

33 Kriesch 1904, 22

34 Kriesch 1904, 24

35 Kriesch 1904, 27.

36 Kriesch 1904, 28

37 Kriesch 1904, 28

38 Kriesch 1904, 29

39 Kriesch 1904, 30

40 Kriesch 1904, 31

41 Péteri 2005, 195 
graph on Thoreau. ${ }^{42}$ This had followed a celebration of the canonical Dutch writer and psychiatrist, Frederik van Eeden (1860-1932), who had himself established the simple commune of artists and peasants near Bussum, in northern Holland, which had been inspired by his own love of Thoreau and Ruskin and which he called Walden..$^{43}$

Rudolf Schlattauer (1861-1915), the Moravian painter, educator and textile artist, provides another practical example of a Czech response to Ruskin comparable with that of Körösföi-Kriesch in Hungary. He was partly motivated by Ruskin and Morris to found, in 1898, his textile workshop in Zašové, near Valašské Meziř́čí, where there was a long history of cottage industries involved in producing textiles. Its direct descendant, the Moravská gobelínová manufaktura (Moravian Tapestry Manufactury) survives today. Ruskin and Morris provided both a philosophical framework and a practical example: Ruskin in the Langdale Linen Industry; Morris in Merton Abbey. Having studied at the Academy of Fine Arts in Vienna, and having spent time as an artist living in Scandinavia, Schlattauer decided to dedicate his life to the weaving and design of tapestries.

As they lived and worked in the area, Körösföi-Kriesch and Nagy simultaneously contributed to the five-volume study of Hungarian national cultural identity, A magyar nép művészete (The Art of the Hungarian People, 19071922) which Péteri has argued was seen as a Ruskinian project. ${ }^{44}$ The art critic, Pál Nádai (1881-1945), writing in Népmívelés (National Education) in 1906, commended Ruskin for advocating an education for all in which appreciation of beauty played a fundamental part, and he considered as vital Ruskin's call to revive craftsmanship as an art. The general view in Hungary, however, was not with Körösföi-Kriesch and Nádai. Most Hungarian intellectuals saw craft revival as utopian at best, and backward at worst, because the expense of hand-made objects made them economically uncompetitive and unaffordable to the poor.

In key respects, the pre-eminent Czech art critic, František Xaver Šalda, unconsciously shared in Körösfői-Kriesch's analysis. Šalda's preface to his translation of Sesame and Lilies, an essay on Ruskin, also presents implicit testimony of Ruskin's influence, though his analysis is not uncritical. ${ }^{45}$ Ruskin, Šalda writes, combined "the enthusiasm of hymns and the intuitiveness of lyricism, whereas professional aesthetics strives to arrive at a few meagre, grey little truths by way of sober induction" ${ }^{46}$ Šalda shared Körösföi-Kriesch's admiration for Ruskin's synthesis of the scientific and artistic, considering it Ruskin's greatest philosophical contribution. Also in common with Körösföi-Kriesch, he fully agreed with Ruskin's criticism of the separation of art and handicrafts, and accepted his analysis that their perfect synthesis had been destroyed by the Renaissance. Although Šalda did not go as far as Körösföi-Kriesch in founding a craft colony, he wrote eloquently and influentially about the importance of Ruskin's enjoinder to revive the applied arts and to put craftsmanship on a par with the fine arts. It was the main distinction between Šalda's analysis and his colleague Jaroš. Šalda made his point most

42 Miloš Seifert (1934) Henry D. Thoreau, filosof prírody (Henry D. Thoreau, Philosopher of Nature).

43 Miloš Seifert (1922). U básníka Frederika van Eedena (To the Poet Frederik Van Eeden). Van Eeden contributed a long and interesting foreword to a Dutch translation of Fors Clavigera: Fors Clavigera. Verzameling van Brieven aan Werklieden (A Collection of Letters to Workmen) transl. by Bertha Koch-Huber (1901). Praha: B. Kočí. See Eagles 2013.

44 Péteri 2005, 195.

45 See Šalda 1901, 3-19.

46 Šalda 1901, 3-4.

407 Fonti, letterature, arti e paesaggi d'Europa | Sources, Literatures, Arts \& Landscapes of Europe 1 Fonti, etterature, artie paesaggi d'Europal Sources, Lteratures, Arts
John Ruskin's Europe. A Collection of Cross-Cultural Essays, $399-412$ 
eloquently in his 1903 article for Volné Smĕry (Free Directions), "Smysl dnešní tzv. renaissance uměleckého průmyslu" (The Meaning of the Present So-Called Renaissance in the Applied Arts), in which he called for a renewed union of art and life in a society held together by a shared joy in beauty. ${ }^{47}$ In his own art theory Šalda blended a Ruskinian appeal to intuition with the ideas of Carlyle, Goethe, Taine and Nietzsche. Where Šalda differed most profoundly from Ruskin and Körösföi-Kriesch was in his modernist rejection of the distinction between art as "truth to nature" and art derived from abstraction, and Šalda was also sceptical about what he perceived to be Ruskin's Christian socialism. ${ }^{48}$

The selection from Ruskin made and translated into Polish by Stanislaw Koszutski, Droga do sztuki (The Road to Art) was approved by the Russian censor for publication on 11 March 1900. Koszutski was a fascinating figure. He trained as a lawyer at the University of Warsaw but was thrown out because of involvement with illegal Marxist organisations, was briefly exiled to the Volga region of Russia, and eventually resumed his studies at the University of Kiev. As a school student he had participated in patriotic, socialist-leaning self-education groups which were proscribed under Russian law, and even edited one of the circle's journals, contributing poems and essays on economics. Returning to Warsaw in 1897, he worked as an assistant lawyer and a journalist. In 1898 he published his first book, about the development of industry in Poland, and with the money he earned he continued his legal and economics studies in Berlin and Par- is. He returned to Poland in 1900 and resumed his career in law and journalism, frequently lecturing and authoring a series of industrial, economic and political studies of Poland, producing important insights into the political geography and historical ethnography of the region. He was also a keen champion of women's rights.

Koszutski's Ruskin anthology is split into 32 sections of unequal length, by far the longest being the first two, consisting of long extracts from the lecture, "The Mysteries of Life and Its Arts" collected in Sesame and Lilies, and "Art and Morality" from Lectures on Art. Other works translated from include A Joy for Ever, Ariadne Florentina, The Crown of Wild Olive, The Eagle's Nest, Fors Clavigera, The Laws of Fesole, Modern Painters, On the Old Road, The Queen of the Air, The Stones of Venice and The Two Paths. The anthology presents Ruskin's broad conception of art, but readers were warned not to look for a "closed systems" in Ruskin's theory, but instead to expect "a great abundance of independent and original ideas, giving fresh inspiration in its bold insights, [and] unexpected turns of thought and phrase". ${ }^{49}$

In the przedmowa tłumacza (translator's preface), Koszutski promises that in his anthology "the reader will find all the most outstanding features of Ruskin's artistic individuality". ${ }^{50}$ He proceeded to give a brief biographical sketch. Above all, Ruskin was a fighter of systems and doctrine engaged "in the endless pursuit of beauty and truth". ${ }^{51}$ With the advent of Pre-Raphaelitism came a fertile example of Ruskin's influence. In addition, Ruskin had inspired "anthems of praise" to Giotto, Fra An-

47 See Volné Smĕry, VII (1903), 137-8.

48 Šalda's views contrasted with the conservative opinion of the art historian, František Xaver Jiř́k (1867-1947), Director of the Museum of Applied Arts in Prague, who emphasised Ruskin's criticisms of the present in support of his view that artists should reach into the past to rediscover its true value. See Jiř́k.

49 Koszutski 1900, 8.

50 Koszutski 1900, 2.

51 Koszutski 1900, 3. 
gelico and Botticelli. ${ }^{52}$ He was not merely the independent aesthetician and "devotee of pure beauty" but a "man of action" who needed "to penetrate the various issues of life" ${ }^{53}$ He came to see that industry, with its division of labour, had created the "czlowieka-tryb maszynowy" (the human-machine). ${ }^{54}$ Infected by greed and the consequences of unnatural factory work, "the inner soul of man" was unable to mirror the outside world. ${ }^{55}$ Ruskin recognised the crucial significance of the "cultural base" to the full development of man, and sought to demolish "modern industry, railroad and [other] inventions" in favour of a revival of "the old era of small industry, the manual and artistic work of the medieval artisans". ${ }^{56} \mathrm{He}$ promoted a "joy of life, of nature, a harmony of physical and spiritual work" that created a culture at the height of which one could "flourish in art - the noblest expression of the human spirit". ${ }^{57}$ For all its politically radical elements, it is striking at how many points Koszutski's analysis agrees with that of Körösföi-Kriesch and Šalda.

\section{Women Interpreters}

The Hungarian teacher, translator and Christian Socialist, Sarolta Geőcze (1862-1928), deserves a special place in the story of Ruskin's reception in central Europe because of her heroic achievement in translating all three volumes of The Stones of Venice. It was a feat she consolidated with the publication in 1903 of her more influential study and anthology, Ruskin élete és tanításai (The Life and Teachings of Ruskin), a book of more than 400 pages. ${ }^{58}$ Split into two sections, the first, comprising 72 pages, focuses on Ruskin's biography (following a short introduction about "the Ruskinian creed"), in which she describes Ruskin boldly as "the saviour of the English nation". Geőcze then presents 32 translated extracts from

Modern Painters (covering 267 pages) and six from The Seven Lamps of Architecture (70 pages). As evidence of thoroughness, this is followed by a list of books by and about Ruskin, explanatory notes, and an index.

Eva Péteri has claimed that Geőcze made "Ruskin's ideas widely available for the whole nation". ${ }^{59}$ If that is an over-statement, it is nevertheless certain that it attracted the attention of an influential artistic elite, and through them, Ruskin's message permeated Hungarian society more widely. Geőcze's study places Ruskin at the centre of turn-of-the-century efforts to change public taste, personal lifestyles and social values, and argues that Ruskin's ethical cult of beauty is socially transform-

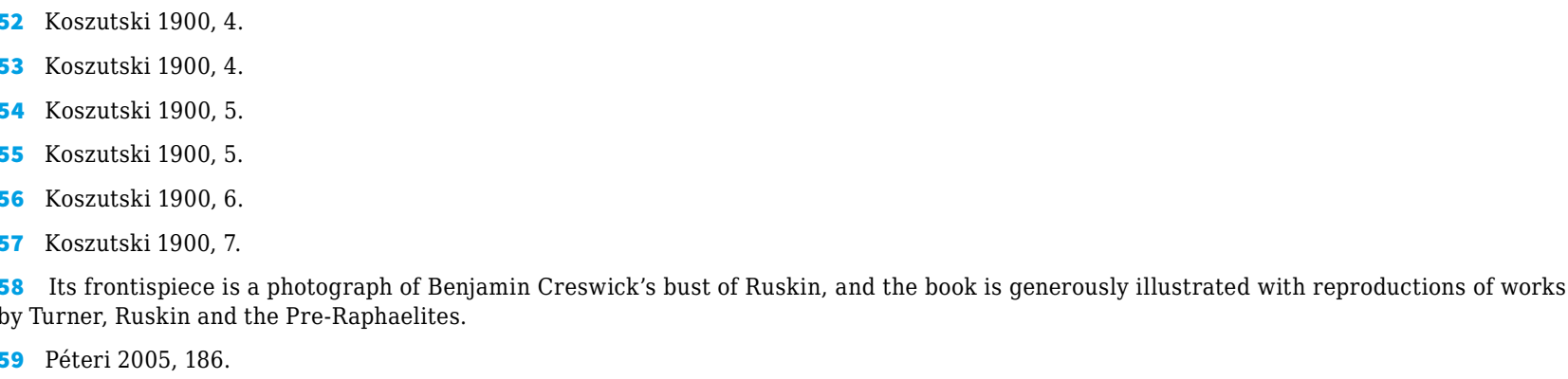


ative. Geőcze emphasises the importance of preserving historic monuments and promoting the ethical qualities of artistic and architectural culture. "I would like", she writes in her biographical sketch, "to invest the Hungarian mind-set with Ruskin's noble idealism. I would like his noble views of life to take root in each Hungarian soul".,60

Geőcze's wider influence in Hungary was significant. No feminist, she nevertheless campaigned for women's self-realisation through education, but she combined it with a strong belief in family values derived in part from Ruskin's lecture, "Of Queens' Gardens", and a belief in the different responsibilities of women and men. She trained as a teacher and worked initially at a girls' school in what today is Romania. When she translated Stones, she was on the board of an international school for girls in Komárom County (western Hungary) which was later named after her. On a tour of Italy in 1895, Ruskin's book in hand, she went to see the places Ruskin had spoken of in Stones. She wrote that she was determined “to grasp Ruskin's message and thus to understand all mankind". ${ }^{61}$ In 1897-1898, she studied pedagogical methods at religious institutions in France, Switzerland and England. In 1898 she began teaching at the Pest Institute of Instruction, and in 1907 was appointed Director of the State Institute of Women School Teachers in Buda, a post she held for ten years. In 1904 she established the Hungarian Christian Women's Workers' Section of the Budapest Christian Workers' Association, defending the rights of the most vulnerable and deprived women. In 1906 she founded the National Association of Christian Women Workers which provided a home for women workers in Budapest. She engaged with Felix Adler's Society for Ethical Culture and staunchly defended Christian family values as the only bulwark against national decline. Her views became increasingly reactionary, and she joined the right-wing Hungarian National Women's Union. The First World War had a profound impact on her and she ended her career back at the Pest Institute where she became an implacable opponent of Bolshevism and a vocal critic of Hungary's wartime territorial losses.

Geőcze was only one of many important women who helped establish Ruskin's reputation in central and eastern Europe. The first major work of the Polish author, Maria Bujno-Arctowa (1877-1952) was her study, John Ruskin i jego poglądy (John Ruskin and His Ideas), published in 1901. Bujno-Arctowa had studied in Warsaw, and then went abroad as a student of foreign languages. She would become well-known in Poland principally for the books she wrote and the magazines she edited for children and young people. The Czech illustrator and graphic artist, Zdenka Braunerová (1858-1934), was inspired by Ruskin's ideas and Morris's practical example in the Kelmscott Press, to revitalise contemporary book design by reviving aspects of Czech tradition influenced by Venice in the Seventeenth and Eighteenth centuries. ${ }^{62}$ In Russia, many of Ruskin's key interpreters at the turn-of-the-century were women (Vengerova, Soloveva, Gertsyk) and one, Lidia Ivanovna Veselitskaya (1857-1936), like Bujno-Arctowa, specialised in stories for children. In 1905 she published an anthology under her pseudonym, V. Mikulich, entitled, Na den' rozhdeniya. Mȳsli na kazhdyi (On the Birthday, Thoughts for Every

60 Peteri 2005, 5. It was in part owing to the success of this study of Ruskin that an appetite grew in Hungary for his work, and it helped prepare the ground for the publication in 1904 of a translation of Unto this Last, under the title ... Az utólsónak is ... annyit, mint neked. The translator was the medic, József Ibos (1864-1945), later - appropriately for the translator of a book that took its title from Christ's Parable of the Vineyard - a director of the Research Institute of Viticulture and Enology in Budapest. The Ruskin translation was Ibos's first book.

61 Szczerski 2015, 336-7.

62 See Szczerski 2015, 305-6. 
Day) based on the The Ruskin Birthday Book, a Selection of Thoughts, Mottoes and Aphorisms compiled by Maude Bateman and Grace Allen in 1883. A novelist, short story writer, memoirist and translator, Veselitskaya studied at the Pavlovsk Institute to be a teacher, like Geőcze, and then completed the St Petersburg pedagogical courses. Her first publications were simple children's stories, and her later novels were frank and insightful (and included a Jamesian novel about Venice). Her short stories often attended to the sufferings of the poor and the problems of social injustice, and were influenced strongly by Tolstoj, whose Moscow home she lived near to and used frequently to visit, and whose publishing house, Psorednik (Intermediary), which published several of Nikiforov's Ruskin publications, she occasionally worked for.

\section{Conclusion}

How deeply Ruskin's message penetrated in central and eastern Europe is difficult to judge in a survey of this brevity. Ruskin was certainly taken seriously by some of the most important theorists and practitioners of art at the turn of the century and helped inspire a wide range of ideas and experiments. Above all, his value in preaching the religion of beauty was understood as a call to reconnect humanity with nature and to democratise the bene-

fits of creative endeavour by revitalising the applied arts and uniting head, heart and hand by crafting beautiful objects for everyday use. The true breadth and significance of Ruskin's reception in Russia, Poland, Hungary and the Czech lands is only beginning to be mapped, and it is to be hoped that a comprehensive study of the continuities and variety of Ruskin's influence across the region commends itself even in a survey as brief and selective as this.

\section{Bibliography of Works by John Ruskin}

References are to volume and page numbers in:

Cook, E.T.; Wedderburn, A. (eds) (1903-1912). The Works of John Ruskin. Library Edition, 39 vols. London: George Allen.

https://www.lancaster.ac.uk/the-ruskin/the-complete-works-of-ruskin/.

Works, 34: The Storm-cloud of the Nineteenth Century, On the Old Road, Arrows of the chace, Ruskiniana.

\section{General Bibliography}

Bogdanovich, L.A. (1900). Dzhon Reskin: 'Apostol religii krasoty' (ocherk) (John Ruskin: 'Apostle of the Religion of Beauty' [An Essay]). Moscow: A.I. Mamontov.

Bogdanovich, T. (ed.) (1900). Robert de la Sizeranne: 'Reskin i religiya krasoty'(Robert de la Sizeranne: 'Ruskin and the Religion of Beauty'). St Petersburg: I.N. Skorokhodova.

Bujno, M. (1901). John Ruskin i jego poglądy (John Ruskin and His Ideas). Warsaw: Michał Arct.

Dömötör, I. (1904). "Ruskin nálunk (Ruskin Here)". Magyar Iparmüvészet (Hungarian Applied Arts), 7(1), 24-34

Eagles, S. (2011). “'For Fear of Bears': Ruskin in Russia (A Biblio-Historical Sketch)”. Nineteenth-Century Prose, 38(2), $157-94$.

Eagles, S. (2013). “Ruskin as 'World-Author': The Netherlands”. Ruskin Review and Bulletin, 9(2), 4-13.

411 Fonti, letterature, arti e paesaggi d'Europa | Sources, Literatures, Arts \& Landscapes of Europe 1

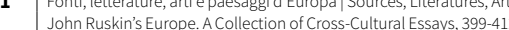


Eagles, S. (2016). Ruskin \& Tolstoj. 2nd edition. York: Guild of St George.

Gertsyk, A. (1899). "Religiya krasoty" (The Religion of Beauty). Russkoe bogatstvo (Russian Treasure), 1, 30-44.

Geőcze, S. (ed.) (1896-1898). John Ruskin: “Velencze kövei”. (John Ruskin: “The Stones of Venice”). 3 vols. Budapest: Magyar Tudományos Akademia.

Geőcze, S. (1903). Ruskin élete és tanításai (The Life and Teachings of Ruskin). Budapest: Athenaeum.

Jaroš, G. [Gamma] (1900). “John Ruskin”. Volné Sméry (Free Directions), 4, 89-96.

Jiřík, F.X. (1905). “John Ruskin a jeho umĕlecké teorie” (John Ruskin and His Theories of Art). Dílo (Artwork), 3, 3-22, 76-9.

Josek, O. (1901). “Apoštol krásy a altruismu” (Apostle of Beauty and Altruism). Osvěta (Enlightenment), 884-90.

Koszutski, S. (1900). John Ruskin: Droga do sztuki (John Ruskin: The Road to Art). Warsaw: M. Borkowski.

Kriesch, A. (1904). Ruskinról s az Angol Preraffaelitákról (On Ruskin and the English Pre-Raphaelites). Budapest: Franklin.

Nádai, P. (1907). "Ruskin mint népnevelő" (Ruskin as a Teacher of the People). Népmívelés (National Education), 4, 44-51, 296-307.

Nesterov, M.V. (1985). Vospominaniya. Moscow: Sovetskii khudozhnik.

Nikiforov, L.P. (1900). Robert de la Sizeranne: 'Reskin i religiya krasoty' (Robert de la Sizeranne: 'Ruskin and the Religion of Beauty'). Moscow: Komkniga, 2007. [Reprint of Moscow: Magazina "Knizhnoe Delo" and I.A. Balandin]

Péteri, É. (2005). "Pre-Raphaelitism in Hungary”. Tobin, T.J. (ed.), Worldwide Pre-Raphaelitism. New York: SUNY Press, 185-214.

Polonsky, R. (1998). English Literature and the Russian Aesthetic Renaissance. Cambridge: Cambridge University Press.

Potocki, A. (ed.) (1898-1899). Robert de la Sizeranne: 'John Ruskin i kult piękna' (Robert de la Sizeranne: 'John Ruskin and the Cult of Beauty’). 2 vols. Lwów: H. Altenberg.

Šalda, F.X. (1901). “John Ruskin”. John Ruskin: ‘Sezam a Lilie. Tri prednasky’ (John Ruskin: ‘Sesame and Lilies. Three Lectures'). Prague: J. Otto, 3-19.

Šalda, F.X. (1902). “Johna Ruskina ‘vyklady o umĕni'” (John Ruskin’s ‘nterpretations of Art'). Volné Sméry (Free Directions), 6, 136.

Seifert, M. (1937). John Ruskin: 'Apostol pravdy a krásy'. Myslenky a dilo (John Ruskin: 'Apostle of Truth and Beauty'. Ideas and Work). Prague: Josef Svoboda.

Sizeranne, R. de la (1897). Ruskin et la religion de la beauté. Paris: Hachette.

Spiegel, F. (1902). "Brit Iparmüvészet Budapesten” (British Applied Art in Budapest). Magyar Iparmüvészet (Hungarian Applied Arts), 5(3), 97-106.

Stibral, K.; Binka, B.; Johanisová, N. (2011). John Ruskin a př́roda (John Ruskin and Nature). Brno; Praha: Masarykova.

Szczerski, A. (2015). Views of Albion: The Reception of British Art and Design in Central Europe, 1890-1918. Bern: Peter Lang.

Ulita, M. (2007). "Ruskin's Echoes in Poland". Ruskin Review and Bulletin, 3(1/2), 64-7.

Vengerova, Z. (1903). Ocherki po istorii zarubezhnǒ literatury (Essays on the History of Foreign Literature). St Petersburg: Prometei. Vybíral, J. (2004). "The Reception of the Arts and Crafts Movement in Bohemia around 1900". Centropa, 4(3), 218-30.

Wiszniowska, M. (1995). "Popularity Against the Odds: Ruskin and Zakopane”. Acta Universitatis Nicolai Copernici: English Studies, 5, $49-57$. Zubrzyck, J.S. (1902). Krótkie myśli z dzieła Ruskina 'Siedm lamp architektury' wraz z uwagami (A Brief Summary of Thoughts from Ruskin's Work the Seven Lamps of Architecture' with Comments). Lviv: Towarzystwa Politechnicznego. 


\section{Appendix}

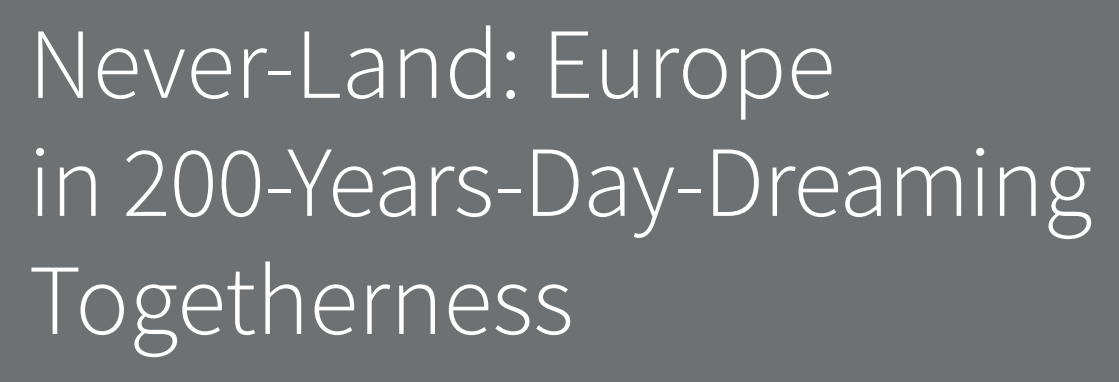

Kate Genever

Steve Pool 

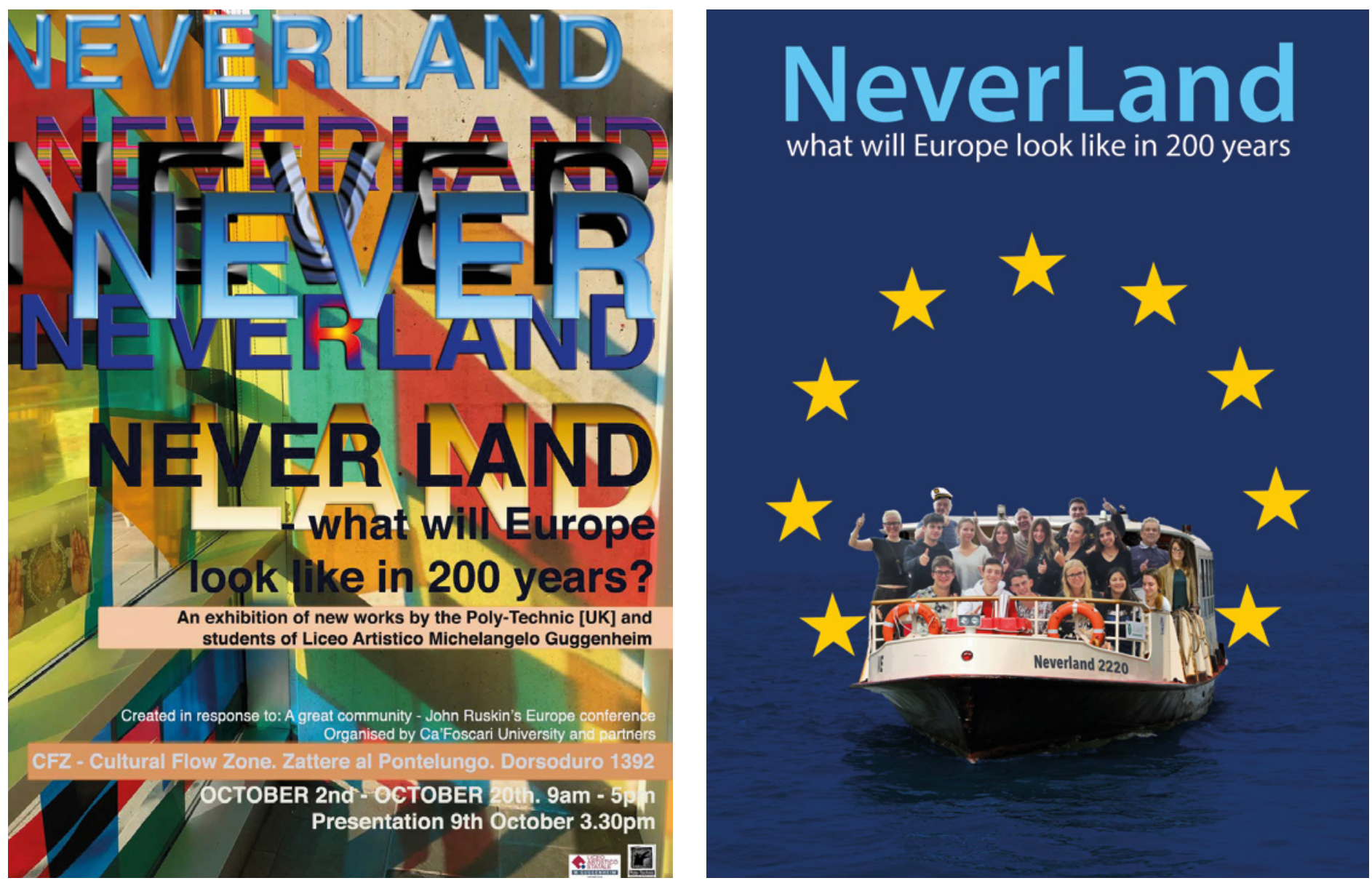

Kate Genever and Steve Pool delivered Never-Land. What will Europe look like in 200 years? In Venice and Sheffield in 2019. They talked about this work as part of the conference. They developed Never-Land in response to the current state of things. They are committed to making with people and are interested in what happens when art is made together. Their ambition is to connect, to challenge and to enquire. 

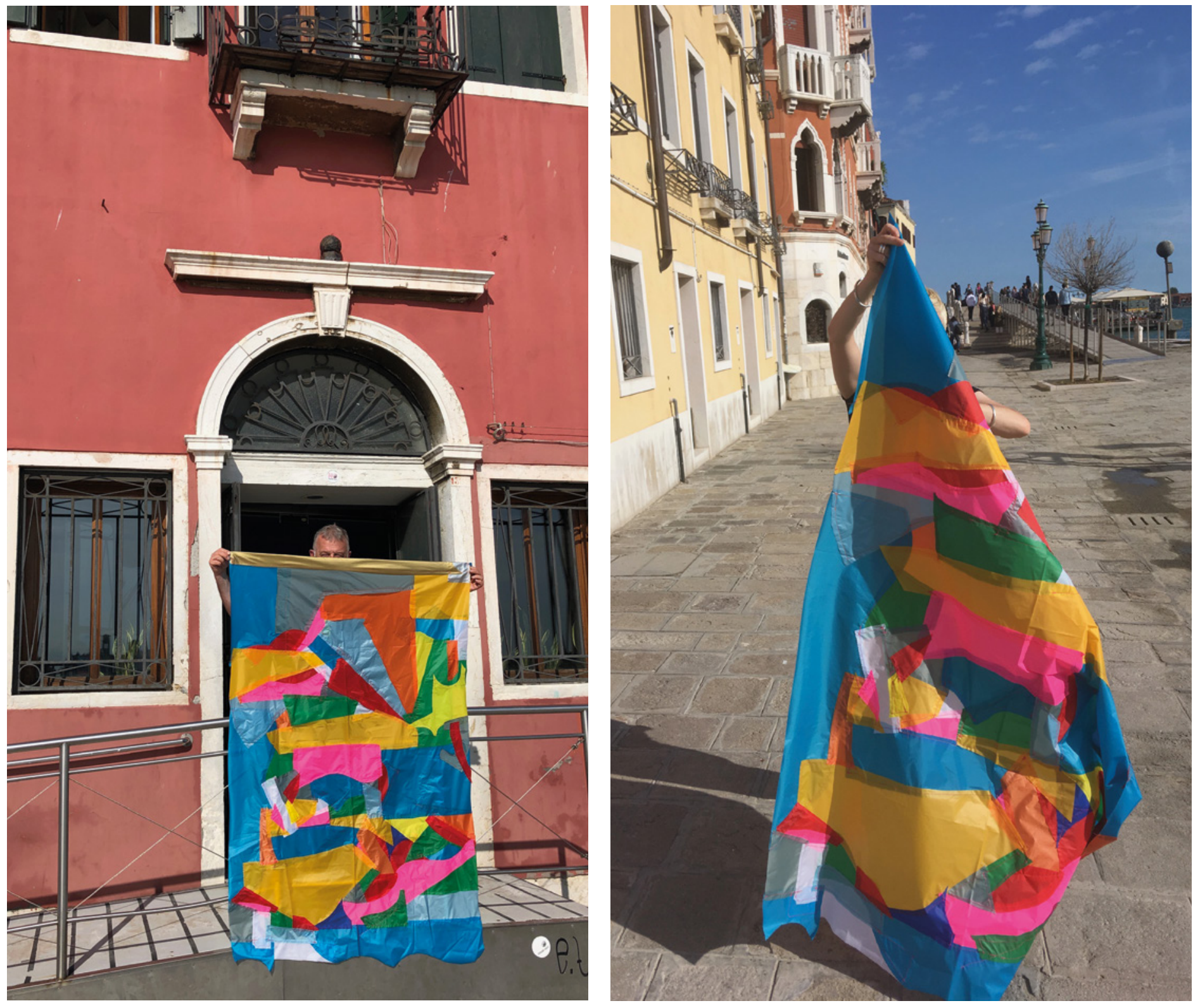

Never-Land builds on We are not an island project from 2018. Funded by The British Council, Making Ways - Sheffield and Ca' Foscari University. This year Never-Land builds on those relationships and carries the work forward. Work travelled between sites and was used to grow ideas and build connections. 


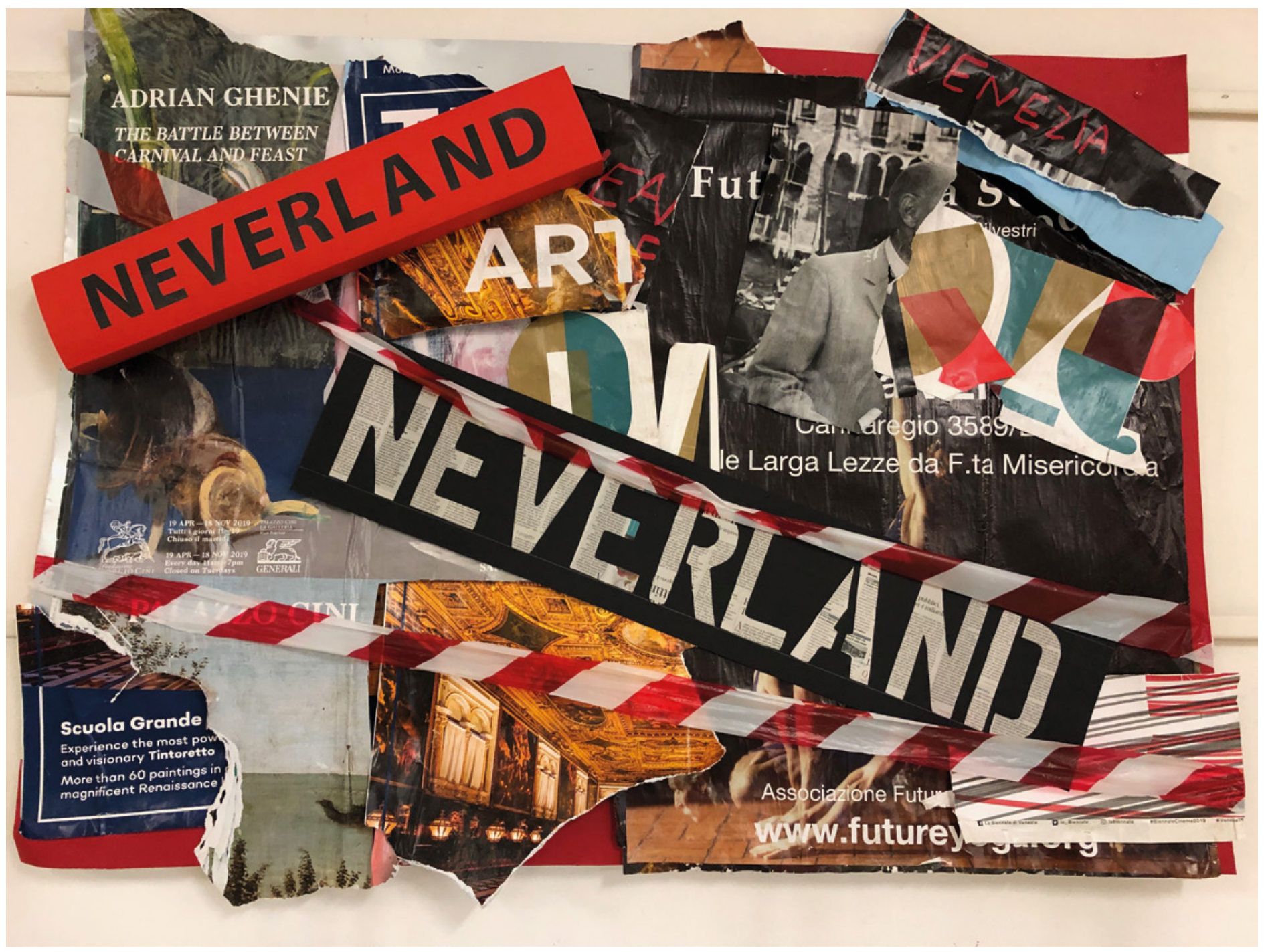

Never-Land is a fictional nowhere. Its is an imagined Europe 200 years from now. John Ruskin was born only 4 years after the battle of Waterloo, he took his first trip to Europe in 1833, the year the British abolished slavery. When the young Ruskin stepped onto land in Calais, the horse and the hand were still the driving force behind human industry. 


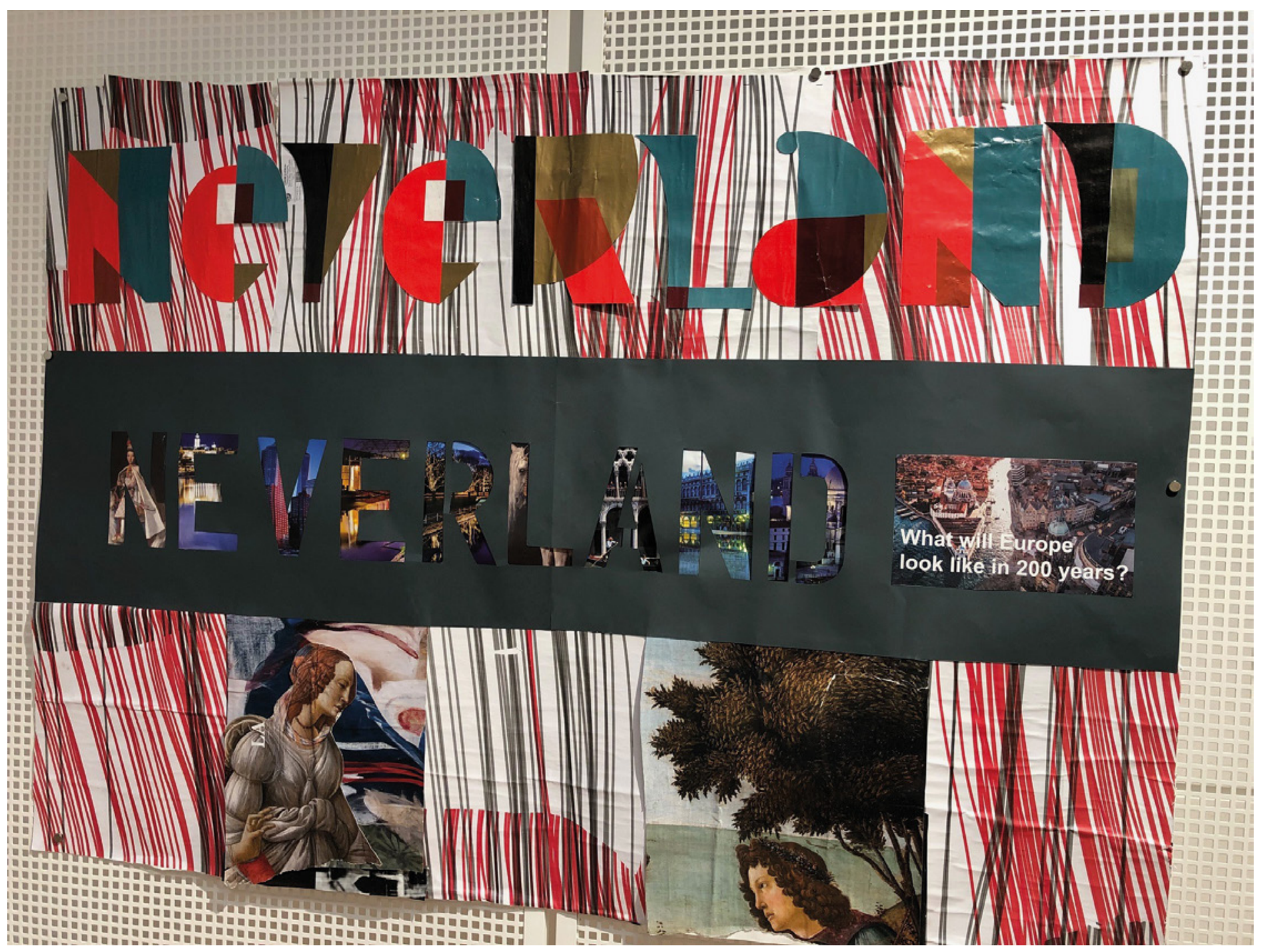

In looking backwards 200 years, Kate and Steve wondered could it have been possible to predict of today. In looking forward they ask: "Will it be a place that acknowledges John Ruskin's love of beauty and nature? Will it value the hand and personal creativity and the importance of collective action? We felt 200 years put you far enough into the future so you weren't tied to what was happening now. The changes of the last 200 years would suggest we can only dream and speculate". 


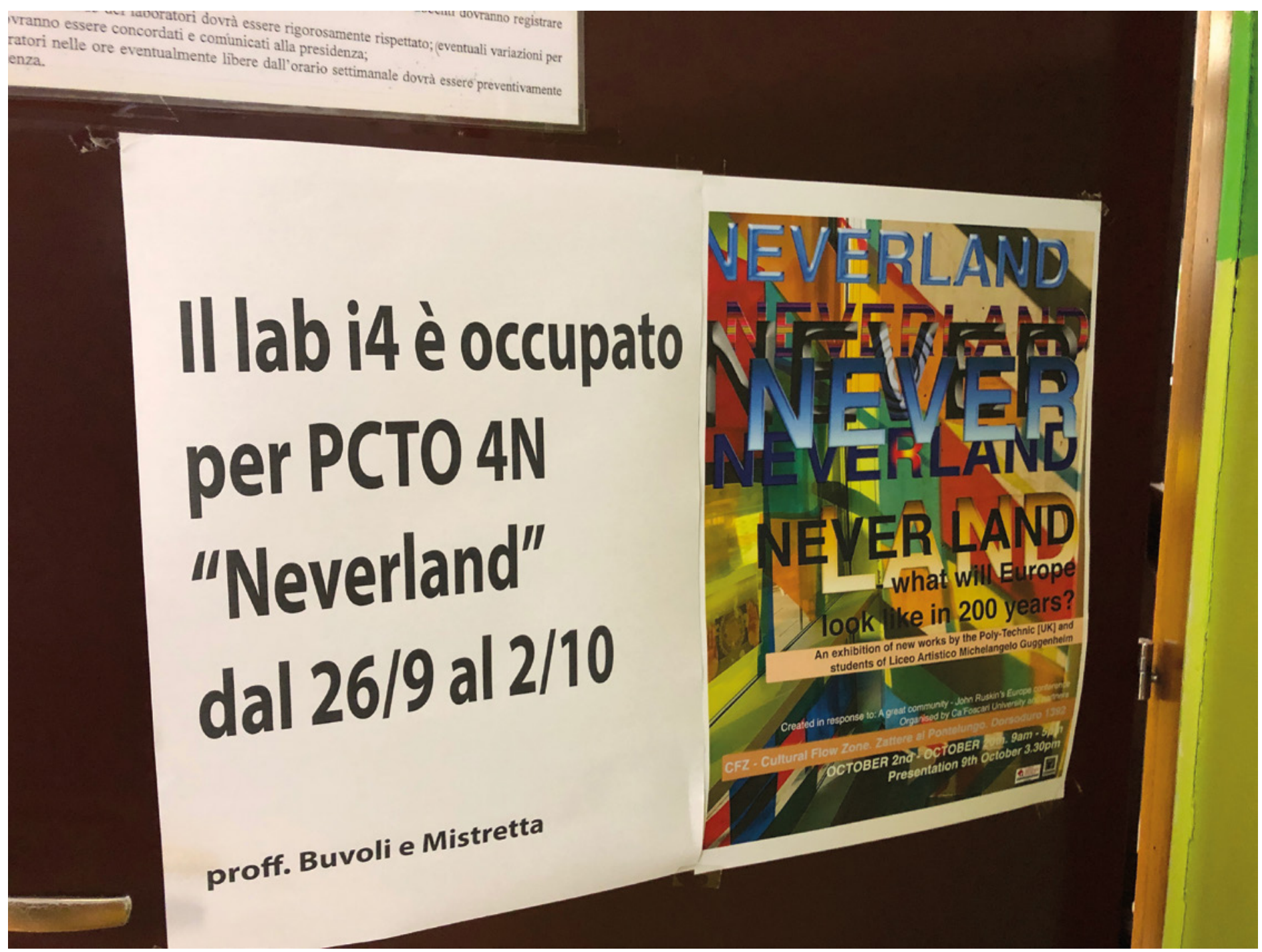

In Sheffield and Venice they established spaces where people came together to share their visions through talking, debate, reflection and making. 


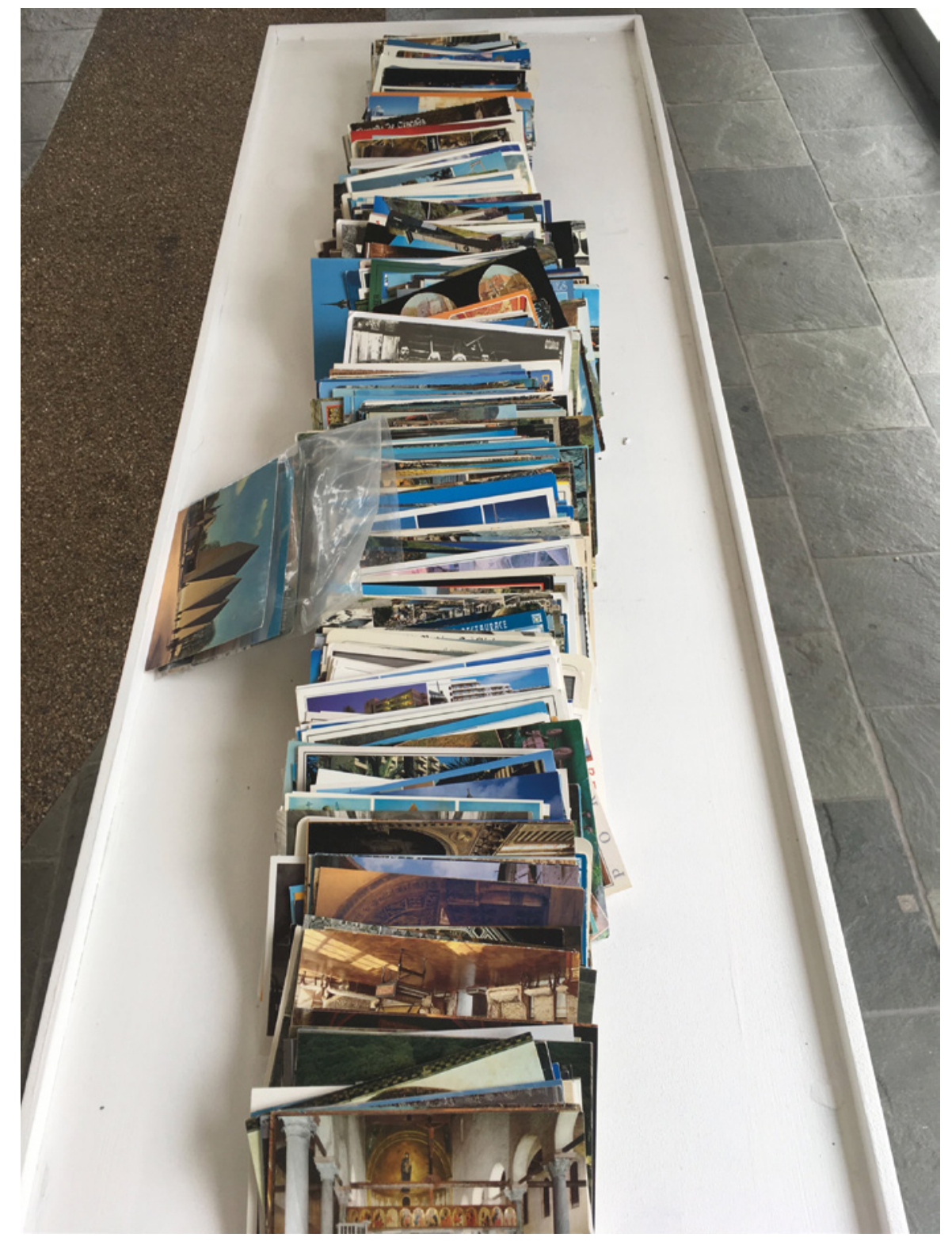

They utilised 100 s of found European postcards and invited people to collage using magazines, imagery, words - to add their vision building on the visions of others. The only rule was that the vision must be hopeful. 


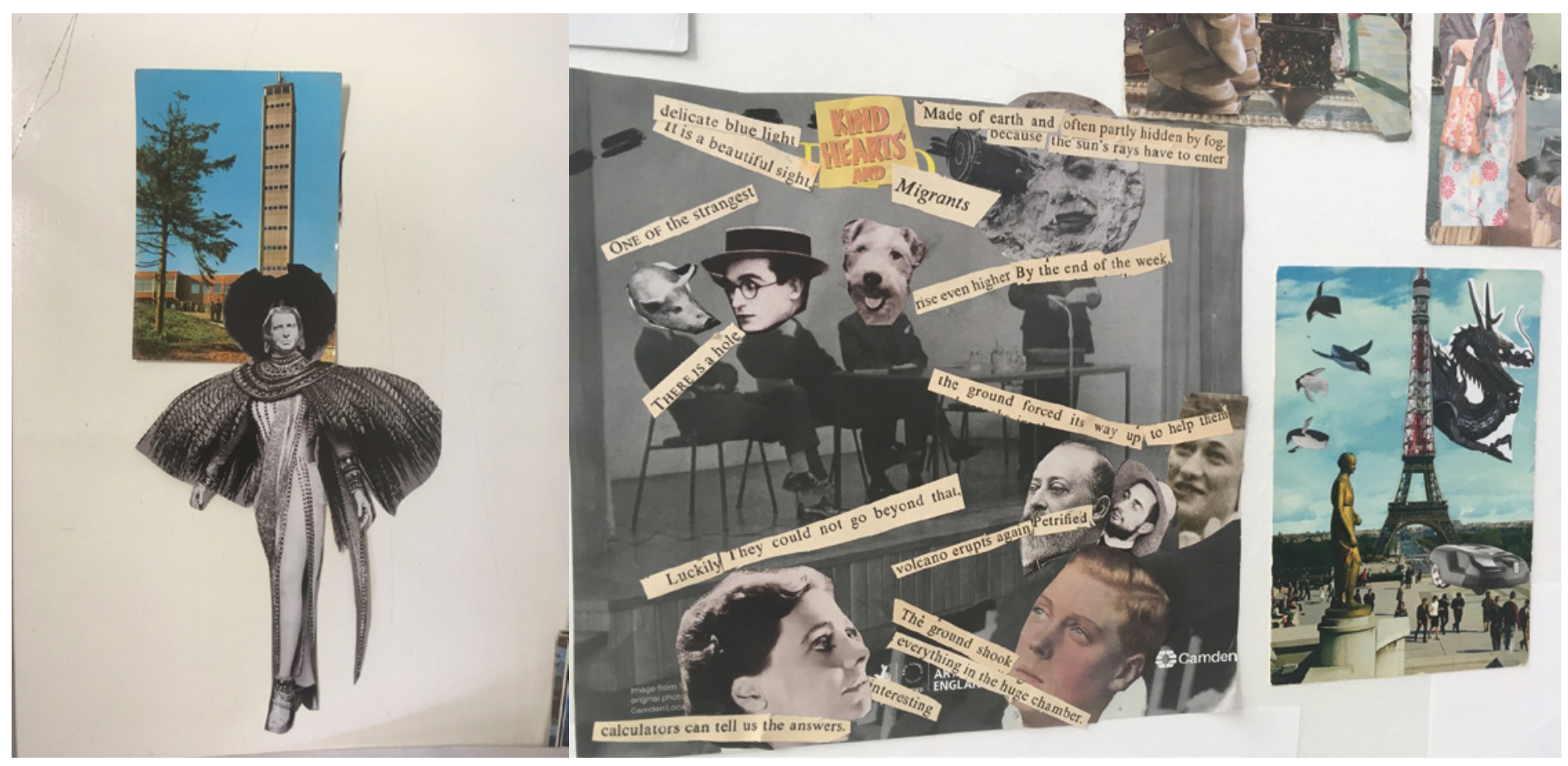

Why Collage? Collage sticks thoughts together it allows you to imagine things differently. Using found imagery does not rely on learnt and practised skills. You can put images together quickly. Collage Emphasises concept and process over end product, collage brings the incongruous into meaningful congress with the ordinary. 

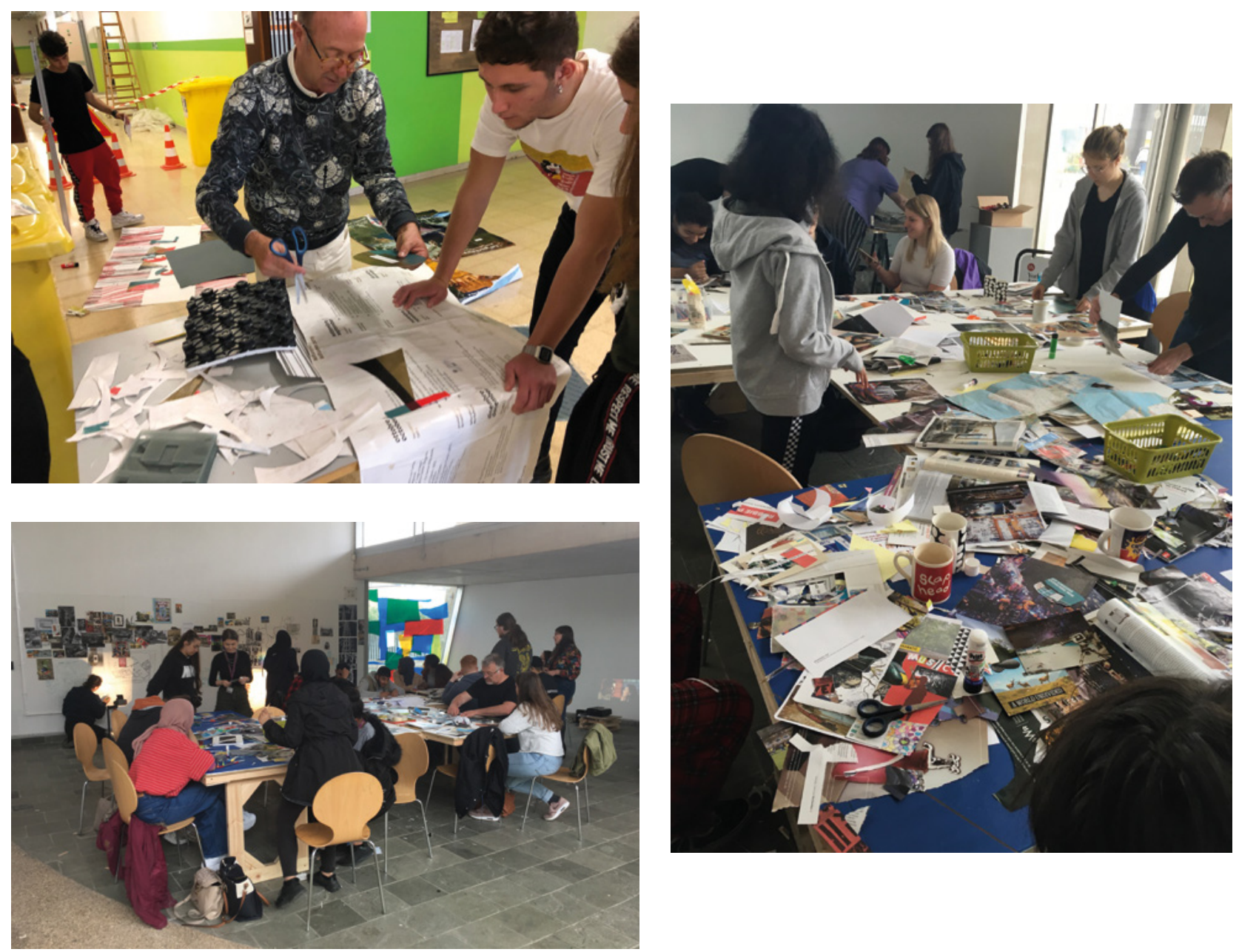

The project supported people to address what is scary and what has potential, what is urgent, what is, and may not be to come. Collage allowed people to warp and make new realities in new ways. Collage is propositional, it's the perfect method to complement and extend our theme.

Never Land is/was a space of resistance and action. Conscious of Brexit and the generation that weren't given a choice on something that would affect their future - Kate and Steve aimed to counter negative thoughts by reframing them and making actions. 

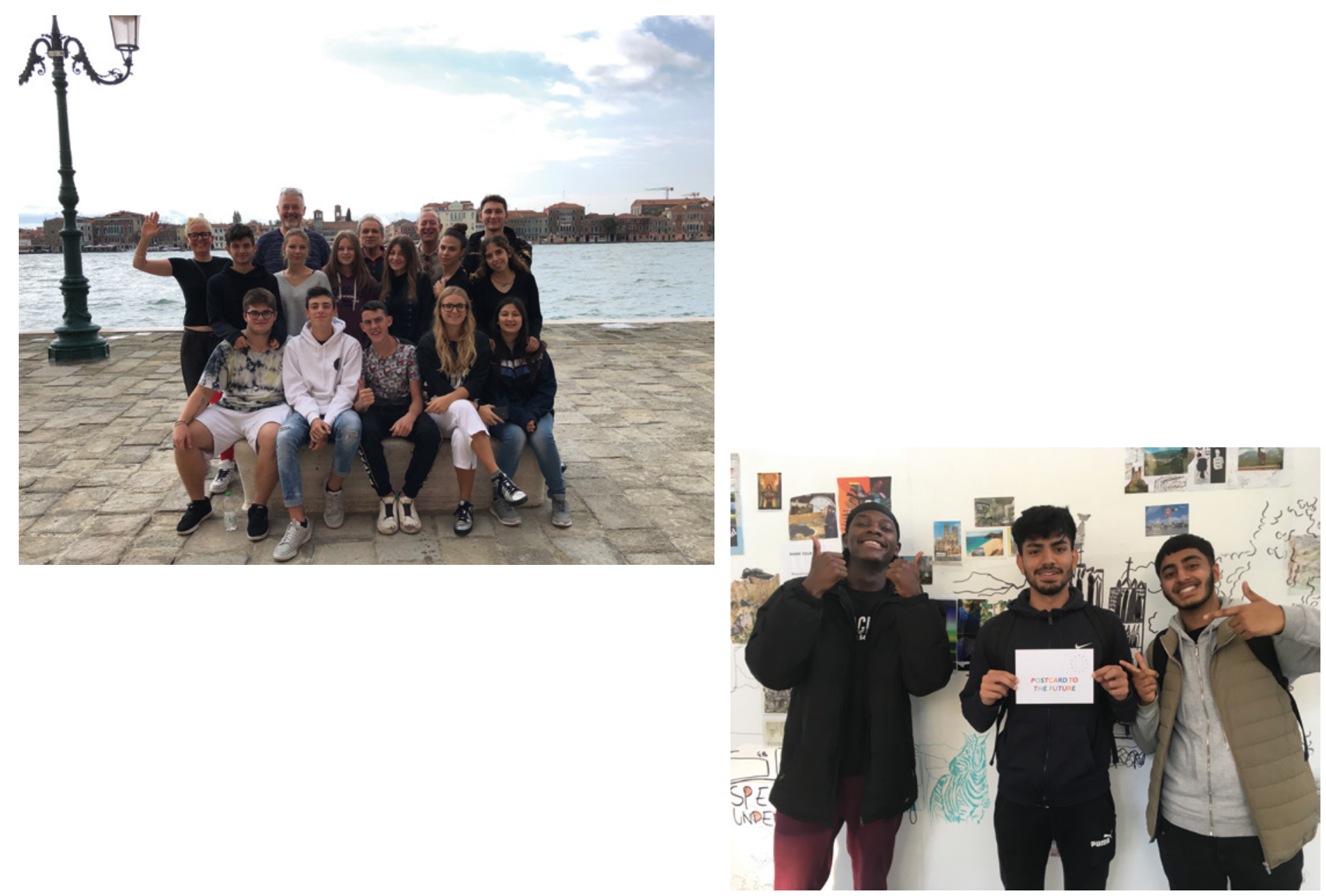

Never Land is a place we can only dream of, it's a place that doesn't exist but in trying the project connected young people across Europe. 

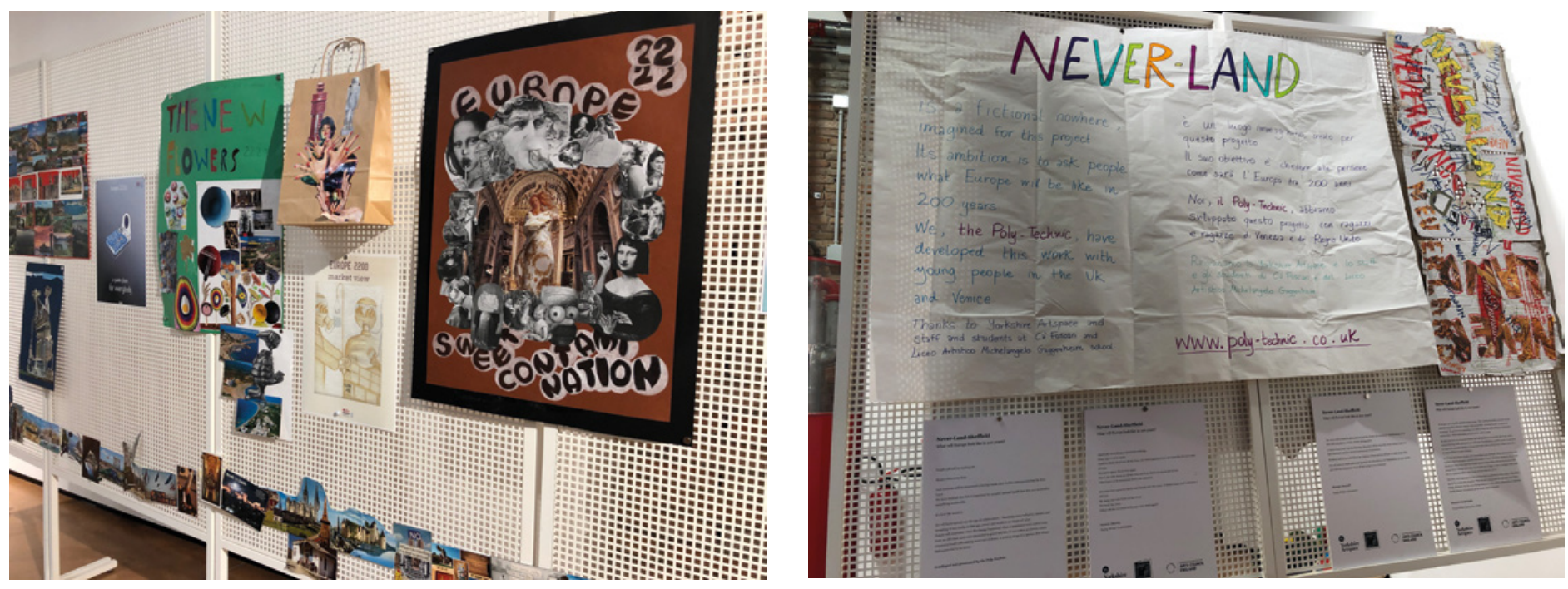

At the final exhibition students were asked what was important. A young woman said: "We were scared and negative at the beginning about the future, but working together has made us more positive - not about the state of the world or Europe, but that through coming together and working ideas come to help". 



\section{Index of Names}

Adam, Alexander 211

Adler, Felix 410

Alberti, Leon Battista $\quad 53,135-6,137 \mathrm{fn}, 141$

Alessandri, Angelo 103, 321

Alexander, Christopher 141

Alexander, Esther Francis (Fanny, Francesca) 100, 319-33

Alexander, Francis 319

Alexander, Lucia Gray 319

Alighieri, Dante (Dante) 179, 271-83, 365-6

Alison, Sir Archibald 176

Allen, George 301fn

Allen, Grace 410

Altenberg, Peter 153, 156

Amendola, Giovanni 368,389

Amoni, Leopoldo 323fn

Andreescu Treadgold, Irina 231

Anthony, Peter D. $\quad 378,380,382,386-7$

Aplin, John 110

Arad, Heidi 184

Arago, François 43

Arnim, Ferdinand von 232

Arnold, Matthew 321

Ashbee, Charles Robert 157

Auden, Wystan Hugh 1424

Augé, Marc 80

Ax, Christine 385-6, 396

Bakhtine, Mikhail 37, 174, 179

Baldoria, Natale $120 \mathrm{fn}, 121$
Balzac, Honoré de 179

Barclay, Thomas 367fn, 379-80, 388, 390

Barlow, Emily 337

Barthes, Roland 44, 179

Bastianelli, Jérôme 312

Bateman, Maude 410

Battaglini, Nicolò 240

Baudelaire, Charles 36, 46, 47, 52, 278

Bayard, Pierre 175

Beauquier, Charles 22

Bebel, August Ferdinand 384

Beever, Susan 248, 256

Bell, Margaret 247

Bellamy, Francis Julius 384

Bellini, Gentile 121fn, 290

Bellini, Giovanni $\quad$ 285, 290, 292

Benjamin, Walter 174

Berenson, Bernard 96

Berg, Maxine 382

Berkeley, George 56

Bernardi di Pian degli Ontani, Beatrice 320-1

Bernini, Gianlorenzo 93

Bissolo, Francesco 291

Blake, William 47

Blatchford, Robert 384

Blau, Tina 155

Bloch, Marc 148

Bloom, Harold 275-7, 279 
Index of Names

Bode, Wilhelm von 226-7, 236-7

Bogdanovich, Lev A. 403

Boni, Giacomo $70,75,100,106-8,110,116-17,120-1,123,126$

$$
\text { 7, 239, } 321
$$

Bonsignori, Francesco 290

Booth, William 384

Bordon, Paris 290

Bosanquet, Bernard $91 \mathrm{fn}$

Bossuet, Jacques-Bénigne 179

Botticelli, Sandro 254, 256, 304, 408

Boullée, Étienne-Louis 141

Boutmy, Émile 331

Bouvier, Nicolas 176

Bramante, Donato 137

Brangwyn, Frank 155

Braunerová, Zdenka 410

Broicher, Charlotte 94

Brown, Rawdon 287, 290

Browning, Robert 286

Brunelleschi, Filippo $\quad 135,138$

Brunetière, Ferdinand 365

Brunhes, Jean 393, 395

Bruni, Luigino 391-2

Bruno, Andrea 80

Bujno-Arctowa, Maria 410

Bunney, John Whalton 100, 103, 110

Buonarroti, Michelangelo (Michelangelo) 93, 135

Burke, Edmund 81

Burne-Jones, Edward 321

Burton, Sir Frederic 286

Byron, George Gordon (Lord Byron) 179, 273

Callegari, Adolfo 242

Calore, Pier Luigi 127

Canal, Antonio (Canaletto) 52

Carlyle, Thomas $179,249,337-8,367-8$

Caröe, William Douglas $100,100 \mathrm{fn}$

Carpaccio, Vittore 209, 285, 290

Carpenter, Edward 353

Carus, Carl Gutav 53

Cassin, Barbara 36

Castiglione, Baldassare 57

Cataldi, Melita 291

Cervantes, Miguel de 179

Cézanne, Paul 46

Chateaubriand, François-René de
Chesterton, Gilbert K. 369

Chimenti, Francesco 388

Chimenti, Giacinto 388

Ciampi, Carlo Azeglio 24

Cicogna, Emmanuele Antonio $\quad 228,229,232$

Clark, Kenneth 63

Clegg, Jeanne 251, 254, 379, 389

Clemens, Olivia 152

Coceva, Giuseppe $120 \mathrm{fn}$

Cole, George D.H. 369

Coleridge, Samuel Taylor 272, 275, 277

Collingwood, William Gershom 173, 318-19, 399

Constable, John 63

Constantin, Antoniade $402 \mathrm{fn}$

Conti, Angelo $361,362 \mathrm{fn}, 365-7,370$

Cook, Edward Tyas 94, 173, 175, 217, 306, 338

Cooper, Davies Arnold 396

Corradini, Enrico 363fn, 364, 366

Corsini, Tommaso 370

Cosgrove, Denis 32, 192

Cosnier, Colette 176

Coste, Bénédicte 219, 393

Courbet, Gustave 55, 56

Cozza, Adolfo 120

Crane, Walter 152fn, 157

Crawley Frederick 45, 251

Creswick, Benjamin 409fn

Croce, Benedetto 20, 21, 22, 55, 363

Cubitt, Joseph 204

Curtius, Ernst 94, 94fn

D'Ancona, Antonio 320

D’Annunzio, Gabriele 364

D'Aquino, Tommaso 37

D'Errico, Ernestina $367 \mathrm{fn}, 388$

da Vinci, Leonardo (Leonardo) 136, 145, 321

Daguerre, Louis 43

David, Lydia $300 \mathrm{fn}$

David, Uwe 387-8

de Honnecourt, Villard 135

de Koepff, Cristiano Federico 226fn, 228

de l'Aveyron, Victor 56

de la Sizeranne, Robert $\quad$ 17, 300fn, 362, 393, 402-3

de Loutherbourg, Philip James 63,76

De Tocqueville, Alexis 324

Delacroix, Charles 46 
della Quercia, Jacopo 208

Dewey, John 376fn

di Breme, Ludovico 273

Di Stefano, Roberto 59

Dickens, Charles 25

Diderot, Denis 56, 179

Duby, Georges 37

Dvořák, Max 127fn

Eckmann, Otto 385

Eco, Umberto 36

Edward, Johnston 347

Eijkman, Pieter 384

Eisemann, Peter 14

Eliade, Mircea 148

Emerson, Ralph Waldo 48

Emison, Patricia A. 24

Engel, Eduard 94

Engels, Friedrich 388

Erasmo da Rotterdam 179

Eschenbach, Wolfram von 335

Evangelista, Stefano 95fn

Faigl, Ludwig 163

Fajardo, Paulino 396

Falcone, Nicola 20

Fapanni, Francesco Scipione 229

Farkas, Klára 402

Faunthorpe, John Pincher $347 \mathrm{fn}$

Federico Guglielmo IV 233, 236

Ferrari, Gaudenzio 290

Fesch, Joseph 126

Fickert, Auguste $156 \mathrm{fn}$

Fiedler, Konrad 55

Fielding, Copley 264

Fielding, Henry 174

Fiocco, Giuseppe 340fn

Fogolari, Gino 290

Forcellini, Annibale 107

Foscolo, Ugo 272fn

Fossati, Gaspare 126

Fossati, Giuseppe $126 \mathrm{fn}$

Fra' Angelico 216, 314, 408

Friedman, Milton 376fn

Friedrich, Caspar David 53

Fry, Roger 106

Fuller, Anna 322
Fumi, Luigi 120

Gadda, Carlo Emilio 24

Gandhi, Mohandas Karamchand 382, 395

Garrett, John 126

Gautier, Théophile 46

Geőcze, Sarolta 409-11

George, Dennis 252fn, 257

George, Henry 384

Georgescu-Roegen, Nicholas 395

Gerard, Emily 152

Gertsyk, Adelaida 403

Gimpel, Jean 263

Giolitti, Giovanni 17, 389

Giotto di Bondone (Giotto) 216, 284, 408

Giuliani, Giambattista 320

Goethe, Wolfgang 37, 179, 271

Gombrich, Ernst 54, 106

Gregory, Lady Augusta 283-95, 319

Gregory, Sir William 284, 289-90

Gregotti, Vittorio 148

Grimm, Jacob Ludwig e Wilhelm Karl 179

Gualtieri, Edwige 320

Guasperi di Bartolomeo Casentino 331

Guerzoni, Guido $\quad 388,391$

Guidobaldi, Federico $\quad 100,126$

Harloe, Katherine 95fn

Harrison, William Henry 106

Hartmann, Ludo 160

Hauser, Gaspar 56

Hay, Robert 337

Hayek, Friedrich August von 376fn

Hebb, John 100

Heidegger, Martin 148

Hill, Andrew 383

Hilton, Tim 38, 287

Hinterberger, Friedrich 385

Hobbs, John (George) 45

Hobson, John Atkinson 362, 382, 391

Hoffmann, Joseph 155

Hoffmannsthal, Hugo von 156

Holme, Charles 153

Honma, Hisao 354

Howell, Charles Augustus 338

Hugo, Victor 37, 179, 217, 279

Hunt, William Henry 249 
Index of Names

Hunt, William Holman 338

Husserl, Edmund 38

Huxley, Aldous 59

Ibos, József 410fn

James, Henry 286

Janin, Jules 44

Jaroš, Gustav 401

Jerusalem, Wilhelm 152

Jiřík, František Xaver 408fn

Jodl, Friederich 152, 160

Jones, Owen 337, 341

Justi, Carl 94, 96

Kant, Immanuel 139fn

Kautsky, Karl 384

Keats, John 179, 273

Kerouac, Jack 174

Ketteler, Wilhelm Emmanuel von 393

Kierkegaard, Søren $146 \mathrm{fn}$

Kinglake, Alexander William 286

Klee, Paul 57

Kleinberg, Ludwig 152

Koolhaas, Rem 260

Körösfői-Kriesch, Aladár $\quad 401,405-9$

Koszutski, Stanislaw 402, 404, 408-9

Kundera, Milan 37, 179

La Touche, Rose $\quad 253,256-7,326,346 f n$

Labriola, Arturo 391

Lamartine, Alphonse-Marie-Louis Prat de 179

Landow, George Paul $147 \mathrm{fn}$

Lanes, Edward 337

Lassalle, Ferdinand 388

Layard, Lady Enid $\quad$ 284-6, 289-92

Layard, Sir Henry $\quad 121 \mathrm{fn}, 284-6$, 289-92

Le Corbusier (Jeanneret-Gris, Charles-Édouard) 141

Le Goff, Jacques 146

Le Keux, John Henry 192fn

Lee, Vernon 369

Leighton, Sir Frederic $\quad 321,338 \mathrm{fn}$

Lenné, Peter Joseph 232

Leopardi, Giacomo $\quad 273,280$

Lesage, Alain-René 174

Lessing, Gotthold Ephraim 91fn

Lethaby, William 100, 126

Levetus, Amelia Sarah 151, 153, 155-61, 163-4

Levi, Donata $17 \mathrm{fn}, 92 \mathrm{fn}$
Lévinas, Emmanuel 148

Lewis, John Frederick 338

Locke, John 56

Lodge, Giles Henry $95 \mathrm{fn}$

Loos, Adolf 155,157

Loria, Achille 391

Lorrain, Claude 59

Lowell, Henry 321

Lubich, Silvia 'Chiara' 391

Luini, Bernardino 285, 290

Lutyens, Sir Edwin Landseer 347

Lux, Joseph August 156

Lyall, Sir Alfred 286

Lynch, Kevin 82

MacDonald, George 249, 251

Mackintosh, Charles Rennie $\quad 155,157$

Mâle, Émile 304

Mallarmé, Stéphane $\quad 36,46,53,54,57$

Malraux, André 36,57

Manet, Édouard 46, 53, 56

Manning, Cardinal Henry Edward 321-4, 369

Mármol, Dolores 396

Marshall, Mary Paley 252

Marx, Karl 368, 384, 388, 391

Mataji, Shri 387

Matisse, Henri 53

Matta-Clarke, Gordon 80

Maurice, Frederick Denison 249

Mayreder, Rosa 156fn

McCarraher, Eugene 381

Merleau-Ponty, Maurice 46, 57

Michéa, Jean-Claude 381

Milbank, Alison 279

Mill, John Stuart 384

Milman, Henry Hart 332

Milton, John 277-8

Minutoli, Alexander von 229

Miyazawa, Kenji 351

Molyneux, William 56

Monet, Claude 306

Mongini, Luigi 388

Montagna, Bartolomeo 290

Montaigne, Michel de 36,171, 179

Montanari, Tomaso 26

Moore, Thomas 337 
Morasso, Mario 364

Moreau, Gustave $\quad 307,309,311$

Morris, William 100,107, 156, 159, 163, 267, 347, 353-6, 369-70, 384, 386, 395, 400

Moschini, Giannantonio 240

Moser, Koloman 155

Moss, Celia $152 \mathrm{fn}$

Müller, Max 338

Mumford, Lewis 386

Murchison, Sir Roderick 250, 257

Murray, Charles Fairfax $\quad 100,108,123,123 \mathrm{fn}$

Murray, John 257

Musil, Robert 157

Musset, Alfred de 179

Nadar (Tournachon, Félix) 44

Nagy, Sándor 406-7

Nerval, Gérard de 36

Nesterov, Mikhail 401

Newman, H.R. 319

Newton, Charles 252, 252fn

Niépce, Joseph-Nichéphore 43

Nietzsche, Friedrich 37,55

Nikiforov, Lev Pavlovich 401, 403

Norton, Charles Eliot $86,96,100,107,256,323 \mathrm{fn}, 347 \mathrm{fn}$

O'Gorman, Francis 346

O'Meara, Kathleen 324

Ogden, Daryl 347

Ojetti, Ugo 364, 370

Olbrich, Joseph Maria 155

Omero 179, 261, 273

Orage, Alfred 368-9

Oriveto, Adolfo $366 \mathrm{fn}$

Orvieto, Angiolo 363fn, 366

Ossola, Carlo 175

Otakar, Josek 404

Otero-Pailos Jorge 82

Ouida (Maria Louise Ramé) 320

Owen, Robert 391

Ozanam, Frédéric 323-4

Ozouf, Mona 176

Pajaro, Francesco 226-7, 229, 231-2

Pamuk, Orhan 25

Pane, Roberto 77

Pannini, Giovanni Paolo 63

Panofsky, Erwin 13, 14, 24
Pareto, Vilfredo 363

Pater, Walter 95fn

Pavese, Cesare 276, 279fn

Peirce, Charles Sanders 217

Peltier, Emile 393

Penty, Arthur 368-9

Péteri, Eva 409

Pfister, Federico 92fn, $93 \mathrm{fn}$

Pilutti Namer, Myriam 123, 242fn

Piovene, Guido 79

Piranesi, Giovanni Battista 126

Platone 38

Polacco, Renato 242

Polo, Marco 249

Potocki, Antoni 403

Presler, Jan 402fn

Previtali, Andrea 290

Prezzolini, Giuseppe 363

Proust, Marcel 36, 39, 47, 50, 94fn, 132fn, 175, 215, 221, 281, 297-301, 303-4, 306-7, 311-12, 316

Prout, Samuel 202, 214, 264

Prutscher, Otto 155

Przychowski, Anna von 385, 387

Rabbeno, Ugo 391

Rakowitz, Michael 80

Rauch, John 80

Rava, Luigi 17, 20

Ravelli, Luigi 120

Richmond, George 215

Riegl, Alois $127 \mathrm{fn}$

Riva, Cecilia 290

Rizzi, Alberto 228

Robert, Hubert 63

Robusti, Jacopo (Tintoretto) 51, 176

Roger, Samuel 264

Rogers, Richard 66

Rosa, Salvatore 59, 216

Rosadi, Giovanni 20, 370

Rossetti, Dante Gabriel 276

Rossi, Pellegrini 273fn

Roth, Joseph 157

Rousseau, Jean-Jacques 179

Runge, Philipp Otto 45fn, 53

Saba, Umberto 273fn

Said, Edward 345 
Index of Names

Sakai, Toshihiko 353

Šalda, František Xaver $\quad 402,404,407-9$

Sand, George 179

Sanzio, Raffaello (Raffaello) 93, 226, 303

Sargent Curtis, Ariana Randolph Wormeley in 295

Sargent Curtis, Daniel 295

Scala, Arthur von $155 \mathrm{fn}$

Scarron, Paul 174

Schelling, Friedrich 81

Schiller, Friedrich 179, 273

Schinkel, Karl Friedrich 232

Schlattauer, Rudolf 407

Schlosser, Julius von $127 \mathrm{fn}$

Schölermann, Wilhelm 153, 156, 385

Schopenhauer, Arthur 52

Schorske, Carl 158fn

Schwiedland, Eugen 152

Scott, Alexander (Sandy) 249

Scott, Baillie 155

Scott, Walter 179

Sdegno, Emma 147fn

Seguso, Lorenzo 227, 237

Seifert, Miloš 406

Settis, Salvatore 34

Severn, Arthur 252, 346

Severn, Joan 14fn, 100, 250-2, 257, 264

Shakespeare, William 179, 273

Shaw, George Bernard 284

Siegel, Steven 80

Smith, Adam 376

Smith, George 375

Snowden, Philip 382

Soloveva, Olga 402

Sontag, Susan 28

Southworth, Michael 82

Sozio, Alberto $116 \mathrm{fn}$

Spartali Stillman, Maria 100

Spiegel, Frygies 404

Spuybroeck, Lars $\quad 181-2,184,186-8,191$

Stanley, Henry Morton 256

Stendhal (Beyle, Marie-Henri) 179

Stevenson, Robert Louis 80

Stoddart, Judith 345

Stoker, Bram 152

Swainson, Harold 126
Szczepanik, Jan 153

Szukiewicz, Wojciech 402

Talbot, William Henry Fox $\quad 43,44$

Tarr, John Charles 347

Tea, Eva $100,100 \mathrm{fn}, 108,110$

Telford, Thomas 206fn

Tennyson, Alfred $\quad 214,273$

Thackeray, William 375-6

Thiesset, Pierre 395

Thomasset, Quentin 395

Tiepolo, Giovanna Battista 295

Tigri, Giuseppe 320

Tolstoj, Lev 159, 353, 365-67, 401, 411

Tommaseo, Niccolò 320

Toniolo, Giuseppe 392

Torelli, Luigi 240

Tramontin, Ludovica 184

Treves, Giacomo 229, 233

Tristram, William Outram 265

Trotter, Lilias 321

Tucker, Paul 17, 92fn, 96fn

Tumiati, Domenico 367

Turner, William $\quad 47,52,53,56,63,66,67,78,173,176,193,202$, 204, 206, 214, 216, 249, 264, 311

Twain, Mark 152, 153

Urquhart, David 338

Uzanne, Octave 152fn

Vaglieri, Dante 120

Valabrègue, Antony 53

Valéry, Paul 44

van Eeden, Frederik 407

Vélazquez, Diego 292

Vendrasco, Luigi $107 \mathrm{fn}$

Vengerova, Zinaida 404

Venturi, Robert 80

Veselitskaya, Lidia Ivanovna 410

Vigny, Alfred de 36

Villani, Felice 390-1

Villegas Alfonso 331

Viollet-le-Duc, Eugène Emmanuel 37

Virgilio (Publius Vergilius Maro) 179

Vitruvius (Marcus Vitruvius Pollio) 137, 141

Voltaire (François-Marie Arouet) 179

von Neumann, Frank 163

Voysey, Chalres Francis Annesley 155 
Waagen, Gustav von 226, 229, 233

Wagner, Otto 155

Wagner, Richard 336

Walker, Emery $\quad 108,110,347$

Walras, Léon 363

Webb, Philip $100,107,110,116-17,119-21,123,126-7$

Wedderburn, Alexander 94, 173, 176, 217, 306, 338, 385

Wey, Francis 55

Wheeler, Michael 29

White, William Hale $110,116 \mathrm{fn}$

Whitehouse, John Howard 389

Wickhoff, Frank $127 \mathrm{fn}$

Wilde, Oscar 295

Wilmer, Clive 380-1, 397

Winckelmann, Johann Joachim 22, 91-4
Wiszniowska, Marta 406

Witkiewicz, Stanisław 159, 406

Wölfflin, Heinrich 52

Woodbridge, Lucy 327

Wordsworth, William 57, 179, 263, 273, 275, 276, 279

Yeats, William Butler 259, 284

Yule, Anna Maria 247

Yule, Henry 252, 256

Zanetti, Vincenzo (abate) 239-40

Zanzotto, Andrea 28

Zawiejski, Jan 403

Zola, Émile 53

Zubrzycki Jan Sas 402

Zumthor, Peter 81

Zweig, Stefan 157 



\section{List of Authors}

\section{Abreu, Pedro Marques de}

Graduated as an architect (1990) from the Faculty of Architecture of the Lisbon University (FAUL), has a Master's Degree in Rehabilitation of Architecture and Urban Cores (1997) and a Doctorate in Theory of Architecture (2007), also from the FAUL, where he lectures in Theory of Architecture, Theory of Places, History of the Architecture of the Modern Age, and Ontology and

\section{Agazzi Michela}

Michela Agazzi graduated in History of Medieval Art (rel. W. Dorigo, Ca' Foscari University of Venice) in 1987. She graduated from the School of Archival Palaeography and Diplomacy of the State Archives of Venice (1983), as well as from the Graduate School in History of Art at the University of Bologna (1992). She is a researcher at Ca' Foscari University of Venice since 2001 and she deals mainly with sculpture, medieval architecture, liturgical furnishing and photographic archives. She ed-
Phenomenology of Architecture and the City. He carries out research especially in the fields of Theory of Architecture (Phenomenology and Hermeneutics in Architecture) and Theory of Architectural Restoration, on which he has several articles published. He is responsible for the specialization course in Church Architecture.

ited the "Corpus of early medieval sculpture" of Venice, Torcello, Murano and Caorle for the Early Middle Ages Study Center in Spoleto. She dealt with Sergio Bettini (two volumes, Marsilio 2011), taking care of the enhancement of his archive, preserved at the Department of Philosophy and Cultural Heritage (DFBC). She teaches History of Medieval Art and Exegesis of Sources for Art History (degree courses in Conservation of Cultural Heritage and History of the Arts). 


\section{Cerasi Laura}

Laura Cerasi is Associate Professor of Contemporary History at Ca' Foscari University of Venice, Department of Linguistics and Comparative Cultural Studies, and member of the editorial board of the journal Studi Storici. Her field of research is the history of political cultures, intellectuals and cultural institutions through the nineteenth and twentieth centuries, with particular attention to: Corporatism and Fascism in the interwar period and beyond; History and representations of empire; Labour and labour cultures as a constitutional foundation of the state. Her most recent publications include: "A contested legacy. Conflicting images of the Roman and British empire in the

\section{Eagles, Stuart}

Dr. Stuart Eagles is a graduate of the University of Oxford. $\mathrm{He}$ has published widely on Ruskin and his legacy. His study, After Ruskin. The Social and Political Legacies of a Victorian Prophet, 1870-1920, published by Oxford University Press (2011), explored Ruskin's social and political influence in Britain between 1870 and 1920. His study, Ruskin and Tolstoj, based on his Ruskin Lecture (2010), was published by the Guild of St

\section{Eells Emily}

Emily Eells is Professor of British literature at the University of Paris at Nanterre. She is author of Proust's Cup of Tea: Homoeroticism and Victorian Culture (Ashgate, 2002) and Two 'Tombeaux' to Oscar Wilde: Jean Cocteau's 'Le Portrait surnaturel de Dorian Gray' and Raymond Laurent's Essay on Wildean Aesthetics (Rivendale, 2010). She is head of her department's
Italian imperialist discourse through Liberal and Fascist era", in Renovatio, inventio, absentia imperii. From the Roman Empire to Contemporary Imperialism, Turnhout, Brepols publishers, 2018, 239-60; "Intellectuals in the Mirror of Fascist Corporatism at the Turning Point of the Mid-Thirties", in Authoritarianism and Corporatism in Europe and Latin America, Crossing Borders, Routledge, 2019, 27-41; Genealogie e geografie dell'anti-democrazia nella crisi europea degli anni Trenta. Fascismi, corporativismi, laburismi, Venezia, Edizioni Ca' Foscari, 2019; Il lavoro corporativo. Cultura politica ed esperienze istituzionali di un sindacalista fascista, Milano, Fondazione Giangiacomo Feltrinelli, 2020.

George (2018). He has contributed papers to book collections, exhibition catalogues, journals and websites, addressing political, economic, cultural, educational, aesthetic and translational questions, and focusing on Britain (especially Sheffield and Manchester), Denmark, the Netherlands, Spain, and most comprehensively, Russia. He was a founding member of the Anglo-Russian Research Network.

research group 'Confluences', which focuses on translation and intermedial transfer. Her work on Ruskin includes an article on Proust's pastiche in Etudes Anglaises and a chapter in the volume on Ruskin and Gender edited by Dinah Birch and Francis O'Gorman (Palgrave, 2002). 


\section{Frangne Pierre-Henry}

Pierre-Henry Frangne is Full Professor of Philosophy of Art and Aesthetics at the Université Rennes 2. He teaches in the Art History Department of this university. He is a specialist in French symbolism, Mallarmé's work, landscape theory and especially mountain photographic landscape. Among the twenty books he has published, the last four are here

\section{Frank Martina}

Martina Frank has studied History of Art at the University of Vienna, where she got her master and PhD degrees and the Habilitation (venia docendi). She is currently Full Professor for History of Architecture at the Università Ca' Foscari of Venice where she teaches also courses on Landscape Architecture and Art Patronage. Previous academic charges brought her to teaching and research experiences in several European and North American institutions. Author of numerous essays and articles concerning the history of art and architecture from the fif-

\section{Genever Kate}

Kate Genever is a UK based artist. She works over extended periods alongside people and communities to consider, reveal and celebrate how they improvise and imagine at times of stress. Outcomes of this co-produced work includes draw-

\section{Hélard André}

André Hélard has taught Classics at classes préparatoires aux grandes écoles in Rennes. He is the author of John Ruskin et les Cathédrales de la terre (2005) and translated John Ruskin, Écrits sur les Alpes (edited by E. Sdegno and C. Reichler, 2013), as well as Tintoret sous le regard de John Ruskin mentioned: L'invention photographique du paysage (with P. Limido), Presses universitaires de Rennes, 2016; Le sublime. Poétique, esthétique et philosophie (with C. Flécheux et D. Laroque), Presses universitaires de Rennes, 2018; Mallarmé philosophe, Manucius, 2018; De l'alpinisme, Presses universitaires de Rennes, 2019. teenth to the twentieth century. Among her books a study of the patronage of the Manin family, a monograph on Baldassare Longhena and the recent work on the representation of gardens in painting. A particular interest in her research activity is devoted to questions of art patronage in a trans-disciplinary perspective, topics discussed in numerous papers in international refereed journals and conferences. Editor of the journal MDCCC 1800. ings, exhibitions, events, participatory programmes, strategic organisational and community development. More examples of Kate's work can be found at kategenever.com. (ed. by E. Sdegno, 2018). One of his main research fields is the Dreyfus affair, on which he has written many articles and books, including Rennes et Dreyfus en 1899, une ville un procès, co-written with Colette Cosnier (1999). 


\section{Hull Howard}

Howard Hull is the Director and Curator of Brantwood, John Ruskin's former home, a position he has held since 1996. From 2000 to 2019 he was also the Director of the Ruskin Foundation which has overseen care of the world's largest archive of Ruskin material, now in the ownership of Lancaster University. Most recently he has curated Incandescence: Turner in Venice and co-curated The Ruskin: Museum of the Near Future. Howard read English at St Peter's College, Oxford 1972-

\section{Kawabata Yasuo}

Yasuo Kawabata is Professor in the English Department, Faculty of Humanities, Japan Women's University, Tokyo. His publications include William Morris and His Legacy (Tokyo, 2016), Haran-o-meguru Bōken (Tokyo, 2013), Orwellian Mother Goose

\section{Morezzi Emanuele}

Emanuele Morezzi, architect, PhD in Cultural Heritage (Politecnico di Torino) is a Researcher in architectural preservation in DAD - Department of Architecture and Design - Politecnico di Torino, where he works as aggregate professor in Theory, History and Restoration course and in Design Studio Ateliers involving the built heritage. Member of the PhD Architectural and Landscape Heritage board, he is involved in researches concerning conservation, preservation, enhancement and
1975. He taught English and Drama at Gordonstoun School before becoming a founding partner of the Support Group, a public relations and event management company, responsible for organizing large scale festivals and public celebrations. In 1996 he moved to Brantwood with his wife, Pamela. Howard is a Fellow of the Royal Society of Arts, Fellow of the Royal Geographical Society, Member of the London Sketch Club and a Director \& Companion of the Guild of St George.

(Tokyo, 1998), He has also translated Ruskin's The Nature of Gothic (2011) into Japanese, as well as some of William Morris's works, including News from Nowhere (2003), The Well at the World's End (2000) and The Ideal Book and Other Essays (1992).

\section{Paribeni Andrea}

Born in Rome, graduated in History of Byzantine Art of La Sapienza University of Rome; PhD in Late Antique and Medieval Archaeology at the University of Bologna; Associate Professor at the University of Urbino Carlo Bo, where he teaches History of the Medieval Art and History of Byzantine Art. His studies include: fifth-sixth century architectural sculpture (with par-

transformation of cultural legacy and heritage. He is author of Perspectives on Architectural Preservation (2020), Terni_LAB, Sperimentazioni didattiche tra restauro e progetto (2019), Post War/Disaster Recovery of historical cities and cultural heritage sites (2019), Riflessioni sulla conservazione del patrimonio archeologico (2017, with E. Romeo and R. Rudiero), Memory, Transformation, Innovation. From compatibility to sustainability in architectural preservation (2016).

ticular reference to the Justinian marbles of the Hagia Sophia); early byzantine religious architecture of the Anatolian regions; the bronzes and the sumptuary art products of the middle and late Byzantine age; the floor and wall mosaics from Classical till Medieval age; and finally the research on excavation and restoration activities of Giacomo Boni. 


\section{Pedone Silvia}

Silvia Pedone has obtained her PhD in History of Art at the Università degli Studi di Roma. She has been postdoctoral researcher at the Università del Salento in Lecce and Adjunct Professor in Medieval History of Art at the Università di Urbino Carlo Bo. She has been a member of the research group on Byzantine marble sculptures of the Ayasofya Müzelerei Institution (whose first outcomes have been published in The Sculptures of Ayasofya Muzesi in Istanbul. A Short Guide, Istanbul 2010). She is currently professor of History of Byzantine Art at Università della Tuscia di Viterbo. For over ten years she has worked at MiBACT, in the National Gallery (Palazzo Barberini Corsini)

\section{Pilutti Namer Myriam}

Myriam Pilutti Namer obtained her BA in Preservation of Cultural Heritage (2007) and a MA in Classical Archaeology (2009) at Ca' Foscari University, Venice. She discussed her PhD at the Scuola Normale Superiore (2013) in History of Art and Archaeology under the supervision of Paul Zanker and Salvatore Settis. She was awarded by post-doctoral fellowships in Venice (Giorgio Cini Foundation; Ca' Foscari University - DSU) and Naples (Istituto Italiano per gli Studi Storici). She has been responsible for the project "Studies on Giacomo Boni" at the Scuola Normale Superiore di Pisa (SAET: Director Prof. Andrea Giardina) from 2016 to 2018. From 2018 she is part of the team of the Getty-project "Mediterranean Palimpsests: Connecting the Art and Architectural Histories of Medieval \& Early Modern Cities" (The Cyprus Institute; The

\section{Reichler Claude}

Claude Reichler, researcher and writer, is Professor Emeritus at the University of Lausanne, where he taught French literature and cultural history. His recent research interests include travel literature as well as landscape theory and history, particularly in the Alps. He has written several books and articles on these subjects (La Découverte des Alpes et la question du paysage, Geneva, Georg, 2002). He led a research project on representations of high altitude air at the Swiss National Sci- in Rome and she is currently conducting her activity at Accademia Nazionale dei Lincei. Together with V. Cantone, she published the Phantazontes. Visions of Byzantine Art volume with a preface by Herbert L. Kessler (Padova 2013), and she edited several volumes of the series Sensibilia. Colloquium on Perception and Experience. Her principal research fields involve problems concerning the perception of the artistic phenomenon in Byzantium; the subject of colour in the byzantine art; Constantinople's topography and its monuments in the documentation of modern age travellers; the status of images in the byzantine visual culture; the issue of the icon and the ritual image.

Getty Foundation). Since 2017 she is Adjunct Professor in Classical Archaeology and Art History at the Ca' Foscari School for International Education in Venice, teaching in the programmes of Harvard (USA), UIBE e Suzhou (China), INHA (South Korea), Sydney (Australia). She is the author of a monograph on the Fondaco dei Turchi in Venice (2016). She is also co-editor of a volume on Giacomo Boni (Venice, 2016), author of a monograph on Boni himself (Rome, 2019), founder and co-editor of the online international journal focusing on 19th c. art history MDCCC1800 (http:// edizionicf.unive.it/index.php/MDCCC). Her research interests include Classical Archaeology, Classical Reception Studies, History of Archaeology, Conservation and Restoration Studies, Digital Humanities, Venetian Art History and Architecture.

ence Foundation, as well as a project on illustrations of travel books, which shows the importance of viatic iconography (Les Alpes et leurs imagiers. Voyage et histoire du regard, Lausanne, PPUR, 2013). With Daniela Vaj, he created the Viatimages database, as well as multimedia products in the form of animated stories and an online curio cabinet: www.unil.ch/wonderalp. In collaboration with Emma Sdegno and André Hélard, he edited the book John Ruskin, Écrits sur les Alpes, Paris, PUPS, 2012. 


\section{Remport Eglantina}

Eglantina Remport is Senior Lecturer in Irish and Modern English Literature at Eötvös Loránd University, Budapest and is one of the founders of the Budapest Centre of Irish Studies. She holds a PhD in English from Queen's University Belfast, Northern Ireland. Her research focuses on the interplay between literature and the visual arts during the period of the Irish Literary Revival, with special attention given to the influence of

\section{Sandrini Giuseppe}

Giuseppe Sandrini teaches Italian Literature at the University of Verona. His research is focused on Giacomo Leopardi, Alessandro Manzoni and the twentieth century. He published essays on the relations of Leopardi with European culture and with the Classics; he is a member of the scientific committee of the Centro Nazionale di Studi Leopardiani. He edited Manzoni's tragedy /l Conte di Carmagnola for the National and European Edition of Manzoni's works. Among the authors of the twentieth century, he studied the influence of Leopardi on Calvino, Landolfi, Buzzati, Delfini (see his book Le avventure della luna. Leopardi, Calvino e il fantastico italiano, Marsilio 2014). He
John Ruskin on the writers of the Revival. She has published on Lady Augusta Gregory and the visual arts in the Irish University Review (2011) and on W.B. Yeats and John Ruskin in the Irish Studies Review (2020). She is the author of Lady Gregory and Irish National Theatre: Art, Drama, Politics (Palgrave Macmillan, 2018). She is currently preparing a new book-length study on Lady Gregory and her connection with Venice.

\section{Sdegno Emma}

Emma Sdegno is Associate Professor of English Literature at Ca' Foscari University Venice. Her field of research is Victorian literature and culture with a major focus on John Ruskin. Her publications are on British travel culture and the construction of place myths: Ruskin, Venice and 19th-century Cultural Travel, edited with Keith Hanley (2010); John Ruskin, Écrits sur les Alpes, edited with Claude Reichler and André Hélard (2012), Looking

wrote essays about poets like Saba, Sereni and Zanzotto (see the collection of critical lectures on Zanzotto's poetry A foglia ed a gemma, Carocci 2016). He edited the diary Guerra del '15 and the short novels Un anno di scuola and L'isola by Giani Stuparich (Quodlibet 2015, 2017, 2019). He also studied the relations between writers and landscape; he edited /l viaggio di Monte Baldo by the 16th Century botanist Francesco Calzolari, Dino Campana's walk diary to La Verna, Antonia Pozzi's letters and photographs (Soltanto in sogno) and John Ruskin's Letters from Verona (Alba Pratalia 2013), written during Ruskin's travel in Verona in 1869

at Tintoretto with John Ruskin. A Venetian Anthology (2018), also published in French and Italian editions. With Kate Genever and Steve Pool (The Poly-Technic) she has run the project Enacting Fors: Ruskin and 21st century Venice, involving highschool and university students and Venetian institutions in workshops and events on the theme of cultural memory and the role of art in society. 


\section{The Poly-Technic}

"With people in places, doing things". The Poly-Technic, established in 2006, is the collaborative arts practice of Steve Pool and Kate Genever. Why Poly-Technic? Poly as in many and Technic as in techniques. The Poly-Technic works in places any place and builds on a history of artists working with communities. Our contemporary social practice creates opportunities for ourselves and others to come together to make, talk,

\section{Tizot Jean-Yves}

Jean-Yves Tizot is Senior Lecturer in contemporary British history at the Department of Foreign Languages and Cultures of the University of Grenoble (France). He teaches UK history, contemporary economic thought and translation at graduate and post-graduate level. His research is mainly in economic and social history and the history of ideas of nineteenth- and twentieth-century Britain, in particular on topics relating to

\section{Tucker Paul}

Paul Tucker teaches History of Art Criticism at the University of Florence, Italy. His research interests include the history of art criticism and collecting, especially in nineteenth-century Britain, and the linguistic analysis of art-critical text. Recent publications include editions of Ruskin's Guide to the Principal Pictures in the Academy of Fine Arts at Venice (1877) (Guida ai

\section{Wildman Stephen}

Stephen Wildman is Emeritus Professor of History of Art and former Director of the Ruskin Library and Research Centre at Lancaster University. He has written widely on nineteenth-century British art, with publications including 'A new and noble think and learn. We stepped, back a few years ago and asked ourselves what can we do that's worth doing or rather - What can art do, that's worth doing? In response we decided to ask tough questions, critically think and open up 'active' spaces. To date these have included discussion groups, artist development, curated programs, radical print workshops, open call commissions and the production of work for exhibition.

inequality and poverty, social and urban policy, planning and utopia, and the ideology of development. Recently he has been working on the intellectual foundations of E. Howard's "Garden City" idea; aspects of contemporary socio-economic inequality in the UK (the recent "Beds in Sheds" epidemic, the problems of the Gini index); "development critique"; and, in connexion with all of these topics, John Ruskin's social theory.

principali dipinti nell'Accademia di Belle Arti di Venezia, trans. E. Sdegno, Milano: Electa, 2014) and A Connoisseur and his Clients: The Correspondence of Charles Fairfax Murray with Frederic Burton, Wilhelm Bode and Julius Meyer (1867-1914), Volume 79 of the Walpole Society (2017).

school': Ruskin and the Pre-Raphaelites (Pallas Athene, London, 2013) and An Instinct to Draw: John Ruskin's Drawings in the Ashmolean Museum (Ashmolean Museum, Oxford, 2021). 


\section{Zaman Mujadad}

Mujadad Zaman is Research Fellow at the Center for Islamic Theology, University of Tübingen. His work specialises in the philosophy of knowledge and education, specifically investigating the interactions between the premodern and contemporary Islamic and European 'Western' world. Interested in how knowledge is transmitted and why we educate, Mujadad has published widely on the contemporary rise of the twenty-first century 'Knowledge Society' and its affects upon classical liberal ideas of learning within universities. He has also published works on aesthetics, contemporary intellectual culture and the history of Islamic education and its interactions with Christian Europe. His forthcoming book, Imagination in the University (Oxford University Press) critically calls upon new ways to conceive the purpose and ends of education. Mujadad holds degrees from the London School of Economics, University of Oxford and a doctorate in the Philosophy of Education from the University of Cambridge. 


\section{List of Figures}

\section{Cover Image}

John Ruskin, Map of Europe. 1829 ca. Drawing. CONRM.1989.540 Map of Europe H Res 3937. Coniston, The Ruskin Museum

\section{John Ruskin: un paysage moralisé per il nostro tempo}

Salvatore Settis

Figura 1 Piero di Cosimo, La scoperta del miele. 1505-1510 ca. Olio su tavola. Worcester (MA), Worcester Art Museum. @ Worcester Art Museum / Bridgeman Images

Figura 2 Nicolò Soggi, Ercole al bivio. 1510 ca. Olio su tavola. Berlino, Bode Museum

Figura 3 Ambrogio Lorenzetti, Gli effetti del Buon Governo, città/campagna (dettaglio). 1338-1340. Affresco. Siena, Palazzo Pubblico. (c) Roberto Testi, Comune di Siena

Figura 4 Ambrogio Lorenzetti, Gli effetti del cattivo Governo, campagna (dettaglio). 1338-1340. Affresco. Siena, Palazzo Pubblico. (c) Roberto Testi, Comune di Siena

Figura 5 John Ruskin, Chamonix: vista dalla mia finestra a Mornex. 1862-1863 ca. Acquerello. Kendal, Cumbria, Abbot Hall Art Gallery. (c) Abbot Hall Art Gallery / Bridgeman Images

Figura 6 Paesaggio antropomorfo, pittore tedesco (?) del primo Seicento. Collezione privata

Figura 7 John Ruskin, Autoritratto. 1861. Matita e acquerello. New York, The Morgan Library and Museum

Figura 8 John Ruskin, Fondaco dei Turchi. 1853 ca. Acquerello su carta. Coniston, Cumbria, Ruskin Museum. (c) Ruskin Museum / Bridgeman Images

Figura 9 John Ruskin, Part of Saint Mark's Basilica, Venice: Sketch after Rain. 1846. Acquerello. University of Oxford, Ashmolean Museum 


\section{Foreword}

Emma Sdegno

Figure 1 John Ruskin, Map of Europe. 1829 ca. Drawing. CONRM.1989.540 Map of Europe H Res 3937. Coniston, The Ruskin Museum

\section{Osservazione e comprensione dal rudere al paesaggio Unità morfologica e verità estetica negli scritti di John Ruskin}

Emanuele Morezzi

Figura 1 Autore Sconosciuto (attribuito ad Andrea del Verrocchio e bottega) (1435-1488), Vergine in adorazione del Bambino ('The Ruskin Madonna'). 1470 ca. Tempera e olio su tela. Questo dipinto, caratterizzato da rovine classiche sullo sfondo, apparteneva alla collezione privata di John Ruskin. National Galleries of Scotland, accession number NG 2338. Acquistato con l'ausilio di Art Fund and the Pilgrim Trust 1975

Figura 2 Joseph Michael Gandy (1771-1843), A Bird's-eye View of the Bank of England. 1830. Acquerello su carta. Museum number: P267. CSir John Soane's Museum, London

Figura 3 John Le Keux (1783-1846), Tintern Abbey, Monmouthshire, Looking West. 1809. Incisione. London, Tate Gallery. CC license

Figura 4 Joseph Mallord William Turner (1775-1851), Tintern Abbey: The Crossing and Chancel, Looking towards the East Window. In Watercolours and Studies Relating to the Welsh and Marches Tours, 1794. London, Tate Gallery. CC license

Figura 5 John Constable (1776-1837), Netley Abbey by Moonlight. 1833 ca. London, Tate Gallery. CC license

Figura 6 Ruderi della cattedrale di Coventry dopo l'attacco aereo dell'esercito tedesco, 16 novembre 1940. Fotografia di George W. Hales/Fox Photos/Getty Images.

Figura 7 Steven Siegel, New York in the 80s n.94. Charlotte street, the Bronx. 1980 ca. Courtesy of the Author

Figura 8 Steven Siegel, New York in the 80s n.190. Central Harlem. Primi anni Ottanta. Courtesy of the Author

Figura 9 Michael Rakowitz, The Flesh is Yours, the Bones are Ours. 2015. Allestimento per la Biennale di Istanbul, 2019

Figura 10 Michael Rakowitz, What Dust Will Rise?. 2012. Courtesy of the Author

Figura 11 Jorge Otero-Pailos, The Ethics of Dust. 2008. Manifesta, Bolzano. Courtesy of Patrick Ciccone

Figura 12 Jorge Otero-Pailos, The Ethics Of Dust: Old United States Mint. 2016. Mostra allestita nel 2016 a San Francisco, Museum of Yerba Buena. Courtesy of the Author

\section{«Aratra Pentelici» di John Ruskin Insegnare l'arte greca dopo Winckelmann \\ Myriam Pilutti Namer}

Figura 1 Afrodite assisa su un cigno. Tondo da una kylix a figure rosse a fondo bianco, dalla tomba F43 a Kameiros (Rodi). Nell'opuscolo «Aratra Pentelici» è indicata come «Aphrodite Urania. Photogravure from a Greek vase» («AP», 337-8). (C) The Trustees of the British Museum

Figura 2 Venus. Quarantatreesima carta dei cosiddetti 'Tarocchi del Mantegna', qui nell'incisione di Hans Ledespelder, con annotazioni, conservata presso il British Museum e datata tra il 1530 e il 1561. Nell'opuscolo «Aratra Pentelici» è indicata come «Aphrodite Urania. Photogravure of an early Florentine engraving» («AP», 336-7). (C) The Trustees of the British Museum 
Figura 3 Esempi di monetazione dell'antica Grecia a cui Ruskin ricorre per esemplificare le fasi artistiche nel loro sviluppo tripartito dall'età arcaica attraverso l'apogeo fino al declino. Nell'opuscolo «Aratra Pentelici» la didascalia riporta: «Archaic, Central, and Declining Art of Greece. Photogravure from Greek coins» («AP», 280)

Figura 4 Frontespizio dell'opera di John Ruskin, Ausgewählte Werke in vollständiger Übersetzung (Bde. 11/12). Moderne Maler (Bde. 1-2): Broicher 1902. Universitätsbibliothek Heidelberg, digital resource. https://doi.org/10.11588/diglit.3877\#0005

\section{La corrispondenza epistolare come rete di conoscenza, dibattito e azione Le riflessioni sulle arti e sulla tutela di Philip Webb, Giacomo Boni e John Ruskin}

Andrea Paribeni, Silvia Pedone

Figura 1 In senso orario: sezione stratigrafica delle fondazioni di Palazzo Ducale a Venezia (Milano ILASL, Archivio Boni-Tea, LXXIII. Calchi e disegni, doc. 2); la ricostruzione della Casa Romuli sul Palatino (Milano ILASL, Archivio Boni-Tea, XXVII/b. Casa Romuli Riproduzioni di case, doc. 1b); disegno di gandasa (utensile agricolo) dalla località di Runkuta (Agra, India), inviato a Giacomo Boni da Eugenia Barnes (Milano ILASL, Archivio Boni-Tea, XXXIX. Epistolario B); lettera di Giacomo Boni a William St Clair Baddeley del 26 agosto 1899 (Milano ILASL, Archivio Boni-Tea, XXXIX. Epistolario B); schizzi di Boni per modelli di contenitori della marmellata di melangolo (Milano ILASL, Archivio Boni-Tea, CXLIX. Marmellata italiana, docc. 8a-b)

Figura 2 Giacomo Boni, disegno (intero e particolare) del portale della Scuola Grande di San Giovanni Evangelista a Venezia, realizzato nel marzo del 1883. Oxford, Ashmolean Museum, inv. WA.RS.RUD.108bis. Fotografia di Andrea Paribeni

Figura 3 Lettera di John Ruskin a Giacomo Boni del 25 marzo 1883. Milano, ILASL, Archivio Boni-Tea, LVII. Epistolario R - Ricci, Ricciardi, Rigobon, Robertson

Figura 4 Lettera di Philip Webb a Giacomo Boni del 18 settembre 1886. Milano, ILASL, Archivio Boni-Tea, LXI. Epistolario U, V, W.

Figura 5 Lettera di Emery Walker a Eva Tea del 3 febbraio 1927. Milano, ILASL, Archivio Boni-Tea, LXI. Epistolario U, V, W

Figura 6 Ritratto fotografico di Emery Walker (1920-1933). London, National Portrait Gallery, Photographs Collection, NPG x31052

Figura 7 Philip Webb, schizzi architettonici interpolati nelle lettere inviate a Boni. Milano, ILASL, Archivio Boni-Tea, LXI. Epistolario U, V, W

Figura 8 Lettera di Philip Webb a Giacomo Boni del 4 gennaio 1890 con dettaglio del portale della chiesa di San Salvatore a Spoleto. Milano, ILASL, Archivio Boni-Tea, LXI. Epistolario U, V, W

Figura 9 Lettera di Philip Webb a Giacomo Boni del 21 giugno 1891. Milano, ILASL, Archivio Boni- Tea, LXI. Epistolario U, V, W

Figura 10 Stalla con colonne di epoca rinascimentale a Pontealto. Dall'album Vicenza Medioevale. Milano, ILASL, Archivio Boni-Tea, CXXXVIII. Vicenza Medioevale, doc. 1.b

Figura 11 Casa colonica a Pontealto. Dall'album Vicenza Medioevale. Milano, ILASL, Archivio Boni-Tea

Figura 12 Località Cattane (Vicenza), Villa Loschi, Zileri Dal Verme: fasi dei cambiamenti e restauri dell'edificio dagli anni Trenta del Novecento ad oggi. Da Istituto Regionale Ville Venete

Figura 13 John Ruskin, Loggia of the Ducal Palace, Venice. 1849-1850. New York, Metropolitan Museum, Roger Fund 1908, inv. 08.227.39 Figura 14 Toro bronzeo del duomo di Orvieto, fotografia dopo il restauro. Fondo Armoni Moretti ICCD D 10361

Figura 15 Venezia, basilica dei Santi Giovanni e Paolo, capitelli bizantini provenienti dal San Polieucto di Costantinopoli (Barsanti, Pilutti Namer 2009, fig. 4)

Figura 16 Lettera di Philip Webb a Giacomo Boni dell'8 marzo 1894 con schizzo del capitello in collezione Mattei. Milano, ILASL, Archivio Boni-Tea, LXI. Epistolario U, V, W

Figura 17 G.B. Piranesi, Incisione del capitello di Palazzo Mattei (Piranesi 1750, tav. 15, fig. 3) 
Figura 18a-b Capitelli dalla chiesa dei Santi Cosma e Damiano a Roma. Lione, Tesoro della Cattedrale (Guidobaldi 1989)

Figura 19 Altare ligneo della John Garrett \& Son su disegno di Philip Webb, 1897. Londra, Victoria \& Albert Museum (V\&A:W.4-2003)

Figura 20 Istanbul, Santa Sofia, particolare di una delle transenne giustinianee riutilizzate nella Hünkâr Mahfili (Loggia del Sultano). Fotografia di Silvia Pedone

Figura 21 Abbazia di San Clemente a Casauria, capitello del portico (da Calore 1891)

Figura 22 Lettera di Philip Webb a Giacomo Boni del 29 marzo 1891. Milano, ILASL, Archivio Boni-Tea, LXI. Epistolario U, V, W

\section{Ruskin's Ontology of Architecture}

Pedro Marques de Abreu

Figure 1 An interpretation of Ruskin's allegory. Fotomontage of Nuno Mesquita according to the instructions of the author

\section{Intermezzo. Amelia Sarah Levetus (1853-1938) e il John Ruskin Club di Vienna dalla sua fondazione fino alla Prima Guerra Mondiale}

Martina Frank

Figura 1 Prima pagina dell'articolo di Amelia Sarah Levetus sul padiglione austriaco alla Prima esposizione internazionale d'arte decorativa moderna tenutasi a Torino nel 1902, apparso nel volume 26 del 1902 della rivista The Studio. https://digi.ub.uni-heidelberg. de/diglit/studio1902b/0059

Figura 2 Prima pagina dell'articolo di Amelia Sarah Levetus sull'esposizione primaverile della Secession a Vienna del 1906, apparso nel volume 36 del 1905 della rivista The Studio. https://digi.ub.uni-heidelberg.de/diglit/international_studio26/0066

Figura 3 Presentazione di Amelia Sarah Levetus del Palais Stoclet a Bruxelles di Josef Hofmann, apparsa nel volume 61 del 1914 della rivista The Studio. https://digi.ub.uni-heidelberg.de/diglit/studio1914a/0195

Figura 4 Cerimonia della posa della prima pietra del Volksheim a Vienna Ottakring il 18 dicembre 1904 con Amelia Sarah Levetus al centro a destra. Fotografia di H. Schumann, Österreichische Nationalbibliothek, Bildarchiv Austria

Figura 5 L'esterno del Volksheim a Vienna Ottakring in una fotografia del 1905. Österreichische Nationalbibliothek, Bildarchiv Austria

Figura 6 Prima pagina di un fascicolo che celebra il venticinquesimo anniversario della fondazione del John Ruskin Club. Annessi una dedica e una fotografia di Amelia Sarah Levetus e il biglietto di donazione del fascicolo alla Ruskin Library di Lancaster

\section{Division, Juncture, System: Bridges and Bridge-Building in the Work of John Ruskin}

Paul Tucker

Figure 1 Joseph Mallord William Turner, Coblenz. 1842. Watercolour, $286 \times 445$ mm. Ohio, Cincinnati Art Museum (CIN6198923). Bequest of Mary Hanna. (c) Cincinnati Art Museum / Bridgeman Images

Figure 2 Joseph Mallord William Turner, Rheinfelden from the North. 1844. Graphite, watercolour and pen on paper, $229 \times 330$ mm (support). From the Rheinfelden Sketchbook. Accepted by the nation as part of the Turner Bequest 1856. London, Tate Britain. Photo $\odot$ Tate Figure 3 John Henry Le Keux after John Ruskin, The Bridge of Rheinfelden. 1860. Etching. Published as PI. 83 in Modern Painters V (1860). Image scanned from 1888 edition. (c) Paul Tucker 
Figure 4 John Henry Le Keux after John Ruskin, Peace [the walls of Rheinfelden]. 1860. Etching. Published as Pl. 84 in Modern Painters $V(1860)$. Image scanned from 1888 edition. () Paul Tucker

Figure 5 Jacob Philipp Hackert, The Ponte a Mare in Pisa. 1799. Oil on canvas, $643 \times 963$ mm. Greifswald, Pomeranian State Museum. (c) Wikimedia Commons

Figure 6 John Ruskin, The Ponte della Pietra, Verona. 1869. Watercolour and bodycolour over graphite on grey, wove paper, $176 \times 261$ mm. Oxford, Ashmolean Museum (WA.RS.ED.295.a). ( ) Ashmolean Museum, University of Oxford

Figure 7 John Ruskin, Ponte Vecchio. 1882. Graphite on cream wove paper, $356 \times 484 \mathrm{~mm}$. Cambridge (MA) Harvard Art Museums/Fogg Museum (1957.192). Gift of Edward W. Forbes. (c) President and Fellows of Harvard College

Figure 8 John Ruskin, Bridge at Lauffenbourg. 1863 [wrongly dated by Ruskin 1868]. Graphite on pale pink wove paper, $137 \times 223$ mm. Harvard Art Museums/Fogg Museum (1926.33.149). Transfer from the Fine Arts Department, Harvard University. ( $)$ President and Fellows of Harvard College

Figure 9 Samuel Prout, Two-arched Bridge, from A Series of Easy Lessons in Landscape Drawing, London: R. Ackermann, 1820. Lithograph, $210 \times 270 \mathrm{~mm}$. Image from copy held by the Fine Arts Library, Fogg Art Museum, Harvard University, digitized by Google and available from HathiTrust Digital Library (www. hathitrust.org)

Figure 10 Joseph Mallord William Turner and Charles Turner, LITTLE DEVIL'S BRIDGE over the RUSS above ALTDORFT, SWISSd, from Liber Studiorum, part IV. 1809. Etching and mezzotint. Photo of Impression in the Metropolitan Museum of Art, New York. @ Wikimedia Commons

Figure 11 Joseph Mallord William Turner, Richmond Hill and Bridge. 1828-1829. Watercolour on paper, $291 \times 435 \mathrm{~mm}$. London, British Museum (1958.0712.435). Bequeathed by Robert Wylie Lloyd. @ Wikimedia Commons

Figure 12a John Ruskin, The Elements of Drawing. In Three Letters to Beginners, London: Smith, Elder, and Co., 1857, 253, fig. 32: woodcut by Miss Byfield after drawing by Ruskin. Image downloaded from copy at University of California digitized by Internet Archive and available from HathiTrust Digital Library (www. hathitrust.org)

Figure 12b John Ruskin, The Elements of Drawing. In Three Letters to Beginners, London: Smith, Elder, and Co., 1857, 268, fig. 34: woodcut by Miss Byfield after drawing by Ruskin. Image downloaded from copy at University of California digitized by Internet Archive and available from HathiTrust Digital Library (www. hathitrust.org)

Figure 12c John Ruskin, The Elements of Drawing. In Three Letters to Beginners, London: Smith, Elder, and Co., 1857, 271, fig. 35: woodcut by Miss Byfield after drawing by Ruskin. Image downloaded from copy at University of California digitized by Internet Archive and available from HathiTrust Digital Library (www. hathitrust.org)

Figure 13 The badge of the London Chatham and Dover Railway, originally part of the first Blackfriars Railway Bridge (1864). Photograph by "SyndVer" (2013). (c) Wikimedia Commons

Figure 14 Joseph Dredge, Thames Bridges, from the Tower to the Source, London: Engineering, [1897], Pl. 6 (Blackfriars Railway Bridge). (c) Wikimedia Commons

Figure 15 George Hawkins, Lithograph showing the second wrought-iron box girder tube of Robert Stephenson's tubular railway bridge at Conwy being floated into position (September 1848), $300 \times 465 \mathrm{~mm}$ (image), $370 \times 547 \mathrm{~mm}$ (paper). Impression digitized by National Library of Wales. (c) Wikimedia Commons

Figure 16 Vittore Carpaccio, The Return of the Ambassadors (detail), from the St Ursula series. 1490s. Tempera and oil on canvas, 2970 $\times 5260 \mathrm{~mm}$ (whole). Venezia, Gallerie dell'Accademia. (c) Wikimedia Commons

Figure 17 Fratelli D'Alessandri, Photograph of the Ponte rotto, Rome. 1875. Print in the Archivio fotografico comunale, Rome. (C) Wikimedia Commons

445 Fonti, letterature artie paesaggi d'Europal Sources, Literatures, Arts \& Landscapes of Europel

John Ruskin's Europe A A Collection of Cross-Cultural Essays, 441-44 


\section{John Ruskin and the Europe of Cathedrals}

Claude Reichler

Figure 1 Édouard Baldus, Amiens, 1855. Albumen print from wet collodion negative. The Cleveland Museum of Art, Andrew R. and Martha Holden Jennings Fund 1986.15. CC license

\section{Il mercato antiquariale nella Venezia di Ruskin L'arte medievale in Germania \\ Michela Agazzi}

Figura 1 Potsdam Friedenskirche, abside, mosaico da San Cipriano di Murano. @ Wikimedia Commons, Ph. Karl Heinz Meurer

Figura 2 Berlino, Staatliche Museen, arcata di ciborio acquistata nel 1841 a Venezia. Raccolta Pajaro, inv. 6. () Staatliche Museen zu Berlin

Figura 3a Murano, Basilica dei santi Maria e Donato, balaustra absidale realizzata con arcata di ciborio altomedievale. Fotografia dell'autrice, fotomontaggio

Figura 3b Ricostruzione dell'arcata di ciborio altomedievale documentata da due frammenti. Murano, Basilica Santi Maria e Donato, balaustra della loggia absidale e cappella Santa Filomena. Autore: Paolo Vedovetto

Figura 4 Ravenna, San Michele in Africisco, disegno di Alexander von Minutoli. 1842. Staatliche Museen zu Berlin, da Nehls 1991, abb. 10. (da Spadoni Kniffitz 2007)

Figura 5 Klein Glienicke, Klosterhof, abside. Stiftung Preußische Schlösser und Gärten Berlin-Brandenburg/Bildarchiv. Su gentile concessione della Stiftung Preußische Schlösser und Gärten Berlin-Brandenburg (SPSG)/Fotograf: Daniel Lindner

Figura 6 Klein Glienicke, Klosterhof, porta di ingresso. Stiftung Preußische Schlösser und Gärten Berlin-Brandenburg/Bildarchiv. Su gentile concessione della Stiftung Preußische Schlösser und Gärten Berlin-Brandenburg (SPSG) / Fotograf: Daniel Lindner

Figura 7 John Ruskin, Archivolt in the Duomo of Murano. In The Stones of Venice, 1874, vol. II, tav. V

Figura 8 Murano, Museo Vetrario (già Museo Civico), allestimento dei pezzi medievali nel portico nel 1947. Venezia, Museo Correr, Archivio Fotografico, inv. M4095. 2021 @ Archivio Fotografico - Fondazione Musei Civici di Venezia. Fotografia Cacco 1947

Figura 9 Torcello, Museo Provinciale, sculture medievali esposte nella loggia. Fotografia Naya Boehm 3732

\section{Intermezzo. Songlines: Ruskin and the Roads of Europe}

Howard Hull

Figure 1 Double brougham carriage built in Camberwell to Ruskin's design in 1875 for the journey between London and Brantwood. Frontal view. Whitehouse Collection, R72, Lancaster University. On display at Brantwood

Figure 2 Double brougham carriage built in Camberwell to Ruskin's design in 1875 for the journey between London and Brantwood. Lateral view. Whitehouse Collection, R72, Lancaster University. On display at Brantwood 


\section{The Stones of Venice: Lady Augusta Gregory and John Ruskin}

Eglantina Remport

Figure 1 John Ruskin, The Four Venetian Flower Orders, from The Stones of Venice, 1853, vol. III, tav. X. Courtesy of the Marciana Library, Venice

\section{From Ruskin's Amiens to Proust's Venice Reflections on the Diapered Screen \\ Emily Eells}

Figure 1 John Ruskin, The Ducal Palace, Venice, 1835. In Works, 35: Pl. 8, facing 182. Scanned image and text by George P. Landow. http://www.victorianweb.org/painting/ruskin/ drawings/14.html

Figure 2 Notre-Dame d'Amiens. Photograph of a detail of the southern portal. Amiens. (c) Wikimedia Commons

Figure 3 Notre-Dame d'Amiens Cathedral, Northern Porch. Detail of Zodiac signs and months of the year. From left to right: Aries, Taurus, Gemini (above); March, April, May (below). ( ) Wikimedia Commons

Figure 4 Gustave Moreau, Les Muses quittant Apollon, leur père, pour aller éclairer le monde. 1868. $292 \times 152$ cm. Paris, Musée Gustave Moreau

Figure 5 Gustave Moreau, Le jeune homme et la Mort. 1865. $215.9 \times 123.2 \mathrm{~cm}$. Cambridge (MA), Fogg Art Museum

Figure 6 Gustave Moreau, Saint Martin partageant son manteau. 1882. Private Collection. (c) Wikimedia Commons

Figure 7 Paul César Helleu (1859-1927), Intérieur de l'église abbatiale de Saint-Denis. 1891 ca. Oil on canvas, $194 \times 155$ cm. Boston (MA), The Isabella Stewart Gardner Museum. The Athenaeum. https://www.gardnermuseum.org/ experience/collection/10732

Figure 8 John Ruskin, Ancilla Domini, frontispiece of Works, 7. Drawing from the painting by Fra Angelico. http://www.victorianweb. org/painting/ruskin/ drawings/33.jpg

\section{Edited by Ruskin: Francesca Alexander's Roadside Songs of Tuscany}

Emma Sdegno

Figura 1 Esther Frances "Francesca” Alexander, La Madonna e il Riccone, title page to The Madonna and the Rich Man. Collection of the Guild of St George, Museums Sheffield

Figura 2 Esther Frances "Francesca" Alexander, Non ho né pan né vin cosa ti posso dar? 381×277 mm. In The Madonna and the Rich Man (Works, 32: 104). Collection of the Guild of St George, Museums Sheffield

Figura 3 Esther Frances "Francesca" Alexander, The Jessamine Window. $389 \times 284$ mm. Works, 32: 142. Collection of the Guild of St George, Museums Sheffield

Figura 4 Francesca Alexander, Roadside Songs of Tuscany, London, George Allen, 1885. E.T. Cook and A. Wedderburn, The Works of John Ruskin, Library Edition, vol. 32, Studies of Peasant Life 


\section{Intermezzo. John Ruskin and Kenji Miyazawa}

An Idea of Nomin-Geijutsu (Peasant Art) and its European Legacy

Yasuo Kawabata

Figure 1 Unknown photographer, Portrait of Kenji Miyazawa (1896-1933). Late 1920s. Kamakura Museum of Literature archives. C Wikimedia Commons

\section{Ruskin in Translation: Versions of Unto this Last in a Few Europeans Languages Toward a Reception History of John Ruskin's Social Thought}

Jean-Yves Tizot

Figures 1a-b Thomas Barclay, The Rights of Labour according to John Ruskin, $1890 \mathrm{ca}$. London: Reeves. Original cover. Free of rights

Figure 2 Horrix, Paul (ed. and transl.). John Ruskin, "Dezen Laatsten Ook" (Unto this Last). Vier schetsen over de beginselen der Staathuishoudkunde. Groningen: G.A. Evers, 1901. Front cover. Free of rights

Figures 3a-b Hugenholtz-Zeeven, M. (transl.). John Ruskin, "De Laatstgekomenen". Vier lezingen over de eerste beginselen der Staathuishoudkunde. Amsterdam: S.L. van Looy, 1901. Front cover. Free of rights

Figure 4 Villani, Felice (ed. and transl.). I diritti del lavoro (Unto this Last). Bari: Laterza e Figli, 1946. Front cover. Free of rights

Figure 5 Brunhes, Henriette and Jean (éds); Peltier, Emile (transl.). Unto this Last. Il n'y a de richesse que la vie. Paris: Gabriel Beauchesne et Cie éditeurs, 1902. Front cover. Free of rights

Figure 6 Brunhes, Henriette and Jean (éds); Peltier, Emile (transl.). Unto this Last. Il n'y a de richesse que la vie. Paris: Gabriel Beauchesne et Cie éditeurs, 1902. Title page. Free of rights

Figure 7 Ciges Aparicio, M. (ed.). Unto this Last (Hasta este último). Libreria de Fernando Fé, Madrid, c. 1910. Free of rights 

Ruskin's work is strongly embedded in the broad European context, marking an important moment in the movement for the establishment of a community culture and spirit. The essays collected here intend to place the theme of Ruskin's fruitful and vital relationship with Europe at the centre of a critical reflection, offering opportunities for an in-depth study and a discussion on issues related to aesthetics, the protection of tangible and intangible heritage, cultural and literary memory. By bringing to the attention of the scientific community the multiple aspects - geographic, historical-artistic, critical-aesthetic, literary, socio-political - of Ruskin's work from inter- and transcultural perspectives, the volume aims to (re)discover a deliberately European Ruskin and to stimulate new research paths.

The volume includes essays by: Pedro Marques de Abreu, Michela Agazzi, Laura Cerasi, Stuart Eagles, Emily Eells, Pierre-Henry Frangne, Martina Frank, Kate Genever, André Hélard, Howard Hull, Yasuo Kawabata, Emanuele Morezzi, Andrea Paribeni, Silvia Pedone, Myriam Pilutti Namer, Claude Reichler, Eglantina Remport, Giuseppe Sandrini, Emma Sdegno, Jean-Yves Tizot, Paul Tucker, Stephen Wildman, Muhadad Zaman.

Università

Ca'Foscari

Venezia 